\title{
NREL Photovoltaic Program FY 1995 Annual Report
}

Stuart Smoller, Coordinator

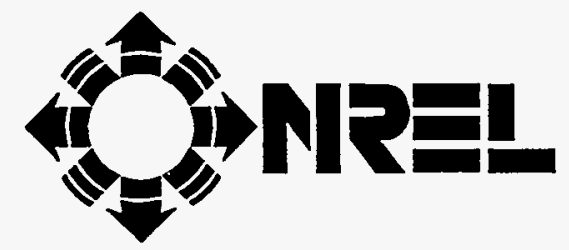

National Renewable Energy Laboratory 1617 Cole Boulevard Golden, Colorado 80401-3393

A national laboratory of the U.S. Department of Energy Managed by Midwest Research Institute for the U.S. Department of Energy under Contract No. DE-AC36-83CH10093 


\section{NREL Photovoltaic Program FY 1995 Annual Report}

Stuart Smoller, Coordinator

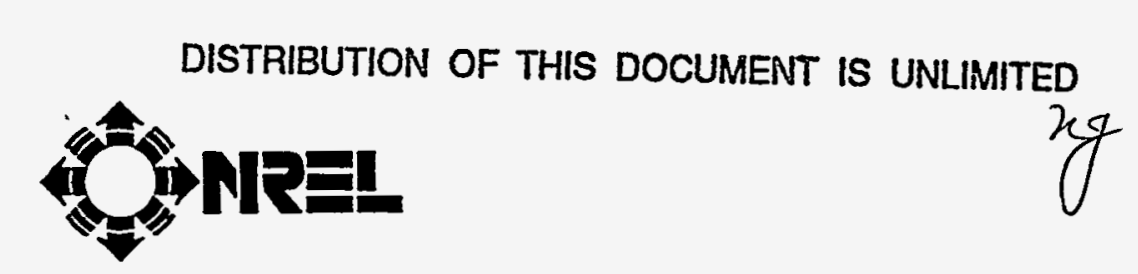

National Renewable Energy Laboratory 1617 Cole Boulevard Golden, Colorado 80401-3393

A national laboratory of the U.S. Department of Energy Managed by Midwest Research Institute for the U.S. Department of Energy under Contract No. DE-AC36-83CH10093

Prepared under Task No. PV610107

June 1996

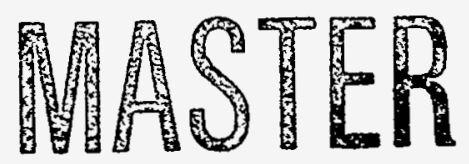




\section{NOTICE}

This report was prepared as an account of work sponsored by an agency of the United States government. Neither the United States govemment nor any agency thereof, nor any of their employees, makes any warranty, express or implied, or assumes any legal liability or responsibility for the accuracy, completeness, or usefulness of any information, apparatus, product, or process disclosed, or represents that its use would not infringe privately owned rights. Reference herein to any specific commercial product, process, or service by trade name, trademark, manufacturer, or otherwise does not necessarily constitute or imply its endorsement, recommendation, or favoring by the United States govemment or any agency thereof. The views and opinions of authors expressed herein do not necessarily state or reflect those of the United States govemment or any agency thereof.

Available to DOE and DOE contractors from:

Office of Scientific and Technical Information (OSTI)

P.O. Box 62

Oak Ridge, TN 37831

Prices available by calling (423) $576-8401$

Available to the public from:

National Technical Information Service (NTIS)

U.S. Department of Commerce

5285 Port Royal Road

Springfield, VA 22161

(703) $487-4650$ 


\section{DISCLAIMER}

Portions of this document may be illegible in electronic image products. Images are produced from the best available original document. 


\section{PREFACE}

This report summarizes the in-house and subcontracted research and development (R\&D) activities under the National Renewable Energy Laboratory (NREL) Photovoltaics (PV) Program from October 1, 1994, through September 30, 1995 (fiscal year [FY] 1995). The NREL PV Program is part of the U.S. Department of Energy's (DOE's) National Photovoltaics Program, as described in the DOE National Photovoltaics Program Plan for 1996-2000. The mission of the DOE National Photovoltaics Program is to make PV a significant part of the domestic economy-as an industry and an energy resource. The two primary goals of the national program are to make PV more costcompetitive with other forms of electrical generation and to make it as reliable and convenient to use as the next best alternative for each application. The NREL PV Program provides leadership and support to the national program toward achieving its mission and goals.

The FY 1995 budget authority for carrying out the NREL PV Program was $\$ 47.87$ million in operating funds and $\$ 0.8$ million in capital equipment funds. Subcontract activities represent a major part of the NREL PV Program, with more than \$27.2 million (nearly 57\%) of the FY 1995 operating funds going to more than 100 subcontractors. Cost sharing by industry added approximately $\$ 12$ million to the subcontract $R \& D$ activities with industry.

The NREL PV Program activities involve conducting basic, applied, and engineering research; managing subcontracted $R \& D$ projects; performing research complementary to subcontracted work; developing and maintaining state-of-the-art measurement and device capabilities; supporting PV manufacturing technology and module development; transferring results to industry; and evolving viable partnerships for $\mathrm{PV}$ systems and market development. The program is carried out under six project areas: PV Program Management, Crystalline Silicon and Advanced Devices, Thin-Film PV Technologies, Photovoltaic Manufacturing Technology, PV Module and System Performance and Engineering, and PV Applications and Markets Development.

Approved for the NATIONAL RENEWABLE ENERGY LABORATORY

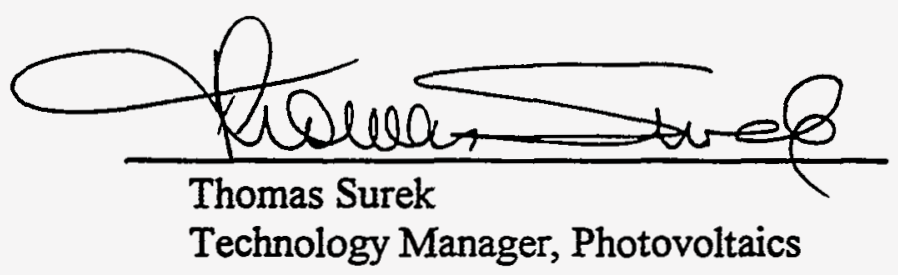

Notice: This publication was reproduced from camera-ready copy submitted by the individual subcontractors. The efficiency values reported by the subcontractors may not have been independently confirmed by NREL or Sandia National Laboratories. 


\section{TABLE OF CONTENTS}

Page

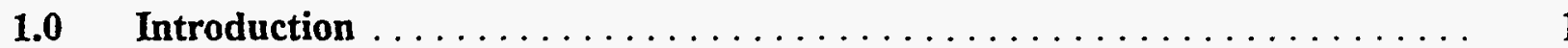

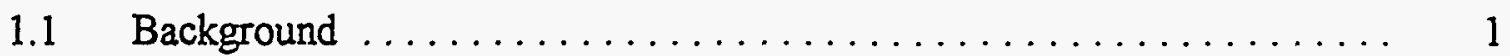

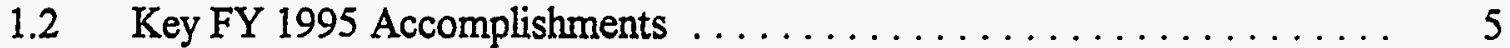

1.3 Technology Transfer .............................. 9

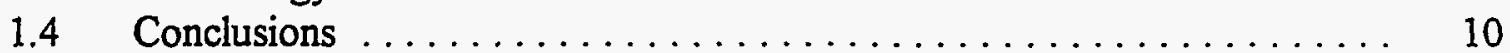

2.0 Crystalline Silicon and Advanced Devices Project $\ldots \ldots \ldots \ldots \ldots \ldots, 13$

\section{Crystalline Silicon and Advanced Devices In-House Research}

Crystalline Silicon Research:

NREL In-House Crystalline Silicon and Advanced Devices Research

Material and Interface Characterization of PV Devices:

NREL In-House Crystalline Silicon and Advanced Devices Research . . . . . . 24

Electro-Optical Characterization:

NREL In-House Crystalline Silicon and Advanced Devices Research

GaInP/GaAs Tandem Solar Cells:

NREL In-House Crystalline Silicon and Advanced Devices Research

Solid State Theory:

NREL In-House Crystalline Silicon and Advanced Devices Research . . . . . . .

\section{Crystalline Silicon and Advanced Devices Subcontracts}

Novel Low-Temperature Substrate Technology for Compound Semiconductor Solar Cells on Low-Cost Substrates:

California Institute of Technology

Influence of Self-Interstitials Injected by Phosphorus Diffusion on Defect Structures and Electronic Properties in Crystalline Silicon:

Duke University

Fundamental Research on Post-Growth Quality Enhancement Techniques:

Georgia Institute of Technology . . . . . . . . . . . . . . . . . . . 55 
Solar Energy Conversion at Dye-Sensitized Nanostructured Electrodes Fabricated by Sol-Gel Processing:

Johns Hopkins University . . . . . . . . . . . . . . . 59

The Role of Point Defects and Impurities in the Processsing Performance of Crystalline Silicon Solar Cells:

Massachusetts Institute of Technology . . . . . . . . . . . . . . . .

Detailed Non-Contact Electrical and Structural Characterization of Photovoltaic Silicon Substrates:

North Carolina State University $\ldots \ldots \ldots \ldots \ldots \ldots \ldots \ldots \ldots \ldots \ldots \ldots \ldots \ldots$

New Approaches for High-Efficiency Solar Cell Effect of Strain and Heavy Doping on $\mathrm{In}_{\mathrm{x}} \mathrm{Ga}_{1-x} \mathrm{P} / \mathrm{In}_{0.51} \mathrm{Ga}_{0.49} \mathrm{P}$ Band Structure for $\mathrm{BSF}$

North Carolina State University . . . . . . . . . . . . . . . . .

Very-High-Efficiency Photovoltaic Cells Based on Fully Organic Multiple Quantum Wells:

Princeton University . . . . . . . . . . . . . . . . . . . . . 75

Development of High-Efficiency GaAs Solar Cells on Polycrystalline Ge Substrates:

Research Triangle Institute

Porous Silicon Solar Cell Development:

Spire Corporation .

Thin-Film Silicon by Constitutional Supercooling:

State University of New York at Buffalo

Theoretical Analysis of Hydrogen-Vacancy-Impurity Formation and Dissociation:

Texas Tech University . .

Identification and Control of Lifetime-Reducing Defects in Polycrystalline Silicon Photovoltaic Materials:

University of California at Berkeley

Ultra-Efficient Epitaxial Liftoff Solar Cells Exploiting Optical Confinement in the Wave Limit:

University of California at Los Angeles

Processing of CuInSe $\mathrm{C}_{2}$-Based Solar Cells: Characterization of Deposition Processes in Terms of Chemical Reaction Analysis:

University of Florida . 
TABLE OF CONTENTS (continued)

Pagé

Fundamental Studies of the Effect of Crystal Defects on CuInSe $/$ CdS

Heterojunction Behavior:

University of Illinois

Experimental Study of Photodegradation in a-Si:H Solar Cells:

University of North Carolina

Optimization of Gettering Processes for Photovoltaic Silicon:

University of South Florida . . . . . . . . . . . . . . . . . . . . 114

Electronic Processes in Thin-Film PV Materials:

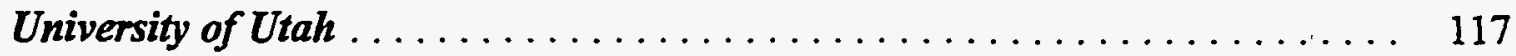

Electrochemical Fabrication of Dye-Sensitized Heterojunctions:

University of Washington

3.0 Thin-Film Technologies Project

Thin-Film Technologies In-House Research

Development of Polycrystalline Thin-Film CdTe Solar Cells Deposited by

Close-Spaced Sublimation:

NREL In-House CdTe Research

Development of Polycrystalline $\mathrm{Cu}(\mathrm{In}, \mathrm{Ga}) \mathrm{Se}_{2}$ Thin Films and Devices:

NREL In-House CIS Research .

Hydrogenated Amorphous Silicon Device Research:

NREL In-House Amorphous Silicon Research

Thin-Film Technologies Subcontracts

Light-Trapped, Interconnected, Silicon-Film Modules:

AstroPower, Inc.

Polycrystalline Thin-Film Cadmium Telluride Solar Cells Fabricated by Electrodeposition: 


\section{TABLE OF CONTENTS (continued)}

Microstructure of Amorphous-Silicon-Based Solar Cell Materials by Small Angle $X-$ Ray Scattering:

Colorado School of Mines ............................ 159

Device Physics of Thin-Film Polycrystalline Cells and Modules:

Colorado State University . . . . . . . . . . . . . . . . . . . . . . . 163

Development of High, Stable-Efficiency Triple-Junction a-Si Alloy Solar Cells:

Energy Conversion Devices, Inc.

CIS Photovoltaic Technology:

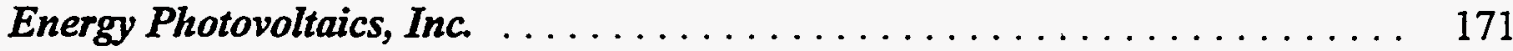

Polycrystalline CuInSe ${ }_{2}$ and CdTe PV Solar Cells:

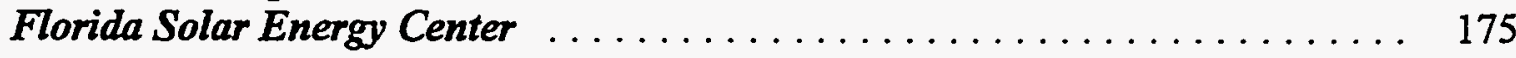

Development of High-Efficiency CdTe Solar Cells:

Georgia Institute of Technology . . . . . . . . . . . . . . . . . . . . . . . . 179

Optimization of Transparent and Reflecting Electrodes for Amorphous Silicon Solar Cells: Harvard University . . . . . . . . . . . . . . . . . . . . . . . . 183

Process Modeling Issues for Thin-Film Solar Cell Devices:

University of Delaware, Institute of Energy Conversion . . . . . . . . . . . 187

Application of CIS to High-Efficiency PV Module Fabrication:

International Solar Electric Technology . . . . . . . . . . . . . . . . 191

Research on Stability and Electronic Properties of a-Si:H and a-Si, Ge):H Devices: Iowa State University . . . . . . . . . . . . . . . . . . . . . 195

Atomic-Scale Characterization of Hydrogenated Amorphous Silicon Films and Devices: National Institute of Standards and Technology . . . . . . . . . . . . . . . . 199

Wide Bandgap Solar Cells with High Stabilized Performance:

Pennsylvania State University . . . . . . . . . . . . . . . . . . . 203

Development and Application of a Computer Model for CdTe- and CuInSe,-Based Solar Cells:

Purdue University .................................... 207 
TABLE OF CONTENTS (continued)

Page

Research on High-Efficiency, Large-Area CuInSe $e_{2}$-Based Thin Film Modules

Siemens Solar Industries . . . . . . . . . . . . . . . . . . . . . 208

Technology Support for Initiation of High-Throughput Processing of Thin-Film CdTe PV Modules:

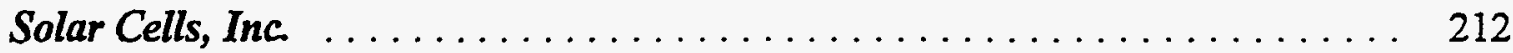

Research on Polycrystalline Thin-Film Submodules Based on CuInSe, Materials:

Solarex Corporation ........................... 216

Research on Wide Bandgap, Amorphous-Silicon-Based Solar Cells:

Syracuse University . .

Amorphous Silicon Research:

United Solar Systems Corporation . . . . . . . . . . . . . . . . . . . . . 224

Photocharge Transport and Recombination Measurements in Amorphous Silicon

Films and Solar Cells by Photoconductive Frequency Mixing:

University of California at Los Angeles

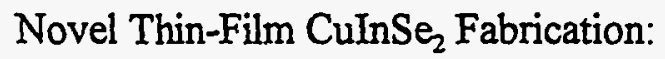

University of Colorado at Boulder

Microscopic Origins of Metastable Effects in a-Si:H and Deep Defect

Characterization in a-Si, Ge:H Alloys:

University of Oregon . . . . . . . . . . . . . . . . . . . . . .

Advanced Processing Technology for High-Efficiency Thin-Film CuInSe $e_{2}$ and CdTe Solar Cells; Heterojunction Development and Optimization in Thin-Film Compound

Semiconductor Solar Cells:

University of South Florida

High-Efficiency Thin-Film Cadmium Telluride Photovoltaic Cells:

University of Toledo

Optimization of Laser Scribing for Thin-Film PV Modules:

University of Toledo

Investigation of Polycrystalline Thin-Film CuInSe, Solar Cells Based on $\mathrm{ZnSe}$ Windows:

Washington State University . 
TABLE OF CONTENTS (continued)

4.0 Photovoltaic Manufacturing Technology (PVMaT) Project

\section{PVMaT Subcontracts}

Large-Area Silicon-Film ${ }^{\mathrm{TM}}$ Manufacturing:

AstroPower, Inc.

Continuous Roll-to-Roll a-Si Photovoltaic Manufacturing Technology:

Energy Conversion Devices, Inc.

PV Metal Roofing and Shingle Modules:

Energy Conversion Devices, Inc.

Photovoltaic Manufacturing Technology (PVMaT) Improvements for ENTECH's

Concentrator Module:

ENTECH, Inc.

High-Efficiency, Large-Area CdTe Panels:

Golden Photon, Inc.

Monolithic Amorphous Silicon Modules on Continuous Polymer Substrates:

Iowa Thin Films Technologies, Inc.

Advanced Development of PV Encapsulants:

Materials Science Corporation

PV Cz Silicon Manufacturing Technology Improvements:

Siemens Solar Industries

High-Throughput Manufacturing of Thin-Film CdTe Photovoltaic Modules:

Solar Cells, Inc.

Cast Polycrystalline Silicon Photovoltaic Module Manufacturing Technology Improvements:

Solarex Corporation

Automated Solar Cell Assembly Teamed Process Research:

Spire Corporation

Amorphous Silicon Photovoltaic Manufacturing Technology:

Utility Power Group 
TABLE OF CONTENTS (continued)

Development of a Low-Cost Integrated 20-kW A.C. Solar Tracking Sub-Array for Grid-Connected PV Power System Applications:

Utility Power Group . . . . . . . . . . . . . . . . . . . . 326

5.0 PV Module and System Performance and Engineering Project $\ldots \ldots \ldots$. . . 329

PV Module and System Performance and Engineering In-House Research

PV Efficiency Measurements - Standard Reporting Conditions:

NREL In-House PV Module and System Performance and

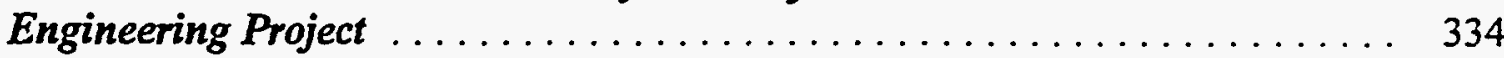

PV Module Materials and Encapsulation Research:

NREL In-House PV Module and System Performance and

Engineering Project

PV Module Qualification Testing and Test Method Development:

NREL In-House PV Module and System Performance and

Engineering Project

Photovoltaic Module and System Technology Validation:

NREL In-House PV Module and System Performance and

Engineering Project

Photovoltaic Component Diagnostics and Failure Analysis:

NREL In-House PV Module and System Performance and

Engineering Project ......................... 356

Photovoltaic Solar Radiometric Measurements and Evaluation:

NREL In-House PV Module and System Performance and

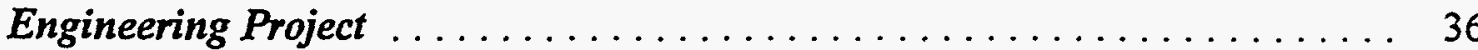

PV Standards and Codes:

NREL In-House PV Module and System Performance and

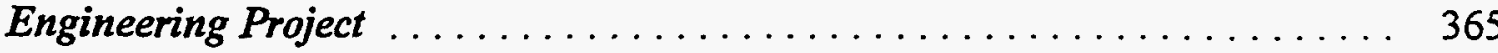

PV Module and System Performance and Engineering Subcontracts

Photovoltaic Module Certification/Laboratory Accreditation Criteria Development:

Arizona State University . . . . . . . . . . . . . . . . . . . . . . . . . . 369 
TABLE OF CONTENTS (continued)

NREL Module Energy Rating Methodology Project:

Endecon Engineering

PV Module Degradation-EVA Pore Volume

Pennsylvania State University

Long-Term Effects on Roof-Mounted Photovoltaic Modules:

Southwest Technology Development Institute

PV Translation Equations and PV Module Energy Rating Concept:

Sunset Technology Corporation

6.0 PV Applications and Market Development.Project

Photovoltaics in Buildings; Design Guidance for Practicing Architects and Design

Professionals:

AIA Research

Solar Resource Utility Load Matching Assessment:

University at Albany at New York, Atmospheric Sciences Research Center . . . 395

Evaluation of DSM Incentive Opportunities for Photovoltaics:

University of Delaware, Center for Energy and Environmental Policy . . . . . 398

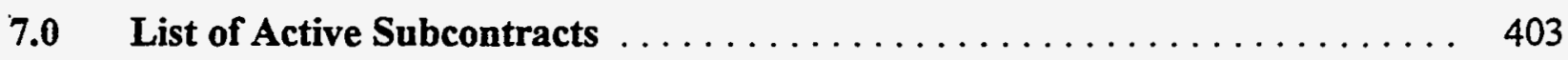

8.0 NREL Photovoltaic Program FY 1995 Bibliography . . . . . . . . . . . 417 


\subsection{INTRODUCTION}

This report summarizes the in-house and subcontracted research and development (R\&D) activities under the NREL PV Program from October 1, 1994, through September 30, 1995 (FY 1995). The NREL PV Program is part of the U.S. Department of Energy's (DOE's) National Photovoltaics Program, as described in the DOE National Photovoltaics Program Plan for 1996-2000. The FY 1995 budget authority for carrying out the NREL PV Program was $\$ 47.87$ million in operating funds and $\$ 0.8$ million in capital equipment funds. The operating funds included $\$ 0.675$ million of non-PV Program funds from DOE. Subcontract activities represent a major part of the NREL PV Program, with more than $\$ 27.2$ million (nearly 57\%) of the FY 1995 operating funds going to more than 100 subcontractors. Cost sharing by industry added about $\$ 12$ million to the subcontract $R \& D$ activities with industry.

\subsection{Background}

The NREL PV Program is part of the DOE National Photovoltaics Program managed by the Photovoltaics Technology Division in the Office of Solar Energy Conversion (OSEC). OSEC is under the Office of Utility Technologies in DOE's Office of Energy Efficiency and Renewable Energy. Major program thrusts in FY. 1995 continued to be implemented based on DOE's National Photovoltaics Program Plan for 1996-2000. The program mission is to:

Work in partnership with U.S. industry to develop and deploy photovoltaic technology for generating economically competitive electric power, making photovoltaics an important contributor to the nation's and the world's energy use and environmental improvement.

The two primary goals of the nation program are to (1) maintain the U.S. PV industry's world leadership in research and technology development, and (2) help the U.S. industry remain a major, profitable force in the world market. The NREL PV Program provides leadership and support to the national program toward achieving its mission and goals.

The DOE strategy over the next few years will be to lay the groundwork for a growing U.S. PV technology and industrial base, with increased emphasis on market and project development activities with industry. To accomplish this, the national program embraces three relatively equal priority activities: (1) technology development and validation, (2) market conditioning, and (3) project venturing. The new strategy continues a strong technology development program, but emphasizes $R \& D$ for the technologies and companies that are positioned to substantially penetrate the market, reduce prices, and scale up manufacturing. Program activities include continuing efforts to form partnerships with manufacturers and utilities (the ultimate benefactors and users), with universities, and with federal and state agencies.

Under the DOE National Photovoltaics Program, the NREL PV Program supports fundamental and applied R\&D, manufacturing development, and systems and market development in PV energy technology. The project also provides services to industry, electric utilities, and other users, and it 
provides overall programmatic support for the National Photovoltaics Program. The NREL subcontract program is responsible for most of the $R \& D$, manufacturing technology development, and some of the systems and market development task areas under the National Photovoltaics Program. It is implemented via competitive public solicitation. One of the most important subcontracting mechanisms is government/industry partnerships, with industry sharing the cost of research with DOE/NREL.

Closer work with industry, utilities, and other end users on PV manufacturing technology, systems, and market needs is being increasingly emphasized. Approaches for this emphasis include mitigating barriers to PV adoption in the utility and international marketplaces, and project venturing with decision makers and organizations representing domestic and international market sectors for PV. As appropriate for the system and market development areas, NREL is supporting activities such as education; technical assistance and training; market, economic, and financial analysis; technology characterizations; regulatory, rate, and value analysis; codes and standards assessment and development; working with customers in project development activities; and co-financing demonstration projects. Under project venturing, DOE is placing particular emphasis on supporting and strengthening programs already in place, such as PV:BONUS (Building Opportunities for the U.S. for Photovoltaics), the Americas' 21st Century Project, and the Utility PhotoVoltaics Group (UPVG).

NREL's PV activities include laboratory research and subcontract project management. We also provide technical support to efforts contracted from the DOE Golden Field Office (GO), such as PV:BONUS and UPVG. The primary research activities are conducted in advanced PV material technologies, including amorphous silicon (a-Si) thin-film materials; polycrystalline thin films, such as copper indium diselenide (CIS), cadmium telluride (CdTe), and their alloys; and high-efficiency crystalline cells, including silicon ( $\mathrm{Si}$ ) and gallium arsenide and their alloys. These activities are conducted in-house and through subcontracts with industry (mostly cost shared) and universities. The research activities are closely coordinated through several NREL/industry/university "team" efforts. Improving PV device manufacturing is vital. We are pursuing two complementary approaches:

- Government/industry partnerships, such as the PVMaT project (which focuses on improving manufacturing processes and products, accelerating manufacturing cost reduction, and laying the foundation for increased production capacity)

- Module development research to evaluate modules (and module performance) and suggest solutions to manufacturers' module problems.

System and market development rounds out the balanced approach pursued. The objective is to create an environment in which system technology, user acceptance, and the PV industry can accommodate the continued expansion of PV into large applications and markets. NREL subcontracts also support the continued influx of new ideas and highly qualified university research teams to expand the current limits of PV technology. Transferring research results into commercial products and applications in a timely and effective manner is another major activity of NREL's PV Program. 
Table 1.1-1. Budget History of the NREL Photovoltaics Program

Fiscal Year

\begin{tabular}{|c|c|c|c|c|c|}
\hline $7 \cdot y^{3}$ & $\begin{array}{l}1978-1991 \\
(\$ M)\end{array}$ & $\begin{array}{l}1992 \\
(\$ M)\end{array}$ & $\begin{array}{l}1993 \\
(\$ M)\end{array}$ & $\begin{array}{l}1994 \\
(\$ M)\end{array}$ & $\begin{array}{l}1995 \\
(\$ M)\end{array}$ \\
\hline \multicolumn{6}{|l|}{ TASK AREA } \\
\hline In-House R\&D & 96.5 & 14.0 & 14.3 & 16.4 & 18.8 \\
\hline Capital Equipment & 15.9 & 1.3 & 5.7 & 3.3 & 0.8 \\
\hline Subtotal (in-house) & 112.4 & 15.3 & 20.0 & 19.7 & 19.6 \\
\hline \multicolumn{6}{|l|}{ Subcontracted R\&D ${ }^{1}$} \\
\hline Amorphous Silicon Thin Films & 76.2 & 3.9 & 3.4 & 4.3 & 4.2 \\
\hline Polycrystalline Thin Films & 49.9 & 4.8 & 5.8 & 6.7 & 8.2 \\
\hline High-Efficiency Concepts & 35.8 & 0.9 & 1.0 & 0.6 & 0.3 \\
\hline Crystalline Silicon & 24.1 & 0.9 & 0.9 & 1.2 & 1.0 \\
\hline New Ideas & $18.8^{2}$ & 0.1 & 0.3 & 0.2 & 0.0 \\
\hline University Participation & 6.9 & 0.7 & 0.8 & 0.8 & 0.8 \\
\hline $\begin{array}{l}\text { Subtotal (R\&D } \\
\text { subcontracts) }\end{array}$ & 211.7 & 11.3 & 12.2 & 13.8 & 14.5 \\
\hline \multicolumn{6}{|c|}{ Manufacturing Technology Development and System and Market Development Subcontracts ${ }^{1}$} \\
\hline PVMaT Project & 8.4 & 10.2 & 10.6 & 16.6 & 10.7 \\
\hline $\begin{array}{l}\text { Module and System Performance } \\
\text { and Engineering Project }{ }^{3}\end{array}$ & 2.0 & 0.1 & 0.5 & 0.6 & 0.6 \\
\hline $\begin{array}{l}\text { PV Analysis and Applications } \\
\text { Development Project }{ }^{4}\end{array}$ & N/A & 0.7 & 2.5 & 2.9 & 3.3 \\
\hline $\begin{array}{l}\text { Subtotal (other } \\
\text { subcontracts) }\end{array}$ & 10.4 & 11.0 & 13.6 & 20.1 & 14.6 \\
\hline Subtotal (subcontracts) ${ }^{1}$ & 222.1 & 22.3 & 25.8 & 33.9 & 29.1 \\
\hline $\begin{array}{l}\text { TOTAL NREL PV } \\
\text { PROGRAM }\end{array}$ & 334.5 & 37.6 & 45.8 & 53.6 & 48.7 \\
\hline
\end{tabular}

\footnotetext{
'Includes about $15 \%-20 \%$ for technical program management, fees, etc.

2 Includes $\$ 9$ million for photoelectrochemical cell research

${ }^{3}$ Significant subcontracted effort initiated in FY 1991

4 Significant subcontracted effort initiated in FY 1992
} 
Subcontracted R\&D is a significant part of the NREL PV Program; nearly two-thirds of the program's budget is allocated yearly to subcontracts. Table 1.1-1 shows the distribution of both subcontract and in-house budgets. From FY 1978 through FY 1994, we awarded more than 1100 subcontracts totalling nearly $\$ 285$ million out of the total operating budget of $\$ 445$ million. In FY 1995, we awarded more than 100 subcontracts with a total funding of $\$ 27.2$ million. Cost sharing by industry subcontractors added about $\$ 12$ million to the $\$ 19.54$ million in NREL funding to 45 industry subcontractors. NREL also subcontracted with 35 universities, with a total funding of $\$ 7.21$ million. Figure 1.1-1 shows the distribution of subcontract funds by business category. Table 1.1-2 shows the contacts for the various NREL PV Program areas.

Figure 1.1-1. Business category distribution of FY 1995 subcontract funds

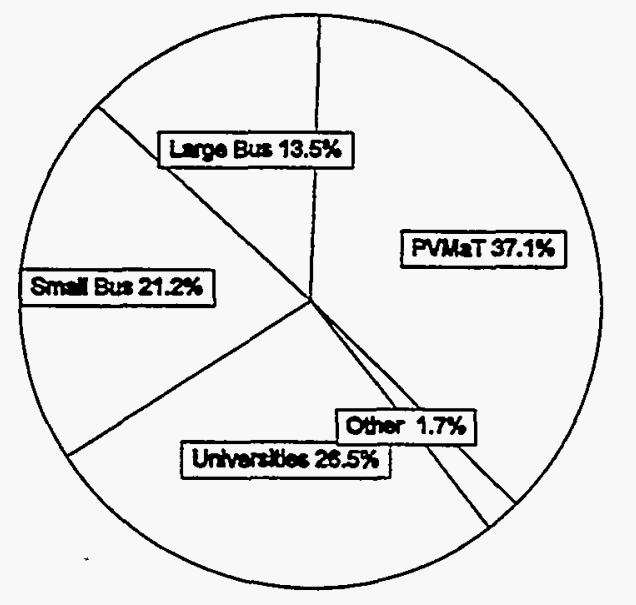

PVMaT

Large Business $\quad 9.4$

Small Business $\quad 27.1$

Other

$37.1 \%$

Total Subcontracts $\quad=\$ 27.2$ million

Cost-share by Industry $\quad=\$ 12.0$ million

\begin{tabular}{|l|l|l|}
\hline \multicolumn{2}{|c|}{ Table 1.1-2. NREL Photovoltaics Program Contacts by Project Area } \\
\hline \multicolumn{1}{|c|}{ Project Area } & \multicolumn{1}{|c|}{ Contact Name } & \multicolumn{1}{c|}{ Telephone } \\
\hline PV Program & $\begin{array}{l}\text { Thomas Surek, Manager } \\
\text { Stuart Smoller }\end{array}$ & $\begin{array}{l}\text { (303) 384-6471 } \\
\text { (303) 275-3716 }\end{array}$ \\
\hline $\begin{array}{l}\text { Crystalline Silicon and } \\
\text { Advanced Devices Project }\end{array}$ & John Benner, Manager & (303) 384-6496 \\
\hline $\begin{array}{l}\text { Thin-Film Photovoltaics } \\
\text { Technologies Project }\end{array}$ & Kenneth Zweibel, Manager & (303) 384-6441 \\
\hline PVMaT Project & Ed Witt, Manager & (303) 384-6402 \\
\hline $\begin{array}{l}\text { PV Module and System } \\
\text { Performance and Engineering }\end{array}$ & Richard DeBlasio, Manager & (303) 384-6760 \\
\hline $\begin{array}{l}\text { PV Applications and Market } \\
\text { Development Project }\end{array}$ & Roger Taylor, Manager & (303) 384-6432 \\
\hline
\end{tabular}




\subsection{Key FY 1995 Accomplishments}

Table 1.2-1 describes some key achievements in the NREL PV Program during FY 1995. The accomplishments are grouped by the six project areas of the program. Sections 2.0 through 6.0 of this report provide further details of the in-house and subcontracted research performed under these projects.

\begin{tabular}{|c|c|}
\hline Research Group & Achievement \\
\hline \multicolumn{2}{|c|}{ PV PROGRAM MANAGEMENT } \\
\hline NREL & $\begin{array}{l}\text { Ninety-four of NREL's projects were reviewed by a } \\
\text { dozen panels of expert reviewers selected by DOE's } \\
\text { Office of Program Analysis with both NREL in- } \\
\text { house projects and NREL subcontractors receiving, } \\
\text { on average, a rating designated as strong project } \\
\text { deserving of continuing priority support }\end{array}$ \\
\hline NREL & $\begin{array}{l}\text { Extensively revised the National PV Program Plan } \\
\text { for } 1996 \text { through } 2000 \text { by working closely with } \\
\text { industry representatives to accurately represent } \\
\text { their interests in program R\&D and } \\
\text { commercialization activities. }\end{array}$ \\
\hline NREL & $\begin{array}{l}\text { Evaluated more than } 15,000 \text { modules, devices, and } \\
\text { PV materials for more than } 150 \text { different research } \\
\text { and industry groups. }\end{array}$ \\
\hline NREL & $\begin{array}{l}\text { Sponsored PV research programs at seven } \\
\text { historically black colleges and universities, } \\
\text { supporting sixteen undergraduate students during } \\
\text { the academic year. }\end{array}$ \\
\hline \multicolumn{2}{|c|}{ CRYSTALLINE SILICON AND ADVANCED DEVICES PROJECT } \\
\hline Research Triangle Institute & $\begin{array}{l}\text { Produced polycrystalline gallium arsenide solar cells } \\
\text { with conversion efficiencies of } 16.6 \% \text {. }\end{array}$ \\
\hline NREL & $\begin{array}{l}\text { Verified a record } 30.2 \% \text {-efficient gallium arsenide } \\
\text { alloy concentrator cell at } 180 \text { suns. }\end{array}$ \\
\hline Johns Hopkins University & $\begin{array}{l}\text { Near } 10 \% \text { efficiency in photoelectrochemical solar } \\
\text { cell. }\end{array}$ \\
\hline
\end{tabular}




\begin{tabular}{|c|c|}
\hline Research Group & Achievement \\
\hline Princeton University & $\begin{array}{l}\text { Grew highly ordered, crystalline organic films with } \\
\text { strong photoconductive signals at low electric field } \\
\text { levels, long minority-carrier lifetimes, and } \\
\text { photoluminescence. }\end{array}$ \\
\hline Massachusetts Institute of Technology & $\begin{array}{l}\text { Showed that the level of iron impurity could explain } \\
\text { poor minority-carrier lifetimes. }\end{array}$ \\
\hline NREL & $\begin{array}{l}\text { Led development of new focus area in Photovoltaic } \\
\text { Materials that will be a major part of the Center for } \\
\text { Synthesis and Processing of Advanced Materials, a } \\
\text { multilab program funded by the DOE OER/ } \\
\text { BES/MSD. }\end{array}$ \\
\hline \multicolumn{2}{|c|}{ THIN-FILM TECHNOLOGIES PROJECT } \\
\hline NREL & $\begin{array}{l}\text { Achieved world-record thin-film cell efficiency of } \\
17.1 \% \text { for copper indium gallium diselenide cell. }\end{array}$ \\
\hline NREL & $\begin{array}{l}\text { Demonstrated nearly stable amorphous silicon } \\
\text { production using hot-wire deposition. }\end{array}$ \\
\hline NREL & $\begin{array}{l}\text { Improved glow-discharge production rate of } \\
\text { amorphous silicon films by hydrogen dilution in the } \\
\text { interface region. }\end{array}$ \\
\hline Solarex & $\begin{array}{l}12.9 \% 40-\mathrm{cm}^{2} \text { module of copper indium gallium } \\
\text { selenium. }\end{array}$ \\
\hline International Solar Electric Technology & $\begin{array}{l}\text { Made copper indium diselenide cells with greater } \\
\text { than } 12 \% \text { efficiency using a non-vacuum method } \\
\text { with materials utilization of nearly unity. }\end{array}$ \\
\hline AstroPower, Inc. & $\begin{array}{l}\text { Made } 10.5 \% \text {-efficient silicon-film device on low- } \\
\text { cost substrate. }\end{array}$ \\
\hline Institute of Energy Conversion & $\begin{array}{l}\text { Made } 13 \% \text {-efficient cell with high (about } 50 \% \text { ) } \\
\text { gallium content leading to a high-band-gap device. }\end{array}$ \\
\hline Colorado School of Mines & $\begin{array}{l}\text { Made } 12 \% \text { cadmium telluride cell using new zinc } \\
\text { telluride contact. }\end{array}$ \\
\hline NREL & $\begin{array}{l}\text { Set up national research teams in a-Si, CIS, CdTe, } \\
\text { and environmental, safety, and health areas. }\end{array}$ \\
\hline
\end{tabular}




\begin{tabular}{|c|c|}
\hline \multicolumn{2}{|c|}{ Table 1.2-1. Key Achievements } \\
\hline Research Group & Achievement \\
\hline \multicolumn{2}{|c|}{ PHOTOVOLTAIC MANUFACTURING TECHNOLOGY (PVMaT) PROJECT } \\
\hline NREL & $\begin{array}{l}\text { Successfully completed several PVMaT contracts } \\
\text { having 3-year commitments, exceeding cost and } \\
\text { production goals of the program. Awarded new } \\
\text { contracts for product-driven system and component } \\
\text { and manufacturing technologies. }\end{array}$ \\
\hline AstroPower, Inc. & $\begin{array}{l}\text { Received R\&D } 100 \text { Award for AP- } 225 \text { solar cell. } \\
\text { Developed four new products and demonstrated a } \\
\text { 4.4-MW/yr production rate for the Silicon-Film } \\
\text { wafer-manufacturing machine. }\end{array}$ \\
\hline Spire Corporation & $\begin{array}{l}\text { Completed development of new automated cell } \\
\text { stringer for module assembly lines. }\end{array}$ \\
\hline Solar Cells, Inc. & $\begin{array}{l}\text { Accomplished a twofold increase in total module } \\
\text { batch throughput. This result will be incorporated } \\
\text { into their 20-MW manufacturing line design. }\end{array}$ \\
\hline Energy Conversion Devices, Inc. & $\begin{array}{l}\text { Demonstrated a 9.5\%-efficient, triple-junction cell } \\
\text { using its serpentine web, continuous roll-to-roll } \\
\text { deposition chamber with potential for reduced gas } \\
\text { utilization, improved module throughput, and } \\
\text { reduced plant floor-space requirements. }\end{array}$ \\
\hline ENTECH, Inc. & $\begin{array}{l}\text { Achieved technology improvements and component } \\
\text { specifications for "fourth generation" concentrator } \\
\text { modules having a } 53 \% \text { cost reduction. }\end{array}$ \\
\hline Solarex Corporation & $\begin{array}{l}\text { Manufacturing process for the commercial } \\
\text { production of } 8-\mathrm{ft}^{2} \text { multijunction amorphous silicon } \\
\text { modules. }\end{array}$ \\
\hline $\begin{array}{l}\text { Springborn Materials Science } \\
\text { Corporation }\end{array}$ & $\begin{array}{l}\text { Development of two new encapsulant formulations } \\
\text { for the PV industry. }\end{array}$ \\
\hline \multicolumn{2}{|c|}{ PV MODULE AND SYSTEM PERFORMANCE AND ENGINEERING PROJECT } \\
\hline NREL & $\begin{array}{l}\text { Completed construction of expanded outdoor } \\
\text { facility for PV module and system performance } \\
\text { testing. }\end{array}$ \\
\hline University of Arizona & $\begin{array}{l}\text { Developed criteria for accrediting laboratories to } \\
\text { perform PV module certification in the United } \\
\text { States. }\end{array}$ \\
\hline
\end{tabular}




\begin{tabular}{|l|l|}
\hline \multicolumn{2}{|c|}{ Table 1.2-1. Key Achievements } \\
\hline Research Group & \multicolumn{1}{|c|}{ Achievement } \\
\hline NREL & $\begin{array}{l}\text { Designed, constructed, and installed a small-systems } \\
\text { test bed employing emerging thin-film technologies. }\end{array}$ \\
\hline NREL & $\begin{array}{l}\text { Deployed a 1-kW CdTe array from Solar Cells, Inc. } \\
\text { and a 2-kW a-Si array from Solarex at NREL's } \\
\text { Outdoor Test Facility. }\end{array}$ \\
\hline NREL & $\begin{array}{l}\text { Continued to lead the coordination of PV standards } \\
\text { and codes involving the Institute of Electrical and } \\
\text { Electronics Engineers, the American Society for } \\
\text { Testing and Materials, the International } \\
\text { Electrotechnical Commission, and the National } \\
\text { Electric Code. }\end{array}$ \\
\hline PV APPLICATIONS AND MARKET DEVELOPMENT PROJECT \\
\hline NREL & $\begin{array}{l}\text { Designed a program for the Chinese province of } \\
\text { Gansu, in cooperation with the Solar Electric Fund. }\end{array}$ \\
\hline NREL & $\begin{array}{l}\text { Helped DOE establish a black-owned business in } \\
\text { South Africa that assembles PV modules. }\end{array}$ \\
\hline NREL & $\begin{array}{l}\text { Designed a pilot project for rural electrification in } \\
\text { India and released a request for quotation for PV } \\
\text { equipment. Completed second phase of a rural } \\
\text { electrification deployment in eight states in Brazil. }\end{array}$ \\
\hline NREL & $\begin{array}{l}\text { Supported the start-up of a 2-MW amorphous } \\
\text { silicon manufacturing line in Moscow, Russia. }\end{array}$ \\
\hline NREL & $\begin{array}{l}\text { Evaluated the match between actual utility load and } \\
\text { projected output of a PV system for more than } \\
\text { 40 utilities across the United States. }\end{array}$ \\
\hline NREL-DOE Golden Field Office & $\begin{array}{l}\text { Coordinated Sunrayce 95, the solar car race for } \\
\text { college students from across the country. Thirty- } \\
\text { eight cars participated in the race from June 20 } \\
\text { through June 29. }\end{array}$ \\
\hline $\begin{array}{l}\text { Prototype products began to emerge from the } \\
\text { second phase of the Building Opportunities in the } \\
\text { United States for Photovoltaics (PV:BONUS) } \\
\text { program. }\end{array}$ \\
\hline $\begin{array}{l}\text { Distributed a PV resource package to 50 North } \\
\text { American schools of architecture. }\end{array}$ \\
\hline New York at Albany of Architects
\end{tabular}




\subsection{Technology Transfer}

Consistent with DOE policy, technology transfer within NREL's PV Program is defined as collaborative $R \& D$ with industry to help industry commercialize products or services. An underlyin'g theme of NREL technology transfer activities is the joint work accomplished by industry and NREL researchers focused on a common R\&D objective. Among government laboratories, seven principal tools effect technology transfer:

- Subcontracted R\&D

- Cooperative R\&D

- Industry-sponsored R\&D

- User facilities (at NREL)

- Technology licenses

- Researcher exchanges

- Information dissemination.

NREL's PV Program conducts its technology transfer primarily through subcontracts, cooperative $R \& D$, and information dissemination.

\section{Subcontracts with Industry}

Nearly $40 \%$ of the more than 100 subcontracts placed in FY 1995 were with the U.S. PV industry. The NREL funding of $\$ 19.54$ million to industry was supplemented by an additional $\$ 12$ million (estimated) of cost sharing by the industry partners. Most industry funding (and cost sharing) was in the PVMaT project (see Figure 1.1-1). Technically knowledgeable NREL research managers participate in defining, evaluating, awarding, and negotiating statements of work submitted by industry researchers in competitive solicitations. Following subcontract awards, NREL subcontract managers direct and evaluate research progress by visiting subcontractor sites and evaluating subcontractor deliverables in the NREL laboratories.

\section{Cooperative R\&D}

NREL in-house researchers frequently perform informal cooperative R\&D with their industrial counterparts working under NREL subcontracts. These interactions have been ongoing since PV research started in 1977 at the Solar Energy Research Institute (now NREL). Most involve performance measurements and materials analyses performed with NREL's large and unique set of capabilities for PV efficiency and materials analysis. Informal cooperative R\&D, as distinguished 
from formal cooperative research and development agreements (CRADAs), is a natural complement to NREL's subcontracted PV program. Informal cooperative R\&D during FY 1995 included more than 15,000 measurements on PV materials, devices, and modules on properties ranging from composition and microstructure to cell and module performance. More than 150 organizations from the worldwide PV community worked with NREL researchers in this fashion.

CRADAs, as authorized by the National Competitiveness Technology Transfer Act of 1989, are formal agreements signed by the NREL Director and his industrial counterparts for conducting joint research projects that involve NREL and industrial researchers. We performed research under eight CRADAs during FY 1995: AstroPower, Inc. (improvement of growth, properties, and device processing of silicon films); Martin Marietta (development and manufacturing of large-area CIS modules); Solar Cells, Inc. (PL-based quality control monitor); Spectrolab, Inc. (GaInP/GaAs tandem cells); UniSil Corporation (support for high-purity float-zone silicon); Labsphere, Inc. (solar cell material and device characterization); Energy Conversion Devices, Inc. (PV module characterization and measurement); and Energy Photovoltaics, Inc. (development of state-of-the-art CIS solar cells).

\section{Information Dissemination}

Effective traditional ways of transferring technology are to report $R \& D$ results to the technical community by publishing in scientific journals and by presenting at technical conferences and meetings. Section 8.0 contains a bibliography of FY 1995 publications, including subcontractor reports. During FY 1995, PV program staff helped organize several conferences and workshops, including the 13th NREL Photovoltaics Program Review Meeting, the fifth annual NREL Silicon Workshop, the NREL Photovoltaic Performance and Reliability Workshop, a Photovoltaic Radiometric Measurements workshop, and the 3rd NREL Photovoltaic Standards and Codes Forum. These gatherings provide important opportunities for industry researchers to exchange technical information with NREL and university researchers.

\subsection{Conclusions}

This report reviews the in-house and subcontracted R\&D activities under the NREL PV Program during FY 1995. Major PV program thrusts during FY 1995 continued to be implemented based on DOE's Photovoltaics Program Plan, FY 1991-FY 1995.

In summary, the activities of the PV Program are to:

- Conduct basic, applied, and engineering research

- Manage subcontracted $R \& D$ projects

- Perform research complementary to subcontracted work

- Develop and maintain state-of-the-art measurement and device capabilities 
- Develop PV manufacturing technology and modules

- Transfer results to industry

- Evolve viable partnerships for PV systems and market development.

This report describes the in-house and subcontracted R\&D activities, many of which encompass close collaborations between NREL and outside researchers.

The research activities under the program are summarized under the five project areas in the following sections: Crystalline Silicon and Advanced Devices Project, Thin-Film PV Technologies Project, PVMaT Project, PV Module and System Performance and Engineering Project, and PV Applications and Market Development Project. The sections include a brief overview that includes the objectives, approaches, and some key developments. Following that are technical summaries of the in-house and subcontract activities. The subcontract sections were, for the most part, provided by the subcontractors themselves. Some were compiled by NREL project managers from various reports submitted by the subcontractors. Section 7.0 provides a list of FY 1995 subcontracts, and Section 8.0 lists major research publications and subcontractor reports. 


\title{
2.0 CRYSTALLINE SLICON AND ADVANCED DEVICES PROJECT
}

\author{
John Benner, Manager
}

The Crystalline Silicon and Advanced Devices project supports basic and applied research to ensure that the U.S. photovoltaic (PV) industry has access to leading-edge technology needed for continuing improvement of silicon cells used in current modules, for definition of next-generation products, and for the achievernent of long-term goals of the DOE Photovoltaics Program. This project develops and maintains capabilities in crystalline silicon, high efficiency, advanced measurement techniques, and exploratory materials and devices that provide cross-cutting support for all photovoltaic technology options. It also supports a directed program in research on crystalline semiconductors in key topics selected to maximize the benefit to the PV program of the national infrastructure in silicon electronics and photonic technologies. These activities are intended to provide manufacturers with new process steps to replace existing procedures, ultimately leading to the replacement of silicon wafers with lower-cost crystalline films that also extend the limit of efficiency potential.

\section{Crystalline Silicon}

Attendees of the Fifth NREL Workshop on Silicon Point Defects and Defect Processes learned the answer to a key question that has nagged the community since the first meeting in this series. Polls of the industry working with multicrystalline silicon have continuously identified the enigma of excessive loss of minority-carrier lifetime in low-resistivity silicon as a key limitation in engineering higher-efficiency solar cells. Work led by L.C. Kimerling at Massachusetts Institute of Technology showed that the iron $(\mathrm{Fe})$ impurity level could explain the poor lifetime. Fe is an impurity closely monitored by the integrated circuit industry, and is even used as a wafer qualification standard. In the solar material environment, the cost of avoiding introduction of impurities demands developing other ways of solving impurity problems where practical. The MIT work showed that the solubility of ionized $\mathrm{Fe}^{+}$is much higher than that of atomic $\mathrm{Fe}$. Thus, the solubility of $\mathrm{Fe}$ increases with increasing boron concentration because of the increased availability of positively charged carriers needed for $\mathrm{Fe}$ ionization. The iron-boron complex forms a shallow defect energy level $0.10 \mathrm{eV}$ above the valence band, providing an effect recombination center. The minority-carrier lifetime then falls off in direct proportion to the increasing boron concentration.

\section{High Efficiency}

In FY 1995, we embarked on the next step in the sequential development of low-cost, highefficiency solar cells. Subcontractors completed their first year of effort on new projects to explore high-efficiency technology in imperfect materials. With very limited funds for the last several years, the high-efficiency PV research focused on understanding the requirements for reaching highperformance cells. This understanding is now being put to use in the next step of the plan, which is to begin to design process trade-offs that can produce low-cost cells without giving up too much efficiency. The Research Triangle Institute (RTI) success in achieving 16.6\%-efficient polycrystalline solar cells during this first year of research made the case for the importance of this approach. Thin films of GaAs are deposited on germanium substrates having millimeter-size grains. Using a cell design that combats losses driven by surface roughness typical of polycrystalline semiconductors, the RTI solar cell minimized a major component of shunt losses. In their first year 
of study of polycrystalline devices, they achieved efficiencies $50 \%$ higher than those achieved in the 1980s, when the program put poly-GaAs research on hold. Related projects at the California Institute of Technology and in-house at NREL are tackling the materials challenge of producing GaAs films of this morphology on glass or other low-cost substrates.

NREL's molecular beam epitaxy (MBE) system within the cluster tool has been commissioned with the growth of its first sample, a 1.5-micron-thick GaAs epilayer grown simultaneously on $\mathrm{GaAs}$ and Ge substrates. This new equipment combines deposition (MBE and MOCVD) and analytical capabilities in a single vacuum system and allows, for the first time, detailed studies on the nucleation and growth of thin-film III-V materials. This path may lead to $30 \%$-efficient solar cell designs making their way into low-cost flat-plate modules.

\section{Advanced Measurement Techniques}

Major efforts during the year were devoted to the completion of the SERF basement measurements and characterization facility. The relocation of the laboratories from Building 16 to the SERF basement facility has been completed. This $5500-\mathrm{ft}^{2}$ laboratory space is the home for 12 members of the Measurements and Characterization Branch, as well as for about $\$ 11$ million in capital equipment. The laboratories have been designed and constructed to meet special environmental needs, including physical and acoustic vibration, temperature control, and security for the facility.

The following major equipment is now in service at the SERF: the Cameca IMS $5 f$ and $3 f$ dynamic SIMS, the Philips STEM, the JEOL electron microprobe, the JEOL SEM, the large-area laser scanner, and the entire Nanoscale Science and Technology Laboratory. In addition, all Measurements and Characterization staff are now located in the SERF facility. Still to be delivered for these facilities are the following new capital purchases: the time-of-flight SIMS, the field emission scanning electron microscope, and the X-ray diffactometer. The scheduling of the equipment arrival was planned in order to save the program any additional fit-up or move costs. The basement microcharacterization facilities were completed under budget, and on the predicted schedule.

\section{Exploratory Materials and Devices}

The project supports the more exploratory investigations for the PV Program. For example, Steven Forrest at Princeton University completed a project awarded through the Novel Ideas Solicitations that investigated organic semiconductors for low-cost photovoltaics. His objective was to determine if a multiple quantum well structure could be used to overcome a basic limitation of organic semiconductors. His work demonstrated growth of highly ordered, crystalline organic films yielding strong photoconductive signals at low electric field levels, long minority-carrier lifetimes and photoluminescence. This combination of properties is essential for design of efficient thin-film solar cells from low-cost organic semiconductors. This is also the first demonstration that shows they can be achieved simultaneously in a single film.

Peter Searson and Gerald Meyer at Johns Hopkins University demonstrated a photoelectrochemical solar cell that achieved nearly $10 \%$ efficiency. It is based upon the photo-sensitization of $\mathrm{TiO}_{2}$ nanostructured electrodes by organic dye molecules. This project was selected through the University Participation Program, and was created to provide an environment free from short-term 
program pressures to ensure that new, potentially breakthrough technologies have a channel into the National Program. A similar program was initiated internally through the joint sponsorship of the Division of Chemical Sciences, Advanced Energy Projects and the PV Program that is expanding in scope to éngineer improved sealing and module technologies.

The integration of research activities across programs within the U.S. Department of Energy is building stronger support for solving fundamental issues in PV. NREL led the development of a new Focus Area in Photovoltaic Materials that will be a major part of the Center for Synthesis and Processing of Advanced Materials, a multi-lab program funded by the DOE Office of Energy Research/Basic Energy Sciences/Materials Science Division (OER/BES/MSD). At the request of MSD, we invited scientists from the PV community and the MSD projects to meet at the NREL PV Program Review Meeting to review existing research and consider the materials science issues associated with PV technology. The principal investigators in projects supported by MSD were encouraged to identify scientifically challenging topics of significance to PV technology development and redirect their investigations toward these. From the large original list of topics, 20 project descriptions were selected and structured into research plans drawing on the collaborative efforts of PV-and MSD-supported investigators. The plan was completed and submitted to MSD in July. Although the summer budget rescission caused a delay in the start of the investigations, MSD has allocated $\$ 300,000$ in FY 1996 to stimulate these collaborations and "glue together" the research efforts redirected by the PIs toward topics of greater relevance to PV. 


\section{Crystalline Silicon and Advanced Devices In-House Research}



Organization: National Renewable Energy Laboratory

Contributors: $\quad$ T.F. Ciszek, principal investigator; B. Sopori, S. Tsuo, R. Schwerdtfeger, R. Burrows, T. Wang, C. Marshall, M. Landry, M. Symko, M. Cudzinovic, R. Murphy, and characterization staff

\section{Introduction}

The objective of this FY 1995 research is to enhance the U.S. position in crystalline-silicon-based photovoltaics (PV) technology through: (1) expansion of the understanding of materials, defects, impurities, and innovative growth and processing possibilities to provide the underpinning for improved U.S. competitiveness in silicon PV technology; (2) technical assistance and character-ization with current industrial silicon materials processes; and (3) critical assessment and identification of new R\&D opportunities that have the potential to further improve the throughput, lower the energy consumption and reduce the cost of crystalline silicon PV manufacturing.

\section{Silicon Materials}

Thin-Laver Silicon Growth

U.S. Patent No. 5,401,331 entitled "Substrate for Thin Silicon Solar Cells" was awarded to Ted Ciszek on March 28, 1995. This new technology utilizes an alloy of boron and metallurgical-grade silicon (MG-Si) containing $0.1 \%$ to $1.3 \% \mathrm{~B}$ and possesses a number of advantageous properties for use as a substrate for subsequent deposition of thin, active silicon layers. It has a resistivity of $<1 \mathrm{x}$ $10^{-3} \mathrm{ohm}-\mathrm{cm}$ (about the same as that of liquid mercury) and thus can form a conductive rear contact for the PV device.

Liquid phase epitaxy (LPE)-grown thin-layer silicon samples with areas as large as $4 \mathrm{~cm}^{2}$ were made on cast MG silicon substrates from $\mathrm{Cu}-\mathrm{Al}-\mathrm{Si}$ growth solutions. Al incorporation into the layers was studied by the multicomponent regular solution model in the system of Cu-Al-Si. Electrical resistivity of the silicon layers is thus controlled from 0.05 to $0.1 \Omega \cdot \mathrm{cm}$ for device fabrication.

\section{Controlled Defect and Impurity Studies}

Nitrogen-doped ingots were grown by float-zoning to investigate $\mathrm{N}$-doping effects on minority charge carrier lifetime and crystal growth. We conclude that it should be possible to conduct Si growth in a partial $\mathrm{N}_{2}$ ambient to take advantage of mechanical strengthening effects, lower purge gas costs, and other process advantages without significant reductions in minority-carrier lifetime.

\section{Silicon Casting/Directional Solidification and Czochralski Crystal Growth}

Installation of all facilities and engineering controls for the Lepel $30-\mathrm{kHz}$ radio frequency (RF) generator is complete. A readiness verification was done to test the electromagnetic field around the RF leads. Experiments were conducted on procedures for silicon nitride coating of graphite crucibles to allow directional solidification of MG-Si in reusable graphite containers. 


\section{Silicon Devices and Processes}

\section{SERE Move-In Activities}

We are continuing the work on upgrading the Tempress high-temperature furnace, which is used for processing crystalline silicon solar cells in a clean-room environment. Customized programs were written using Paragon TNT process monitoring and control software with a touch screen for user input. The hardware interface for process input and output (IO) is done using commercially available Opto 22 IO modules. Additionally, over-temperature protection and IO watchdog functions are hardwired into the system for added safety.

The Zeiss Axiomat microscope was moved from 16/103 to SERF C123. The plasma-enhanced chemical vapor deposition system intended for thin-layer silicon deposition was disassembled and moved from Building 16 to the SERF for storage.

The spreading-resistance-measurement system was moved to SERF Lab C121. We ordered new probes and qualification-testing-alignment samples and recalibrated the system with these new parts. The system is now fully operational.

The Kaufman ion-beam system was rebuilt and put into operation for hydrogen passivation experiments.

\section{Wave Theory Medeling}

The wave theory modeling of thin solar cells, based on the Finite Difference Time Domain (FDTD) method, was applied to a structure consisting of a thin $(1 \mu \mathrm{m})$ silicon film on glass. The resulting data of the wavelength dependence of reflectance and the interference effects are consistent with those from standard analyses. The FDTD analysis uses basic Maxwell's equations, suitable for a multi-layer configuration (including any absorbing media), and seeks field solutions based explicitly on Yee's interleaving algorithm. This model takes into account complete interface geometry of the device and is not limited to planar surfaces. Due to memory and speed limitations, the current working model is two-dimensional.

\section{High-Elux Solar Fumace Processing (Supported by NREL Directer's Development Fund)}

A patent application on "High-Flux Solar Furnace Processing of Silicon Solar Cells" by Y.S. Tsuo, J.R. Pitts, and M.D. Landry was filed in December 1994.

We developed a novel extrinsic gettering method that utilizes the large surface areas produced by a porous Si etch. The annealing was done using NREL's high-flux solar furnace (HFSF), which provides the high temperatures as well as the required injection of silicon interstitials. We used MG-Si prepared by directional solidification casting as the starting material. The porous Si layers (about $2 \mu \mathrm{m}$ thick) were prepared using a $\mathrm{HNO}_{3} / \mathrm{HF}(1: 100)$ etching solution and characterized by photoluminescence spectral emission and decay-time measurements. The porous Si etched MG-Si wafers were annealed in a HFSF for 15 to $30 \mathrm{~min}$. at a sample temperature of $1000^{\circ} \mathrm{C}$. Depth-profiling measurements by secondary ion mass spectroscopy showed that the HFSF annealing treatments caused impurities in the bulk of the wafers to diffuse and segregate at the porous Si etched surfaces. Strong gettering effects were observed for all the impurities measured, including $\mathrm{Al}, \mathrm{B}, \mathrm{Fe}, \mathrm{Cu}, \mathrm{Cr}$, and $\mathrm{Ti}$. 
Laser Scriber

The 486 PC, LabVIEW ${ }^{\circledast}$ software, and video monitor for the laser scriber have all been delivered and tested. The X-, Y-, Z-, and $\theta$-motion-control software and hardware were delivered, installed and tested using LabVIEW ${ }^{*}$. We have also used LabVIEW to write a Q-switch driver for the laser. We have decided to use the HPGL file protocol for programming the laser scriber and have identified commercially available programs that can translate DXF (used by most CAD programs) and GDS2 (used by L-edit) files into HPGL files.

\section{Cell Fabrication}

We fabricated LPE-Si layers deposited on MG silicon substrates. The front junctions of the cells were diffused using spin-on dopants and HFSF heating. The LPE-Si cells have low conversion efficiencies so far due to very low shunt resistances ( $<200 \mathrm{ohms}$ ), possibly caused by impurity precipitates in the junction region. Hetero-junction structures of ITO/n-type a-Si:H/intrinsic a-Si:H/LPE Si/MG-Si have also been used to test the quality of LPE-Si layers.

\section{Silicon Characterization}

We have established the calibration curves between photoluminescence intensity ratio and the dopant concentrations in silicon. Based on the calibration, several samples from UniSil have been measured to determine the absolute $\mathrm{P}$ and $\mathrm{B}$ doping level. The complete result was reported to UniSil so that company could improve crystal purity by selecting proper feedstock and avoiding possible contamination sources in its float-zoning process.

The far-infrared, cryogenic analysis technique used for high-resistivity UniSil wafers was also used in the analysis of several Crystal Systems, Inc. (CSI), polycrystalline silicon samples for B and P. It was found that $B$ is present at fairly high concentrations in these samples.

Several silicon samples from Brookhaven National Laboratory (BNL) with resistivities between $3 \times 10^{3}$ and $5 \times 10^{3} \Omega \cdot \mathrm{cm}$ were characterized by photoluminescence for $\mathrm{B}$ and $\mathrm{P}$ content. This analysis helped the BNL researchers to understand the degree of compensation in their Si substrates used in Si microstrip detectors.

\section{Silicon Industry Interaction}

\section{Workshop on Point Defects in Silicon}

The Fifth Workshop on Point Defects in Silicon was held in August 1995.

\section{UniSil Cooperative Research and Development Agreement (CRADA)}

Resistivity measurements were performed on a series of UniSil silicon samples, which were then compared to silicon ingots grown in NREL's float-zoner. We refurbished UniSil's bulk-ingot minority charge carrier lifetime tester. A complete computer data acquisition system was configured, interfaced, and programmed based on LabVIEW for UniSil to monitor lifetime in its high-purity-silicon production line. We also designed and performed photoluminescence and Fourier transform infrared spectroscopy experiments to evaluate $\mathrm{B}$ and $\mathrm{P}$ content at parts-per-trillion atomic levels in ultra-highpurity-silicon single crystals for UniSil. 
Crystal Systems. Inc.

NREL and CSI signed a CRADA intended to assist in the development of technology to further reduce the cost of polycrystalline-silicon-ingot-based solar cells.

\section{Labsphere}

The first unit of SDMS_LBIC-Ref was shipped to NREL for evaluation and testing. The second unit was shipped to Georgia Institute of Technology.

\section{Materials Research Group. Inc. (MRG)}

As part of a collaborative project with Materials Research Group, Inc., we crystallized, using NREL's HFSF, a 10-micron-thick a-Si:H film deposited by MRG on a TiB ${ }_{2}$-coated graphite substrate provided by Georgia Institute of Technology. The solid-phase crystallization happened while the sample was heated to around $1100^{\circ} \mathrm{C}$ by concentrated sunlight for about 10 seconds in an argon atmosphere. The crystallized film has no visible bumps or pinholes. We have also succeeded in crystallizing 10-micronthick a-Si:H films deposited on our own cast MG silicon substrates using the HFSF. The main advantage of using an MG silicon substrate is the expitaxial growth that preserves the large grain size of the substrate.

\section{Solarex and Astropower}

Ted Ciszek visited Solarex Corpuration and AstroPower, Inc., in December 1995 and presented talks on "Effects of Nitrogen Doping on Microdefects and Minority Charge Carrier Lifetime of High-Purity, Dislocation-Free and Multicrystalline Silicon" and "Growth Kinetics Studies of Si LPE from Metal Solutions."

\section{Publications}

Benner, J.P., and B.L. Sopori, "Impurity and Defect Engineering Approaches for Realization of $18 \%$ Commercial Silicon Solar Cells," Proceedings of the $I^{\text {st }}$ WCPEC, December 1994, p. 1591.

Ciszek, T.F., "Controlled Samples for Silicon Defect and Impurity Studies," Proceedings of the Fitth Workshop on Point Defects in Silicon, August 1995.

Ciszek, T.F., (NREL) and A. Eyer (Fraunhofer Institute for Solar Energy Systems), "Silicon Growth Technology for Photovoltaics," a half-day tutorial at the $1^{\text {st }}$ WCPEC.

Ciszek, T.F., T.H. Wang, R.W. Burrows, T. Bekkedahl, M.I. Symko, and J.D. Webb, "Effect of Nitrogen Doping on Microdefects and Minority Charge Carrier Lifetime of High-Purity, DislocationFree and Multicrystalline Silicon," Proceedings of the $1^{s t}$ WCPEC, December 1994, p.1343.

Ciszek, T.F., T.H. Wang, R.W. Burrows, T. Bekkedahl, M.I. Symko, and J.D. Webb, "Nitrogen Effects on Silicon Growth, Defects, and Carrier Lifetime," Proceedings of the Fifth Workshop on Point Defects in Silicon, August 1995.

Mao, D., K.J. Kim, Y.S. Tsuo, and A.J. Frank, "Improved Efficiency and Stability of Silicon Photocathodes by Electrochemical Etching," Journal of Physical Chemistry, Vol. 99, 1995, p. 3643. 
Sopori, B.L., "Defect Engineering in Solar Cell Manufacturing and Thin Film Solar Cell Development," Proceedings of the Fifth Workshop on Point Defects in Silicon, August 1995.

Sopori, B.L., X. Deng, and J.P. Benner, "Hydrogen in Silicon: Current Understanding of Diffision and Passivation Mechanisms," Proceedings of the $1^{s t}$ WCPEC, December 1994, p. 1615.

Tsuo, Y.S., P. Menna, R. Pitts, M. Al-Jassim, S. Asher, and T.F. Ciszek, "Porous Silicon Gettering," Proceedings of the Fifth Workshop on Point Defects in Silicon, August 1995.

Tsuo, Y.S., J.R. Pitts, M.D. Landry, C.E. Bingham, A. Lewandowski, and T.F. Ciszek, "High-Flux Solar Furnace Processing of Silicon Solar Cells," Proceedings of the $1^{s t}$ WCPEC, December 1994, p. 1307.

Wang, T.H., and T.F: Ciszek, "Growth Kinetics Studies of Silicon LPE from Metal Solutions," Proceedings of the $1^{\text {st }}$ WCPEC, December 1994, p. 1250.

Wang, T.H., T.F. Ciszek, S. Asher, and R. Reedy, "Reduced Cu Concentration in CuAl-LPE-Grown Thin Si Layers," Proceedings of the Fitth Workshop on Point Defects in Silicon, August 1995.

Wang, T.H., T.F. Ciszek, and C.R. Schwerdtfeger, "Macroscopically Smooth Si Layer Grow:th by IPE on Cast Metallurgical-Grade Silicon Substrates," $13^{\text {th }}$ NREL PV Program Review Meeting, AIP Conference Proceedings 353, May 1995, p. 503. 
Title: Material and Interface Characterization of PV Devices

\section{Organization: NREL}

Contributors: M. Al-Jassim, S. Asher, M. Bode, A. Franz, F. Hasoon, K. Jones, A. Mason, R. Matson, D. Niles, and R. Reedy.

\section{Introduction}

The Materials Characterization task has provided extensive and crucial support for both our inhouse and subcontract groups. A wide variety of techniques was utilized to characterize PV materials and devices. Compositional measurements are performed by fully computerized electron probe microanalysis using either energy dispersive or wavelength dispersive X-ray analysis. Structural and defect analyses are carried out by transmission electron microscopy (TEM). A project for the enhancement of light element analysis on thin foils in the TEM was completed during this year, enabling us to detect carbon and oxygen in various PV materials such as silicon. Additionally, scanning electron microscopy (SEM) in its various modes, such as secondary electron, CL and EBIC imaging, is providing very wide support for measuring the topographical, luminescent and microelectrical properties of PV materials and devices.

In addition to these measurements, the Materials Characterization task encompassed a powerful array of Surface and Interface Analysis techniques. This includes Auger electron spectroscopy (AES), X-ray photoelectron spectroscopy (XPS), ultraviolet photoelectron spectroscopy (UPS), and secondary ion mass spectrometry (SIMS). XPS and AES spectroscopies are related techniques for. determining the composition and chemistry of surfaces. The new field-emission AES and small spot XPS systems were commissioned last year and have been used extensively this year to support the $\mathrm{CdTe}$ and CIS device development tasks. Furthermore, a new dynamic SIMS instrument (Cameca 5f) was commissioned during 1995. Capabilities of this instrument include improved sensitivity for trace element analysis, better mass resolution, and significantly improved secondary ion imaging capabilities.

Most of the research activities carried out by this task are planned through other in-house and subcontractors activities. The following is a brief description of some of the highlights of our work during 1995 . These will be divided according to the material technology.

\section{CIS}

The Materials Characterization task continued to support in-house as well as outside research and development on CIS with a large number of analytical studies on various material aspects. More than 700 analyses were performed using the electron microprobe facility, and over 200 devices and films were analyzed by SIMS, XPS and AES. In addition, a large number of selected devices were characterized by SEM and TEM. Many of these devices and films were of a proprietary nature, showing the facility's value to in-house and industrial developers.

Aside from short-term characterization of CIS-technology-related materials, much time was concentrated on more fundamental aspects of materials growth. One example of such a study is the 
investigation of thin-film CdS. CdS is the usual heterojunction partner in CIS solar cells. Researchers at NREL currently use two different methods to deposit CdS onto CIS: physical vapor deposition (PVD) and chemical bath deposition (CBD). A significant effort under the Materials Characterization task has been to understand the differences between depositing CdS onto CIS by PVD and CBD. Through the use of XPS and AES, researchers in the Materials Characterization task have shown that:

- CIGS has $\sim 20$ at.\% Na contamination

- The Na contamination is wrapped up in a Na-Se-0 complex

- The chemical bath removes most of the Na-Se-0 complex

- The chemical bath first deposits $\sim 20$ at.\% $\mathrm{Cd}$ to the surface

- $\mathrm{Cd}$ is bound to $\mathrm{Se}$

- S deposition occurs subsequent to $\mathrm{Cd}$ deposition in the chemical bath.

Figure 1 shows $\mathrm{X}$-ray photoelectron spectra that prove many of these points. The bottom spectrum shows the native CIS surface, whereas the top spectrum shows the same surface after insertion into the chemical bath. One can see a large $\mathrm{Cd}$ signal after insertion into the chemical bath.

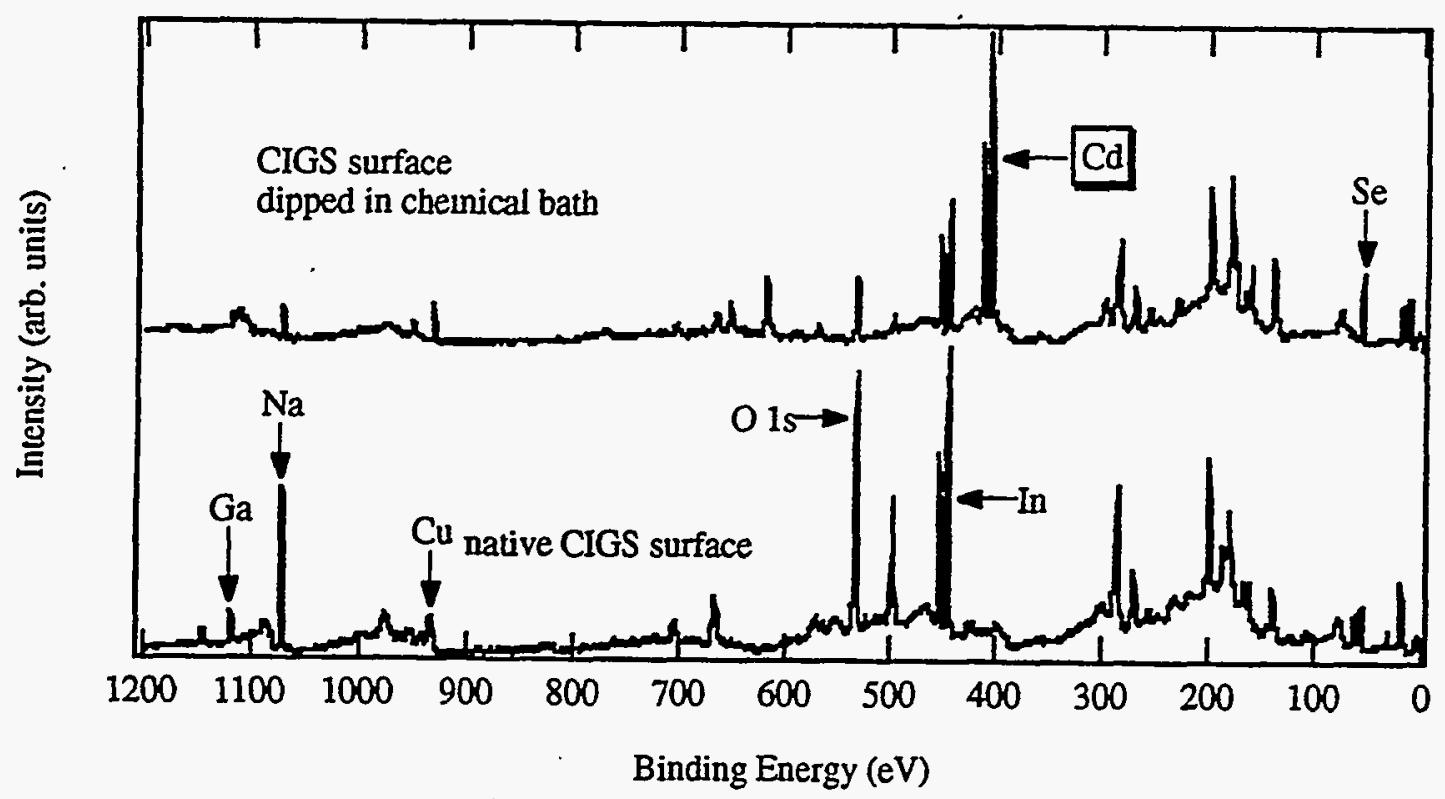

Figure 1. XPS spectra of a native CIS surface, and the same surface after insertion into the chemical bath

These observations prove that the CBD process produces a CdS/CIS interface that is inherently different from the PVD process. These differences may help to explain why devices made with the CBD process tend to outperform those made with the PVD process. 
The effects of the $\mathrm{Ga}$ concentration and $\mathrm{Ga}$ distribution on junction development in thin-film $\mathrm{ZnO} / \mathrm{CdS} / \mathrm{CuIn}_{\mathrm{x}} \mathrm{Ga}_{1-x} \mathrm{Se}_{2}$ devices were investigated this year. Using charge collection (or electronbeam-induced current [EBIC]) microscopy to obtain charge collection efficiency depth distributions, or profiles, of cleaved thin-film $\mathrm{ZnO} / \mathrm{CdS} / \mathrm{CuIn}_{x} \mathrm{Ga}_{1-x} \mathrm{Se}_{2}$ (CIGS) photovoltaic devices, we observed considerable variation in the position of the junction the width of the space charge region, and the minority-carrier diffusion lengths on the submicron scale as a function of the overall concentration (ranging from 0 to $100 \% \mathrm{Ga}$ substitution for $\mathrm{In}$ ) and depth distribution of the $\mathrm{Ga}$ as determined by Auger depth profiles. Variations in the charge collection efficiency profiles were also observedprimarily within individual devices with $\mathrm{Ga}$ concentrations less than $30 \%$. The primary findings from the work were 1) that the systematic addition of $\mathrm{Ga}$ to the $\mathrm{p}$-CuInSe $\mathrm{e}_{2}$ matrix affected the conductivity type of the material, thereby creating an increasingly abrupt (narrow), uniform, and shallower junction near the heteroface, 2) that the corresponding device behavior (corroborated by the EBIC profiles and quantum efficiency measurements) was an increasing $\mathrm{V}_{\mathrm{oc}}$ with a decrease in the $\mathrm{J}_{\mathrm{so}} \mathrm{FF}$, and overall conversion efficiency with $\mathrm{Ga}$ concentrations greater than $\sim 30 \%$, and 3 ) that the Auger depth profiles indicated increased $\mathrm{Ga}$ concentrations near the Mo and CdS interfaces without yielding any definitive clue as to why the junction evolved in the way that it did. Finding 1) was corroborated with earlier single-crystal $\mathrm{CuIn}_{\mathrm{x}} \mathrm{Ga}_{1-x} \mathrm{Se}_{2}$ studies. Further study is under way, but now adding the effect of the concentration and distribution of $\mathrm{Na}$ on formation due to the unintended diffusion of $\mathrm{Na}$ from the glass.

\section{Blue Book}

Members of the Materials Characterization task made significant contributions to the CIS ThinFilm Partnership Program in FY 1995. This included most of the materials analysis contained in the Absorber and Junction Team Materials Analysis Compilation, "blue book," distributed in May 1995. Analytical results from AES, EPMA, SEM, SIMS, and XPS measurements were all represented in the book.

\section{CdTe}

An area of focus for this task in the CdTe arena has been the development of a model for backcontact formation to CdTe. NREL's baseline process involves dipping CdTe devices into a concentrated solution of nitric and phosphoric acids before application of the back contact. The model developed last year through the use of X-ray photoelectron and Auger electron spectroscopies is based on the observation that the acid etch produces a pure Te layer that promotes charge flow through the CdTe surface into the metallic back contact.

The acid etch is not amenable to large-scale device production at minimum cost for several reasons, including the disposal of copious quantities of Cd-laden acidic waste. A substantial effort between the CdTe Device Development Task and the Materials Characterization task this year has involved the development of a process to evaporate $\mathrm{Te}$ on the CdTe surface, thereby eliminating the need for the acid etch. Figure 3 shows a comparison of I-V profiles for two devices: one made with NREL's baseline process (control contact), and one made without the assistance of the acid etch (evaporated $\mathrm{Te}$ ). The performance of the devices is identical. 

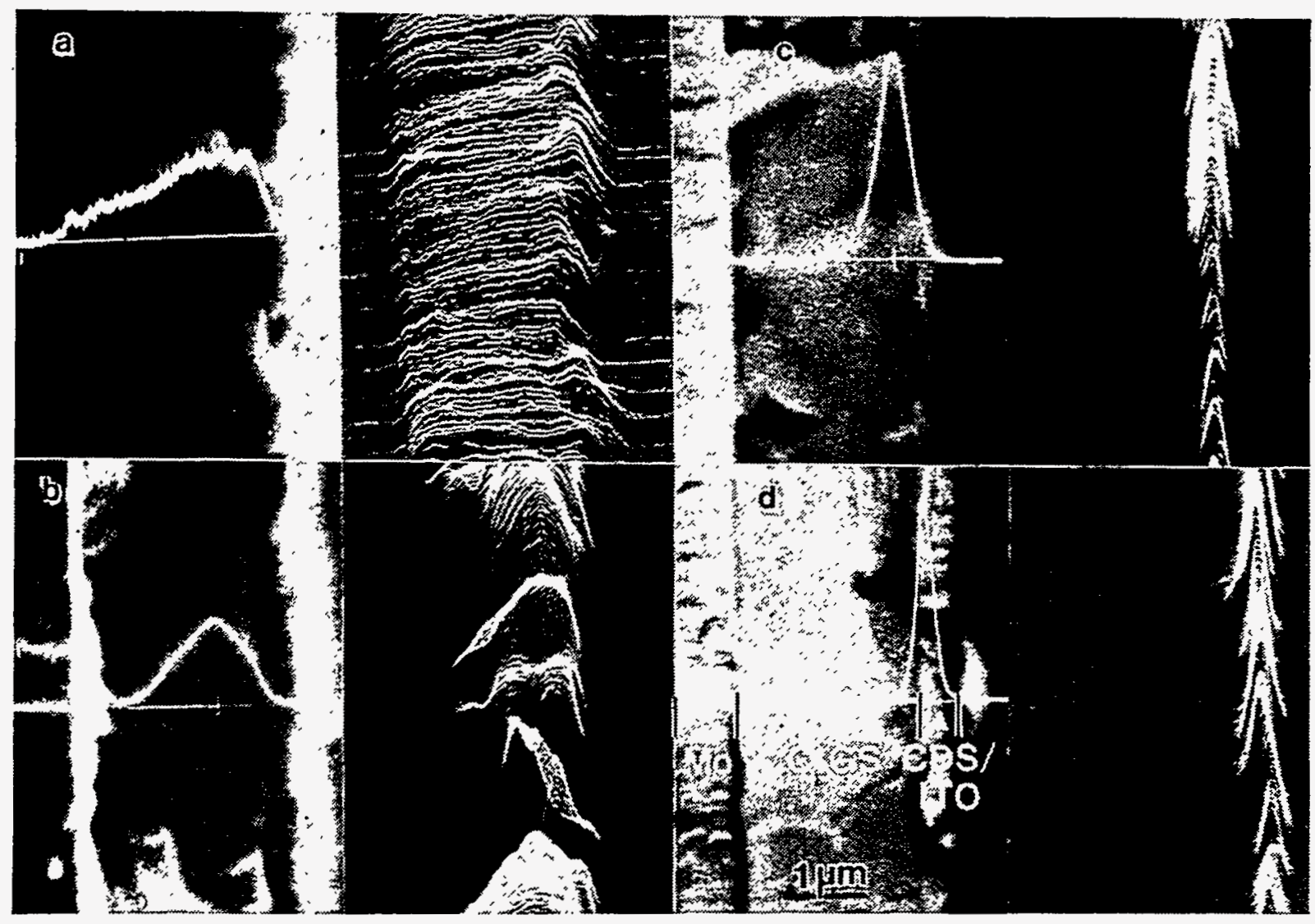

Figure 2. Comparison of the JEBIC profile for the 0 (a), 30 (b), 50 (c), and $100 \%$ (d) Ga substitution cases superimposed on their corresponding topography.

I-V measurements on 10 similar devices proved that evaporated Te would make as good a contact as the Te layer made by etching, thereby substantiating the model proposed last year and eliminating the need for the acid etch.

Another topic we investigated this year is the chemistry of the CdS/CdTe interface. Understanding the interdiffusion at this interface is critical to understanding the role of the CdS window layer. Measurements of this interface are complicated by the native roughness of the CdTe film surface and by sputtering related artifacts. The CdTe surface roughness is affected by both the growth conditions and the film thickness. This makes it difficult to separate features of diffusion at the interface from topographically induced broadening of the ion signals. We have developed two sample preparation methods for reducing the roughness of CdTe films prior to depth profile analysis. 


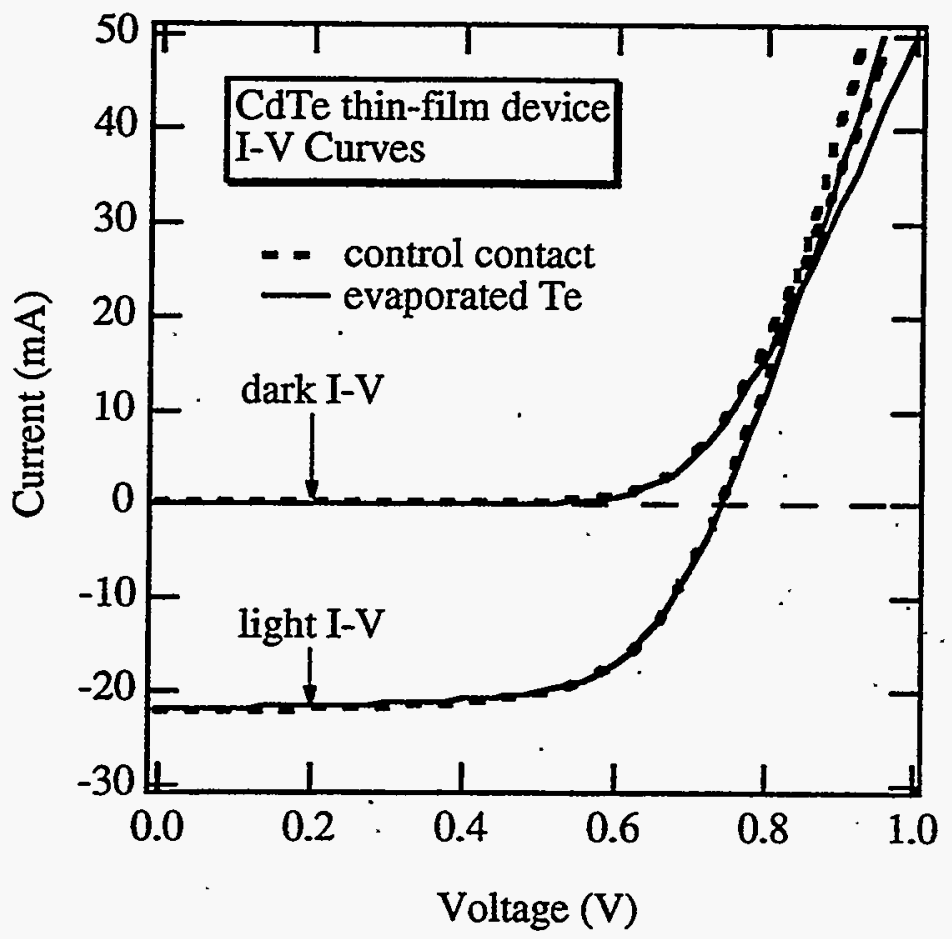

Figure 3. A comparison of I-V profiles for two devices: one made with NREL's baseline process (control contact), and one made without the assistance of the acid etch (evaporated Te).

The first method developed relies on mechanical polishing of the CdTe film with a surfaces dimpler and $0.05-\mu \mathrm{m}$ polishing compound. This method produces surfaces with average roughnesses of $<60 \AA$. Initial surface roughnesses in these samples were as great as $6000 \AA$. The depth profiles in Figures 4 and 5 illustrate depth resolution obtained with this method. Figure 4 shows a SIMS depth profile from an as-deposited CdTe/CdS film. The average surface roughness of this film was $4900 \AA$. In this profile, the CdS layer appears to be absent or greatly broadened. The low signal level from the $S$ tends to support the notion that the CdS layer has been consumed (diffused) and is no longer present. Figure 5 is a SIMS depth profile from the center of a polished area on the same sample. In the polished area the average surface roughness was $58 \AA$ prior to analysis. This profile clearly shows the presence of a thin CdS layer under the CdTe. The small amount of topography generated by the ion beam during the analysis, from $58 \AA$ to $143 \AA$, does not obscure the layer. This result is extraordinary since the thickness of the CdTe film is $>4.5 \mu \mathrm{m}$ and the thickness of the underlying CdS layer is $\sim 1000 \AA$. The signal levels obtained from the bulk of the CdTe, however, appear to be unaffected by the surface roughness. 


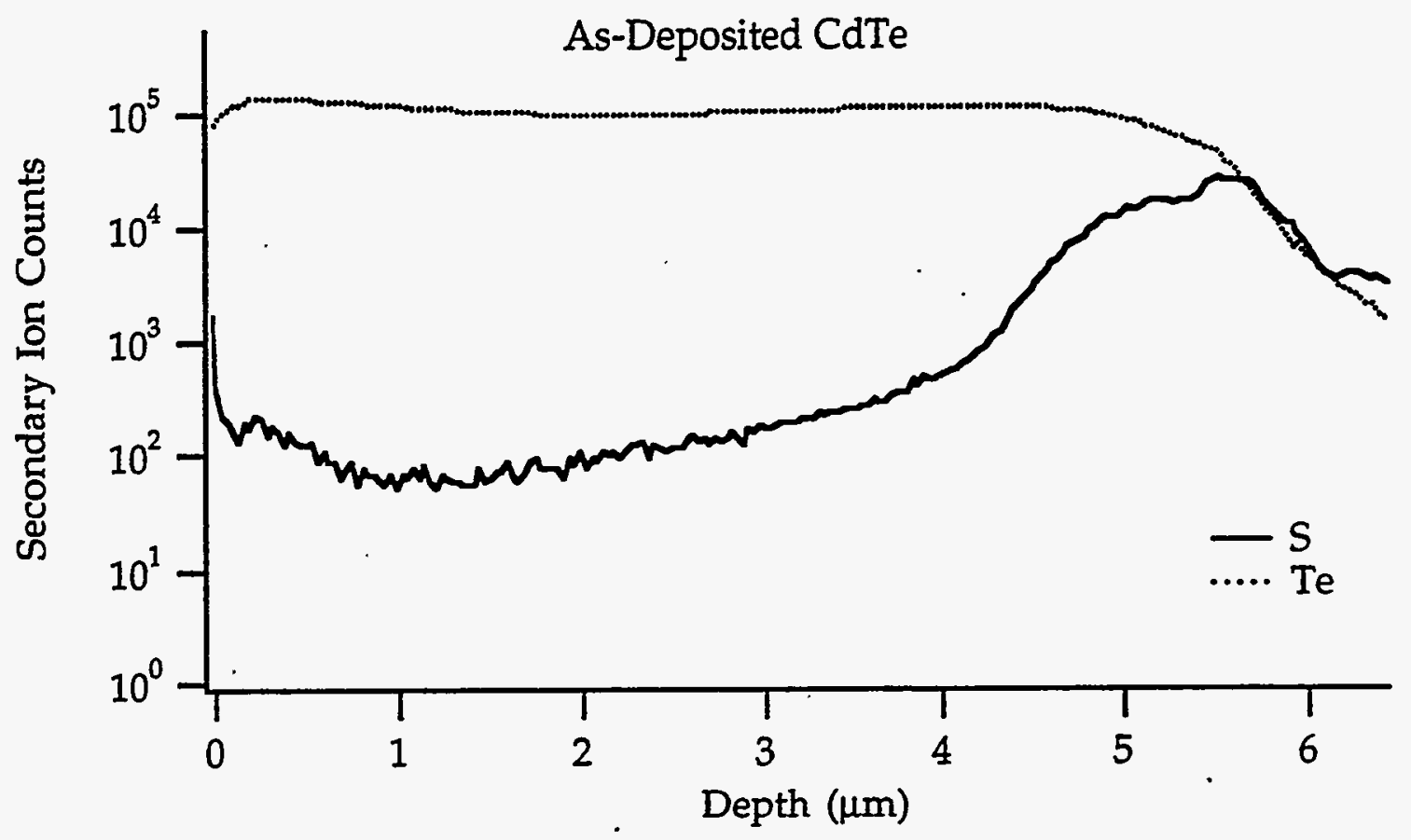

Figure 4. SIMS depth profiles from an as-deposited CdTe/CdS film.

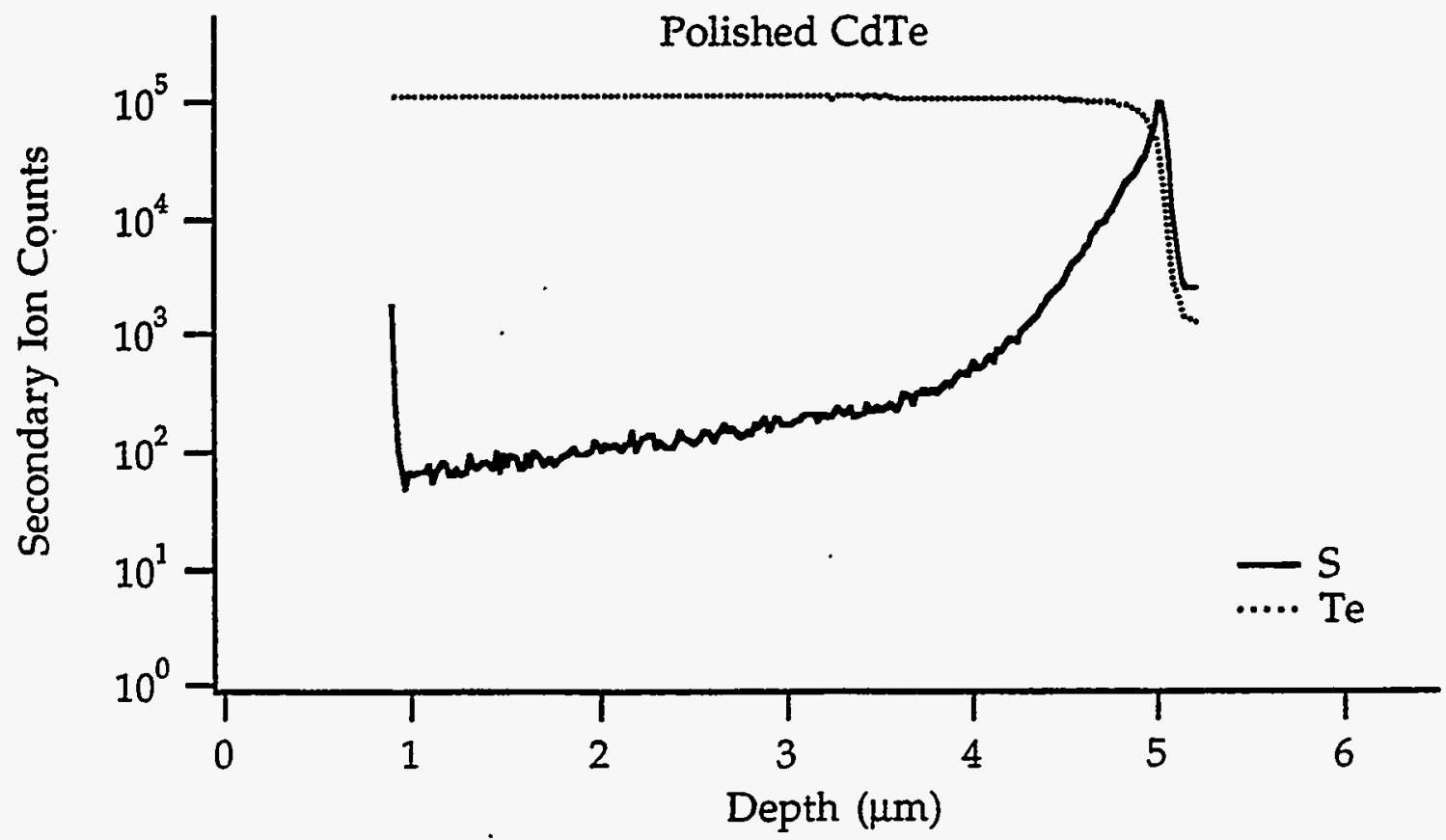

Figure 5. SIMS depth profiles from the center of a polished area on the same sample in Figure 4. 
The second method of sample preparation developed uses a solution of Br:Methanol to chemically polish the CdTe surface prior to depth profiling. A $1 \%$ by volume solution of $\mathrm{Br}$ dissolved in methanol was found to minimize and in some cases remove the surface topography from CdTe films. This method yields surfaces with average roughnesses comparable to the mechanical polishing. The depth profiling results from these samples are also similar to those from mechanically polished case.

In the past, it has been difficult to compare depth profiles from CdTe/CdS devices grown by different methods. It has also been difficult to compare the results from films with different surface morphologies. This lack of standardization has made it difficult to understand the optimum configuration of the heterointerface. The sample preparation methods we developed will reduce the effects of surface topography, allowing for better understanding of the CdTe/CdS interface in structures deposited by different techniques.

\section{III-Vs}

Our efforts in this area were concentrated on supporting the commercialization of NREL's highefficiency tandem cell. This included supporting the in-house High Efficiency task and Spectrolab in their effort to grow the high-efficiency tandem cell on Ge substrates. 


\title{
Title: $\quad$ Electro-Optical Characterization
}

\author{
Qrganization: National Renewable Energy Laboratory \\ Golden, Colorado 80401
}

Contributors: R. K. Ahrenkiel, B. M. Kèyes, D. L. Levi, F. Abou-Elfotouh, A. Mascarenes, J. Webb

A great deal of progress was made in research supporting the thin-film polycrystalline and silicon programs. Technique development has added two new, very powerful techniques for looking at the dynamics of light absorption and recombination by photovoltaic semiconducting materials.

The new femtosecond titanium sapphire system was purchased under the SERF capital budget in FY 1994. That system has been upgraded by the addition of additional components that were purchased by capital funds from the Basic Energy Sciences program. The upgrades include an optical parametric amplifier (OPA) and a high-power argonne ion pump laser. These were used to extend the measurement capability in the time and frequency domain. The OPA provides laser wavelengths from the ultra-violet wavelengths $(-400 \mathrm{~nm})$ to the infrared wavelengths $(\sim 2.0 \mathrm{~mm})$. This new facility provides diagnostics for measuring the dynamic effects of light absorption and the recombination kinetics of free carriers that were not before measurable. One of the highlights was the measurement of not only CdS recombination lifetimes but the interaction of electrons and holes with the controlling impurities in the CdS thin-film system.

The second technique developed during the course of FY 1994, and greatly improved in FY 1995, is radio frequency photoconductive decay (RFPCD). The RFPCD system is a contactless technique based on a balanced VHF ( $430 \mathrm{MHz}$ ) bridge. The bridge is unbalanced by excess carrier induced by a laser pulse. That excess conductivity is amplified and detected by a phase-sensitive detector. That signal decays with the recombination or minority-carrier lifetime.

RFPCD provides the means for measuring minority-carrier lifetime in non-light emitting materials such as silicon and germanium. This technique is also very useful for measuring lifetime in various InGaAs that are being developed for the thermophotovoltaic (TPV) program. During FY 1995, RFPCD was significantly modified to improve sensitivity and linearity of response (high fidelity). The phase II RFPCD system has been recommended by the NREL patent committee for patent application. The technique has become very popular in the silicon industry and measurements of solar cell lifetimes were made with the improved RFPCD that were not attainable by any other methods. The latter includes the measurement of minority-carrier lifetime in the AstroPower samples that were used for the TPV program.

\section{Highlights of the RFPCD Technique}

\section{Industrial Silicon Support}

Measurements were completed on a large group of polycrystalline samples provided by AstroPower. RFPCD appears to be the only contactless technique that has the sensitivity to measure lifetime in these samples because of the complications of grain boundary trapping. As is frequently found in small-grain polycrystalline samples, the decay spectra are complex, providing a considerable 
small-grain polycrystalline samples, the decay spectra are complex, providing a considerable challenge to interpretation. We are trying to establish the relationship between a variety of processing steps and changes in the minority-carrier lifetime of the polysilicon. In addition, a number of RFPCD lifetime measurements were also made on single crystal calibration wafers provided by AstroPower. These measurements are to be compared with their in-house microwave reflection technique.

Measurements successful of the minority-carrier lifetime in heavily doped, large grain polysilicon provided by Solarex have been very successful. Here the relationships between various processing steps and lifetime were established.

Recombination lifetime measurements were made, using RFPCD, on the EFG samples (ASE Americas) processes by A. Rohatgi at Georgia Tech. We measured the recombination lifetime as a function of the injection level in order to ascertain the concentrations of deep-level impurities. Also, some RFPCD lifetime measurements were performed on silicon wafers provided by Spire Corporation in support of their solar cell program.

RFPCD lifetime measurements were performed on thin films provided by Richard Wallis of SUNY, Buffalo. These measurements supported a subcontract to grow thin-film silicon on foreign substrates.

\section{Other Materials}

For the first time, lifetime measurements were performed on thin films of CIS and CIGS using the RFPCD technique. A series of RFPCD measurements have been made on NREL-produced thin-film CIS and CIGS. These measurements show lifetimes in the $30 \mathrm{~ns}$ to $60 \mathrm{~ns}$ time range. These lifetimes may be recombination times or trap emission times similar to those found in poly CdTe. However, higher speed recombination events could not be identified with the technique. These results were presented by Brian Keyes at the 10th International Conference on Ternary and Multinary Compounds.

The phase II RFPCD system established a breakthrough in measurement technology. This technique was so improved that we were able to measure minority-carrier lifetimes in uncut ingots of FZ silicon that contained various concentrations of iron impurity. These ingots were about $20 \mathrm{~cm}$ in length and $3 \mathrm{~cm}$ in diameter. These results of these measurements will be presented in a paper by $\mathrm{T}$. Ciszek at the Photovoltaic Specialists Conference in Washington D.C.

Using the phase II RFPCD system, measurable samples range from thin films of approximately $1 \mu \mathrm{m}$ thick by several $\mathrm{mm}$ wide to the above wafers.

\section{Time-Resolved Photoluminescence}

Time-resolved photoluminescence (TRPL) has become a routine diagnostic technique for much of the ongoing research in polycrystalline CdTe-based materials. We performed over $90 \mathrm{PL}$ and TRPL measurements for materials characterization for researchers at NREL, Golden Photon, Solar Cells Incorporated, and the Colorado School of Mines.

Time-resolved photoluminescence data were obtained on a series of ordered GaInP samples (Y. Zhang, NREL). Data were taken at various temperatures between $4 \mathrm{~K}$ and $300 \mathrm{~K}$. Results 
indicate appreciable nonradiative recombination at the lower temperatures. Time-resolved photoluminescence data were obtained on AlGaInP-passivated GaInP double heterostructures (J. Olson, NREL). These data were used to assist this group in monitoring the material quality of their growth system. These specific samples are quantifying the effect of changing the purifier location. Time-resolved photoluminescence data were obtained on AlGaAs passivated, highly doped $\left(\mathrm{p}-8 \times 10^{19} \mathrm{~cm}^{-3}\right) \mathrm{GaAs}$ double heterostructures provided by Purdue University. Our results indicate lifetime values ranging from 20 to $50 \mathrm{ps}$. The effect of substrate growth temperature and interface recombination was investigated.

\section{Energy-Resolved Photoluminescence}

The PL system has performed up to 100 runs per month for two ongoing studies on the CdTe/CdS material system. In one study, $300 \mathrm{~K}$ and $4 \mathrm{~K} \mathrm{PL}$ measurements were taken on polycrystalline CdS/CdTe solar cells (R. Dhere, NREL). These PL measurements support the idea of a lowerband-gap CdTeS region at the CdTe/CdS growth interface. This work was completed by lowtemperature PL as well as time-resolved PL measurements. In the second study, $300 \mathrm{~K} \mathrm{PL}$ and time-resolved PL data taken on as-deposited films and processed CdS/CdTe solar cells are being correlated with other characterization techniques. This study investigated the effect of different nucleation temperatures on film and device quality. These results were summarized and presented at the PV Program Review Meeting in May.

\section{Fourier Transform Infrared Spectroscopy (FTIR)}

We measured the FT-PL spectra of many sets of two $\mathrm{CI}(\mathrm{G}) \mathrm{S}$ films, with one film in each set deposited on glass, and the other on molybdenum/glass substrates. The $\mathrm{CI}(\mathrm{G}) \mathrm{S}$ films on glass substrates exhibited PL maxima at significantly lower energies than those on molybdenum/glass substrates. Some of the $\mathrm{CI}(\mathrm{G}) \mathrm{S}$ films deposited on glass substrates also exhibited bimodal PL band shapes. These data suggest that systematic differences in deposition temperature related to the two substrate types may explain the differences in the PL spectra.

An InSb detector was installed on our FT-Raman spectrophotometer to extend the low-energy limit of FT-PL measurements from the $0.74 \mathrm{eV}$ obtainable with our Ge detector to $0.5 \mathrm{eV}$. The InSb detector was used to determine an $\mathrm{E}_{\mathrm{g}}$ of $0.63 \mathrm{eV}$ from the FT-PL spectrum of a GaInAs epitaxial film. FT-PL measurements were also done on a GaAsGe alloy film from Spire Corp. having an $E_{g}$ of $0.8 \mathrm{eV}$ with both the $\mathrm{Ge}$ and InSb detectors to compare their sensitivity. These analyses showed that the $\mathrm{Ge}$ detector is about $500 \mathrm{x}$ more sensitive than the InSb detector at $0.8 \mathrm{eV}$. This sensitivity difference precludes using the InSb detector for long-wavelength FT-Raman measurements, and the 0.92-eV high-energy response limit of the InSb detector will prevent its use for FT-PL measurements on $\mathrm{CI}(\mathrm{G}) \mathrm{S}$ films. However, the long-wavelength capability of the InSb detector should be useful in room-temperature FT-PL characterization of strong, low-band-gap PL emitters such as the III-V films. 


\section{Other Industrial Support}

Considerable effort has recently gone into the technique development of a nondestructive thin-film thickness measurement system. The catalyst for this work was Golden Photon's need to quickly and easily monitor their CdS film thickness. The technique being developed is an optical transmission measurement that is designed to monitor the CaS thickness to within $\pm 4 \%$. Golden Photon will be purchasing the necessary equipment in the near future. In addition to the development and design of this system, NREL will provide technical assistance in constructing and calibrating the system in their production environment.

\section{Summary}

A substantial number of contributions were made under this task in the areas of research support and characterization, industrial manufacturing diagnostics, and technique development. Continued effort in the forthcoming year will focus on the above with increased emphasis on new technique development. 
Title:

Organization:

Contributors:
GaInP/GaAs Tandem Solar Cells

National Renewable Energy Laboratory, Golden, CO

J.M. Olson, Principal Scientist

S.R. Kurtz, Senior Scientist

K.A. Bertness, Senior Scientist

D.J. Friedman, Staff Scientist

A.E. Kibbler, Staff Scientist

C. Kramer, Research Technician

\section{Concentrator Cell Development}

The monolithic two-terminal GaInP/GaAs solar cell ${ }^{1}$ is a promising candidate in terrestrial concentrator power systems. Efficiencies in excess of $30 \%$ for concentrations in the range of 100-300 suns ${ }^{2}$ have been measured under simulated AM1.5D conditions. In collaboration with J.B. Lasich and co-workers at the Solar Research Corporation (Victoria, Australia), the GaInP/GaAs concentrator device has been adapted for and tested in a prototype "real-world" parabolic-reflector, concentrator power system. The requirements for a device usable in the concentrator system are more stringent than those for devices measured on a simulator. For our previous work, a device size of $0.1 \mathrm{~cm}^{2}$ was used, and tests were performed at the standard reporting temperature of $25^{\circ} \mathrm{C}$. For this study, practical considerations, as well as signal-to-noise requirements of the measurement system, called for the use of $1 \mathrm{~cm}^{2}$ devices. The efficiencies were measured at the actual system operating temperature, which in these measurements ranged from $32^{\circ} \mathrm{C}$ to $50^{\circ} \mathrm{C}$ for concentration ratios of approximately $80-400$ suns. Despite the larger area and higher measurement temperatures, the GaInP/GaAs solar cell achieved an on-sun efficiency of $27 \% \pm 1 \%$ over the concentration range of 80-400 suns. A number of ways for improving this device performance were identified and are currently being examined.

\section{Commercialization of the GaInP/GaAs Tandem Solar Cell}

A vital link in the development of a new terrestrial PV power generation system is the establishment of large-scale production capability. Because of its high efficiency and radiation hardness, the monolithic two-terminal GaInP/GaAs solar cell has attracted the attention of several companies in the space power community. As a result, NREL, over the last several years, has had several technology transfer agreements with Applied Solar Energy Corporation and Spectrolab to develop a production capability for this cell.

Over the last year, in collaboration with Spectrolab, we have achieved a number of important milestones. Heretofore, most of the work and all of the world-record solar cells were grown on expensive and fragile $\mathrm{GaAs}$ substrates in research-scale metalorganic chemical vapor deposition (MOCVD) reactors. In collaboration with Spectrolab, near state-of-the-art GaInP/GaAs dual junction solar cells were grown on Ge substrates. The AMO efficiency of these devices was $24.2 \%$, which is $94 \%$ of the highest efficiency achieved to date on GaAs. These devices were grown in the NREL MOCVD research reactor. More importantly, tandem cells grown in a production-scale. reactor on $50 \mathrm{~mm} \mathrm{Ge}$ wafers yielded average AM0 efficiencies of $22.0 \%$ and $21 \%$ on $75 \mathrm{~mm} \mathrm{GaAs}$ 
wafers for $1 \mathrm{~cm} \times 1 \mathrm{~cm}$ and $2 \mathrm{~cm} \times 2 \mathrm{~cm}$ devices, respectively. These results, while still in the preliminary stages of the development efforts, strongly suggest that this tandem cell technology, despite its complexity, is manufacturable. At first glance, this effort might appear to be a diversion since it does not focus directly on terrestrial PV power. But it should be pointed out that this is a legitimate interim market for the tandem cell. It provides industry with a manufacturable product in a profitable market. At the same time, the lessons learned can easily be applied to the developing terrestrial market. As the space power market shits more from military to civilian applications, cell manufacturing costs become increasingly important, and it is expected that this market will help drive down the costs of high-performance tandem cells for terrestrial applications. We project that within a few years the GaInP/GaAs tandem solar cells on inexpensive Ge substrates will be available at a cost of about $\$ 5 / \mathrm{cm}^{2}$ (or about $\$ 0.20 /$ watt at $1000 \mathrm{X}$ ) with an industry capacity of more than $1 \mathrm{GW} /$ year.

\section{Laboratory Development}

A major portion of time and effort during FY 1995 was devoted to the design, construction and qualification of three new deposition systems: a stand-alone MOCVD system to replace the former Lab 16/103 system, a cluster system consisting of a low-pressure MOCVD system, and a molecular beam epitaxy (MBE) system attached to a linear transfer line and an analytical chamber. A schematic of the cluster system is shown in Fig. 1.

The MBE system was commissioned in early November 1995. It is charged with elemental sources of $\mathrm{Ga}, \mathrm{In}, \mathrm{As}$, and $\mathrm{P}$ and has the capacity for five additional sources. Substrate diameters up to 3 inches can be accommodated. The As and P sources are valved ovens from EPI, Inc., and obviate many of the problems associated with the use of $\mathrm{PH}_{3}$ and $\mathrm{AsH}_{3}$. The growth chamber is pumped with a $400 \mathrm{l} / \mathrm{s}$ ion pump and is equipped with a $15 \mathrm{keV}$ electron gun for in situ reflection high-energy electron diffraction (RHEED) studies. The system will be used for basic studies of the electronic properties of PV materials both in single and polycrystalline form.

The low-pressure MOCVD should be commissioned sometime during the second quarter of 1996. This system will be used for the growth of devices of interest to industry and for basic studies of MOCVD growth processes. As such, it has been designed with two main objectives: (1) uniform, large-area growth of III-V PV materials and (2) in situ optical spectroscopy at both glancing and normal incidence. Both objectives are achieved by the use of the same 3-inch substrate heater manipulator that is used in the MBE system. This manipulator allows one to mount the sample in an "upside-down" configuration over an optics table configured for reflectance difference spectroscopy and spectroscopic ellipsometry. With careful tuning of the growth reactor environment (pressure, $\mathrm{H}_{2}$ flow, etc.), uniform growth over the 3-inch wafer should be achievable.

The analytical chamber is equipped with an ultra-high vacuum (UHV) scanning tunneling/atomic force microscope (STM/AFM) and a low-energy electron diffraction (LEED) system. Both of these instruments are sensitive probes of surface structure and will be used to study the similarities and differences between MOCVD- and MBE-grown surfaces.

As shown in Fig. 1, both of these systems are connected to a common linear transfer line. This will allow us to transfer substrates between the two growth chambers and capitalize on the strengths of both growth techniques (e.g., monolayer capability of MBE for growth of abrupt heterojunctions combined with the higher growth rate and superior material quality of MOCVD). Samples can be 


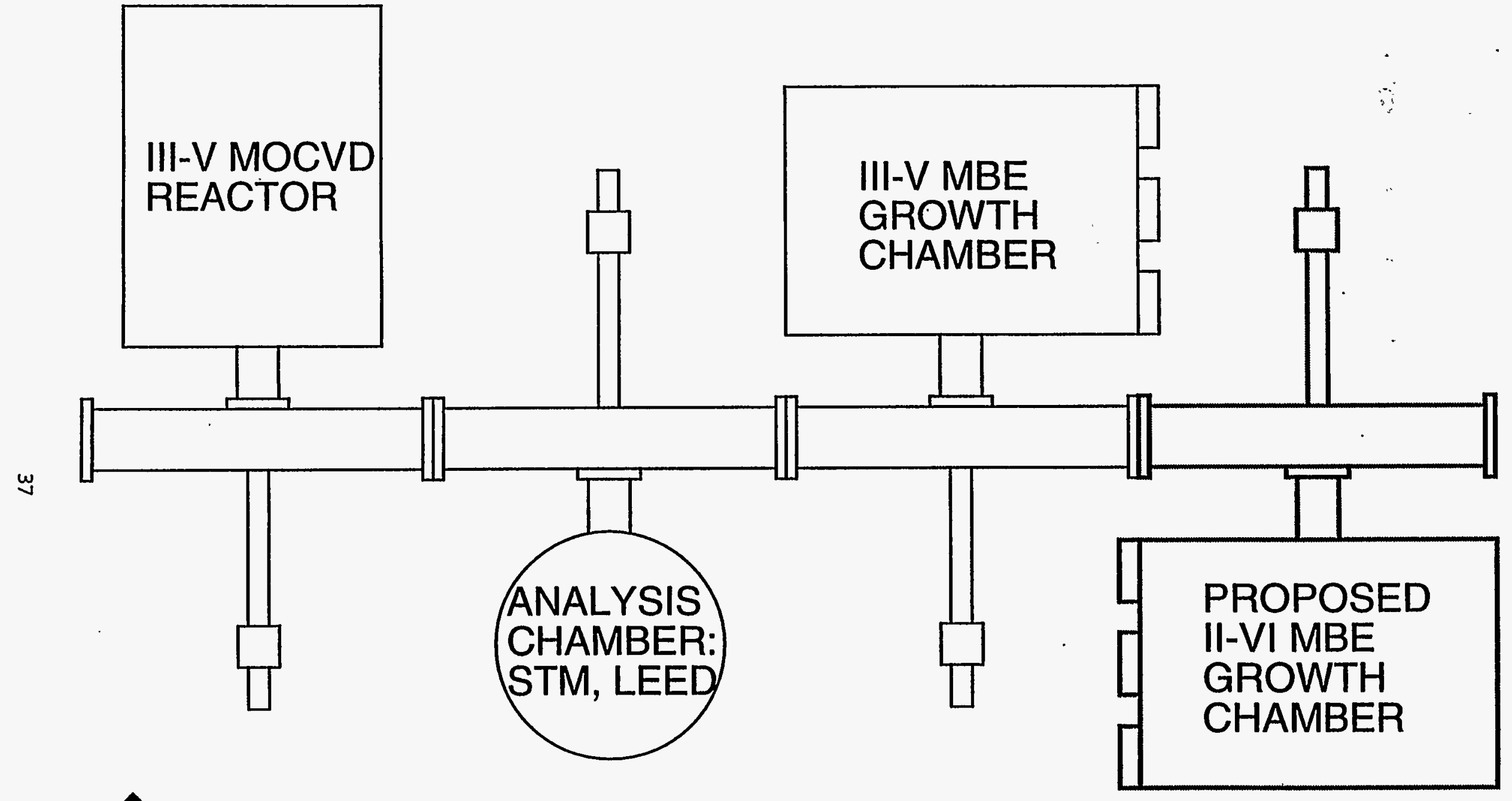

Figure 1. Schematic of MBE cluster system 
transferred between the MBE and MOCVD systems and the analytical chamber without being exposed to atmosphere, permitting direct examination of the as-grown sample surface without the requirement for post-growth sample surface preparation that can skew or hide important features.

The new stand-alone MOCVD system was completed and brought on-line in February 1995. Software was written to allow control of the valves from the computer using the mouse. The manual switching of valves that was done in 16/103 is now handled by the computer, improving reliability and decreasing work for the operator. Although most of the calibration work was straightforward, several problems were encountered that took significant amounts of time.

A new quantum efficiency (QE) system was designed and built. Compared to the previous system, it can measure samples more quickly and over a broader spectral range. Also, several new and very useful features were added. The reflectivity data are now used to calculate the optical thickness of the layers. Although this seldom gives an absolute, quantitative thickness because of the presence of multiple layers, it does give a precise relative value that can detect variations of $1 \%$. Also, a model capability for the top cell is included in the software. This allows us to check to see whether a diminished red response is caused by reduced base thickness or poor material properties. A calculation of the spectral correction factor is also included, allowing us to make corrections for differences in the reference cells' responses.

Progress was made toward the development of a two-source simulator that can be used for measuring tandem cells on a routine basis. The tungsten light source that was originally purchased did not deliver adequate power. The solar simulator experts at Spectrolab recommended using a narrow-focus spotlight and this proved to work better. Andrew Tennant of NREL's CIS Group has helped to develop software to control the two lamps so that the computer can control the relative illumination on the two junctions. When completed, this will allow us to quickly measure not only the one-sun IV curve, but also curves that show the performance of the top and bottom cells. We hope to complete this work in the next year.

\section{References}

1. K. A. Bertness, S. R. Kurtz, D. J. Friedman, A. E. Kibbler, C. Kramer, and J. M. Olson, "29.5\%-Efficient GaInP/GaAs Tandem Solar Cells," Appl. Phys. Lett. 65, 989-991 (1994).

2. D. J. Friedman, S. R. Kurtz, K. A. Bertness, A. E. Kibbler, C. Kramer, J. M. Olson, D. L. King, B. R. Hansen, and J. K. Snyder, in GaInP/GaAs Monolithic Tandem Concentrator Cells, Waikoloa, Hawaii, 1994 (IEEE), pp. 1829-1832. 


\title{
SOLID STATE THEORY
}

\author{
Alex Zunger (PI), S.-H. Wei, and S. Froyen \\ National Renewable Energy Laboratory, Golden, Colorado
}

In this fiscal year, our work concentrated on three directions:

(1) Analysis of the properties of III-V materials and alloys

(2) Theory of chalcopyrite and defect chalcopyrite semiconductors

(3) Developing novel electronic structure methods and algorithms for mesoscopic systems.

This report provides a succinct summary of activities in these three areas. The reader interested in further detail can consult the original journal publications cited at the end of each subsection.

\section{ANALYSIS OF THE PROPERTIES OF III-V MATERIALS AND THEIR ALLOYS}

\section{a. Theory of reflectance difference spectroscopy in III-V alloys}

Spontaneous CuPt-like ordering in III-V alloys causes an anisotropy in the intensities of the transitions between the split valence-band maximum states and the conduction-band minimum. This optical anisotropy has been detected in ordered III-V alloys using modulated reflectivity, and more recently, using reflectance-difference spectroscopy (RDS). We derive here a general formula relating the ordering-induced bulk RDS intensity with the degree of long-range order. We show that the previously neglected coupling between the spin-orbit split-off band and the crystal-field split-off band is crucial for determining the RDS intensity of the $\mathrm{Ga}_{x} \mathrm{In}_{1-x} \mathrm{P}$ alloy. This work provides an easy-to-use link between RDS measurements on the $E_{0}$ transition and the degree of ordering in a sample. See Wei and Zunger, Phys. Rev. B, 52, 14,110 (1995).

\section{b. Interpreting the high-energy $\left(E_{1}, E_{2}, E_{0}^{\prime}\right)$ transitions in GaInP}

We investigated theoretically the ordering-induced change of the $E_{1}, E_{2}$, and, $E_{0}^{\prime}$ transitions in $\mathrm{Ga}_{0.5} \mathrm{In}_{0.5} \mathrm{P}$ using symmetry arguments and first-principles band-structure calculations. We show that upon $<111>$ superlattice ordering these transitions are altered dramatically-some states shift up in energy, some shift down, and new "ordering-induced" transitions, absent in the disordered phase, now become allowed. Experimental observation of these changes will serve as new fingerprints of ordering. We have also studied the pressure dependence of the energies of $X_{1 \mathrm{c}}$ and $L_{\mathrm{lc}}$ derived states in the ordered superlattice. The recent experimental observation of the " $X_{\mathrm{lc}}$-like" state at higher pressure is identified as a mixture of the folded $L_{1 \mathrm{c}}$ and $X_{1 \mathrm{c}}$ states. A microscopic explanation is given. See Wei, Franceschetti, and Zunger, Phys. Rev. B, 51, 13,097 (1995).

\section{c. Surface segregation and ordering in III-V alloys}

Using the first-principles total-energy pseudopotential method, we have studied the formation energy of the (001) $\mathrm{Ga}_{1-x} \mathrm{In}_{x} \mathrm{P}$ alloy surface as a function of composition and reconstruction. The results are presented as $T=0$ surface stability diagrams that show the lowest energy reconstruction and cation occupation pattern as functions of the chemical potentials. The stability diagrams show a pronounced asymmetry between the $\mathrm{Ga}$ - and In-rich regions. For surfaces in equilibrium with the bulk, we find a strong dependence of surface segregation on the surface reconstruction, and we 
This result suggests a special way to achieve abrupt interfaces in semiconductor heterostructures. For surfaces not in equilibrium with the bulk, we identify regions in the stability diagram where surface-induced CuPt ordering (both type A and type B) occurs. See Froyen and Zunger, Phys. Rev. $B, 53,4570$ (1996).

\section{d. Effect of ordering on the effective masses of GaInP*}

The conventional eight-band $\mathbf{k} \cdot \mathbf{p}$ model predicts a decrease of the electron effective mass and no dependence of the (001) strain band-gap deformation potential with the degree $\eta$ of long-range order in $\mathrm{Ga}_{0,5} \mathrm{In}_{0.5} \mathrm{P}$ alloys. We show that a complete band-structure approach predicts instead that (i) the electron effective mass in the ordering direction increases from $0.092 m_{0}$ for $\eta=0$ (random alloy) to $0.133 m_{0}$ for $\eta=1$ (ordered alloy), and (ii) the strain deformation potential decreases in magnitude from $8.26 \mathrm{eV}$ for $\eta=0$ to $6.34 \mathrm{eV}$ for $\eta=1$. These two effects are caused by the mixing of the conduction-band minimum with the $L$-derived conduction band, neglected in the standard eight-band model. This work provides another experimental way to detect ordering. See Franceschetti, Wei, and Zunger, Phys. Rev. B, 52, 13,992 (1995). *EE-OER collaboration.

\section{e. Properties of the thermo-PV alloy system InAsSb}

InAsSb alloys are used in far IR detectors, and are also promising thermo-PV materials. Using firstprinciples band-structure calculations we have studied alloys band gap $v s$ composition and the valence-band alignment of $\operatorname{InAs} / \mathrm{InSb}$, deducing also the offset at the $\operatorname{InAs} s_{1-x} \mathrm{Sb}_{\mathrm{x}} / \operatorname{In} \mathrm{As}_{1-\mathrm{y}} \mathrm{Sb}_{\mathrm{y}}$ heterostructure. We find the following: (i) pure InAs/InSb has a "type-II broken gap" alignment both with and without strain, (ii) for Sb-rich $\operatorname{InAs}_{1-x} \mathrm{Sb}_{x} / \mathrm{InSb}$ heterostructures, the unstrained band alignment is type II; both epitaxial strain and CuPt ordering enhance the type-II character in this $\mathrm{Sb}$ rich limit, and (iii) for As-rich $\operatorname{InAs/InAs}{ }_{1-x} \mathrm{Sb}_{x}$ heterostructures the top of the valence band is always on the alloy layer while the conduction-band minimum can be localized either on the alloy layer (typeD) or on the InAs layer (type-II), depending on the balance between concentration, strain, and degree of ordering/phase separation. In this case, epitaxial strain enhances the type-II character, while ordering enhances the type-I character. Our results are compared with recent experimental observations. See Wei and Zunger, Phys. Rev. B, 52, 12,039 (1995).

\section{f. How do surface reconstructions decide the characteristics of MBE or MOCVD grown GaInP?}

Spontaneous ordering of III-V alloys has now been extensively observed and characterized in many semiconductors. The most frequently seen spontaneous ordering consists of monolayer superlattice alternation along the $\langle 111\rangle$ cubic body diagonals. Of the four bulk-equivalent body diagonals, ordering has been seen to take place along only two directions [i111] and [1ī1] (called $\mathrm{CuPt}_{\mathrm{B}}$ variants), while, until recently ordering along the other two directions [111] and [111], (called $\mathrm{CuPt}_{A}$ variants) was absent. Two significant experimental observations were recently made in this respect. First, Gomyo et al discovered a triple period ordering of $\mathrm{Al}_{0.5} \mathrm{In}_{0.5} \mathrm{As} / \mathrm{InP}(001)$ under MBE growth conditions favoring an anion-rich $2 \times 3$ surface reconstruction. Second, Gomyo et al. discovered a $\mathrm{CuPt}_{A}$ ordering of $\mathrm{Al}_{0.5} \mathrm{Jn}_{0.5} \mathrm{P} / \mathrm{GaAs}(001)$ under $\mathrm{MBE}$ growth conditions favoring an anion-terminated $2 \times 2$ reconstruction (with $1 \times 2$ basic building blocks). In contrast, the conventional higher growth temperature $\mathrm{T} \sim 560^{\circ} \mathrm{C}$ produces the $2 \times 1$ reconstruction that leads to $\mathrm{CuPt}_{B}$ ordering. Both the $2 \times 2$ and $2 \times 3$ surface reconstructions occur at low-growth temperatures $\left(520^{\circ} \mathrm{C}\right.$ and $420^{\circ} \mathrm{C}$, respectively) and are more anion-rich than the $2 \times 1$ surface. Such reconstructions with heavy excess $>1$ monolayer) of anions were not theoretically studied before since the nature of the surface structure under heavy anion coverage became clear only recently. Using a valence force field (VFF) approach 
and $a b$ initio pseudopotential calculations, we have examined the role of subsurface strain in the ordering of $\mathrm{Ga}_{0.5} \mathrm{In}_{0.5} \mathrm{P}$ alloys. We found that, depending on the orientation of surface $\mathrm{P}$ dimers, these alloys can have: (i) a CuPt ordering for $1 \times 2$ or $\mathrm{c}(4 \times 4)$ reconstruction, (ii) $\mathrm{a} \mathrm{CuPt}_{\mathrm{B}}$ ordering for $2 \mathrm{xI}$ or $\beta 2(2 \times 4)$ reconstruction, and (iii) a triple period ordering for $2 \times 3$ or $c(8 \times 6)$ reconstruction. These results are in good agreement with recent experiments of Gomyo et al. See Zhang, Froyen, and Zunger, Appl. Phys. Lett., 67, 3,141 (1995).

\section{g. A new III-V alloy system-GaAsN}

$\mathrm{GaAs}_{1-x} \mathrm{~N}_{\mathrm{x}}$ alloy is the new candidate material for strong absorber and light-emitting devices with gaps between 1.5 and $3 \mathrm{eV}$ (i.e., either absorber or window). It can be integrated on $\mathrm{Si}$ if sufficient $\mathrm{GaN}$ can be mixed into GaAs. It is therefore interesting to study the miscibility of the alloy and the change of the band gap as a function of composition $x$. The deviation of band gap $\mathrm{E}_{\mathrm{g}}(x)$ of an $\mathrm{A}_{1-x} \mathrm{~B}_{\mathrm{x}}$ alloy from the average gap of the constituents solids A and $B$ is described in an "optical" bowing coefficient $b$. Previous measurements and calculation for isovalent semiconductor alloys show that $b$ is typically a fraction of an $\mathrm{eV}$ and composition independent. We show here, via first-principles local density approximation calculations, that in $\mathrm{GaAs}_{1 \cdot x} \mathrm{~N}_{\mathrm{x}}$ alloys $b$ is giant $(16 \mathrm{eV})$ and composition-dependent: it increases rapidly as the nitrogen concentration decreases. Our analysis reveals that both anomalies are due to the existence of sharply localized band-edge states in the alloy system. Our calculated bowing parameter at low concentration $(b=16 \mathrm{eV}$ ) agrees with recent experimental determination by Kondow et al. From our total energy calculation we have estimated the miscibility of $\mathrm{GaN}$ and $\mathrm{GaAs}$ using the regular solution model finding a miscibility gap temperature of $T_{c}=11024 \mathrm{~K}$. These values show that at the typical growth temperature of $\sim 800 \mathrm{~K}$ the equilibrium solubility is essentially zero while the spinodal limit is about 1.9\%. These indicate that the experimental samples of Kondow et al and Weyers et al. were grown in a metastable region. Since $\sim 20 \% \mathrm{GaN}$ is required to be lattice match to $\mathrm{Si}$, non-equilibrium techniques are needed to grow high nitrogen concentration $\mathrm{GaAs}_{1 \cdot x} \mathrm{~N}_{x}$ alloys. See Wei and Zunger, Phys. Rev. Lett., 76, 664 (1996).

\section{THEORY OF CHALCOPYRITE AND DEFECT CHALCOPYRITE SEMICONDUCTORS}

\section{a. Band offsets and alloy bowing in chalcopyrite alloys}

Using first-principles band-structure theory we have systematically calculated the (i) alloy-bowing coefficients, (ii) alloy mixing enthalpies, and (iii) interfacial valence- and conduction-band offsets for three mixed-anion (CuIn $X_{2}, X=S, S e, T e$ ) and three mixed-cation (CuMSe $, M=\mathrm{Al}, \mathrm{Ga}, \mathrm{In}$ ) chalcopyrite systems. The calculated bowing coefficients are in good agreement with the most recent experimental data for stoichiometric alloys. Results for the mixing enthalpies and the band offsets are provided as predictions to be tested experimentally. Comparing our calculated bowing and band offsets for the mixed-anion chalcopyrite alloys with those of the corresponding $\mathrm{Zn}$ chalcogenide alloys ( $\mathrm{ZnX}, \mathrm{X}=\mathrm{S}, \mathrm{Se}, \mathrm{Te}$ ), we find that the larger $p$ - $d$ coupling in chalcopyrite alloys reduces their band offsets and optical bowing. Bowing parameters for ordered, $\mathrm{Zn}$-based II-VI alloys in the $\mathrm{CuAu}$, $\mathrm{CuPt}$, and chalcopyrite structures are presented: we find that ordered $\mathrm{Zn}_{2} \mathrm{SeTe}$ has bowing coefficients of 1.44 and $3.15 \mathrm{eV}$ in the $\mathrm{CuAu}$ and CuPt structures, while the random $\mathrm{ZnSe}_{x} \mathrm{Te}_{1-x}$ alloy has a bowing of $1.14 \mathrm{eV}$. The band alignments between $\mathrm{CuInSe}_{2}$ and $\mathrm{CuInSe}_{2}$-derived ordered vacancy compounds are also presented. See Wei and Zunger, J. Appl. Phys., 78, 3846 (1995). 


\section{b. Properties of a new window material-Mn- and Zn-hased II-VI alloys}

We have previously suggested that $\mathrm{Mn}$-based alloys (e.g., $\mathrm{ZnMnSe}$ ) could form a useful alternative to CdS as a window material, since $\mathrm{ZnMnSe}$ is cubic, lattice-matched to CIGS, contains a common anion (Se) and is non-toxic. We now calculated some properties of $\mathrm{Mn}$ - and $\mathrm{Zn}$-based II-VIs. Calculation of the unstrained band offsets between conventional zinc-blend II-VIs ( $\mathrm{ZnS} / \mathrm{ZnSe} / \mathrm{ZnTe}$ ), or between magnetic II-VIs ( $\mathrm{MnS} / \mathrm{MnSe} / \mathrm{MnTe})$, or combinations thereof, ( $\mathrm{MnX} / \mathrm{ZnX})$ shows that: (i) the range of offsets spanned by different magnetic II-VIs is compressed by a factor of two relative to the range of offsets spanned by conventional II-VIs, (ii) the distance between the Mn $d$ band and the valence band maximum in $\mathrm{MnX}$ depends weakly on $X$, while in conventional II-VIs (e.g., $\mathrm{Zn} 3 d$ in $\mathrm{ZnX}$ ) there is a wider spread, and (iii) unlike the case for conventional common-anion II-VIs, the mixed offset $\Delta E_{v}(\mathrm{ZnX} / \mathrm{MnX})$ depends strongly on $X$. We show that all three effects have a simple and common physical origin. See Wei and Zunger, Phys. Rev. B, Rapid Communication 53, 1045 (1996).

\section{c. Verification of CdS/CIS band offset}

Wei and Zunger have previously predicted that the conduction band minimum (CBM) of CdS is above that of CIS (by $\Delta E_{c}=0.3 \pm 0.1 \mathrm{eV}$ ), while all device models have assumed that the CdS CBM is below the CIS CBM. The predicted valence band discontinuity was $\triangle E_{v} \sim 1.07 \pm 0.1 \mathrm{eV}$ (Wei and Zunger, APL, 63, 2549 [1993]). The January issue of J. Appl. Phys. contains a publication of Löhr et al of the Hahn-Meitner Institute of Berlin (Vol. 77, p. 731) reporting a soft X-ray photoemission study of the crystalline $\mathrm{CdS} / \mathrm{CuInS} \mathrm{e}_{2}$ interface. The measured valence band offset was $\Delta \mathrm{E}_{\mathrm{v}}=0.8 \pm 0.2$ $\mathrm{eV}$, in reasonable agreement with our prediction. The ensuing conduction band offset is $\Delta \mathrm{E}_{\mathrm{c}}=\mathrm{Eg}(\mathrm{CdS})-\mathrm{Eg}(\mathrm{CuInSe})-(0.8 \pm 0.2)=0.6 \pm 0.2 \mathrm{eV}$ in reasonable agreement with the calculated value. A successful photovoltaic action will require $\Delta E_{c}<0$. The fact that both measured and calculated conduction band discontinuities for CdS/CIS show $\Delta \mathrm{E}_{c}>0$ could suggest that in the actual device the electrically active interface is not between CdS and CIS. Indeed, we find that the CISderived ordered vacancy compound (OVC) observed at the CdS/CIS interface has a slightly lower CBM than CIS, thus strongly supporting the idea that the actual $p-n$ junction is between CIS/OVC. See Zunger and Wei, AIP Conf. Proc., 353, 155 (1996).

\section{DEVELOPING NOVEL ELECTRONIC STRUCTURE METHODS AND ALGORITHMS FOR MESOSCOPIC SYSTEMS}

Photovoltaic applications often use "quantum well" structures. For very thin structures traditional atomistic quantum mechanics can be used, but thicknesses of technological importance are usually far beyond the reach of current direct methods and are usually treated by the $k \cdot p$ method. This approach has serious deficiencies: (i) it usually is applicable only to direct-gap materials, (ii) predicted bands are correct only for an (unknown) range near the zone center, (iii) predicted properties are quite sensitive to the number of (bulk) states retained in the description; spurious bands often occur, and (iv) unwarranted assumptions, with an unknown regime of validity, are generally made. We are developing (a) a code which uses existing band structure calculations to produce a legitimate and systematically improvable set of basis functions for complete $k \cdot p$ calculations and, (b) a code to use the output of (a) to predict bands for quantum wells (and eventually other geometries). Restrictions for the validity of our new approach are easily met for film thicknesses at and beyond the scope of atomistic approaches. Moderately thick $(\mathrm{GaAs})_{\mathrm{n}}(\mathrm{AlAs})_{\mathrm{n}}$ superlattices provide an excellent example of the potential of the proposed method. For $n=20$ a direct calculation using empirical pseudopotentials (EPM) takes 38 hours on an RS/6000; a corresponding $\mathrm{k} \cdot \mathrm{p}$ calculation (using the 
conventional, rather than the proposed, approach) takes under one minute. The new method should provide a flexible and natural way to bridge the gap between bulk materials and the size domain beyond the reach of current direct quantum mechanical (atomistic) approaches, at the expense of a relatively modest increase in computational effort. 


\section{Crystalline Silicon and Advanced Devices Subcontracts}





\begin{tabular}{ll} 
Title: & $\begin{array}{l}\text { Novel Low-Temperature Substrate Technology } \\
\text { for Compound Semiconductor Solar Cells } \\
\text { on Low-Cost Substrates }\end{array}$ \\
Organization: & $\begin{array}{l}\text { California Institute of Technology } \\
\text { Contributors: }\end{array}$ \\
\hline & $\begin{array}{l}\text { Harry A. Atwater, Principal Investigator, } \\
\text { C.M. Yang and C.M. Chen }\end{array}$
\end{tabular}

\section{OBJECTIVES}

The objectives of this program are to develop low-temperature solid phase growth approaches to obtaining large-grained $(20-100 \mu \mathrm{m})$ crystalline Ge films on low-cost amorphous (e.g., glass) substrates, which serve as templates for GaAs growth for thin film GaAs solar cells.

\section{BACKGROUND AND TECHNICAL APPROACH}

The overall approach for fabrication of large-grained Ge films on glass is depicted in Fig 1. Work on selective nucleation of crystalline Ge seeds in amorphous Ge utilizes reactions with a patterned film of material deposited on top of the amorphous Ge film. Subsequent thermal anneals at temperatures below $475^{\circ} \mathrm{C}$ produce large $(20-30 \mu \mathrm{m})$ grains via lateral solid phase epitaxial crystal growth from the seed regions. These largegrained Ge microstructures are to be used as templates for epitaxial growth of GaAs thin films.

\section{RESULTS}

I. Mechanical Masking for Lithography-Free Patterning

We have performed low-cost lithography-free patterning of evaporated thin films using freestanding $\mathrm{Ni}$ membrane mechanical masks which were formed by electroplating. We have found that these masks are well-suited to our purpose, and yield patterns with sharply-defined features, even on the micron size scale. Since the deposited films are much thinner than the mask feature sizes, the masks are quite readily reusable, and mask cleaning, if necessary, can be done using electrochemical techniques.

II. Impurity-Induced Selective Nucleation

We have surveyed the use of various eutectic-forming and binary compound forming alloys with $\mathrm{Ge}$, including $\mathrm{Sn}, \mathrm{In}, \mathrm{Ni}, \mathrm{Co}, \mathrm{Pd}, \mathrm{Cu}$ and $\mathrm{Al}$ as agents to induce selective nucleation[1,2]. Films of amorphous Ge $50 \mathrm{~nm}$ thick were coated with 5-20 nm thick metal films, and these structures were vacuumed annealed at temperatures ranging from $225^{\circ} \mathrm{C}$ to $475^{\circ} \mathrm{C}$. Each of these materials promoted crystallization of amorphous Ge at temperatures up to $250^{\circ} \mathrm{C}$ below the typical temperature for thermal crystallization of pure $\mathrm{Ge}$. In the case of $\mathrm{Pd}$ and $\mathrm{Cu}$, transmission electron diffraction patterns indicated the presence of metal-germanide phases in addition to the diamond cubic structure for crystalline $\mathrm{Ge}$. In the case of $\mathrm{Al}$, no intermetallic phase was found (as expected, since 
this is a simple eutectic system). Eventually, In was chosen as the selective nucleation agent to be used in all subsequent experiments, since it both enabled selective nucleation and it does not pose significant problems for subsequent GaAs epitaxial growth, unlike some of the other impurities studied. To date, the seed regions produced by selective nucleation are polycrystalline, rather than a single-grained. Work focussed at synthesis of single-crystal seed regions is underway.

III. Lateral Solid Phase Epitaxy to Achieve Large Ge Grains

We have employed a two-step anneal procedure to achieve large Ge grains by first inducing selective nucleation and then promoting lateral solid phase epitaxy from the nucleation seed regions $[1,2]$. Nucleation is performed by an anneal at $350^{\circ} \mathrm{C}$ for $20 \mathrm{~min}$ for a $50 \mathrm{~nm}$ a-Ge film on $\mathrm{SiO}_{2}$ with a $20 \mathrm{~nm}$ thick patterned $\mathrm{In}$ film on top of it. Lateral solid phase epitaxy occurs from these nucleation regions during anneals at temperatures from $425<T<475^{\circ}$, for various times. As expected, lower anneal temperatures results in less random nucleation in the unpattern a-Ge regions during lateral solid phase epitaxy. A typical result is illustrated in Fig. 2(a). In all experiments, crystalline Ge grains grow radially outward from the nucleation sites, yielding elongated crystals whose size is ultimately limited by impingement with grains from adjacent nucleation sites at low temperatures. Grain sizes of 5-20 $\mu \mathrm{m}$ are seen in samples that have undergone lateral solid phase epitaxy in undoped Ge films.

\section{Dopant-Enhanced Lateral Solid Phase Epitaxy of Ge}

Doping of the a-Ge starting materials with electronic dopants significantly enhances the solid phase epitaxy rate. This is an effect which had been previously observed in $\mathrm{Si}$, but this is the first observation of this effect in Ge. Doping of a-Ge was performed by ion implantation of $\mathrm{B}(8 \mathrm{keV})$ and $\mathrm{P}(25 \mathrm{keV})$ to yield peak atomic concentrations of 0.3 at. $\%$ and 0.6 at. $\% B$ and $P$, respectively. It was found that 0.6 at. $\% \mathrm{~B}$ caused an increase in the solid phase epitaxy rate by approximately 2 times relative to undoped films. Remarkably, 0.6 at. \% P caused an increase in the solid phase epitaxy rate by at least a factor of 5 times[3]. This resulted in grain sizes in P-doped Ge films of as large as 20-30 $\mu \mathrm{m}$, which is a factor of 600 times the film thickness. This is illustrated by the TEM images of Figs. 2(a) and 2(b) comparing in undoped and P-doped Ge films, respectively. Figure 2(b) illustrates the growth of large grains in fully-crystallized Ge films, with grains from adjacent nucleation centers impinging on each other at the midpoints between these centers. Figure 3 are a close-up image and diffraction patterns of one seed region in Fig 2(b) illustrating the grain size and density in the nucleation region and outside the nucleation region, where lateral solid phase epitaxy occurred. The wavy line features seen in the TEM image of Fig. 3 are bend contours, which are characteristic contrast features seen in TEM images of large single crystals. 
Figure 1: Process schematic for patterning of nucleation seed regions, selective nucleation of seed regions, lateral crystal growth of large grains from seed regions, and subsequent GaAs growth on the large-grained $\mathrm{Ge} /$ glass template. All Ge growth steps are performed at temperatures $\leq 475^{\circ} \mathrm{C}$.
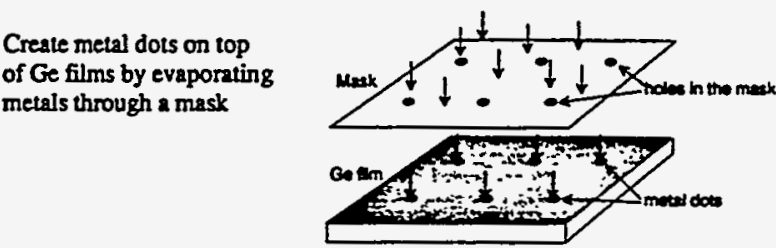

Upon annealing, Ge crystals nucleate selectively undemeath the metals dots.
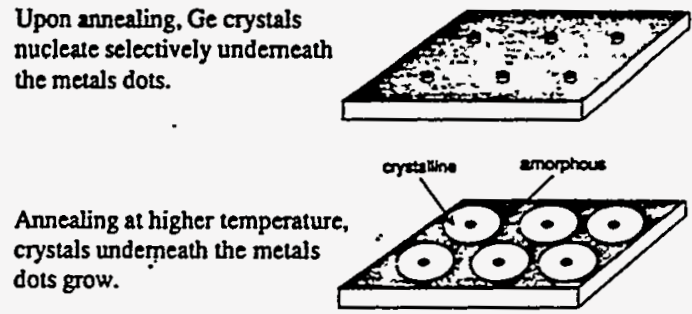

Crystals keep growing until they impinge on each other.

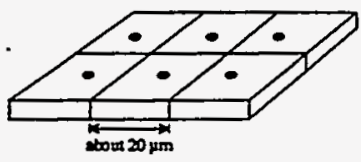

The large grained Ge films can be used as epitaxial templates for GaAs films.

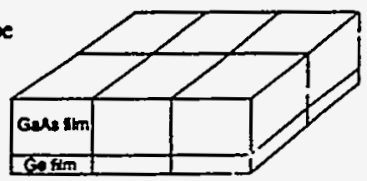

\section{CONCLUSIONS}

The selective nucleation and lateral solid phase epitaxy approach for fabrication of largegrained Ge films on glass appears to be very promising. Ge grain sizes of $20-30 \mu \mathrm{m}$ have been achieved in an unoptimized process, and 50-100 $\mu$ mgrain sizes appear feasible. Doping of Ge films with $\mathrm{P}$ and $\mathrm{B}$ at concentrations of $\leq 1$ at.\% significantly enhances (up to 5 times) the lateral solid phase epitaxy rate without enhancing the random nucleation rate, leading to even larger grain microstructures. Future efforts will be focussed on (i) achieving single-grained seed regions and (ii) GaAs epitaxy on the Ge/glass template films as well as (iii) initial work on extensions to other materials systems, such as crystalline Si thin films.

\section{REFERENCES}

[1]. "Low Temperature Approaches to Large-Grained Si and Ge on Glass Thin Films", H.A. Atwater and C.M. Yang, presented at 13th NREL Photovoltaics Program Review, Lakewood, CO,May 16-19, 1995.

[2]. "Selective Nucleation and Solid Phase Growth of Large-Grained $\mathrm{Ge} / \mathrm{SiO}_{2}$ Films at Low Temperatures", C.M. Yang and H.A. Atwater, to be published in Appl. Phys. Lett., 1996.

[3]. "Dopant Effects on Ge Solid Phase Epitaxy", C.M. Yang and H.A. Atwater, submitted to Appl. Phys. Lett. 

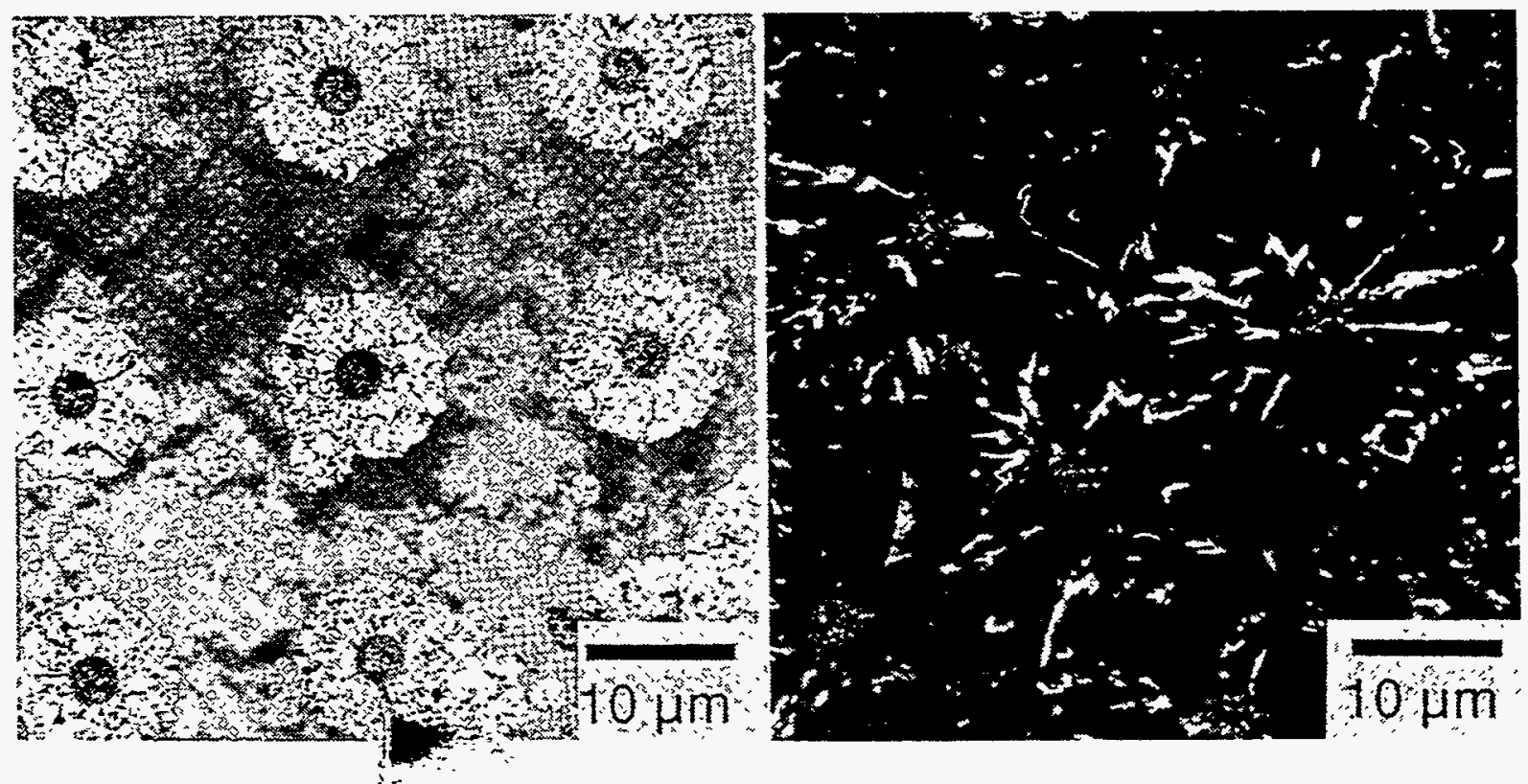

Figure 2: In (a), bright field transmission electron micrograph illustrating selective nucleation at In seed regions and crystal growth of large-grained Ge after annealing a 50 $\mathrm{nm}$ thick undoped a-Ge film at $350^{\circ} \mathrm{C}$ to induce nucleation, and $425^{\circ} \mathrm{C}$ for $2 \mathrm{hr}$ to enable crystal growth. In (b), dark field transmission electron micrograph illustrating enhanced crystal growth rate in $\mathrm{P}$ doped $(0.6$ at. $\% \mathrm{P})$ a-Ge film after annealing at $350^{\circ} \mathrm{C}$ to induce nucleation, and $475{ }^{\circ} \mathrm{C}$ for $10 \mathrm{~min}$ to induce crystal growth.

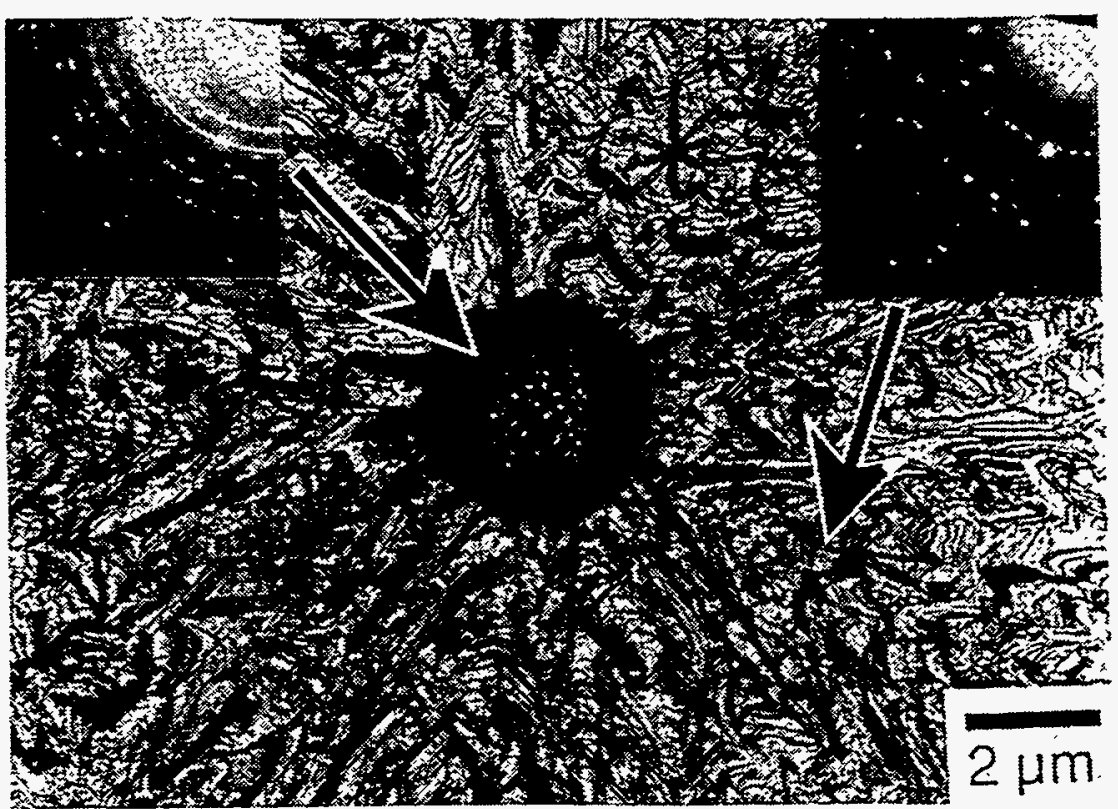

Figure 3: A closer view of a seed region in P-doped films in bright field. Insets are selected area diffraction patterns indicating small grains in seed region and very large grains outside of seed region. 
Title: $\quad$ Influence of Self-Interstitials Injected by

Phosphorus Diffusion on Defect Structures and

Electronic Properties in Crystalline Silicon

Organization: School of Engineering, Duke University, Durham, North Carolina

Contributors: T. Y. Tan and U. M. Gösele, principal investigators;

R. Gafiteanu and S. Joshi, graduate students

\section{Objective}

The overall objective is to identify means of effective gettering processes for improving crystalline Si minority carrier lifetimes so as to improve Si solar cell efficiencies. Presently our research focuses on the simulation of the metal atom precipitation process, the precipitate dissolution process, and the gettering efficiency of dissolved metal atoms versus that involving also metal precipitates. The present study is stemmed by the fact that, in polycrystalline solar grade $\mathrm{Si}$, there exist the so called "good" and "bad" regions or grains, possessing respectively fairly long and very short minority carrier diffusion lengths. It is known that the carrier diffusion lengths in the good regions can often be further improved by a gettering treatment such as using an evaporated Al layer [1], while the same gettering process usually has no effect on the carrier diffusion lengths in the bad regions. These bad regions contain high densities of dislocations observable by a variety of methods. It is possible that these regions also contain a high density of metal silicide precipitates that are very small in size, and hence difficult to observe. A plausible reason that the quality of the bad regions is difficult to improve by a gettering treatment is that precipitate dissolution is involved, which requires a much more extensive treatment than the gettering of dissolved metal atoms. The purpose of the present study contains two aspects. First, it is to provide a formulation that will allow to quantitatively model the processes of metal precipitation, precipitate dissolution, and the gettering of metals in both atomic and precipitated forms. Second, it is to quantitatively check whether it is indeed difficult to getter precipitated metals from $\mathrm{Si}$.

\section{Formulation of the Problem}

In the present formulation, diffusion limited precipitate growth and dissolution processes are assumed. The precipitate nucleation process is negiected. Also neglected is the possibility that the precipitate growth and/or dissolution processes may be controlled by a reaction barrier. This means that, in our model, the metal precipitate growth as well as dissolution rates are the attainable upper limits. This is particularly important for the precipitate dissolution process in the sense that, for a given set of experimental conditions, the calculated dissolution rate cannot be exceeded. The precipitate dissolution rate is one dominant factor limiting the effectiveness of gettering of the precipitated metals.

The precipitate growth/dissolution rates are described by

$$
\frac{d r_{p}}{d t}=\Omega \mathrm{J}^{i} .
$$

where $r_{p}$ is the precipitate radius, $\Omega$ is the volume of a silicide molecule containing one metal atom, and $\mathrm{J}^{\mathrm{i}}$ is the metal atom flux at the precipitate-Si interface, given by

$$
J^{i}=D \frac{C^{\infty}-C^{i}}{r_{p}} .
$$

where $D$ is the metal diffusivity, $C^{\infty}$ and $C^{i}$ are respectively the metal atom concentration at the far field matrix and at the precipitate-Si interface. The quantity $C$ is given by 


$$
C^{i}=C^{e q} \exp \left(\frac{2 \Omega \sigma}{r_{p} k_{B} T}\right)
$$

where $C^{\text {eq }}$ is the thermal equilibrium metal concentration in $\mathrm{Si}$, and $\sigma$ is the precipitate-Si interfacial energy density. The matrix metal concentration change rate is given by

$$
\left(\frac{\mathrm{dC}}{\mathrm{dt}}\right)_{\mathrm{g} / \mathrm{d}}=-4 \pi \mathrm{r}_{\mathrm{p}}^{2} \rho \mathrm{J}^{\mathrm{i}}
$$

where $\rho$ is the precipitate density. Equations (1)-(4) are valid for both precipitate growth and dissolution processes. Whether the precipitate is growing or dissolving is determined by the sign of $\mathrm{J}^{\mathrm{i}}$, which is in turn determined by the relative magnitude of $C^{\infty}$ and $C i$ via Eq. (2).

To treat the gettering problem, the diffusion-segregation equation (DSE), given by [2]

$$
\frac{\partial C}{\partial t}=\frac{\partial}{\partial x}\left[D\left(\frac{\partial C}{\partial x}-\frac{C}{m} \frac{\partial m}{\partial x}\right)\right]
$$

where $m$ is the impurity segregation coefficient, is used for the dissolved metal atom cases. For cases involving precipitate dissolution, the DSE is modified to read

$$
\frac{\partial C}{\partial t}=\frac{\partial}{\partial x}\left[D\left(\frac{\partial C}{\partial x}-\frac{C}{m} \frac{\partial m}{\partial x}\right)\right]+\left(\frac{d C}{d t}\right)_{g / d},
$$

where the term $(\mathrm{dC} / \mathrm{dt})_{\mathrm{g} / \mathrm{d}}$ is given by Eq. (4) for which the value is positive due to the fact that $\mathrm{J}^{\mathrm{i}}<0$, which is in turn because in Eq. (2) now $\mathrm{C}>C^{\infty}$ holds.

\section{Simulation Results}

In the present simulations, the model system selected is that of Fe. For precipitation/precipitatedissolution studies, it is assumed that $\mathrm{Fe}$ has been introduced into a $200 \mu \mathrm{m}$ thick Si wafer at $900^{\circ} \mathrm{C}$ to the solubility limit of $\sim 4.26 \times 10^{13} \mathrm{~cm}^{-3}$. The precipitation temperature is assumed to be $700^{\circ} \mathrm{C}$, while the after-precipitation precipitate-dissolution temperature is assumed to be $900^{\circ} \mathrm{C}$. Similar conditions were assumed for the simulation of the gettering processes. The getterer is assumed to be a $2 \mu \mathrm{m}$ thick Al-Si liquid layer at the backsurface of the Si wafer and the gettering temperature is $700^{\circ} \mathrm{C}$. The Fe solubility in the Al-Si liquid layer is assumed to be 1 at\%. These simulation conditions were selected to be compatible with the use of polycrystalline solar grade $\mathrm{Si}$ substrates in solar cell fabrications. For simplicity, in this simulation study the effect the precipitate-Si interfacial energy $\sigma$ has been neglected. Simulations were carried out using the partial differential equation solver ZOMBIE [3].

Simulation results of the Fe precipitation and precipitate dissolution processes have shown that the processes take a reasonably short time to complete, i.e., to reach steady-states, for reasonably high precipitate density cases, e.g., a few min for $\rho=10^{11} \mathrm{~cm}^{-3}$. However, considerably longer times are needed for cases with lower precipitate densities.

Figure 1 shows the simulation results of gettering $\mathrm{Fe}$ by the $\mathrm{Al}-\mathrm{Si}$ liquid layer at $700^{\circ} \mathrm{C}$ for two wafers, respectively containing dissolved Fe of concentrations of $4.26 \times 10^{13}$ and $1 \times 10^{11} \mathrm{~cm}^{-3}$, corresponding to $\mathrm{Fe}$ solubility values respectively at 900 and $700^{\circ} \mathrm{C}$. It is seen that for both cases the effect of getteirng has become quite noticeable after a gettering time of $\sim 10 \mathrm{~min}$ and the gettering processes are complete after $-3 \mathrm{~h}$. Figure 2 shows the simulation results of the same gettering process carried out for a wafer which initially contain $4.26 \times 10^{13} \mathrm{~cm}^{-3}$ of Fe, but before gettering tak- 
ing place the $\mathrm{Fe}$ precipitation process has occurred at $700^{\circ} \mathrm{C}$ to completion with the precipitate density being $1 \times 10^{11} \mathrm{~cm}^{-3}$. Thus, at $700^{\circ} \mathrm{C}$, this wafer contains $10^{11} \mathrm{~cm}^{-3}$ dissolved $\mathrm{Fe}$, with $4.25 \times 10^{13} \mathrm{~cm}^{-3} \mathrm{Fe}$ existing in precipitates. It is seen from 2(a) that for a gettering time of $3 \mathrm{~h}$, for which the getteirng processes are already complete for the cases shown in Fig. 1, there is hardly any change in the dissolved Fe concentration in the Si matrix. In fact, the effect of gettering did not become readily noticeable until after a gettering time of $\sim 50 \mathrm{~h}$, while the gettering process is complete only after $\sim 60 \mathrm{~h}$. Compare to the cases shown in Fig. 1, it is apparent that it is indeed very difficult to getter the wafer containing Fe precipitates by the same process. The physical reason for this phenomenon to occur is that, for the wafer containing Fe precipitate: (i) the magnitude of the metal flux moving toward the gettering region is small; and (ii) the matrix region removed $\mathrm{Fe}$ is continuously replenished by the precipitate dissolution process, which takes a long time to complete.

The present results suggest that, in order to more effectively getter metal contaminants from the bad grain regions, it may be advantageous to use two gettering steps: one at a higher temperature and another at a lower temperature. This is because we have found that precipitate dissolution occurs fairly fast at a higher temperature, e.g., $900^{\circ} \mathrm{C}$ in the present examples.

\section{Summary}

In summary, we have carried out the formulation of the problems of modeling impurity precipitation, precipitate dissolution, and getteimg in the presence of dissolving precipitates. Our preliminary simulation results have shown that, once precipitated, it is extremely difficult to getter the impurities out from Si substrates to a gettering region. This provides a possible explanation to the fact that the low carrier diffusion length "bad" grain regions in solar grade polycrystalline Si substrates does not respond to a typical gettering treatment.

\section{References}

1. S. M. Joshi, U. M. Gösele and T. Y. Tan, "Improvement of diffusion length in single crystal Si by Al gettering", accepted for J. Appl. Phy. 77, 3858 (1995).

2. T. Y. Tan, R. Gafitsanu, and U. M. Gösele, "Diffusion-Segregation Equation and Simulation of the Diffusion-Segregation Phenomena" in Semiconductor Silicon 1994, eds. H. R. Huff, W. Bergholz, and K. Sumino (The Electrochem. Soc., Pennington, PA, 1994) p. 920.

3. W. Jüngling, P. Pichler, S. Selberherr, E. Guerrero, and H. W. Pötzl, IEEE Trans. Electron. Devices ED-32, 156 (1985).

\section{Figure Captions}

Fig. 1. Simulated Fe concentrations after gettering by $\mathrm{Al}$ at $700^{\circ} \mathrm{C}$. (a) With initial Fe concentration of $1 \times 10^{11} \mathrm{~cm}^{-3}$, which is $\mathrm{C}^{\mathrm{eq}}\left(700^{\circ} \mathrm{C}\right)$. (b) With initial Fe concentration of $4.26 \times 10^{13} \mathrm{~cm}^{-3}$, quenched-in from $900^{\circ} \mathrm{C}$ (which is $\mathrm{C}^{\mathrm{eq}}\left(900^{\circ} \mathrm{C}\right.$ )).

Fig. 2. (a) Simulated Fe concentrations after gettering treatment by $\mathrm{Al}$ at $700^{\circ} \mathrm{C}$ for the case of a wafer containing $\mathrm{Fe}$ of $4.26 \times 10^{13} \mathrm{~cm}^{-3}$, precipitated to completion at $700^{\circ} \mathrm{C}$. At the gettering temperature the $\mathrm{Fe}$ concentration is that of $\mathrm{C}^{\mathrm{eq}}\left(700^{\circ} \mathrm{C}\right)$, i.e., $1 \times 10^{11} \mathrm{~cm}^{-3}$, while the rest of $\mathrm{Fe}$ are in precipitated form. Noticed that the gettering process is very ineffective compared to those shown in Fig. 1. (b) Simulated results of the corresponding precipitate radii during gettering. The precipitate density is assumed to be $1 \times 10^{11}$ $\mathrm{cm}^{-3}$. The precipitate radius of $2 \AA$ represents dissolved $\mathrm{Fe}$ atoms. 

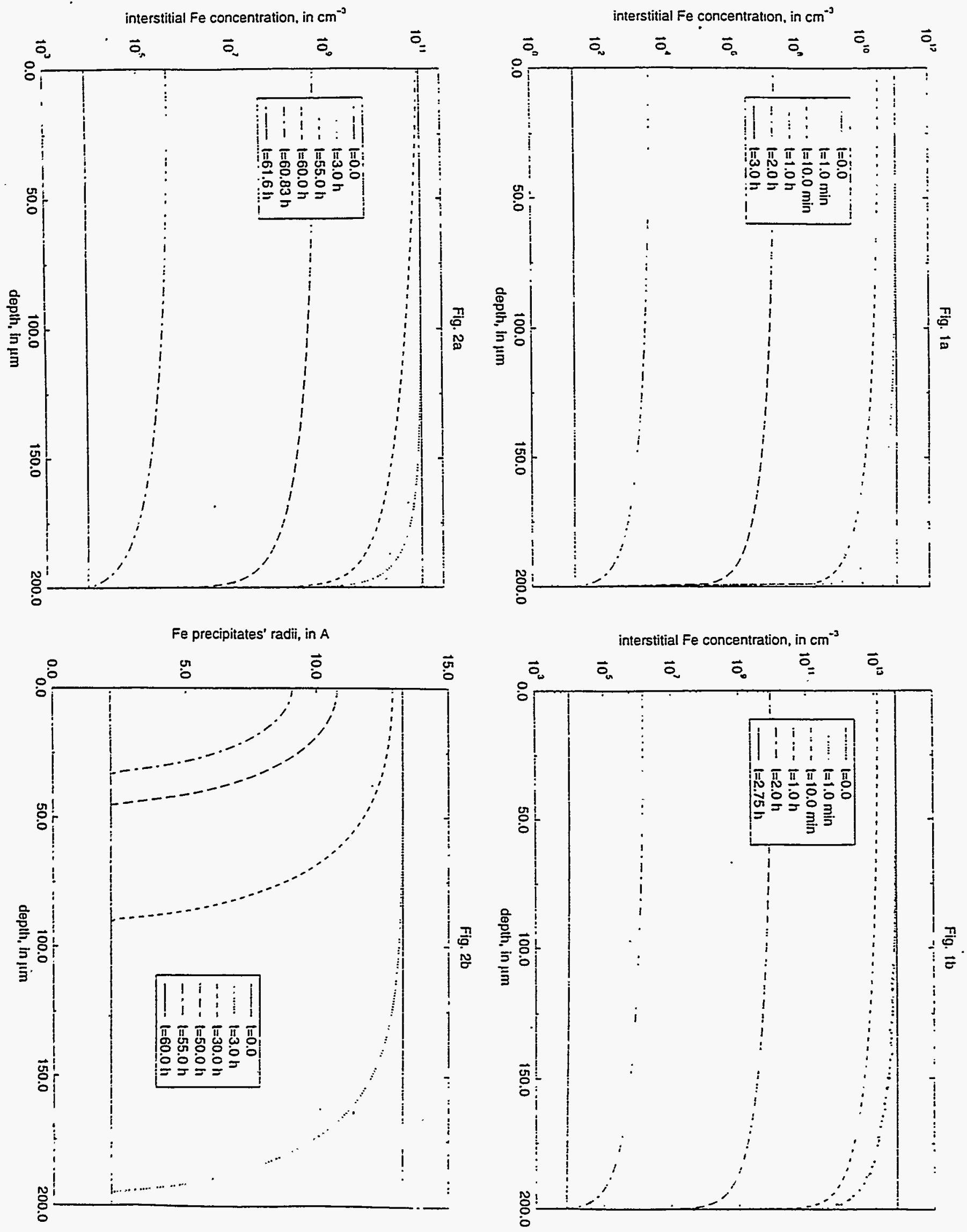
Organization:

Contributors:

\author{
School of Electrical and Computer Engineering \\ Georgia Institute of Technology, Atlanta, Georgia 30332
}

Objective

The objective of this program is to investigate quality enhancement techniques, such as $\mathrm{PECVD} \mathrm{SiO}_{2} / \mathrm{SiN}$ deposition for defect passivation, phosphorus and aluminum treatments for defect and impurity gettering, and integration of gettering and passivation techniques to achieve high-efficiency multicrystalline silicon (mc-Si) cells.

\section{Understanding and Optimization of PECVD Process for Bulk and Surface Passivation}

Surface and bulk defect passivation by hydrogenation also plays an important role in achieving high efficiency PECVD-coated silicon cells. We have shown elsewhere [1] that a $\mathrm{PECVD} \mathrm{SiO}_{2} / \mathrm{SiN}$ double layer AR coating annealed at $350^{\circ} \mathrm{C}$ in an RTP system, increases the bulk lifetime by a factor of 1.1 to 1.8 , depending on the multicrystalline material. In order to investigate the impact of this post-PECVD rapid thermal anneal (RTA) on cell performance, cells were fabricated on EFG mc-Si with PECVD coatings. The RTA conditions were: (1) $350^{\circ} \mathrm{C} / 20$ min., (2) $500^{\circ} \mathrm{C} / 5 \mathrm{~min}$., and (3) $650^{\circ} \mathrm{C} / 1.5 \mathrm{~min}$. The anneal time was reduced for high temperature anneals to prevent or retard contact shunting. EFG material was selected because it responds dramatically to hydrogenation. Fig. la shows that the initial $350^{\circ} \mathrm{C} / 20 \mathrm{~min}$. post-PECVD RTA improves the short and long wavelength response. Subsequent higher temperature anneals $\left(>400^{\circ} \mathrm{C}\right)$ continue to increase the long wavelength response, but this comes at the expense of a decrease in short wavelength response (fig. lb).

Fig. 2 shows the emitter reverse saturation current density $\left(\mathrm{J}_{\alpha c}\right)$ as a function of RTA temperature. $J_{\infty c}$ was measured using the PCD technique [2] on high-resistivity Si samples diffused and passivated (both sides) in the same way as the EFG cells. The etched-back, emitters had a sheet resistance of 100-140 $\Omega / s q$. with a $\sim 10 \mathrm{~nm}$ thick oxide grown prior to the $\mathrm{SiN} / \mathrm{SiO}_{2}$ depositions. The sharp drop in $\mathrm{J}_{\mathrm{oe}}$ after the initial $350^{\circ} \mathrm{C}$ anneal indicates that the significant increase in short wavelength response is due to improved surface passivation. However, since $J_{o e}$ does not increase by subsequent high temperature anneals, the decrease in short wavelength response cannot be attributed to degradation in surface passivation. The decrease in the short wavelength response at higher annealing temperatures is explained on the basis of increased refractive index (fig. 2) accompanied by increased absorption in the SiN film. Independent spectral ellipsometry measurements confirm that the extinction coefficient increases significantly in the short wavelength $(<0.55 \mu \mathrm{m})$ with the increase in SiN refractive index.

The enhanced long wavelength response is attributed to bulk defect passivation by hydrogen trapped in the as-deposited SiN film. This hypothesis was confirmed by the observation that the EFG cells without a SiN coating did not show any appreciable increase in cell performance after undergoing the same RTAs. In addition, cells made on materials that do not respond to hydrogen passivation, such as HEM mc-Si, actually showed a decrease in cell performance due to the loss in the short wavelength response and the effects of shunting which become apparent at RTA temperatures above $500^{\circ} \mathrm{C}$. Similarly, it is important to recognize that not all $1 \mathrm{~cm}^{2}$ EFG cells, show enhanced long wavelength response by RTA. This is not surprising given the multicrystalline nature of this material which leads to inherent non-uniformity in the bulk. In this experiment, $\sim 40 \%$ of the EFG cells did not exhibit evidence of hydrogenation. Thus, the bulk passivation effect the post-PECVD RTA is material and site specific.

To further examine the cells which did respond favorably to the PECVD/RTA treatment, FTIR and EBIC characterization was performed to explain the enhanced long wavelength response of certain EFG cells. FTIR measurements revealed a consistent decrease in the $\mathrm{Si}-\mathrm{H}$ bond concentration in the SiN film with increasing RTA temp., supporting-but not rigorously establishing-hydrogenation of bulk defects. Therefore, EBIC characterization was performed to investigate the electrical activity of the defects after PECVD/RTA. EBIC measurements (fig. 3) following the $\mathrm{PECVD} / 350^{\circ} \mathrm{C}$ RTA procedure confirmed significant improvements in the bulk quality especially in the intragrain response. 


\section{Effect of Oxygen Content on Recombination and PECVD Passivation of Extended Defects in mc-Si}

Electron Beam Induced Current (EBIC) was used to investigate the effects of oxygen on extended defect recombination. Two EFG samples with high and low oxygen concentrations were examined by EBIC and EBIC maps were converted to diffusion length maps. Since the intragrain diffusion length is controlled by the high dislocation density, the EFG material showed an increase in bulk diffusion length when moderate amounts of oxygen are present (compare figs. 4 and 5). Furthermore, when a grain boundary surface recombination velocity (GB-SRV) is determined as a function of injection level (see fig. 6), the low oxygen samples show a strong injection level dependence where as the high oxygen sample shows almost no injection level dependence. This same trend was observed in the bulk diffusion length, which are dominated by dislocations. The combination of these two observations, lower GB-SRV for higher oxygen and less injection level dependence for the higher oxygen sample, indicates that the density of interface states profiles of the higher oxygen samples contain less midgap states than does the low oxygen samples. PECVD passivation showed less improvement on oxygen rich samples because of lower density of midgap states.

\section{Aluminum and Phosphorus Gettering of HEM mc-Si}

To understand the effects of process-induced gettering on HEM mc-Si material, a sample lot with a resistivity of $0.65 \Omega-\mathrm{cm}$ was analyzed. The average as-grown lifetime in this lot was found to be $12 \mu \mathrm{s}$. Samples from the same wafer (or from wafers which were found consecutively in the ingot) were cleaned and then subjected to 30 minute $\mathrm{POCl}_{3}$ diffusions in the temperature range from $850^{\circ} \mathrm{C}$ to $950^{\circ} \mathrm{C}$. After the diffusions, the emitters were etched from the samples, and their bulk lifetime determined from photoconductive decay (PCD) analysis. As fig. 7 reveals, an optimum gettering temperature in the vicinity of $900^{\circ} \mathrm{C}$ was determined for the $0.65 \Omega$-cm HEM material, and samples diffused at higher temperatures showed a decrease in bulk lifetime.

Preliminary results from aluminum gettering experiments show that this process does not improve the bulk lifetimes in HEM mc-Si beyond the high levels achieved by effective phosphorus gettering. In fact, at times aluminum gettering tends to lower the high lifetimes achieved by effective phosphorus gettering. More experiments dealing with aluminum gettering and forming gas anneals are presently underway to increase the performance of HEM solar cells further.

\section{Fabrication of High-Efficiency HEM mc-Si Solar Cells}

Solar cells were fabricated on two different lots of HEM material. Lot 1 had an average bulk resistivity of $1.3 \Omega-\mathrm{cm}$ and an average as-grown bulk lifetime of $1.4 \mathrm{~ms}$. The same parameters for Lot 2 were $0.80 \Omega$-cm and 6.3 ms. Table 1 shows the performance of typical solar cells made from these two lots.

The process sequence for these cells involved a 40 minute phosphorus diffusion at $930^{\circ} \mathrm{C}$ followed by a 30 minute Al back surface field (BSF) drive at $850^{\circ} \mathrm{C}$. The final step was a 30 minute forming gas anneal at $400^{\circ} \mathrm{C}$.

Table 1 - Cell Data from two different lots of HEM mc-Si

\begin{tabular}{|c|c|c|c|c|c|c|c|}
\hline $\begin{array}{c}\text { Cell } \\
\text { ID }\end{array}$ & $\begin{array}{c}\text { Res. } \\
(\Omega-\mathrm{cm})\end{array}$ & $\begin{array}{c}\text { Voc } \\
(\mathrm{mV})\end{array}$ & $\begin{array}{c}\text { Jsc } \\
\left(\mathrm{mA} / \mathrm{cm}^{2}\right)\end{array}$ & $\begin{array}{c}\text { Eff. } \\
(\%)\end{array}$ & $\begin{array}{c}\text { Leff } \\
(\mathrm{mm})\end{array}$ & $\begin{array}{c}\text { As-grown } \\
\mathrm{t}_{\mathrm{b}}(\mathrm{us})\end{array}$ & $\begin{array}{c}\text { Processed } \\
\mathrm{t}_{\mathrm{b}}(\mathrm{us})\end{array}$ \\
\hline Cell 15 & 1.30 & 610 & 35.1 & 17.1 & 270 & 1.4 & 200 \\
\hline Cell 16 & 0.80 & 627 & 34.1 & 17.3 & 275 & 6.3 & 30 \\
\hline
\end{tabular}

The gettering caused by the individual process steps was investigated by a combination of internal quantum efficiency (IQE) and PCD measurements. IQE data was acquired after cell fabrication. As shown in Table 1, the bulk lifetime in the $1.30 \Omega-\mathrm{cm}$ material increased from $1.4 \mathrm{~ms}$ to $200 \mathrm{~ms}$, and the lifetime in the $0.80 \Omega$-cm material rose from $6.3 \mathrm{~ms}$ to $30 \mathrm{~ms}$. Table 1 also shows that the cell made from the $0.80 \Omega$-cm material with bulk lifetime of $30 \mathrm{~ms}$ gave approximately the same efficiency as the cell made from the $1.3 \Omega$-cm material with bulk lifetime of $200 \mathrm{~ms}$. This suggests that the bulk lifetime is not the limiting factor for these HEM mc-Si solar cells in the efficiency 
range of $17.0 \%-18.0 \%$. Instead, the design of the cell in general tends to cap the efficiencies that are achieved, as discussed below.

In order to determine the effects of cell design on limiting the device performance, the $17.1 \%$ and $17.3 \%$ solar cells listed in Table 1 were modeled using PC-1D. The bulk lifetimes listed in Table 1 were used as the basis for the model calculations. In both cases, the $L_{d}$ values were at least as long as the thickness of the devices. Hence, matching the model simulations to the cell parameters of Table 1 required accurate selection of $S_{b}$. The modeling revealed that Cell 15 from the $1.3 \Omega$-cm wafer has an $S_{b}$ of approximately $6500 \mathrm{~cm} / \mathrm{s}$, and Cell 6 from the $0.80 \Omega-\mathrm{cm}$ wafer has an $\mathrm{S}_{\mathrm{b}}$ of approximately $4000 \mathrm{~cm} / \mathrm{s}$. After matching the cell data through modeling, the effects of bulk lifetime and $S_{b}$ on the performance of the devices were analyzed by extending the model calculations. These calculations in fig. 8 support the finding that an $S_{b}$ value of $4000 \mathrm{~cm} / \mathrm{s}$ together with a $30 \mu \mathrm{s}$ bulk lifetime in the case of the $0.8 \Omega$-cm material is sufficient to produce cells with efficiencies above $17.0 \%$. Fig. 8 also reveals that the 1.3 $\Omega-\mathrm{cm}$ material requires only a $75 \mu \mathrm{s}$ bulk lifetime (instead of the measured lifetime of $200 \mu \mathrm{s}$ ) to reach the $17: 0 \%$ mark. Again, this point corroborates the claim that the performance of this HEM mc-Si cell is limited by $\mathrm{S}_{b}$ and not the bulk lifetime which results after completion of the fabrication process. More recently, a combination of effective gettering and improved cell design has resulted in a record high HEM mc-Si solar cell efficiency of $18.2 \%$ on 0.65 $\Omega-\mathrm{cm}$ material. The light $\mathrm{I}-\mathrm{V}$ data for this cell shows a $\mathrm{V}_{\mathrm{oc}}$ of $636 \mathrm{mV}, \mathrm{a} \mathrm{J} \mathrm{J}_{\mathrm{sc}}$ of $35.5 \mathrm{~mA} / \mathrm{cm}^{2}$, and a fill factor of 0.808 . These results were officially tested at NREL.

The authors would like to thank Dr. G. Jellison and Oak Ridge National Laboratories for spectral ellipsometry measurements.

\section{References}

[1] Z. Chen, A. Rohatgi, D. Ruby, in 24th IEEE PVSC, Waikoloa, Hawaii, 1994, p. 1331.

[2] D. E. Kane, R. M. Swanson, in I8th IEEE PVSC, Las Vegas, 1985, p. 578.

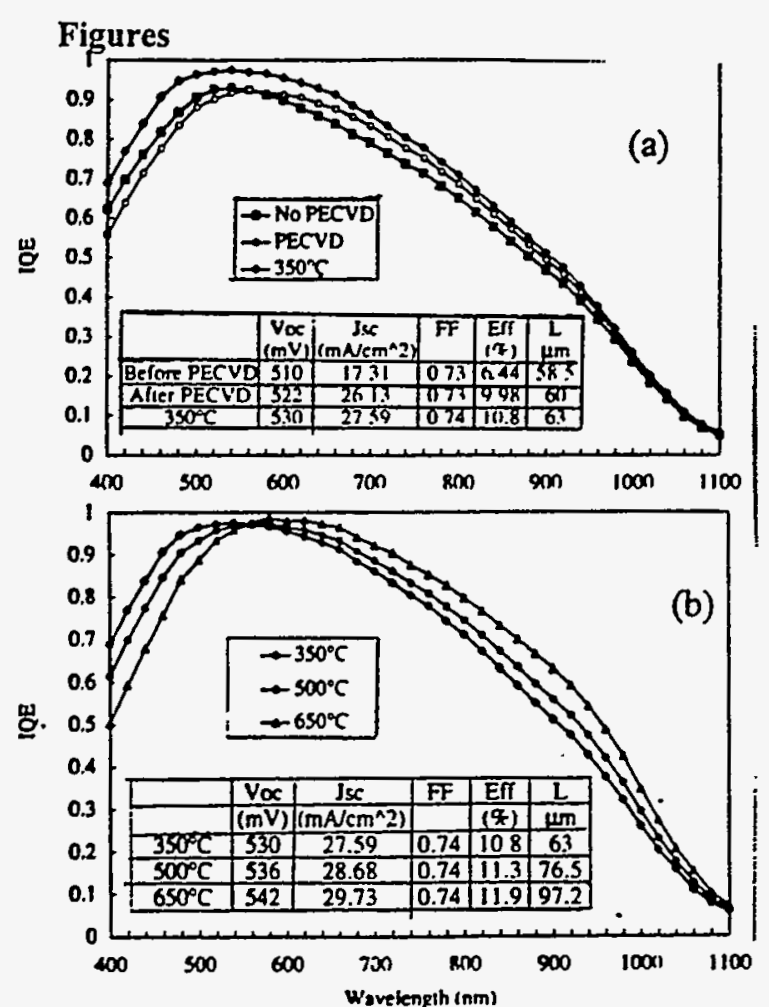

Fig 1. Effect of post-PISCVD RTA cn EFG cell performance \& IQE: (a) Low-terop, RTA improved shor and $\log g \lambda$ response as well as cell performance.

(b) High-temp. RTA significantiy enhanced long $\lambda$ and cell performance, but at the expense of short $\lambda$ response.

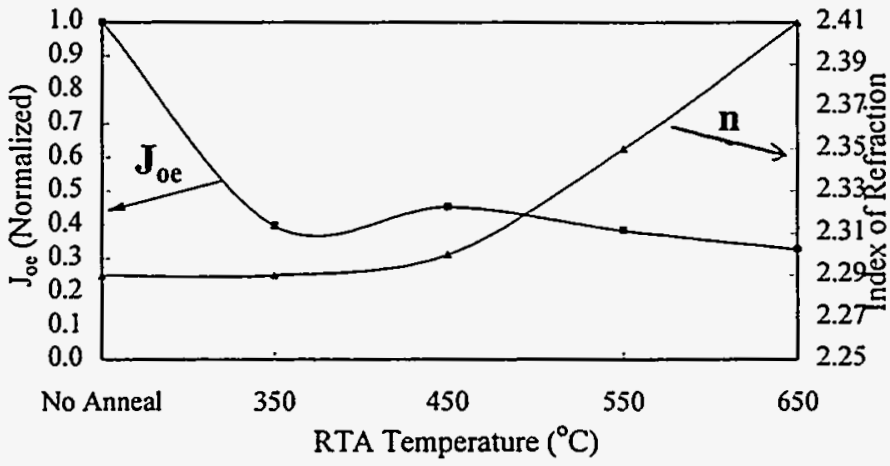

Fig. 2. Joe and SiN refractive index as a function of post-PECVD RTA temperature indicating improved surface passivation but increased index accompanied by increased parasitic absorption in the film.

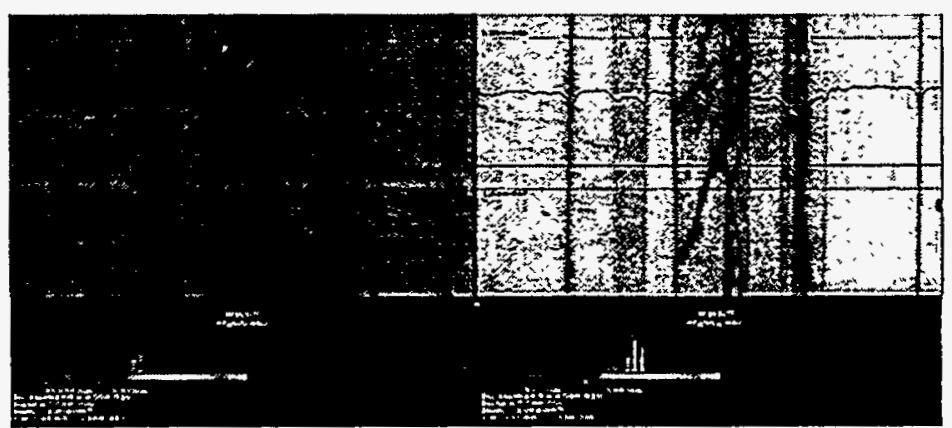

Fig. 3. EBIC image of an EFG sample before (left) and after (right) the RTA at $350^{\circ} \mathrm{C} / 20 \mathrm{~min}$. Lighter regions represent greater EBIC response. 


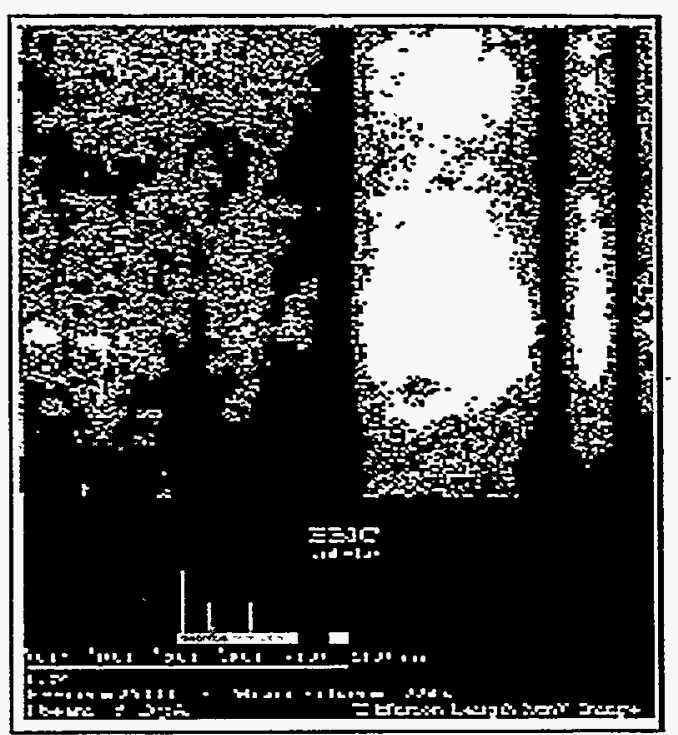

Fig. 4. Diffusion Length image of low oxygen EFG material at $54.3 \mathrm{pA}$ injection level.

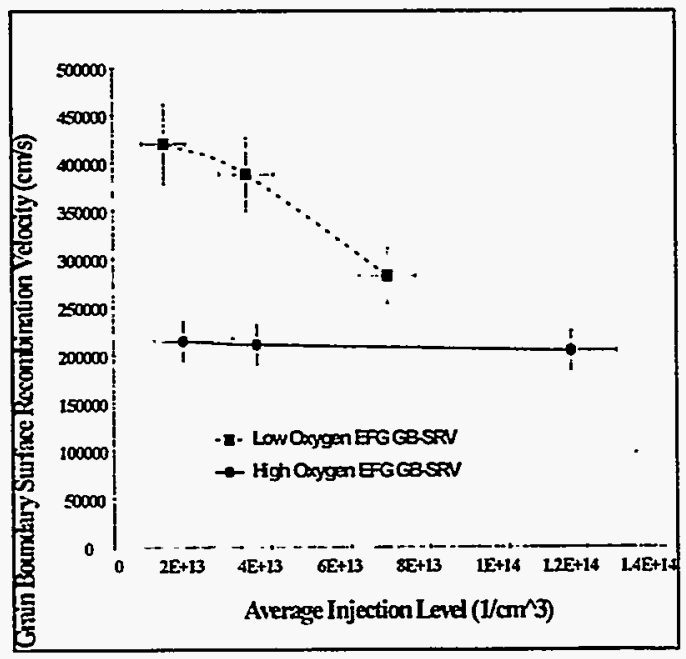

Fig. 6. GB-SRV versus injection level for low and high oxygen EFG material.

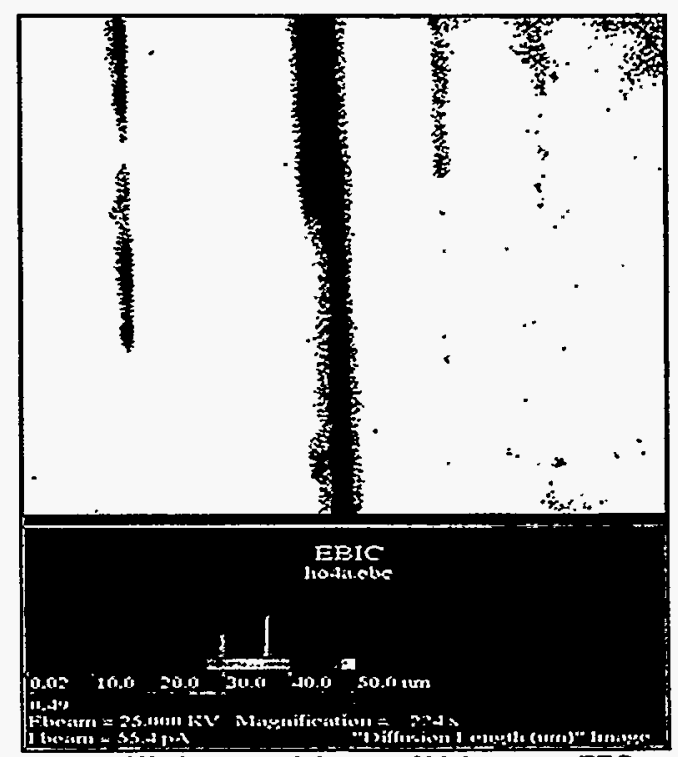

Fig. 5. Diffusion Length image of high oxygen EFG material at $55.4 \mathrm{pA}$ injection level.

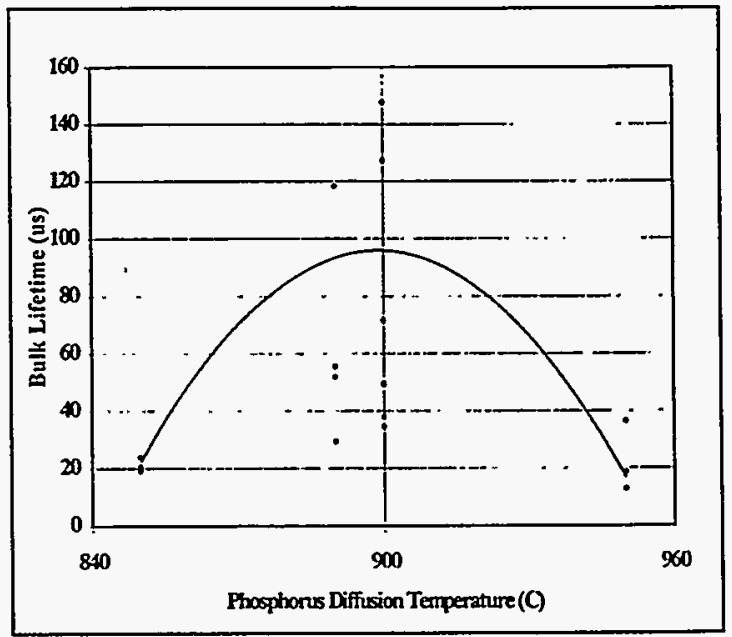

Fig.7. The effects of phosphorus gettcring on IIEM mc-Si material for various temperatures between $850 \mathrm{C}$ and $950 \mathrm{C}$.

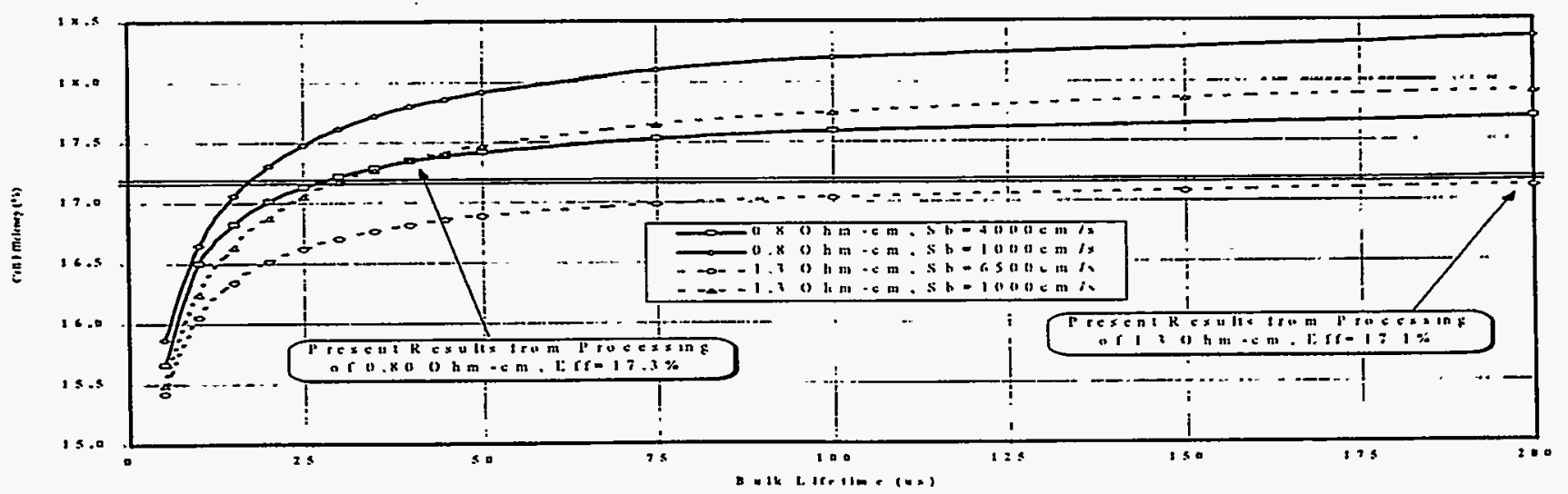

Fig. 8. Model calculations which show the effects of bulk lifetime and Sb on cell efficiency for base resistivities of $1.3 \mathrm{Ohm}-\mathrm{cm}$ and $0.8 \mathrm{Ohm}-\mathrm{cm}$. 
Title: $\quad$ Solar Energy Conversion at Dye Sensitized Nanostructured Electrodes Fabricated by Sol-Gel Processing

Organization: The Johns Hopkins University, Baltimore, MD 21218

Contributors: 'P. C. Searson and G. J. Meyer, Principle Investigators

F. Cao, T.A. Heimer, J.M. Stipkala, L. Friedman, G. Oskam.

\section{Objectives}

In this program we are exploring the use of nanostructured materials as substrates for dyemediated light-to-electricity conversion in a photoelectrochemical cell. The objective of current work is to develop new materials, electrolytes, and molecular sensitizers which increase solar energy efficiency and stability. Materials processing and synthesis is carried out in conjunction with characterization of cell performance, detailed investigation of operating mechanisms, and modeling of charge transfer and transport in these systems.

\section{Results}

We recently organized a symposium on nanostructured materials in electrochemistry. The symposium took place at the 187th Meeting of the Electrochemical Society in Reno Nevada. Valuable presentations and discussions of the photoelectrochemical properties of materials related to this program were presented and a proceedings volume was recently published. ${ }^{1}$

The optical and electrochemical properties of nanocrystalline $\mathrm{TiO}_{2}$ electrodes prepared from commercially available DeGussa material have been explored by cyclic voltammetry, UV/Vis/NIR attenuance, EPR, photocurrent spectroscopy, and electrochemical impedance measurements. At negative applied potentials the $\mathrm{TiO}_{2}$ films turn black and an EPR spectrum appears consistent with the formation of $\mathrm{Ti}$ (III) states. The electrochemical data is well described by a model in which the reduction of $\mathrm{TiO}_{2}$ leads to band edge unpinning. ${ }^{2}$

The previously unknown ligand, bpy-acac shown below, and $\mathrm{Ru}$ (II) sensitizers

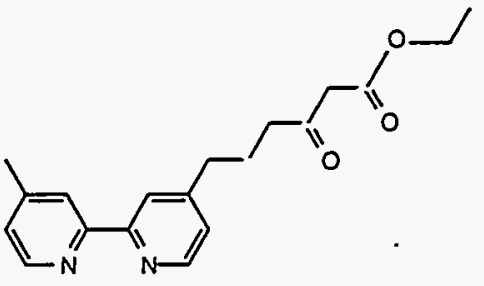

derived from it were recently prepared in our laboratory. This ligand was designed with a acetyl acetonate (acac) derivative which is known to chelate hard transition metals such as $\mathrm{Ti}(\mathrm{IV})$. We find that sensitizers based on this ligand are as efficient as those based on (dcbH) $=\left(4,4^{\prime}-(\mathrm{COOH})_{2}-2,2^{\prime}\right.$-bipyridine $)$. This indicates that intimate electronic coupling between the 
surface binding group and the bpy ring is not required for efficient sensitization. The enhanced stability which may result from the chelate effect is currently under study.

Cis- $\mathrm{Ru}(\mathrm{dcbH})_{2}(\mathrm{CN})_{2}$ efficiently converts photons to electrons when anchored to nanocrystalline $\mathrm{TiO}_{2}$ in a regenerative photoelectrochemical cell while the analogous Os(II) sensitizer does not. ${ }^{3}$ Time resolved diffuse reflectance measurements allow us to directly measure the recombination rate of electrons in the solid with the oxidized sensitizer. The data observed is modeled by a single exponential process and the recovered kinetics are $-10^{6} \mathrm{~s}^{-1}$ in the absence of iodide in a variety of organic solvents. Interestingly, recombination kinetics to the Os-based sensitizer were consistently twice as fast as those for the Ru sensitizer despite a $300 \mathrm{mV}$ smaller driving force. The key observation was that the recombination kinetics for the Os sensitizer were not effected by addition of $0.5 \mathrm{M} \mathrm{I}^{-}$indicating that a slow halide oxidation rate is responsible for the low photocurrent efficiency observed for this sensitizer. For surface attached $\mathrm{Ru}(\mathrm{dcbH})_{2}(\mathrm{CN})_{2}$ an iodide oxidation rate of $4 \times 10^{7} \mathrm{M}^{-1} \mathrm{~s}^{-1}$ was measured.

Photo-induced charge separation processes across nanocrystalline $\mathrm{TiO}_{2}$ interfaces was explored with two sensitizers: $\mathrm{Ru}\left(4,4^{\prime}-\left(\mathrm{CO}_{2}-\right)_{2}-2,2^{\prime} \text {-bipyridine }\right)_{2}\left(4-\mathrm{CH}_{3}, 4^{\prime}-\mathrm{CH}_{2}-\mathrm{PTZ},-2.2^{\prime}\right.$

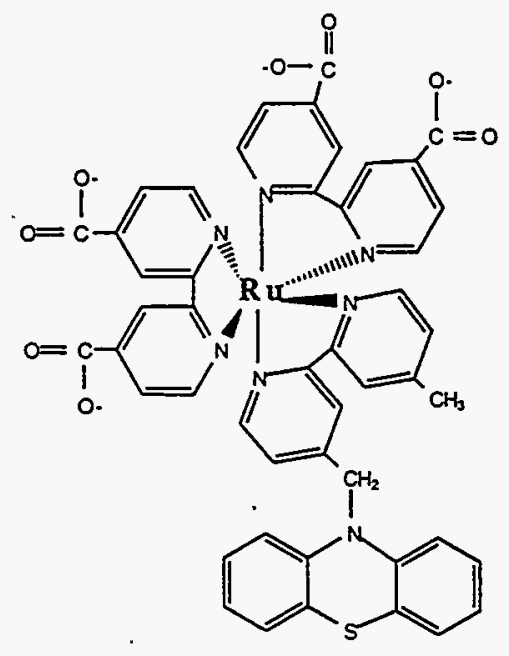

-bipyridine)], (Ru(dcb) ${ }_{2}\left(\right.$ bpy-PTZ) ${ }^{2-}$ shown above where $\mathrm{PTZ}$ is phenothiazine) and $\left[\mathrm{Ru}\left(4,4^{\prime}-\left(\mathrm{CO}_{2}-\right)_{2}-2,2^{\prime} \text {-bipyridine }\right)_{2}\left(4,4^{\prime}-\left(\mathrm{CH}_{3}\right)_{2}-2,2^{\prime}\right.\right.$-bipyridine $\left.)\right],\left(\mathrm{Ru}(\mathrm{dcb})_{2}(\mathrm{dmb})^{2-}\right) .^{5}$ Excitation of surface attached $\mathrm{Ru}(\mathrm{dcb})_{2}(\mathrm{bpy}-\mathrm{PTZ})^{2-}$, leads to rapid intramolecular electron transfer from PTZ to the ruthenium metal center efficiently translates the hole away from the chromophoric unit to the pendant PTZ group. The net result is the formation of a long-lived charge separated pair which lives for $\sim 300$ microseconds. For $\mathrm{Ru}(\mathrm{dcb})_{2}(\mathrm{dmb})^{2-}$, the chargeseparated pair lives for $\sim 0.1$ microsecond. The increased lifetime directly results in an increased open circuit photovoltage for $\mathrm{Ru}(\mathrm{dcb})_{2}$ (bpy-PTZ) ${ }^{2-}$ in quantitative agreement with a model developed for solid state photovoltaics. 
Sensitized nanostructured $\mathrm{TiO}_{2}$ films give rise to remarkably efficient photocurrents in the visible range. Since the band bending in the film is very small, due to the small particle size, the charge separation must be kinetically determined and the carriers have to diffuse to the back contact to be collected. Lindquist and co-workers proposed a theoretical model for the carrier transport in microporous semiconductor films under steady state conditions assuming that carrier transport occurs through diffusion. While this model is consistent with the photocurrent action spectrum and the current-voltage curves for sensitized photoelectrochemical cells, the steady state experiments may not reveal all the details of the carrier transport mechanism.

We have studied photocurrent transients of dye sensitized $\mathrm{TiO}_{2}$ photoelectrochemical cells under monochromatic illumination. ${ }^{6}$ The photocurrent rise is exponential and dominated by a single time constant at short times (up to $\tau$ ). The time constant is inversely proportional to illumination intensity, as shown in Figure 1. We have also used Intensity Modulated Photocurrent Spectroscopy (IMPS) to study the non-steady state behavior of the cells. In this technique, the frequency at the maximum imaginary quantum efficiency corresponds to the time constant. The time constants for the $\mathrm{TiO}_{2}$ cells obtained from this technique are generally slightly smaller than that measured with conventional transient technique. ${ }^{7}$

To model the transient behavior of the cell we have assumed that electron transport in the film is diffusion limited and that the diffusion coefficient is independent of concentration, following Lindquist's approach. We have solved the time dependent diffusion equation for the electrons in the porous film. Although the solution to this equation is consistent with the characteristic features of the photocurrent rise, as shown in Figure 2, the dependence of the time constant on light intensity cannot be explained. In the simple diffusion model, in order for the time constant to decrease with increasing light intensity, the diffusion coefficient has to increase with light intensity. This could be due to the diffusional barrier at the $\mathrm{TiO}_{2}$ particle contact which will decrease at higher electron injection rates. The impurity induced trapping and detrapping mechanism in the film will also complicate the diffusional process. The difference of time constants obtained from the transient experiments and IMPS also imply the carrier concentration dependence of the diffusional coefficient. We have made some progress in modifying the model to account for the intensity dependance of the time constant.

\section{References:}

1. Meyer G.J.; Searson, P.C. Eds. "Nanostructured Materials in Electrochemistry", Electrochemical Society, Pennington, NJ (1995).

2. Cao, F.; Oskam, H.; Searson, P.C.; Stipkala, J.M.; Heimer, T.A.; Farzhad, F.; Meyer, G.J. "The Electronic and Optical Properties of Nanostructured $\mathrm{TiO}_{2}$ Films" J. Phys Chem. $1995,99,11974$. .

3. a) Heimer, T.A.; Meyer, G.J. "Molecular Level Photovoltaics: The Electro-Optical Properties of Metal Cyanide Complexes Ancored to Titanium Dioxide" J. Phys. Chem. 1993, 97, 11987. b) Argazzi, R.A.; Bignozzi, C.A.: Heimer, T.A.; Castellano, P.N.: 
Meyer, G.J. "Enhanced Spectral Sensitivity from Ru(II) Modified Photovoltaic Devices" Inorg. Chem. 1994, 33, 5741.

4. a) Heimer, T.A.; Meyer, G.J. "Efficient Ruthenium Diimine Modified Nanocrystalline $\mathrm{TiO}_{2}$ Photoanodes" Proc. Electrochem. Soc. 1995, 121, 141. b) Heimer, T.A.; Meyer, G.J. "Electron Injection Rates in Sensitized Nanostructured $\mathrm{TiO}_{2}$ Photovoltaic Devices" Proc. Electrochem. Soc. 1995, 121, 312.

5. Argazzi, R.A.; Bignozzi, C.A.; Heimer, T.A.; Castellano, P.N.; Meyer, G.J. "Long-Lived Charge Separation Across Nanostructured $\mathrm{TiO}_{2}$ Interfaces" J. Am. Chem. Soc. 1995, $.117,11815$.

6. a) Cao, F.; Oskam, G.; Searson, P.C. "A Solid State, Dye Sensitized Photoelectrochemical Cell", J. Phys. Chem., 1995, 99, 17071. b) Cao, F.; Oskam, G.; Searson, P.C. "A Quasi Solid State, Dye Sensitized $\mathrm{TiO}_{2}$ Photoelectrochemical Cell", Proc. Electrochem. Soc. 1995, 121, 180.

7. Oskam, G.; Cao, F.; Searson, P.C. "Charge Transport in Nanostructured $\mathrm{TiO}_{2}$ Films", Proc. Electrochem. Soc. 1995, 121, 98.

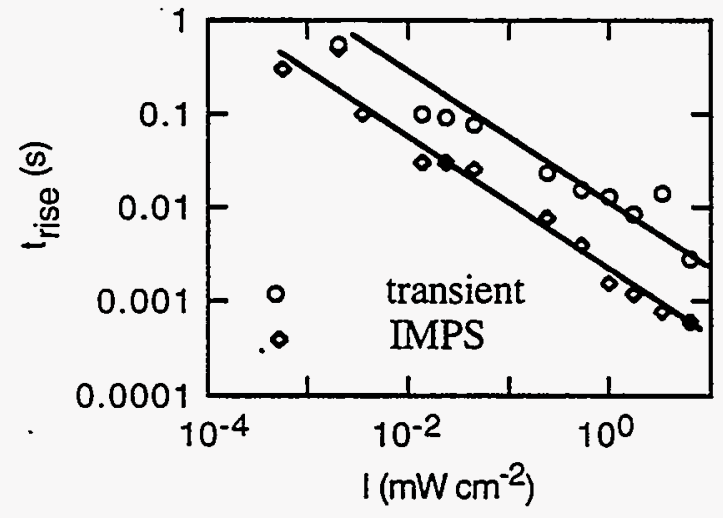

Figure 1. Rise times of a cell under monochromatic illumination $(528 \mathrm{~nm})$ from transient and IMPS measurements.

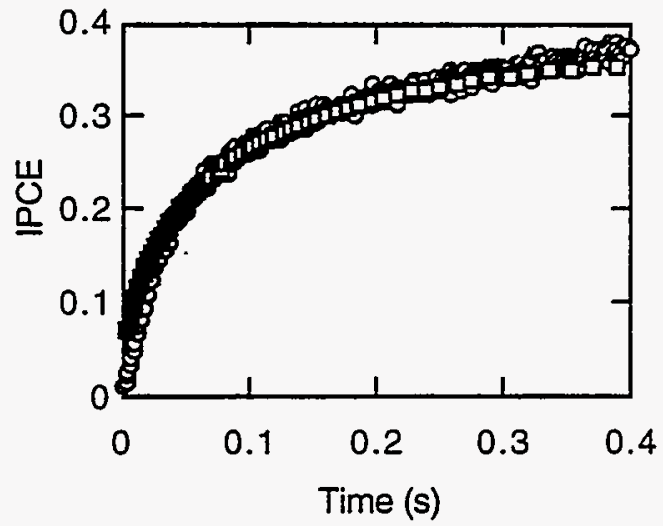

Figure 2. Incident Photon to Current Efficiency (IPCE) of the cell as function of time under monochromatic light $(528 \mathrm{~nm})$ intensity of $0.17 \mathrm{~mW} \mathrm{~cm}^{-2}$. Simulation parameters ( ): film thickness $\mathrm{d}=10 \mu \mathrm{m}$, absorbance length $\alpha=3 \mu \mathrm{m}$; diffusion coefficient $D=10^{-7} \mathrm{~cm}^{2} \mathrm{~s}^{-1}$; average electron life time, $\tau=0.33 \mathrm{~s}$.) 
Title:

The Role of Point Defects and Impurities in the Processing Performance of Crystalline Silicon Solar Cells

Organization: Massachusetts Institute of Technology

Contributors: L. C. Kimerling, Principal Investigator; J. Michel, L. Chalfoun, G. Norga, S. Zhao

\section{Introduction}

Our purpose is to develop and demonstrate processes that will enhance the yield and performance of crystalline silicon solar cells by the discovery of critical factors in defect control. Studies of wafer handling and Al backside contact formation have yielded 1) a predictive model for contamination during etching to guide specification of chemical purity; 2) an in-situ monitor of process and materials quality; 3) a model for gettering efficiency of transition metal contaminants by $\mathrm{Al}$ contact formation; 4) determination of the highest diffusion length process window (time, temperature, lifetime, interface morphology) for backside Al contact formation; and 5) theory and experiment to explain lifetime instabilities in iron contaminated cells.

\section{Experimental Results}

\section{Baseline Materials Handling and Defect Analysis}

The objective is evaluation of starting material and materials handling, and correlation with cell performance. The key technical issues are identification of persistent lifetime killers, and development of a methodology for monitor control of materials quality during processing. The materials metric for cell performance is minority carrier lifetime. The philosophy is to monitor lifetime for control and to use spectroscopic diagnostics only when the process is out of control.

We used our Radio Frequency Photoconductance Decay (RFPCD) tool as an in-situ contamination monitor. Research focused on etching and wafer handling steps to develop contamination resistant processes. Our paper entitled "Metal Removal from Silicon Surfaces in Wet Chemical Systems" won the Best Student Paper Award from the Journal of Electronic Materials. The paper presents complete thermodynamic and kinetic models for metal adsorption and desorption for silicon in a variety of standard cleaning solutions. The model predicts the dominant reactions based on known chemical potentials, and provides guidelines for process design with engineering phase diagrams.

We have enhanced our RFPCD monitor to provide single wafer mapping using a commercial light emitting diode. The method has the unique advantage of providing a noninvasive evaluation of material quality and no measurement overhead when integrated with the wafer transport mechanism. Our approach is summarized in the paper entitled "Microdefect Analysis of Silicon: Tools and Strategies."

We have characterized the stability of silicon surfaces following cleaning in HF solutions. Wafers in a $\mathrm{N}_{2}$ ambient maintain a clean, chemically inert surface. Exposure to trace amounts of $\mathrm{O}_{2}$ degrades the surface passivation immediately. RFPCD allows detection of oxygen chemisorption at $<1 \mathrm{ppm}$ monolayer. A two step degradation process is observed, which consists of rapid attack of defect sites by oxygen followed by a slower chemisorption process. An activation barrier of $1.2 \mathrm{eV}$ was estimated for transformation of $\mathrm{Si}-\mathrm{H}$ to Si-O. The sticking coefficient for oxygen on a hydrogenated silicon surface at 300 $\mathrm{K}$ was determined to be $3.5 \times 10^{-18}$. 
We have examined, in detail, metal contamination from HF solutions by RFPCD, $x$-ray fluorescence (TXRF) and atomic force microscopy (AFM) (see Figure 1). Copper deposits on silicon by electrochemical outplating as $\mathrm{Cu}$ is reduced from solution while $\mathrm{Si}$ is oxidized (etched). Metal adsorption is detected as an increase in the surface recombination velocity, $\mathrm{S}$. For $[\mathrm{Cu}]<10^{10} \mathrm{~cm}^{-2} \mathrm{~S}$ changes in inverse proportion to the adsorbed $\mathrm{Cu}$ concentration with the constant of proportionality representing the minority carrier capture cross section: $\sigma_{\mathrm{n}}(\mathrm{Cu})=5 \times 10^{-17} \mathrm{~cm}^{2}$ and $\sigma_{\mathrm{p}}(\mathrm{Cu})=8 \times 10^{-17} \mathrm{~cm}^{2}$. AFM measurements show nucleation of metal islands at $[\mathrm{Cu}]>10^{11} \mathrm{~cm}^{-2}$. The islands form by surface diffusion, and the dependence of $\mathrm{S}$ on [Cu] is reduced. For $\mathrm{HF}$ solutions, $\mathrm{Au}, \mathrm{Ag}$ and $\mathrm{Cu}$ are likely contaminants; for basic peroxide solutions, $\mathrm{Al}, \mathrm{Fe}, \mathrm{Zn}, \mathrm{Ni}$, and $\mathrm{Cu}$ are likely contaminants.

\section{Defect Reactions in Procéssing}

This project addresses stability of passivation and gettering during contact formation. RFPCD served as the monitor. Research has focused on determination of gettering efficiency for metal impurities and process optimization for the Al backsides contact. Gettering efficiency depends on a driving force represented by the impurity supersaturation $\ln \left(\mathrm{C}_{S} / \mathrm{C}_{0}\right)$ and kinetics controlled by impurity diffusivity $\mathrm{D}$. We rank the tendency for metal outdiffusion to molten $\mathrm{Al}$, or to Al-doped $\mathrm{Si}$, by the growth rate factor $\mathrm{C}_{s}{ }^{2 / 3} \mathrm{D}$ at the process temperature. Figure 2 shows the ranking. A value of $10^{5} \mathrm{~s}^{-1}$ clearly separates haze (surface precipitate) formers, confirming the validity of the model.

We evaluated the gettering effectiveness of the $\mathrm{Al}$ alloy process by coating both sides of wafers with evaporated Al. After alloying, the negative surface acceptor charge reduces surface recombination by repelling minority carrier electrons. The lifetime measured after stripping metallic $\mathrm{Al}$ represents the quality of the bulk silicon (see Figure 3 ). Heat treatment at $850 \mathrm{C}$ yields little effect after 3 minutes, but a five-fold improvement in lifetime after 120 minutes. Heat treatment for 3 minutes at $1050 \mathrm{C}$ shows an improvement similar to the longer times at $850 \mathrm{C}$. The higher temperature gives a higher throughput process due to the faster diffusion and more heavily doped silicon.

The morphology of the Al-doped silicon was examined by SEM-EBIC on $n$-type substrates. The backside field (BSF) junction is highly irregular for $\langle 100\rangle$ oriented substrates (see Figure 4). Substrates with (111) orientation show flat, faceted interfaces.

\section{Modeling and Simulation}

Process models were constructed from our experimental data to confirm, understand and guide cell fabrication. Fe is the most common bulk contaminant in solar grade silicon. We have constructed a model of the solubility of $\mathrm{Fe}$ which includes pairing with the boron dopant. The Fe solubility increases with $[\mathrm{B}]$ at low temperatures $(\mathrm{T}<700 \mathrm{C})$. The FeB pairs are metastable, giving rise to instabilities in $\tau$ and $\mathrm{L}_{\mathrm{D}}$. We have completed a model that describes our observations of pair formation, binding energies and electronic state energies. We are working with Siemens Solar to transfer this understanding to solar grade $\mathrm{CZ}$ material.

\section{Conclusion}

This program addresses the limiting factors in cell performance with low cost manufacturing. We have created tools to monitor cleanliness; process to specify chemistries for handling; and fundamental formalisms to guide process development. The understanding and development of robust process technologies are key to PV energy sources. 


\section{References}

- Gerd J. Norga and Lionel C. Kimerling, "Metal Removal from Silicon Surfaces in Wet Chemical Systems," Journal of Electronic Materials 24, 397, (1995).

- Lionel C. Kimerling, Jurgen Michel, H. M'saad, and Gerd J. Norga, "Microdefect Analysis of Silicon: Tools and Strategies," Semiconductor Characterization: Present Status and Future Needs, eds.W. M. Bullis, D. G. Seiler, A. C. Diebold, (AIP Press, 1995) 97.

- J. Michel, M. R Black, G. J. Norga, K. A. Black, H. M'saad, and L. C. Kimerling, "In -Situ Wafer Contamination Detection through RF-PCD Measurements," Proceedings of the SPIE Conference on Microelectronic Manufacturing, eds. J. K. Lowell, R. T. Chen, J. P. Mathur, 2638, 256 (1995).

- J. Palm,"Defects and Future Silicon Technology," Proceedings of the MRS Spring Meeting, San Francisco, CA, April 17-21, 1995, INVITED TALK.

- S. Zhao, L. V. C. Assali, and L. C. Kimerling, "The Structure and Bonding of Iron-Acceptor Pairs in Silicon," Proceedings of the 18th International Conference on Defects in Semiconductors, ICDS, Sendai, Japan, July 23-28, 1995.

- G. J. Norga, M. R. Black, K. A. Black, H. M'Saad, J. Michel, and L. C. Kimerling, "High Sensitivity Detection of Silicon Surface Reactions By Photoconductance Decay," Proceedings to the 18th International Conference on Defects in Semiconductors, ICDS, Sendai, Japan, July 23-28, 1995.

- G. J. Norga, K. A. Black, M. R. Black, J. Michel, and L. C. Kimerling, "In-Line Monitoring of Wet Cleaning Processes Using Radio Frequency Photoconductance Decay," Proceedings of the 4th International Symposium on Cleaning Technology in Semiconductor Device Manufacturing, Chicago, Illinois, October 8-13, 1995.

- L. Chalfoun, G. Norga, S. Zhao, L. C. Kimerling, "In-Line Materials Quality Monitor for Crystalline Silicon Solar Cell Fabrication," Proceedings of 13th NREL Photovoltaics Program Review, eds. H. Ullal and C.E. Witt, 353, 535, (1995).
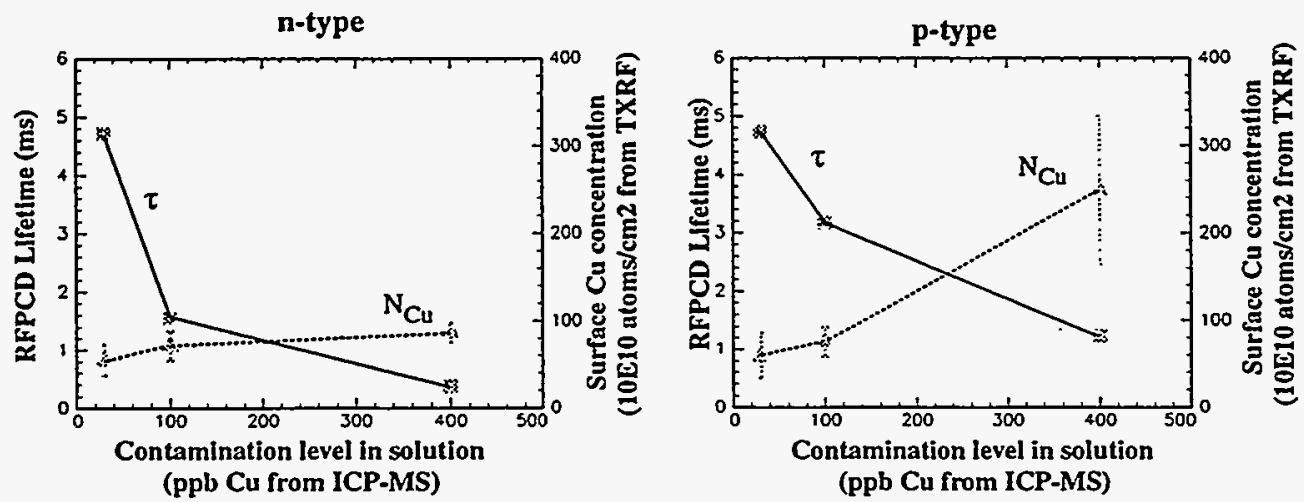

Figure 1: Effect of spiking level on copper surface concentration and measured lifetime in $48 \% \mathrm{HF}$ for (a) n-type, and (b) p-type CZ silicon. The error bars of the TXRF data are the standard deviations over the 5 analysis points.
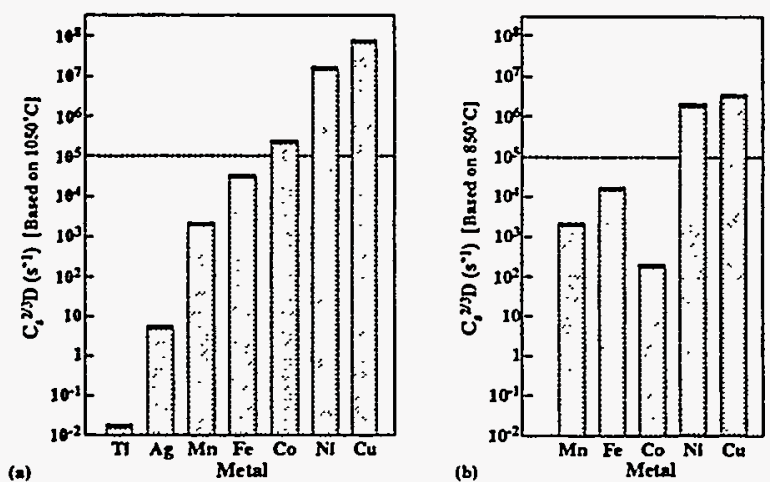

Figure 2: At high temperatures $\mathrm{Co}, \mathrm{Ni}$ and $\mathrm{Cu}$ readily outdiffuse and can be gettered (a). Gettering of $\mathrm{Fe}$ may be possible. At $850 \mathrm{C} \mathrm{(b)} \mathrm{gettering} \mathrm{of} \mathrm{Ni}$ and $\mathrm{Cu}$ will occur but is not expected for $\mathrm{Co}$. 


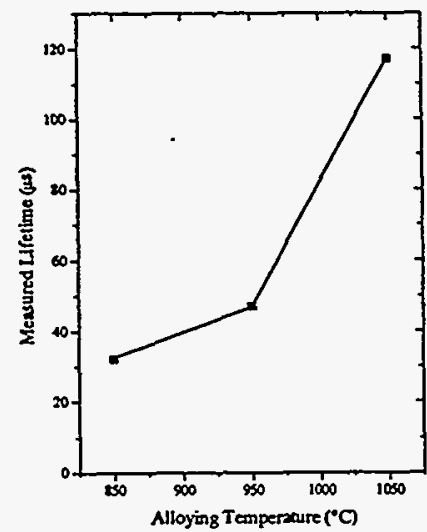

(a)

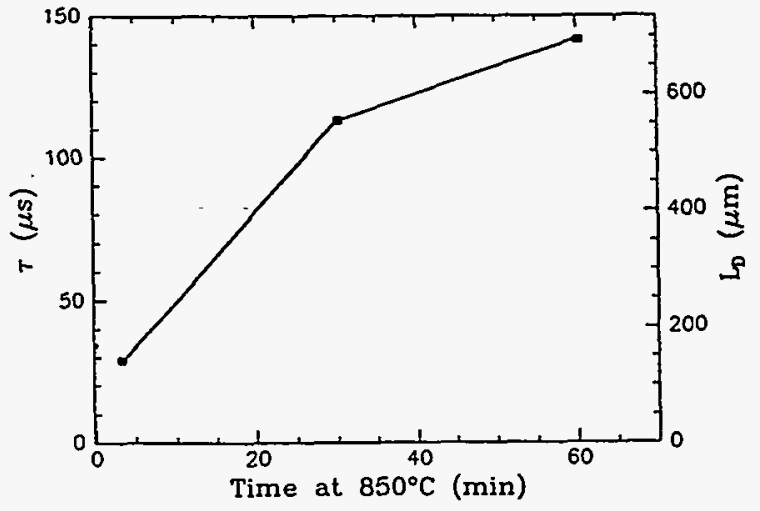

(b)

Figure 3: (a) Average measured lifetimes after the alloying step at different temperatures $(850 \mathrm{C}, 950 \mathrm{C}$, and $1050 \mathrm{C}$ ). The alloying time was constant at 3 minutes in an $\mathrm{N}_{2}$ ambient. The average initial lifetime was $35 \mu \mathrm{s}$. Faster diffusion and high solubilities at $1050 \mathrm{C}$ cause significant gettering to occur in the short annealing time. (b) Maximum lifetime measured after alloyed step for the various times indicated. Lifetimes higher than original values indicate gettering as well as the effectiveness of the $p+$ layer in reducing the effect of surface recombination.
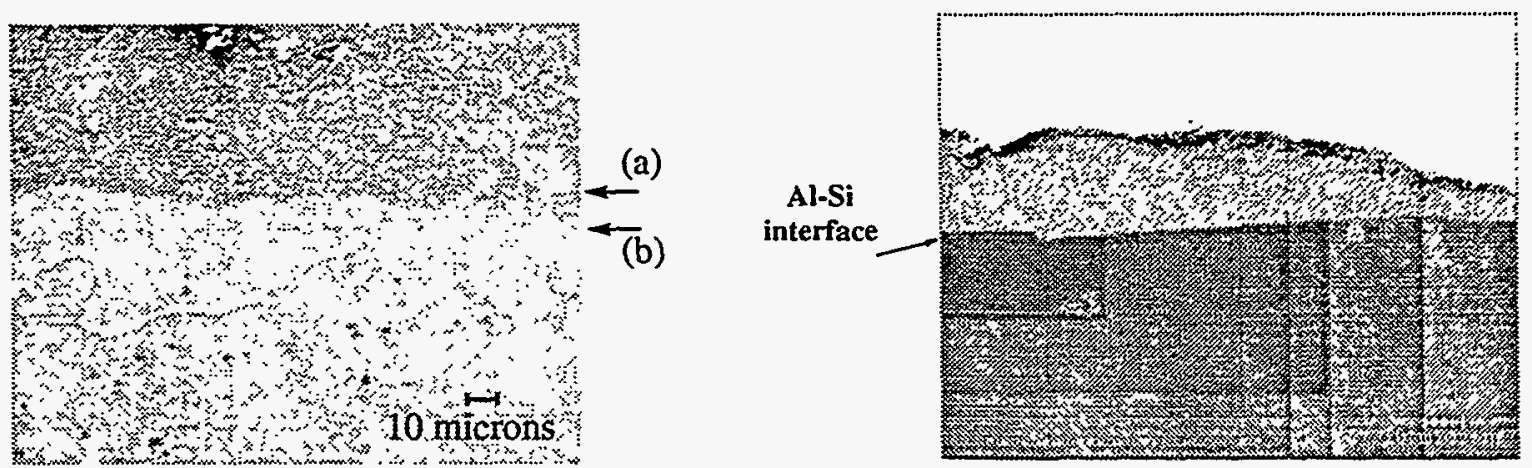

Figure 4: (Left) Combined SEM and EBIC image ( $3 \mathrm{kV}$ ) of the BSF junction region. This sample has an n-type base and was annealed at $950 \mathrm{C}$ for 30 minutes. The sample was beveled (4 degrees) and shows the following: (a) the Si-Al interface, and (b) the pt-n junction. The roughness reduces the effectiveness of the backside field in retarding surface recombination. (Right) The SEM micrograph of Al on Si after alloying $(850 \mathrm{C}, 1 / 2$ hour, $N 2$ ) shows the preference for faceting along the low energy (111) surfaces. This is a cross section of a (111) wafer offcut by 4 degrees. 
Title:

Organization:

Contributors:

\section{Detailed Non-Contact Electrical and Structural Characterization

Department of Materials Science and Engineering

North Carolina State University

Raleigh, NC 27695-7916

B.Sopori, program manager: G.A.Rozgonyi, principal investigators; $A$. Romanowski and J. Raebiger

\section{Introduction}

The objectives of the research program at NCSU are (i) improve nondestructive photoconductance methods for evaluating wafer electrical parameters, (ii) to the find optimal conditions for gettering and hydrogenation processes for low cost silicon wafers, and (iii) understand the physical processes during passivation/hydrogenation. In this work phosphorus diffusion gettering (PDG), and hydrogenation thermal annealing have been investigated. Each process was followed by nondestructive microwave lifetime measurements. The laser microwave photoconductive (LM-PCD) lifetime characterization is especially suitable for this purpose since it is very sensitive to the presence of extended defects and metal impurities and allows one to estimate surface recombination and bulk lifetime from the PCD decay signal. However, it is difficult to accurately measure and analyze nonuniform processes at the end of the laser pulse [1]; therefore, we present a new method where the excitation source is sine modulated $[2,3]$. This technique allows the excitation source level and equilibrium conditions at the wafer to be placed under better control than the pulse/time decay method.

\section{Frequency Resolved IR/LED Microwave Technique}

Illuminating a sample with an AC optical source generates excess carriers which change the conductivity of the sample. The AC conductivity change results in a variation of the low frequency $A C$ microwave reflection coefficient $R_{\mathrm{ac}}$ [3] according to:

$$
R_{u c} \propto \int_{V}\left(\mu_{p} p+\mu_{n} n\right) d V
$$

where $\mathrm{n}, \mathrm{p}$ are the $\mathrm{AC}$ electron/hole concentrations and $\mu$ is the carrier mobility. The volume $\mathrm{V}=$ $S \delta$ depends on the microwave penetration depth $\delta$, which is $\approx 500 \mu \mathrm{m}$ for $\mathrm{f}=10 \mathrm{Ghz}$ and $\rho=1$ $\Omega \mathrm{cm}$. Because the dielectric relaxation time $\tau_{\mathrm{D}}=\rho \varepsilon_{\mathrm{s}} \approx 1 \mathrm{ps}$ is lower than the high frequency microwave electrical field quasi, neutral conditions can be assumed for the excess free and trapped carriers.

The minority carrier diffusion equation can be solved explicitly for a homogenous sample. Assuming that $\delta$ is much larger than $L_{n}$ and $1 / \alpha$, where $L_{n}$ is the electron diffusion length and $\alpha$ is the absorption coefficient, the reflection coefficient will vary linearly with the $\mathrm{AC}$ minority canrier concentration $\mathrm{N}$ :

$$
R_{a c} \propto \frac{\beta+\gamma+\tau_{n}^{-1}+i \omega}{\beta+\gamma+\tau_{p}^{-1}+i \omega} N, N=\frac{G_{t} \tau_{n}}{L_{n}^{2} k_{n}^{2}\left(1+\alpha / k_{n}\right)}\left(1+\frac{\alpha / k_{n}}{1+S / k_{n}}\right)
$$

where $S=v_{s} L / D, v_{s}$ is the surface recombination velocity, $G_{t}$ is the total electron/hole generation coefficient, and $\tau_{n}, \tau_{p}$ are the electron/hole lifetimes, respectively. The reflection coefficient is a complex number and its imaginary part has a gaussian shape on a semilog plot which can be 
described by the minimum frequency $f_{o}$ and halfwidth $\Sigma$. Numerical simulation has shown that Im $R_{a c}$ depends on the product of the trap concentration $N_{T}$ and electron cross section $\sigma_{a}$ (or the electron lifetime $\tau_{\mathrm{n}}$ ); therefore, $\sigma_{\mathrm{p}}, \sigma_{\mathrm{n}}, \tau_{\mathrm{n}}$ and $\mathrm{E}_{\mathrm{T}}$ describe a single level recombination center. Both parameters $f_{o}$ and $\Sigma$ depend strongly on $E_{T}$ and $\tau_{n}$, and weakly on diffusivity. A linear dependence has been found between $\log \left(f_{\mathrm{o}}\right)$ and $\log \left(\tau_{\mathrm{n}}\right)$.

In the measurement set up the original Lifetech 88 microwave system has been used with a high brightness IR LED in place of a laser. The photodiode has been placed underneath the wafer at the microwave mirror. The beam size is equal to $5 \mathrm{~mm}$. The DC and AC LED currents are controlled with a PC through the GPIB interface. Figure 1 shows the nomalized ratio $r=I m R_{a c} /\left(I m R_{a c}\right)_{\max }$ spectrum characteristic as measured for several p-type $S i \quad\left(N_{A}=1 E 15 \mathrm{~cm}^{-3}\right)$ wafers with different $\mathrm{Fe}$ concentrations ranging from $1 E 10$ to $1 \mathrm{E} 12 \mathrm{~cm}^{-3}$. The solid line corresponds to a theoretical characteristic with $E_{\mathrm{T}}=0.1 \mathrm{eV}, \sigma_{\mathrm{n}}=1 \mathrm{E}-14 \mathrm{~cm}^{2}$ and $\sigma_{\mathrm{p}}=1 \mathrm{E}-16 \mathrm{~cm}^{2}$. The lifetime has been estimated from the best fitting using a MATLAB program. The $\tau_{\mathrm{n}}$ lifetime accurately matched the LM-PCD log lifetime. Figure 2 shows spectrum characteristics of the p-type $(1 \Omega \mathrm{cm}) \mathrm{CZ}<100>$ Siemens wafers after PDG treatments. The-spectrum characteristics are not well described by the theoretical characteristic (solid line). The discrepancy is attributed to the presence of a multiple trap recombination process. In this case the lifetime $\tau_{\mathrm{n}}$ was determined after fitting the data at the maximum value of the spectrum characteristic. The contribution of additional trapping centres is seen in Fig.3, where spectrum characteristics are measured for the different DC LED currents $(7,17,21$ and $25 \mathrm{~mA})$, with a constant $\mathrm{AC}$ current of $5 \mathrm{~mA}$. In the case of an $\mathrm{Fe}$ contaminated wafer (Fig.3b) $f_{0}$ does not depend on the DC current. A model which will accommodate a distributed AC carrier concentration (non-uniform quasi Fermi level distribution) is under development.

\section{Hydrogenation Passivation process of PV silicon wafers}

Hydrogenation passivation processes have been carried out on two sets of Siemens PV wafers ( hereafter Set 1 and Set 2). The Set 1 wafers were p-type, boron doped with $\rho=0.5 \Omega \mathrm{cm}$. The best lifetime was achieved with a wafer using a RTA and PDG procedure, (the effective lifetime changed from 2.3 to $4 \mu \mathrm{s}$ ). The hydrogenation process improved the lifetime (about 30\%) for gettered and non-gettered samples; however the largest lifetime of $7 \mu \mathrm{s}$ was obtained for the RTA - PDG wafer. The Al segregation gettering procedure improved the lifetime only for the PDG processed wafer. Because of the lifetime enhancement of the RTA - PDG wafers another experiment with several PDG and RTA conditions was done.

In the Set 2 p-type $(1 \Omega \mathrm{cm}) \mathrm{CZ}<100>$ textured wafers were used. The PDG processes were carried out at different temperatures and times. The wafers were divided into seven pieces (G1, G2,..,G7). A LM-PCD reference (G1) relaxation time equal to $2.3 \mu$ s was enhanced after long time $(1,2 \mathrm{~h})$ and high temperature $\left(1000,1100^{\circ} \mathrm{C}\right)$ PGD processing (wafer G4, G5, G6 and G7). However, an SPV diffusion length degradation occurred with increasing anneal temperature and time (see Table I). The hydrogenation process improved the lifetime only for RTA (G2) and RTA/POCl (G7) wafers. The lifetime enhancement of the G2 wafer is due to hydrogenation of the metal impurities. The G2 spectral characteristic has a gaussian shape due to $\mathrm{Fe}$ impurities. The data in the Table I shows the lifetime degradation of the non-annealed RTA/POCl ${ }_{3}$ wafer. However, the spectrum has a gaussian shape characteristic with maximum signal consisted with Fe. Frequency resolved lifetime $\tau_{\mathrm{n}}$ increases from 3.57 to $4.27 \mu \mathrm{s}$ after hydrogenation. We conclude that in both cases hydrogenation increases the lifetime. A puzzling effect is seen for the wafer G5 with annealing time $1 \mathrm{~h}$ at $1100^{\circ} \mathrm{C}$; namely, the lifetime is two times lower with respect to the other PDG 
(G4 and G6) wafers. The spectrum characteristic for this wafer is much weaker than for the others and is probably due to a high bulk $\mathrm{SiO}_{2}$ precipitate concentration. The $\mathrm{G} 5$ frequency resolved lifetime is large (about $60 \mu \mathrm{s}$ ). Almost the same frequency resolved lifetime value was measured for the G7 wafer with high temperature PDG but without RTA. We speculate that in both cases the lifetime is controlled by trapping centers related to the $\mathrm{SiO}_{2}$ interfaces. The wafers $\mathrm{G} 4$ and $\mathrm{G} 6$ have large relaxation lifetime (about $40 \mu \mathrm{s}$ ) due to oxide related trapping centers. These traps are not active in the frequency resolved technique $\left(\tau_{n} \approx 10 \mu s\right)$ because of the DC illumination.

\section{Conclusions}

New possibilities of a microwave contactless technique are described. The frequency modulated method is better than the pulse-laser method for controlling the DC quasi Fermi level. However, in this case numerical methods have to be used. Multilevel-recombination centers have to be included to analyze Siemens wafer. Since multi-parameter fitting procedures are less reliable, temperature and DC current dependent measurements are under investigation. Process variable lifetime enhancements are seen after phosphorus diffusion gettering (PDG) treatment because of trapping effects at the oxide related defects. Hydrogenating may degrade the bulk lifetime because of oxide hýdrogen complexes which reduces oxygen related trapping centers.

Table I

\begin{tabular}{|c|c|c|c|c|c|c|}
\hline \multirow{3}{*}{$\begin{array}{l}\text { Wafers } \\
\text { No. }\end{array}$} & \multicolumn{4}{|c|}{ time resolved lifetime } & \multirow{2}{*}{\multicolumn{2}{|c|}{$\begin{array}{l}\text { frequency resolved lifetime } \\
\qquad \tau_{n}[\mu s]\end{array}$}} \\
\hline & & $\tau 1[\mu s]$ & & $\tau 2[\mu s]$ & & \\
\hline & non & hyd & non & hyd & non & hyd \\
\hline$\overline{G 2(11)}$ & 0.42 & 0.89 & 2.02 & 7.56 & 4.76 & 5.85 \\
\hline G3(27) & 0.37 & 0.41 & 1.9 & 1.97 & 3.57 & 4.27 \\
\hline G4(18) & 1.23 & 1.36 & 44.98 & 49.63 & 10.9 & 10 \\
\hline $\mathrm{G} 5(16)$ & 3.91 & 2.14 & 25.33 & 14.12 & 54.9 & 78.9 \\
\hline G6(31) & 2.22 & 2.02 & 45.56 & 35.09 & 9.41 & 16.35 \\
\hline G7(2) & $1 . \overline{42}$ & 0.62 & 9.63 & 13.67 & 85.7 & 45.5 \\
\hline
\end{tabular}

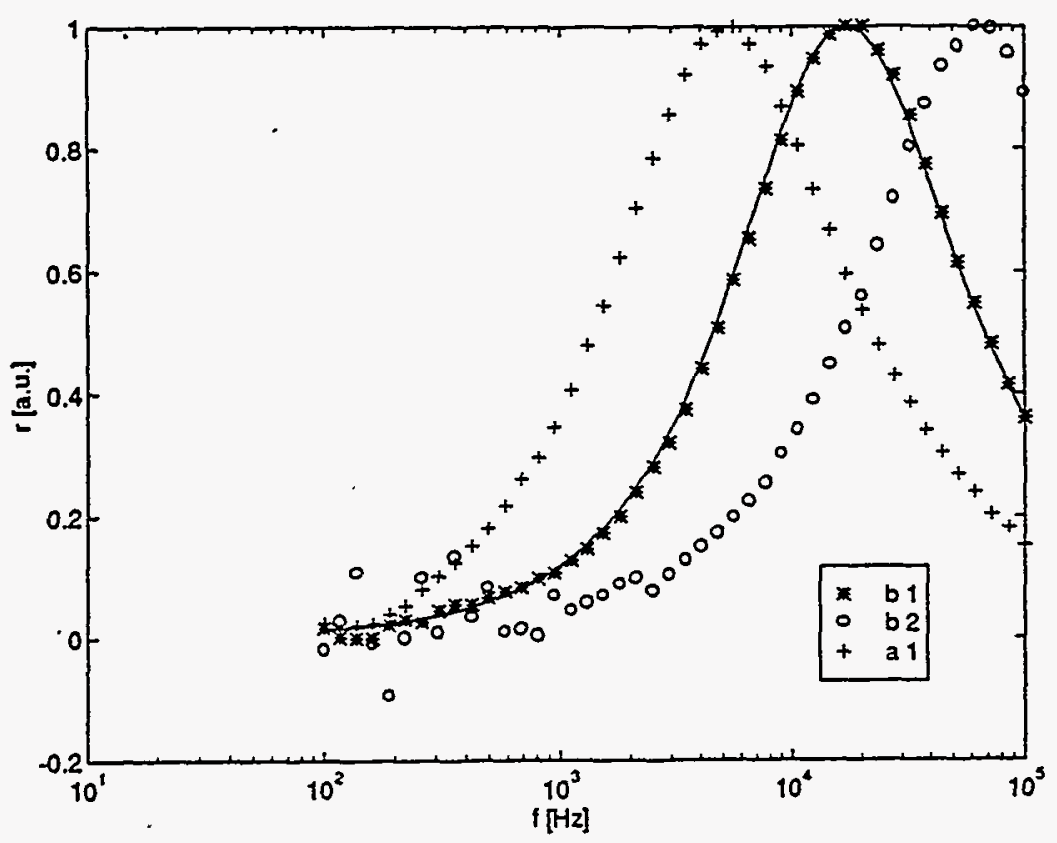

Fig. 1. Theoretical (solid line) and experimental spectrum characteristics for p-Si wafers. 


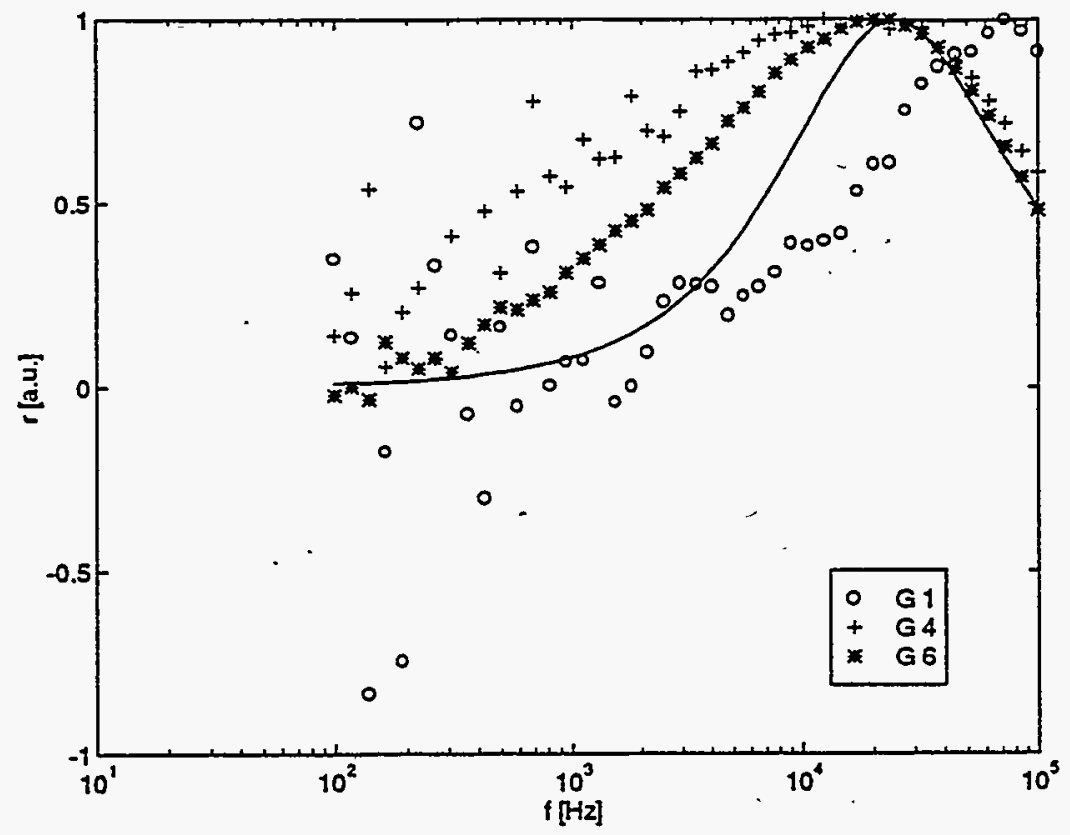

Fig. 2. Theoretical (solid line) and experimental spectrum characteristics for Siemens wafers where $\mathrm{Gl}(6)$ is the reference wafer.
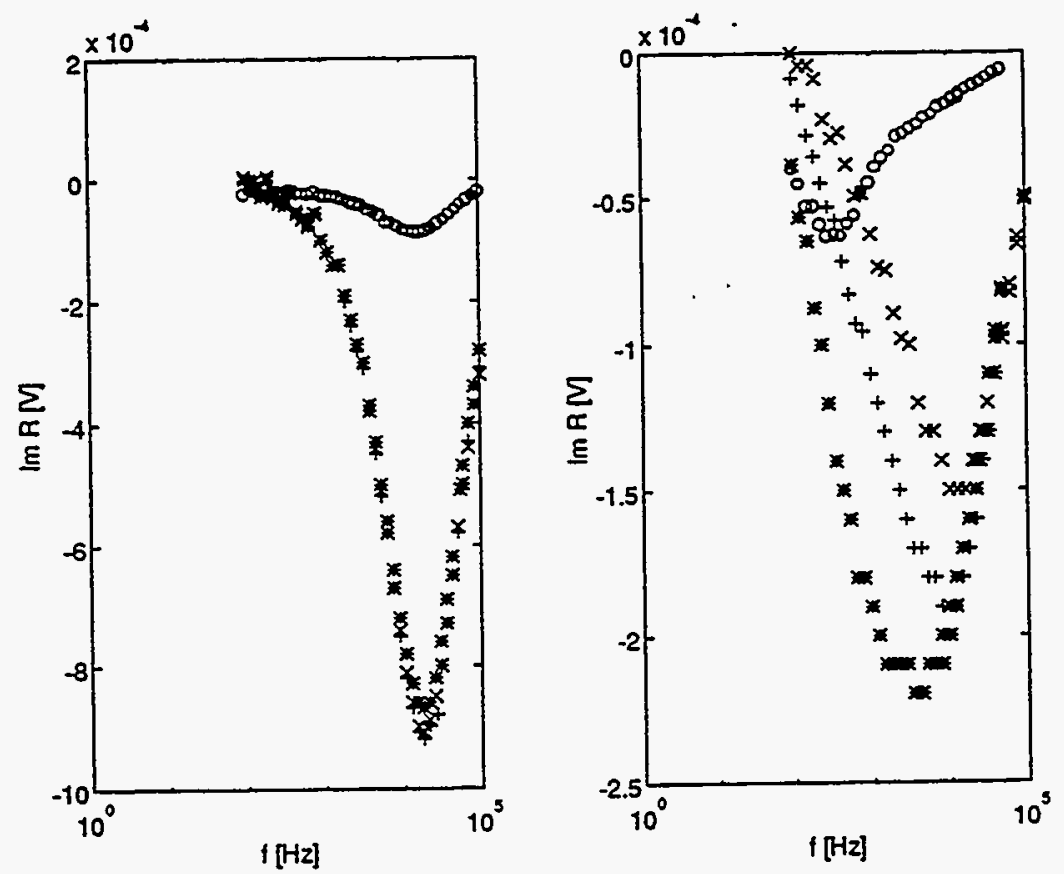

Fig. 3. Spectrum characteristics for the different DC LED currents.

\section{References}

1. M. Schofthaler, and R. Brendel, J.Appl.Phys.77, 3162(1995).

2. T. Otaredian, Contactless Microwave Lifetime Measurement, Thesis, Technische Universiteit Delft, 1992.

3. A. Romanowski, A. Buczkowski, N. Sukidi, and G.A. Rozgonyi, AIP Conference Proceedings 353, 545(1995) Woodbury, New York. 
Title:

New Approaches for High Efficiemcy Solar Cell

Effect of Strain and Heavy Doping on

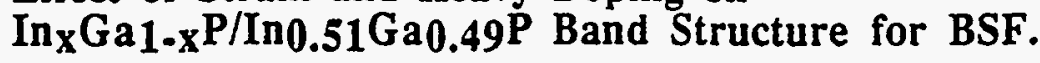

Organization: Electrical and Computer Engineering Department

North Carolina State University

Raleigh, North Carolina 27695-7911

Contributors: $\quad$ S. M. Bedair and N. A. El-Masry

\section{Objective}

We reported last year on the use of GaInP ternary alloy as back surface field (BSF) in the GaAs/InGaP multijunction solar cell. Results showed that the abrupt strained GaInP BSF outperforms the disordered GaInP ones. Also solar cell parameter such as ISC \& Voc and ff were improved for the $\mathrm{p} / \mathrm{n} / \mathrm{n}^{+}$case, however, similar improvements were not observed for the $\mathrm{n} / \mathrm{p} / \mathrm{p}^{+}$solar cell structure. We believe that these results can be explained by studying the bandgap structure line up between strained $\mathrm{In}_{\mathrm{x}} \mathrm{Ga}_{1-\mathrm{x}} \mathrm{P}$ and unstrained In $0.51 \mathrm{Ga} 0.49 \mathrm{P}$, and the effect of doping on the barriers generated to establish this backsurface field.

\section{Approach}

We have carried out bandgap calculations to study the bandgap line-up in the $\mathrm{Ga} 0.5 \operatorname{In} 0.5 \mathrm{P} / \mathrm{Gax} \operatorname{In} 1-\mathrm{x} P$ material system. The goal is to determine $\Delta \mathrm{E}_{\mathrm{c}}$ and $\Delta \mathrm{E}_{\mathrm{V}}$ and thus the potential applications of $\operatorname{Gax}_{\mathrm{x}} \operatorname{In} 1-\mathrm{x} P(x>0.5)$ as $B S F$ and for $\mathrm{p} / \mathrm{n}$ and both $\mathrm{n} / \mathrm{p}$ structures. In these calculations the following factors were considered:

i) effect of strain

ii) effect of heavy doping

Strain effects were studied using both Van de Walle's and Hrivnak's models. Effective mass data were obtained using Krijn approach. These calculations were first applied to the strained GaInAs ternary aloy and results were compared favorably to the available experimental data for this system.

In Van der Walle's Model which is also called, the model-solid theory, the energy band levels are calculated as follows. First we calculate the valence band position of the unstrained material with respect to a reference level then add to it the experimental value of the bandgap to obtain the conduction band position. The calculations of the valence band are based on the so called the average potential $E_{v, a v}$ which is the average energy over the three uppermost valence bands at $\Gamma$. These three bands are the light hole, $E_{\mathrm{ylh}}$, the heavy hole, Evhh, hole valence bands and the spin orbit split-off, Evso, valence band. The details of this model are explained in Reference (1).

Hrivnak's Model does not locate the energy levels $\left(E_{\mathrm{c}}, E_{\mathrm{V}}, \ldots\right)$ in each material alone (the epitaxial material and the substrate). Howevere, it calculates the unstrained or strained energy gap of each material as a function of lattice constants and electron and light-hole effective masses. Details of this model are given in reference (2).

The bandgap narrowing was calculated using the common expression for $\mathrm{p}$ or $\mathrm{n}$ doped material(3):

$$
\mathrm{BGN}=\mathrm{A} \mathrm{N}^{1 / 3}
$$


where, $B G N$ is the energy gap narrowing in ev, $N$ is the doping concentrationin $\mathrm{cm}^{-3}$ and $A$ is a constant. The $A$ values for the Gax $\operatorname{In}_{1-X} P$ were taken from Jain(3). However, Jain suggested more terms to be added to equation (1). We have used the common exprenssion (equation (1)) following the approach of several published works.

Harmon(4), Chung(5) and Jain(3) also suggested expression for the effect of degeneracy on the bandgap narrrowing which leads to an apparent bandgap narrowing, $B \mathrm{BN}^{*}$, given by,

$$
B G N^{*}=B G N+K T \ln \left[F_{1 / 2}\left(E_{f} / K T\right)\right]-E_{f}
$$

where, $\mathrm{f}_{1 / 2}$ is the Fermi-Dirac integral, and Ef is the Fermi level position with respect to majority band edge in ev. Band gap narrowing leads to shifts in both the conduction band and the valence band positions. Jain (2) suggested that the shifts of the two bands (conduction and valence) divided by their effective masses are equal, in the limit of high > $10^{18} \mathrm{~cm}^{-3}$ carrier concentrations. He assumes that the heavy and light hole bands move together into the gap due to heavy doping.

A comparison between Van der Walle's model and Hrivnak's model was done, they are comparable but not exact matching specially at high strain values. The calculations of the bandgaps and energy levels' shifts due to strain, were applied to the GaInAs material and to the GaInP material. The effect of heavy doping on the strained materials was also studied. A discussion will be presented to analyze the experimental results through the theoritical strain and heavy doping study.

\section{Results and Discussion}

Figure (1) shows the band levels of the Ga.51In.49P / Ga.56In .44P / GaAs structure. this is a typical structure of the (base/back surface field region (BSF)/substrate) of the experimentally grown solar cells at RTI. Figure (1a) shows the three regions for the the case Ga0.56In0.44P layer unstrained and undoped. This Ga0.56In0.44P region looks as a good choice as a BSF to our cells from the discontinuity values between it and the Ga.51In.49P base. The bandgap discontinuity between the BSF and the base is about 73 $\mathrm{m}$ ev that is divided as $53.5 \mathrm{~m}$ ev in the conduction band and $18.7 \mathrm{~m}$ ev in the valance band. This makes the structure a good candidate to be used in solar cells. However, when the effect of strain is added i.e for the strained Ga0.56In0.44P case the results are shown in figure (1b). The energy gap offset between G.56In.44P (strained) and Ga.51In.49P (unstrained) was reduced to $36 \mathrm{~m}$ ev and lies mainly in the conduction band $\sim 33.4 \mathrm{~m}$ ev. From these resuts we can predict that this structure may work as a BSF with a barrier for electrons in $\mathrm{n} / \mathrm{p} / \mathrm{p}^{+}$structure but not for holes in $\mathrm{p} / \mathrm{n} / \mathrm{n}+$ structure

The effect of heavy doping on the bandgap has to be taken into consideration. For an n-type Ga.56In.44P doped with $2 \times 10^{18} \mathrm{~cm}^{-3}$, an additional narrowing to the energy gap takes place due to this heavy doping as shown in figure (1c). This leads to a conversion of the barrier in the valence band interface to a sink. This result shows that the existance of the heterojunction BSF can be worse than a normal Ga.51In.49P homojunction. However, this heavy doping, will also lead to a shift in the fermi level inside the conduction band and the material becomes degenerate. This leads to an apparant widening in the band gap that opposes the shrinkage effects i.e. with the potential of cancelling this shrinkage effect. Our results indicate that the net result with both effects are considered as widening of the band gap as shown in figure (1d). In this figure the sink in the valence band is changed to a barrier again and this figure looks similar to that in figure (1b) even with slightly higher barrier. For the p-type Ga.56In.44P material with $2 \times 10^{18} \mathrm{~cm}^{-3}$ doping, does not reach the degeneracy limit and only band gap shrinking in the bandgap is the only result of heavy doping as seen in figure (1e) and (1f). We have a sink in the 
valence band interface and a reasonable barrier in the conduction band interface. This can work as a good structure for p-type BSF.

In our experimental measurement for the GaInP cells with strained Ga.56In.44P BSF on the cell parameters is not as expected from a heterojunction BSF as is apparent in Figure (7-10d). At the valence band interface between the base and the BSF, a slight, negligible barrier to minority holes in a $\mathrm{p} / \mathrm{nn}^{+}$structure exists. This negligible barrier is not enough to enhance the short circuit current of such a cell. However, at the conduction band interface, there is a reasonable barrier to the electron majority carriers in a $\mathrm{p} / \mathrm{n} / \mathrm{n}^{+}$which is not the case for homojunction BSF or no BSF case. This barrier for majority carriers in a $\mathrm{p} / \mathrm{n} / \mathrm{n}+$ structure may lead to a reduction in the majority carrier's losses at that interface. This leads to a reasonable increase in the dark current, with no reasonable enhancement in the short circuit current leads directly to a reduction in the open circuit voltage. This prediction agrees with our experimental results measured from our cells. For $n / p / p^{+}$structure, the barrier in the conduction band can enhance the short circuit current over the no BSF case. In this case, there is no barrier for the majority carriers that allows an enhancement in the open circuit voltage and of course in the fill factor.

\section{Conclusion}

This theoritical prediction can be used to explain the experimental results(6). Mainly, GaInP strained back surface field is a good choice and works fairly well in improving cell parameter for $\mathrm{p} / \mathrm{n}$ structure. This BSF does not seems to help in $\mathrm{n} / \mathrm{p}$ case as being experimentally observed, that agrees with our current prediction.

\section{References}

1. C. G. Van de Walle and R. M. Martin,"Theoritical Calculations of Semiconductor Heterojunction Discontinuities," J. Vac. Technology B, 4 (4), p.8154 (1986).

2. L. Hrivnak,"The Relations for Electron Effective Masses of Strained InxGa1-xAs Layers," Phys. Stat. Sol. (A), 123, p. K133 (1991)

3. S. C. Jain, J. M. McGregor and D. J. Roulston,"Band-gap Narrowing in Novel III-V Semiconductors," J. Appl. Phys., 68(7), p. 3747 (1990).

4. E. S. Harmon, M. R. Melloch, M. S. Lundstrom and M. L. Lovejoy,"Experimental Determination of the Effects of Degenerate Fermi Statistics on Heavily p-Doped GaAs," Appl. Phys. Lett., 58 (15), p.1647 (1991).

5. H. L. Chang, P.D. DeMoulin, M. E. Klausmeier-Brown, M. R. Melloch and M. S. Lundstrom,"Evidence for Band-gap Narrowing Effects in Be-doped, p-p+ GaAs Homojunction Barriers," J. Appl. Phys., 64(11), p. 6361(1988).

6. N. H. Rafet, S. M. Bedair, P. R. Sharps, J. S. Hill, J. A. hancock and M. L. Timmons, "Back Surface Fields for $n / p$ and p/n GaInP Solar Cells," Int. Photo Voltaic Conf. Dec 1994, Hawaii. 


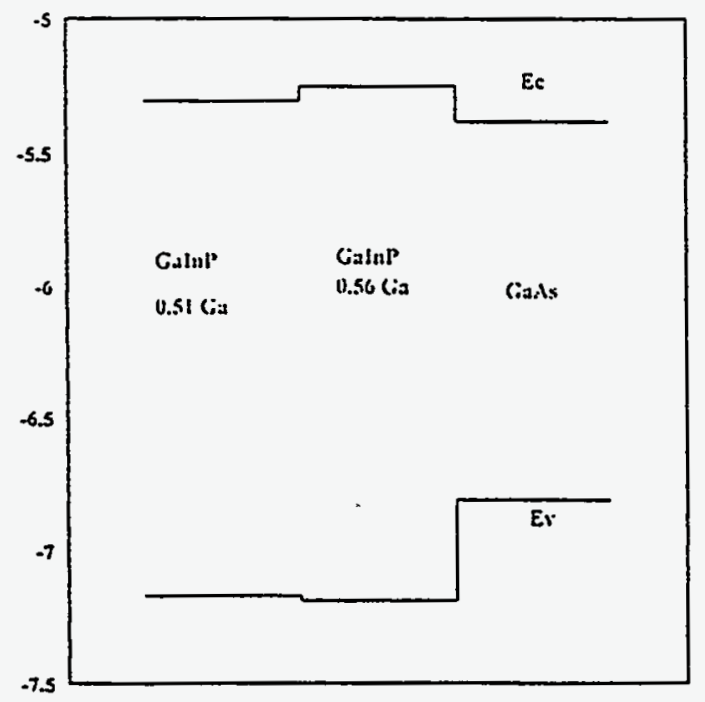

Fig. 1-n The band levels and the interface dicontinuities between GaAs, Ga.56In.44P, and 'G.51Ln.49P materisk, No strin or doping effects

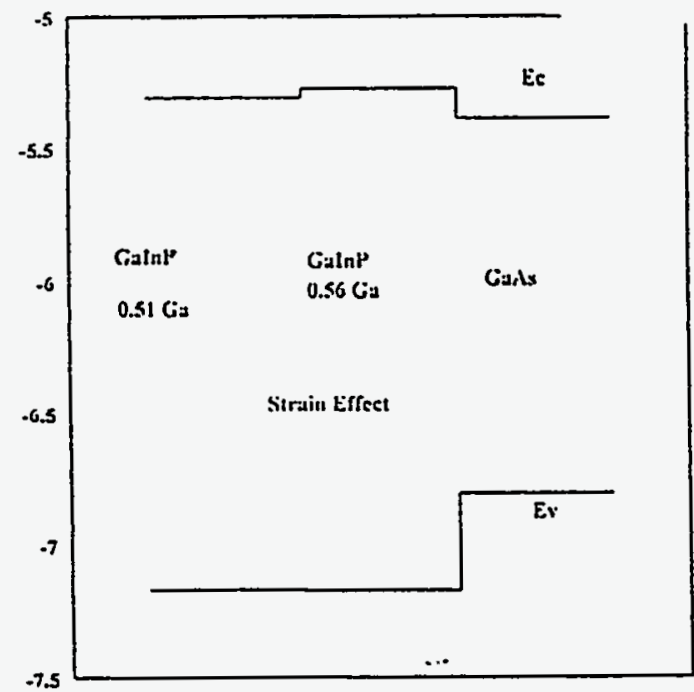

Fig.l-b The bend levels and the interface diecontiouitie between G2As, GaS6In.44P, and G2.51ln.49P materinls. Strin effect is included

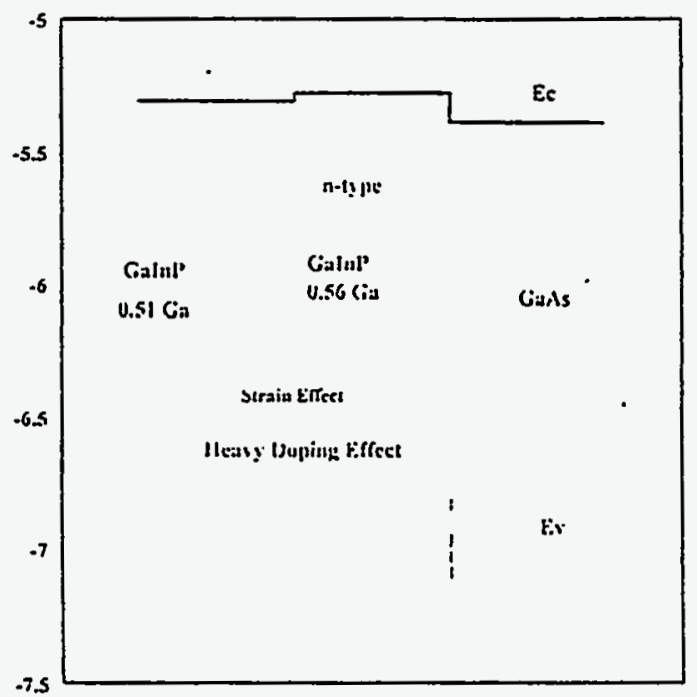

Fig l-c The band levels and the interfuce discontinuities between GaAs, Gz.56ln 44P, and 'Gs SIIn.49P materinle Strin effect and beavy doping effect are included (n-type material).

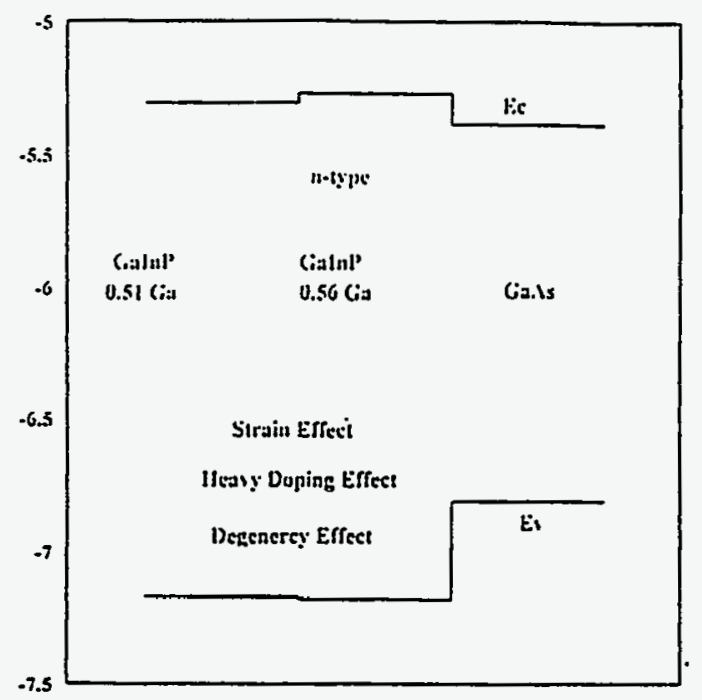

Fig. 1-d The band levels and the interfice discontinuitiea between GaAs, Ga.56In.44P, and 'Ga.5IIn.49P materiais. Strin effect heavy doping effect and degeneracy effect are included(n-type

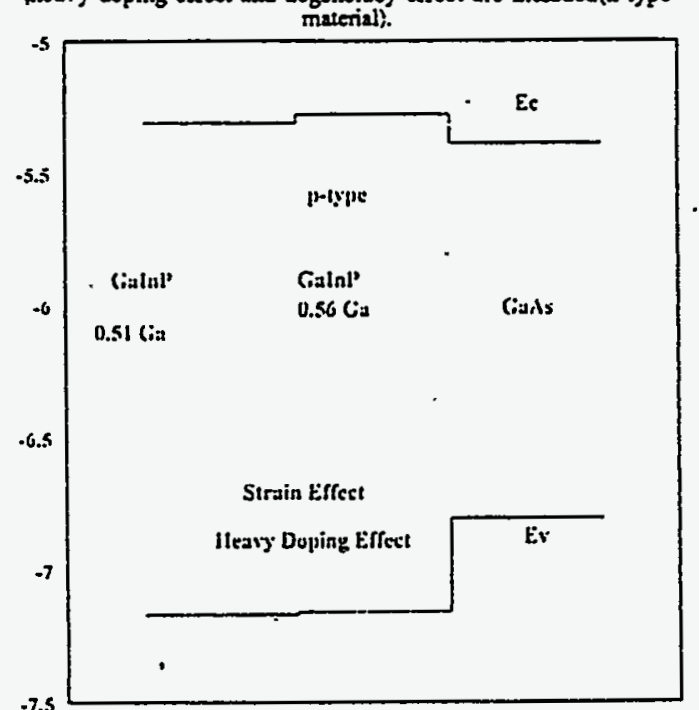

Fig. 1-e The band levels and the interface discontinuities between GAAs, Ge.56In.44P, and 'GesIln.49P malerials. Strin effect and

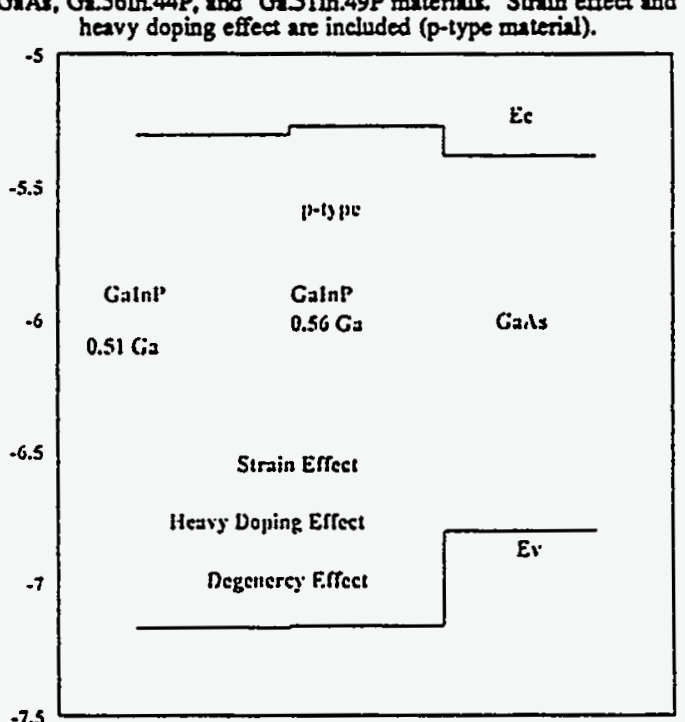

Fig 1-f The band levels and the interface diecontinuities between GaAs, Ga.56In.44P, and "Ga.51In.49P muleriale. Strin effect heavy doping effect and degenency effect are included(n-type material). 
Title:

\title{
Very High Efficiency Photovoltaic Cells Based on Fully Organic Multiple Quantum Wells
} ,

\author{
Organization: \\ Department of Electrical Engineering \\ Center for Photonics and Optoelectronic Materials (ATC/POEM) \\ EQUAD J301 \\ Princeton University \\ Princeton, NJ 08544
}

Contributors:

Stephen Forrest, Principal Investigator, Vladimir Bulovic, Dr. Dmitri Garbuzov

\section{Project Objectives:}

The principal project objective is to demonstrate relatively high solar conversion efficiency using extremely low cost thin film technology based on crystalline organic multiple quantum well photovoltaic cells. We base our work on recent observations both in our lab and elsewhere that have indicated the quantum efficiency of organic photoconductors based on vacuum deposited thin films can be increased by at least two orders of magnitude (to at least $10 \%$ ) if the organic films are grown in a highly ordered manner, and if organic multiple quantum wells are used in the absorption region. Thus, we are investigating the physical origin of this phenomenon, and are growing thin film MQW cells which demonstrate relatively high quantum efficiencies to determine the practicality of crystalline organic thin film cells for solar power applications. The investigations are based on a unique, ultrahigh vacuum organic molecular beam deposition system in our laboratory.

\section{Results:}

It has long been recognized that organic materials provide a promising means for solar energy conversion due to the potential for very low cost manufacture of solar cells employing such materials. In particular, crystalline organic semiconductors such as the phthalocyanines (Pc's), perylenes and other relatively low atomic weight polyacenes have been regarded as having the greatest potential due to their ability to be controllably deposited via conventional vacuum techniques, their relatively high purity, and high mobilities (hence relatively low series resistance). In spite of considerable research having been pursued in investigating both organic $p-n$ junction and Schottky barrier crystalline organic photovoltaic (PV) cells over the past 20 years, however, there has yet to be a demonstration of such a cell whose characteristics are adequate for even the most undemanding of solar conversion applications. Their poor performance can be ascribed principally to the following three causes: 1. Due to the intrinsic nature of photoconductivity in crystalline organic materials, where free electron-hole pairs are generated in a second order process following absorption and exciton generation, the efficiencies realized to date are very low (typically <1\% to AM0 - AM2 illumination). 2.

Due to the limited $\pi$-orbital overlap between adjacent molecules in an organic crystalline stack, and due to the presence of numerous defects in the crystalline order in the deposited materials, the free carrier mobilities in many organics are very low (typically $<10^{-3} \mathrm{~cm}^{2} / \mathrm{V}$ s). This leads to high film resistance, and hence low power conversion efficiency. 
While these problems still prevent the realization of fully organic, low cost PV cells, several significant advances in the growth and processing technology of such materials have been made in our own lab, and by other researchers worldwide. These advances suggest that we are at a turning point in the technology of crystalline organic semiconductor PV cells. Indeed, recent experiments indicate that there are novel and effective means for growing nearly perfect, multiple quantum well (MQW) crystalline structures which can significantly increase the quantum efficiency and carrier lifetime in organic materials. Furthermore, materials which are grown with a high degree of crystalline perfection have enhanced $\pi$-orbital overlap, thereby decreasing bulk layer resistance. Finally, recent work in our laboratory has shown that very high stability photodetectors can be obtained using certain organic molecules based on perylene and naphthalene derivatives.

The crystal structure of PTCDA, many metal phthalocyanines (Pc's), and other related compounds consists of molecules which, when deposited under high vacuum conditions, form very extended, nearly perfect stacks. For such structures, the charge mobility (and hence the conductivity) along the stacks is high, leading to significant charge delocallization in this direction. In the case of PTCDA, the stacking axis is perpendicular to the film plane, hence allowing for charge to be easily transported between contacts made to the top and bottom film surfaces.

In general, low $\eta$ in organic semiconductors is attributed to the intrinsic nature of the photoconductivity process. That is, absorbed solar energy first results in the highly efficient generation of excitons in the film bulk (described by the process $S_{0}+h v \rightarrow S_{0}{ }^{*}$, where $S_{0}$ and $S_{0}{ }^{*}$ denote the molecular ground and excited states, respectively). These excitons then diffuse to an impurity site (denoted $\mathrm{M}$ ), crystal defect or interface where they ionize into a free electron and hole pair, via $S_{0}{ }^{*}+M \rightarrow e+h$. Typically, exciton dissociation (prior to recombination) and charge collection are very low efficiency $(<1 \%)$ processes. Although the ionization process is not fully understood, it is often attributed to exciton dissociation in the presence of the built-in electric field surrounding the crystal defect. If this dissociation occurs in an otherwise neutral region of the film, the free electron-hole pairs generated in the ionization event are still localized near the defect where they recombine without being collected in the external circuit. Hence, to significantly increase $\eta$ as is the objective of our Phase I research, two conditions must be met: 1) The film must be relatively free of random defects which generate local electric fields, and 2) The exciton and free carrier diffusion lengths must be sufficiently long such that these particles can migrate to regions of the film where the built-in electric field from an adjacent junction is high enough to separate the free electron-hole pairs prior to recombination.

The work in this program has therefore been to determine the ultrahigh vacuum growth conditions, structures and materials combinations which result in the highest $\eta$ for use in fully organic MQW PV cells. The investigations has been to study the dependence of $\eta$ on MQW layer thickness ( $\leq 10 \AA$ to $500 \AA$ ) and composition grown in this system. To assess materials quality, photoconductor structures were fabricated in both the substratenormal (using In and ITO contacts) and substrate-parallel (using interdigitated electrodes) geometries. We found that under the appropriate growth conditions, very long exciton diffusion lengths could be obtained $(220 \mathrm{~nm})$ due to the extended stacking of the molecules. Furthermore, substantial photoconductive efficiencies (approaching 1\%) were achieved at what is apparently the lowest applied fields ever reported in organic thin films $\left(\sim 10^{4} \mathrm{~V} / \mathrm{cm}\right)$. These results are strongly indicative of the high structural ordering and purity of the thin films grown in our laboratory. Indeed, the low fields necessary to 
achieve high carrier collection efficiency, along with the long exciton diffusion lengths are necessary preconditions to achieving high PV cell efficiencies. These experiments have shed a fundamental new light on charge generation and transport in organic thin films, and suggest that PV cells of reasonable power efficiencies can be obtained using very tightly $\pi$ stacked organic structures such as PTCDA.

Typical MQW materials combinations investigated include $\mathrm{CuPc/PTCDA}$ and NTCDA/PTCDA multilayers. Due to the absorption of all of these materials in the visible, good spectral coverage of solar radiation by these combinations is anticipated. Rectifying isotype heterojunctions consisting of PTCDA/CuPc or PTCBI/CuPc (PTCBI is another perylene derivative closely related to PTCDA) can be used to create $V_{b i}$ across the MQW stacks. We have found that the $\mathrm{V}_{\mathrm{bi}}$ of these heterojunctions are $0.5 \mathrm{~V}$ and $0.7 \mathrm{~V}$, respectively. On the other hand, rectifying $p-n$ heterojunctions consisting of, for example, PTCDA/CuPc MQWs in contact with PBD will be attempted. In another approach, blocking contacts such as Au/PTCDA junctions can be used to form Schottky barriers which deplete the underlying MQW stack. Typically, the absorption coefficient of these organics is $>10^{5} \mathrm{~cm}^{-1}$ in their absorption band, implying that fully depleted MQW stacks of only a few thousand Ångstroms thickness are needed to obtain very high $\eta$. Given the very low carrier concentrations of materials such as PTCDA and PTCBI (where $N_{d}=5 x$ $10^{14} \mathrm{~cm}^{-3}$ ), these $V_{b i}$ are adequate to achieve such depletion region widths.

In corollary work, we have identified several organic phosphors which, when deposited directly onto Si PV cells, can increase their UV collection efficiencies by a substantial amount. The process involved film absorption in the UV and then near 100\% re-emission efficiency in the green, where the Si PV cell is quite sensitive. Application of these re-emitting films can increase the AMO efficiency of UV-enhanced PV cells by nearly $3 \%-5 \%$. While similar techniques have been used for enhancing the sensitivity of CCD imaging arrays, to our knowledge, our particular technique of optimizing the film for both anti-reflection in the visible and UV regions while enhancing the UV sensitivity of PV cells is unique. We have submitted a patent application covering this work.

Beyond those issues covered above, we have accomplished the following:

1. Established growth methods for achieving high quality crystalline films of PTCDA, NTCDA and PTCBI using OMBD.

2. Established measurement procedures and constructed experimental set-up for modified Shockley-Haynes measurements.

3. Trained student in the experimental and analysis procedures to be employed in the experiment.

4. Measured carrier velocities in PTCDA bulk and MQW films

5. Measured of absorption coefficients of PTCDA and PTCBI

6. Currently measuring quantum efficiency of as-grown films and MQWs

\section{References:}

1. "Excitons in crystalline thin films of $3,4,9,10$ perylenetetracarboxylic dianhydride studied by photocurrent response", V. Bulovic and S. R. Forrest, Chem. Phys. Lett., 238 88 (1995).

2. "Excitons in an ordered molecular semiconductor thin film studied using photocurrent response at low electric fields", V. Bulovic and S. R. Forrest, Chem. Phys., submitted. 
Title:

Organization: $\quad$ Research Triangle Institute, RTP, NC 27709

Contributors: $\quad$ R. Venkatasubramanian, Principal Investigator

B.C. O'Quinn, J.S. Hills, M.L. Timmons, and D.P. Malta,

Development of High-Efficiency GaAs Solar Cells on Polycrystalline Ge Substrates

\section{Introduction}

High-efficiency single-junction GaAs solar cells with an efficiency of $25.7 \%$ under AM1.5G [1] and tandem $\mathrm{GaInP} / \mathrm{GaAs}$ solar cells with efficiencies of $29.5 \%$ under AM1.5G [2] and 30.2\% under 140-180 suns [3], have been demonstrated on singlecrystal GaAs substrates. It is important to investigate and effect a transition of these highefficiency cell results on to potentially low-cost, large-area substrates for flat-plate (1-sun and low-concentration ) applications.

Several alternatives to single-crystal GaAs substrates have been considered in the past for the demonstration of high-efficiency GaAs solar cells. Large-area, cast, poly-Ge substrates, with an average grain size of $1 \mathrm{~cm}$, are commercially available and appear as potential low-cost substrates for GaAs cell growth. Our initial attempt at developing GaAs solar cells on such poly-Ge substrates was successful. Under AM1.5G simulation, a total-area efficiency of $15.8 \%$ [4] was obtained and no degradation of efficiency was observed in going from $0.25 \mathrm{~cm}^{2}$ to $1 \mathrm{~cm}^{2}$ cells. Further, the use of large-area poly-Ge substrates with well-established large-scale MOCVD growth will allow cell processingcosts to be reduced.

The objective of this program, therefore, is to further develop the performance of GaAs solar cells on poly-Ge substrates. We describe material and device-structure optimization studies that have led us to achieve a open-circuit voltage of $\sim 1$ Volt in a GaAs solar cell on poly-Ge substrate and also improve the best cell efficiency to $18.2 \%$ for a $4-\mathrm{cm}^{2}$ cell.

\section{GaAs Growth on Poly-Ge and Minority-Carrier Lifetimes}

First, we investigated the effect of various polishing procedures prior to the MOCVD growth of GaAs layers on poly-Ge substrates. It was observed that conventional chemical-polishing of substrates resulted in a non-flat poly-Ge substrate from varying etch-rates of the different grains in the poly-Ge substrate. This uneven surface especially on the backside of the substrate can cause portions of the wafer to be not in thermal contact with the susceptor, thereby leading to a non-uniform temperature distribution across the poly-Ge wafer during MOCVD. This can result in poor surface morphology of GaAs-AlGaAs layers on the poly-Ge substrate. In contrast, a chem-mechanical polishing of the poly-Ge substrate resulted in a significant improvement in "across-the grains" morphology of GaAs-AlGaAs layers. We typically obtain uniform specular growth across 85 to $90 \%$ of area of a 2" poly-Ge wafer, with tens of different crystalline orientations. 
With the morphology of GaAs-AlGaAs layers on poly-Ge substrates under control, we conducted an optimization study of the minority-carrier properties of $\mathrm{GaAs}$ layers. Several GaAs-Al ${ }_{0.8} \mathrm{Ga}{ }_{0.2} \mathrm{As}$ double-hetero $(\mathrm{DH})$ structures were grown at a growth temperature of $670^{\circ} \mathrm{C}$, to obtain minority-carrier lifetimes by photoluminescence (PL) decay. All the $\mathrm{DH}$ structures were grown with a $2-\mu \mathrm{m}$ GaAs buffer to minimize the effects of Ge auto-doping in the active region, although $5-\mu \mathrm{m}-\mathrm{GaAs}$ buffer has been found to effectively eliminate any auto-doping.

The lifetime-spread across various grains can be reduced through the use of lower doping for the $\mathrm{Al}_{0.8} \mathrm{Ga}_{0.2} \mathrm{As}$ confinement layers, as shown in Table 1. It is worth noting here that under less-than-optimized buffer-thickness, we obtain minority-carrier lifetimes in the range of $7.0 \mathrm{nsec}$ in GaAs DH structures on optical-grade poly-Ge substrates. These lifetimes are comparable to typical values obtained in $\mathrm{GaAs} \mathrm{DH}$ structures, for similar buffer layer thickness, grown on single-crystal Ge substrates. Thicker $(>2.0 \mu \mathrm{m}) \mathrm{GaAs}$ buffer layers have not been grown, to possibly improve the lifetimes further, as we have seen negligible or only nominal improvement in GaAs solar cell performance on $\mathrm{Ge}$ substrates with thicker buffer layers.

TABLE 1. Summary of PL-Decay Lifetimes on GaAs- $\mathrm{Al}_{0.8} \mathrm{Ga}_{0.2}$ As DH Structures on Poly-Ge

\begin{tabular}{lcccc}
\hline Sample \# & $\begin{array}{c}\text { Growth Rate } \\
(\mu \mathrm{m} / \mathbf{m i n})\end{array}$ & $\begin{array}{c}\text { Window } \\
\text { Doping Level }\end{array}$ & $\begin{array}{c}\text { Thickness of } \\
\text { Active Region } \\
(\mu \mathrm{m})\end{array}$ & $\begin{array}{c}\text { Lifetime Spread } \\
(\mathbf{n s})\end{array}$ \\
\hline $1-2181$ & 0.045 & Medium & 2.2 & $0.8-1.0$ \\
$1-2183$ & 0.045 & Medium & 3.0 & $0.2-7.0$ \\
$1-2184$ & 0.045 & Low & 2.2 & $1.0-4.0$ \\
\hline
\end{tabular}

Electron-beam-induced-current (EBIC) scans of $\mathrm{p}^{+} \mathrm{n}$ GaAs junctions grown on poly-Ge substrate indicate hole diffusion lengths of $\sim 1.2 \mu \mathrm{m}$ in the base region, near the depletion layer. This diffusion length is consistent with the typical lifetimes discussed above. EBIC images of $\mathrm{p}^{+} \mathrm{n} \mathrm{GaAs}$ junctions, in plan-view, were studied as a function of accelerating voltage. Plan-view EBIC images taken at $7 \mathrm{kV}, 15 \mathrm{kV}$ and $25 \mathrm{kV}$, giving an estimated depth penetration of $0.5 \mu \mathrm{m}, 1.6 \mu \mathrm{m}$ and $3.7 \mu \mathrm{m}$, respectively, are shown in Fig. 1. The defect densities, in the layers (revealed by dark spots) and grain boundaries/dislocations (revealed by dark lines) are a function of depth of the epi-film. An undersatnding of this behaviour is being pursued for further optimization of the performance of the $\mathrm{p}^{+} \mathrm{n}$ GaAs junction on poly-Ge susbtrate.

\section{GaAs Solar Cell Device Optimization on Poly-Ge Substrates}

We have initiated preliminary device optimization of GaAs solar cells on poly-Ge. Shown in Fig. 2, is a schematic of a GaAs solar cell device structure that we have developed [4] for use on poly-Ge substrates. The undoped spacer between the base and the emitter has been shown [4] to reduce the dark-current considerably, thereby improving the cell open- 
circuit voltage $\left(\mathrm{V}_{\mathrm{oc}}\right)$ and fill-factor. However, short-circuit current density $\left(\mathrm{J}_{\mathrm{sc}}\right)$ has been found to be less sensitive to spacer thickness.

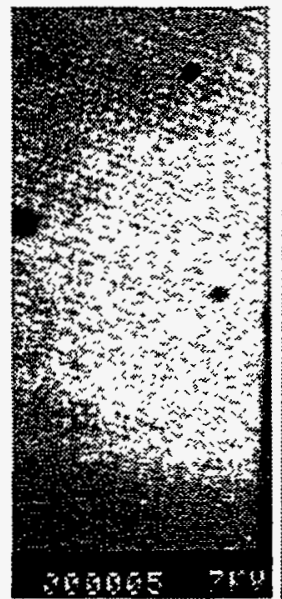

$7 \mathrm{kV}$

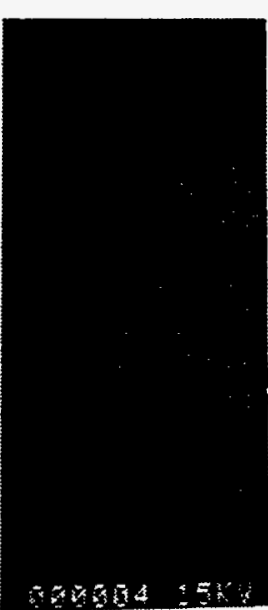

$15 \mathrm{kV}$

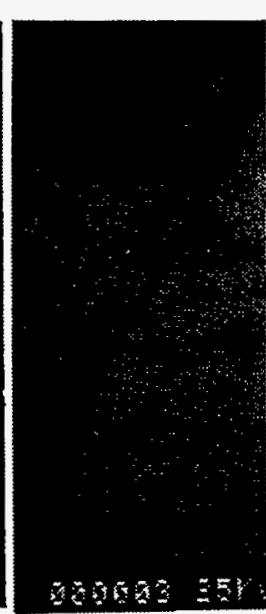

$25 \mathrm{kV}$

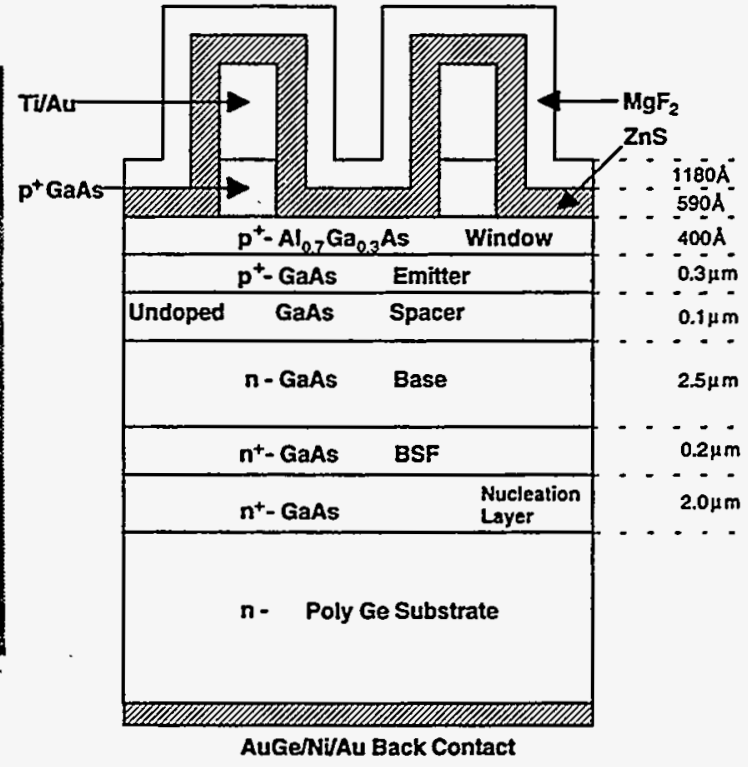

Fig. 2. Schematic of a p ${ }^{+} n$ GaAs solar cell device structure on poly-Ge.

junction at various accelerating voltages.

In contrast to the beneficial effect of spacer on $V_{o c}$ and fill factor values, we have observed that substrate-polishing defects such as dents/ledges/steps are detrimental to GaAs solar cell performance. The GaAs solar cell data in' Table 2 illustrate this point. Here, we observe that the cell $\mathrm{V}_{\mathrm{oc}}$ values do improve with larger grain-sizes, for a given spacer thickness of $0.12 \mu \mathrm{m}$. However, macro-polishing defects such as dents, ledges and steps in the cell active-area tend to reduce the $\mathrm{V}_{\text {oc }}$ much more strongly than grainboundaries. We believe this to be a possible result of higher electric-fields (in the $\mathrm{p}^{+} \mathrm{n}$ junction) at the vicinity of these steps and ledges, causing higher leakage currents. Once again, the $J_{s c}$ is less sensitive to the density of grain boundaries or macro-polishing defects.

\section{Advances in Performance of GaAs Solar Cells on Poly-Ge}

The optimization procedures for improving the GaAs cell performance have included the use of lower growth-rate to grow the cell structure, lower doping in the back-surface-field (BSF) region and an optimum spacer thickness of $\sim 0.12 \mu \mathrm{m}$, all for obtaining improved $\mathrm{V}_{\text {oc }}$ values. The series resistance associated with the back contact to n-poly Ge substrate needs to be minimized to obtain improved fill factor. AuGe/Ni/Au metallization appears suitable for the back contact.

Initial NREL-measured I-V data on GaAs solar cells on poly-Ge, with typical cell efficiency of $16.6 \%$, indicated a roll-off in the blue spectral-response. We have remedied this problem a little, through the use of thinner emitter plus improved AlGaAs-window 
growth. This has resulted in an improved $\mathrm{J}_{\mathrm{sc}}$ of $\sim 23 \mathrm{~mA} / \mathrm{cm}^{2}$ and an AM1.5 efficiency of 18.2\%. The NREL-measured data is shown in Fig 3. This cell also indicates a $V_{\text {oc }}$ near 1.0 Volt and is impressive for a GaAs solar cell on optical-grade poly-Ge substrate. This $\mathrm{V}_{\text {oc }}$ is almost $70 \mathrm{mV}$ higher than on our previously-reported best cell [4]. We also note that this $18.2 \%$ efficiency is obtained on a $4-\mathrm{cm}^{2}$-area cell compared to the previous best of $15.8 \%$ on a $1-\mathrm{cm}^{2}$-area cell.

\begin{tabular}{|c|c|c|c|c|c|}
\hline $\begin{array}{c}\text { Cell } \\
\#^{\circ}\end{array}$ & $\begin{array}{l}\text { Area } \\
\mathrm{cm}^{2}\end{array}$ & $\begin{array}{c}\text { Average } \\
\text { Grain } \\
\text { Size }\end{array}$ & $\begin{array}{l}\text { Sub- } \\
\text { strate } \\
\text { Polish } \\
\text { Dents }\end{array}$ & $\begin{array}{l}v_{o c} \\
\text { (v) }\end{array}$ & $\begin{array}{c}\mathrm{J}_{\mathrm{sc}} \\
\mathrm{mA} / \mathrm{cm}^{2}\end{array}$ \\
\hline 1 & 0.25 & $\begin{array}{l}1-2 \\
\mathrm{~mm}\end{array}$ & None & 0.97 & 26.3 \\
\hline 2 & 0.25 & $1 \mathrm{~mm}$ & Yes & 0.87 & 25.8 \\
\hline 3 & 0.25 & $1 \mathrm{~mm}$ & None & 0.91 & 25.7 \\
\hline 4 & 0.25 & $\begin{array}{c}0.5-1 \\
\mathrm{~mm}\end{array}$ & None & 0.90 & 24.9 \\
\hline 5 & 0.25 & $2 \mathrm{~mm}$ & None & 0.94 & 24.9 \\
\hline
\end{tabular}

TABLE 2. Effect of grain size and other substrate-polishing defects on device performance.

\section{RTI GaĀs on poly Ge}

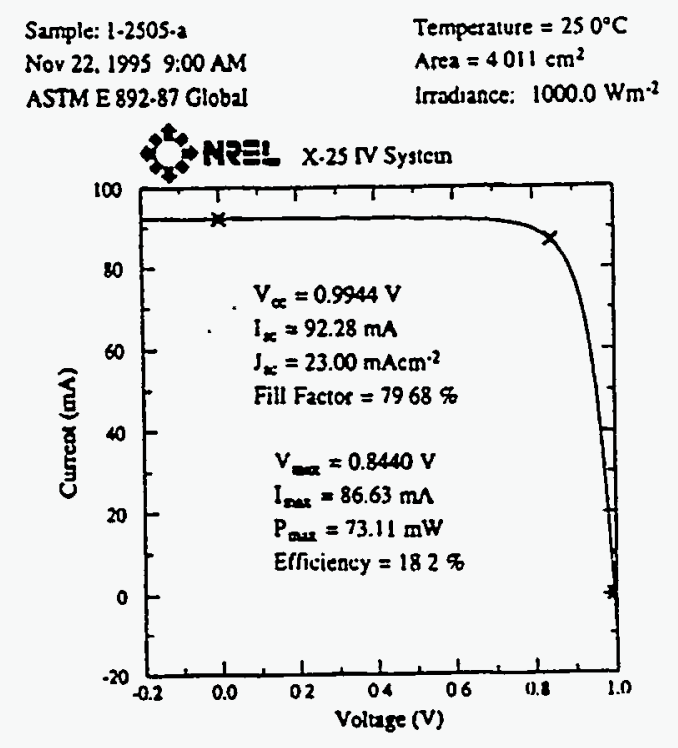

Fig. 3 I-V characteristic of a 18.2\%efficient, 4- $\mathrm{cm}^{2}$-area $\mathrm{GaAs}$ cell on polyGe with a $V_{o c}$ of $\sim 1$ Volt.

\section{Acknowledgemenits}

This work has been performed under Subcontract No. YAL-3-1-3357-03 from NREL, with Dr. John Benner as the technical monitor. We acknowledge Dr. Keith Emery and Dr. Halden Field of NREL for cell measurements and Dr. Richard Ahrenkiel and Dr. Brian Keyes for lifetime measurements.

\section{References}

1. S.R. Kurtz, J.M. Olson, and A. Kibbler, Proc. of 21st IEEE Photovoltaic Specialists Conf. (IEEE Press, NY, 1990) p.138.

2) J.M. Olson, S.R. Kurtz, A.G. Kibbler, and P. Faine, Proc. of the 21 st IEEE Photovoltaic Specialists Conf. (IEEE Press, NY, 1990) p.24.

3) D.J. Friedman, K.A. Bretness, S.R. Kurtz, C. Kramer, A.E. Kibbler, and J.M. Olson, AIP Conf. Proceedings, Ed. by H. Ullal and C.E. Witt, 1995, p. 150.

4) R. Venkatasubramanian, M.L. Timmons, P.R. Sharps, and J.A. Hutchby, Proc. of the 23rd IEEE Photovoltaic Specialists Conf. (IEEE Press, NY, 1993) p.691. 
Title:

Organization:

Contributors:
Porous Silicon Solar Cell Development

Spire Corporation, Bedford, MA 01730-2396

S.M. Vernon, Principal Investigator

Nader M. Kalkhoran, Project Scientist

\section{Introduction}

The goal of this research is to establish technology to produce very-high-efficiency solar cells for terrestrial photovoltaic applications. Our approach involves the formation of a layer of porous $\mathrm{Si}$ on a conventional Si substrate. In FY94 we demonstrated photovoltaic action in a porous $\mathrm{Si} \mathrm{p} / \mathrm{n}$ junction for the first time; this year we studied methods of forming improved ohmic contact to the porous Si material. Last year we also showed that porous Si can form an antireflection (AR) coating and front-surface passivation for single-crystal Si solar cells; this year we demonstrated that a porous Si surface layer can form an AR/surface passivation layer on polycrystalline material. Structures studied are shown in Figure 1.

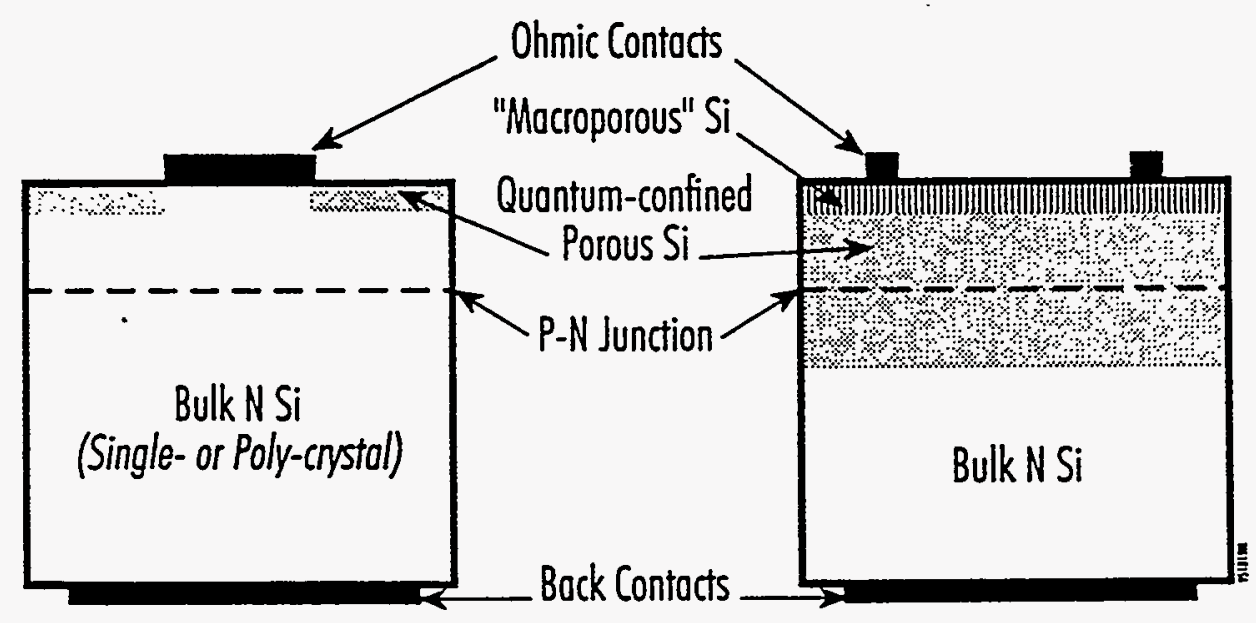

a) b)

Figure 1 (a) Cell structure with shallow porous Si surface layer; metal contact is to the bulk Si (single- or poly-crystalline) which contains the junction. (b) Cell structure with deep porous Si layer, containing the $\mathbf{p} / \mathbf{n}$ junction. Metal grid contact is to the porous Si. 


\section{Porous Si p/n Junction Solar Cells}

We made porous Si solar cells by ion-implanting boron into N-type crystalline Si wafers; junction depth is $\approx 0.75 \mu \mathrm{m}$. After annealing to remove implant damage, the Si was made porous, to a depth of several microns, (deep enough to penetrate the junction), by anodic etching under UV illumination. Top metallization was then applied, using either shadow-mask dots or photolithographically defined evaporated grid lines.

Although a photovoltaic effect was clearly observed (see Figure 2), several problems were noted. For the porous Si device shown, a photocurrent of only $2 \mu \mathrm{A}$ was generated; the light source was uncalibrated microscope illumination. The largest $V_{o c}$ measured was $\approx 0.3$ volts. The low value of $\mathrm{V}_{\mathrm{oc}}$ is consistent with the small photocurrent.

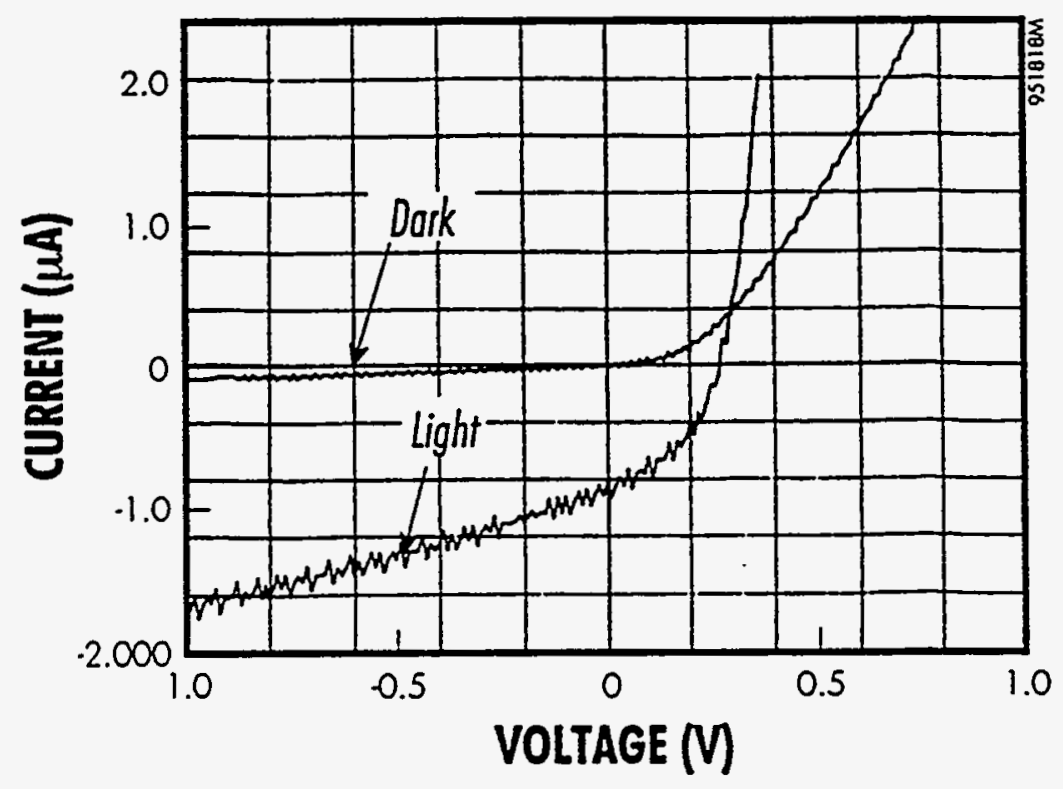

Figure 2 Current-voltage characteristics of porous Si diode exhibiting photovoltaic characteristics. Top ohmic contact consists of grid lines on the porous $\mathrm{Si}$ surface. Noise on curves is instrumental artifact.

\section{Improved Ohmic Contacts to Porous Si Junction with Electroplated Indium}

The starting wafers were $\mathrm{P}+\mathrm{Si}(\approx 0.01 \mathrm{ohm}-\mathrm{cm})$, with a thick $\mathrm{N}$ region $(\approx 0.1 \mathrm{ohm}-\mathrm{cm})$ grown epitaxially. The porous $\mathrm{Si}$ was made using deep electrochemical etching which penetrated the $\mathrm{p} / \mathrm{n}$ junction. During porous Si formation, a very thin layer of indium was deposited on the surface by in-situ electroplating. This layer coats all surfaces of the porous Si structure and improves lateral conduction. Onto this, $\approx 2000 \AA$ transparent indium-tin-oxide (ITO) was deposited (by ion-beamassisted deposition) as $6 \mathrm{~mm}\left(0.28 \mathrm{~cm}^{2}\right)$ round dots through a shadow mask. Back contact was made with full-area metallization. The $5-\mu \mathrm{m}$ junction depth is misleading. With a surface having deeply 'etched "pores," much of the junction area is a lot closer to the actual surface than $5 \mu \mathrm{m}$. 
Figure 3 shows an I-V measurement of this device. The "light" curve was tested under uncalibrated microscope illumination. As reported previously, the light and dark curves cross under forward bias, indicative of a voltage-dependent photogenerated current. The dark I-V curve shows an increase in current with increasing reverse bias, indicating a barrier to charge collection.

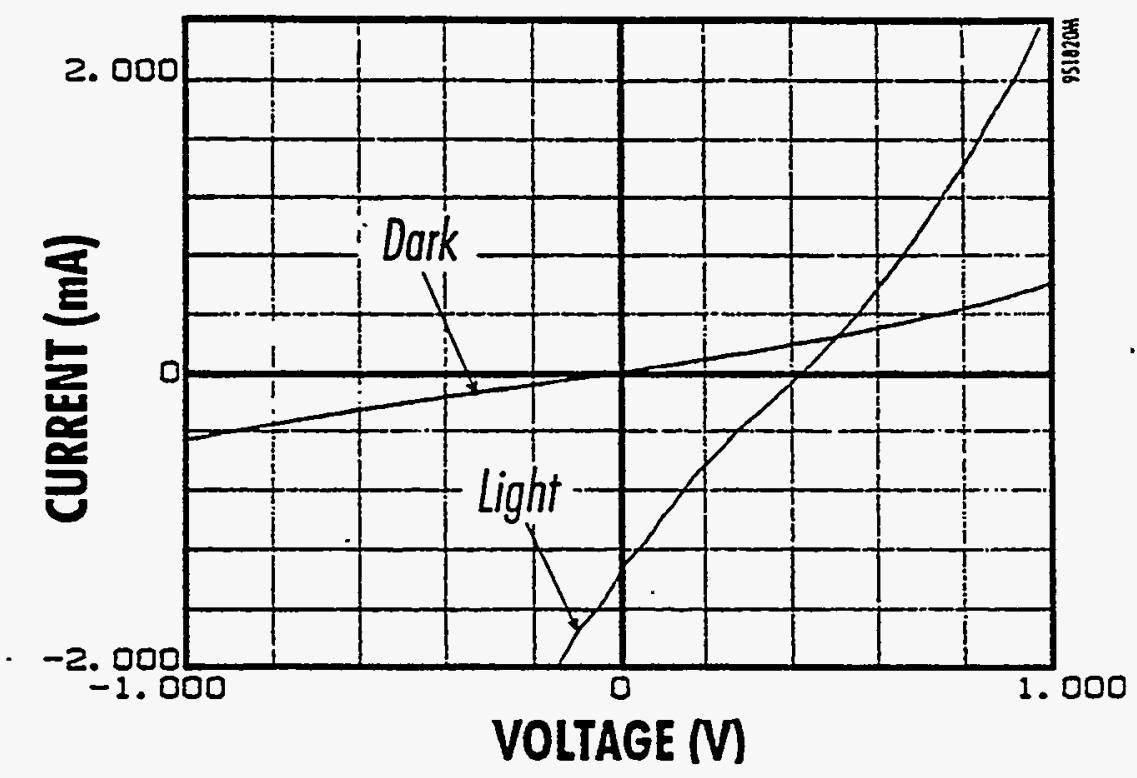

Figure 3 Dark and light I-V measured data for an ITO-contacted porous Si solar cell with a thin layer of electroplated In.

We see that the open-circuit voltage is 0.414 volts, and the short-circuit current is $1.4 \mathrm{~mA}$, or $5 \mathrm{~mA} / \mathrm{cm}^{2}$ current density. This is a three orders-of-magnitude increase over devices made previously without the electroplated indium layer. This large increase in photocurrent is attributed to improved conduction properties of the In-coated Si "wirelike" features. Similarly, the open-circuit voltage has increased from a previous value $\approx 0.3$ volts to $\approx 0.4$ volts.

\section{Porous Si AR Coating on Polycrystalline Si Solar Cells}

Polycrystalline $\mathrm{Si}$ is an important solar cell material, and texture etching is a significantly needed technology. Texture etching of polycrystalline $\mathrm{Si}$ cells to form an AR coating is not possible by conventional means; these etches rely on well-defined crystal directions to achieve the anisotropy which produces the textured, light-trapping surface morphology. To address this need, we have performed experiments to achieve a non-reflective, light-absorbing surface on polycrystalline $\mathrm{Si}$. The material used in our study is cast and sliced P-type polycrystalline Si. The average grain size is $\approx$ $5 \mathrm{~mm}$. A thin, porous, texturized surface layer is formed by chemical etching in hydrofluoric acid (HF) - nitric acid - water (1:3:5), or alternatively by electrochemical etching in HF-ethanol with an applied bias. The most nonreflective surface was made by electrochemical etching at a current density of $\approx 100 \mathrm{~mA} / \mathrm{cm}^{2}$ for a few minutes. Etching for a longer time at lower current density results in a less black surface. 
Preliminary experiments showed that we can greatly reduce surface reflectivity on polycrystalline $\mathrm{Si}$ wafers by forming a thin porous-Si layer by chemical etching. Next we showed that a porous surface layer on a polycrystalline solar cell actually improves performance by acting as an ARcoating/surface-passivation layer.

A polycrystalline Si solar cell without AR coating was used in this trial. On the wafer, a porous surface was formed, being careful not to penetrate the junction. This shallow etch did improve all solar cell parameters, but only slightly; the very shallow etch made the surface slightly less reflective than the bare wafer, but not nearly as black as for a longer etch treatment.

We have learned that the porous-Si surface treatment needs to be $\approx 0.5 \mu \mathrm{m}$ deep to make a good AR coating with low reflectivity. For this design, the junction depth needs to be initially $\approx 1 \mu \mathrm{m}$; on such cells, we feel that we could optimize the porous surface process to yield reasonable efficiency gains. - By consuming the top $\approx 0.5 \mu \mathrm{m}$ with the porous etch treatment, the final junction depth would remain at essentially $0.5 \mu \mathrm{m}$, thus preserving the basic cell design.

\section{Conclusions/Summary}

We have shown that porous $\mathrm{Si}$ is an interesting material for solar cell applications. We have seen photovoltaic action in porous Si p/n junctions, with short-circuit current density up to $5 \mathrm{~mA} / \mathrm{cm}^{2}$ and open-circuit voltage over 0.4 volts (with uncalibrated microscope illumination). The major aspects that we have demonstrated are the following:

- Porous silicon active layers have been made to show photovoltaic action

- Porous silicon surface layers can act as $\mathrm{AR}$ coatings to improve the performance of single-crystal silicon solar cells

- Porous silicon surface layers can act as AR coatings on polycrystalline silicon solar cells

\section{Publications}

"High Performance Porous Silicon Solar Cell Development," S.M. Vernon, N.M. Kalkhoran, and W.D. Halverson, Proc. of $1^{\text {st }}$ World Conference on Photovoltaic Energy Conversion, Wailkoloa, HI, December 1994.

"High Performance Porous Silicon Based Photovoltaic Cells," N.M. Kalkhoran, S.M.Vernon, H.P. Maruska, and W.D. Halverson, presented at 1994 Materials Research Society Meeting, Symposium on Microcrystalline and Nanocrystalline Semiconductors, Boston, MA, December 1994. 
Title:

Organization:

Contributors:
Thin Film Silicon by Constitutional Supercooling

State University of New York at Buffalo

Center for Electronic and Electro-optic Materials

Department of Electrical and Computer engineering

Bonner Hall, Amherst, New York 14260

W.A. Anderson, program manager; D.D.L. Chung, J. Coleman, K. Etemadi, principal investigators; X. Gu, N. Sridhar, R. Wallace and $\mathrm{J}$. $\mathrm{Y}_{1}$, research assistants

\section{Objective}

This project was conducted to design and fabricate a low-cost, thin-film, silicon solar cell having an efficiency of about $15 \%$. Thin-film Si solar cells have been of interest for many years due to the availability of $\mathrm{Si}$, the non-toxic nature of $\mathrm{Si}$ and the advanced knowledge about $\mathrm{Si}$. a-Si:H is a "true" thin-film approach but suffers from instability and relatively low efficiency. Our goal is to develop a thin-film Si-formation method and a solar cell design providing high efficiency, low-cost and long-term stability.

\section{Approach}

Firstly, the a-Si:H/crystalline (c)-Si solar cell was designed by the PC1D code. This design was then fabricated using glow discharge-deposition of a-Si:H and later, d.c. sputtering of a-Si:H. Perfection of this process is to be followed by application to thin-film poly-Si.

Secondly, thin-film poly-Si is deposited onto Mo-metal by compositional supercooling (CS). This involves depositing an $\mathrm{SiO}_{2}$ buffer layer, a $\mathrm{Sn}$ or $\mathrm{In} / \mathrm{Ti}$ seeding layer, and then sputtering $\mathrm{Si}$ from a wafer target. With the substrate at $600^{\circ} \mathrm{C}$, the Si-metal melt remains on the surface while poly-Si grows on the substrate as shown in Figure 1.

Several related tasks include hydrogenation to passivate grain boundaries, laser processing to improve electrical properties of thin Si films, annealing studies to understand hydrogen bonding and various test procedures.

\section{Results}

Thin film poly-Si has been grown on Mo substrates by Compositional Supercooling (CS) growth [1,2]. The method, illustrated in Figure 1, has produced large-grain poly-Si having carrier mobility exceeding $100 \mathrm{~cm}^{2} / \mathrm{V}$-s and carrier lifetime of $8 \mu \mathrm{s}$. These data are contained in Table 1 . The process is very controllable as evidenced by mobility variation with prelayer thickness of Figure 2, mobility variation with substrate temperature of Figure 3 and conductivity change with prelayer thickness. The carrier lifetime plot gives a value of $8.5 \mu \mathrm{s}$. Table 1 shows the control of the poly-Si film by changing the seeding layer ( $\mathrm{Sn}$ vs. In/Ti). Some films are sensitive to ECR hydrogenation as shown in Figure 4. At this point, use of the film is hindered by non-uniformity and inclusion of some seeding metal as an impurity in the $\mathrm{Si}$. Other seeding layers need to be considered. 
Heterojunction solar cell fabrication has been done on crystalline wafers to develop the a$\mathrm{Si}: \mathrm{H} /$ poly-Si fabrication process. The PC1D model predicts a $17 \%$ efficiency for $0.1 \mu \mathrm{m}$ a-Si:H on $30 \mu \mathrm{m}$ poly-Si thin film. Thickness of the a-Si is important as illustrated in Figure 5 . The a-Si:H has been deposited by glow discharge in the past and more recently by d.c. magnetron sputtering. The substrate must be dipped in HF prior to a-Si deposition to passivate dangling bonds and remove oxides. This is illustrated in Table 2 which shows progress made during this year. The photovoltaic data of Figure 6 illustrate some of the good results obtained recently. An A/R coating was not used here. We have found that sputtering conditions greatly influence results. More work is needed in applying the solar cell fabrication process to CS thin-film Si.

Progress has also been made on crystallizing a-Si:H/Mo using iso-thermal furnace anneal, anneal in a vacuum or laser scan[3]. A laser scan technique was developed which causes $H$ to be retained in the grain boundaries [4]. Much has been learned about hydrogen evolution upon heating of a$\mathrm{Si}: \mathrm{H}$. The low temperature heating of a-Si:H has led to improved photovoltaic performance.

\section{Conclusions and Recommendatinos}

Fabrication of the heterojunction a-Si:H/c-Si cell is well on the way to achieving the predicted efficiency of $17 \%$. Key fabrication steps are an $\mathrm{HF}$ dip of the c-Si prior to a-Si:H deposition and a thin $(0.03 \mu \mathrm{m})$ a-Si:H layer. The CS growth technique has produced thin-film poly-Si on Mo with carrier mobility exceeding $100 \mathrm{~cm}^{2} / \mathrm{V}$-s and lifetime of $8 \mu \mathrm{s}$. Properties of the poly-Si can be controlled by the deposition process and grain size can exceed $20 \mu \mathrm{m}$. Electrical properties may be improved by ECR hydrogenation. We have developed a laser crystallization method which retains hydrogen in the grain boundaries.

Further studies in heterojunction fabrication are needed to further improve the a-Si:H/c-Si interface and apply the design to CS silicon. For the CS process, other seeding metals need to be explored to improve the photoconductivity of the CS film.

\section{Acknowledgment}

Financial support was provided by National Renewable Energy Laboratory (John Benner) and New York State Energy Research and Development Authority (Jennifer Harvey). Ken Jones and Richard Ahrenkiehl from NREL assisted with some diagnostics.

\section{References}

1. R.L. Wallace and W.A. Anderson, "Thin Film Polycrystalline Si by CS Solution Growth Technique", Materials Research Society Fall Meeting, Boston, Nov. 28-Dec. 2, 1994.

2. R.L.Wallace and W.A. Anderson, "Thin Film Polycrystalline Si by A Novel Solution Growth Technique", First World Conference on Photovoltaic Energy Conversion, Waikoloa, Hawaii, Dec. 5-9, 1994.

3. J. Yi, R.L. Wallace, B. Jagannathan, X. Gu, K. Etemadi and W.A. Anderson, "An Analysis of Thin Film Silicon for Photovoltaic Application", First World Conference on Photovoltaic Energy Conversion, Waikoloa, Hawaii, Dec. 5-9, 1994.

4. N. Sridhar, D.D.L. Chung and W.A. Anderson, "Effect of Deposition Temperature on the Photoresponse of Crystallized Hydrogenated Amorphous Silicon Films", Materials Research Society Fall Meeting, Boston, Nov. 28-Dec. 2, 1994. 


\section{Table 1. PROPERTIES OF CS THIN FILM SILICON}

Conductivity type

Grain size $(\mu \mathrm{m})$

Carrier concentration $\left(\mathrm{cm}^{-3}\right)$

Dark conductivity $(\Omega-\mathrm{cm})^{-1}$

Darrier mobility $\left(\mathrm{cm}^{2} / \mathrm{V}-\mathrm{s}\right)$

Carrier mobility $\left(\mathrm{cm}^{2} / \mathrm{V}-\mathrm{s}\right)$

after hydrogenation

Lifetime $(\mu \mathrm{s})$

Photoconductivity $(\Omega-\mathrm{cm})^{-1}$ @ $35 \mathrm{~mW} / \mathrm{cm}^{2}$
Sn Prelaver

$\mathrm{N}$

$\leq 1$

$10^{18}$

$1-10$

$60 @ 300 \mathrm{~K}$

140@300K

$\sim 8$
$10^{-3}$ $\underline{\text { In/Ti Prelaver }}$

$\mathrm{P}$

20 or more

$10^{16}$

$0.1-2$

$100 @ 300 \mathrm{~K}$

$100 @ 300 \mathrm{~K}$

$\sim 0.3$

$10^{-5}$

Table 2. PROGRESS IN HETEROJUNCTION SOLAR CELL FABRICATION*

\begin{tabular}{|l|l|c|c|c|c|}
\hline Sample & Substrate & $\begin{array}{c}\text { Special Design } \\
\text { or Fab-Condition }\end{array}$ & $\begin{array}{c}\mathbf{V}_{\mathrm{oc}} \\
(\mathrm{V})\end{array}$ & $\begin{array}{c}\mathrm{J}_{\mathbf{s e}} \\
\left(\mathrm{mA} / \mathrm{cm}^{2}\right)\end{array}$ & $\begin{array}{c}\text { Fill } \\
\text { Factor }\end{array}$ \\
\hline$\# 1 \mathrm{n}^{+}-\mathrm{i}-\mathrm{p}$ & $1-10 \Omega-\mathrm{cm}(100)$ & $1000 \AA$ i-layer & 0.46 & 1.58 & 0.36 \\
\hline$\# 2 \mathrm{n}^{+}-\mathrm{i}-\mathrm{p}$ & $1-10 \Omega-\mathrm{cm}(100)$ & $1000 \AA$ i-layer HF dip & 0.48 & 23.5 & 0.39 \\
\hline$\# 3 \mathrm{i}-\mathrm{p}$ & $1-10 \Omega-\mathrm{cm}(100)$ & $300 \AA$ i-layer HF dip & 0.53 & 30.0 & 0.60 \\
\hline
\end{tabular}

NOTE: \#1 was by glow discharge of a-Si:H

\#2 was by glow discharge of a-Si:H following the $\mathrm{HF}$ dip

\#3 was sputtered a-Si:H. HF dip used just before depositing a-Si:H

* All data without an antireflection coating.

1. Prelayer is formed on substrate surface, about 400nm thick.

2. Temperature is stabilized, and the Si spurtering starts. The solution layer quickly saburates, excess Si precipitates onto substrate surface.

3. Si sputtering continues until desired film thickness is obtained. Residual meit is removed by wet etching, leaving the film exposed.

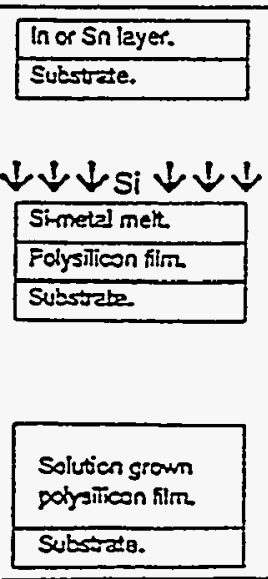

Figure $1 \mathrm{CS}$ growth mechanism.

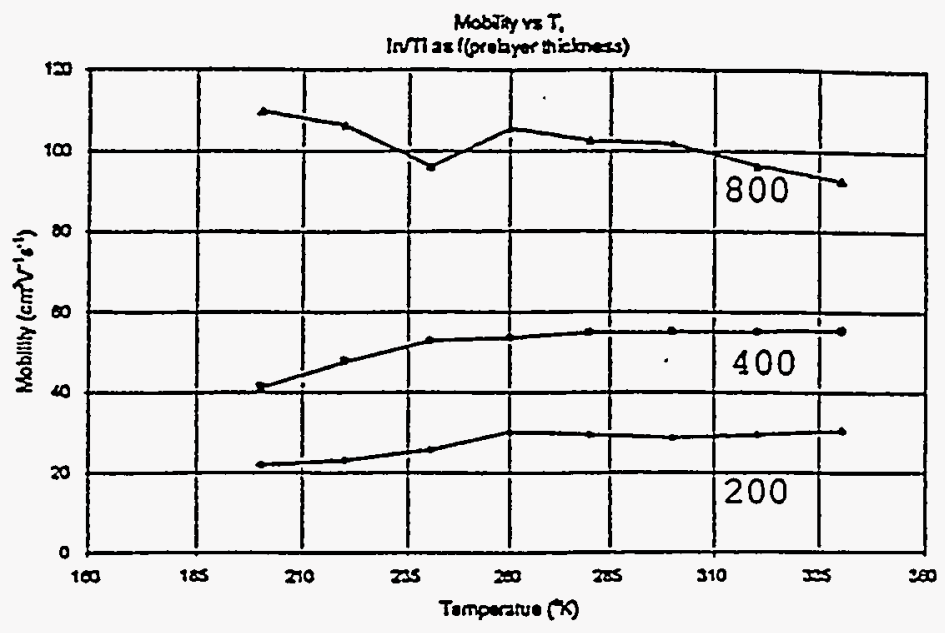

Figure 2 Hall mobility $v s$. T. for increasing In prelayer thicknesses $(200,400,800 \mathrm{~nm})$. 


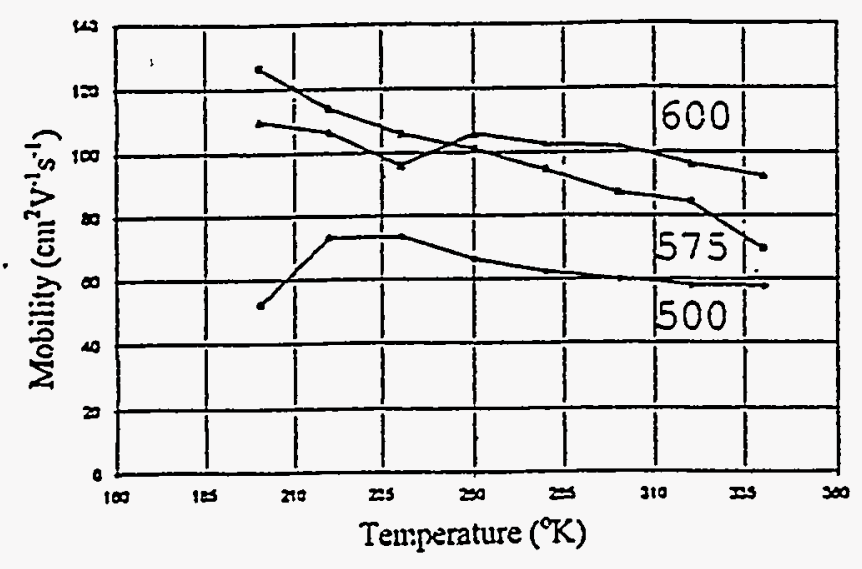

Figure 3 Increase in mobility with increased deposition temperature $\left(500,575,600^{\circ} \mathrm{C}\right)$ for $\operatorname{In} / \mathrm{Ti}$.

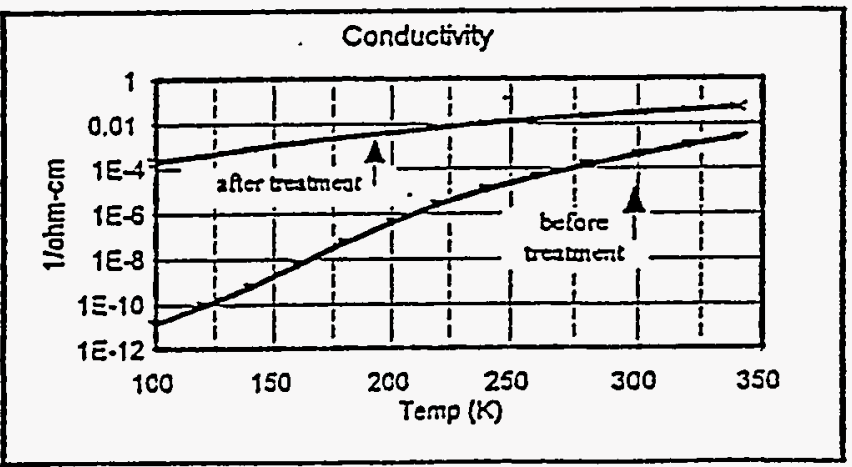

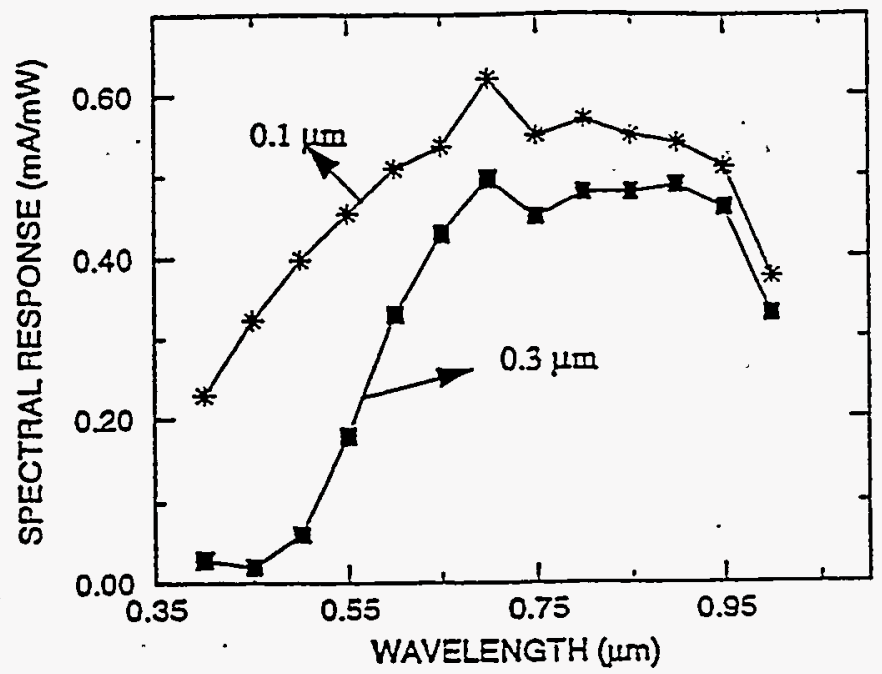

Figure 5 Spectral response $v s$ thickness for a-Si:H/c-Si with an area of $0.36 \mathrm{~cm}^{2}$.

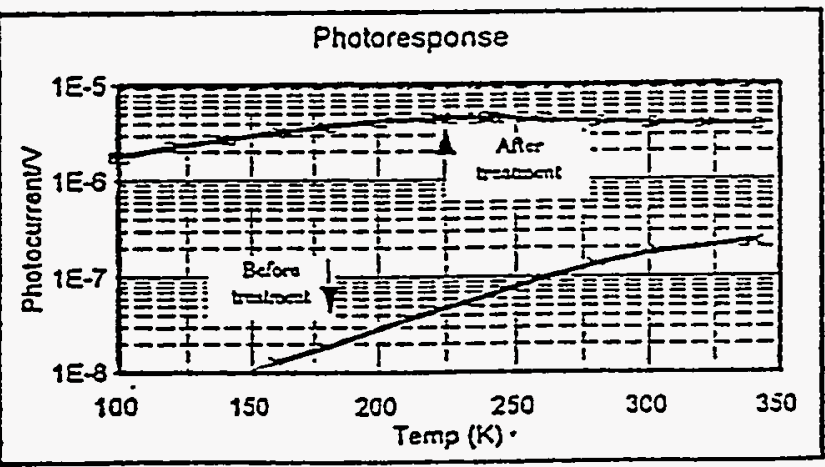

Figure 4 Effect of microwave hydrogenation on film conductivity and photoresponses.

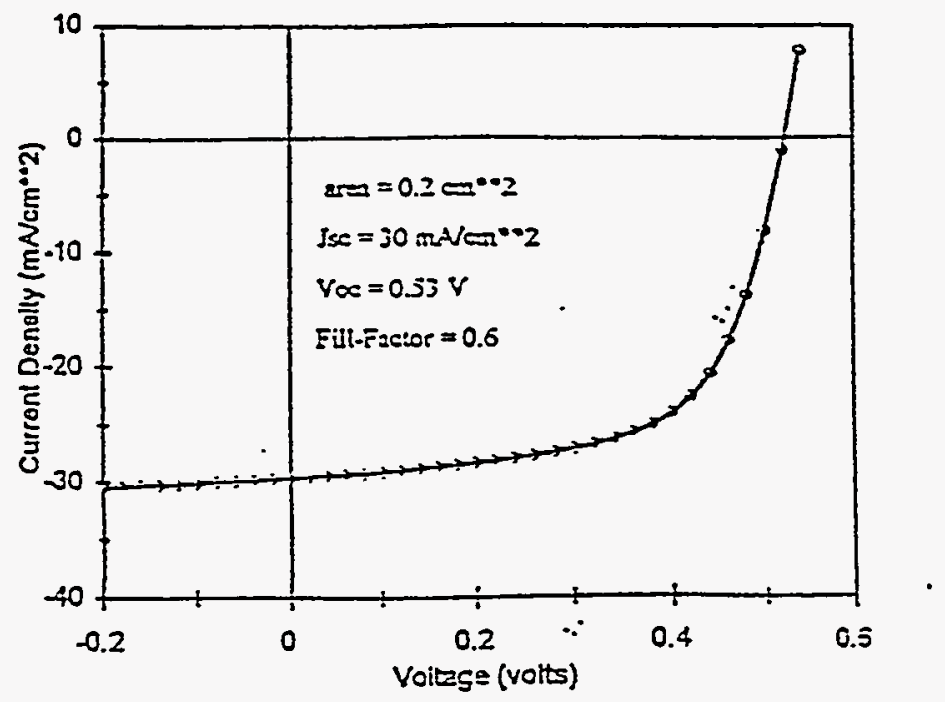

Figure 6 Photoresponse of the a-Si/c-Si cell fabricated at a sputter power of $90 \mathrm{w}$. 
Title: THEORETICAL ANALYSIS OF
HYDROGEN-VACANCY-IMPURITY
FORMATION AND DISSOCIATION

Organization:

\author{
Physics Department, Texas Tech University \\ Lubbock, TX 79409-1051
}

Contributors:

Stefan K. Estreicher, principal investigator

\title{
Objectives
}

Poly-Si solar cells often benefit substantially from the passivation by $\mathrm{H}$ of dangling bonds, at vacancies, dislocations, grain boundaries, and other such defects. While the passivation reaction itself is well understood ( $H$ binds to a dangling bond, thus removing its electrical activity), the processes leading to such a reaction are not. The diffusivity of $\mathrm{H}$ in vacancy-rich material is much greater[1-3] than in $\mathrm{FZ}$ - or $\mathrm{CZ}-\mathrm{Si}$, suggesting that vacancies play an active role in the diffusion of $H$. Further, Kaufmann source implantation around 220 to $250^{\circ} \mathrm{C}$ results in more efficient passivation[4] with $\mathrm{NH}_{3}$ than $\mathrm{H}_{2}$ ions, suggesting that $\mathrm{N}$ may also be important.

The objectives of this research is to investigate theoretically the details of a number of interactions involving $\mathrm{H}$, vacancies, and potentially relevant impurities such as $\mathrm{N}$. The results obtained in previous years dealt with $\mathrm{H}$-vacancy complex formation and diffusion, and the calculation of IR active vibrational modes for experimental identification. $[5,6]$ Ongoing research focuses on vacancy-vacancy interactions and on the role of $N$ in the passivation process.

\section{Theoretical Approach}

The equilibrium geometries, electronic configurations, dissociation energies, diffusion paths, and activation energies for diffusion are calculated using approximate $a b$-initio Hartree-Fock (HF), $a b$-initio HF, and density functional (DF) methods in molecular clusters containing up to 44 host atoms. The dynamics of complex formation and dissociation, the vibrational frequencies, and the diffusion properties are obtained from the $a b$-initio tight-binding molecular dynamics (MD) method developed by Sankey et al. $[7,8]$ In the MD approach, the host crystal is represented by periodic supercells of 64 atoms. Calculations using substantially larger cells are in the testing stage. For details about the theoretical techniques, their strengths and limitations, see Ref. [9].

The HF and MD codes ran on a dedicated IBM RS6000/375 at Texas Tech University as well as on various machines at the Swiss Center for Scientific Computing.

\section{Stretching Vibrational Modes of the Hydrogen-Vacancy Complexes}

Following last year's study of the equilibrium and dynamic properties of the vacancy and the four $\mathrm{H}$-vacancy complexes, we have calculated the $\mathrm{H}$ and $\mathrm{D}$ stretching vibrational modes using MD simulations (at $300 \mathrm{~K}$ ). [6] Subsequent measurements[10] of these modes (at $77 \mathrm{~K}$ ) have confirmed that our approach is appropriate. The calculated values systematically 
overestimate the $H$ stretching modes by $110 \mathrm{~cm}^{-1}$ and the $D$ ones by $30 \mathrm{~cm}^{-1}$. Table I summarizes our results. It also contains the results of a static calculation by $R$. Jones.[10]

TABLE I: Comparison of the experimental[10] (at $77 \mathrm{~K}$ ) and theoretical H-vacancy and D-vacancy stretching vibrational modes, in $\mathrm{cm}^{-1}$. The calculations are (a) by $\mathrm{R}$. Jones (static density functional calculations), [10] and (b) our results (MD simulations at $300 \mathrm{~K}$ ).

\begin{tabular}{l|cllllllll}
\hline defect & $\left\{\mathrm{V}, \mathrm{H}_{1}\right\}$ & $\left\{\mathrm{V}, \mathrm{H}_{2}\right\}$ & $\left\{\mathrm{V}, \mathrm{H}_{3}\right\}$ & $\left\{\mathrm{V}, \mathrm{H}_{4}\right\}$ & $\left\{\mathrm{V}, \mathrm{D}_{1}\right\}$ & $\left\{\mathrm{V}, \mathrm{D}_{2}\right\}$ & $\left\{\mathrm{V}, \mathrm{D}_{3}\right\}$ & $\left\{\mathrm{V}, \mathrm{D}_{4}\right\}$ \\
\hline theory (a) & 2248 & 2316 & 2318 & 2319 & 1613 & 1658 & 1661 & 1664 \\
theory (b) & 2168 & 2268 & 2301 & 2334 & 1550 & 1601 & 1618 & 1634 \\
experiment & 2068 & 2144 & 2191 & 2222 & 1507 & 1564 & 1594 & 1617 \\
\hline
\end{tabular}

Ongoing MD studies include vacancy-vacancy interactions, which will be followed by calculations of IR active $\mathrm{H}$ and $\mathrm{D}$ vibrational modes in divacancies and larger complexes. The immediate goal is to determine which are the energetically most favorable vacancy aggregates and how $\mathrm{H}$ interacts with them.

\section{Nitrogen-Hydrogen Pair Diffusion}

Interstitial $\mathrm{N}$ in $\mathrm{Si}$ is a very slow diffusing deep-level impurity which tends to form pairs with a variety of impurities. Substitutional $N$ should be a trap for interstitial $\mathrm{H}$ since it will be 3-fold coordinated, leaving one Si dangling bond. I therefore considered the possibility that it is a molecule containing $\mathrm{N}$ and/or $\mathrm{H}$ atoms could diffuse. The most likely candidates are $\mathrm{H}_{2}, \mathrm{~N}_{2}$, and $\mathrm{NH}$. The latter is the smallest closed-shell molecule containing both $\mathrm{N}$ and $\mathrm{H}$. Its equilibrium configuration, electronic structure, diffusion path, and activation energy were calculated at three levels of theory: approximate $a b$-initio HF, ab-initio HF, and DF in molecular clusters. The properties of $\mathrm{NH}$ were compared to those of $\mathrm{H}_{2}$ and $\mathrm{N}_{2}$. The calculations show that $\mathrm{NH}$ has a doubly occupied deep level in the gap. Therefore, the calculations were repeated for $\mathrm{NH}^{++}$, which is the likely charge state in $p$-type material.

I caution the reader that many calculations are still under way. Only preliminary results are given below. Further, the calculations do not address the issues of the solubility of $\mathrm{NH}$ in $\mathrm{Si}$ or the surface conditions required for the molecule to penetrate into the bulk.

$\mathrm{NH}^{0}$ and $\mathrm{NH}^{++}$form a 2-electron $\mathrm{N}-\mathrm{H}$ bond. The $\mathrm{N}$ atom has another four (two) valence electrons in the neutral (doubly positive) charge state. In $\mathrm{NH}^{0}$, two electrons form a (non-bonding) lone pair and the other two show only a weak chemical activity with the host crystal. In the case of $\mathrm{NH}^{++}$, the $\mathrm{N}$ atom also forms a two-electron bond with $\mathrm{H}$ and shows a more pronounced covalent overlap with several host atoms. In both charge states, the molecule is not on the trigonal axis but moves considerably off-center to maximize its overlap with the crystal. Figure 1(a) shows two adjacent lowest-energy configurations of $\mathrm{NH}^{0}$ and (b) the saddle point. $\mathrm{NH}^{++}$has $\mathrm{N}$ even farther from the $\mathrm{T}$ site. In contrast, $\mathrm{N}_{2}$ and $\mathrm{H}_{2}$ remain along the tetrahedral - hexagonal - tetrahedral (T-H-T) axis. Note that the molecules were not assumed to be in the $\{110\}$ plane, but 3-dimensional gradient optimizations with no symmetry assumption found them to lie in that plane. 
FIGURE I: Two adjacent equilibrium (a) and the saddle point (b) configurations of $\mathrm{NH}^{0}$ in the $\{110\}$ plane in $\mathrm{Si}$. The $\mathrm{T}$ (triangles) and $\mathrm{H}$ (squares) interstitial sites are shown, and the $\mathrm{T}-\mathrm{H}-\mathrm{T}$ axis is dotted. The host atoms are at their perfect lattice positions. $\mathrm{NH}^{++}$has $\mathrm{N}$ farther from the $\mathrm{T}$ site.
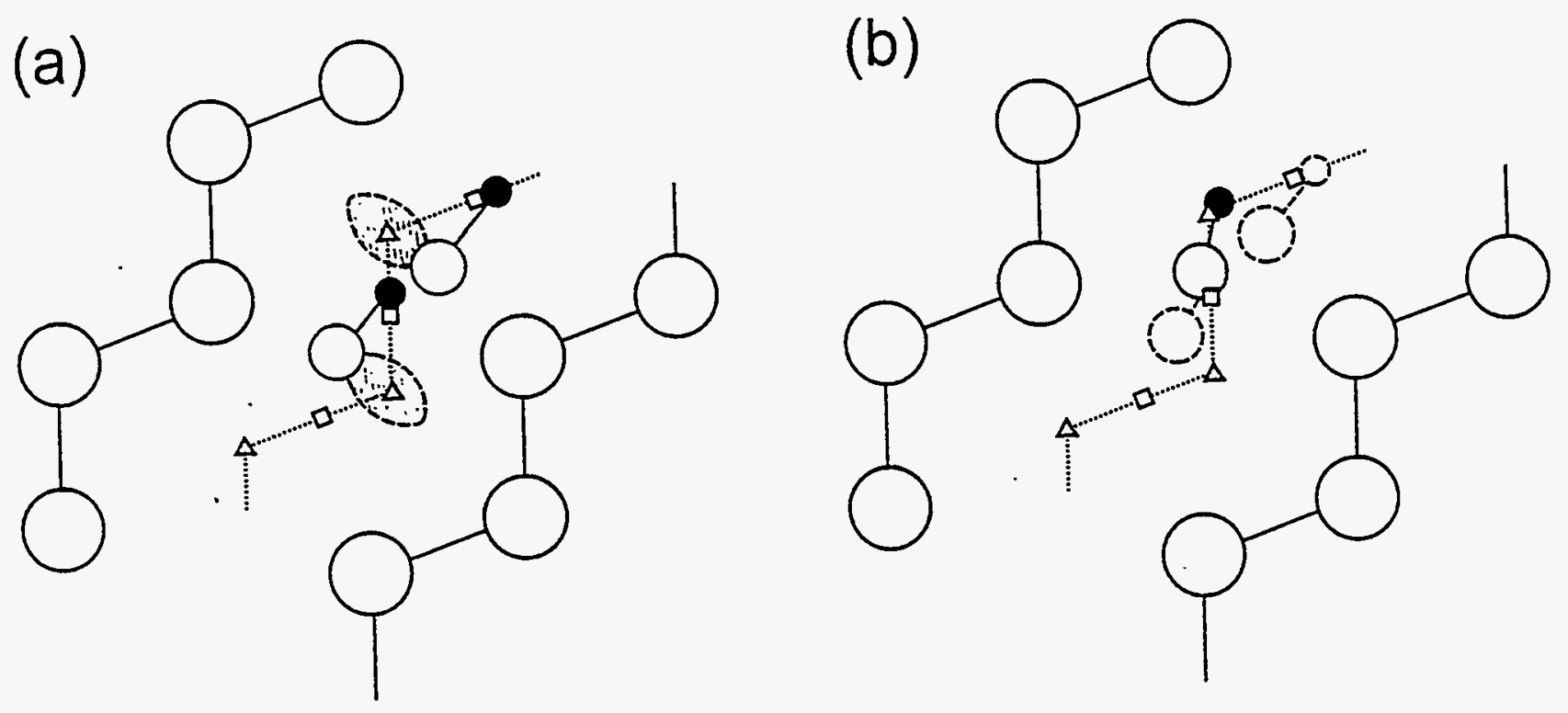

The saddle point for diffusion of $\mathrm{NH}$ was obtained as follows. A line connecting the coordinates of $\mathrm{N}$ atoms at two adjacent equilibrium sites was drawn. As $\mathrm{N}$ was moved along this line, its coordinates were optimized on the bisector to that line, and those of $\mathrm{H}$ optimized with no restrictions. This leads to the lowest-energy path between adjacent equilibrium minima of $\mathrm{NH}$ with the restriction that the saddle point has $\mathrm{N}$ in the $\{110\}$ plane.

Both $\mathrm{H}_{2}$ and $\mathrm{N}_{2}$ move along the $\mathrm{T}-\mathrm{H}-\mathrm{T}$ axis. Their diffusion involves two activation energies. First, the molecule must move across the $\mathrm{H}$ site, then it must rotate by $\mathrm{a}$ tetrahedral angle to face the next $<111>$ direction. The activation energy for translation is the higher of the two for both molecules considered here and is the only one given below.

Table II gives the activation energies for diffusion of $\mathrm{H}_{2}, \mathrm{~N}_{2}$, and $\mathrm{NH}^{0}$ calculated at the $\mathrm{HF}$ and DF levels in an unrelaxed 30 host-atom cluster. Preliminary calculations indicate that the activation energy for diffusion of $\mathrm{NH}^{++}$is lower than that of $\mathrm{NH}^{0}$. Note that these activation energies are upper limits for three reasons. First, all the calculations used relatively small (split-valence) basis sets: It is known that increasing the basis set size benefits more the saddle point than the equilibrium configuration. Second, the motion of the molecules was restricted to the $\{110\}$ plane: Although the equilibrium configurations are indeed in that plane, it is possible that the true saddle point lies somewhat off of if, which would results in lower activation energies. Third, lattice relaxations and distortions were not allowed: They (almost) always lower the calculated activation energies (if a few $\mathrm{Si}$ atoms adjacent to $\mathrm{NH}^{0}$ in the saddle point configuration are allowed to relax by a 0.05 to $0.08 \AA$, the activation energy drops to around 0.65 to $0.70 \mathrm{eV}$ ). 
TABLE II: Activation energies (in eV) for diffusion of $\mathrm{H}_{2}, \mathrm{~N}_{2}$, and $\mathrm{NH}^{0}$ in the rigid crystal (see text) and equilibrium bond lengths (in $\AA$ ). Ongoing calculations suggest that the activation energy of $\mathrm{NH}^{++}$is lower than that of $\mathrm{NH}^{0}$.

\begin{tabular}{|c|c|c|c|c|c|c|}
\hline & \multicolumn{2}{|c|}{$\mathrm{H}_{2}$} & \multicolumn{2}{|c|}{$\mathrm{N}_{2}$} & \multicolumn{2}{|c|}{$\mathrm{NH}$} \\
\hline theory & $\mathrm{H}-\mathrm{H}$ & $E_{a}$ & $\mathrm{~N}-\mathrm{N}$ & $E_{a}$ & $\mathrm{~N}-\mathrm{H}$ & $\overline{E_{a}}$ \\
\hline $\begin{array}{l}\text { Hartree-Fock } \\
\text { density-functional }\end{array}$ & $\begin{array}{l}0.70 \\
0.73\end{array}$ & $\begin{array}{l}1.61 \\
1.44\end{array}$ & $\begin{array}{l}1.11 \\
1.20\end{array}$ & $\begin{array}{l}2.55 \\
2.42\end{array}$ & $\begin{array}{l}1.05 \\
1.06\end{array}$ & $\begin{array}{l}0.96 \\
0.82\end{array}$ \\
\hline
\end{tabular}

\section{-Passivation of Vacancies with NH}

When placed near a vacancy, the $\mathrm{NH}^{0}$ molecule dissociates (with zero activation energy). The $\mathrm{N}$ atom binds to three $\mathrm{Si}$ dangling bonds and $\mathrm{H}$ to the fourth one, pointing toward the center of the vacancy. This contrasts with the case of the $\{P, H\}$ pair, which has $\mathrm{H}$ antibonding to the host atom. The complex with $\mathrm{N}$ and $\mathrm{H}$ in the vacancy has no deep level in the gap. Thus, $\mathrm{NH}^{0}$ should passivate vacancies very efficiently since it takes four $\mathrm{H}$ interstitials to achieve the same result. This is particularly relevant to vacancy-rich material such as poly-Si. The interactions of $\mathrm{NH}^{++}$with vacancies have not yet been calculated.

\section{References}

1. B.L. Sopori, MRS Proc. 262, 407 (1992).

2. B.L. Sopori, K. Jones, and X.J. Deng, Appl. Phys. Lett. 61, 2560 (1992).

3. B.L. Sopori, X.J. Deng, J.P. Benner, A. Rohatgi, P. Sana, S.K. Estreicher, Y.K. Park, and M.A. Roberson, Proc. First World PV conf. vol.II, IEEE Press (Piscataway, NJ, 1995), p. 1915.

4.' F. Wald, private communication.

5. M.A. Roberson and S.K. Estreicher, Phys. Rev. B 49, 17040 (1994).

6. Y.K. Park, S.K. Estreicher, C.W. Myles, and P.A. Fedders, Phys. Rev. B 52, 1718 (1995).

7. O.F. Sankey and D.J. Niklewski, Phys. Rev. B 40, 3979 (1989).

8. O.F. Sankey, D.J. Niklewski, D.A. Drabold, and J.D. Dow, Phys. Rev. B 41, 12750 (1990).

9. S.K. Estreicher, Mat. Sci. Engr. R 14, 319-412 (1995).

10. B. Bech Nielsen, L. Hoffmann, M. Budde, R. Jones, J. Goss, and S. Öberg, Proc. $18^{\text {th }}$ Int. Conf. Defects Semic., Mat. Sci. Forum (in press), and B. Bech Nielsen, E-MRS Proc., Spring 1995, paper N-VII.5 (in press). 
Title:

Organization:

Contributors:
Identification and Control of Lifetime-Reducing Defects in Polycrystalline Silicon Photovoltaic Materials

\author{
Department of Materials Science and Mineral Engineering \\ University of California, Berkeley \\ Berkeley, California
}

Eicke R. Weber, principal investigator;

S.A. McHugo and $\mathrm{H}$. Hieslmair

\section{Introduction}

Our past work has focused on identification of lifetime-limiting defects as well as phosphorus gettering of as-grown and iron contaminated solar grade silicon. This work indicated dislocations and microdefects are the lifetime-limiting defects. Additional research has shown the minority carrier recombination activity of both dislocations and microdefects increases with metallic impurity decoration and/or precipitation. Based on these results, we had pursued gettering studies of solar grade silicon. The preliminary results of the phosphorus gettering studies demonstrated that low lifetime as-grown multisilicon regions, as well as intentionally contaminated multisilicon, responds poorly to gettering treatments.

Our most recent experiments have been primarily directed towards aluminum gettering in solar grade silicon. Aluminum backside segregation gettering has been shown 1,2,3 to increase minority carrier diffusion lengths and PV cell efficiencies. These increases have been attributed to the gettering of impurity metals in the aluminum layer as supported by other studies ${ }^{4}$. Therefore, we had hoped to demonstrate improvement in solar grade silicon with this treatment and investigate a relationship between gettering response, structural defects and as-grown lifetime. Our results provide evidence that gettering in solar grade silicon is significantly different than in Integrated Circuit (I.C.) grade silicon. The results suggest gettering of solar grade silicon is limited by two separate mechanisms which are characterized by their gettering response. The first gettering response is distinguished by a lifetime degradation with increasing gettering temperature. This is most likely due to a high as-grown contamination level in these regions. The second type of gettering response is that of invariance to gettering temperature. Here, lifetime is not limited by some undetermined mechanism, possibly slow diffusing impurities or undecorated microdefects.

\section{Results \& Discussion}

We have conducted a series of quantitative gettering experiments. Aluminum backside gettering of as-grown and iron contaminated float-zoned (FZ) silicon, I.C. grade CZ silicon, PV grade CZ silicon, and PV grade ribbon and cast ingot silicon samples were investigated. The minority carrier diffusion length, as measured by the surface photovoltage (SPV) technique, has been used to quantify the gettering response of these materials. Structural characterization was determined with a Schimmel etch 5 .

$2.5 \mu \mathrm{m}$ of $\mathrm{Al}$ was sputtered onto each sample under high vacuum, $\approx 2 \times 10^{-7}$ torr. $\mathrm{Al}$ gettering was performed at $800,850,900$ and $950^{\circ} \mathrm{C}$ in a nitrogen ambient for 1,3 or 5 hours on a number of samples from each material type. The gettering was performed in a clean furnace used for daily I.C. device fabrication. Figure la shows the gettering response of I.C. grade single crystal silicon at various temperatures for 3 hours. We see an optimal gettering temperature for this gettering treatment. This is as predicted by the Kang and Schroder ${ }^{6}$ model which anticipates the gettering response to be limited by two mechanisms, producing an optimal gettering temperature. The first mechanism is impurity diffusion which produces an increased gettering response with increasing temperature. The second mechanism originates from the temperature dependence of the segregation coefficient between the gettering layer and the silicon which yields an increased response with decreasing temperature. The temperature dependent segregation coefficient goes as: 


$$
S=\frac{1}{K} \propto 1+C \cdot \exp \left[\left(E_{S i}^{i m p}-E_{\text {getser }}^{i m p}\right) / k T\right]
$$

Eq. 1

However, as shown in Figure $1 b$, a much different gettering response is observed for single crystal solar grade silicon. Some silicon materials degrade with increased gettering temperature while others remain invariant, depending on the manufacturer. Preferential etching of these samples
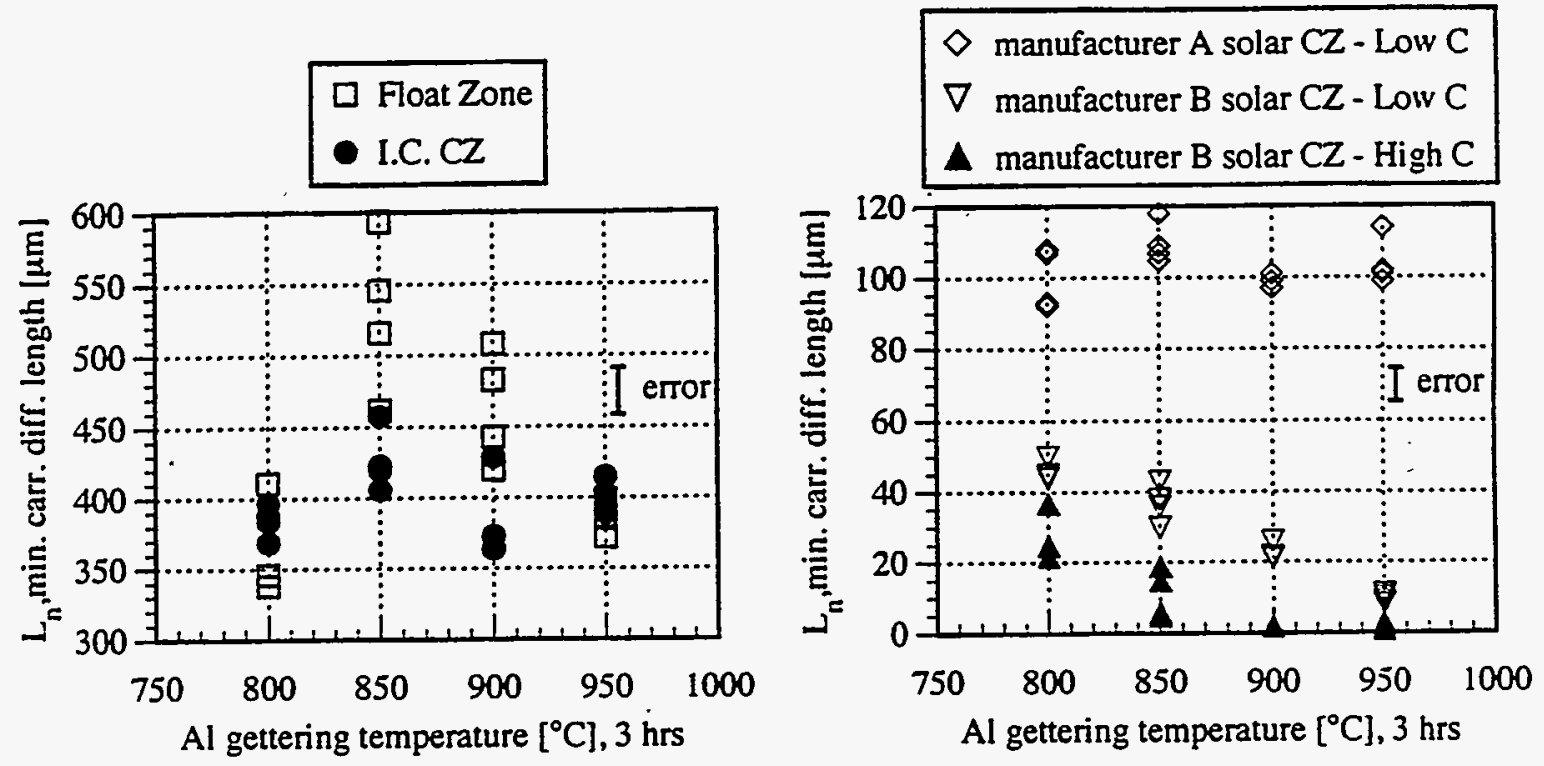

Figure 1: a) Gettering response of Float Zone and I.C. CZ with as-grown $\mathrm{Ln}$ values of $-425 \mu \mathrm{m}$ and $\approx 155 \mu \mathrm{m}$ respectively. $\mathrm{b})$ Gettering response of manufacturer $A$ and $B$ 's solar grade CZ. Asgrown $\mathrm{Ln}$ values are: $\mathrm{A}-$ low $\mathrm{C}:=110 \mu \mathrm{m}, \mathrm{B}-$ low $\mathrm{C}: \approx 72 \mu \mathrm{m}$ and $\mathrm{B}-$ high $\mathrm{C}:=70 \mu \mathrm{m}$.
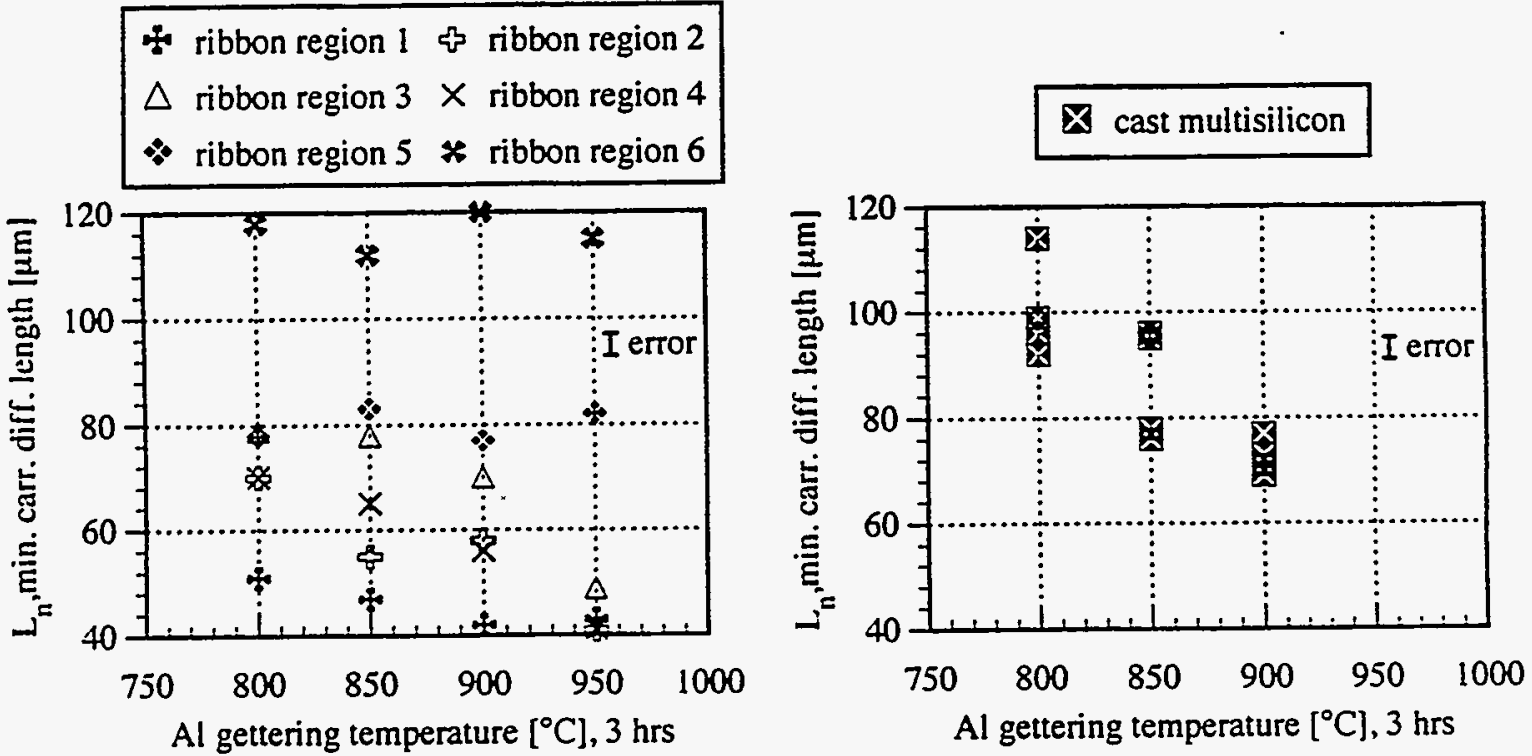

Figure 2: a) Gettering response of ribbon multisilicon with as-grown $\mathrm{Ln}$ values of: region 1 : $\approx 36 \mu \mathrm{m}$, region $2: \approx 43 \mu \mathrm{m}$, region $3:=48 \mu \mathrm{m}$, region $4: \approx 33 \mu \mathrm{m}$, region $5: \approx 75 \mu \mathrm{m}$ and region $6:$

$\approx 115 \mu \mathrm{m}$. b) Gettering response of cast multisilicon with as-grown $L$ n values of $\approx 27 \mu \mathrm{m}$ 
revealed similar microdefect densities and no dislocations. The same type of response is observed in multicrystalline silicon, Figure 2, where dislocated regions (regions $1-4$ in the ribbon material and all cast samples) degrade with increased temperature and undislocated regions remain invariant.

Gettering was also carried out at 1,3 and 5 hours at $900^{\circ} \mathrm{C}$ on the solar grade materials. An "equilibrium" gettering response (minority carrier diffusion length) was reached after 1 hour in all materials. This indicates either all impurities are removed within this hour or an "equilibrium" steady state flux of impurities has been established after one hour. The former is not consistent with the observation of gettering response degradation of both single crystal and multisilicon with increasing gettering temperature.

Considering these results we believe the following to occur. In material/regions which are invariant to gettering, no gettering of impurities occurs and diffusion length is limited by means other than dislocations. Possible causes could be slow diffusing impurities or microdefects. In materials/regions with a degrading response, these materials have a high degree of metallic impurity contamination which is precipitated in the as-grown state. These impurities are released during the gettering treatment but are not fully gettered to the Al layer due to inadequate gettering time. In essence, the gettering treatment disperses the impurities throughout the material's thickness while gettering occurs. The diffusion length degradation at higher temperatures can be ascribed to a greater amount of impurity left in the bulk upon completion of the gettering treatment. Approximate calculations of impurity flux from the silicon to the gettering layer, with these experimental conditions, show that aluminum gettering can be flux limited (the product of solubility and diffusivity) with high initial impurity concentrations of even fast diffusing impurities such as iron or chromium. Thus, high levels of impurities, as may be found in some PV grade materials, may require unrealistically long gettering times.

\section{Future Work}

Our future work will concentrate on three topics. First we will focus on an unexpected result from this year's work, specifically, the invariance of certain single crystal and multicrystalline regions to gettering. This may be a result of slow diffusing impurities, or structural defects which limit the minority carrier lifetimes while being unaffected by gettering treatments. Our work will determine the source of this response with the intention of discovering alternative treatments for these materials. Additionally, we will partake in the round-robin characterization of multisilicon materials organized by the National Renewable Energy Laboratory (NREL). This includes SPV measurements, Deep Level Transient Spectroscopy (DLTS) studies and gettering experiments.

Finally, as a continuance of our aluminum gettering study, we will determine the actual aluminum/silicon segregation coefficient for iron. Impurity segregation in the aluminum layer, is a result of the difference in solubilities of iron in the aluminum layer and in the silicon bulk. The segregation coefficient, $S$, has been estimated by Joshi ${ }^{2}$ et. al. to be in the order of $1 \times 10^{4}$. Apel et. al. ${ }^{4}$ estimated a lower bound of the segregation coefficient for $\mathrm{Co}$ to be $1 \times 10^{4}$. The literature suggests the segregation coefficient of iron between silicon and aluminum apparently remains large even at high temperatures $\left(=950^{\circ} \mathrm{C}\right)$. Thus aluminum gettering, in principle, should be able to significantly reduce metal impurity concentrations in silicon at any gettering temperature. Yet no study has determined an effective segregation coefficient. The amount of impurity reduction, that is the effective gettering coefficient, depends not only on the segregation coefficient, but also on the silicon and aluminum layer thicknesses. This relationship goes as;

$$
\frac{C_{f i n u l}^{i m p}}{C_{i n i t i a l}^{i m p}}=\frac{1}{(1+S / X)} \text { where } X=\frac{V_{s i}}{V_{A l}}
$$

Figure 3 shows the equilibrium concentration reduction in the silicon as a function of aluminum thickness and segregation coefficients. For a segregation coefficient of 104 , and a 
silicon/aluminum thickness ratio of 300 , the impurity concentration in the bulk silicon is expected to drop by a factor of 0.03 .

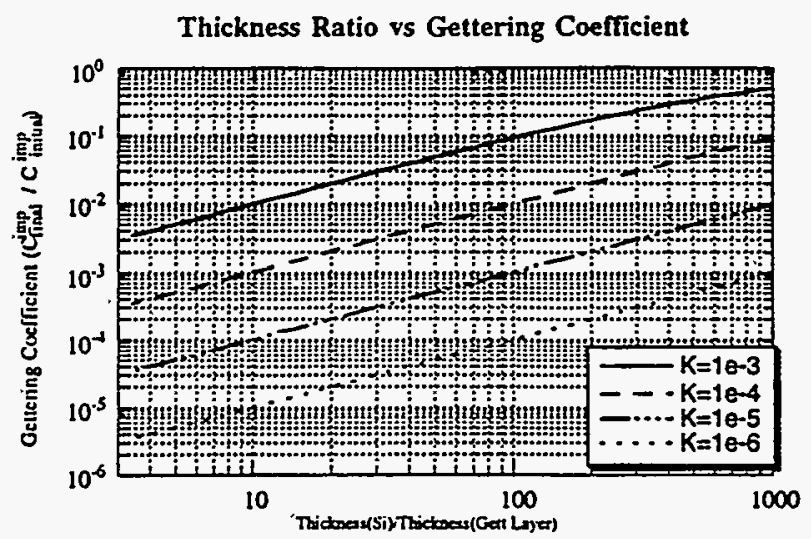

Figure 3: The gettering layer/silicon wafer thickness ratio vs. the gettering coefficient for various segregation coefficients. Note: $S=1 / K$

From this graph, one clearly sees the importance of accurately knowing the segregation coefficient in order to properly model gettering. A simple experiment with intentionally iron contaminated $\mathrm{FZ}$ silicon will yield information on the segregation coefficient of iron in the silicon/aluminum system. An effective segregation coefficient, coupled with a kinetic model, will provide exact time-temperature information for aluminum gettering. With this knowledge, aluminum gettering can be better optimized for different types of solar grade materials with varying amounts of as-grown impurities.

\footnotetext{
1 A. Rohatgi, P. Sana, M.S.Ramanachalam. J. Salami and W. B. Carter, Investigation of the Effects of Aluminum Treatment on Silicon Solar Cells, Proc. of Twenty Third IEEE Photovoltaic Specialists Conference-1993, IEEE, Louisville, KY, May 10-14, 1993, pp. 52-57.

2 S. M. Joshi, U. M. Gösele and T. Y. Tan, Improvement of minority carrier diffusion length in Si by Al gettering, Journal of Applied Physics., Vol. 77, , pp. 3858-63.

${ }^{3}$ P. Sana, A. Rohatgi, J. P. Kalejs and R. O. Bell, Gettering and hydrogen passivation of edge-defined film-fed grown multicrystalline silicon solar cells by Al diffusion and forming gas anneal, Applied Physics Letters., Vol. 64, 1 , Jan 3, 1994, pp. 97-99.

${ }^{4}$ M. Apel, I. Hanke, R. Schindler and W. Schröter, Aluminum gettering of cobalt in silicon, Journal of Applied Physics., Vol. 76, , pp. 4432-33.

5D.G. Schimmel. J. Electrochem. Soc., Vol. 126, No. 3, 1979, pp. 479

6J. S. Kang and D. K. Schroder, Gettering in silicon, Journal of Applied Physics., Vol. 65. 8,15 April 1989, pp. 297485.
}

[HHO] 
Title:

Organization:

Contributors:
Ultra-Efficient Epitaxial Liftoff Solar Cells Exploiting Optical Confinement in the Wave Limit

\author{
Department of Electrical Engineering \\ University of California, Los Angeles \\ Los Angeles, CA
}

E. Yablonovitch, principle investigator; A. K. Verma, C. Q. Wu,

R. Ranganathan, M. Goertemiller, and M. Boroditsky.

\section{Introduction}

In recent years, III-V solar cell technology has been actively pursued for use in space applications. This is due, in large part, to the superior efficiency of these materials, compared to silicon. Attention has also been given to the fabrication of thin film cells, due to their potential for reduced weight and cost, and improved efficiency. As a result, III-V cells show significant potential for terrestrial use as well. The primary focus of our research efforts over the last year has been the fabrication of $\mathrm{GaAs} / \mathrm{AlGaAs}$ thin film solar cells using the epitaxial liftoff (ELO) technique. This technique allows the transfer of these cells onto non-absorbing glass substrates, and makes possible light-trapping operation with enhanced performance. A second major goal of our work has been the development of a room-temperature photoluminescence measurement for non-destructive material evaluation and process monitoring.

\section{Fabrication of Thin Film Solar Cells Using Epitaxial Liftoff ${ }^{[1]}$}

The structure used in our experiment (Figure 1) was grown using metal organic chemical vapor deposition. It consists of an $\mathrm{n}$-GaAs active region sandwiched between $\mathrm{Al}_{0.3} \mathrm{Ga}_{0.7} \mathrm{As}$ window/passivation layers, and capped by a $\mathrm{p}^{+} \mathrm{GaAs}$ contact layer. The entire structure is grown on top of a $500 \AA$ thick sacrificial layer of AlAs, which is subsequently etched to release the device from its substrate. A thin GaAs layer above the AlAs serves to protect device layers during liftoff.

Devices were fabricated by first depositing $\mathrm{Cr} / \mathrm{AuZn} / \mathrm{Au}$ p-type contacts onto the sample. This was followed by a non-selective mesa etch down to the n-type active region using $\mathrm{H}_{2} \mathrm{SO}_{4}: \mathrm{H}_{2} \mathrm{O}_{2}: \mathrm{H}_{2} \mathrm{O}$ (1:8:500). After the evaporation of $\mathrm{AuGe} / \mathrm{Ni} / \mathrm{Au}$ contacts onto the active region, the sample was annealed at $385^{\circ} \mathrm{C}$, to make the contacts ohmic. The junction quality was monitored after each process step using nondestructive photoluminescence and open-circuit voltage $\left(\mathrm{V}_{\mathrm{oc}}\right)$ testing. No reduction in $\mathrm{V}_{\mathrm{oc}}$ was observed during the process sequence, indicating minimal parasitic leakage in the junction.

After device fabrication, the thin film solar cell was removed from its substrate using epitaxial liftoff (ELO) ${ }^{[2]}$. The sample was first covered with black wax, after which the thin film was lifted off of its substrate by selectively etching the AlAs layer in HF acid solution. The film was then attached to a glass substrate with UV-curing polyurethane. Finally, the black wax was dissolved using trichloroethylene (TCE), after which conductive brass wires were attached to the $\mathrm{p}$ and n-type contacts. 
Electrical measurements were then performed on the lifted-off solar cell. The dark currentvoltage (I-V) characteristic is virtually identical to that obtained before liftoff, indicating little material degradation during that step. This plot exhibits a diode factor of $\mathrm{n}=2.3$ for voltages between $0.2 \mathrm{~V}$ and $0.9 \mathrm{~V}$, which corresponds to nonradiative recombination current. However, the $\mathrm{n}=1$ radiative recombination current regime, which we expect to encounter at higher voltages, is obscured in this measurement by series resistance.

I-V curves were also obtained under an illumination condition of 1 sun (AM0) at Spectrolab, Inc. (Sylmar, CA). The above solar cell is bifacial, in that it can be illuminated either through the glass slide (the "front" side) or from the contact ("back") side. The results of both measurements are shown in Figure 2. Illumination from the backside yields $\mathrm{V}_{\mathrm{oc}}=0.89 \mathrm{~V}$ and a short-circuit current $I_{\mathrm{sc}}$ of $0.21 \mathrm{~mA}$. At an operating voltage of $0.69 \mathrm{~V}$, the fill factor of this characteristic is $69 \%$. When illuminated from the front side, the I-V characteristics of the cell improve to $\mathrm{V}_{\mathrm{oc}}=$ $0.93 \mathrm{~V}$ and $\mathrm{I}_{\mathrm{sc}}=0.48 \mathrm{~mA}$. An operating voltage of $0.73 \mathrm{~V}$ yields a fill factor of $68 \%$. The improved performance in this configuration is due to the fact that incident light is not obstructed from the active region by metal contact pads. Moreover, reflection from the pads on the backside may enhance performance by recycling unabsorbed photons.

\section{Non-Destructive Photoluminescence Testing ${ }^{|3|}$}

Non-destructive photoluminescence (PL) measurements can be used to evaluate the internal quantum efficiency of solar cell structures, as well as to predict their I-V characteristics. The experimental procedure employed in our work was similar to that in Reference 4. An AlGaAs laser $(780 \mathrm{~nm}$ ) was used as the pump source, and photoluminescence at $880 \mathrm{~nm}$ was collected by a Si-photodetector through a $830 \mathrm{~nm}$ long pass color filter. The external quantum efficiency $\eta_{\text {ext }}$ was determined by referencing the PL from the active layer against the scattered light from a reflective Lambertian white surface placed in the identical optical set up (Figure 3), taking into account the appropriate correction factors. The internal quantum efficiency $\eta_{\text {int }}$ is related to $\eta_{\text {ext }}$ by the equation $\eta_{\text {int }}=4 n^{2} \eta_{\text {ext }}$. For a plane surface, the escape cone of only $16^{\circ}$, imposed by Snell's law, covers a solid angle of only $\approx\left(1 / 4 n^{2}\right) \times 4 \pi$ steradians. Because the refractive index $\mathrm{n}_{\mathrm{GAAs}} \approx 3.5$ at $880 \mathrm{~nm}, \eta_{\text {ext }}$ would be $\approx 2.0 \%$ for $\eta_{\mathrm{int}}=100 \%$. From these measurements, the internal efficiency was found to be $\geq 90 \%$ which indicates the good quality of the sample.

From the luminescence efficiency versus pump intensity, we can determine the I-V curve. The PL signal decreases below $50 \%$ when the excitation photo-current density is $J_{c} \approx 5-50 \mathrm{~A} / \mathrm{cm}^{2}$. $\mathrm{J}_{\mathrm{c}}$ represents the cross-over transition from radiative recombination to non-radiative recombination. Electrically, the transition point from the electrical diffusion regime (lowinjection) to the recombination regime is $\mathrm{J}_{\mathrm{c}}=\left(\mathrm{J}_{02}\right)^{2} / \mathrm{J}_{01}$, where $\mathrm{J}_{01}$ is the pre-factor of the $\mathrm{n}=1$ radiative current and $\mathrm{J}_{02}$ is the pre-factor of the $\mathrm{n}=2$ non-radiative current. The narrow-base diffusion model gives us a saturation current of diffusion $\mathrm{J}_{01} \approx 1.3 \times 10^{-20} \mathrm{~A} / \mathrm{cm}^{2}$. Therefore, plugging $\mathrm{J}_{01}$ and $\mathrm{J}_{\mathrm{c}}$ into the above equation, we obtain optical $\mathrm{J}_{02} \approx 2.5 \times 10^{-10}-8.0 \times 10^{-10} \mathrm{~A} / \mathrm{cm}^{2}$. For comparison, we obtain $\mathrm{J}_{02} \approx 4.3 \times 10^{-10} \mathrm{~A} / \mathrm{cm}^{2}$ from the dark I-V curve discussed in the 
previous section. This value lies within the range predicted from the PL data, indicating good qualitative agreement between the optical and electrical measurement techniques.

Another simple application of the PL measurement is the calibration of etch rates and epi-layer thicknesses. We rely upon the efficiency drop when the wide bandgap hetero-layers are removed by etching. To characterize the sample, we used $\mathrm{H}_{2} \mathrm{SO}_{4}: \mathrm{H}_{2} \mathrm{O}_{2}: \mathrm{H}_{2} \mathrm{O}(1: 8: 500)$ to non-selectively etch a small piece cut from the wafer. The PL intensity versus etching time is shown in Figure 4. The final drop in this plot is due to the loss of electrical confinement when the cladding layer ( $p$ $\mathrm{AlGaAs}$ ) is etched and removed.

\section{Discussion and Future Work}

From the preceding discussion, it can be seen that the operating point of our cell lies in the undesirable $\mathrm{n}=2$ region of the $\mathrm{I}-\mathrm{V}$ characteristic. This can be partially attributed to the high series resistance $(\approx 350 \Omega)$ in this diode, the primary source of which is the sheet resistance of the lightly doped n-type active region. Another critical factor is the bandgap of the $\mathrm{Al}_{0.3} \mathrm{Ga}_{0.7} \mathrm{As}$ window layers. These layers were made with a low Al-content, in order to prevent their degradation during the sacrificial etch step. Consequently, a large amount of solar radiation is absorbed and lost in these layers before reaching the active region. The result is a reduction in both $V_{o c}$ and $I_{s c}$, which also tends to move the operating point into the $n=2$ regime. Work is currently underway to fabricate ELO solar cells with wide bandgap window layers such as $\mathrm{Al}_{\mathrm{x}} \mathrm{Ga}_{1-\mathrm{x}} \mathrm{As}(\mathrm{x}>0.6)$ and $\mathrm{In}_{0.5}(\mathrm{GaAl})_{0.5} \mathrm{P}$.

In addition, we continue to perfect a surface texturing technique which employs "natural lithography." We have shown experimentally that texturing the surface of a thin GaAs film leads to a $50 \%$ increase in light trapping. Numerical analysis shows that an even greater increase is possible. Therefore, by incorporating a textured surface onto the backside of thin film ELO solar cells, we anticipate a significant enhancement of $V_{o c}$, which should allow us to approach record values for $\mathrm{V}_{\mathrm{oc}}$ and cell efficiency.

\section{Summary}

In summary, we have integrated a working thin film GaAs solar cell onto a glass substrate, using the epitaxial liftoff technique. This configuration allows illumination through the non-contact side of the cell, which increases its collection area and short-circuit current. The cell exhibits $\mathrm{V}_{\mathrm{oc}}=0.93 \mathrm{~V}$ and $\mathrm{I}_{\mathrm{sc}}=0.48 \mathrm{~mA}$. In addition, we have performed contactless photoluminescence characterization of GaAs solar cells. Besides the calibration of absolute internal quantum efficiency, $\mathrm{J}_{02}$ from $\mathrm{PL}$ characterization is found to be $2.5 \times 10^{-10}-8.0 \times 10^{-10} \mathrm{~A} / \mathrm{cm}^{2}$, compared

with $4.3 \times 10^{-10} \mathrm{~A} / \mathrm{cm}^{2}$ from the electrical I-V curve. These preliminary results show that contactless absolute PL characterization is a useful tool for process development of solar cells.

\section{References}

[1] A. K. Verma, C. Q. Wu, M. Goertemiller, E. Yablonovitch, J. Eldridge and D. Lillington, "Thin Film GaAs Solar Cells on Glass Substrates by Epitaxial Liftoff," submitted to the 1996 IEEE Photovoltaic Specialists Conference. 
[2] E. Yablonovitch, T. J. Gmitter, J. P. Harbison, and R. Bhatt, "Extremely Selectivity in the Lift-Off of Epitaxial GaAs Films," Appl. Phys. Lett., vol. 51, pp. 2222-2224, 1987.

[3] C. Q. Wu, A. K. Verma, R. Ranganathan, and E. Yablonovitch, "Non-Destructive Testing by Absolute Room Temperature Photoluminescence Quantum Efficiency of GaAs Solar Cells," submitted to the 1996 IEEE Photovoltaic Specialists Conference.

[4] I. Schnitzer, E. Yablonovitch, C. Caneau, and T. J. Gmitter, "Ultrahigh Spontaneous Emission Quantum Efficiency, 99.7\% Internally and 72\% Externally, from AlGaAs/GaAs/ AlGaAs Double Heterostructures", Appl. Phys. Lett., vol. 62, pp. 131-133, 1993.

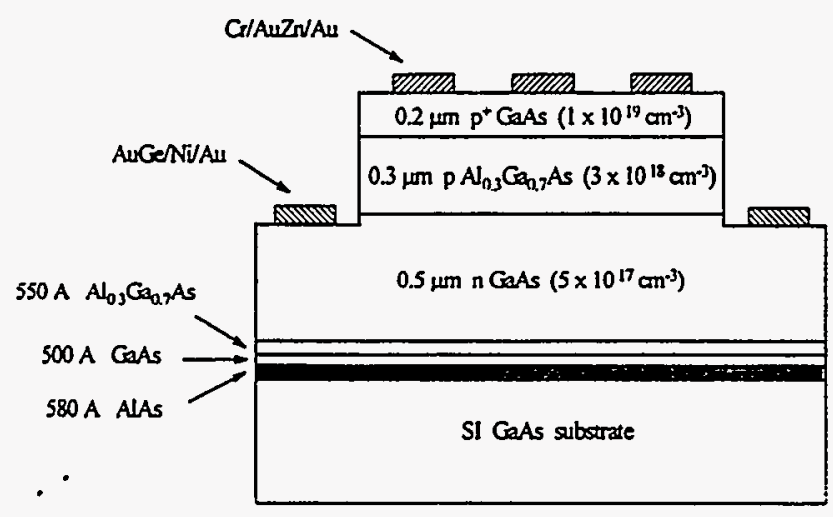

Figure 1: Structure of $\mathrm{p}-\mathrm{n}$ GaAs solar cell

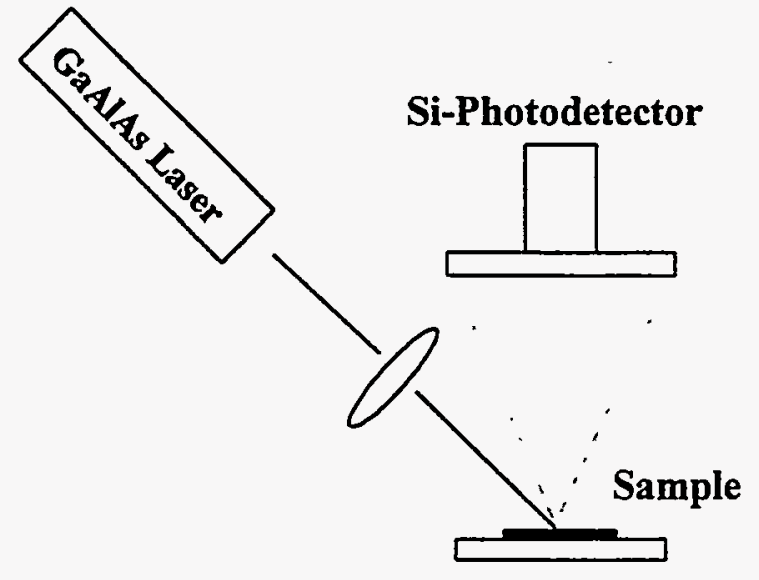

Figure 3: Experimental PL set-up

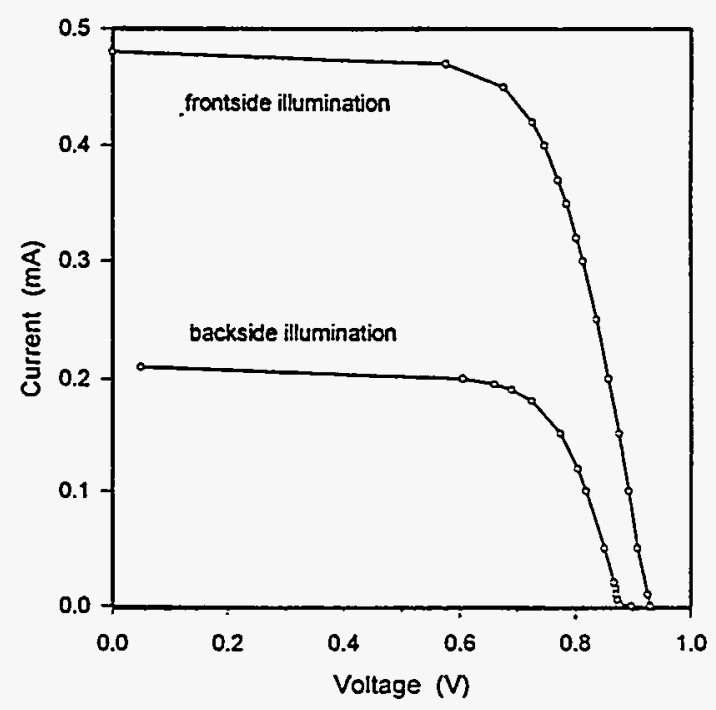

Figure 2: $I-V$ curve under 1-sun (AM0) illumination from front and back

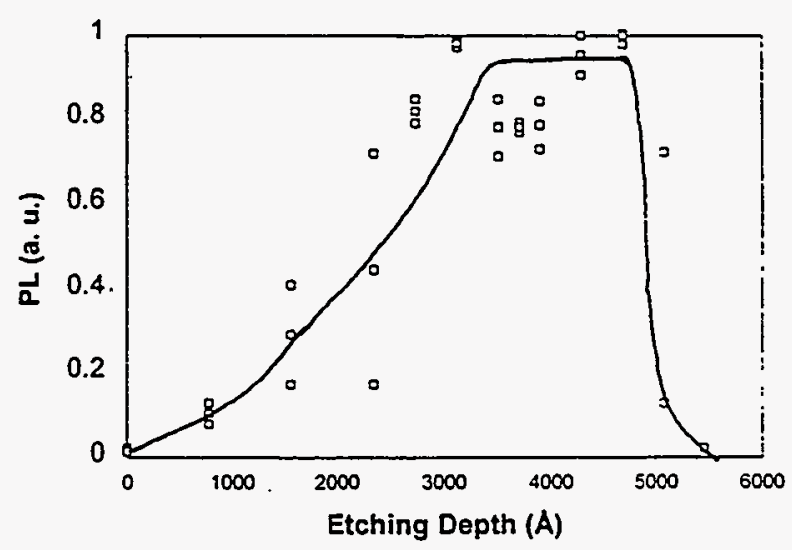

Figure 4: PL measurements of the GaAs heterostructure during the etching. 
Title:

Organization:

Contributors:
Processing of CuInSe 2 -Based Solar Cells: Characterization of Deposition Processes in Terms of Chemical Reaction Analysis

Chemical Engineering, Electrical Engineering, and Materials Science and Engineering Departments, University of Florida, Gainesville, FL

Tim Anderson, principal investigator; Paul Holloway, Kevin Jones and Sheng $\mathrm{Li}$, co-principal investigators; B. Stanbery, C. H. Chang, N. Park, J. Desroches, L. Rieth, graduate research assistants

\section{Objectives}

This new research effort was initiated in May 1995 to develop a novel high-rate processing technique for device quality thin films of CIS-based materials. Fundamental studies of the role of an ordered vacancy compound on junction formation, and the effects of contaminants such as sodium on the kinetics of the growth and recrystallization processes are key components of the research program.

\section{Approach}

An interdisciplinary team of researchers is developing a high-rate processing technique to produce device-quality thin films of $\mathrm{CI}(\mathrm{G}) \mathrm{S}(\mathrm{Su})$. A comprehensive $\mathrm{CI}(\mathrm{G}) \mathrm{S}(\mathrm{Su})$ device fabrication capability is being established that includes thermal evaporation and plasma assisted deposition of $\mathrm{Cl}(\mathrm{G}) \mathrm{S}(\mathrm{Su})$, rapid thermal processing, and $\mathrm{DC}$ sputtering of both undoped and doped $\mathrm{ZnO}$. Insight into the materials processing issues will be gained through assessment of the thermochemistry and phase equilibria of $\mathrm{CI}(\mathrm{G}) \mathrm{S}(\mathrm{Su})$ system, single crystal growth studies, studies of possible sodium surfactant effects, and detailed chemical, structured and electrical characterization, including development of a new nondestructive contactless optical technique for measurement of excess minority carrier lifetimes.

\section{High Rate Processing}

Deposition of the mixed-phase $\mathrm{CI}(\mathrm{G}) \mathrm{S}(\mathrm{Su})$ precursor films for subsequent RTP will be performed in a custom rotating disc deposition system designed at Boeing and transferred to the University of Florida. At high susceptor rotation rate, the system is capable of depositing single phase films in an atomic layer-by-layer growth mode (Migration Enhanced Epitaxy), while multilayer films can be deposited at low rotation rate. Selenium is supplied to the system by a microwave ECR plasma source and/or a thermal cracking source. The 2-step precursor deposition process begins with the growth of a thin layer of large-grain single-phase $\mathrm{CuInSe}_{2}$ to act as a nucleation layer. Various approaches to the growth of this layer, including direct lowrate deposition of the CuInSe 2 phase and both thermal as well as plasma-activated processes will be investigated. The remainder of the reactants required to form the desired film thickness and composition will then be deposited in situ on the seed layer. By controlling evaporation rate profiles, rotation speed, and selenium source parameters, it will be possible to widely vary the elemental and phase compositions of this layer, including the formation of metastable phases, to optimize it for the subsequent thermal recrystallization process.

The structures deposited by the process described above will be processed by RTP with various temperature profiles and times to react the precursor phases and obtain large-grain single-phase $\mathrm{CuInSe}_{2}$ with the desired surface phase. The temperature ramp, maximum temperature, soak time and ambient vapor will be varied to optimize the material properties and to minimize the 
thermal stresses. The various samples before and after annealing will be characterized by a variety techniques, including SIMS depth profiling for $\mathrm{Na}$ of liquid $\mathrm{N}_{2}$ cooled samples.

\section{Interfaces}

It is hypothesized that the enhanced grain growth reported when soda-line glass substrates are used is a consequence of a surfactant effect. Specifically the segregation of $\mathrm{Na}$ to the surface of $\mathrm{CIS}$ films enhances the surface mobility of $\mathrm{Cu}$ and $\mathrm{In}$ to produce larger grain sizes and better PV performance. It is planned to test this hypothesis by comparing the results of heat treated samples that included $\mathrm{Na}$ deposition at the quartz/CIS interface, after deposition of the top CIS layer, or ion implanting $\mathrm{Na}$ in the CIS films. Another approach is to examine the interaction between $\mathrm{Na}$ and CIS when grown on nearly lattice matched (111) $\mathrm{SrF}_{2}$ single crystal substrates. This represents a model system where $\mathrm{CuInSe}_{2}$ can be grown subject to modest tensile stress under conditions expected to produce 3D island growth. Comparison of the growth habitat of films grown with or without intentional dosing of the substrate with $\mathrm{Na}$ prior to initiation of deposition should indicate whether a surfactant effect or compressive strain effect is responsible for the enhanced grain size.

It also is planned to clarify the role of ordered vacancy compounds in CIS devices by the growth and characterization of single crystal structures ( $\mathrm{SrF}_{2}$ substrate). Characterization of single crystal films permit electronic effects at the junction to be separated from those of grain boundaries. HRTEM, HRXRD, SIMS and DBOM will be used to determine the structure of the $\mathrm{OVC}$, the extent of Cd diffusion, and the recombination lifetime at the CIS/OVC interface.

\section{Thermochemistry and Phase Equilibrium of the $\mathrm{Cu}$-In-Se System}

A critical assessment of the $\mathrm{Cu}$-In-Se thermochemical and phase diagram data using standard CALPHAD procedures is being performed. The outcome of this assessment will produce useful information, including equilibrium vapor compositions (required annealing ambients, Se composition from effusion cells), phase diagrams (conditions to form a eutectic melt during annealing), chemical potentials (driving forces for diffusion and chemical reactions), and consistent solution models (extents of solid solutions and consistent point defect structures). The assessment work combines thermodynamic data from the literature with models that describe solution behavior in a thermodynamically consistent manner to estimate solution model parameters and unknown thermodynamic properties.

A systematic approach has been taken with the Se unary system examined first. Mass spectrometric measurements have shown the presence of Se molecules from the monomer to the $\mathrm{Se}_{10}$ species. Assessment results predict that the predominant gas phase species above $1000 \mathrm{~K}$ is the dimer while at lower temperature the $\mathrm{Se}_{5}$ through $\mathrm{Se}_{7}$ species are the major species in the equilibrium mixture. It is interesting to note that these trends are consistent with changes in evaporated CIS film characteristics as the source temperature is varied; $\mathrm{Se}_{2}$ is apparently a more efficient growth species.

The next segment of this study was an assessment of the In-Se system. The four intermediate solid phases were modelled as line compounds and a Redlich-Kister expression was used to describe the liquid phase. The literature thermodynamic and phase equilibria data were found to be reasonably consistent. As an example of the type of output that can be obtained, the calculated In-Se phase diagram is shown in Figure 1.

\section{ZnO Window Layers}

Transparent Conducting Oxide (TCO) films are essential as "window" layers for high performance CIS devices, but currently employed techniques for $\mathrm{ZnO}$ film deposition are either 
too expensive for PV manufacturing or result in films with inadequate quality for efficient cells. The cost criterion favors thin, high conductivity films deposited from inexpensive raw materials with low capital cost equipment. Effective dopant activation is important to permit high mobility at degenerate doping levels, and has been observed empirically to improve as lighter group III elements are used (boron > aluminum), and possibly by the use of group IV elements (e.g., silicon). We are developing a potentially lower-cost, manufacturable process for conductive, transparent $\mathrm{ZnO}$ deposition based on DC sputtering from metallic zinc targets using boron or silicon doping.

\section{Measurement of Carrier Lifetimes}

A nondestructive contactless dual-beam optical modulation (DBOM) technique has been developed for the purpose of characterizing the excess carrier lifetimes and the surface recombination velocities in $\mathrm{CuInSe}_{2}$ (CIS) films deposited on Mo-coated soda-lime glass substrates formed by RTP. The DBOM technique is based on the modulation of transmission intensity of an infrared (IR) probe beam (hv $<E_{g}$ ) by a pump beam (hv $\geq E_{g}$ ) via free carrier absorption in the CIS film. A theoretical model suggests that the fractional change in transmitted probe beam intensity is directly related to the excess carrier lifetime and the surface recombination velocity in the CIS films, and that the surface recombination velocity is measureable only if it is comparable to the ratio of film thickness to excess carrier lifetime.

The experimental setup for the DBOM technique includes a tungsten lamp passing through an IR filter (e.g., Ge wafer). This is used as the probe beam which is focused to a beam size of $4 \mathrm{~mm}$ in diameter. The IR filter is employed to ensure that free carrier absorption does not take place in the CIS film due to the probe beam. A $1.5 \mathrm{~mW} \mathrm{He-Ne} \mathrm{laser}(\lambda=44 \mathrm{~nm})$ is used as a pump beam. The pump beam is focused to a beam size of $2 \mathrm{~mm}$ in diameter and is aligned with the probe beam during the measurements. This would eliminate the $3-\mathrm{D}$ effect due to the spatial distribution of nonequilibrium electrons and holes. Both the probe and pump beams are chopped at $405 \mathrm{~Hz}$ for the synchronous detection by using a lock-in amplifier.

To determine the excess carrier lifetimes and surface recombination velocities in the CIS films, the transmitted IR intensity (I) is first measured by turning on the chopper and the tungsten lamp, and the resulting signal is measured by using a lock-in amplifier. The IR intensity modulation $(\Delta \mathrm{I})$ is next measured by turning on a chopped He-Ne or He-Cd laser pump beam superimposed on the IR probe beam. Figure 2 shows the linear relation obtained from the measured $\Delta I$ versus I plot for both the $\mathrm{He}-\mathrm{Ne}$ and $\mathrm{He}-\mathrm{Cd}$ lasers. The surface recombination velocities and the excess carrier lifetimes were calculated by using the measured values of $\mathrm{I}$ and $\Delta \mathrm{I}$.

Characterization of the excess carrier lifetimes and the surface recombination velocities was performed on a 2" x 2" CIS sample provided by D. L. Morel at the University of South Florida: The sample was prepared by growing a $2.5 \mu \mathrm{m}$ thick CIS film on a $100 \AA$ thick Mo-coated soda lime glass substrate. The excess carrier lifetimes determined by the DBOM measurements on this sample ranged from 2 ns to 17 ns with an average value of 10 ns within the 2 " $\times 2$ " wafer. A two-dimensional mapping of the excess carrier lifetimes in this CIS sample showed that the lifetime variation was consistent with the non-uniformity in film color observed on the surface of the sample. Values of surface recombination velocities of this CIS sample ranged from $\sim 10^{4}$ to $10^{5} \mathrm{~cm} / \mathrm{sec}$. These high values of surface recombination velocities were attributed to the large surface roughness of the CIS film. The results showed that regions with large surface recombination velocity also yield low excess carrier lifetime in the film.

The new DBOM technique can be applied to study the effect of CBD CdS films on the surface recombination velocity and excess carrier lifetimes in the CIS films and could shed new light on the interface properties of the CdS/CIS film formed under different processing conditions. 


\section{Conclusions and Future Directions}

Considerable progress has been achieved in the first half year of this new program towards the establishment of a comprehensive program in CIS photovoltaics. A research-scale highthroughput CIS deposition system has been transferred from Boeing and modified. This system will begin to produce films at the beginning of the new year. Other experimental systems that have been concurrently installed include a DC-sputtering system for ZnO, a RTP system that includes a proximity-cap annealing susceptor, CBD ZnCdS system, and a If-sputtering system for Mo. With these growth and processing systems operational, the proposed experimental program summarized above will be initiated.

A promising new nondestructive contactless technique (DBOM) has been developed and demonstrated for the characterization of excess carrier lifetimes and surface recombination velocities in CIS films. A detailed study of the effect of depositing thin CBD CDS films on the surface recombination velocity and excess carrier lifetime is currently being conducted. The thermodynamic assessment effort has produced critically evaluated phase diagrams for the Se and In-Se systems. In the next year the $\mathrm{Cu}$-In and $\mathrm{Cu}$-Se systems will be completed, and position the team to address the important $\mathrm{CuSe} 2-\mathrm{In}_{2} \mathrm{Se}_{3}$ pseudobinary system.

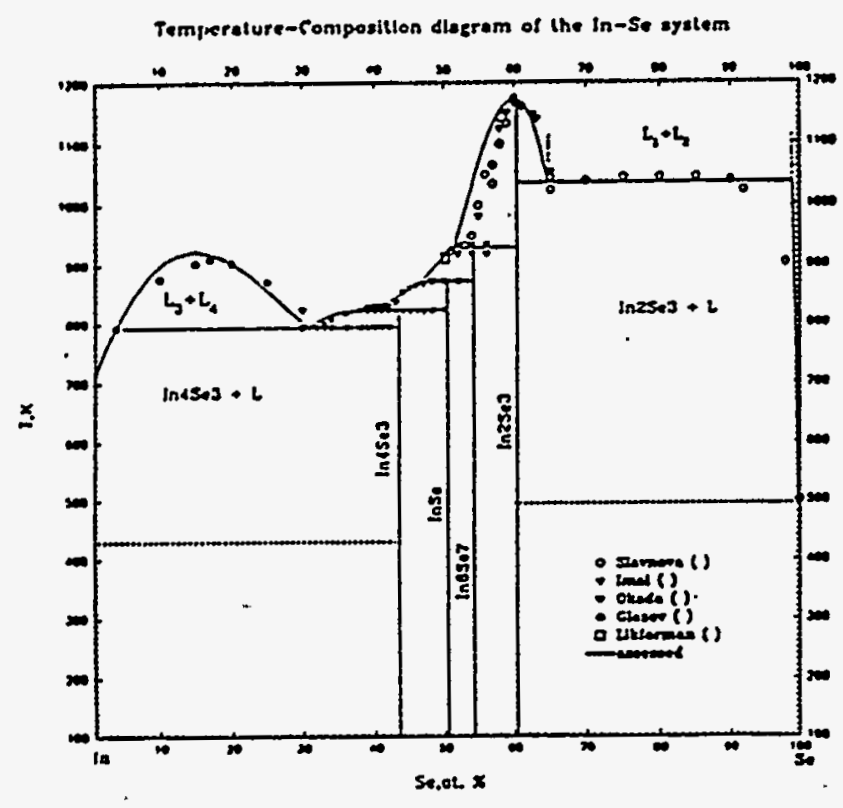

Figure 1 The assessed In-Se phase diagram.

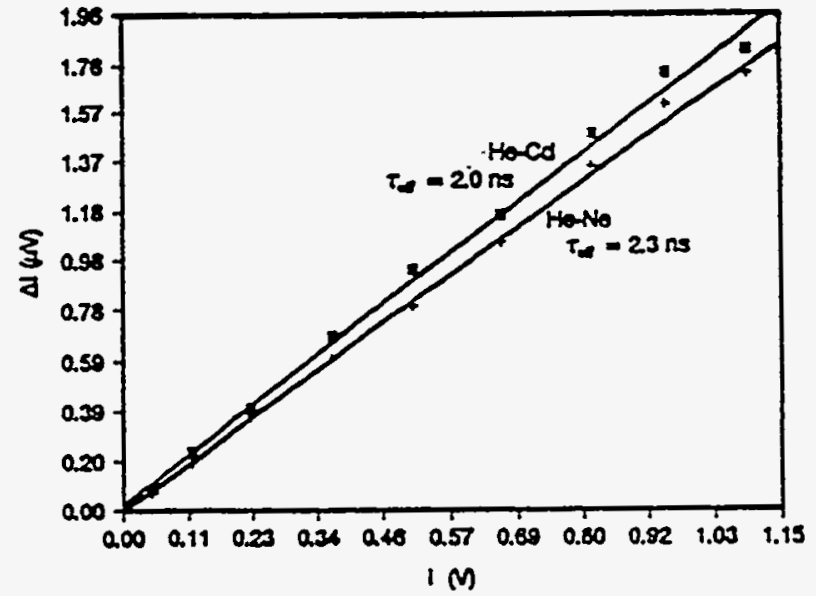

Figure $2 \Delta I$ versus I plots using the $\mathrm{He}-\mathrm{Cd}$ and $\mathrm{He}-\mathrm{Ne}$ lasers as pump beams in the DBOM measurements on CIS sample. 
Title:

Organization:

Contributors:
Fundamental Studies of the Effect of Crystal Defects on $\mathrm{CuInSe}_{2} / \mathrm{CdS}$ Heterojunction Behavior

Department of Materials Science and Engineering University of Ilinois, Urbana, $\mathbb{L}$

J. Benner, program manager; A. Rockett, principal investigator, J. Hernandez and Jiangang Chen, visiting scientists; D. Schroeder, G. Berry, John Belanger, graduate research assistants; Benjamin French, undergraduate research assistant.

\section{Objectives}

The project seeks to determine the source of sample-to-sample variability in $\mathrm{CuIn}_{1-\mathrm{x}} \mathrm{Gax}_{\mathrm{x}} \mathrm{Se}_{2}$ (CIGS)-based solar cell performances and the mechanisms restricting carrier collection at large voltages in CIGS/CdS heterojunctions. The detailed objectives involve determining the relative importance of structural and chemical defects in the material and how they affect electronic properties and hence device performances. A byproduct of the project is fundamental data on the properties of CIGS alloys as a function of processing conditions and composition. This is important to improving device modelling. Finally, as a university research program the goals include the training of graduate students and post-doctoral researchers and the exposure of as many individuals as possible to renewable energy research (see contributors list).

\section{Approach}

The basic approach is to grow the highest quality CIGS under a variety of conditions and to measure the morphology, microstructure, and properties of the resulting layers. Electrical properties have been measured extensively this year for correlation with compositional and structural data. Except as noted, the results described were recorded for CIGS deposited in a single step from magnetron sputtered $\mathrm{Cu}$ and In fluxes and evaporated molecular Se. The single crystal CuIn 1-x $\mathrm{Ga}_{x} \mathrm{Se}_{2}$ layers were deposited directly on "epi-ready" GaAs (001) or (111) As wafers. Deposition temperatures were $\sim 650^{\circ} \mathrm{C}$. In addition, a collaborative study of polycrystalline CIGS grown by Lars Stolt and coworkers at the University of Uppsala in Sweden was undertaken. The samples were grown by open boat multiple source evaporation on sodalime glass or other substrates. Some substrates were coated with $\mathrm{Al}_{2} \mathrm{O}_{3}$ as a diffusion barrier to prevent $\mathrm{Na}$ from passing from the substrate into the film. Na could also be intentionally added to the films.

Microstructural studies were carried out by X-ray diffraction and by scanning and transmission electron microscopies. Composition analyses used secondary ion mass spectrometry (SIMS), and by energy-dispersive spectroscopy. Scanning ion images were also obtained using the Cameca IMS-5f SIMS. Hall-effect measurements were obtained as a function of temperature. Electroluminescence measurements were made on solar cells from the University of Delaware, the National Renewable Energy Laboratory (NREL), and Siemens Solar Industries. Emission from forward-biased solar cells was studied as total intensity and as a function of wavelength and bias current. Spectral response was also measured for each device in the same instrument.

Results (10/1/94 - 9/30/95)

\section{Characterization of hole transport in CIGS}

In FY 1994 we demonstrated growth of CIGS with the highest hole mobilities ever recorded. During FY 1995 we have characterized the transport of holes in this material and demonstrated 
that ionized defect scattering dominates at low temperatures with mobility increasing with temperature $T$ as $T^{1.2}$. At high temperatures the mobility decreases as $T^{-2.1}$, probably as a result of acoustic and non polar optical phonon scattering. Simulation results for one sample giving various contributions to mobility are presented in Figure 1. The ionized defect scattering process varies significantly from sample to sample. The temperature and maximum mobility of the peak in the mobility as a function of temperature curve is determined primarily by this variation. Because the room temperature mobility is primarily determined by phonon scattering there is relatively little variation in room temperature mobility of the layers. Furthermore, because the scattering is dominated by phonons, the analysis suggests that high levels of impurities will be required before the room temperature mobility will be significantly degraded. This will be explored during FY 1996.

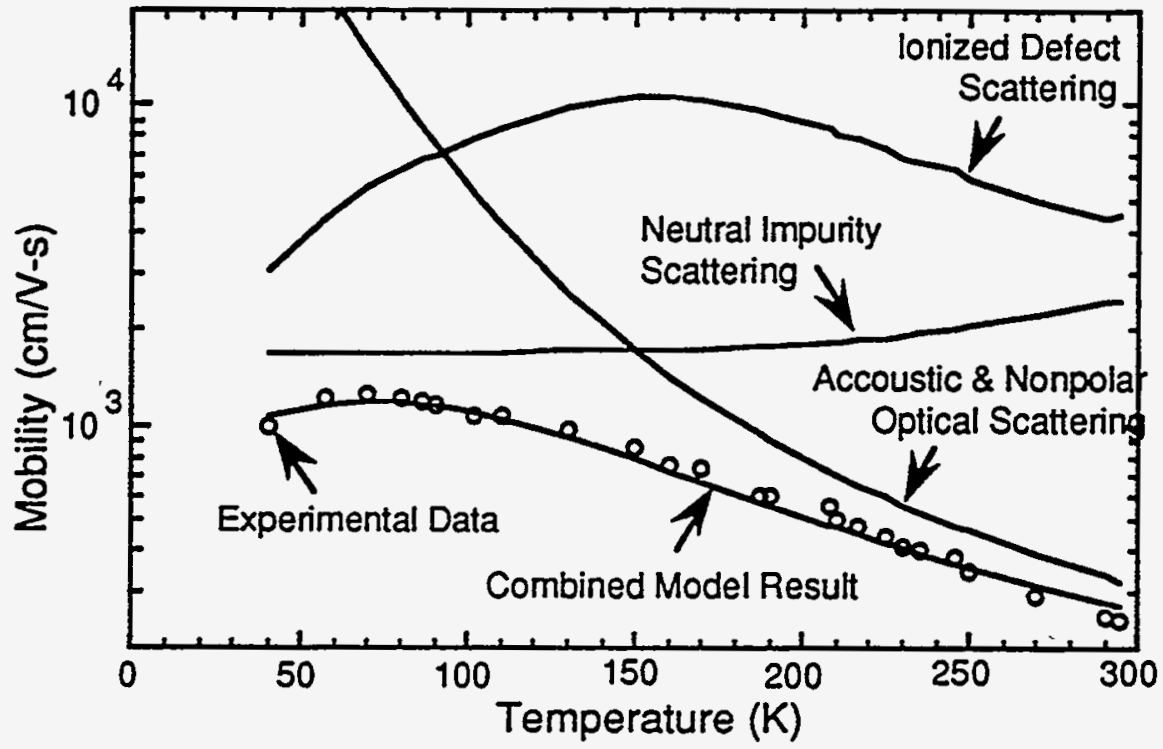

Figure 1. Hall mobility experimental data (open circles) and the fit contributions to the total mobility showing the three major mechanisms considered in the fit.

\section{Ga Diffusion in CIGS}

One feature of epitaxial growth of CIGS on GaAs is that Ga diffuses out of the substrate at a rate sufficient to compensate for missing $\mathrm{Cu}$, yielding a nearly stoichiometric film in most cases. This provides us with the opportunity to determine the Ga diffusivity in CIGS as a function of composition. Single crystal epitaxial layers with $\mathrm{Cu} / \mathrm{In}$ ratios ranging from 0.4 to 1.4 were deposited on $\mathrm{GaAs}(111)_{\text {As. All }}$ All compositions lay close to a "tie-line" connecting $\mathrm{Cu}_{2} \mathrm{Se}$ and $\left(\operatorname{In}_{1-\mathrm{x}} \mathrm{Ga}_{x}\right)_{2} \mathrm{Se}_{3}$ on a pseudo-ternary phase diagram. Composition depth profiles for the layers demonstrated nearly constant $\mathrm{Ga}$ concentrations throughout the films when significant excess $\mathrm{Cu}$ was present in the growth fluxes. However, when the layers were near stoichiometry, diffusion of $\mathrm{Ga}$ into the film followed a nearly perfect error-function behavior. A solution to Fick's second law with a moving boundary and accounting for $\mathrm{Ga}$ loss at the surface was used to determine the diffusion coefficient of $\mathrm{Ga}$ in the films with error function behaviors. This gave a diffusion coefficient $4.3 \times 10^{-13} \mathrm{~cm}^{2} / \mathrm{s}$ for films with a $\mathrm{Cu} / \mathrm{In}$ ratio of $\sim 1$ at the growth temperature. (See Figure 2.) The diffusion coefficient increased dramatically for $\mathrm{Cu} / \mathrm{In}$ ratios away from 1.0 in either direction. These data in combination with carrier types and estimated defect energies show: 1) the types of native point defects required to enhance or allow the diffusion of $\mathrm{Ga}, 2$ ) that diffusion passes through a minimum at a $\mathrm{Cu} / \mathrm{In}$ ratio of 1 , and 3 ) that the diffusion coefficient is similar and large in the case of large deviations on either side of $\mathrm{Cu} / \mathrm{In}=1$. 


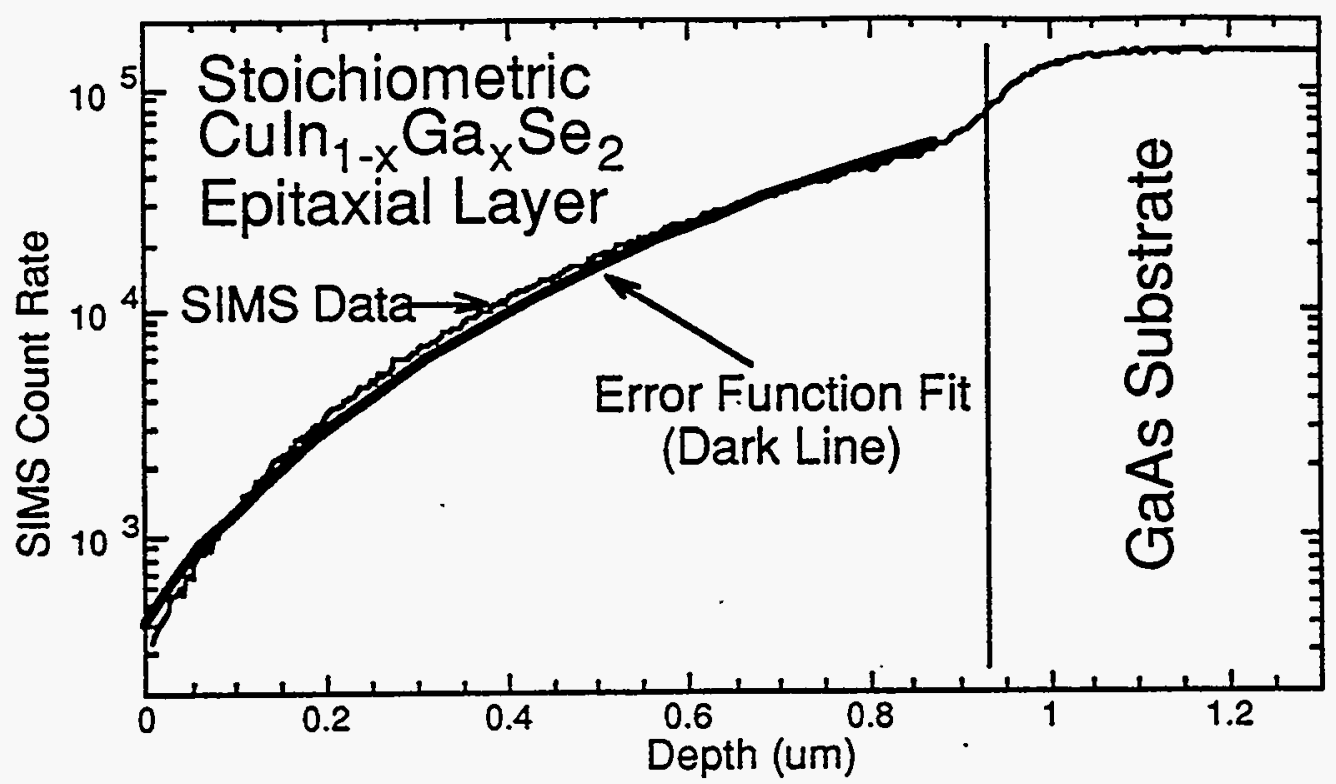

Figure 2: SIMS data showing outdiffusion of $\mathrm{Ga}$ and an error function curve fit for a near stoichiometric CuInSe2 epitaxial layer on a GaAs substrate.

\section{Cu-rich Second Phases and In-rich Ordered Vacancy Compounds}

We have analyzed by SIMS the formation of In-rich and $\mathrm{Cu}$-rich second phases in polycrystalline CIGS thin films grown at Uppsala. While the conventional wisdom has generally suggested that device quality In-rich films include a thick (up to $50 \mathrm{~nm}$ ) layer of $\mathrm{CuIn}_{3} \mathrm{Se}_{5}$ on the surface, we have shown that device quality materials so not necessarily include such a layer at thicknesses of $>5 \mathrm{~nm}$. Furthermore, there is no significant evidence of a composition-dependent thickness of this near surface layer in In-rich layers. This is important because it suggests that most of the excess In is not accommodated in a surface segregated layer as generally presumed. Rather, there must be distributed point defects throughout the layer. This is consistent with the $\mathrm{Ga}$ diffusion data in single crystals described above. We have imaged $\mathrm{Cu}$-rich precipitates in $\mathrm{Cu}$ rich CIGS using SIMS. The results are consistent with moderate sized $(\sim 0.5 \mu \mathrm{m}) \mathrm{Cu}_{2} \mathrm{Se}$ second phases distributed uniformly throughout the film with a volume fraction that accounts for the excess $\mathrm{Cu}$ in the layer.

\section{The Effect of Na on CIGS Devices}

We have analyzed layers containing $\mathrm{Na}$ that diffused out of $\mathrm{Na}$-containing substrates, which was ion implanted into layers after growth, and which was deposited on the surface of substrates that did not contain Na. We have shown that Na diffuses rapidly in CIGS and tends to segregate: out of the film to the front and back surface, into the grain boundaries of sputtered Mo back contacts or into Na-rich second phases. This demonstrates that the improved device performance generally associated with $\mathrm{Na}$ is not primarily due to $\mathrm{Na}$ acting as a dopant in the CIGS itself. Rather, it apparently acts primarily through changing the microstructure of the CIGS. 


\section{Electroluminescence}

We have characterized the electroluminescence of forward biased solar cells based on CIGS alloys and have demonstrated significant emission with a peak near the minimum direct energy gap of the CIGS. The emission efficiency in very good solar cells is comparable to a commercial light emitting diode driven at very low current densities. However, the commercial diode improves in efficiency faster than the solar cells do as the current density is increased. The solar cells are not currently capable of withstanding the current densities used in light emitting diodes. However, the results do suggest that with some effort, polycrystalline CIGS-based light emitting diodes may be practical. A typical emission spectrum and spectral response curve for a NREL device containing $\mathrm{Ga}$ are shown in Figure 3. The electroluminescence efficiency is on the order of $10^{-3}$ photons per injected electron at the highest current densities tested.

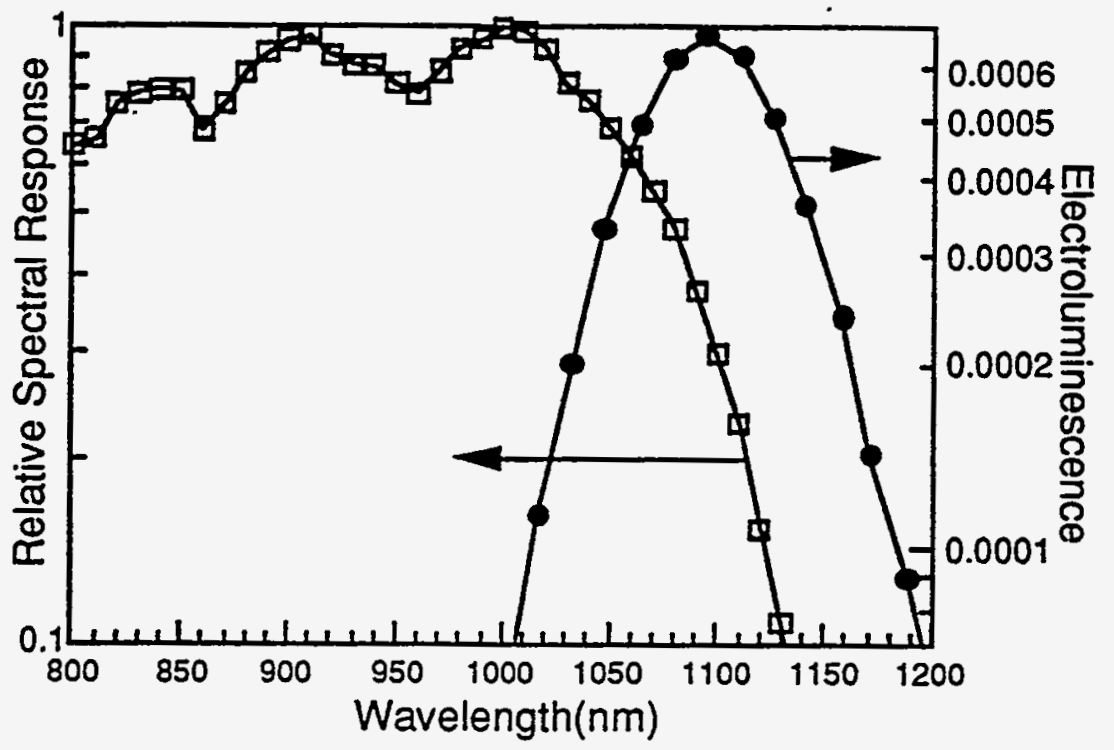

Figure 3. Shows electroluminescence results and spectral response data for an $-15 \%$ efficient CIGS solar cell fabricated at NREL. Short wavelength variations in spectral response are due to interference effects in the device layer.

\section{Conclusions}

The results obtained this year resolve two key long standing debates (about the effect of $\mathrm{Na}$ and the ordered defect compound layer). Data obtained concerning $\mathrm{Ga}$ diffusivity will simplify interpretation of results on technologies requiring interdiffusion and control thereof. It also sheds new light on native point defects in CIGS. New information about recombination and scattering processes should improve fundamental device modelling that is crucial to controlling failures in devices and in developing novel devices. 
Organization:

Department of Physics \& Astronomy, University of North Carolina at Chapel Hill, Chapel Hill, North Carolina

Contributors: $\quad$ Daxing Han, principal investigator; L. E. McNeil, co-principal investigators; K. D. Wang, and C.N. Yeh

\section{Objective and Outline of the approaches}

Our objective is to understand recombination and metastability in amorphous silicon alloys, especially in $\mathrm{p}-\mathrm{i}-\mathrm{n}$ device structures. Our research project is primarily aimed at determining the position and precursor states involved in the SWE, and to gain a better understanding of the relationship between material characteristics and solar cell performance.

During the contract year of 1995 , we continued our experiments on electroluminescence (EL) and transient forward bias current as well as photogain measurements before and after light soaking. We have set up the equipment for electronic profile measurements and started to calibrate the system. We are pursuing vigorous collaborative efforts : with Dr. Liyou Yang on photodegradation studies of H-diluted and Non-diluted solar cells by EL spectroscopy; with Prof. Adriaenssens, on the SWE on charge transport in $p-i-n$ cells by transient forward current; with Prof. McNeil, we studied bulk and/or interface effects by comparing PL to EL spectroscopy. Besides, in collaboration with Prof. Yue Wu in our department we have started a new project to study the microstructure of high stability hot-wire samples by NMR in this year.

Six papers have been published and two abstracts have been submitted as a result of this year's research. $1-8$

\section{The Most Interesting \& Important Research Results}

\section{EL}

One of the goals of our team is to find the relation between the material parameters and the solar cell performance. We have cooperated with Dr. Yang at Solarex to study how the hydrogen dilution affects material parameters and how it relates to the solar cell performance.

Yang and Chen at Solarex found 10 that there are two types of defects, characterized as "fast" and "slow" defects according to their respective time constants for solar cell degradation. The "fast" and "slow" defects co-exit in solar cells made with pure silane. Cells made with $\mathrm{H}_{2}$-dilution appear to have predominantly the "fast" defects only resulting in much faster saturation of photodegradation. We further characterize the nature of such defects using EL spectroscopy to measure the energy distribution of defects and their photodegradation kinetics.

The two types of cells studied have similar initial energy conversion efficiencies, but under AM1.5 light soaking those made with $\mathrm{H}_{2}$-dilution stabilized after about $100 \mathrm{~h}$ and those made without $\mathrm{H}_{2}$-dilution did not until after $1000 \mathrm{~h}$.

The EL spectra from the same type of cells are quite uniform as shown in Fig. 1 for cells with and without $\mathrm{H}$-dilution, respectively. This means that the EL spectrum is a meaningful feature for the diode quality. One can see that the $\mathrm{H}$-dilution cell shows a narrow $0.9 \mathrm{eV}$ defect band domination and the non-dilution cell shows a 0.75 and $0.9 \mathrm{eV}$ double-peaked-defect band.

\footnotetext{
${ }^{\times N R E L}$ Subcontract No. Xan-4-13318-09
} 


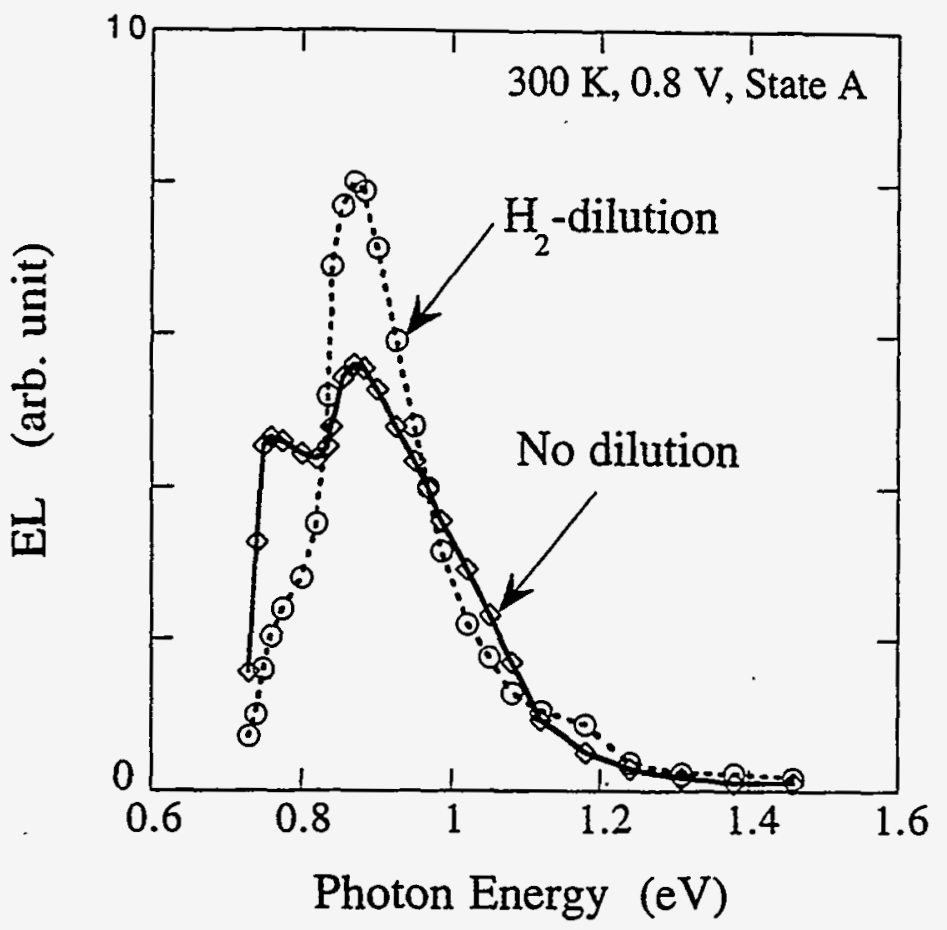

Fig. 1 EL spectra of a-Si:H p-i-n cells made with and without $\mathrm{H}$ dilution under $0.8 \mathrm{~V}$ at $300 \mathrm{~K}$. Both cells were in an initial state (State A).

Since the defect band luminescence provides direct information on the defect electronic structure of the material including its density as well as energy distribution, it is very interesting to study the evolution of the defect properties as the material is being light-soaked. We have exposed both types of cells to $200 \mathrm{~mW} / \mathrm{cm}^{2}$ white light through the p-layer. The light-soaking condition used was similar to the performance photodegradation studies of the same types of solar cells. The EL spectra were measured at various stages of light-soaking for samples made without $\mathrm{H}_{2}$ dilution. As the solar cells are being light-soaked, the intensity of the EL main-band (1.0-1.4 eV) decreases and that of the defect-band increases due to the increase of the density of defect states. ${ }^{5}$ To investigate the creation kinetics for individual components in the defect band, we have analyzed the intensities of the two peaks at $\sim 0.9 \mathrm{eV}$ and $\sim 0.75 \mathrm{eV}$, respectively. The results are plotted in Figs.2a and $2 b$ for cells made with and without $\mathrm{H}_{2}$-dilution, respectively.

As shown in Fig. 2a, the intensity of the dominant peak at $\sim 0.9 \mathrm{eV}$ for the $\mathrm{H}_{2}$-diluted cell increases first, but rapidly stabilizes after only $\sim 10$ hours of light-soaking. No significant change in the low-energy shoulder can be resolved for this type of device. In contrast, for the nondiluted cell as shown in Fig. $2 \mathrm{~b}$ the peak at $0.75 \mathrm{eV}$ is much more pronounced and the intensity for both components increases significantly with light-soaking. More interestingly, while the $0.9 \mathrm{eV}$ peak also stabilizes after $10-100$ hours of light-soaking, the $0.75 \mathrm{eV}$ peak continues to rise and no sign of saturation is seen even beyond $1000 \mathrm{hr}$. of light-soaking. These observations for both types of cells could be entirely consistent with the results of the solar cell degradation studies, 10 if we assume that the $0.9 \mathrm{eV}$ and $0.75 \mathrm{eV}$ peaks in the defect band are associated respectively with the "fast" and "slow" defects identified from cell degradation kinetics. Thus, the EL measurements have not only confirmed the existence of the two kinetically different 
defect states in certain a-Si:H, depending on the deposition condition, but also provided their unique energy characteristics in the bandgap.
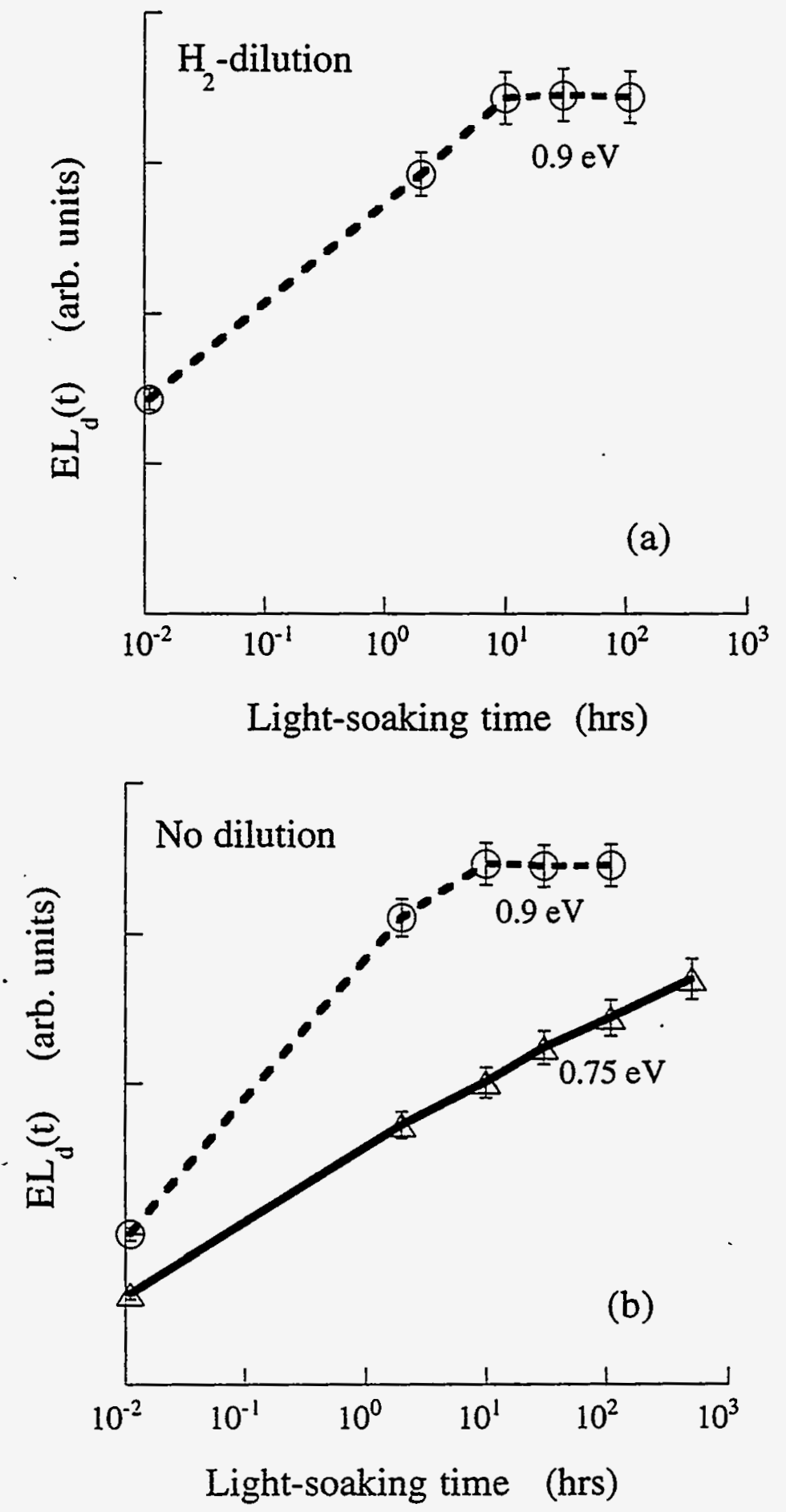

Fig. 2 EL intensities of defect components at $0.9 \mathrm{eV}$ and $0.75 \mathrm{eV}$ as a function of lightsoaking time (a) cell made with $\mathrm{H}_{2}$ dilution and (b)without dilution. 
Interestingly, a-Si:H solar cells with better stability (fast stabilize) made at ECD also show a single narrow EL peak around $0.9 \mathrm{eV}$. It seems that there is a correlation between a narrow EL defect band and the better stability. There are several possibilities to explain the defect energy distribution and their degradation kinetics, such as relaxed and unrelaxed defects, impuritycomplex, etc. based on the standard DOS model. Alternatively, according to the defect-pool model both the type of defects and their energy position depend on the formation energy or the position of Fermi-level, so the features of EL bands could be related with the internal electric field profile. More investigations are needed to find the corresponding microstructure origins.

\section{Nuclear magnetic resonance (NMR)}

Concerning the new material studies, we have preliminary NMR results for the structure of hotwire material that has shown improved stability. The microstructures of $2-3 \mathrm{H}$ at.\% hot-wire and 8-10 $\mathrm{H}$ at.\% glow-discharge (GD) a-Si:H films were characterized by ${ }^{1} \mathrm{H}$ NMR. Significant differences were found between these two types of a-Si:H films. Among other things, the broad resonance line in the $2-3 \mathrm{H}$ at.\% hot-wire a-Si:H is about $55 \mathrm{kHz}$ wide, which is much broader than that observed in GD a-Si:H film. Moreover, approximately 90 percent of the hydrogen atoms give rise to the $55 \mathrm{kHz}$ line and only a very few percent of the hydrogen atoms give rise to the much narrower resonance line. Our NMR results imply that the high stability a-Si:H contains different type of microstuctures. Further details were investigated by multiple quantum NMR.

\section{Future Studies}

Future work will focus on (1) photodegradation effect on EL spectral line shape in highperformance cells, such as inserting a H-diluted thin layer in $p / i$ interface; (2) internal electric field profile $E(x)$ in $p-i-n$ structures and the photodegradation effect on $E(x)$ studied by nullcurrent method, and (3) the $\mathrm{H}$ distributions in new materials with improved stability studied by NMR.

\section{References}

1. Daxing Han and Keda Wang, "A distinct recombination regime in amorphous silicon diodes under double injection", Appl. Phys. Lett. 66, 879 (1995).

2. Daxing Han and Keda Wang, "Electroluminescence from hydrogenated amorphous silicon p-i-n diodes" J. Non-Cryst. Sol. 190, 74 (1995).

3. Regis Vanderhaghen and Daxing Han, "Interface effects on double injection current and photocurrent in a-Si:H n-i-p and p-i-n diodes", J. Non-Cryst. Sol.190, 85 (1995).

4. B. Yan, G.J. Adrianenssens, A. Eliat, and D. Han, "Forward current transients in amorphous silicon p-i-n structures", J. Non-Cryst. Sol. 190, 95 (1995).

5. Keda Wang and Daxing Han, "Relation of the EL measurements to solar cell parameters", in Amorphous Silicon Technology, edited by E.A. Schiff, M. Hack, A. Madam, M. Powell, A. Matsuda, MRS Symp. Proc. (1995).

6. C.N. Yeh, D.X. Han, K.D. Wang and L.E. McNeil, "Carrier recombination in a-Si:H p-i-n devices studied by PL and EL spectroscopies", in Amorphous Silicon Technology, edited by E.A. Schiff, M. Hack, A. Madan, M. Powell, A. Matsude, MRS Symp. Proc. (1995)

7. Daxing Han, Keda Wang, and Liyou Yang, "Electroluminescence spectroscopy of amorphous silicon solar cells made with and without hydrogen dilution", submit to MRS-96.

8. C.N. Yeh, Qi Wang, and Daxing Han, "The effect of hydrogen dilution near the $\mathrm{p} / \mathrm{i}$ interface region on DOS profile in a-Si:HG p-i-n solar cells", submit to MRS-96.

9. J. T. Stephen, Daxing Han, Harv Mahan, and Yue Wu, "Hydrogen distribution in high stability a-Si:H prepared by the hot wire technique", submit to MRS-96.

10. L. Yang and L. Chen, Appl. Phys. Lett. 63400 (1993). 


\section{Title: $\quad$ Optimization of Gettering Processes for Photovoltaic Silicon}

Organization: Center for Microelectronics Research, University of South Florida, Tampa, Florida

Contributors: $\quad$ B.Sopori, program manager;

L.Jastrzebski, principal investigator,

S.Ostapenko, A.U.Savchouk, J.Lagowski

\section{Objectives}

Our effort was focused on optimization and development of new gettering approaches to improve diffusion length (L) of commercial bulk polycrystalline silicon (poly-Si) for PV application. The novel technique of defect engineering based on the ultrasound treatment (UST) was applied to polycrystalline silicon (poly-Si) commercial solar cells. In order to assess changes in defect characteristics on microscale after UST, we developed and applied the photoluminescence (PL) mapping technique.

\section{UST Effect on Room-Temperature Photoluminescence Mapping}

The UST approach is based on generation of low-power ultrasound vibrations into poly$\mathrm{Si}$ wafer, which activates the interaction of point defects (heavy metals, hydrogen) and extended defects (dislocations, grain boundaries, precipitates). Ultrasonic vibrations are generated into poly-Si by external piezoelectric transducers, while UST parameters (sample temperature and ultrasonic power) can be controlled "in-situ" with non-contact infrared detector and miniature acoustic wave detector. A schematic of UST apparatus is shown in Figure 1, details of UST method were described in the previous NREL reports. Using surface photovoltage technique, we observed previously that minority carrier lifetime can be improved by factor of ten after UST in poly-Si regions with low $L$ value $(10$ to $15 \mu \mathrm{m})[1,2]$. This was interpreted as UST induced improvement of recombination properties and/or reduction of crystallographic defect concentration or heavy metal precipitates.

We used PL spectroscopy and PL mapping as diagnostic technique to monitor microscopic distribution of recombination centers in cast and EFG poly-Si wafers, or solar cells. This technique was developed in order to obtain a distribution of recombination centers on microscale with resolution of $10 \mu \mathrm{m}$. In Figure 2 the PL spectra at $4.2 \mathrm{~K}$ and room temperature of EFG polysilicon are presented. The line of boron bound exciton $(\mathrm{B}+\mathrm{TO})$ dominates at $4.2 \mathrm{~K}$. With the temperature increasing to $300 \mathrm{~K}$, the exciton line is quenched and two broad PL bands persist and can be resolved. The maximum at $1.1 \mathrm{eV}$ corresponds to band-to-band recombination in $\mathrm{Si}$, and its intensity is related to concentration of non-radiative recombination centers. The second PL band with the maximum at $0.8 \mathrm{eV}$ is a "defect" luminescence attributed to oxygen precipitates [4]. We used both these bands for PL mapping of poly-Si with high spacial resolution limited by focused laser spot. PL mappings were performed on as-grown polysilicon, as well as wafers at different stages of processing, including the final solar cells. In Figure 3 two histograms of band-to-band PL intensity are presented before and after UST for solar cell from ASE Americas. The intensity of band-to-band luminescence is strongly inhomogeneous across the wafer, which corresponds to distribution of non-radiative recombination centers. This is consistent with our previous SPV mapping study [2]. We also found that the distribution of "defect" PL band does not correlate with distribution of the band-to-band luminescence. Therefore, we concluded 
that oxygen precipitates are distributed also inhomogeneously across poly-Si wafer, but in different manner compared to non-radiative centers.

We applied UST to poly-Si wafers and compared PL histograms before and after UST treatment. As shown in Figure 3, the average band-to-band PL intensity was increased after UST by $60 \%$. The UST caused the reduction in concentration of non-radiative recombination centers, which is consistent to our previous UST findings $[1,2,3]$.

The UST of commercial solar cells (Solarex and A.S.E.) have been shown to increase a fill factor and efficiency at low excitation level (Figure 4). With increasing of illumination intensity this UST induced improvement was reduced. The origin of this phenomenon is presently not clear and its understanding requires further study. We found that the UST effect at the low excitation level is accompanied with changes of ideality factor ( $n$ ) of solar cells. At the low excitation level the value of $n$ is close to 2 , which means that the recombination at space-charge region of solar cell dominates. After UST the n-value is reduced by $10 \%$ due to a reduction of the recombination rate in the space-charge region. We suggest that concentration or/and capture cross section of nonradiative recombination centers in the space-charge region are reduced after the UST. This is confirmed by our PL mapping results at the solar cells, which are similar to that presented in Figure 3.

\section{Conclusions}

We demonstrated in our previous study that the classical gettering approaches: $\mathrm{Cl}$ and $\mathrm{P}$ gettering, can improve diffusion length in regions of poly-Si with large L [5]. The mechanism is related to gettering of dissolved heavy metals, which control minority carrier lifetime in these regions. An additional improvement of $\mathrm{L}$ can be achieved by the novel processing techniques: non-equilibrium Al gettering during rapid thermal annealing [4] and the ultrasound treatment. The UST effect on minority carrier diffusion length and band-to-band PL intensity improvement is explained as reduction of non-radiative recombination. Based on the results of the $\mathrm{PL}$ mapping one can conclude that the recombination in a low lifetime regions is not controlled by the oxygen precipitates. Our data on the UST of solar cells suggest that adversary effects of deep centers located in the space charge region of $p-n$ junction could be reduced by UST. In the future efforts, we plan to investigate the origin of these centers and to study possibility of their enhanced passivation in solar cells using UST enhanced hydrogenation processing [6-9] as well as hydrogenation with "in-situ" ultrasound treatment.

\section{References}

1. S.Ostapenko, L.Jastrzebski, J.Lagowski and B.Sopori, Appl.Phys.Lett. 65, 1555 (1994).

2. S.Ostapenko, L.Jastrzebski, and B.Sopori, Semicond.Sci.Technol. 10, 1494 (1995)

3. L.Jastrzebski, S.Ostapenko, W.Henley, D.Schielieu, G.Nowak, and J.Lagowski, AIP Conference Proceedings 353, ed.Harin S.Ullal, C.E.Witt (Woodbury, NY 1995), p.135

4. M.Tajima, H.Takeno, M.Warashina, and T.Abe, Mater.Sci.Forum 143 , 147 (1994)

5. Annual Report, Photovopltaic Program, FY 1994 (September 1994).

6. S.Ostapenko, L.Jastrzebski, J.Lagowski amd R.Smeltzer, Appl.Phys.Lett., 1995, in press.

7. Thin Film Transistor Technology II, ed. by Y.Kuo(1995) v.94-35, p.61.

8.Defect and Impurity Engineered semiconductors and Devices, ed.by S.Ashok,

J.Chevallier, I.Akasaki, N.Johnson, B.Sopori, (1995), v.378, p.405

9. S.Ostapenko and L.Jastrzebski, Abstracts of 5th Workshop on the Role of Impurities and Defects in Si Device Processing, (Colorado, 1995) p.152. 


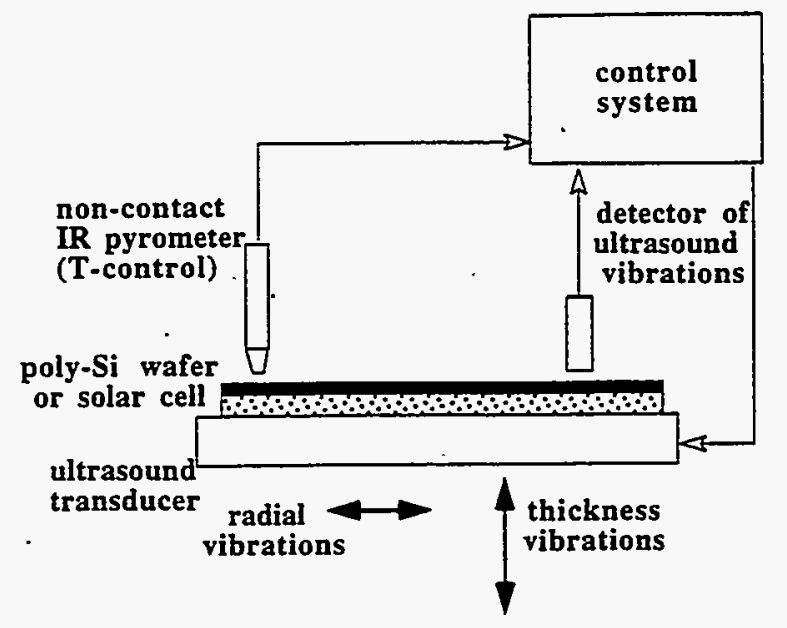

Fig.1 Schematic of ultrasound treatment unit for poly-silicon wafers and solar cells

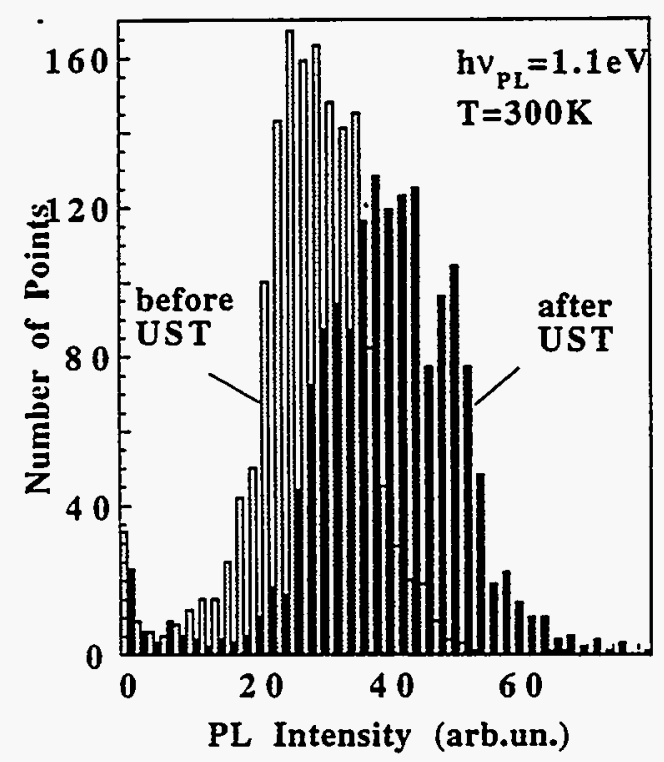

Fig.3 Increasing of solar cell PL intensity after UST $\left(80^{\circ} \mathrm{C} / 1 \mathrm{hr} / 25 \mathrm{kHz}\right)$

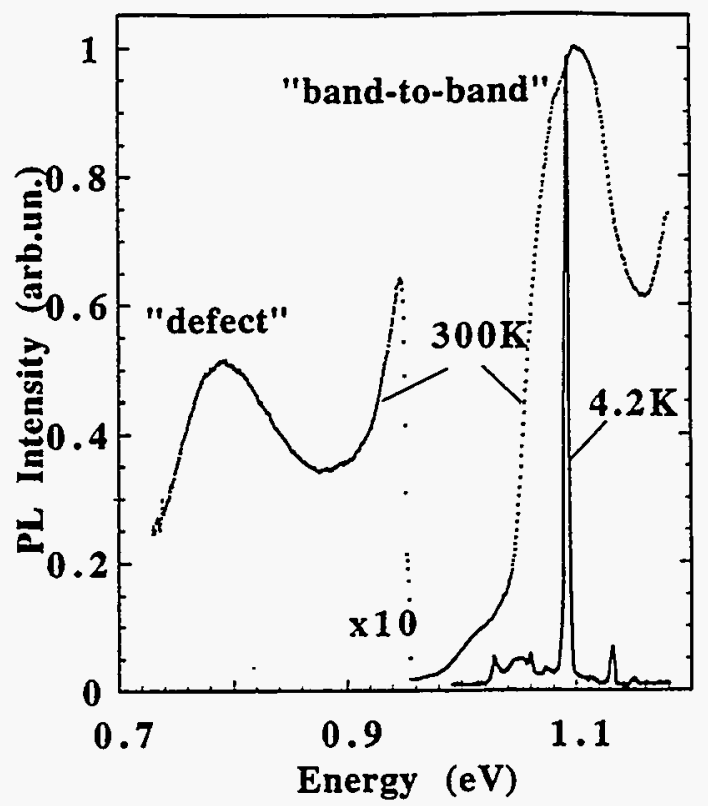

Fig.2 PL spectra of EFG poly-Si; excitation $514 \mathrm{~nm}, 40 \mathrm{~mW}$

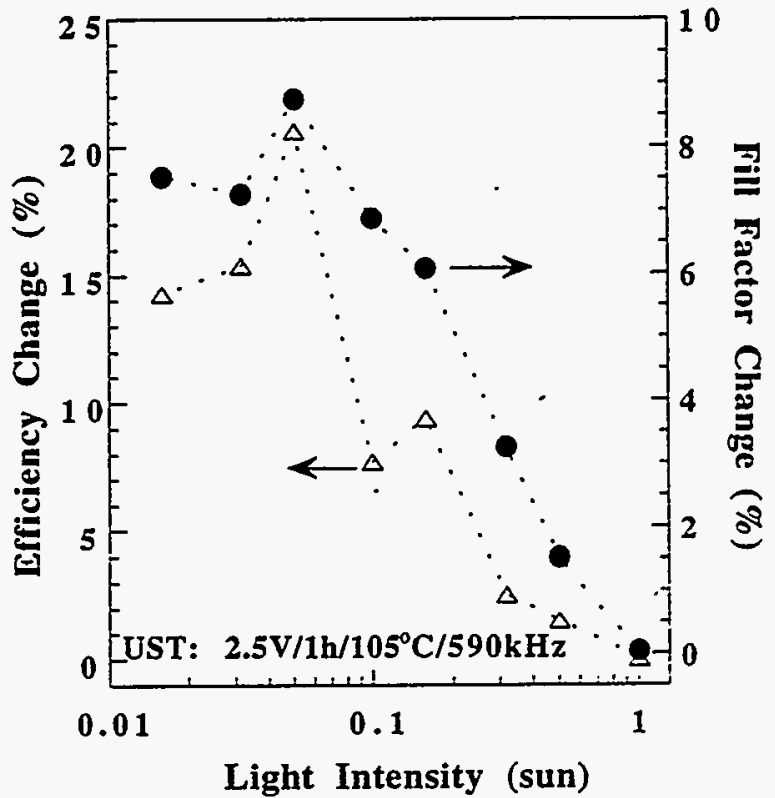

Fig.4 Change of solar cell efficiency and fill factor due to UST 
Contributors: $\quad$ P.C. Taylor, principal investigator; G.A. Williams, W.D. Ohlsen, J.M. Viner, D. Chen, P. Hari, S. L. Chen, D. Mao

The major objectives of this subcontract are (1) to identify metastabilities caused by carrier recombination or by frozen-in departures from equilibrium, and to determine if the metastabilities that plague devices are "intrinsic" and therefore not correctable or "extrinsic" and therefore capable of being eliminated in future devices, (2) to characterize the important defects and impurities in the bulk and at surfaces and interfaces because interfaces are becoming more important with the advent of tandem cells with several heterojunctions, and (3)to measure the motion of hydrogen in a-Si:H and related alloys on a microscopic scale because the motion of hydrogen is thought to play an important role in many of the metastable effects that occur in a-Si:H and related alloys and the microscopic mechanism for this motion is not known.

\section{Approaches}

Two new approaches, that have not been employed in the past, have been emphasized: (1) below-gap spectroscopy, such as optical absorption, photoluminescence (PL), PL excitation, electron spin resonance and other spectroscopies using primarily a tunable $T i$ sapphire laser system to excite carriers well below the optical gap (down to $-1.1 \mathrm{eV}$ ). (2) novel nuclear magnetic resonance (NMR) techniques such as measurements of the spin-lattice relaxation in a dipolar field $\left(T_{1 D}\right)$, to probe local hydrogen motion in doped and intrinsic a-Si:H and selected alloys.

\section{Research Results}

The subcontract is divided into several tasks. One task is the growth and characterization of doped and undoped a-Si:H and related alloys. Ditertiarybutylsilane has been added to silane to vary the bandgap of a- $\mathrm{Si}_{\mathrm{x}} \mathrm{C}_{\mathrm{l}-\mathrm{x}}: \mathrm{H}$ alloys from $\mathrm{E}_{04}$ of about 1.9 to about $3 \mathrm{eV}{ }^{\mathrm{l}}$ Samples of a-Si $\$$ :H have been made using mixtures of silane and hydrogen sulfide. ${ }^{2}$ Samples with large sulfur concentrations ( $>1$ at. \%) exhibit the phenomenon of persistent photoconductivity. In samples with small sulfur concentrations the sulfur acts as an inefficient $n$-type dopant that can in some cases be passivated by hydrogen. ${ }^{3,4}$ These measurements of n-type doping due to sulfur represent the first investigations of group VI "double donors" in a-Si:H.

A second task concerns below gap spectroscopy in a-Si:H and related alloys. We have been using Photoluminescence (PL) excitation spectroscopy (PLE), time resolved PL and optically detected magnetic resonance (ODMR) to probe defects which produce absorption below the gap in a-Si:H. ${ }^{5}$ Photoluminescence in a-Si: $\mathrm{H}$ has been investigated using optical excitation energies $\mathrm{E}_{\mathrm{x}}$ varying from 0.95 to $2.0 \mathrm{eV}$ (See Fig. 1). As shown in Fig. 1 recent measurements on the PL that occurs at energies above the exciting-light energy indicate that two-step excitation processes through the silicon dangling-bond states are important, even in device-quality material. ${ }^{6}$ The most dramatic feature of the data in Fig. 1 is the fact that the PL spectra are very similar independent of the excitation energy and independent of whether the $\mathrm{PL}$ is observed below or above the excitation energy.

A third task involves-studies of local diffusion of hydrogen in a-Si:H using NMR techniques. We measured $\mathrm{T}_{I D}$, the dipolar spin lattice relaxation time, whose inverse $\left(\mathrm{T}_{\mathrm{ID}}^{-1}\right)$ gives a measure of the local motion of hydrogen. $7,8,9$ The $T_{I D}$ values for doped. compensated and undoped a-Si:H exhibit trends that are similar 
to the variation of the macroscopic diffusion (as measured by secondary ion mass spectroscopy or SIMS) of hydrogen with respect to various doping levels, but the details of the local motion are very different from those of the macroscopic diffusion. For example, the rates of local motion are many orders of magnitude faster than those inferred from the macroscopic diffusion experiments, and the activation energies for local motion are much less than those for macroscopic motion. Light soaking also produces an increase in the local hydrogen motion as measured by $T_{1 D}^{-1}$, but the interpretation of this result is not clear as yet.

A fourth task involves the study of interfaces, defects and impurities in a-Si:H and related alloys, including the metastabilities that are related to such interfaces, defects and impurities. The measurement of PL and ESR in nitrogen-rich hydrogenated amorphous silicon nitride $\left(\mathrm{a}-\mathrm{SiN}_{1.6}: \mathrm{H}\right)$ films has provided a probe of optically-induced metastabilities in these films. ${ }^{10,11,12,13,14}$ Recent ESR experiments ${ }^{14}$ have discovered a new nitrogen-related defect in a-SiN ${ }_{1.6}: \mathrm{H}$. Previous ESR experiments in device-quality a-SiN. $: \mathrm{H}$ observed only the silicon dangling bond center, known as the $\mathrm{K}$ center. Our recent experiments ware able to extract the nitrogen-related center from the overlapping $\mathrm{K}$ center resonance by performing experiments at low temperatures $(4 \mathrm{~K})$ and high microwave powers. This discovery suggests the presence of negativelycharged nitrogen dangling bonds that are compensated by positively-charged silicon dangling bonds in this material. The discovery that device-quality a-SiN ${ }_{1.6}: \mathrm{H}$ contains compensating positively- and negativelycharged defects requires that the existing models of defects in this material be refined.

In $\mathrm{a}-\mathrm{SiS}_{\mathrm{x}}: \mathrm{H}$ the sulfur acts as an inefficient donor ${ }^{3,4,15,16}$ which is partially compensated by nearby hydrogen. Some of these passivated donors can be optically activated by exciting with above-band-gap light. ${ }^{3,4}$ This optical activation of inefficient sulfur donors in a-SiS ${ }_{x}: \mathrm{H}$ represents a new optically-induced metastability whose consequences for photoconductivity are the opposite of those produced by the usual Staebler -Wronshi effect. As shown in Fig. 2 there is some evidence that these sulfur-doping effects may reduce the degradation in solar cells

In intrinsic a-Si:H recent optically-detected ESR studies have shown that the so-called enhancing and quenching signals can be effectively separated using time-resolved techniques. ${ }^{17}$

\section{Conclusions}

Major accomplishments of the previous year include (1) the characterization of $\mathrm{a}-\mathrm{Si}_{\mathrm{x}} \mathrm{C}_{1-\mathrm{x}}: \mathrm{H}$ alloys, which have been grown using liquid organic sources mixed with silane, and a-SiS ${ }_{x}: H$ alloys(2) the measurement of various optical processes in a-Si:H using excitation energies well below the optical band gap (down to $0.8 \mathrm{eV}$ ), (3) correlation of local and long-range motion of hydrogen in doped, compensated and intrinsic a-Si:H, and (4) the measurement of optical and ESR properties of a-Si $\mathrm{N}_{1-x} \cdot \mathrm{H}$ alloys, and in particular the discovery of a new nitrogen-related ESR center in a-SiN ${ }_{1.6}: \mathrm{H}$, and (5) the discovery of inefficient sulfur doping in a-SiS $\mathrm{x}: \mathrm{H}$ which can be modified by optical activation of passivated donors. Future directions for the research include (1) measurements on a-SiSe $\mathrm{x}_{\mathrm{H}} \mathrm{H}$ to obtain a better understanding of the role of group VI donors in a-Si:H, (2) continuation of below-gap spectroscopy of a-Si:H to identify the important absorption and degradation mechanisms, and (3) completion of the NMR measurements of local hydrogen motion in a-Si:H.

\section{References}

1. "Electronic and Optical Properties of $\mathrm{a}_{-} \mathrm{Si}_{1-\mathrm{x}} \mathrm{C}_{\mathrm{x}}: \mathrm{H}$ Films Produced From Admixtures of Silane and Ditertiarybutylsilane" ( K. Gaughan, J.M. Viner and P. C.Taylor), in Amorphous Silicon Technology - 1994, edited by E. A. Shiff, M. Hack, A. Madan, M. Powell and A. Matsuda (Materials Research Society, Pittsburgh, 1994), Vol. 336, p. 553. 
2. "Electronic and Optical Properties of a-Si:H Films Alloyed with Sulfur" (S.L. Wang, Z. H. Lin, J.M. Viner and P. C. Taylor), in Amorphous Silicon Technologv - 1994, edited by E. A. Shiff, M. Hack, A. Madan, M. Powell and A. Matsuda (Materials Research Society, Pittsburgh, 1994), Vol. 336, p. 559.

3. "Optical Activation of Inefficient Sulfur Dopants in Hydrogenated Amorphous Silicon: A New Metastable Effect" (S.L. Wang and P. C. Taylor), Solid State Commun. 95, 361 (1994).

4. "Light Induced Metastable Changes in a-SiS ${ }_{x}: H$," (S.L. Wang and P. C. Taylor), in Amorphous Silicon Technology - 1995, edited by M. Hack, E. A. Shiff, A. Madan, M. Powell and A. Matsuda (Materials Research Sociery, Pittsburgh, 1995), Vol. 377, 307.

5. "Below-Gap Excitation of Photoluminescence in a-Si:H," (X. Yin, M.E. Raikh and P. C. Taylor) , in Amorphous Silicon Technology - 1995, edited by M. Hack, E. A. Shiff, A. Madan, M. Powell and A. Matsuda (Materials Research Society, Pittsburgh, 1995), Vol. 377, p. 167.

6. "Photoluminescence Above the Excitation Energy in a-Si:H" (X. Yin, J.M. Viner, S. Q. Gu, M.E. Raikh and P. C. Taylor), Phys. Rev. B 49, 5073 (1994).

7. "Effect of Light Soaking on the Local Motion of Hydrogen in Hydrogenated Amorphous Silicon" (P. Hari, P. C. Taylor and R. A. Street), in Amorphous Silicon Technologv - 1994, edited by E. A. Shiff, M. Hack, A. Madan, M. Powell and A. Matsuda (Materials Research Society, Pittsburgh, 1994), Vol. 336, p. 329.

8. "Local and Long-Range Hydrogen Motion in a-Si:H," (P. Hari, P. C. Taylor and R. A. Street), in Amorphous Silicon Technologv-1995, edited by M. Hack, E. A. Shiff, A. Madan, M. Powell and A. Matsuda (Materials Research Society, Pittsburgh, 1995), Vol. 377, 185.

9. "Hydrogen Motion in Hydrogenated Amorphous Silicon (a-Si:H)" ( P. Hari, P. C. Taylor and R.A. Street), J. Non-Cryst. Solids (1995), in press.

10. "Temperature Dependence of the Electron Spin Resonance in Nitrogen-rich Amorphous Silicon Nitride" (D. Chen, J.M. Viner, P. C. Taylor and J. Kanicki), Phys. Rev. B 49, 13420 (1994).

11. "Photobleaching of PL and Temperature Dependence of ESR in Nitrogen-Rich Amorphous Silicon Nitride Films" (D. Chen, J.M. Viner, P. C. Taylor and J. Z. Kanicki), in Amorphous Silicon Technology - 1994, edited by E. A. Shiff, M. Hack, A. Madan, M. Powell and A. Matsuda (Materials Research Society, Pittsburgh, 1994), Vol. 336, p. 619.

12. "Photoluminescence and Electron Spin Resonance in Nitrogen-rich Amorphous Silicon Nitride" (D. Chen, J.M. Viner, P. C. Taylor and J. Kanicki), J. Non-Cryst. Solids 182, 103 (1995).

13. "New Nitrogen-Related Defects in Nitrogen-Rich a-Si ${ }_{x} N_{1-x}: H$ " (with D. Q. Chen and J.M. Viner), J. Non-Cryst. Solids (1995), in press.

14. On the Nature of the Paramagnetic Defects in Hydrogenated Amorphous Silicon Nitride" (D. Q. Chen, J. M. Viner and P. C. Taylor), Phys. Rev. Lett. (1995), submitted.

15. "Sulfur Doping in a-SiS $: \mathrm{H}^{\prime \prime}$ (S.L. Wang, J.M. Viner and P. C. Taylor), J. Non-Cryst. Solids (1995), in press.

16. "Inefficient Sulfur Doping in Hydrogenated Amorphous Silicon" (S.L. Wang and P. C. Taylor), PV Program Review, Golden CO, May 1995, AIP Conf. Proc. (1995), in press.

17. "Optically Detected ESR Studies of Recombination Processes in a-Si:H (D. Mao and P. C. Taylor), J. Non-Cryst. Solids 190, 48 (1995).

18. M. S. Bennett, J. L. Newton and K. Rajan. Proc. of 7th European PV Conf., edited by A. Goetzberger, W. Palz and G. Willeke (D. Reidel, Dordrecht, The Netherlands, 1986), p. 544. 


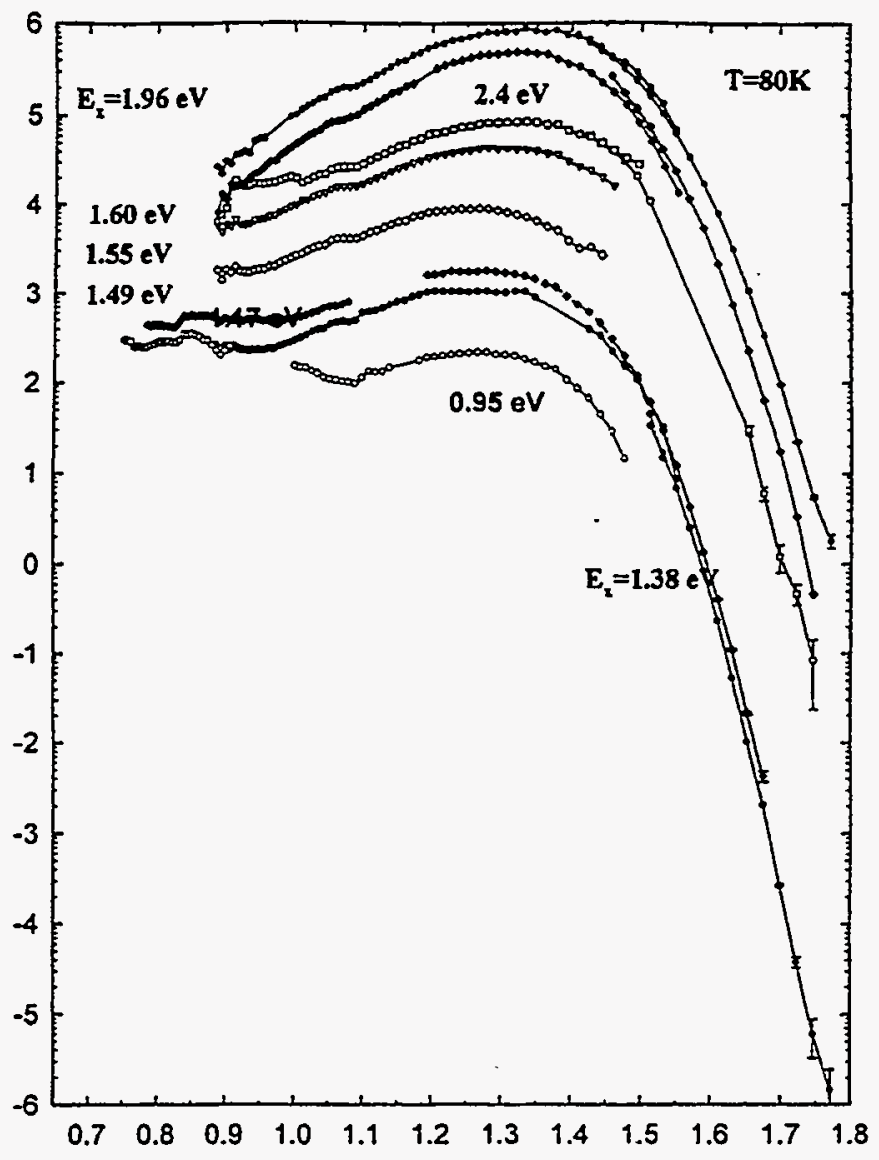

Fig. 1. PL intensities (logarithmic scale) at $80 \mathrm{~K}$ as functions of energy for different values of excitation energy, $E_{x}$, as labeled on the figure. Selected error bars are shown at low signal levels. Breaks in the data occur near $E_{x}$ because of the influence of the strong laser exciting light on the measurement of the much weaker PL signals. Data below about $1.5 \mathrm{eV}$ were taken using a Ge detector and those above about $1.5 \mathrm{eV}$ with a GaAs detector.

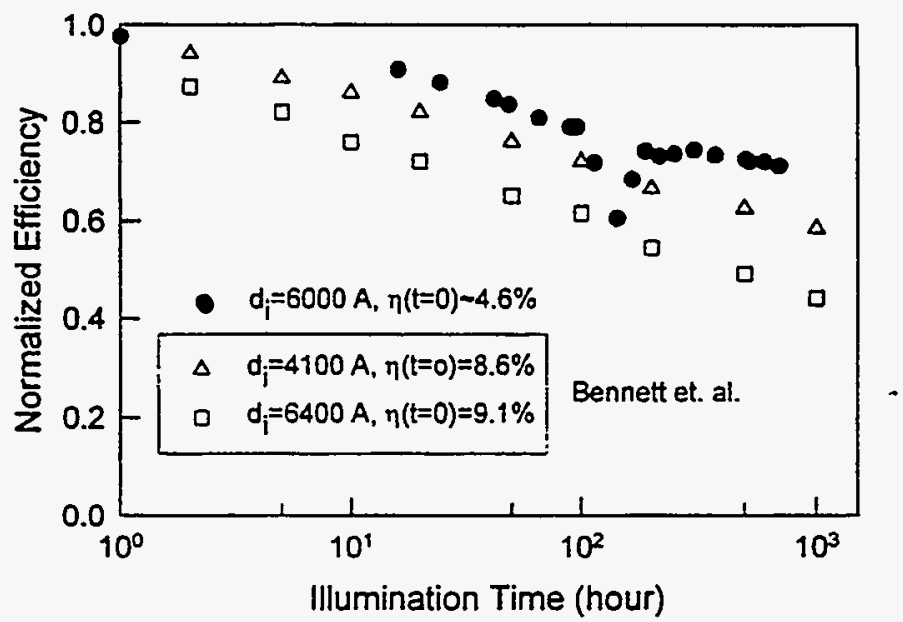

Fig. 2. Relative change in the conversion efficiency of $\mathrm{a}-\mathrm{SiS}_{\mathrm{x}}: \mathrm{H}$ homojunction solar cells with lightly S-doped $\mathrm{a}-\mathrm{Si}: \mathrm{H}$ as the I layer. The degradation was carried out at room temperature using $100 \mathrm{~mW} / \mathrm{cm}^{2}$ of white light. Included also are the data from Ref. 18 using heterojunction cells and carried out at an illumination temperature of $40^{\circ} \mathrm{C}$. 
Title:

Organization:

Contributors:

\section{Electrochemical Fabrication of Dye-Sensitized Heterojunctions}

Department of Chemical Engineering, University of Washington, Seattle, WA.

Daniel T Schwartz, Principal Investigator, Brian O'Regan, Graduate Assistant.

\section{Objectives and Approach:}

The objective of this research is to fabricate a solid state version of the dye sensitized photo electrochemical (DSPEC) solar cell recently developed by Grätzel et al.[1] The DSPEC cell is based on high-surface-area (nano-porous) thin films of $\mathrm{TiO}_{2}$, sensitized with a visible light absorbing dye, and perfused with a redox electrolyte. To make a solid state analog to the DSPEC cell the electrolyte must be replaced with a suitable solid hole conductor. The approach taken in this research is replace the electrolyte with a wide-band-gap p-type semiconductor. The resulting structure consists of a porous n-type semiconductor $\left(\mathrm{TiO}_{2}\right)$ whose pore structure is filled with a different p-type semiconductor, with a monolayer of dye separating the two semiconductors at all points. This structure is thus a high surface area dye sensitized heterojunction (DSH).

Prior research has identified CuSCN as a promising p-type semiconductor that shows high quantum efficiency in liquid-junction dye-sensitization experiments. [2,3] Our results have also shown that planar (non-porous) dye sensitized heterojunctions of $\mathrm{TiO}_{2} /$ dye/CuSCN can show high quantum efficiencies for converting absorbed photons into electrons (APCE), and reasonable open circuit voltages.[4] To form a high-surface-area $\mathrm{DSH}$ the pores of the $\mathrm{TiO}_{2}$ film must be completely filled with CuSCN. Optimally, one would impregnated the $\mathrm{TiO}_{2}$ with liquid CuSCN. Unfortunately the melting point of CuSCN is too high for this process to be used. Electrodeposition may also be used to fill solid structures with another solid. CuSCN may be electrodeposited from solutions of $\mathrm{Cu}$ (II) and $\mathrm{SCN}^{\top}$.[5] We are investigating the growth of electrodeposited films of CuSCN inside porous $\mathrm{TiO}_{2}$ films on conductive substrates.

\section{Results}

Work on this subcontract began in September 1995. Since that time we have confirmed that planar $\mathrm{TiO}_{2} / \mathrm{CuSCN}$ DSHs can show high internal quantum efficiency. We have fabricated planar $\mathrm{TiO}_{2} /$ dye/CuSCN interfaces with a ruthenium bipyridyl dye adsorbed to the $\mathrm{TiO}_{2}$ via a phosphonate moiety. The action spectra of the resulting DSH resembles that of the dye in the visible region (Fig. 1). Based on the surface area and the expected dye coverage the APCE is $\geq 50 \%$. The open circuit potential is $\sim 450$ $\mathrm{mV}$. 
We have also confirmed that the electrodeposition of CuSCN can at least partially fill the pore structure of a $\mathrm{TiO}_{2}$ film. We are investigating the degree to which the pores are filled by measuring the porosity of the $\mathrm{TiO}_{2}$ films $(\sim 50 \%)$ and the change in weight after CuSCN deposition. SEMs show that the CuSCN grows out from the back of the TiO2 film as desired, as opposed to growing randomly throughout the film. However, the conductivity of the CuSCN in the pore structure seems to be lower than that of CuSCN electrodeposited on non-porous substrates. This may indicate that solvent inclusions form gaps in the CuSCN or that the nano-structured nature of the porous structure inhibits charge transport.

\section{References}

1. M. K. Nazeeruddin, A. Kay, I. Rodicio, R. Humphry-Baker, E. Müller, P. Liska, N. Vlahopoulos, and M. Grätzel, J. Am. Chem. Soc. 115, 6382 (1993).

2. K. Tennakone, M. Kahanda, C. Kasige, P. Abeysooriya, R. H. Wijayanayaka, and P. Kaviratna, J. Electrochem. Soc. 131, 1574 (1984).

3. B. O'Regan and D. T. Schwartz, Chem. Mater. 7, 1349 (1995).

4. B. O'Regan and D. T. Schwartz, submitted to J. Appl. Phys.

5. B. O'Regan and D. T. Schwartz, in Symposium on Nanostructured Materials in Electrochemistry; edited by G. J. Meters and P.C. Season (Electrochemical Society, Penning ton, NJ, 1995), p. 208.

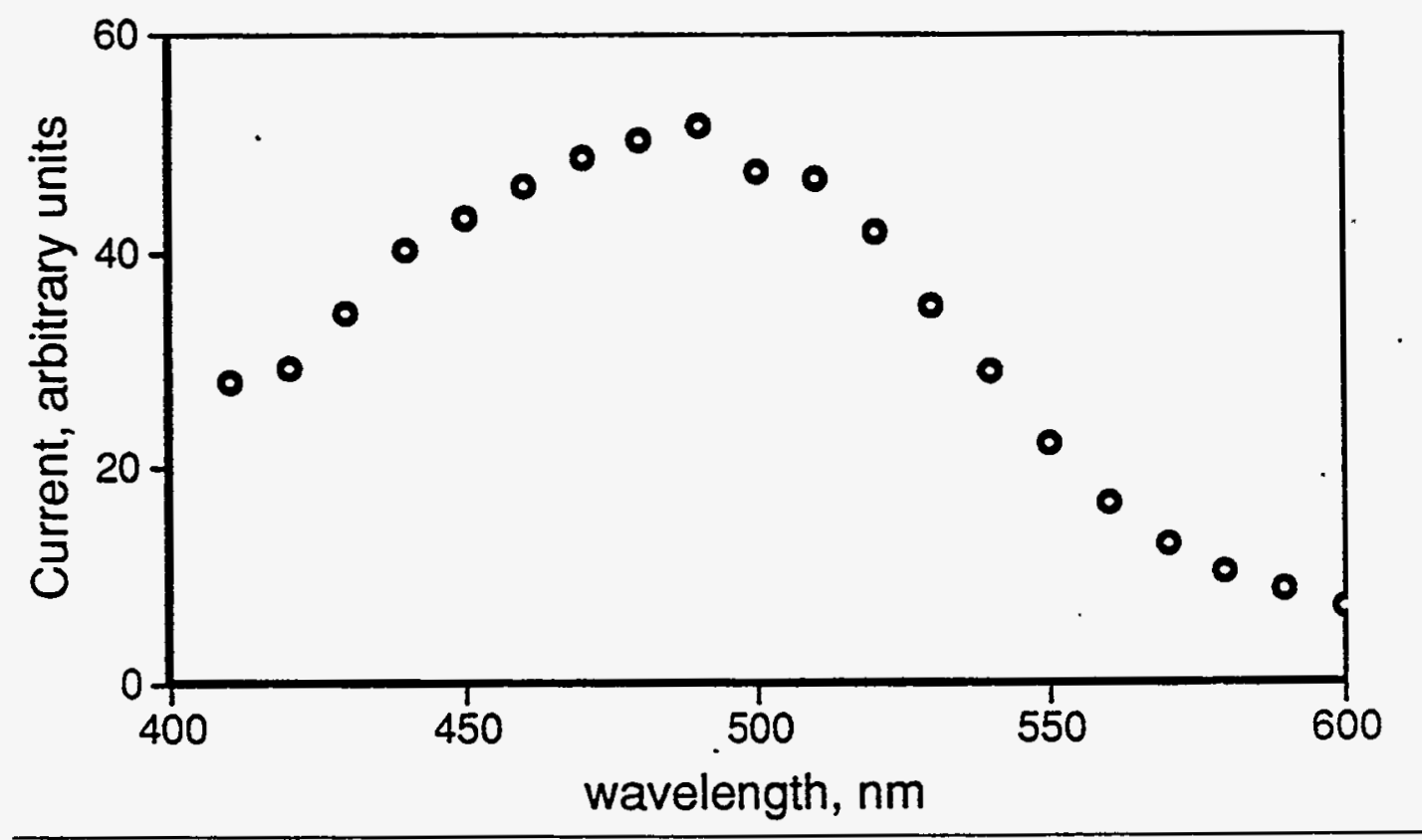

Figure 1. Action spectra of a $\mathrm{TiO}_{2} /$ dye/CuSCN dye sensitized heterojunction. The peak of the dye absorption spectrum is also $490 \mathrm{~nm}$. 


\title{
3.0 THIN-FILM TECHNOLOGIES PROJECT
}

\author{
Ken Zweibel, Manager
}

The Thin-Film Technologies Project encompasses a-Si and polycrystalline thin films of CIGS, $\mathrm{CdTe}$, and Si. Work within the Thin-Film Partnership Program has been organized into teamed research with other organizations around working groups in the various thin films. New teams were formed in CIGS, CdTe, and ES\&H. They joined the existing "model" team in a-Si, which has been in place for 3 years.

Progress in improved cell, submodule, and module efficiencies continued during the year. Figure 3-1 shows the ongoing progress in cell efficiencies. NREL once again raised the world record for cell efficiency above its previous high, now to $17.1 \%$-a level of performance starting to approach the record efficiency $(17.8 \%)$ for wafer-based polycrystalline Si. Such efficiencies were once thought impossible for thin films. Meanwhile, submodule and module efficiencies also improved (Table 3-1), as did power output and size. Several companies announced firm plans for new production or are completing new thin-film facilities. Companies such as United Solar Systems Corporation (USSC) and Solarex-Enron announced plans for significant new production of a-Si modules (greater than 20-MW planned capacity), and a new 5-MW Energy Conversion Devices (ECD)-USSC plant is nearing completion in Troy, Michigan.

Solar Cells, Inc. (SCI), or Toledo, Ohio, began delivering significant levels of CdTe modules to demonstrations throughout the United States (Toledo Edison, and the U.S. Navy at China Lake). Meanwhile, Golden Photon, Inc. (GPI), moved rapidly toward initial production at its new 2-MW facility in Golden, Colorado. By the end of the year, GPI sales had reached nearly $100 \mathrm{~kW}$ and production was approaching capacity.

In CIGS, companies moved to meet the significant challenges of CIGS scale-up. Siemens Solar Industries (SSI) of Camarillo, California, worked intensively on smaller-area (about $100 \mathrm{~cm}^{2}$ ) submodules to solve critical yield issues. SSI successfully improved efficiencies, significantly tightened yield, and developed a new, more forgiving set of processing techniques. Meanwhile, Energy PV of Princeton, New Jersey, moved rapidly from cell experimentation to submodule and module fabrication. They have begun larger-area $\left(6 \mathrm{ft}^{2}\right)$ module experiments and commercialization. Another company, ISET, developed an important new low-cost CIS deposition process, demonstrating cell efficiencies of over $12 \%$ using unalloyed CIS. 


\section{Figure 3-1. Polycrystalline Thin Film Solar Cell Efficiencies}

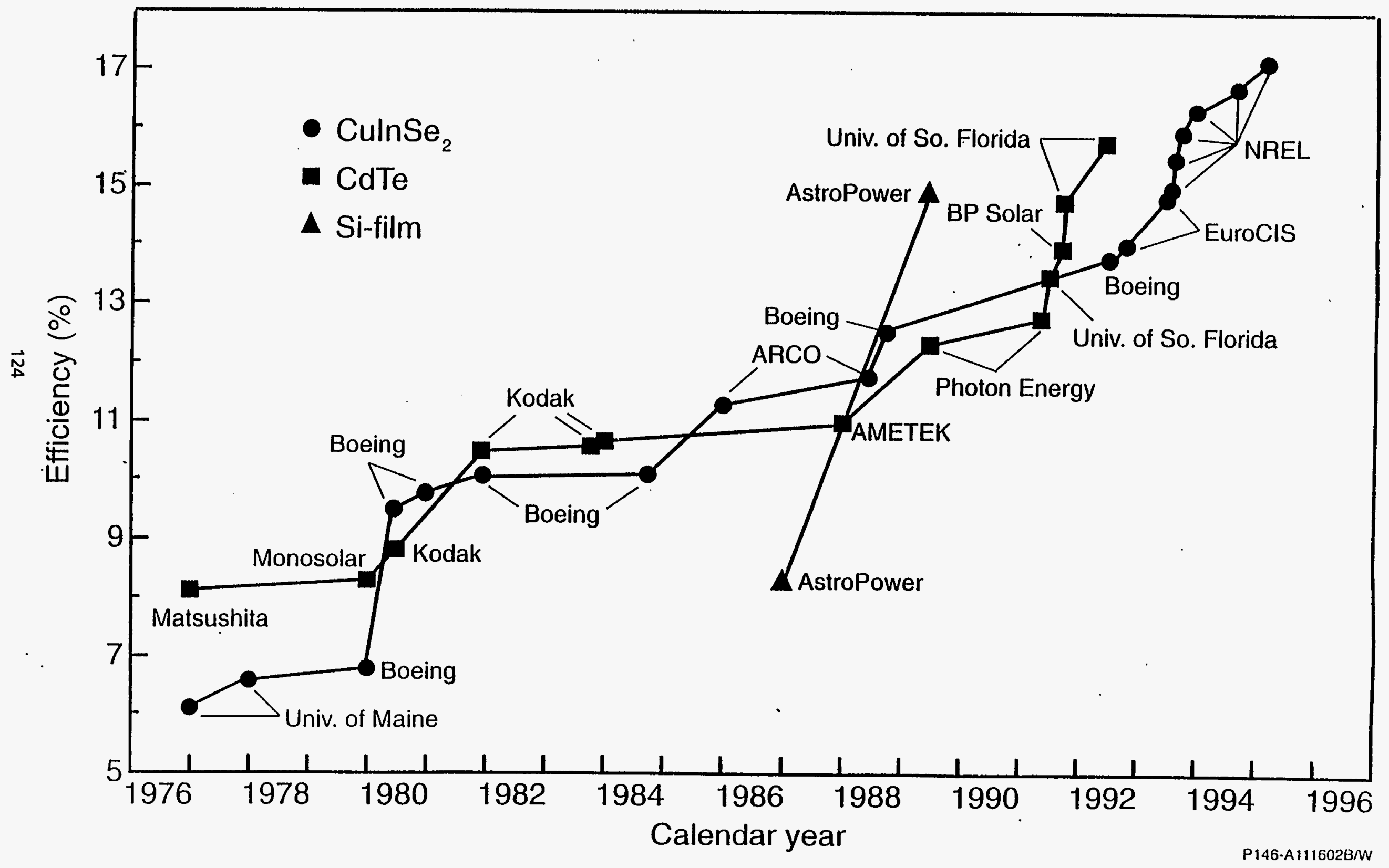


Table 3-1. Best Thin-Film Modules

(after preliminary light-soaking for a-Si)

\begin{tabular}{||l|c|c|c|c||}
\hline \multicolumn{1}{|c|}{ Company } & Material & Area $\left(\mathrm{cm}^{2}\right)$ & Efficiency & Power \\
\hline SCI & CdTe & 7200 & $8.4 \%$ & $60.3 \mathrm{~W}$ \\
\hline APS & a-Si/a-Si & 11,522 & $4.6 \%$ & $53.0 \mathrm{~W}$ \\
\hline SSI & $\mathrm{CIS}$ & 3832 & $11.2 \%^{1,2}$ & $43.1 \mathrm{~W}$ \\
\hline SSI & $\mathrm{CIS}$ & 3859 & $10.2 \%$ & $43.1 \mathrm{~W}$ \\
\hline BP Solar & CdTe & 4540 & $8.4 \% 1.3$ & $38.2 \mathrm{~W}$ \\
\hline ECD & $\begin{array}{c}\text { a-Si/a-Si/ } \\
\text { a-SiGe }\end{array}$ & 3906 & $7.8 \%$ & $30.6 \mathrm{~W}$ \\
\hline Golden Photon & CdTe & 3528 & $7.7 \%$ & $27.5 \mathrm{~W}$ \\
\hline Solarex & a-Si/a-SiGe & 3676 & $7.8 \%$ & $26.9 \mathrm{~W}$ \\
\hline USSC & a-Si/a-Si & 3676 & $6.2 \%$ & $22.8 \mathrm{~W}$ \\
\hline Fuji & a-Si/a-Si & 1200 & $8.9 \%$ & $10.7 \mathrm{~W}$ \\
\hline SSI & CIS & 938 & $11.1 \%$ & $10.4 \mathrm{~W}$ \\
\hline Matsushita Battery & CdTe & 1200 & $8.7 \%$ & $10.0 \mathrm{~W}$ \\
\hline USSC & a-Si/a-Si/ & 903 & $10.2 \%$ & $9.2 \mathrm{~W}$ \\
\hline BP Solar & CdTe & 706 & $10.1 \%$ & $7.1 \mathrm{~W}$ \\
\hline \hline
\end{tabular}

Note: Updated December 1995

'Not measured at NREL

${ }^{2}$ Unencapsulated

${ }^{3}$ Not monolithic 
Table 3-2. Thin-Film Partnership Highlights FY 1995

\begin{tabular}{|c|c|c|}
\hline Who & What & Why \\
\hline NREL CIS Task & $\begin{array}{l}\text { World-record thin-film cell } \\
\text { efficiency (17.1\%) for a CIGS cell }\end{array}$ & $\begin{array}{l}\text { Establishes the potential of } \\
\text { thin films to achieve long-term } \\
\text { efficiency goal (15\%) for } \\
\text { modules }\end{array}$ \\
\hline $\begin{array}{l}\text { NREL Amorphous Silicon } \\
\text { Task }\end{array}$ & $\begin{array}{l}\text { Established 'hot-wire' deposition } \\
\text { as a viable alternative to glow- } \\
\text { discharge (GD) a-Si deposition }\end{array}$ & $\begin{array}{l}\text { Appears to be a possible route } \\
\text { to nearly stable a-Si devices } \\
\text { (Staebler-Wronski }<10 \% \text { ) and } \\
\text { much higher deposition rates } \\
\text { (more than an order of } \\
\text { magnitude higher). Work } \\
\text { done cooperatively with } \\
\text { United Solar. }\end{array}$ \\
\hline $\begin{array}{l}\text { NREL Amorphous Silicon } \\
\text { Task }\end{array}$ & $\begin{array}{l}\text { Showed viability of making } \\
\text { improved films by hydrogen } \\
\text { dilution that is limited to the } \\
\text { interface region }\end{array}$ & $\begin{array}{l}\text { Hydrogen dilution is used to } \\
\text { make more stable GD a-Si; } \\
\text { this method allows it to be } \\
\text { used for a very limited } \\
\text { duration, speeding up GD } \\
\text { deposition rates substantially }\end{array}$ \\
\hline Solarex (Amoco-Enron) & $\begin{array}{l}\text { Joint venture to manufacture low- } \\
\text { cost a-Si modules }\end{array}$ & $\begin{array}{l}\text { First major commitment to } \\
\text { manufacture }(10 \mathrm{MW}) \text { low- } \\
\text { cost PV modules based on an } \\
\text { advanced thin-film technology }\end{array}$ \\
\hline United Solar & $\begin{array}{l}\text { Building 5-MW a-Si plant in } \\
\text { Michigan }\end{array}$ & $\begin{array}{l}\text { Major step to commercialize } \\
\text { their innovative flexible- } \\
\text { substrate modules }\end{array}$ \\
\hline Solarex-NREL CIS Task & $\begin{array}{l}\text { World-record thin-film } \\
\text { minimodule }\left(12.9 \%, 40-\mathrm{cm}^{2}\right. \\
\text { CIGS) }\end{array}$ & $\begin{array}{l}\text { Establishes Solarex as new, } \\
\text { active company in leading CIS } \\
\text { development (work done } \\
\text { closely with NREL) }\end{array}$ \\
\hline Solar Cells, Inc. & $\begin{array}{l}\text { World's largest CdTe arrays (two } \\
10-\mathrm{kW} \text { arrays; Toledo Edison and } \\
\text { PVUSA) }\end{array}$ & $\begin{array}{l}\text { Largest commercial CdTe } \\
\text { arrays; initial grid-connected } \\
\text { systems of a key, new thin- } \\
\text { film technology }\end{array}$ \\
\hline Golden Photon, Inc. & $\begin{array}{l}\text { Shakedown tests of new 2-MW } \\
\text { CdTe production facility }\end{array}$ & $\begin{array}{l}\text { Largest production facility of } \\
\text { a key, new thin-film } \\
\text { technology }\end{array}$ \\
\hline
\end{tabular}


Table 3-2. Thin-Film Partnership Highlights FY 1995 (Concluded)

\begin{tabular}{||l|l|l||}
\hline \multicolumn{1}{|c|}{ Who } & \multicolumn{1}{|c|}{ What } & \multicolumn{1}{|c|}{ Why } \\
\hline ISET & $\begin{array}{l}\text { High-efficiency CIS cells (>12\%) } \\
\text { made by unique, low-cost process }\end{array}$ & $\begin{array}{l}\text { Only high-efficiency process } \\
\text { in CIS in which precursors are } \\
\text { delivered by a low-cost, } \\
\text { nonvacuum method }\end{array}$ \\
\hline AstroPower, Inc. & $\begin{array}{l}\text { Highest efficiency film x-Si on a } \\
\text { low-cost substrate (10.5\%) }\end{array}$ & $\begin{array}{l}\text { First successful growth of film } \\
\text { silicon on a truly low-cost } \\
\text { substrate; opens up possibility } \\
\text { of competing with direct-gap } \\
\text { thin films }\end{array}$ \\
\hline Institute of Energy \\
Conversion & $\begin{array}{l}\text { High Ga content. High-band-gap } \\
\text { CIGS cell with efficiency over }\end{array}$ & $\begin{array}{l}\text { First demonstration that a } \\
\text { CIGS cell with Ga content } \\
\text { about 50\% can be efficient; } \\
\text { opens up possibility of } \\
\text { redesigning CIGS module } \\
\text { technologies for better } \\
\text { outdoor performance }\end{array}$ \\
\hline Colorado School of Mines & $\begin{array}{l}\text { High-efficiency (>12\%) CdTe cell } \\
\text { made with ZnTe contact }\end{array}$ & $\begin{array}{l}\text { Establishes new, potentially } \\
\text { more stable contact to CdTe } \\
\text { (an important thin-film } \\
\text { technology) }\end{array}$ \\
\hline \hline
\end{tabular}




\section{Thin-Film Technologies In-House Research}



Title: $\quad$ Development of Polycrystalline Thin-Film CdTe Solar Cells Deposited by CloseSpaced Sublimation

Organization: National Renewable Energy Laboratory

Golden, Colorado

Contributors: P. Sheldon (Principal Investigator), T.A. Gessert, D.S. Albin, R.G. Dhere, X. Li, D. Rose, D. Niles, S.A. Asher, D. Levi

\section{Objectives:}

- $\quad$ Complete infrastructure necessary to support a strong CdTe research program.

- Develop reproducible processes for high-efficiency CdS/CdTe devices. Develop a baseline CdS/CdTe device process necessary to critically evaluate new processes and obtain a fundamental understanding of the physics and chemistry of the device structure.

- $\quad$ Provide industrial partner support through cooperative research and development agreements (CRADAs) and materials and device characterization.

\section{Technical Approach:}

In FY 1995 the CdTe task focused on CdS/CdTe devices fabricated using chemical-bath-deposited $\mathrm{CdS}$ and close-spaced-sublimation (CSS)-deposited CdTe. The in-house CdTe research program consists of an integrated effort, focused on five key areas: (i) Completion of an infrastructure development plan, initiated in FY 1994, to develop deposition hardware to improve process control and productivity; (ii) Development of a robust and reproducible baseline process, necessary to critically evaluate alternate processes and device structures; (iii) Development of an alternate highperformance, manufacturable ohmic contact; (iv) Development of enhanced characterization techniques necessary to critically evaluate thin films and devices; and (v) Provide industrial partner support. Progress in each of these areas is outlined below.

\section{Results:}

\section{Infrastructure Development:}

In FY 1994 the CdTe team initiated an aggressive infrastructure development plan. Our goal was to develop hardware that yields highly reproducible films and devices (a necessary component for establishing a baseline process). In FY 1995, the bulk of this plan was completed with the design, construction, and commissioning of three deposition systems. The comerstone of this project was the development of two state-of-the-art CSS deposition systems (2m- and 3m-CSS deposition systems). These CSS systems offer process control capabilities that have not been realized in other CSS deposition systems. These features include: (i) A fully automated process control system for improved run-to-run repeatability and temperature profiling; (ii) Fully automated and modular gas introduction system; (iii) Complete data-logging capability for all temperature and pressure process variables; and (iv) Significantly improved source and substrate temperature uniformity $\left( \pm 1^{\circ} \mathrm{C}\right)$ over a $25-\mathrm{cm}^{2}$ area. The CSS $3 \mathrm{~m}$ system, shown in Figure 1, was commissioned in 3/95, and produced a $>10 \%$ device after only the fourth deposition run. The final phase of this infrastructure 
development plan will be realized in FY 1996 with the installation of a low-pressure chemical vapor deposition (CVD) system for transparent conducting oxide (TCO) development work.

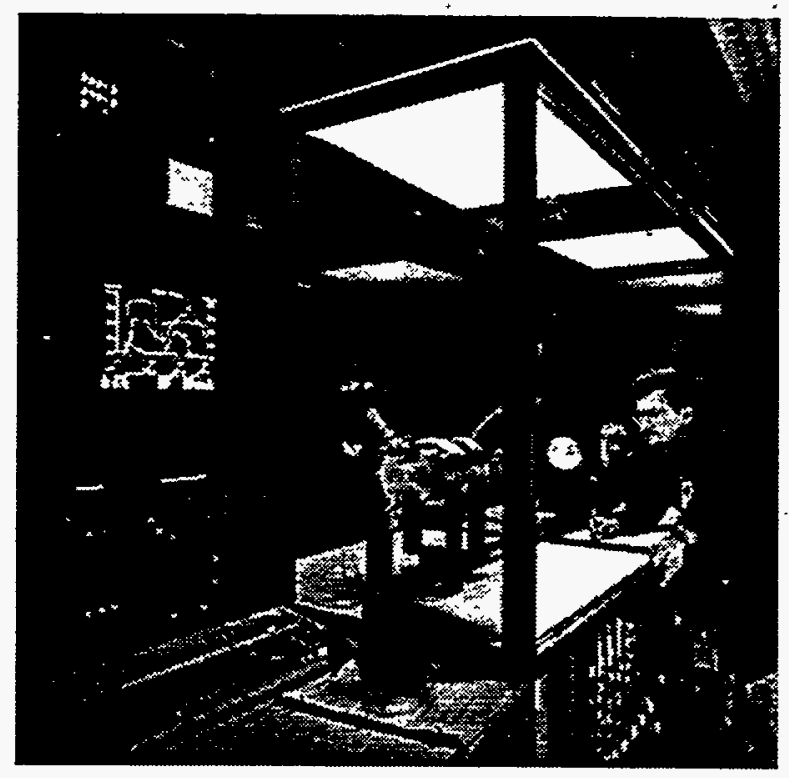

Figure 1. CdTe CSS $3 \mathrm{~m}$ reactor completed and commissioned in FY 1995.

CdS/CdTe Baseline Process Development:

One of the CdTe task's FY 1995 milestones was to develop a 7\% CdS/CdTe baseline process. Establishing a reproducible baseline process allows us to critically evaluate alternate processing schemes, alternate ohmic back-contact sţretures (an induștry priority), and alternate device configurations. The data shown in Figure 2 are device efficiencies from over 250 depositions performed over the past year in the old-, $2 \mathrm{~m}$ - and $3 \mathrm{~m}$-CSS deposition systems. These devices had an $i$-SnO $\mathrm{O}_{2}$ layer of $\sim 1500 \AA$, a CdS layer thickness of $800<\mathrm{t}<1000 \AA$, and a CdTe layer thickness of $5<\mathrm{t}<15 \mu \mathrm{m}$. It is important to note that the deposition conditions in these runs were not kept constant in all cases. In fact, these depositions used a variety of temperature profiles and processing conditions which significantly influence film properties. In spite of this, we were able to identify postdeposition processing conditions which yielded device efficiencies of greater than $7 \%$ in almost all cases. This figure also demonstrates that we have successfully transferred our process to the new deposition systems (the $2 \mathrm{~m}$ and $3 \mathrm{~m}$ systems), which are producing material that is better than that from the old CSS system.

A second critical CdTe task milestone (due $1 / 96$ ) was to improve the $7 \% \mathrm{CdS} / \mathrm{CdTe}$ baseline process to a $>10 \%$ baseline process. Using the enhanced control available with the new deposition systems described above, we have successfully demonstrated a $>11 \%$ baseline process (milestone met 06/95). Experiments were designed to evaluate the statistical repeatability of several baseline processes developed for fabricating CdS/CdTe solar cells. A series of 28 devices were fabricated using three different CdTe source plates, with otherwise identical processing conditions. Substrates consisted of doped tin-oxide 7059 substrates coated with an intrinsic $i$-SnO ${ }_{2}$ layer $(\sim 1400 \AA$ thick) and a thin (900-1000 $)$ chemical-bath-deposited CdS layer. 


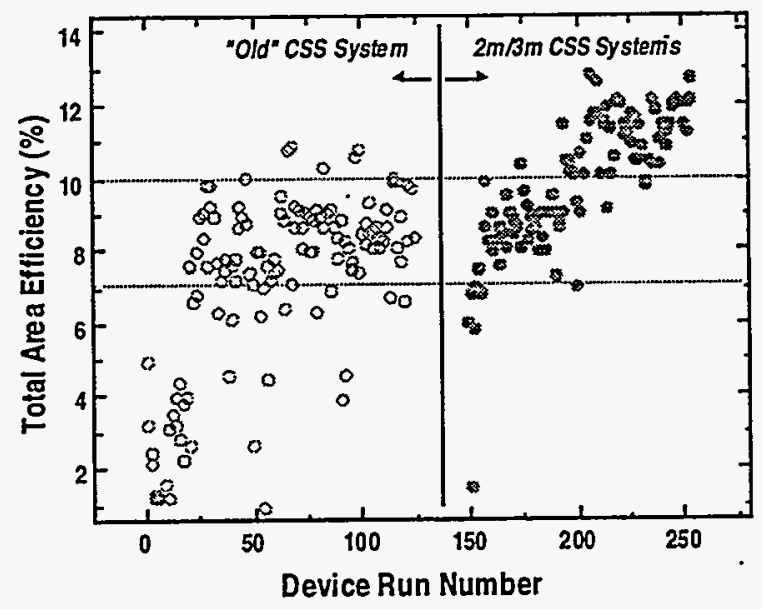

Figure 2. Compilation of device efficiencies from over 250 depositions performed over the past year in the old-, $2 \mathrm{~m}-$, and $3 \mathrm{~m}-\mathrm{CSS}$ deposition systems.

The repeatability of the process was then quantified by determining the statistical mean and standard deviation of these device parameters for the 28 processed devices. Upper and lower control limits for our process were then calculated using two standard deviations from the mean. These limits represent a 95\% confidence interval for random variations of the particular parameter mean. A process control chart for device efficiency for all 28 devices is shown in Figure 3 (data in this figure are based on XT-10 solar simulator measurements, which are typically lower relative to confirmed measurements). The unbiased data for the entire set of samples yielded a process with a statistical mean of $11.13 \%$. This work has led to several devices with NREL-verified efficiencies of $12.8 \%$.

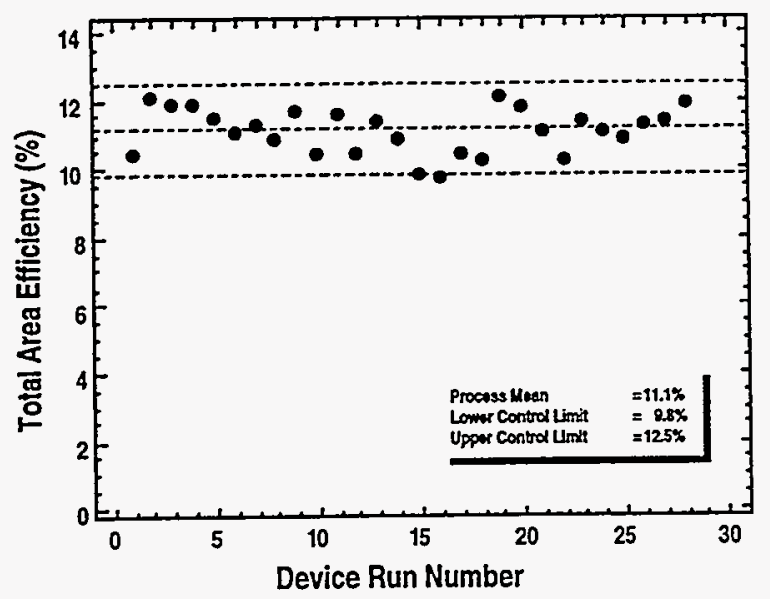

Figure 3. Device efficiencies for 28 devices deposited and processed under identical conditions. 
As part of this investigation, we have also made significant progress in understanding how oxygen -influences CSS CdTe depositions. Previously, this work was not possible because material deposited in our old CSS system was relatively insensitive to variations in oxygen partial pressures. In the old system, we found that hot graphite was efficiently converting $\mathrm{O}_{2}$ to $\mathrm{CO}$ and $\mathrm{CO}_{2}$, which, unlike $\mathrm{O}_{2}$, is not efficiently incorporated in the deposited film. In the new deposition systems, this reaction is nearly eliminated; therefore, we are able to explore the effect of oxygen on material quality and device performance. For many years the presence of oxygen during. CSS of CdTe has been regarded as necessary for high-efficiency CdS/CdTe solar cells because it was thought to increase the p-type carrier density in the absorber. We found that oxygen has many other effects, some of which are more pronounced than its effect on doping and some of which are, in fact, detrimental to device performance and/or manufacturability.

We found that oxygen significantly influences the nucleation and growth of CdTe films. This is clearly seen in the scanning electron microscopy (SEM) micrographs shown in Figures $4 \mathrm{a}$ and $\mathrm{b}$. The film in Figure 4a was deposited in $15 \mathrm{Torr} \mathrm{He}\left(\mathrm{no} \mathrm{O}_{2}\right)$, whereas the film in Figure $4 \mathrm{~b}$ was deposited in 2 Torr $\mathrm{O}_{2}: 13$ Torr He. Both films were grown at a substrate temperature of $600^{\circ} \mathrm{C}$ and a source temperature of $660^{\circ} \mathrm{C}$ (deposition times were adjusted to compensate for the reduced sublimation rate realized at higher oxygen partial pressures). Increasing the oxygen partial pressure from 0 to 2 Torr increases the number of nucleation sites, thereby suppressing pin-hole formation and reducing defects at the junction. However, as seen in Figure 4, increasing oxygen levels also tends to decrease grain size and faceting in films. We also found that oxygen ambient effects the minority-carrier lifetime in CdTe. We used time-resolved photoluminescence (PL) and PL spectroscopy to probe $\mathrm{CdS} / \mathrm{CdTe}$ samples made at a range of oxygen partial pressures. Laser excitation at $600 \mathrm{~nm}$ allowed us to selectively probe within $1 \mu \mathrm{m}$ of the surface from either the front or back of the cell. Our measurements indicate that the bulk CdTe (near the back of the cell) has lower nonradiative recombination when grown in the absence of oxygen. The relative PL intensities from the bulk CdTe, before $\mathrm{CdCl}_{2}$ heat treatments, were $1700,900,775$ and $610 \mathrm{ps}$ for films deposited in oxygen partial pressures of $0,1,2$ and 8 Torr, respectively. In contrast, the intermixed CdSTe layer (near the front of the cell) has the best quality when grown in 1 to 2 Torr of oxygen.
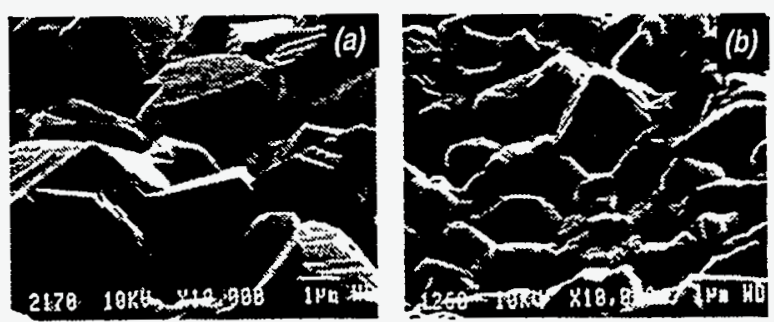

Figure 4. SEM micrographs of CSS CdTE films deposited with 0 Torr oxygen (a) and 2 Torr oxygen (b) at a substrate temperature of $600^{\circ} \mathrm{C}$ 
Combining these ideas we have successfully fabricated a cell, without using oxygen at any stage of the production, with an NREL-verified efficiency of $12.8 \%\left(\mathrm{~V}_{\mathrm{oc}}=0.804 \mathrm{~V}, \mathrm{~J}_{\mathrm{sc}}=22.0 \mathrm{~mA} / \mathrm{cm}^{2}\right.$, and $\mathrm{FF}=72.4 \%$ ). These results clearly indicate that oxygen significantly influences material quality and device performance, and thus, must be carefully controlled during deposition. Work in this area is ongoing.

\section{Alternative Ohmic Contacts to CdTe (Ni/ZnTe:Cu/CdTe):}

Efficiency results exceeding $10 \%$ have been confirmed for NREL-fabricated CSS CdS/CdTe solar cells processed with a $\mathrm{ZnTe}: \mathrm{Cu} / \mathrm{Ni}$ contact. These results represent an encouraging benchmark in the ongoing developmental work aimed at incorporating the $\mathrm{ZnTe}$-based contact into the baseline process of the CdTe project. Processing advantages of this contact (compared to the presently used $\mathrm{Hg}$ doped graphite contact) include the ability to produce test devices from smaller samples, improved device active-area definition, greatly improved reproducibility of contact characteristics, and improved manufacturability. The performance parameters of the highest efficiency device $\left(0.11 \mathrm{~cm}^{2}\right)$ were: $\mathrm{V}_{\mathrm{cc}}=749 \mathrm{mV}, \mathrm{J}_{\mathrm{sc}}=20.8 \mathrm{~mA} / \mathrm{cm}^{2}, \mathrm{FF}=67 \%, \eta=10.4 \%$. Except for the $\mathrm{J}_{\mathrm{sc}}$, these parameters are lower than those of the control device produced with the baseline $\mathrm{Hg}$-doped graphite process. However, it should be noted that this study to optimize NREL devices has just begun (only three NREL CSS devices have been produced with the $\mathrm{ZnTe}$-based contact), and the process is not yet optimized for these devices. Furthermore, based on the experience gained from similar studies pertaining to the recently completed CRADA between NREL and Solar Cells, Inc. (SCI), small changes in the $\mathrm{ZnTe}$ and Ni deposition parameters, as well as optimization of the surface treatments used prior to contacting, will likely lead to significant improvement in $\mathrm{V}_{\infty}$ and FF for the NREL devices.

\section{Characterization Technique Development:}

We have discovered that sputter depth profile measurements (i.e., secondary ion mass spectrometry [SIMS] and Auger analysis) of polycrystalline $\mathrm{CdTe} / \mathrm{CdS}$ interfaces are complicated by the native roughness of the CdTe film surface. The CdTe surface roughness is affected by both the growth conditions and the film thickness. This makes it difficult to separate features of diffusion at the interface from topographically induced broadening of the ion signals. A method has been developed at NREL for polishing the surface of CdTe films prior to depth profile analysis. This method produces surfaces with an average roughness of $<60 \AA$ (initial surface roughness in these samples was as great as $6000 \AA$ ). The two depth profiles shown in Figure 5 illustrate the significant improvement in depth resolution obtained with this method. Figure 5a shows a SIMS depth profile from an as-deposited CdTe/CdS film. The average surface roughness of this film was $4900 \AA$. In this profile, the CdS layer appears to be absent or greatly broadened. The low sulfur signal tends to support the conclusion that the CdS layer has been consumed (diffused) and is no longer present. Figure $5 b$ is a SIMS depth profile from the center of a polished area on the same sample. In the polished area the average surface roughness was $58 \AA$ prior to analysis. This profile clearly shows the presence of a thin CdS layer under the CdTe. The small amount of topography generated by the ion beam during the analysis (increasing from 58 to $143 \AA$ ) does not obscure the layer. This result is extraordinary since the thickness of the CdTe film is $>4.5 \mu \mathrm{m}$ and the thickness of the underlying CdS layer is $\sim 1000 \AA$ The signal levels obtained from the bulk of the CdTe, however, appear to be unaffected by the surface roughness. 


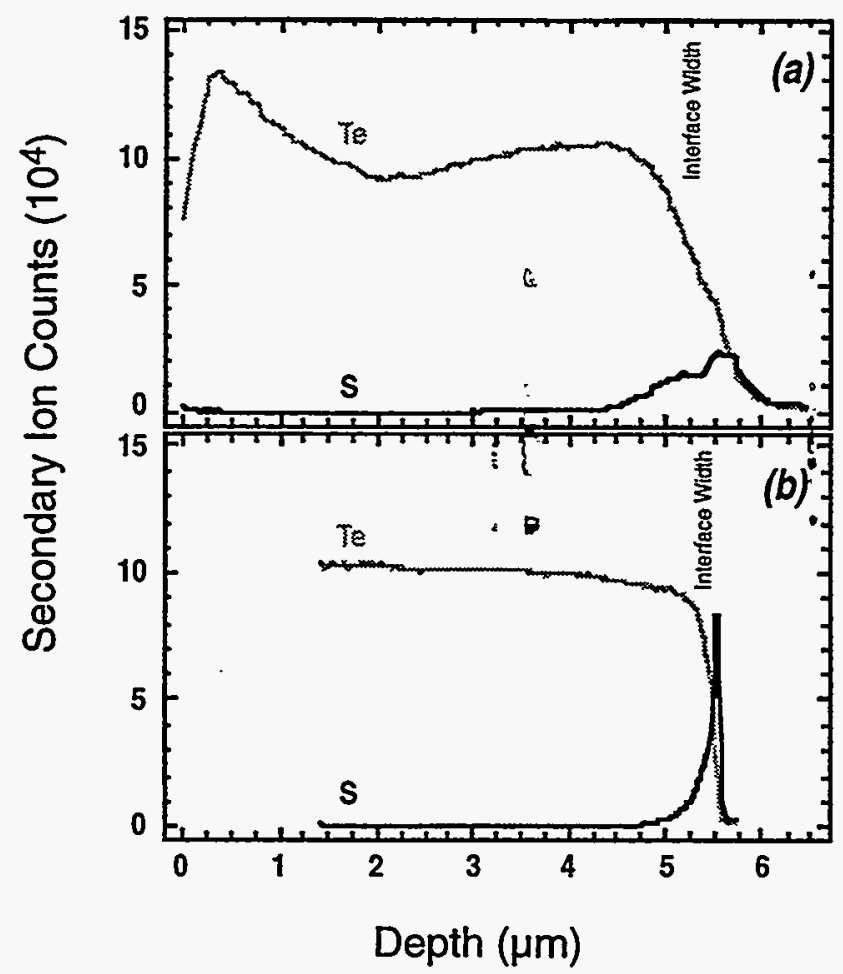

Figure 5. SIMS depth profiles from the same CSS CdTe film. Profile (a) is acquired from the as-deposited surface and (b) is acquired from a polished surface.

In the past, it has been difficult to compare depth profiles from $\mathrm{CdTe} / \mathrm{CdS}$ devices grown by different methods. Or even to compare the results from films with different surface morphologies. This lack of standardization has made it difficult to understand the optimum configuration of the heterointerface. The ability to produce a uniform surface for depth profile analysis will allow experiments to be performed where growth parameters are varied without complications from the resulting changes in surface morphology. It will also facilitate the study of different post-deposition treatments, such as $\mathrm{CdCl}_{2}$, on the interface. It will allow the comparison of materials grown by different methods. Work is ongoing.

\section{Industrial Partner Support:}

$: \begin{array}{ll}c \\ : c\end{array}$

Under a CRADA signed 8/94 with SCI, the NREL CdTe task has developed a PI-based diagnostic monitor to evaluate the uniformity and quality of $\mathrm{CdS} / \mathrm{CdTe}$ modules. This diagnostic system, shown in Figure 6, was tested at NREL using a variety of material supplied by SCI, and preliminary results are encouraging. The prototype system was delivered to $\mathrm{SCI}$ on $8 / 95$ to evaluate using material from their pilot production line. SCI will assess whether this system could be used as an in-line quality control monitor in a production environment. In FY 1996 the focus of this effort will shift towards developing a fundamental understanding of how the spectral emission from the CdS and intermixed 
$\mathrm{CdS}_{\mathrm{x}} \mathrm{Te}_{1-\mathrm{x}}$ layer correlates with device performance. The goal of this work is to apply this information to the PL monitor sent to SCI and develop an improved "next generation" characterization technique.

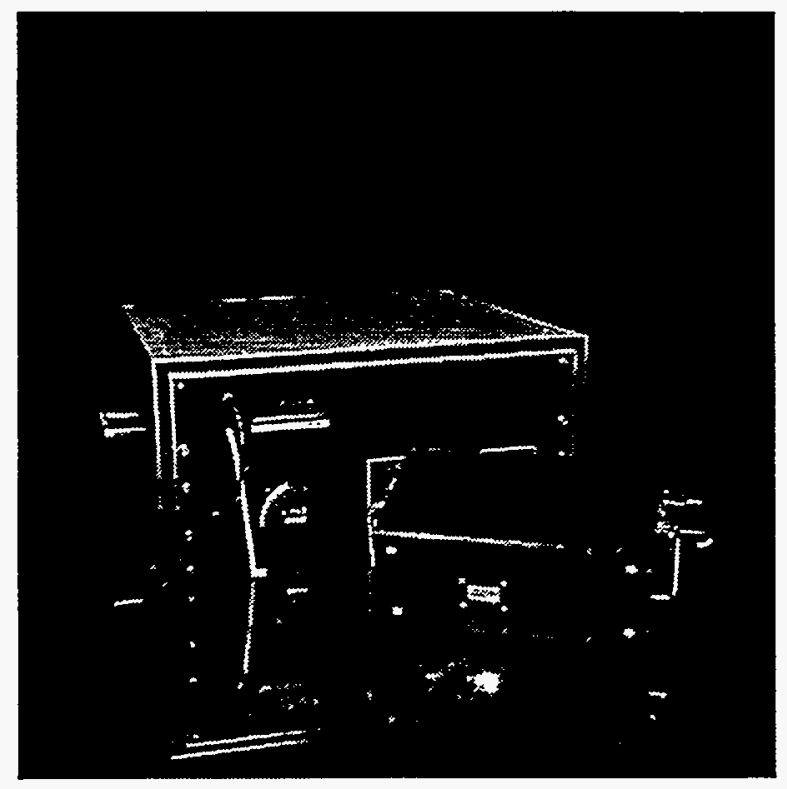

Figure 6. Prototype PL-based diagnostic monitor developed for and delivered to Solar Cells, Inc., in FY95

\section{Future Directions:}

In FY 1996, the emphasis will shift from our FY 1995 goal of developing a baseline process, to using the baseline process to explore alternate processes. This will be accomplished while maintaining our underlying theme of developing a fundamental understanding of the physics and chemistry of these processes. In FY 1996 we will complete our infrastructure development plan with the commissioning of a low-pressure CVD system for routine deposition of TCO films and for advanced TCO development research. We will also develop a dry CSS-based CdS deposition process as well as a vapor-based $\mathrm{Cl}$ treatment to replace the wet $\mathrm{CdCl}$ process. As described previously, we will continue work initiated in other areas, including alternate contact development, understanding the role of oxygen, and collaborative projects initiated with our industrial and team partners. 


\section{Development of Polycrystalline $\mathrm{Cu}(\mathrm{In}, \mathrm{Ga}) \mathrm{Se}_{2}$ Thin Films and Devices}

\section{Contributors:}

\section{Full time}

R. Noufi, Principal Investigator and Task Leader

J.R. Tuttle, Senior Scientist

K. Ramanathan, Senior Scientist

M. Contreras, Staff Scientist

A. Tennant, Assoc. Staff Scientist

J. Dolan, Master Technician

J. Keane, Master Technician

A. Gabor, Visiting Professional, EPV

L. Wang, Visiting Professional, CSM

K. Lindahl, Graduate Student, CSM

T. Berens, Graduate Student, CSM

\section{Part time}

S. Asher, Senior Scientist

R. Matson, Senior Scientist

M. Bode, Senior Scientist

D. Niles, Senior Scientist

R. Bhattacharya, Staff Scientist

B. Keyes, Staff Scientist

A. Franz, Assoc. Staff Scientist

A. Mason, Master Technician

J. Alleman, Master Technician

\section{Objective}

Advance the development of thin-film $\mathrm{Cu}(\mathrm{In}, \mathrm{Ga}) \mathrm{Se}_{2}$ (CIGS)-based photovoltaic technology by:

(1) Improving the material and device quality through diagnostics and process control to advance cell conversion efficiency.

(2) Developing alternative cell fabrication processes to present options for manufacturing.

(3) Addressing key technical issues in partnership with industry which will support their plans for near-term commercialization.

\section{Technical Approach}

We tackle the objectives in the following manner:

(1) Examine and optimize all components of absorber and balance-of-cell processing. This leads to enhanced baseline performance and improved understanding of the solar cell operation.

(2) Utilize device modeling expertise to suggest improvements in cell component design. This includes profiling the band gap of the absorber and understanding the role of interfaces in cell operation.

(3) Investigate physical and chemical nature of existing fabrication processes in order to evolve working models that facilitate the development of alternative processes for manufacturing environments.

(4) Work closely with industry on transferring this knowledge and developing new prototype technology. 


\section{Results}

Fundamental Studies. This effort has focused on problem solving when a specific need arises. One such example is our experiences with the Mo/soda-lime glass substrate and its influence on the properties and performances of films and devices. Secondary ion mass spectrometry analysis of impurity concentrations within the absorber bulk suggests that the Mo interlayer impacts the impurity migration process from the substrate to the absorber in a manner independent of the Mo bulk film properties. We do conclude, however, that the nature of the Mo surface, and its reactivity with Se, can substantially influence the characteristics of the absorber and the performance of the device. Future work will focus on characterizing the Mo surface and identifying the characteristics that lead to this performance.

$\mathrm{X}$-ray photoelectron spectroscopy (XPS) surface analysis, on the other hand, does indicate a relationship between Mo structural defects and Na migration to the absorber surface (Fig. 1). We have performed XPS analysis of dark spots which occasionally appear on our CIGS films. We have examined $\mathrm{Cu}$-rich and $\mathrm{Cu}$-poor films and noticed that, over several months time, dark spots appeared on the film that was $\mathrm{Cu}$-rich. The spots are visible to the naked eye, having a typical size of several tenths of a millimeter. Using the mapping capabilities of the new small-spot XPS system, we determined that the spots contain Na. The Na surface concentration is $\sim 20$ at.\% in these dark spots. High-resolution spectra indicate that the $\mathrm{Na}$ compound is $\mathrm{Na}_{2} \mathrm{SeO}_{3}$. Areas adjacent to the spot, as well as $\mathrm{Cu}$-poor films, did not contain any $\mathrm{Na}$, at least to the detection limit of XPS $(\sim 1$ at.\%).

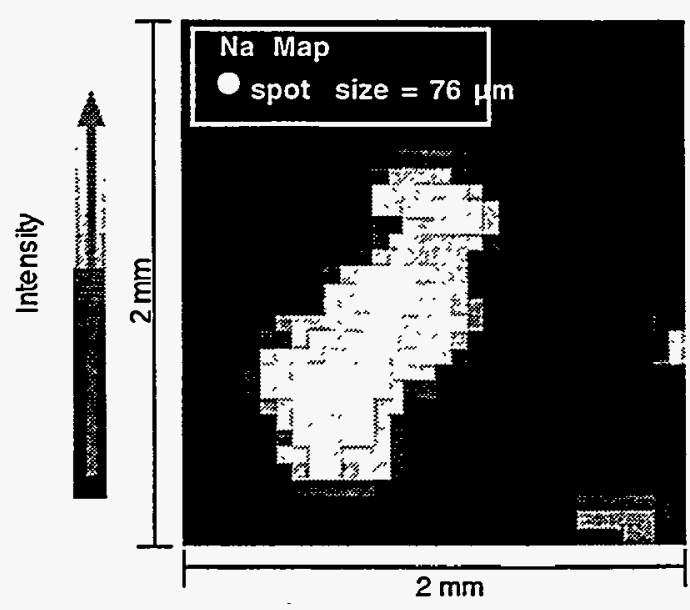

Figure 1. Surface Na map of visible defect region on CIGS absorber.

Device Development. The CuInSe ${ }_{2}$ (CIS) team once again raised the world-record efficiency for CIGS-based solar cells one more notch. We confirmed a $17.1 \%$ total-area efficient glass $/ \mathrm{Mo} / \mathrm{CIGS} / \mathrm{CdS} / \mathrm{ZnO} / \mathrm{MgF}_{2}$ solar cell with the following parameters: $\mathrm{V}_{\infty}=0.654$ volts, $\mathrm{J}_{\mathrm{sc}}=33.96 \mathrm{~mA} / \mathrm{cm}^{2}$, and $\mathrm{FF}=77.2 \%$ (see Table 1). The improved performance with respect to our $16.8 \%$ device is due to improved collection of carriers generated from weakly absorbed photons just above the band edge (1000-1200 nm). We attribute the improved spectral response to a $1.7-\mathrm{eV}$ $\mathrm{CuGaSe}_{2}$ layer that is deposited prior to the CIGS absorber. It serves as a back-surface field to carriers generated away from the field region. The CIGS absorber was fabricated by a simple two- 
stage process whereby a $\mathrm{Cu}$-rich precursor is converted to photovoltaic-quality material by exposure to $(\mathrm{In}, \mathrm{Ga}, \mathrm{Se})$ activity at $500^{\circ} \mathrm{C}$.

Table 1. Summary of device performance for champion cells made with different absorber structures by various processes. All I-V data are derived from official NREL measurements.

\begin{tabular}{||c|c|c|c|c|c|l||}
\hline $\begin{array}{c}\text { Sample } \\
\#\end{array}$ & $\begin{array}{c}\text { Area } \\
\left(\mathbf{c m}^{2}\right)\end{array}$ & $\begin{array}{c}\mathbf{V}_{\mathrm{oc}} \\
(\mathbf{m V})\end{array}$ & $\begin{array}{c}\mathbf{J}_{\mathrm{sc}} \\
\left(\mathbf{m A} / \mathbf{c m}^{2}\right)\end{array}$ & $\begin{array}{c}\text { FF } \\
(\%)\end{array}$ & $\begin{array}{c}\text { Total- } \\
\text { Area } \\
\eta\end{array}$ & \multicolumn{1}{|c|}{ Comments } \\
\hline M1201 & 0.395 & 484 & 36.3 & 75.1 & 13.2 & (a) CIS \\
\hline S573 & 0.413 & 552 & 37.1 & $72.1^{\circ}$ & 14.8 & (b) CIS/CGS \\
\hline C362 & 0.437 & 652 & 33.2 & 77.4 & 16.8 & (c) CIGS/CGS \\
\hline S578 & 0.413 & 654 & 33.9 & 77.1 & 17.1 & (d) CIGS/CGS \\
\hline C371 & 1.025 & 678 & 32.0 & 75.8 & 16.4 & CIGS/CGS \\
\hline C371 & 4.85 & 657 & 31.1 & 74.7 & 15.3 & CIGS/CGS \\
\hline C364 & 0.074 & 767 & 671.4 & 75.2 & 17.2 & 22-sun, CIGS/CGS \\
\hline \hline
\end{tabular}

Process Development. In this effort, we have focused on learning all we can about our standard laboratory processes in order to take them to the next step where process simplicity and reproducibility are the critical factors. To this end, we have focused on decoupling the absorber from the Mo/soda-lime glass substrate, on fabricating high-quality absorbers by non-evaporative techniques, and on replacing the chemically deposited CdS window with non-Cd, non-aqueous, in situ processing.

The critical absorber process parameters have been considered and a generic flowchart developed for the fabrication of CIGS absorbers (see Fig. 2). By breaking the process down into three or four independent stages, there are clear opportunities to use a variety of deposition techniques, separate and reduce the time for high-temperature process segments, and introduce intelligent process control. We have focused first on using evaporation to emulate processes consistent with this flowchart. This year, we demonstrated a 15.3\%-efficient device made by lowering the first-stage processing temperature, and then subjecting the low-quality precursor to a short, high-temperature anneal in a reactive environment. Analysis of these cells indicates voltage loss associated with film quality, and current loss associated with reductions in diffusion length. We can hypothesize that the timetemperature influence on recrystallization is reduced relative to the standard approach. 


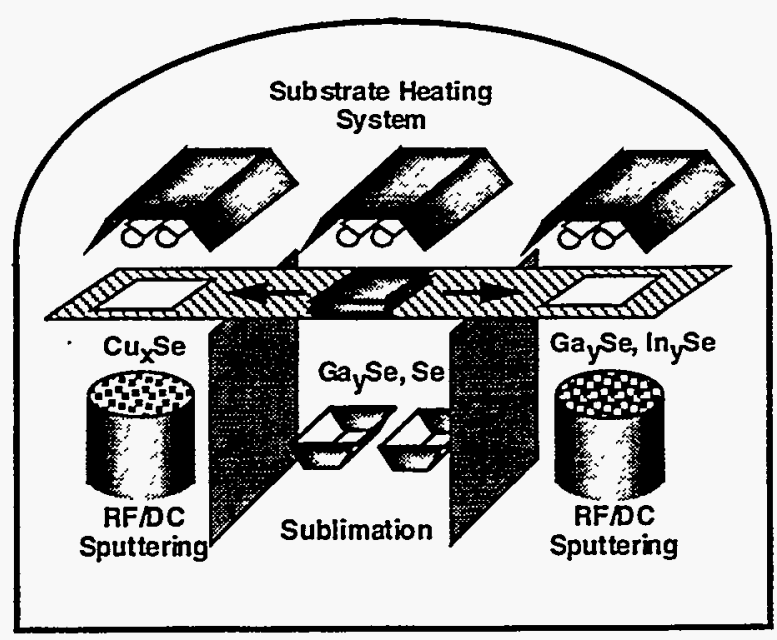

Figure 3. Schematic of hybrid sputtering system.

Table 2. Device parameters resulting from alternative processing of CIGS absorbers

\begin{tabular}{|l|c|c|c|c|c|c|}
\hline \multicolumn{1}{|c|}{ Process } & $\begin{array}{c}\text { Area } \\
\left(\mathrm{cm}^{2}\right)\end{array}$ & $\begin{array}{c}\mathbf{V}_{\mathrm{oc}} \\
(\mathrm{mV})\end{array}$ & $\begin{array}{c}\mathbf{J}_{\mathrm{se}} \\
\left(\mathrm{mAsm}^{2}\right)\end{array}$ & $\begin{array}{c}\text { FF } \\
(\%)\end{array}$ & $\begin{array}{c}\text { Total-Area } \\
\eta\end{array}$ & Comments \\
\hline Evaporation & 0.430 & 623 & 32.9 & 74.7 & 15.3 & $\begin{array}{c}\text { CIGS } \\
(\mathrm{Cu}, \mathrm{Se})+(\mathrm{In}, \mathrm{Ga}, \mathrm{Se})\end{array}$ \\
\hline Evaporation & 0.430 & 605 & 31.4 & 73.3 & 13.9 & $\begin{array}{c}\mathrm{CIGS}: \mathrm{CS} \\
\text { Se+(In,Ga,Se })\end{array}$ \\
\hline Electro-deposition & 0.413 & 419 & 35.9 & 63.5 & 9.4 & $\begin{array}{c}\text { CIS:CS } \\
\text { Se+(In,Se) }\end{array}$ \\
\hline Sputtering & 0.413 & 380 & 21.4 & 67.2 & 5.5 & $\begin{array}{c}\text { CIS:CS } \\
(\mathrm{Cu}, \mathrm{Se})+(\mathrm{In}, \mathrm{Se})\end{array}$ \\
\hline
\end{tabular}

Qther Developments. In addition to the above-mentioned focus areas, there are several other highlights regarding activities from this past year. The following is a list of some of the issues explored:

(1) NREL and Lockheed-Martin (LMA, formerly Martin Marietta) entered into the second year of a cooperative research and development agreement (CRADA) targeting the application of NREL processes to flexible substrates with process temperature limitations. The benefits include an improved understanding of the relationship between processing temperatures and device performance. LMA personnel participated in the fabrication of NREL's world-record $17.1 \%$ device.

(2) NREL and Energy Photovoltaics (EPV) entered into the first year of a CRADA focused on applying NREL processing to EPV's manufacturing equipment. Dr. Andrew Gabor, a former Association of Western Universities graduate student who did his research at NREL, joined EPV and performed research on the CRADA. 
(3) A 4-chamber "cluster" tool vacuum system was designed and scheduled for delivery in the 1st quarter of 1996. The purpose of this tool is to explore manufacturing-friendly deposition processes, including sputtering from compound targets, sublimation, and "in-line" evaporation. The substrate size will be 6 in. $\times 6$ in.

\section{References}

1. "Prescriptions for the Fabrication of High-Efficiency $\mathrm{Cu}(\mathrm{In}, \mathrm{Ga}) \mathrm{Se}_{2}$-Based Thin Films and Devices," J.R. Tuttle, M.A. Contreras, K.R. Ramanathan, A.L. Tennant, S.A. Asher, D. Niles, R. Matson, J. Keane, and R. Noufi, Proceedings of the 13th European Photovoltaic Solar Energy Conference; Nice, France, Oct. 23-27, 1995 (in press).

2. "Absorber Processing Issues in High-Efficiency Thin-Film $\mathrm{Cu}(\mathrm{In}, \mathrm{Ga}) \mathrm{Se}_{2}-\mathrm{Based}$ Solar Cells," J.R. Tuttle, A.M. Gabor, M.A. Contreras, A.L. Tennant, K.R. Ramanathan, A. Franz, R. Matson, and R. Noufi, Proceedings of the 13th NREL Photovoltaic Program Review; Lakewood, Colorado, May 17-19, 1995, p. 47.

3. "Thin-Film Cu(InGa)Se ${ }_{2}$ Materials and Devices: A Versatile Material for Flat-Plate and Concentrator Photovoltaic Applications," J.R. Tuttle, M.A. Contreras, J.S. Ward, A.L. Tennant, K.R. Ramanathan, J. Keane, and R. Noufi, Proceedings of the SPIE 40th Annual Meeting; July 9-14, 1995, SPIE Vol. 2531, p. 194.

4. "A 17.1\%-Efficient $\mathrm{Cu}(\mathrm{In}, \mathrm{Ga}) \mathrm{Se}_{2}$-Based Thin-Film Solar Cell," J.R. Tuttle, M.A. Contreras, T.J. Gillespie, K.R. Ramanathan, AL. Tennant, J. Keane, A.M. Gabor, and R. Noufi, Progress in Photovoltaics, July-Aug. 1995, Vol. 3, pp. 235-238.

5. "A Perspective on High-Efficiency $\mathrm{Cu}(\mathrm{In}, \mathrm{Ga}) \mathrm{Se}_{2}-\mathrm{Based}$ Thin-Film Solar Cells Fabricated by Simple, Scalable Processes," J.R. Tuttle, M.A. Contreras, A.M. Gabor, K.R. Ramanathan, A.L. Tennant, D.S. Albin, J. Keane, and R. Noufi, Progress in Photovoltaics, Nov.-Dec. 1995 (in press).

6. "High-Efficiency Graded Bandgap Thin-Film Polycrystalline $\mathrm{Cu}(\mathrm{In}, \mathrm{Ga}) \mathrm{Se}_{2}$-Based Solar Cells," M.A. Contreras, J.R. Tuttle, A.M. Gabor, A.L. Tennant, K.R. Ramanathan, S. Asher, A. Franz, J. Keane, L. Wang, and R. Noufi, Solar Energy Materials and Solar Cells (in press).

7. "The Influence of $\mathrm{Ga}$ on the Properties of $\mathrm{CuIn}(\mathrm{Ga}) \mathrm{Se}_{2}-\mathrm{Based}$ Thin Films and Devices," B.M. Keyes, J. Tuttle, J. Sites, A. Tennant, S. Asher, M. Contreras, K. Ramanathan, A. Gabor, J. Webb, R. Ahrenkiel, and R. Noufi, Proceedings of the 10th ICTMC (in press). 
Title: $\quad$ Hydrogenated Amorphous Silicon Device Research

Organization: National Renewable Energy Laboratory

Golden, $\mathrm{CO}$

Contributors; R. Crandall, principal investigator, Q. Wang, Y. Xu, A.H. Mahan, B.P. Nelson, E. Iwaniczko, E.C. Molenbroek, H. Branz

Objective: To improve the stability, efficiency, and manufacturing efficiency of amorphous siliconbased solar cells.

Role of Hydrogen Dilution in a-Si:H Solar Cells Performance, Stability, and Manufacturing Speed

Recently, many groups reported more stable solar cells made from hydrogen-diluted hydrogenated amorphous silicon (a-Si:H). We investigated the effect of $\mathrm{H}$-dilution on a-Si:H solar cells performance and stability, and concluded that $\mathrm{H}$ dilution enhances initial device performance and stability. In addition, we demonstrated that inserting a thin layer of $\mathrm{H}$-diluted $\mathrm{a}-\mathrm{Si}: \mathrm{H}$ at the p/i interface also yields an improvement in solar cell performance and stability similar to that from using $\mathrm{H}$-dilution in the entire i-layer. This finding is remarkable. It not only shows that the role of $\mathrm{H}$-dilution dominates at the doped/intrinsic interface, but also that it reduces manufacturing time and increases throughput. In general, $\mathrm{H}$-dilution reduces deposition rate, which is a big drawback in terms of manufacturing. Is there a way to keep all the good parts of using H-dilution material and also speed up the process? The results of this study demonstrate that it is suitable not only for substrate, but also for superstrate structure devices.

Table 1 shows solar cell performance and stability of four superstrate $\mathrm{p}$-i-n cells with their structures donated at the bottom. All the cells have the same thickness of $4500 \AA$. The first cell is a normal a-Si:H layer cell. The second one is an $\mathrm{H}$-diluted a-Si:H i layer cell. And the third one is a normal a-Si:H cell with an $\mathrm{H}$-diluted a-Si:H layer between the $\mathrm{p}$ and $\mathrm{i}$ interface. All cells have a similar initial efficiency, but $\mathrm{H}$-diluted cells have a higher final value after $600 \mathrm{hr} 1 \mathrm{Sun}$ light soaking. We find no degradation in open-circuit voltage for $\mathrm{H}$-diluted cells after $600 \mathrm{hr}$ light soaking. Once again, we proved that $\mathrm{H}$-dilution improves cell performance and stability. In addition, we find the importance of $\mathrm{H}$-dilution at the doped/intrinsic interface. Inserting an $\mathrm{H}$-diluted a-Si:H thin layer at the doped interface has the same effect on device performance and stability as using $\mathrm{H}$-diluted materials in the entire i layer.

We find similar results of the effect of $\mathrm{H}$-dilution using the substrate structure. In this case, we investigated Schottky structures and found that it is necessary to use at least a thin (200- $\AA$ ), hydrogen-diluted $i$ layer following the $n$ layer. Doing this results in an increase in the fill factor from 0.60 to 0.69 for a 4500 - $\AA$-thick ni Pd Schottky device.

\section{Hot-Wire-Deposited Hydrogenated Amorphous Silicon Solar Cells}

We describe recent device results obtained using the hot-wire (HW) deposition technique. Using this technique, we previously found that, by raising the substrate temperature (Ts) to values beyond those used for standard a-Si:H material, device-quality a-Si:H could be deposited for the first time 
with bonded $\mathrm{H}$ contents as little as 1 at. \%, and that the saturated defect densities of these high Ts, low $\mathrm{H}$ content films were significantly lower than those reported for the standard glow-discharge (GD) a-Si:H films commonly used in cells and modules. Thus, a project has been undertaken to incorporate this low $\mathrm{H}$ content material into a solar cell. The first cell structure chosen for this study was the glass/TCO/p-i-n/metal, or superstrate cell. Although reasonable cell efficiencies were reported, we were unable to incorporate the low $\mathrm{H}$ content material into these devices because of damage to the $\mathrm{p}$ layer resulting from the high deposition temperature, and as a result the light-soaking behavior of these cells was found to mirror that of the standard GD devices. Thus, we undertook to develop cells using the substrate structure beginning with the SS/n-i-p/Pd substrate cell approach. By necessity, since the learning curve for this structure involves metal/n-i-/Schottky barrier structure optimization, and since the treatment of the top surface of the HW i layer during cooling was found to be crucial to device performance, a large part of the results are focused on this structure.

Table 1. Summary of a-Si:H $p-i-n$ single-junction solar cell properties

\begin{tabular}{cccc}
\hline \hline Cell Structure* & & $\mathrm{V}_{\alpha}(\mathrm{V})$ & $\mathrm{FF}$ \\
\hline $\mathrm{p}-\mathrm{i}-\mathrm{n}$ & Initial & 0.85 & 0.70 \\
& After 600 hr & 0.82 & 0.60 \\
& Degradation & $3.5 \%$ & $14.3 \%$ \\
& & & \\
& Initial & & 0.69 \\
& After 600 hr & 0.85 & 0.60 \\
$\mathrm{p}-\mathrm{i}_{\mathrm{H}}-\mathrm{n}$ & Degradation & 0.85 & $13.0 \%$ \\
& Initial & $0.0 \%$ & 0.66 \\
& After 600 hr & 0.85 & 0.59 \\
$\mathrm{p}-\mathrm{i}_{\mathrm{H}}-\mathrm{i}-\mathrm{n}$ & Degradation & 0.85 & $10.6 \%$ \\
& Initial & $0.0 \%$ & 0.66 \\
& After 600 hr & 0.86 & 0.57 \\
$\mathrm{p}-\mathrm{i}_{\mathrm{B}}-\mathrm{i}-\mathrm{n}$ & Degradation & 0.84 & $13.6 \%$ \\
& - & $2.3 \%$ & \\
\hline \hline
\end{tabular}

*p denotes p-layer; $\mathrm{i}$ denotes an intrinsic a-Si:H layer; $\mathrm{i}_{\mathrm{H}}$ denotes an $\mathrm{H}$-diluted a-Si: $\mathrm{H}$ layer; $\mathrm{i}_{\mathrm{B}}$ denotes a a-SiC:H buffer layer; and $\mathrm{n}$ denotes n-layer.

We present preliminary results obtained in incorporating low $\mathrm{H}$ content, high substrate temperature, $\mathrm{HW}$-deposited amorphous silicon material into a substrate solar cell structure. By necessity, since the leaming curve for this complete structure involves metal/n-i/Schottky barrier structure optimization, a large part of the results are focused on this (partial) structure. We have found that the treatment of the top surface of the HW i layer during cooling is crucial to device performance. Without any particular attention paid to the treatment of this surface while the sample is cooling from its high deposition temperature, a significant amount of $\mathrm{H}$ diffuses out of the sample during the cooling process, particularly near the surface, resulting in devices with very poor photovoltaic properties. By designing a surface treatment to address this problem, we have been able to deposit HW Schottky structures with device characteristics nearly as good as the GD devices produced in 
our laboratory. We present data concerning these surface treatments, and how they influence the $\mathrm{H}$ content at the $\mathrm{i} / \mathrm{Pd}$ interface.

The HW films were deposited in a deposition apparatus using conditions described elsewhere. The polished SS substrates were supplied to us by United Solar Systems Corp. as part of the a-Si teaming effort. No texturing (light-trapping) layers were used in this feasibility study. The $\mathrm{n}$ layers were deposited either by GD or by HW. The use of a GD n layer necessitated an air break between the GD $\mathrm{n}$ and HW i layers, while the HW n-i structure was deposited in the same chamber, with a purging procedure developed to ensure negligible dopant contamination of the $i$ layer. Various Ts's were used for both types of $n$ layers, but typically the values matched those of the $i$ layers, and were not found to be crucial for device performance. In addition, the interchangeability of the GD and HW $n$ layers was examined, and was found to be satisfactory. After the HW i layer deposition, the partial device was cooled, and either a GD p layer $\left(T s=175^{\circ} \mathrm{C}\right.$ ) was deposited followed by an evaporated $\mathrm{Pd}$ top contact, or, in the case of the Schottky barrier investigations, only the Pd contact. A Pd thickness of $\sim 300 \AA$ ( $\sim 20 \%$ transmission) was initially used to minimize sheet resistivity. The GD devices, needed for comparison purposes, were deposited under standard conditions, with air breaks at interfaces as needed to match the procedure used for the HW devices. All i layer thicknesses were typically $4000-4500 \AA$. The light soaking was done under $100 \mathrm{~mW} / \mathrm{cm}^{2}$ ELH light at $\sim 40^{\circ} \mathrm{C}$.

Figure 1 shows a comparison of the light-soaking behavior (fill factor [FF], $\mathrm{V}_{\mathrm{oc}}$ ) of two devices, a standard GD device with the $\mathrm{i}$ layer deposited at $250^{\circ} \mathrm{C}$ and a hybrid device with the (low $\mathrm{H}$ content) $\mathrm{HW} i$ layer deposited at $375^{\circ} \mathrm{C}$. Both devices were deposited with an air break at the $\mathrm{i} / \mathrm{p}$ interface, and no back reflector/light-trapping structures were used at the substrate surface. The initial FF of the GD device was 0.65 , which is typical of n-i-p devices fabricated at NREL with an air break at the $\mathrm{i} / \mathrm{p}$ interface. To increase the FF of the HW device to its initial value (0.55), it was necessary to subject the HW $\mathrm{i}$ layer to an $\mathrm{H}$ plasma before deposition of the GD $\mathrm{p}$ layer. This treatment increased the FF by $\sim 0.05$ compared to a device made without this plasma treatment. The degradation of the GD device over a 1200 -hr light soak is $\sim 20 \%$, a value typically observed in our laboratory (5) for GD p-i-n devices of this thickness and AM1 light-soaking times of 100-200 hr. The HW device, on the other hand, shows negligible degradation in either $\mathrm{FF}$ or $\mathrm{V}_{\mathrm{oc}}$ upon light soaking, which has been extended for this sample to $>4000 \mathrm{hr}$. These data, although preliminary, are consistent with our earlier findings that the low $\mathrm{H}$ content $\mathrm{HW}$ material is more stable than the standard GD material.

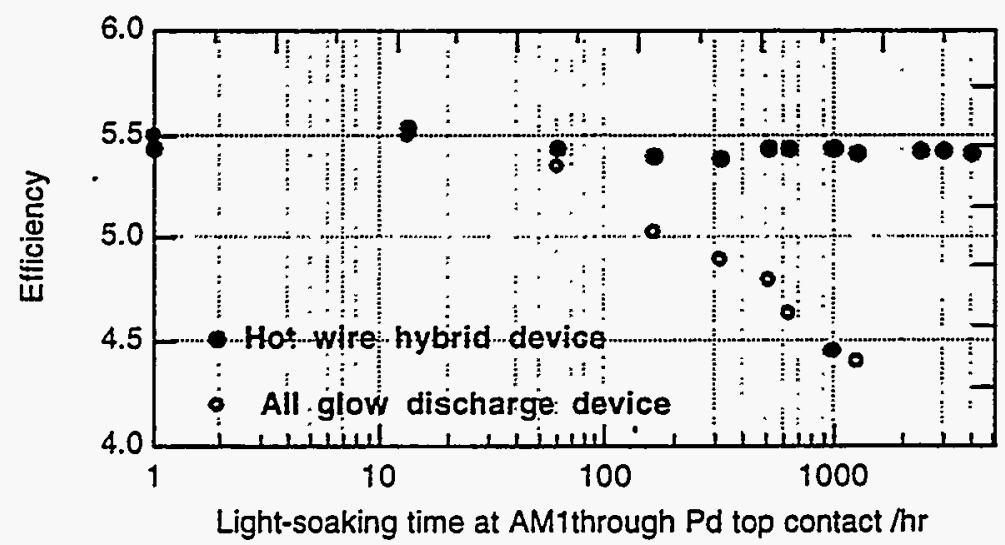

Figure 1. Light-soak data for an all GD-deposited device, and a device containing a low $\mathrm{H}$ content, HW-deposited i layer. The device currents were corrected for a Pd transmission of $\sim 20 \%$. 
However, to establish a truly viable solar cell using the HW deposition method, it is necessary to demonstrate an improved stability with better initial device characteristics (FF, $\mathrm{V}_{\mathrm{oc}}$ ). Hence, we backtracked and examined Schottky barrier devices to see if we could determine what factor, or factors, were limiting our HW n-i-p device efficiencies. First, we made an all GD Schottky barrier device, and then on successive depositions progressively substituted the HW $i$ layer for the GD $i$ layer while keeping the total device thickness the same. These results suggested to us that the Schottky barrier interface could be the major factor limiting device performance. Therefore, we decided to vary the surface treatment of the HW $i$ layer as we cooled it from its high deposition temperature and to correlate these surface treatments with device performance. A partial summary of these results is shown in Table 2. As can be seen, stopping the growth while cooling produces a device with a very low FF, while various surface treatments during cooling yield a significant FF improvement. SIMS investigations showed that an untreated surface is depleted of hydrogen for nearly $1000 \AA$, and this is presumably the cause of the low fill factor. Our best $\mathrm{HW}$ results to date for our low $\mathrm{H}$ content, stable $\mathrm{HW}$ material yield a FF of 0.62 and $a \mathrm{~V}_{\mathrm{cc}}$ of 0.54 . However, this is still not good enough for a high-efficiency device, so we are pursuing other means to improve the surface regions.

Table 2. Typical FF values of HW-deposited devices as a function of $i$ layer surface treatment while the complete device is cooled from its high Ts. The $\mathrm{i}$ layer thickness of all devices is $\sim 4000 \AA$.

\begin{tabular}{|l|l|c|}
\hline \hline Sample & \multicolumn{1}{|c|}{ Surface Treatment } & Fill Factor \\
\hline \hline TH ni \#12 & Cool in Vacuum & FF $=0.27$ \\
\hline TH ni\#31 & Cool in Low Pressure of Silane & FF $=0.46$ \\
\hline TH ni\#54 & Deposit Thin Lower T CAP w/o Breaking Vacuum & FF $=0.50$ \\
\hline TH ni\#21 & Very Slow Deposition While Cooling & FF $=0.52$ \\
\hline TH ni\#46 & Cool During Entire I-Layer Deposition & FF $=0.57$ \\
\hline TH ni\#96 & Present Surface Treatment & FF $=0.64$ \\
\hline
\end{tabular}

\section{References}

Crandall, R. S. and E. A. Schiff, in Proceedings of the 12th NREL Photovoltaic Program Review, edited by H. S. Ullal and C. E. Witt, AIP Conference Proceedings No. 353 (American Institute of Physics, Denver, CO, 1995), 481.

Mahan, A. H., J. Carapella, B. P. Nelson, R. S. Crandall, and I. Balberg, "Hot Wire Deposited Amorphous Silicon," J. Appl. Phys. 69, 6728 (1991).

Mahan, A. H., B. P. Nelson, E. Iwaniczko, Q. Wang, E. Molenbroek, S. A. Asher, R. C. Reedy, and R. S. Crandall, in Proceedings of the 12th NREL Photovoltaic Program Review, edited by H. S. Ullal and C. E. Witt, AIP Conference Proceedings No. 353 (American Institute of Physics, Denver, CO, 1995), 67. 
Mahan, A. H. and M. Vanecek, "A Reduction in the Staebler-Wronski Effect Observed in Low H Content ...," Amorphous Silicon Materials and Solar Cells, edited by B. L. Stafford (American Institute of Physics (AIP), New York, 1991), 195.

Nelson, B. P., E. Iwaniczko, R. I. Schropp, A. H. Mahan, E. C. Molenbroek, S. Salamon, and R. S. Crandall, "Amorphous Silicon Solar Cells Incorporating Hot-Wire Deposited Intrinsic Layers," Proceedings of the I2th International E.C. Photovoltaic Solar Energy Conference, edited by R. Hill, W. Palz, and P. Helm (H.S. Stephens and Associates, 1994), 575-578.

Wang, Q., Y. Xu, and R. S. Crandall, in Proceedings of the 12th NREL Photovoltaic Program Review, edited by H. S. Ullal and C. E. Witt, AIP Conference Proceedings No. 353 (American Institute of Physics, Denver, CO, 1995), 473. 


\section{Thin-Film Technologies Subcontracts}



Title: $\quad$ Light-Trapped, Interconnected, Silicon-Film Modules

Organization: AstroPower, Inc.

Newark, Delaware

Contributors: J.E. Cotter and J.A. Rand, principal investigators; R.B. Hal! program

manager; A.M. Barnett, D.H. Ford, A.E. Ingram, and C.J. Thomas

\section{PROGRAM OVERVIEW}

AstroPower is developing a photovoltaic module manufacturing technology based on the thin-film Silicon-Film ${ }^{\text {TM }}$ sheet growth process. As a Technology Partner in the Thin-Film Partnership Program sponsored by NREL and DOE, AstroPower will apply the Silicon-Film ${ }^{\mathrm{TM}}$ sheet growth technology [1] toward an advanced thin-film, 400 watt, $4 \mathrm{ft} \times 8 \mathrm{ft}\left(2.97 \mathrm{~m}^{2}\right)$ photovoltaic panel product for use in power applications.

Crystalline silicon offers many advantages as a photovoltaic material; it is abundant, benign, electrically stable and there is a well established technology base. Thin-film solar cells incorporate additional features which enable high-performance and low-cost to be achieved which can lead to a commercially successful photovoltaic module technology [2]. The benefits of thin silicon solar cells are becoming widely recognized, as evidenced by the growing number of researchers in Europe, Japan and Australia developing thin-layer crystalline silicon solar cell technologies [3-7].

\section{KEY RESULTS OF PHASE I}

The key results of the first year of this program are:

- a confirmed $12.2 \%$ efficient, thin solar cell fabricated on a low-cost substrate.

- demonstration of films grown on barrier-coated substrates which, after being subjected to a phosphorous gettering process, exhibited diffusion lengths greater than $250 \mu \mathrm{m}$,

- development of a new, low-cost substrate and barrier coating which does not contaminate the thin film of silicon during the growth process,

- observation of optical confinement in $60-\mu \mathrm{m}$ thick films with $80 \mu \mathrm{m}$ diffusion lengths.

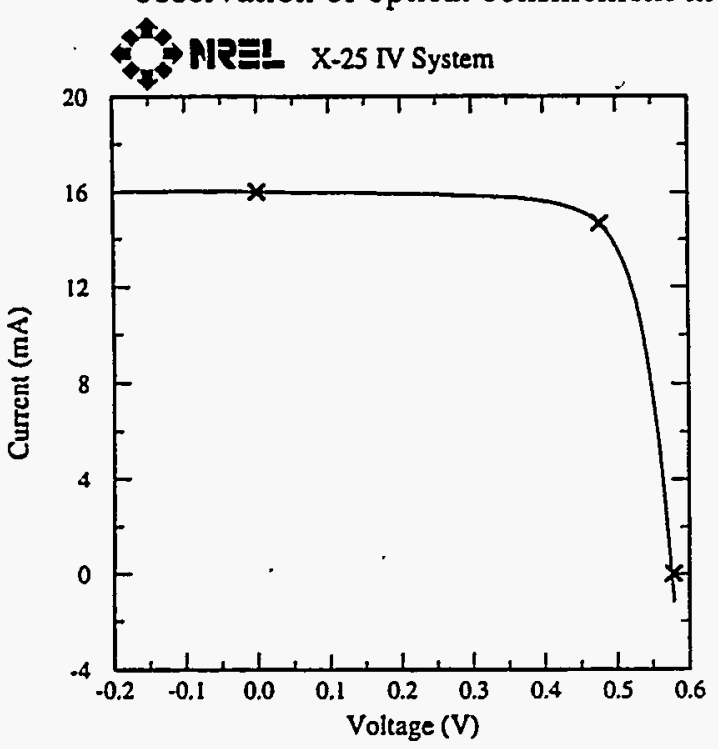

\begin{tabular}{|ll|}
\hline Sample 2 \#3 & Nov. 22, 1995 \\
ASTM E 892-87 Global & Area $=0.573 \mathrm{~cm}^{2}$ \\
$\mathrm{~V}_{\mathrm{oc}}=0.578 \mathrm{~V}$ & $\mathrm{~V}_{\max }=0.477 \mathrm{~V}$ \\
$\mathrm{I}_{\mathrm{sc}}=16.01 \mathrm{~mA}$ & $\mathrm{I}_{\max }=14.65 \mathrm{~mA}$ \\
$\mathrm{~J}_{\mathrm{sc}}=27.94 \mathrm{~mA} / \mathrm{cm}^{2}$ & $\mathrm{P}_{\max }=6.984 \mathrm{~mW}$ \\
Fill Factor $=75.5 \%$ & Eff $=12.2 \%$
\end{tabular}

Figure 1. I-V characteristic of a thin-layer solar cell fabricated on a new barrier-coated substrate. 
A new barrier-coated substrate has been pivotal to the Phase I program. It has enabled, for the first time, diffusion lengths greater than the thickness of the device to be measured in low-cost films of silicon. This has led to a solar cell efficiency of $12.2 \%$ (confirmed at NREL; $J_{\mathrm{sc}}=27.9 \mathrm{~mA} / \mathrm{cm}^{2}$, $\mathrm{V}_{\mathrm{oc}}=578 \mathrm{mV}, \mathrm{FF}=75.5 \%$, see Figure 1). This is the first efficiency greater than $12 \%$ achieved with a layer of polycrystalline silicon grown by a low-cost process on a barrier-coated substrate. The following section summarizes the key results of this Phase I program.

\section{KEY RESULTS OF THE PHASE I PROGRAM}

Substrate and Barrier Layer. A new substrate and barrier layer has been developed which has enabled the deposition and growth of high-quality photovoltaic films. The new substrate has the potential to be low-cost as it uses low-cost precursor materials and is fabricated by a demonstrated lowcost, high-throughput manufacturing process. Several related dielectric barrier materials and deposition techniques are being explored.

The new barrier and substrate have resulted in a dramatic improvement in the quality of grown films of silicon. The films have exhibited very large grain sizes $(>1 \mathrm{~mm}$ in diameter $)$ in thin $(<100 \mu \mathrm{m})$ films. Cross-sectional analysis indicate that the grain structure extends through the thickness of the film and is columnar or elongated, depending on the growth conditions [8].

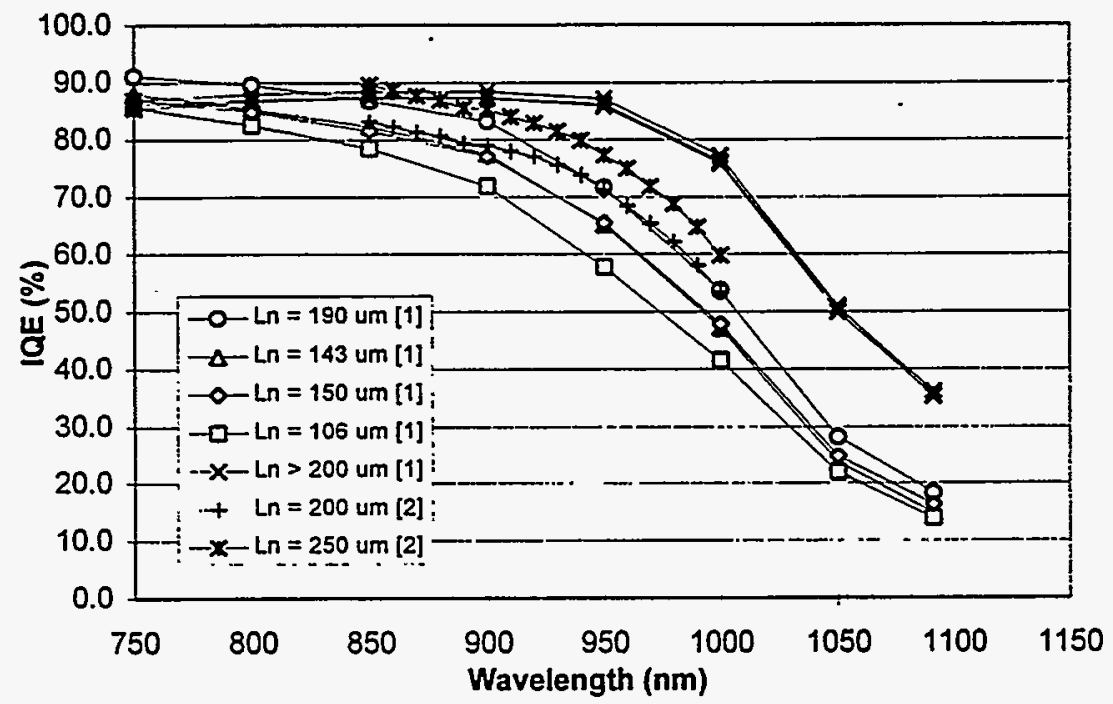

Figure 2. IQE of recently tested solar cell devices indicating exceptional minority carrier diffusion length (tested at [1] AstroPower, [2] Georgia Institute of Technology).

Electrical quality is also dramatically improved. On previous barrier-coated substrates, contamination of the photovoltaic layer during the growth process severely limited diffusion length to below $10 \mu \mathrm{m}$ with no response to post growth treatments such as gettering and hydrogenation. With the new substrate and barrier, minority carrier diffusion lengths are typically between 35 and $50 \mu \mathrm{m}$ in asgrown films. The diffusion length is improved to well over $100 \mu \mathrm{m}$ with the application of an external phosphorous gettering process (based on $\mathrm{POCl}_{3}$ ) [9]. Figure 2 shows the quantum efficiency of several high-diffusion length solar cell devices. Two small-area test devices measured at the Georgia Institute of Technology had diffusion lengths of 200 and $250 \mu \mathrm{m}$. Further development and optimization of the phosphorous gettering process is taking place at the Georgia Institute of Technology.

Modeling. A spread-sheet model has been developed that allows the sub-element losses to be predicted based on solar cell parameters and geometry. This model has been adapted for the design of 
small-area $\left(\sim 0.5 \mathrm{~cm}^{2}\right)$, laboratory-scale devices. Due to the insulating substrate/barrier, all electrical contacts are placed on the top of the silicon layer. In this configuration, resistive losses of the base layer can be significant, especially for thin base layers. The resulting solar cell is rectangular with the base contact forming a 'window frame' around the emitter. This allows for a short conduc: ion path from all points within the base layer to the base contact.

PC-1D is the primary modeling tool used to design and analyze the solar cell structure. This tool has been especially useful as a post-fabrication analysis technique to identify factors limiting device performance. Material and device quantities extracted from quantum efficiency, current-voltage and capacitance-voltage data were used in conjunction with PC-1D to identify and solve several problems. For example, low voltage and fill factor due to a local ' $n=2$ '-like defect formed during layer growth was identified through PC-1D modeling. Subsequently, an appropriate post-growth treatment was developed, resulting in improved material quality. This tool is expected to become increasingly useful during the next two Phases of this program

Light Trapping. The light trapping effectiveness of existing barrier structures was analyzed[8]. Some evidence of light trapping has been observed in thin films $(60 \mu \mathrm{m})$. with good diffusion lengths. IQE analysis of long-wavelength light was used to infer the minority carrier diffusion length $(80 \mu \mathrm{m})$, the effective optical path length $(Z=20)$ and the effective back surface reflection $(>28 \%)$. This is encouraging as only minimal effort has been applied to developing the light-trapping properties of the new barrier layer. Techniques for the analysis of light-trapping and passivation effectiveness are being developed at the University of Delaware's Institute of Energy Conversion under AstroPower sponsorship.

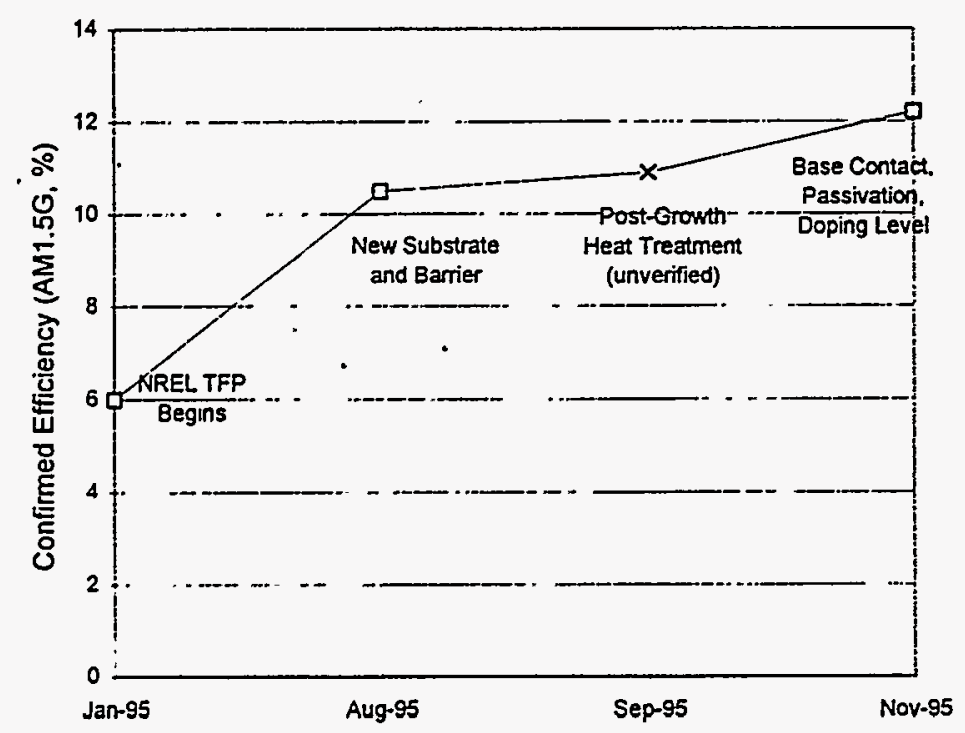

Figure 3. Progress in laboratory-scale solar cell devices during the Phase I program.

Benchmark Efficiency Solar Cells. Laboratory solar cells have been fabricated on thin films of silicon grown on the new barrier/substrate materials. The fabrication process is similar to the standard practice for silicon solar cells, including phosphorous gettering, emitter etch-back, PECVD oxide passivation, evaporated contacts, and a double layer anti-reflection coating.

An efficiency of $12.2 \%$ (verified, NREL) was achieved. Figure 1 illustrates the I-V characteristic of the best solar cell of this set of devices. Figure 3 shows efficiency progress during the Phase I program as key technical issues related to film growth and device fabrication were identified and solved.

Manufacturing Scale-Up. Work carried out during the Phase I program addressed the issues related to the fabrication of a commercial-scale substrates and growth of thin layers using manufacturing technology developed under the PVMaT program for the AP-225 [1] solar cell. Presently, we are capable of forming the substrate in a $15-\mathrm{cm}$ wide format and growing $15-\mathrm{cm}$ wide layers of silicon on these substrates. Future work will enable the incorporation of the metallurgical barrier, light trapping, and back-surface enhancement features. It is envisioned that the entire substrate including its enhancement features will be formed on a continuous sheet. 


\section{SUMMARY AND PHASE II EXPECTED RESULTS}

The Phase I program has been a success; all technical milestones and deliverabies were achieved. A new barrier-coated substrate has enabled, for the first time, the growth of high-quality, thin-layer polycrystalline silicon to be grown on a low-cost, barrier-coated substrate. High diffusion lengths have been measured after external phosphorous gettering. This has led to a $12.2 \%$ efficiency measured in a laboratory-scale solar cell device.

In the Phase II program we will begin developing the substrate for light-trapping and passivation properties. Although light-trapping has been observed in silicon layers grown on the new barrier-coated substrate, it has not been optimized for such. Conversion efficiencies exceeding $15 \%$ are expected as the properties of the substrate are further developed. Basic technologies related to the formation of the monolithically integrated sub-module will also be developed during the Phase II program. This includes techniques for sub-element isolation, interconnection, and conductivity enhancement. Finally, manufacturing technologies will be adopted or developed for key fabrication processes.

\section{REFERENCES}

[1] A.M. Barnett, et.al., "Polycrystalline Silicon-Film" Thin-Film Solar Cells: Present and Future", Progress in Photovoltaics, 2(2), (1994), pp. 163-170.

[2] J.E. Cotter, et.al., "Polycrystalline Silicon-Film" TM Thin-Film Solar Cells: Advanced Products", Progress in Photovoltaics, 3(5), (1995), pp. 351-358.

[3] A. Takami, S. Arimoto, H. Morikawa, S. Hamamoto, T. Ishihara, H. Kumabe and T. Murotani, Proc. 12th European Photovoltaic Solar Energy Conference, Amsterdam, The Netherlands, 1994, pp. 5962.

[4] J.H. Werner, J.K. Arch, R. Brendel, G. Langguth, M. Konuma and E. Bauser, Proc. 12th European Photovoltaic Solar Energy Conference, Amsterdam, The Netherlands, 1994, pp. 1823-1826.

[5] A.B. Sproul, Z. Shi, J. Zhao, A. Wang, Y.H. Tang, F. Yun, T. Young, Y. Huang. S. Edmiston. S.R. Wenham and M.A. Green, First World Conference on Photovoltaic Energy Conversion, Waikoloa, Hawaii, 1994, pp. 1410-1412.

[6] H. Nussbaumer, et. al., Proc. 13th European Photovoltaic Solar Energy Conference, Nice, France, 1995, to be published.

[7] A. Nakajima, et. al., Proc. 13th European Photovoltaic Solar Energy Conference, Nice, France, 1995, to be published.

[8] J.E. Cotter, et. al., "Advanced Silicon-Film" Product Development", 13th PV Program Review Meeting, (May, 1995), to be published.

[9] J.E. Cotter, et.al., "Phosphorous and Aluminum Gettering in Silicon-Film Product II Material", $5^{\text {th }}$ Workshop on the Role of Impurities and Defects in Silicon Device Processing, NREL/SP-413-8250, (August, 1995), pp. 129-132. 
Title:

Polycrystalline Thin Film Cadmium Telluride Solar Cells

Fabricated by Electrodeposition

Organization: Department of Physics, Colorado School of Mines

Golden, Colorado 80401

Contributors: $\quad$ J.U.Trefny, D. Mao, co-principal investigators; D.L. Williamson, R. Collins, T. Ohno, T.E. Furtak, L. Feng, Y. Zhu, W. Song, and J. Tang.

\section{Objectives}

The objective of this project is to develop improved processes for the fabrication of $\mathrm{CdTe} / \mathrm{CdS}$ polycrystalline thin film solar cells. The technique we use for the formation of CdTe, electrodeposition, is a non-vacuum, low-cost technique that is attractive for economic, large-scale production.

\section{Technical Approach}

During the past year, our research and development efforts have focused on several steps that are most critical to the fabrication of high-efficiency CdTe solar cells. These include the optimization of the CdTe electrodeposition process, the pre-treatment of CdS substrates, the post-deposition annealing of $\mathrm{CdTe}$, and back contact formation using $\mathrm{Cu}$-doped $\mathrm{ZnTe}$. Systematic investigations of these processing steps have lead to better understanding and improved device performances of the CdTe-based solar cells.

\section{Results}

\section{Optimization of CdTe absorber layer and the pre-treatment of CdS}

We have investigated the effect of CdTe thickness on the structural properties of CdTe films and on the photovoltaic performance of the resulting cells. The CdTe thickness was varied from 1.5 $\mu \mathrm{r}$, to $4 \mu \mathrm{m}$. We found that an increase in the CdTe thickness from $2 \mu \mathrm{m}$ to $3.5 \mu \mathrm{m}$ led to a consistent improvement in $\mathrm{V}_{o c}$ by $40-60 \mathrm{mV}$. The shunt resistance of the cells was also increased. As a result, $V_{o c}$ greater than $800 \mathrm{mV}$ and $R_{\text {sh }}$ greater than $1000 \Omega \mathrm{cm}^{2}$ were regularly obtained. SEM measurements indicated that the average $\mathrm{CdTe}$ grain size after $\mathrm{CdCl}_{2}$ treatment and annealing was much larger for the thicker CdTe films (Figure 1). XRD studies indicated less CdTe/CdS interdiffusion for thicker CdTe. The sulfur content in a $3.5 \mu \mathrm{m} \mathrm{CdTe}$ is less than $2 \%$, whereas that in a $2 \mu \mathrm{m}$ film is typically $3 \%$ or higher. The relative proportion of (111)-oriented and (220)oriented grains was also found to vary for annealed CdTe films of different thicknesses.

We have investigated the electrical and structural properties of CdS films and the resulting cell performances as a function of the amount of $\mathrm{CdCl}_{2}$ applied on CdS films prior to $\mathrm{CdS}$ annealing. SEM measurements indicated an increase in the grain size when the $\mathrm{CdCl}_{2}$-spray time on CdS was increased from 20 to $40 \mathrm{~s}$ (Figure 2). The lateral electrical conductivity of CdS films was also found to increase with $\mathrm{CdCl}_{2}$-treatment and annealing. For relatively thick CdS films (>2000 $\AA$ ), the opencircuit photovoltage of the $\mathrm{CdTe} / \mathrm{CdS}$ cells was improved by $20-30 \mathrm{mV}$ when the $\mathrm{CdCl}_{2}$-spray time 


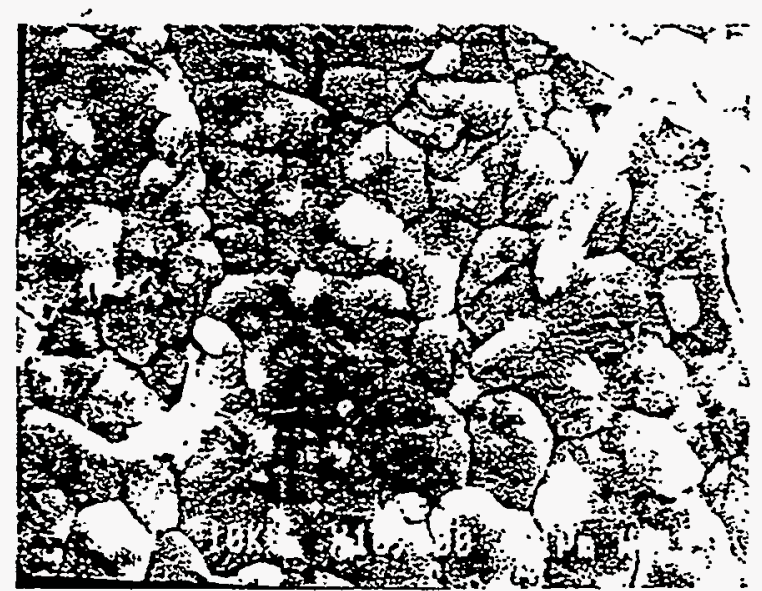

(a) $2 \mu \mathrm{m}$

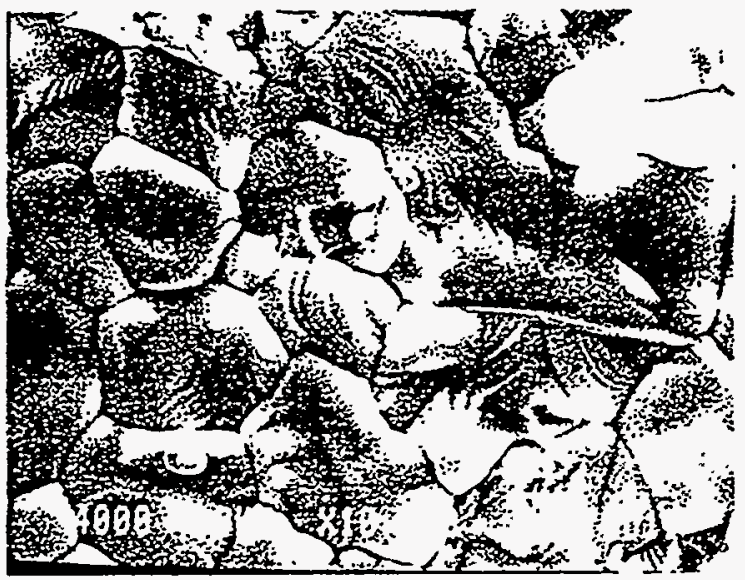

(b) $3.5 \mu \mathrm{m}$

Figure 1. SEM images of CdTe films after $\mathrm{CdCl}_{2}$-treatment and $410^{\circ} \mathrm{C}$-annealing.

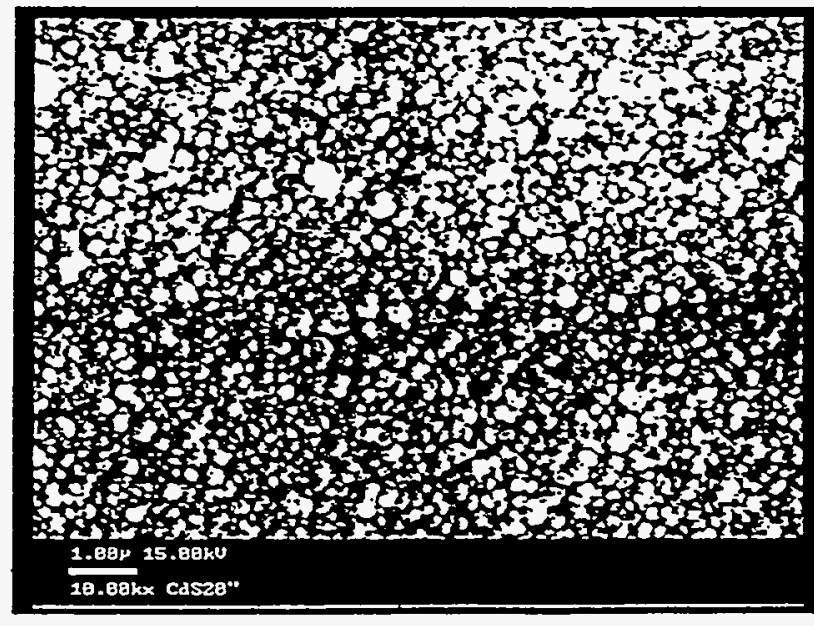

(a) $20 \mathrm{~s}$

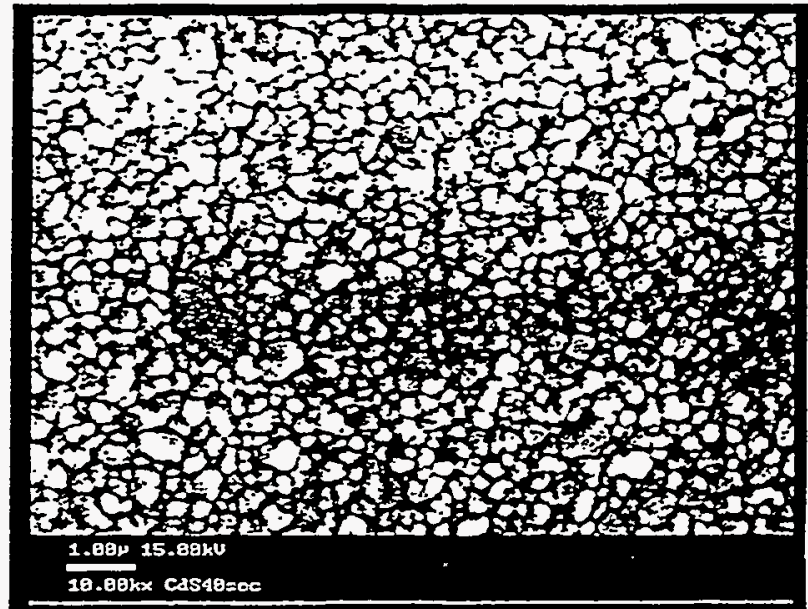

(b) $40 \mathrm{~s}$

Figure 2. SEM images of annealed CdS films. The $\mathrm{CdCl}_{2}$-spray time was varied to monitor its effect on the CdS recrystallization.

was increased. The investigation on the effect of $\mathrm{CdCl}_{2}$-treatment of thin $\mathrm{CdS}$ films is underway.

\section{Studies of improved back contact formation using $\mathrm{Cu}$-doped $\mathrm{ZnTe}$}

The formation of stable, low-resistance back contacts to polycrystalline CdTe is the most critical step in the development of high efficiency CdTe thin film solar cells. ZnTe, which has a small valence band offset with $\mathrm{CdTe}$ and can be doped degenerately with $\mathrm{Cu}$, is a prominent candidate for this purpose. We have investigated the electrical properties of $\mathrm{Cu}$-doped $\mathrm{ZnTe}$ films and the optimization of $\mathrm{ZnTe}$ processing conditions for the formation of low-resistance contact. The $\mathrm{Cu}$ doped $\mathrm{ZnTe}$ films were grown by vacuum co-evaporation. 
We have studied the effect of $\mathrm{Cu}$ concentration, the substrate temperature during $\mathrm{ZnTe}$ deposition, and the post-deposition annealing conditions on the structural and electrical properties of $\mathrm{ZnTe}$ films and on the photovoltaic performances of the $\mathrm{ZnTe} / \mathrm{CdTe} / \mathrm{CdS}$ cells. A Cu concentration of 6 at. \% and an annealing temperature of $\sim 180^{\circ} \mathrm{C}$ yielded the highest fill factors and efficiencies for the $\mathrm{ZnTe} / \mathrm{CdTe} / \mathrm{CdS}$ cells. Higher conductivity $\mathrm{ZnTe}$ films can be obtained at higher $\mathrm{Cu}$ concentrations or higher annealing temperatures. However, cells fabricated under these conditions show much inferior behavior because of the interaction between $\mathrm{ZnTe}$ and $\mathrm{CdTe}$. The substrate (CdTe) temperature during $\mathrm{ZnTe}$ deposition was also found to have a significant impact on the cell performance. Unheated substrates yielded the highest performance. In contrast, indirect heating caused by the thermal radiation from the sources led to large fluctuations in the performance of the resulting cells.

We have studied the effect of metal contacts ( $\mathrm{Au}, \mathrm{Ni}$, and $\mathrm{Co}$ ) to $\mathrm{ZnTe}$ on the $\mathrm{ZnTe} / \mathrm{CdTe} / \mathrm{CdS}$ cell performances. We found that the open-circuit photovoltage of the cells is critically dependent on the processing conditions if $\mathrm{Ni}$ is used as the contact metal to $\mathrm{ZnTe}$. In many cases, $\mathrm{V}_{\infty}$ often decreases after $\mathrm{Ni}$ deposition. In contrast, deposition of $\mathrm{Au}$ on the $\mathrm{ZnTe} / \mathrm{CdTe} / \mathrm{CdS}$ structure leads to consistent improvement of $\mathrm{V}_{\mathrm{co}}$. Table 1 shows the results for a set of data which correspond to a Cu concentration of 5.5 at. \% in $\mathrm{ZnTe}$. Cells made with $\mathrm{Au} / \mathrm{ZnTe}$ show consistently higher $\mathrm{V}_{\infty}$ than those with $\mathrm{Ni}$ contacts. The series resistances are comparable in both cases. The difference in $\mathrm{FF}$ originates mainly from the difference in $\mathrm{V}_{\mathrm{oc}}$.

Table 1. Effect of $\mathrm{ZnTe}$ annealing temperature and back contact metal on cell performances. Each data point is the average value obtained from 5 small-area cells.

\begin{tabular}{cccccc}
\hline Back Contact & Annealing temperature $\left({ }^{\circ} \mathrm{C}\right)$ & $\mathrm{V}_{\propto}(\mathrm{V})$ & $\mathrm{J}_{\mathrm{sc}}\left(\mathrm{mA} / \mathrm{cm}^{2}\right)$ & $\mathrm{FF}(\%)$ & Eff. (\%) \\
\hline \multirow{6}{*}{$\mathrm{Au}$} & 155 & 0.77 & 21.6 & 0.72 & 11.9 \\
& 168 & 0.77 & 21.4 & 0.73 & 12.1 \\
& 185 & 0.76 & 20.2 & 0.68 & 10.4 \\
& & & & & \\
$\mathrm{Ni}$ & 155 & 0.65 & 22.8 & 0.65 & 10.5 \\
& 168 & 0.68 & 23.1 & 0.70 & 11.1 \\
& 185 & 0.70 & 22.4 & 0.67 & 10.5 \\
\hline
\end{tabular}

The mechanism that leads to the observed difference in cell performances was investigated using $\mathrm{C}-\mathrm{V}$ measurements. Cells with Ni/ZnTe back contacts show Mott-Schottky behavior that is typical of a p-i-n cell. At zero bias, the depletion layer width is comparable to the CdTe film thickness. The slope in the forward bias region yields doping concentrations in the low $10^{14} \mathrm{~cm}^{-3} \mathrm{range}$. Cells with $\mathrm{Au} / \mathrm{ZnTe}$ show higher doping concentrations $\left(2 \times 10^{15} \mathrm{~cm}^{-3}\right)$. This higher doping concentration may contribute to a higher diffusion potential at the junction and lead to the higher $\mathrm{V}_{\mathrm{c}}$ observed. At zero bias, the width of the depletion region is much smaller than the CdTe film thickness, suggesting that the $\mathrm{CdTe} / \mathrm{CdS}$ cells using $\mathrm{ZnTe} / \mathrm{Au}$ as back contacts are more appropriately described as $\mathrm{p}-\mathrm{n}$ junction devices. 
As a result of our systematic investigation and optimization of the processing conditions, we have improved the efficiency of $\mathrm{CdTe} / \mathrm{CdS}$ cells using the ZnTe back contact. The light J-V curve of a CdS/CdTe/ZnTe cell, which yielded a $\mathrm{V}_{\propto}$ of $0.778 \mathrm{~V}, \mathrm{a} \mathrm{J}_{s c}$ of $22.4 \mathrm{~mA} / \mathrm{cm}^{2}$, an FF of $74.0 \%$, and an efficiency of $12.9 \%$ is shown in Figure 3 . In terms of individual parameters, we have obtained $V_{\propto}$ of over $0.8 \mathrm{~V}$, and FF of $76 \%$ on other cells. The diode quality factor and series resistance of these cells were derived from light J-V measurements. The series resistance obtained from cells with the ZnTe back contact is less than $0.1 \Omega \mathrm{cm}^{2}$, much smaller than that obtained without the $\mathrm{ZnTe}$ contact.

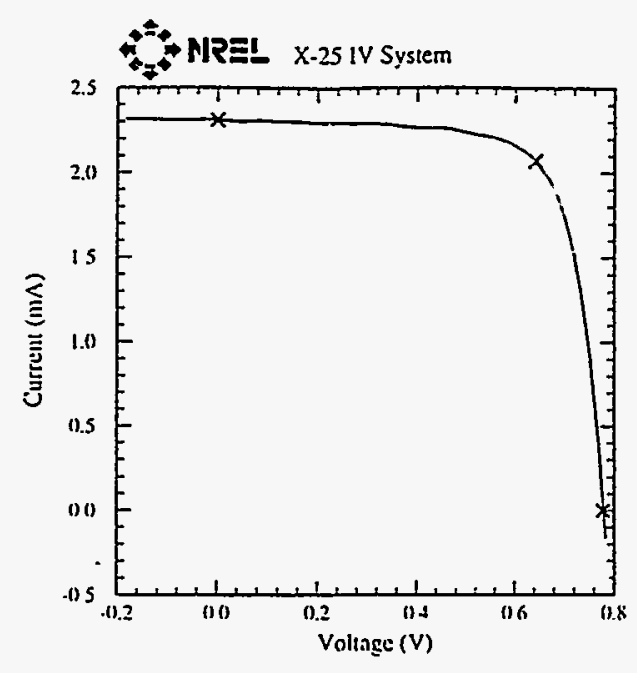

$v_{u}=0.777 .5 \mathrm{~V}$
$J_{w}=2.30 \mathrm{~mm}$
$J_{4}=22.3 \mathrm{~m} \mathrm{mAcm}:$
Iill bus(ur $=74.04 \%$

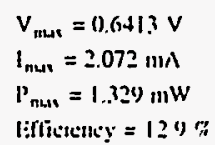

Figure 3. Light J-V curve of a $\mathrm{ZnTe} / \mathrm{CdTe} / \mathrm{CdS}$ cell.

\section{Summary}

We have made significant progress in the fabrication of low-cost, high-efficiency CdTe/CdS thin film solar cells. Improvements of both $\mathrm{V}_{\propto}$ and shunt resistance have been obtained by optimizing the thickness of the electrodeposited CdTe thin films. Systematic investigations of $\mathrm{Cu}$-doped $\mathrm{ZnTe}$ used as the back contact layer on electrodeposited $\mathrm{CdTe}$ have resulted in very low series resistances and high fill factors (0.76). Cells with efficiencies as high as $12.9 \%$ have been demonstrated using the electrodeposited CdTe with an optimized ZnTe back contact.

\section{Publications}

1. "Effect of Annealing on Microstructure, Residual Stress, and Photovoltaic Characteristics of Electrodeposited CdTe Films", D. Kim, B. Qi, D.L. Williamson, and J.U. Trefny, Proc. 24th IEEE PVSC, 338 (1994).

2. "Polycrystalline Thin Film CdTe Solar Cells Fabricated by Electrodeposition", D. Kim, S. Pozder, Y. Zhu, and J.U. Trefny, Proc. 24th IEEE PVSC, 334 (1994).

3. "Interdiffusion in Polycrystalline Thin Film CdS/CdTe Solar Cells", D. Mao, L.H. Feng, Y. Zhu, J. Tang, W. Song, R. Collins, D.L. Williamson, and J.U. Trefny, 13th NREL Photovoltaic Program Review, AIP Conference Proceedings 353, Harin S. Ullal and C. Edwin Witt, ed. p.320.

4. "Effect of Annealing on Microstructure, Residual Stress, and Photovoltaic Characteristics of Electrodeposited CdTe Films", B. Qi, D. Kim, D.L. Williamson, and J.U. Trefny, J. of Electrochem. Soc. (in press).

5. "The Structural, Optical, and Electrical Properties of Vacuum Evaporated Cu-doped ZnTe Polycrystalline Thin Films", L.H. Feng, D. Mao, J. Tang, R. Collins, and J.U. Trefny, J. Electron. Materials (submitted). 


\title{
Title: Microstructure of Amorphous-Silicon-Based Solar Cell Materials by Small-Angle X-ray Scattering
}

\author{
Organization: Department of Physics, Colorado School of Mines, Golden, Colorado
}

Contributors: D.L. Williamson, principal investigator; D. Min and S. Johnston

\section{Objectives}

The general goals are to establish details of the microstructure of a-Si:H and related alloys presently being incorporated into solar cells and to help determine whether particular microstructural features are correlated with photovoltaic behavior. If certain features are found to be detrimental to solar cell efficiency and stability, then optimization efforts will be carried out with the film growers and device makers of the various NREL Amorphous Silicon Teams to improve existing deposition methods or consider alternate methods and materials.

\section{Approach}

The direct structural characterization method of small-angle $x$-ray scattering (SAXS) is being used to study microstructural features ranging in size from the atomic up to approximately 30 nm. Features such as microvoids, hydrogen-rich clusters, columnar-like growth, microcrystallites, surface roughness, and alloy composition fluctuations or segregation can all be detected with a high degree of sensitivity. For example, microvoids of volume fraction $0.1 \%$ are readily seen and their average size can be determined. Atomic-scale structure can be inferred from the X-ray diffuse scattering intensity. Anisotropy in the SAXS, as detected by a sensitivity of the SAXS to sample orientation relative to the $\mathrm{x}$-ray beam, is direct evidence of oriented microstructural features such as columnar-like growth.

The approach involves collaboration with the NREL Low-gap, Mid-gap, and High-gap Teams, particularly the film-making and device-making groups that supply relevant, systematic sets of samples to help address specific issues within the above objectives. We also collaborate with several groups around the world not supported directly by NREL but recognized as experts in the field. SAXS-suitable substrates are supplied by us to all collaborating groups. SAXS measurements made at a central facility allow accurate comparisons of the microstructure of materials prepared by different deposition methods, conditions, and systems. Other in-house measurements complementing our SAXS studies include Raman spectroscopy, $x$-ray diffraction and flotation density. A high-quality annealing system is also available.

\section{Results}

During FY95 several significant results emerged. The discussion will be separated according to materials appropriate to the low-gap, mid-gap, and high-gap components of a multijunction solar cell. The latter is the structure being developed to meet the long-range goal of $15 \%$ stabilized efficiency with the amorphous silicon thin-film technology. 
Low-Bandgap Material (a-Si $1-\mathrm{x} \mathrm{Ge}_{\mathrm{x}}: \mathrm{H}$ )

The effect of $94 \%$ versus $0 \%$ hydrogen dilution of silane and germane source gases during PECVD growth for alloys prepared by IACS (Indian Association for the Cultivation of Science) with $x$ up to 0.7 is to reduce the amount of SAXS-detected microstructure and its degree of preferred orientation. The photoconductivity, $\eta \mu \tau$ product, and Urbach energy are all improved by the hydrogen dilution for $x>0.2$. The results support the concept that the growth chemistry, film microstructure, and opto-electronic properties can be modified by hydrogen dilution [1].

Use of unusually high hydrogen dilution versus more standard dilution in films with $x \sim 0.3$ at USSC yields highly oriented microstructural features about $7 \mathrm{~nm}$ in diameter and more than 20 times this dimension in length [2]. These objects could not be detected by TEM. Their orientation parallel to the solar cell transport direction and their relatively low density as determined by SAXS are apparently not detrimental since this material is currently producing the best low-bandgap devices at USSC [3]. Aside from these highly elongated features, the material is otherwise relatively homogeneous in that the nanovoids found in poor-device-quality a-Si:H are not detected.

New alloys on the Ge-rich side $(x>0.5)$ prepared at Harvard University have unusually low amounts of SAXS-detected microstructure and little preferred orientation. Microvoid volume fractions are below $0.03 \%$ in some samples. This was accomplished by more ion bombardment during deposition and suitable hydrogen dilution. The opto-electronic properties are significantly improved compared to earlier results for similar $\mathrm{x}$. The defect density of these materials as determined by drive-level-capacitance at the University of Oregon was found to be unusually low [4].

Films with $\mathrm{x}=1$ (no $\mathrm{Si}$ ) prepared by sputtering at Kaiserslautern University have extremely low SAXS-detected microstructure and large differences in surface roughness depending on the substrate used. Crystalline $\mathrm{Si}$ substrates yield smoother surfaces compared to the Al-foil substrates and microvoid fractions are well below $0.01 \mathrm{vol} . \%$. These results correlate with low Urbach energies $(<50 \mathrm{meV})[5]$.

Analysis of the diffuse SAXS intensity from all the a-SiGe:H alloys studied to date yields no evidence for short-range ordering or clustering on the basis of comparison to the theoretical Laue monotonic scattering prediction [5].

The group at IACS has reported a-Si:H films with unusually low bandgap (1.5eV) using a He dilution technique [6]. SAXS evaluation shows such films to have essentially homogeneous nanostructure. USSC prepared cells with He-diluted films but found no evidence of a reduction in the open-circuit voltage or in the optical bandgap [3]. It was suggested that there may be differences in geometrical factors of the electrodes in each deposition system leading to the differences in bandgap found by the two groups [3].

Mid-Bandgap Material (a-Si:H)

A new absolute intensity calibration of the SAXS system has allowed new determinations of the microvoid densities in a-Si:H films without the need of the earlier flotation density method of void calibration. Results from current device quality a-Si:H prepared by NREL, USSC, Solarex, 
APS, and IACS show void fractions near the present detection limit of about 0.01 vol.\%. Remaining SAXS-detected features can be classified as large-scale structure $(\geq 20 \mathrm{~nm})$. The amount of this structure and its preferred orientation varies remarkably among the samples from the different groups. Surface roughness or residual columnar-like microstructure are the two likely causes. Microvoid fractions in the range of 0.1 to 1 vol.\% with very small sizes of the order of 1-2 $\mathrm{nm}$ are correlated with poorer device performance [2].

Hotwire films prepared at NREL under conditions that yield low $\mathrm{H}$ contents and improved stability have much lower SAXS intensities than previously studied hotwire material that contained more $\mathrm{H}$. The microvoid densities in these new films are comparable to those of the best PECVD a-Si:H [2]. Device studies of the hotwire a-Si:H are underway at NREL in collaboration with USSC.

A series of a-Si: $\mathrm{H}$ films prepared under different amounts of Ar dilution by IACS yields improved device-quality behavior and the SAXS is remarkably low for such high Ar dilution (98\%) and such high $\mathrm{H}$ content ( -18 at.\%). Densification of these films relative to those made with no Ar dilution is attributed to bombardment of the growing surface by excited Ar atoms. In contrast, earlier Ar dilution in the VHF-PECVD method at Neuchatel University led to strong SAXS intensities and films with degraded opto-electronic properties.

The effect of ion bombardment during depostion of pure a-Si via magnetron sputtering by the group at University of Illinois is under investigation. Understanding of such effects without $\mathrm{H}$ should help improve understanding the behavior when $\mathrm{H}$ is added. Clear differences in SAXSdetected microstructures are detected for different ion bombardment conditions.

Unusual deposition conditions explored at Ecole Polytechnique involving either helium dilution or hydrogen dilution yield low SAXS-detected microstructures at high deposition rates (1.5 $\mathrm{nm} / \mathrm{s})$ or low substrate temperatures $\left(100^{\circ} \mathrm{C}\right)$. These a-Si:H films have low defect densities $\left(\sim 1 \times 10^{16} \mathrm{~cm}^{-3}\right)$ and low Urbach energies $(-50 \mathrm{meV})$.

High-Bandgap Material (a-Si $\left.{ }_{1-x} \mathrm{C}_{\mathrm{x}}: \mathrm{H}\right)$

Based on the new absolute SAXS intensites, the Solarex a-SiC:H films prepared for study by the High-bandgap Team are all found to contain nanovoid volume fractions of the order of $1 \%$ or greater. This fraction is two orders-of-magnitude larger than typical device-quality a-Si:H and occurs even for the smallest additions of carbon $(x=0.06)$. Hydrogen dilution reduces the nanovoid density and improves the opto-electronic properties.

A series of B-doped a-SiC:H alloys prepared by reactive magnetron sputtering at University of Illinois have nanovoid volume fractions that vary from 0.3 to $3.6 \%$ depending on hydrogen dilution and carbon content. A maximum optical bandgap (Tauc) of only $1.85 \mathrm{eV}$ was achieved for carbon contents of about 20 at.\%. Films prepared with no hydrogen dilution yielded the lowest void fraction and the highest flotation density yet observed for a-SiC:H $(2.34 \mathrm{~g} / \mathrm{cm} 3)$, but the optical gap of this film was only $1.43 \mathrm{eV}$. 


\section{Summary}

Nanovoids or $\mathrm{H}$-rich clusters with 1 to $4 \mathrm{~nm}$ sizes in a-Si:H that show little or no anisotropic SAXS correlate with poor solar cell and opto-electronic behavior. Larger-scale structures due either to surface roughness or residual columnar-like features are found in present state-of-theart device material. Ge alloying above $x=0.2$ typically leads to an oriented nanostructure indicative of columnar-like growth while $\mathrm{C}$ alloying typically induces a random nanostructure consisting of a narrow size distribution of $1-\mathrm{nm}$-sized objects that may be correlated in position via some interaction. Recent device-quality $\mathrm{a}-\mathrm{Si}_{0.7} \mathrm{Ge}_{0.3}: \mathrm{H}$ prepared by PECVD under high hydrogen dilution has shown an extremely oriented structure that can be modelled with ellipsoidal objects that have major-to-minor axis ratios in excess of 20 . These oriented objects and those in the a-Si:H are parallel to the solar cell transport direction so it may be reasonable that such features may not be detrimental if they are sufficiently sparse, as indeed indicated by the SAXS analyses. Conditions of enhanced ion-bombardment during PECVD growth of highGe-content alloys $(x>0.5)$ recently led to films with reduced oriented nanostructure and very weak SAXS signals that are comparable to those from the best a-Si:H. Unusually low-gap a$\mathrm{Si}: \mathrm{H}$ prepared by $\mathrm{He}$ dilution yields very weak SAXS but an attempt to make devices from similarly-prepared material did not yield lower open-circuit voltages.

Information on static disorder and atomic-scale composition fluctuations in these materials is being extracted from the diffuse component of the SAXS. Results from the a-Si $\mathrm{i}_{1-\mathrm{x}} \mathrm{Ge}_{\mathrm{x}}: \mathrm{H}$ alloy system do not reveal any evidence of $\mathrm{Si}-\mathrm{Ge}$ short-range clustering. Similar studies of a-Si:H with a wide range of $\mathrm{H}$ content and of a-SiC: $\mathrm{H}$ alloys are underway.

\section{References}

1. A.R. Middya, S. Ray, S.J. Jones, and D.L. Williamson, J. Appl. Phys. 78 (1995) 4966.

2. D.L. Williamson, Mater. Res. Soc. Symp. Proc. 377 (in press).

3. S. Guha, Annual Report. Amorphous Silicon Research. FY 1995 (October 1995) NREL/TP-411-20205, 51 pp. Available NTIS: Order No. DE95013124.

4. P. Wickboldt, D. Pang, W. Paul, J.H. Chen, F. Zhong, J.D. Cohen, Y. Chen, D.L. Williamson, ICAS 16, Kobe, Japan, 1995 (in press).

5. D.L. Williamson, Annual Report, NREL Subcontract No. XAN-4-13318-04 (April 1995).

6. S. Hazra, A.R. Middya, and S. Ray, J. Appl. Phys. 78 (1995) 581. 
Title:

Organization:

Contributors:
Device Physics of Thin-Film Polycrystalline Cells and Modules

Department of Physics, Colorado State University, Fort Collins, Colorado

J. R. Sites, principal investigator, I. L. Eisgruber, J. E. Granata, X. X. Liu, G. Stollwerck, J. A. Sharp, B. F. Murphy, and K. E. Schmidt

\section{Objectives}

The objectives of this program are (1) the separation and quantification of individual losses in specific thin-film solar cells, (2) the detailed characterization of small modules, and (3) the presentation of a viable model for the forward-current loss mechanism.

\section{Cell Analysis}

Cell-analysis projects involving solar cells fabricated by ten collaborating organizations were completed during 1995. The effect of a secondary barrier related to the back contact of CdTe cells was analyzed and presented at the European PV meeting [1], and a summary of the dependence of CdTe response on bias history was given at the NREL Performance and Reliability Workshop [2]. A more extensive CdTe project has been the comparison of performance among cells fabricated with varying thicknesses of CdS by different deposition techniques on different types of glass/TCO superstrates. it was found (see Fig. 1) that losses for photon energies above the CdS bandgap follow generally expected behavior. It was also found, however, that the absorption loss at longer wavelength and the $\mathrm{CdS}$ thickness below which junction quality deteriorates vary significantly with the glass/TCO combination used.

Work on $\mathrm{CuIn}_{1-x} \mathrm{Ga}_{x} \mathrm{Se}_{2}$ (CIGS) cells included collaborative projects with ISET on the CIGS cells made by selenization [3] and with NREL on CIGS cells made by the interdiffusion of a variable-thickness CGS layer into a CIS top layer [4]. One modeling project using ADEPT software explored the effect of a CdS/CIS conduction band offset on cell performance [5]. It was found that a room temperature offset between -0.3 and $+0.3 \mathrm{eV}$ had little effect on performance and that range was not significantly affected by a thin indium-rich layer at the CIS surface. A second modeling project in collaboration with Solarex and Penn State has explained a distortion seen in the current-voltage curves of cells made with certain chemical-bath deposition parameters.

\section{Module Analysis}

Much of the module analysis program has involved collaboration with the large-scale laser-scanning facility at NREL. A technique was developed to separate individual-cell photocurrents and shunting resistances in an encapsulated module by varying the laser-beam chopping frequency [6]. Fig. 2 shows a 14-cell module scan that primarily reflects shunting variations at low frequency and photocurrent variations at higher frequency. Other module issues addressed are localized defects. 
the consequences of distributed series resistance, light scattering onto adjacent cells, voltage distribution among cells in a forward-biased module, and the evolution of EVA browning.

\section{Studies of Thin-film Polycrystalline Cells}

Two updates were given to our quantification of loss mechanisms in the best thin-film polycrystalline cells and the comparable situation with single-crystal cells of comparable bandgap $[7,8]$. The primary difference continues to be forward current in polycrystalline cells, attributed to electron-hole recombination at grain boundaries in the depletion region, that is approximately two orders of magnitude larger than that of the crystalline cells.

\section{References}

1. "Analysis of CdTe Back-Contact Barriers," G. Stollwerck and J. R. Sites, Proc. 12th European Photovoltaic Solar Energy Conf. (1995).

2. "Dependence of CdTe Response on Bias History," J. R. Sites, R. A. Sasala, and I. L. Eisgruber, NREL/CP-4,11-20379, p. 15 (1995).

3. " $\mathrm{Cu}(\mathrm{In}, \mathrm{Ga}) \mathrm{Se}_{2}$ Thin Films and Solar Cells Prepared by Selenization of Metallic Precursors," B. M. Basol et al., submitted to J. Appl. Physics.

4. "The Influence of $\mathrm{Ga}$ on the Properties of $\mathrm{CuIn}(\mathrm{Ga}) \mathrm{Se}_{2}$-Based Thin Films and Devices," B. M. Keyes et al., Proc. 10th International Conf. on Ternary and Multinary Compounds (1995).

5. "Calculated Effect of Conduction-Band Offset on CuInSe ${ }_{2}$ Solar-Cell Performance," X. X. Liu and J. R. Sites, AIP Conf. Proc. $\underline{353}, 444$ (1995).

6. "Extraction of Individual-Cell Photocurrents and Shunt Resistances in Encapsulated Modules Using Large-Scale Laser Scanning," I. L. Eisgruber and J. R. Sites, Progress in Photovoltaics 4 , xxx (1996).

7. "Recent Efficiency Gains for CdTe and $\mathrm{CuIn}_{1-\mathrm{x}} \mathrm{Ga}_{\mathrm{x}}$ Solar Cells: What Has Changed?" J. R. Sites and X. X. Liu, Solar Energy Materials and Solar Cells (1995).

8. "Performance Comparison Between Polycrystalline Thin-Film and Single-Crystal Solar Cells," J. R. Sites and X. X. Liu, Progress in Photovoltaics $\underline{3}$, 307.(1995). 


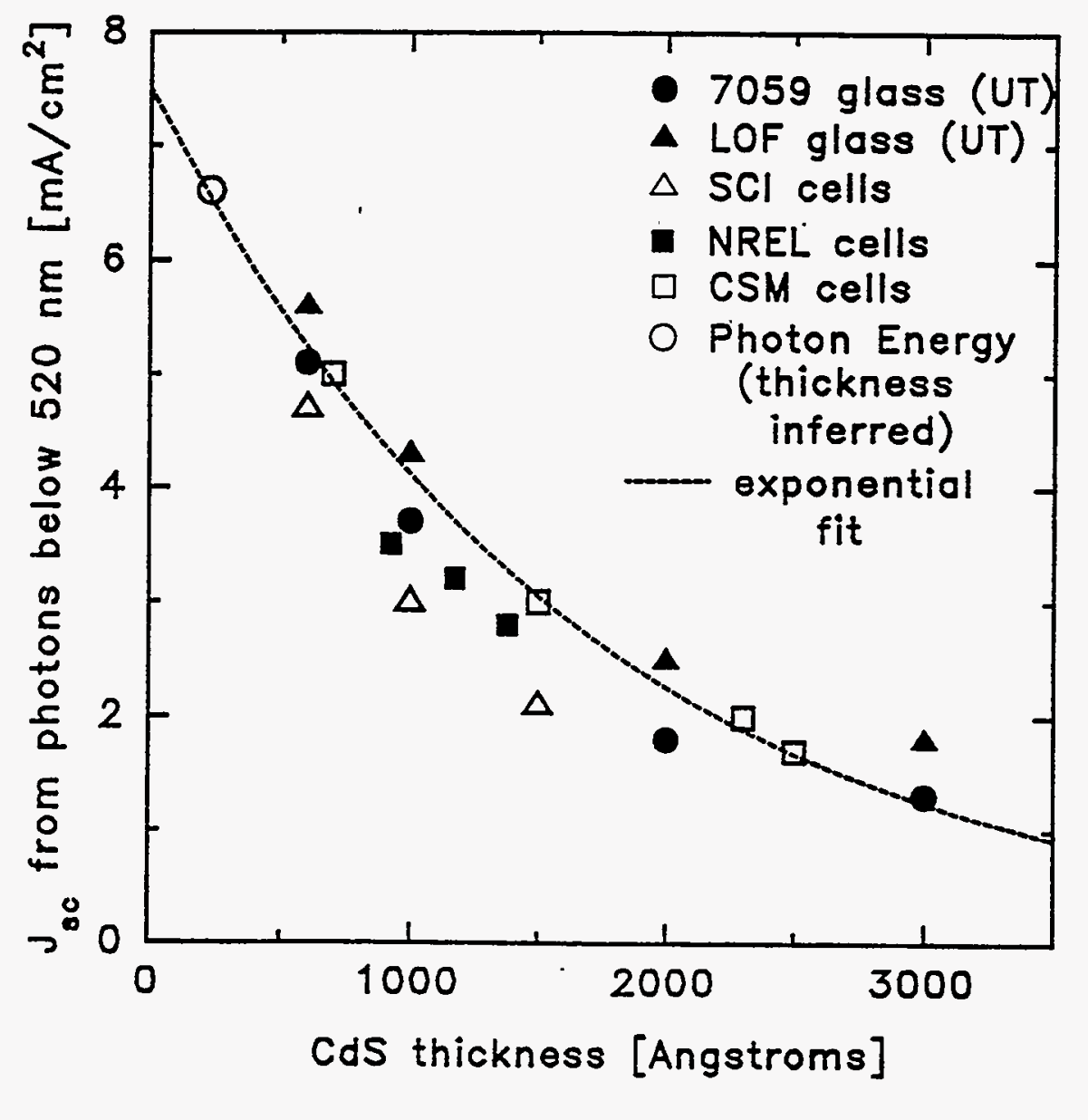

Fig. 1. Blue-light contributions to CdS/CdTe photocurrent as a function of CdS thickness. 


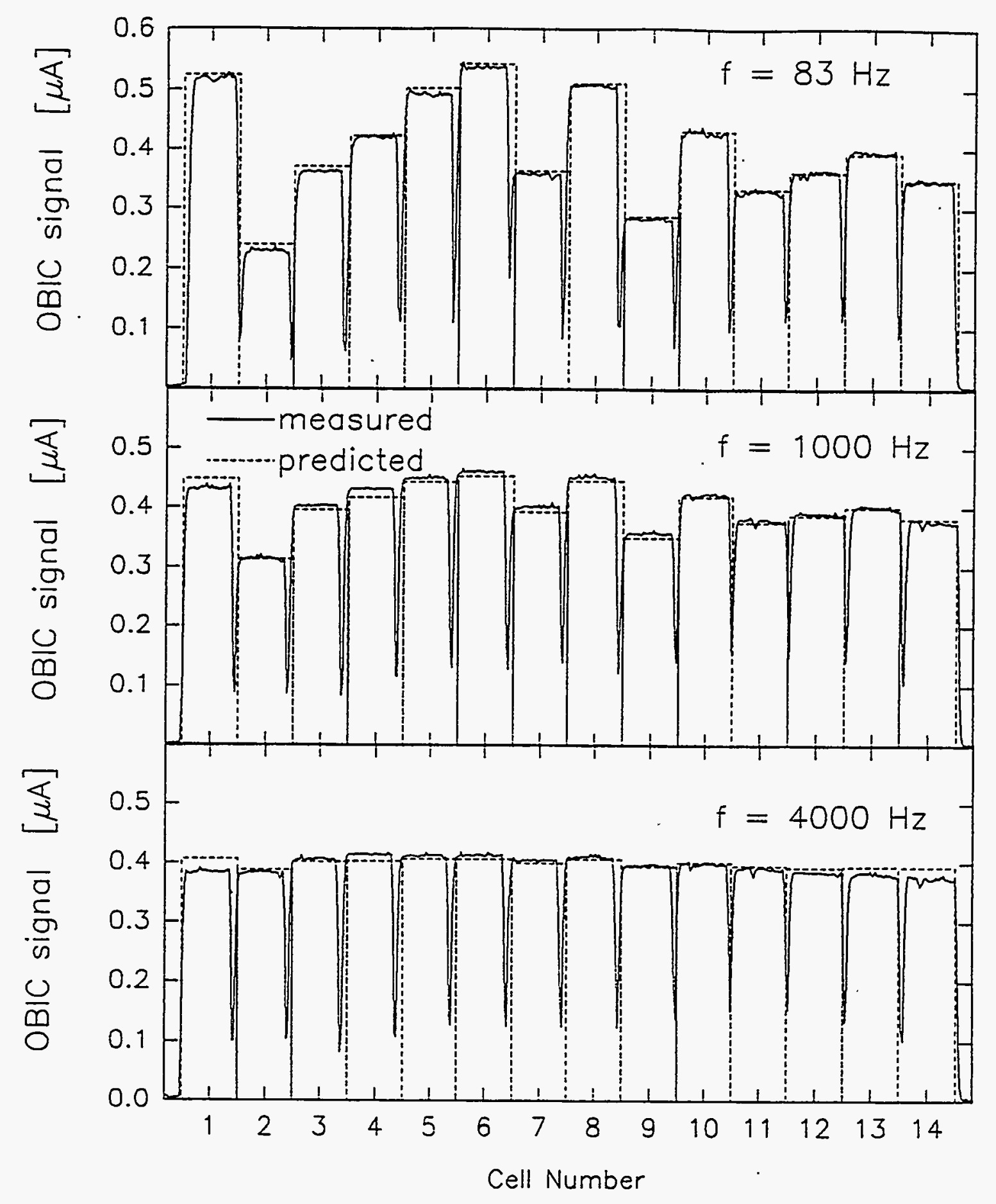

Fig. 2. Chopping-frequency response of OBIC signal used to separate individual-cell photocurrent and shunt resistance in an encapsulated module. 
Title: Development of Bigh, Stable-Efficiency

Triple-Junction a-Si Alloy Solar Cells

Organization: Energy Conversion Devices, Inc.

Troy, Michigan 48084

Contributors: X. Deng, Principal Investigator; S. J. Jones, T. Liu, M. Izu

and S. R. Ovshinsky

\section{Objectives}

The overall objective of this three-year, cost-shared, amorphous silicon research program is to develop advanced technologies and to demonstrate stable $14 \%$ efficient triple-junction $\leftleftarrows-S i$ alloy solar cells. The technologies developed in this program will then be incorporated inio ECD's continuous roll-to-roll deposition process to further enhance ECD's photovoltaic manufacturing technology.

\section{Technology}

In ECD's solar cell design, triple-junction a-Si alloy solar cells are deposited onto stainless steel substrates coated with $\mathrm{Ag} / \mathrm{ZnO}$ back-reflector layers. This type of cell design enables us to use a continuous roll-to-roll deposition process to manufacture a-Si PV materials in high volume at low cost [1-5]. Using this cell design, ECD has previously achieved $13.7 \%$ initial solar cell efficiency [1] using the following features: 1) a triple-junction two-bandgap, spectrum-splitting solar cell design; 2) a microcrystalline silicon p layer; 3) a bandgap profiled a-SiGe alloy as the bottom cell $\mathrm{i}$ layer; 4) a high performance $\mathrm{Ag} / \mathrm{ZnO}$ back-reflector; and 5) a high performance tunnel junction between component cells. We have also applied the technology into ECD's $2 \mathrm{MW}$ a-Si production line and achieved the manufacturing of $4 \mathrm{ft}^{2} \mathrm{PV}$ modules with $8 \%$ stable efficiency [2].

\section{Approaches}

In this program, we are further advancing ECD's existing PV technology toward the goal of $14 \%$ stable solar cells by performing the following four tasks:

1. Improving the Stability of the Intrinsic a-Si Alloy Materials

2. Improving the Quality of Low Bandgap a-SiGe Alloy

3. Improving $\mathrm{p}^{+}$Window Layers

4. Development of High Stable Efficiency Triple-junction a-Si Alloy Solar Cells

\section{Major Accomplishments in Phase I}

1. We have demonstrated, in an n-i-p solar cell, the improved stability of a-Si:H:F materials when deposited using a new fluorine precursor. Single junction n-i-p solar cells with $3000 \AA$ to 3500 A a-Si:H:F intrinsic layers were deposited onto $\mathrm{Ag} / \mathrm{ZnO}$ back-reflectors in one of our multichamber deposition systems (LL1). The initial efficiency of the a-Si:H:F device is around 
$9 \%$, which is the same as the baseline unfluorinated a-Si:H n-i-p solar cells deposited in this machine. After 1680 hours of one sun light soaking at $50{ }^{\circ} \mathrm{C}$, the a-Si:H:F devices showed a degraded efficiency of around $8 \%$. The degradation in efficiency with light soaking is $12-13 \%$, which is lower than the $16-17 \%$ drop for the baseline a-Si:H n-i-p device with a $3000 \AA$ thick i layer after the same amount of light soaking. Table 1 shows the light soaking degradation data for a-Si:H:F single junction solar cell devices and a-Si:H reference devices. The improved stability of a-Si:H:F devices meets the Phase I milestone under Task 1.

2. We have investigated an $\mathrm{C}-\mathrm{Si}\left(\mathrm{n}^{\dagger}\right) / \mathrm{a}-\mathrm{Si}$ alloy/Pd Schottky barrier device as an evaluation tool for the photovoltaic performance of a-Si alloy materials [3]. In this device, the intrinsic a-Si alloy materials to be studied are deposited onto a heavily doped $n^{*}$ crystalline silicon wafer and a thin layer of $\mathrm{Pd}$ is subsequently evaporated on top to complete the device structure. The fill factor of such a device under weak red-light reflects the minority carrier transport properties. We compared the I-V characteristics of these Schottky barrier devices with standard a-Si and a-SiGe $\mathrm{n}$-i-p solar cells having the same intrinsic layer, and established a good correlation between the fill factor of these two type of devices, as is shown in Figure 1. Due to the simplicity of the device structure, it can be fabricated without multichamber solar cell deposition facilities. The c-Si( $\left.\mathrm{n}^{+}\right) / \mathrm{a}-$ $\mathrm{Si}$ alloy/Pd device offers a convenient technique to reliably evaluate the material performance in a solar cell device.

3. We have optimized the deposition of the intrinsic a-Si layer and the boron doped $\mu \mathrm{c}-\mathrm{Si} \mathrm{p}^{+}$ layer to improve the top component cell $\mathrm{V}_{\mathrm{oc}}$. As a result, a $\mathrm{V}_{\mathrm{oc}}$ of $1.053 \mathrm{~V}$ was achieved for a single junction a-Si:H n-i-p solar cell (Figure 2). After 2500 hours of light soaking, the $\mathrm{V}_{o c}$ of these devices is stabilized at $1.014 \mathrm{~V}$ (Figure 3). This high $\mathrm{V}_{o c}$ is achieved by depositing the intrinsic a-Si layer with high hydrogen dilution at relatively low temperature [4]. The high stable $\mathrm{V}_{o c}$ of these devices meets the phase I milestone under Task 3.

4. We designed a novel structure for the $\mathrm{p}-\mathrm{n}$ tunnel junction (recombination layer) in a multiple junction solar cell and demonstrated the improved tunnel junction in the solar cell $\mathrm{V}_{\mathrm{oc}}$ and $\mathrm{FF}$ using an n-i-p-n device structure.

5. We have installed, at ECD's expense, a new solar cell deposition system (LL2). After initial optimization, we have obtained a baseline of $10.6 \%$ initial efficiency for single junction solar cell and $11.2 \%$ initial efficiency for triple junction solar cells.

6. We have collaborated with Harvard University to evaluate the Harvard a-SiGe material in an n-i-p solar cell devices. These devices were then compared with devices having intrinsic layers deposited using a conventional deposition technique.

7. We have optimized the deposition parameters with high Ge content. A bandgap profiled aSiGe alloy solar cell demonstrates a maximum power output of $3.6 \mathrm{~mW} / \mathrm{cm}^{2}$ measured under a Xenon solar simulator with a $610 \mathrm{~nm}$ cut-on optical filter. After 200 hours of light soaking, the $P_{\max }$ is degraded to $3.0 \mathrm{~mW} / \mathrm{cm}^{2}$. This device is expected to stabilize at higher than $2.5 \mathrm{~mW} / \mathrm{cm}^{2}$, which is the Phase I milestone under Task 2. 


\section{References}

1. J. Yang, R. Ross, T. Glatfelter, R. Mohr, G. Hammond, C. Bernotaitis, E. Chen, J. Burdick, M. Hopson and S. Guha, Proc. 20th IEEE PV. Spec. Conf. 241 (1988).

2. M. Izu, X. Deng, A. Krisko, K. Whelan, R. Young, H.C. Ovshinsky, K.L. Narasimhan and S.R. Ovshinsky, Proc. 23rd IEEE PV Spec. Conf., 919 (1993).

3. X. Deng, S. J. Jones, K. L. Narasimhan, J. Evans, M. Izu and S. R. Ovshinsky, Proc. of MRS Spring Meeting, April 1995.

4. X. Deng, K. L. Narasimhan, J. Evans, M. Izu and S. R. Ovshinsky, Proc. of first World Conference on Photovoltaic Energy Conversion (WCPEC), p678, (1995).

5. X. Deng, ECD Annual Subcontract Progress Report to NREL under subcontract ZAN-413318-11, (1995).

Table 1 Light soaking degradation of an a-Si:H:F single-junction solar cell and an a-Si:H reference single-junction solar cell

$\begin{array}{lllllll}\begin{array}{l}\text { Solar cell } \\ \text { i-layer }\end{array} & \begin{array}{l}\text { Light } \\ \text { soaking }\end{array} & \begin{array}{l}\mathrm{V}_{\mathrm{oc}} \\ (\mathrm{V})\end{array} & \begin{array}{c}\mathrm{J}_{\mathrm{sc}} \\ \left(\mathrm{mA} / \mathrm{cm}^{2}\right)\end{array} & \mathrm{FF} & \begin{array}{l}\text { Eff } \\ (\%)\end{array} & \begin{array}{l}\text { Drop in } \\ \text { Efficiency }\end{array} \\ \text { a-Si:H:F } & 0 \mathrm{hrs} & 0.932 & 15.37 & 0.638 & 9.14 & - \\ \text { on Ag/ZnO } & 380 \mathrm{hrs} & 0.896 & 15.10 & 0.592 & 8.01 & 12.3 \% \\ & 1680 \mathrm{hrs} & 0.887 & 15.15 & 0.589 & 7.92 & 13.3 \% \\ \text { a-Si:H } & 0 \mathrm{hrs} & 0.951 & 10.86 & 0.601 & 6.21 & - \\ \text { on SS } & 380 \mathrm{hrs} & 0.916 & 10.29 & 0.554 & 5.22 & 15.9 \% \\ & 620 \mathrm{hrs} & 0.910 & 10.38 & 0.550 & 5.20 & 16.3 \%\end{array}$

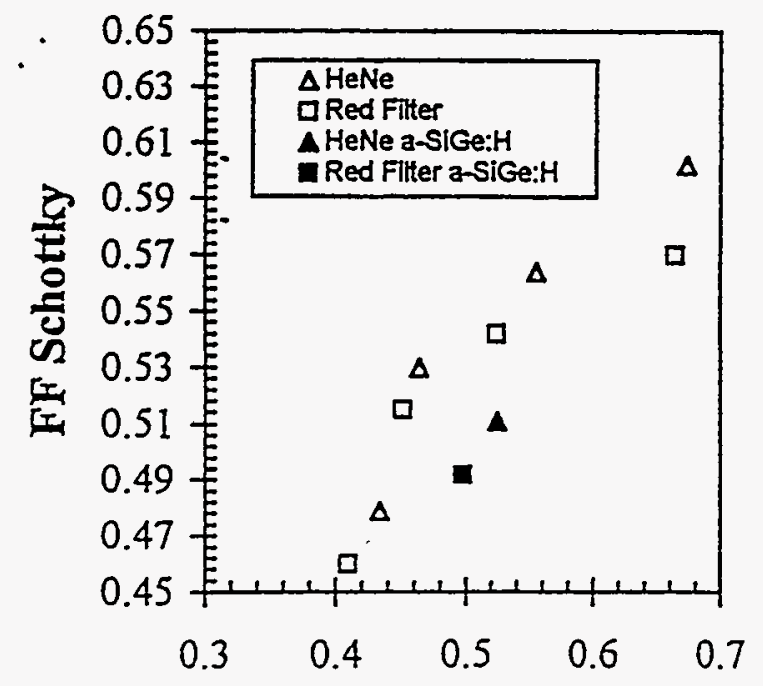

FF NIP

Figure $1 \mathrm{FF}$ of Schottky barrier devices as a function of that of the reference n-i-p solar cells with the same intrinsic layer. 


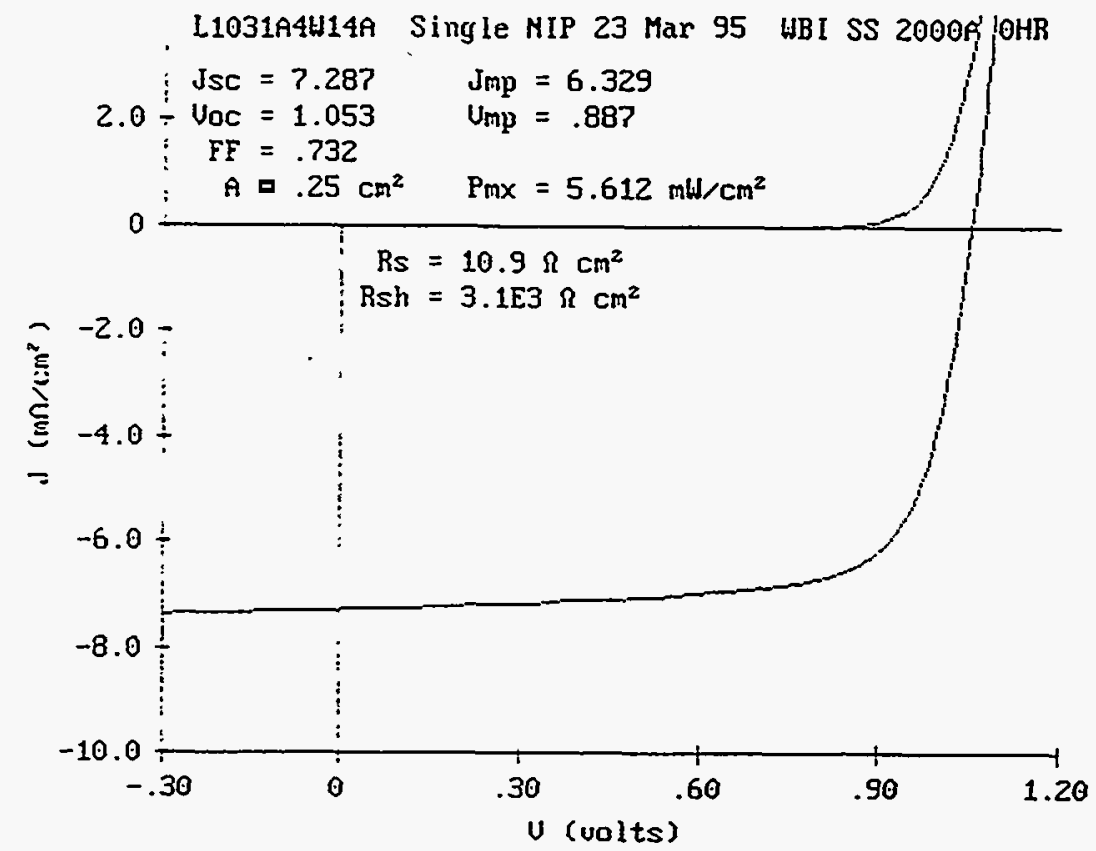

Figure $2 \mathrm{~J}-\mathrm{V}$ curve of a single-junction solar cell with a wide bandgap a-Si i-layer and the improved p-layer, showing $1.053 \mathrm{~V}$ open circuit voltage.

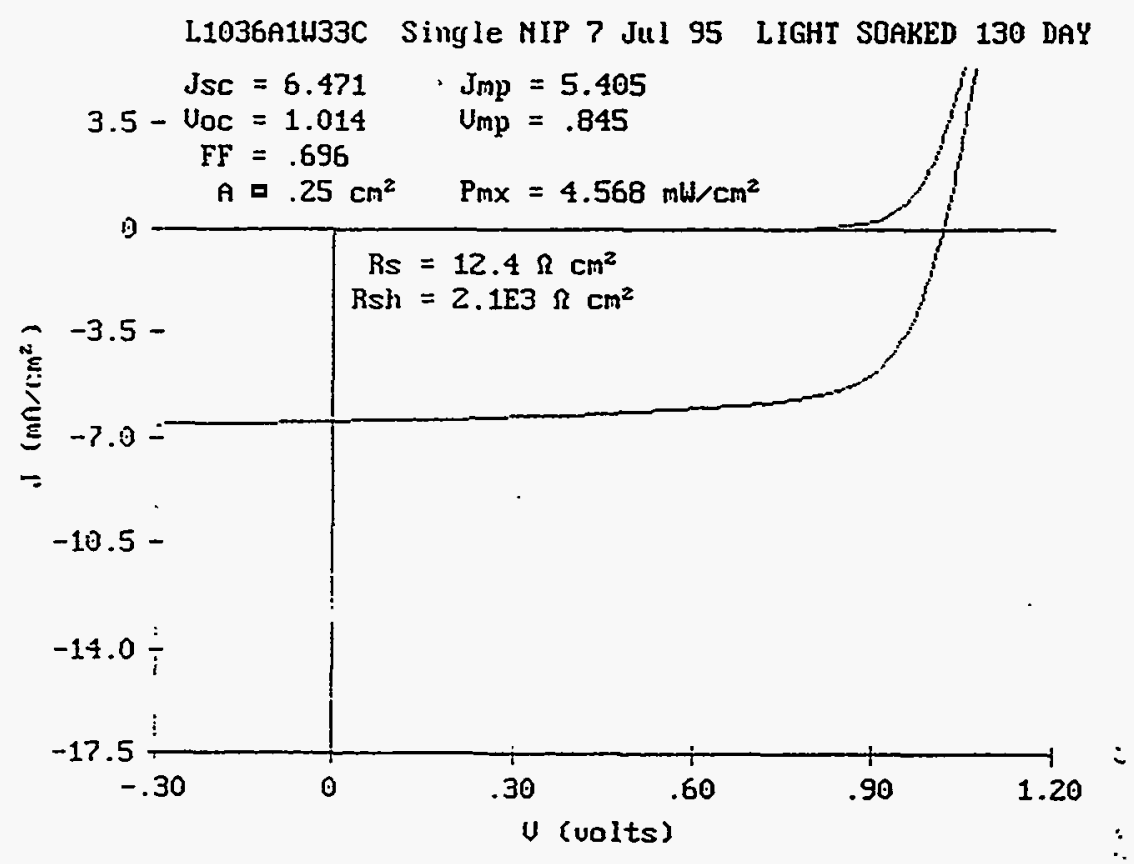

Figure $3 \mathrm{~J}-\mathrm{V}$ curve of a high $\mathrm{V}_{\propto}$ single junction a-Si solar cell after 2500 hours of one-sun lightdegradation at $50^{\circ} \mathrm{C}$, showing $1.014 \mathrm{~V}$ stabilized $\mathrm{V}_{\mathrm{oc}}$. 
Title:

Organization:

Contributors:
CIS Photovoltaic Technology

Energy Photovoltaics, Inc. (EPV)

Princeton, New Jersey

Alan Delahoy, principal investigator; Jeff Britt, co-principal investigator; Andrew Gabor, scientist; Zoltan Kiss, program manager; G. Butler, M. Cherny, M. Doroba, J. Esler, F. Faras, G. McComiskey, A. Sizemore, F. Ziobro.

\section{- Introduction}

Energy Photovoltaics, Inc. (EPV) has pioneered the production of $\mathrm{Cu}(\mathrm{In}, \mathrm{Ga}) \mathrm{Se}_{2}$ photovoltaic modules using vacuum processing but without the use of $\mathrm{H}_{2} \mathrm{Se}$. An efficiency of $9.0 \%$ has been achieved for unencapsulated modules of about $200 \mathrm{~cm}^{2}$, and a pilot line for the production of $0.43 \mathrm{~m}^{2}$ encapsulated modules has been completed [1]. The heart of the pilot line is a computer-controlled large area compound formation machine (Zeus) in which CIGS is formed at temperatures close to the softening point of glass without glass warpage. The machine consists of a deposition and reaction chamber containing a novel dual linear source for material delivery, a load-lock chamber, means for heating and transporting the glass, and appropriate sensors. Sequential CIGS formation schemes have been defined that are better suited to manufacturing than co-deposition of the elements involved.

\section{Objectives}

The overall project goal is the demonstration of encapsulated and qualified large area CIGS photovoltaic modules prepared in prototype manufacturing equipment, and with powers of about $40 \mathrm{~W}$. Another significant goal concerns the development of alternative materials and techniques for contact layers and encapsulation that would result in useful cost reductions if transferred to manufacturing. Modeling and performance monitoring will be used to fully characterize CIGS modules.

\section{Approach}

The tasks most crucial to the fabrication of high performance CIGS modules are:

1. The preparation of a CIGS active layer (absorber) capable of yielding high efficiency devices over the entire $96.5 \mathrm{~cm} \times 44.5 \mathrm{~cm}$ substrate.

2. The preparation of a $\mathrm{ZnO}$ transparent conductor over similar areas with $10 \mathrm{ohms} / \mathrm{square}$ sheet resistance and minimal absorption.

3. Patterning of the layers to effect serial interconnection without defects and losses.

4. Robust encapsulation and lead out of the CIGS plate.

Absorber recipe development is being carried out in three programs: $R \& D$ using equipment scaled for $930 \mathrm{~cm}^{2}\left(1 \mathrm{ft}^{2}\right)$ substrates, a CRADA in collaboration with NREL, and process development carried out directly on the $0.43 \mathrm{~m}^{2}$ equipment (Zeus and in-line sputtering system). Different absorbers are judged on the basis of device performance, uniformity, adhesion and reproducibility. In the area of uniformity alone, a significant diagnostics effort has been established to monitor the uniformity of the constituent layers, the thickness and compositional 
uniformity of the CIGS layer, and device performance as a function of position across the substrate. In parallel with these activities, in-house engineering continues to improve the reliability and reproducibility of the Zeus hardware.

High quality doped $\mathrm{ZnO}$ is being produced over $930 \mathrm{~cm}^{2}$ by $\mathrm{RF}$ sputtering from a $\mathrm{ZnO}: \mathrm{Al}_{2} \mathrm{O}_{3}$ target. For large area depositions, $\mathrm{DC}$ sputtering of $\mathrm{ZnO}: \mathrm{Al}_{2} \mathrm{O}_{3}$ is being performed and improved to satisfy short-term needs, while in the long-term $\mathrm{ZnO}$ will be produced by high rate $\mathrm{DC}$ reactive sputtering from a metallic target.

For patterning, EPV's previously reported process has been scaled up to large areas and is performing satisfactorily. Meanwhile, research is underway to demonstrate an all-laser approach in order to minimize area loss. For encapsulation, a vacuum lamination process using sheet EVA has been developed, although lower cost processes are envisaged in the future.

Finally, module design is being tailored to fit specific alloy properties and module end uses. This is accomplished through calculation of exact module I-V curves as a function of cell and module parameters, and through calculation of integrated energy delivery based on irradiance and temperature distribution functions [2].

\section{Results}

A particular recipe category that is eminently suitable for manufacturing has been extensively investigated. Implemented on EPV's large scale equipment it involves delivery of $\mathrm{Cu}$ by $\mathrm{DC}$ magnetron sputtering, delivery of indium and gallium selenides by a linear evaporation source, and Se by a second and similar linear source [3]. Closely related recipes have been explored under the CRADA, resulting in demonstration of a $13.9 \%$ CIGS device. The I-V curve of this device (no A/R coating) is shown in Figure 1 , the photovoltaic parameters being $V_{o c} 633 \mathrm{mV}, \mathrm{J}_{\mathrm{sc}} 31.5$ $\mathrm{mA} / \mathrm{cm}^{2}$, and $\mathrm{FF} 69.6 \%$.

Diligent optimization of both hardware and processing in the Zeus system has resulted in the production of CIGS with good compositional uniformity along both the short and long directions of the plate. Factors influencing uniformity across the short dimension include sputtering system design and operation, and the emission pattern and source-substrate distance for the linear source. Relevant film profiles were recently published in [3]. The composition of CIGS along the long dimension of a plate, as measured by EPMA at $20 \mathrm{kV}$, is shown in Figure 2.

Despite the compositional uniformity of the absorber material over $0.43 \mathrm{~m}^{2}$, the material has not yet achieved the efficiency level demonstrated in Figure 1. Figure 3 shows an open-circuit voltage map for a $96.5 \times 44.5 \mathrm{~cm}^{2}$ plate prior to final patterning. Although voltages are seen to be reasonable, the absorber currently suffers from a current density that may reach, for example, only $20 \mathrm{~mA} / \mathrm{cm}^{2}$. The root cause of this problem is under intense scrutiny using tools such as SEM, Auger, SIMS, device maps, impurity control, systematic variation of recipe parameters, etc. We are confident that this problem will be solved in the near future.

A complete encapsulation and termination process has been developed for CIS plates. Thus, following final patterning, aluminum lead-out foils are attached and emerge through a hole in the substrate. A glass cover plate is vacuum laminated using EVA, and a low-profile silicone-rubber boot is formed from which the module's insulated output cables emerge. A $1 \mathrm{~cm}$ wide film-free border around the periphery of the module minimizes leakage currents to the active circuit during operation, thereby allowing the module to pass the hi-pot test. 
To address the prohibitive target cost and low deposition rate associated with the use of ceramic $\mathrm{ZnO}$ sputtering targets we have embarked on the development of reactive $\mathrm{DC}$ magnetron sputtering of $\mathrm{Zn}$ to prepare transparent, conducting $\mathrm{ZnO}$. While some high quality films (16 ohms/sq., 90\% transmission) have been prepared in a static mode using a Zn:Al target, it is in general apparent that an active feedback system will be required to maintain the partial pressure of oxygen at the desired level. Figure 4 shows the uncontrollable runaway and strong hysteresis loop of discharge voltage (and hence target oxidation state) versus oxygen flow that can occur in some system configurations. It is notable that the approach $\mathrm{BC}$ to the transition $\mathrm{CD}$ of Figure 4 is heralded by an easily observable increase in the optical emission of excited $\mathrm{Zn}$ atoms.

By compressing a vast amount of solar irradiance data into a compact irradiance distribution function we have been able to calculate the annual and seasonal energy delivery (in $\mathrm{kWh} / \mathrm{m}^{2}$ ) of CIS modules in an efficient manner [2]. We have further shown that, because of the significant quantities of sunlight present at low irradiances, thin film cell widths could reasonably be increased $20 \%$ to $30 \%$ beyond the width that gives maximum module power under standard test conditions. Figure 5 shows the distribution of energy contributions from a CIS module (having a cell width of $0.6 \mathrm{~cm}$, and deployed in Newark, New Jersey) arising from irradiances assigned to $100 \mathrm{~W} / \mathrm{m}^{2}$ wide bins. The overall energy contribution for irradiances less than $600 \mathrm{~W} / \mathrm{m}^{2}$ (namely $\left.17.5 \mathrm{kWh} / \mathrm{m}^{2}\right)$ exceeds that generated by irradiances greater than $600 \mathrm{~W} / \mathrm{m}^{2}\left(15.8 \mathrm{kWh} / \mathrm{m}^{2}\right)$. This result serves as a reminder that attention must be paid to the low light performance of modules.

\section{Conclusions and Future Plans}

We have demonstrated that CIGS can be prepared over large areas with good compositional uniformity by methods compatible with manufacturing. Future emphasis on improved efficiency, control, and low cost processing (especially of the zinc oxide) should enable thin film CIGS modules to compete with crystalline $\mathrm{Si}$ and enter the commercial marketplace.

\section{Acknowledgements}

The collaboration of R. Hulstrom and D. Myers of The National Renewable Energy Laboratory in performing the solar radiation data analysis is gratefully acknowledged.

\section{References}

1. A.E. Delahoy, J.S. Britt, A.M. Gabor, and Z.J. Kiss "Large-Area, . Thin-Film CIS Deposition and Module Fabrication" Presented at the 13th NREL Photovoltaic Program Review Meeting, May 16-19, 1995, Lakewood, Colorado. AIP Conf. Proc. 353, (H.S. Ullal and C.E. Witt, Eds.) 1996, pp. 3-11.

2. A.E. Delahoy "Energy Delivery for Various PV Technologies Based on Realistic Irradiance and Temperature Distributions: Implications for Module and System Design" Presented at the 13th European Photovoltaic Solar Energy Conference, Nice, France, October 23-27, 1995.

3. J.S. Britt, A.M. Gabor, A.E. Delahoy, R. Noufi, and Z.J. Kiss "The Formation of $\mathrm{Cu}(\mathrm{In}, \mathrm{Ga})(\mathrm{S}, \mathrm{Se})_{2}$ Thin Films by Sequential Deposition Stages: Large-area Photovoltaic Module Fabrication" Presented at the 13th European Photovoltaic Solar Energy Conference, Nice, France, October 23-27, 1995. 


\section{EPV ZnO/CdS/CIGS}

junole: M1 $394-i-1$

Vor 3. $1995 \div 58$ PM

i.STM E 392.87 Giobal

HEE X-3iv System

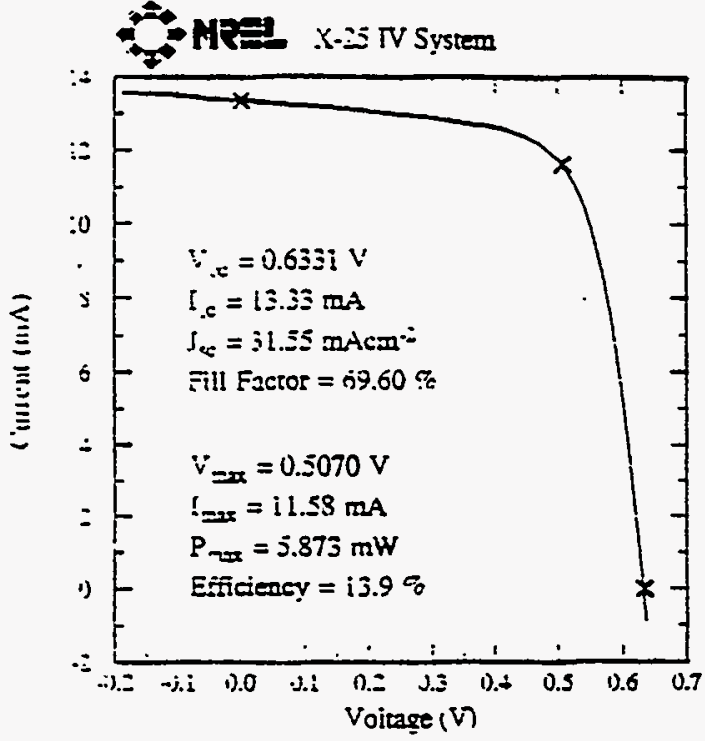

Figure 1. I-V carve for a $13.9 \%$ CIGS cell

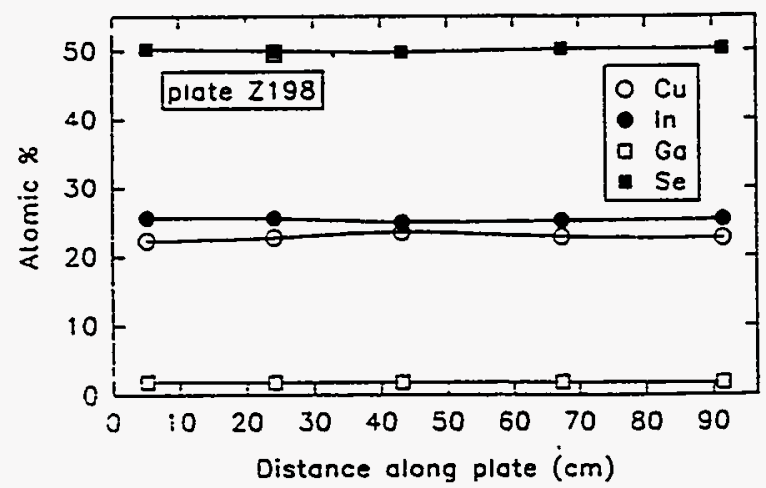

Figure 2. CIGS composition along the long axis of a $96.5 \mathrm{~cm} x+4.5 \mathrm{~cm}$ module

\begin{tabular}{|lllll|}
\hline 480 & 520 & 500 & 510 & 470 \\
485 & 510 & 465 & 510 & 470 \\
465 & 440 & 485 & 490 & 510 \\
420 & 440 & 425 & 430 & 480 \\
400 & 440 & 395 & 395 & 400 \\
\hline
\end{tabular}

Figure 3. Open-circuit voltage map across a $96.5 \mathrm{~cm} \times 44.5 \mathrm{~cm}$ CIGS plate

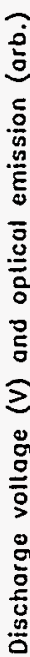

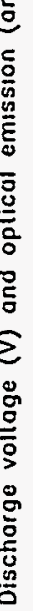

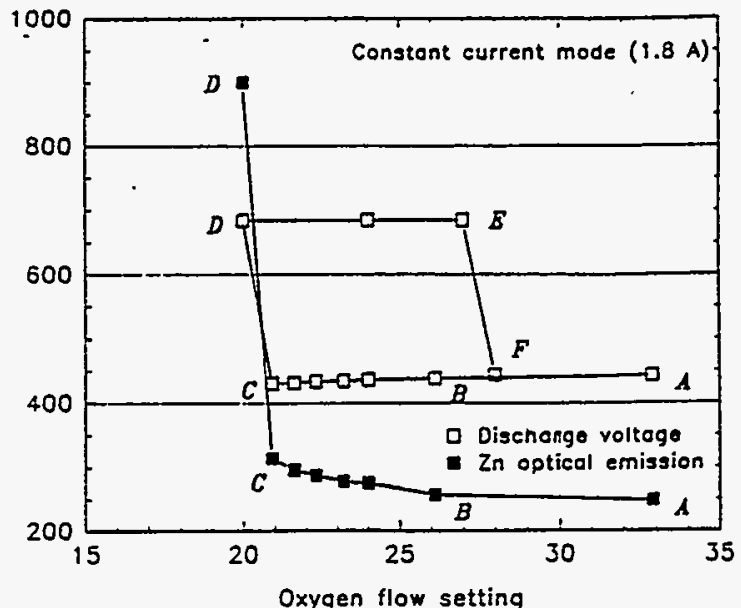

Figure 4. Discharge voltage and optical emission from excited $Z n$ as a function of oxygen flow during reactive sputtering of $\mathrm{Zn}$.

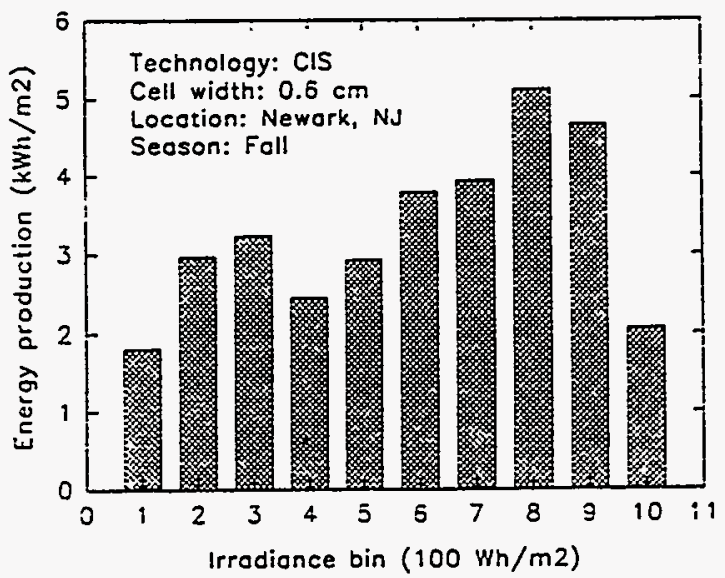

Figure 5. Contributions to the energy produced by a CIS module during the Fall in Newark, New Jersey arising from irradiances falling within $100 \mathrm{~W} / \mathrm{m}^{2}$ wide irradiance bins. 
Title:

Organization:

Contributors:
Polycrystalline CuInSe 2 \& CdTe PV Solar Cells

Florida Solar Energy Center, University of Central Florida, Cocoa, Florida 32922-5703

Neelkanth G. Dhere, Principal Investigator, Kevin Lynn, Mark Wollam, Lucy Orozco, and George Freeman

\section{Objectives}

The overall objective of the research project is to develop novel, low-cost scalable processes for the fabrication of stable and efficient copper-indium-gallium-diselenide, $\mathrm{CuIn}_{1-x} \mathrm{Ga}_{x} \mathrm{Se}_{2}$ and cadmiumtelluride, CdTe polycrystalline-thin-film solar cells for economic, large-scale manufacture.

\section{Technical Approach}

Two-selenizations process and magnetron sputtering from $\mathrm{Cu}-\mathrm{Ga}$ alloy targets have been developed for the preparation of well-adherent, large-compact-grain $\mathrm{CuIn}_{1-x} \mathrm{Ga}_{x} \mathrm{Se}_{2}$ thin films and solar cells, by non-toxic Se vapor selenization of $\mathrm{Cu}-\mathrm{In}$-Ga thin films. Magnetron-sputtered $\mathrm{Cd}$ and $\mathrm{Te}$ layers are being studied for CdTe-solar-cell fabrication. Liquid or liquid-like behavior of indium-gallium with a low-temperature eutectic point at $15.7^{\circ} \mathrm{C}$ and gallium with a melting point of $29.8^{\circ} \mathrm{C}$ would destroy the structural integrity of the precursor. These problems were resolved by depositing gallium using a $\mathrm{Cu}-\mathrm{Ga}$ alloy target. A single selenization step cannot benefit from the fluxing action of $\mathrm{Cu}_{2}$. ${ }_{x}$ Se having a melting point of $\sim 523^{\circ} \mathrm{C}$ for the growth of compact, large-grain films from initial $\mathrm{Cu}$ rich deposits, as has been done in the preparation of recent high-efficiency $\mathrm{CuIn}_{1-x} \mathrm{Ga}_{x} \mathrm{Se}_{2}$ solar cells. The novel two-selenizations process using Se vapor developed at FSEC utilizes initially a stack of $\mathrm{Cu}$-rich precursor for achieving recrystallization during the first selenization followed by a stack of $\mathrm{Cu}$-poor precursor for conversion of $\mathrm{Cu}_{2-x}$ Se phase at the surfaces and grain boundaries during the second selenization. In earlier studies, gallium content remained low even though $\mathrm{Cu}: \mathrm{Ga}$ target composition $\mathrm{Ga}$ was $\sim 22 \%$. Selenium evaporation rates and temperature-ramps during the selenizations have been optimized to enhance the gallium content.

\section{$\mathrm{CuIn}_{1-x} \mathrm{Ga}_{\mathbf{x}} \mathrm{Se}_{2}$ Polycrystalline-Thin-Film Solar Cells}

Mo back-contact thin-films and $\mathrm{Cu}$-In-Ga metallic precursor films were magnetron-sputter-deposited on soda-lime, float-glass substrates in a 18" diameter and 24" height bell jar. Selenizations were carried out in high vacuum by evaporating Se from a Knudsen-cell type molybdenum boat on samples mounted under a ceramic plate heater. Selenization temperatures were measured with a thermocouple attached to one of the substrates using a high-temperature glue. Initially selenium evaporation rates in the range $25-300^{\circ} \mathrm{C}$ and selenization temperatures in the range $450-565^{\circ} \mathrm{C}$ were utilized. Reproducible results were obtained when the selenium vapor incidence rate were maintained in the range $35-50 \AA \mathrm{sec}^{-1}$ and the selenization temperature was maintained in the range $550-565^{\circ} \mathrm{C}$. A two-selenizations process using selenium vapor has been developed to prepare $\mathrm{CuIn}_{1}$. ${ }_{x} \mathrm{Ga}_{x} \mathrm{Se}_{2}$ thin films. It utilizes a Cu-rich first precursor to take advantage of the fluxing action of $\mathrm{Cu}_{2}$. ${ }_{x} \mathrm{Se}$ for the growth of compact, large-grain films. A process sequence developed at FSEC solved the 
problems of incorporation of gallium, low-melting point precursors, and deleterious interaction between indium and gallium by using a single $\mathrm{Cu}-\mathrm{Ga}(22$ at.\%) alloy target and by capping of the indium layer with a $\mathrm{Cu}-\mathrm{Ga}$ layer [1]. $\mathrm{Cu} /(\mathrm{In}+\mathrm{Ga})$ ratio in $\mathrm{CuIn}_{1-x} \mathrm{Ga}_{\mathrm{x}} \mathrm{Se}_{2}$ thin films was varied systematically over a wide range of $0.60-0.92$. The thicknesses of individual layers given below correspond to those necessary to obtain optimum ratios of $\mathrm{Cu} /(\mathrm{In}+\mathrm{Ga})$ tending to 0.88-0.92. The recent two-selenizations process sequence for obtaining a $2.5 \mu \mathrm{m}$ thick $\mathrm{CuIn}_{1-x} \mathrm{Ga}_{\mathrm{x}} \mathrm{Se}_{2}$ layer is shown in figure 1. In an earlier series of experiments, the proportion of indium in the first stack of precursor was lower i.e $25 \%$ of the total indium thickness. More recently the indium proportion in the first precursor has been increased to $55 \%$ of the total indium thickness. In this process, arrived at empirically, firstly a $2210 \AA \mathrm{Cu}-\mathrm{Ga}$ layer i.e. $60 \%$ of the total $\mathrm{Cu}-\mathrm{Ga}$ thickness of $3682 \AA$ was sputter-deposited on molybdenum followed by depositions of a $2158 \AA$ of indium i.e. $55 \%$ of the total In thickness of $3924 \AA$ (in the earlier experiments $981 \AA$ of In i.e. $20 \%$ of the total In thickness), and an additional $736 \AA \mathrm{Cu}-\mathrm{Ga}$ layer i.e $20 \%$ of the total $\mathrm{Cu}-\mathrm{Ga}$ thickness. The thin $\mathrm{Cu}-\mathrm{Ga}$ overlayer was intended to avoid globule formation of In and In-Ga. In the earlier experiments, Cu-In-Ga films were homogenized by in situ heat treatment at $80-90^{\circ} \mathrm{C}$ for $10 \mathrm{~min}$. This has been discontinued in the more recent experiments so as to simplify the process. The $\mathrm{Cu} /(\mathrm{In}+\mathrm{Ga})$ ratios in the first precursor were -2.25 and $\sim 1.5$ in the earlier and recent experiments respectively. The indium excess in the second precursor compensated for the copper excesses in the first precursor. Reduction of the extent of copper excess in the first precursor resulted in films free of pits. For the various copper excesses in the first precursor, the gallium content $\mathrm{x}$ in the formula $\mathrm{CuIn}_{1 \cdot \mathrm{x}} \mathrm{Ga}_{\mathrm{x}} \mathrm{Se}_{2}$, as measured by electron probe microanalysis (EPMA) and X-ray photoelectron spectroscopy (XPS), was low (<0.06). Rutherford back-scattering analysis showed that magnetron-sputtered $\mathrm{Cu}-\mathrm{Ga}$ layers maintained the composition of the $\mathrm{Cu}-\mathrm{Ga}$ sputtering target. Maintaining consistently high selenium evaporation rates of 35-50 $\AA \mathrm{s}^{-1}$, including during the cool-down to $300^{\circ} \mathrm{C}$, improved the gallium content to $\mathrm{x} \approx 0.1$. Multiple selenizations or maintaining the samples at $\sim 475^{\circ} \mathrm{C}$ for $15 \mathrm{~min}$ during the selenizations prior to reaching the maximum selenization temperature of $\sim 565^{\circ} \mathrm{C}$ reduced the gallium content by $4 \%$. EPMA measurements of these films showed a lower gallium content when a lower e-beam energy was utilized. In EPMA, a shallower region is analyzed at lower e-beam energies. The lower gallium content near the surface region may have resulted from the tendency of gallium to diffuse towards the Mo back contact possibly because of the residual stress' in Mo films. The principal factor in controlling the gallium content was found to be the rate of temperature rise during the selenizations. Increasing the rate of temperature rise to $50-60^{\circ} \mathrm{C} \mathrm{min}^{-1}$ during the selenizations improved the overall gallium content, $x$ to 0.17-0.2. The lower gallium content in selenized CuIn $\mathrm{n}_{1-x} \mathrm{Ga}_{x} \mathrm{Se}_{2}$ may be attributed to the loss of gallium possibly in the form of $\mathrm{Ga}_{2} \mathrm{Se}$. The higher rate of temperature rise may be reducing the gallium loss possibly because the formation of the volatile gallium compound may be favored at lower temperatures. The best cell prepared using films with $\mathrm{x} \approx 0.17$ gave an open-circuit voltage, $\mathrm{V}_{o c}$ of $451.8 \mathrm{mV}$, a short-circuit current density, $\mathrm{J}_{\mathrm{sc}}$ of $34.5 \mathrm{~mA}$, a fill factor of $57.87 \%$, and a total-area efficiency of $9.02 \%$ (Figure 2). Increasing the gallium content from $\mathrm{x}<0.6$ to $\mathrm{x} \approx 0.17$ increased the open-circuit voltage from $\sim 380 \mathrm{mV}$ to $\sim 450 \mathrm{mV}$. However, the shift in longer-wavelength spectral-response edge towards higher bandgaps was not significant (Figure 3).

\section{CdTe Polycrystalline-Thin-Film Solar Cells}

Experiments on the chemical-bath deposition (CBD) of CdS layers, magnetron-sputter deposition 
of $\mathrm{Cd}$ and $\mathrm{Te}$ layers, and their heat treatment in various ambients using different temperature ramps and maximum temperatures for fabrication of CdTe solar cells have been continued.

\section{New PY Materials Laboratory}

The Photovoltaic Materials Laboratory was moved to the new FSEC site. Various equipments and systems, and the support facilities, such as plumbing for chilled water and compressed air, electrical connections, distilled and deionized water, and chemical work stations have been installed in a semiclean-room area with double-door entry. The move has brought all the equipments and systems which were scattered over different locations in one contiguous space. Moreover, the Hydrogen Laboratory which is another major FSEC laboratory where some of the analyses such as scanning electron microscopy, optical spectroscopy, etc are carried out is now located across the PV Materials Laboratory. This has provided a considerably improved set-up for the research on thin-film solar cells and materials.

\section{References}

1. N. G. Dhere, "CuIn ${ }_{1-x} \mathrm{Ga}_{\mathrm{x}} \mathrm{Se}_{2}$ and CdTe PV Solar Cells", AIP Conference Proceedings 353 of the 13th NREL Photovoltaic Program Review, Lakewood, CO, 1995, pp. 428-435.

2. N. G. Dhere and K. W. Lynn "CuIn ${ }_{1-x} \mathrm{Ga}_{x} \mathrm{Se}_{2}$ Thin Film Solar Cells by Two-Selenizations Process Using Se Vapor", To be published in Solar Energy materials and Solar Cells (accepted).

\begin{tabular}{|c|c|c|}
\hline & \multicolumn{2}{|c|}{ Total thickness } \\
\hline & CuGa & $3682 \AA$ \\
\hline & In & $3924 \AA$ \\
\hline \multirow{4}{*}{$\begin{array}{l}20 \% \\
45 \%\end{array}$} & Selenization II & $450-560^{\circ} \mathrm{C}$ \\
\hline & CuGa & $736 \AA$ \\
\hline & In & $1766 \dot{A}$ \\
\hline & Selenization I & $450-560^{\circ} \mathrm{C}$ \\
\hline \multirow{5}{*}{$\begin{array}{l}20 \% \\
55 \% \\
60 \%\end{array}$} & CuGa & $736 \AA$ \\
\hline & In & $2158 \AA$ \\
\hline & CuGa & $2210 \dot{A}$ \\
\hline & \multicolumn{2}{|c|}{ Molybdenum } \\
\hline & \multicolumn{2}{|c|}{ Glass } \\
\hline
\end{tabular}

Fig. 1. Process sequence for preparation of $2.5 \mu \mathrm{m} \mathrm{CuIn} n_{1-x} \mathrm{Ga}_{x} \mathrm{Se}_{2}$ layer. 


\section{Florida Solar Energy Center CdS/CIS}

Sample: ST1.1\#1

May 30, 1995 10:08 AM

ASTM E 892-87 Global
Temperature $=25.0^{\circ} \mathrm{C}$

Area $=0.3824 \mathrm{~cm}^{2}$

Irradiance: $1000.0 \mathrm{Wm}^{-2}$

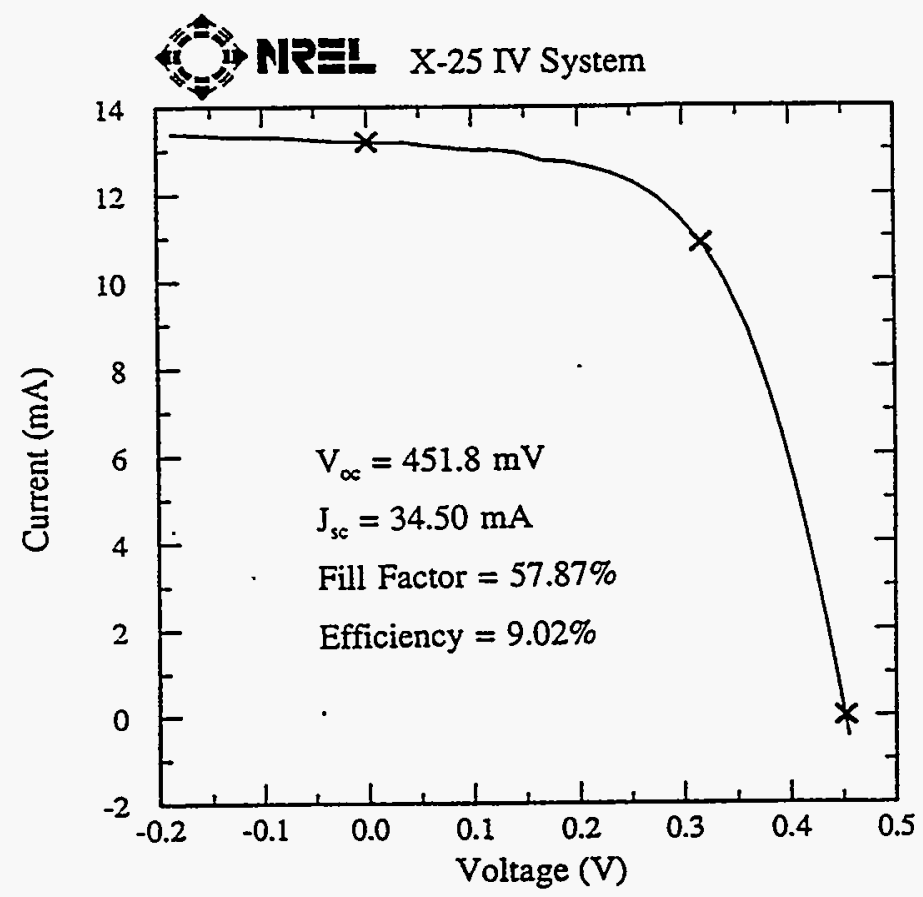

Fig. 2. IxV characteristic of a $\mathrm{CuIn}_{1-x} \mathrm{Ga}_{x} \mathrm{Se}_{2}$ thin-film solar cell

Florida Solar Energy Center CdS/CIS

Sample: ST1.1\#1

May 22, 1995 8:52 AM

Temperature $=25.0^{\circ} \mathrm{C}$
Device Area $=0.3824 \mathrm{~cm}^{2}$

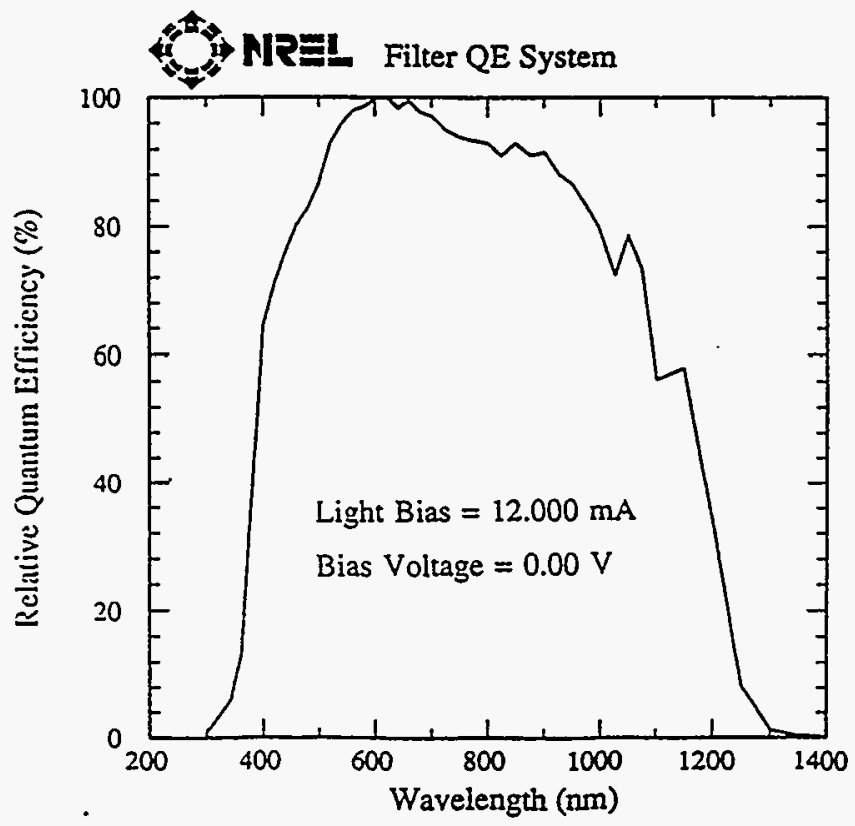

Fig. 3. Relative quantum efficiency curve of a $\mathrm{CuIn}_{1-x} \mathrm{Ga}_{x} \mathrm{Se}_{2}$ thin-film solar cell 
Title: Development of High Efficiency CdTe Solar Cells

\section{Organization: School of Electrical and Computer Engineering, Georgia Institute of Technology, Atlanta, Georgia.}

Contributors: A. Rohatgi, director; H.C. Chou, graduate research assistant, and S. Kamra, postdoctoral fellow

\section{Objectives}

The objective of this program is to improve the basic fundamental understanding of efficiency limiting mechanisms in polycrystalline CdTe cells, fabricate high efficiency CdTe cells, and provide guidelines for achieving higher efficiency CdTe cells.

\section{Film Growth and Cell Fabrication}

CdS films (1000-2000 $\AA$ ) were deposited on textured $\mathrm{SnO}_{2}$ /glass substrates, using solution growth starting from $\mathrm{CdCl}_{2}$, thiourea, $\mathrm{NH}_{4} \mathrm{Cl}$, and $\mathrm{NH}_{4} \mathrm{OH}$. The $\mathrm{CdS} / \mathrm{SnO}_{2} /$ glass substrates were treated with a $\mathrm{CdCl}_{2}: \mathrm{CH}_{3} \mathrm{OH}$ solution and annealed in the furnace at $450^{\circ} \mathrm{C}$ in $\mathrm{N}_{2}$ ambient for $50 \mathrm{~min}$. Polycrystalline CdTe films with a thickness of $2.6 \mu \mathrm{m}$ were grown by MOCVD on the annealed $\mathrm{CdS} / \mathrm{SnO}_{2} /$ glass substrates at a temperature of $400^{\circ} \mathrm{C}$ in $\mathrm{N}_{2}$ ambient for $70 \mathrm{~min}$. After the CdTe deposition, $\mathrm{CdTe} / \mathrm{CdS}$ structures were treated in a $\mathrm{CdCl}_{2}: \mathrm{CH}_{3} \mathrm{OH}$ solution followed by an air anneal at $400^{\circ} \mathrm{C}$ for $30 \mathrm{~min}$. Ohmic back contacts were formed on the CdTe surface by sequential evaporation of $100 \AA \mathrm{Cu}$ and $400 \AA$ $\mathrm{Au}$. After the metallization, a contact anneal was performed at $150^{\circ} \mathrm{C}$ in $\mathrm{Ar}$ ambient for $90 \mathrm{~min}$. Cell fabrication was completed by a $0.1 \% \mathrm{Br}_{2}: \mathrm{CH}_{3} \mathrm{OH}$ etch, followed by a DI water rinse and $\mathrm{N}_{2}$ blow-dry. The final $\mathrm{CdTe}$ solar cell structure consists of $\mathrm{Au} / \mathrm{Cu} / \mathrm{CdTe} / \mathrm{CdS} /$ glass structures.

\section{Material and Device Characterization}

The morphology and grain structure of the polycrystalline CdTe films were studied by SEM. Measurements techniques, such as SIMS, C-V, and I-V were performed to probe the Cu migration and its influence on doping density, shunts and cell performance. PL and XRD measurements were performed to assess lattice mismatch-induced changes in lattice constant and defects.

\section{Results and Discussion}

In the past we have reported that the $\mathrm{CdS}$ and $\mathrm{SnO}_{2}$ thickness have a significant impact on the $\mathrm{CdTe}$ cell performance and a Te-rich MOCVD growth ambient allows more interdiffusion at the CdS-CdTe interface, and increases carrier lifetimes in the CdTe. However, in order to further enhance our cell performance we need to understand and optimize some other efficiency limiting mechanisms, including the role of chemical and heat treatments, the effect of cu migration in CdTe and investigation of new process techniques.

\section{Effects of Chemical and Heat Treatments on CdTe Microstructure and CdTe/CdS Interface} Properties

The $\mathrm{CdCl}_{2}$ concentration and annealing conditions were varied to investigate the effect of chemical and heat treatments on CdTe films and cells. It was found that $\mathrm{CdCl}_{2}$ treatment of $\mathrm{CdTe}$ films tends to densify the film, changes the surface morphology, makes the grains flat and less faceted, and serves as a flux to recrystallize the CdTe structure (Fig.1). This reduces the effective grain boundary surface area 
per unit volume $\left(\mathrm{S}_{\mathrm{vl}}\right)$ or decreases the grain boundary conduits for impurity or metal migration, which results in enhanced cell performance. Light I-V and PL measurements (Tab.1 and Fig.2) revealed optimum $\mathrm{CdCl}_{2}$ concentration in the range of $50 \%$ to $75 \%$ for our $\mathrm{MOCVD}$ cells, because higher $\mathrm{CdCl}_{2}$ concentration gives rise to higher $\mathrm{Cl}$-related defect density and degrades the cell performance. Therefore, even though $\mathrm{CdCl}_{2}$ treatment is essential for high efficiency CdTe cells, it could place an upper limit on the practically achievable cell efficiency. Rapid thermal processing was performed on $\mathrm{CdS} / \mathrm{CdTe}$ structure to achieve grain regrowth in the CdTe films with lower $\mathrm{CdCl}_{2}$ concentrations, or even without any $\mathrm{CdCl}_{2}$ treatment. It was found that $\mathrm{RTP}$ conditions produced significant changes in the

CdTe cell parameters (Tab.2). A cell with $10.7 \%$ efficiency was achieved using the $700^{\circ} \mathrm{C}, 5$ second RTP without any $\mathrm{CdCl}_{2}$ treatment. SEM (Fig.1c) measurement showed that the microstructure in the $\mathrm{CdTe}$ after the $\mathrm{RTP}$ was similar to the $\mathrm{CdTe}$ cell with conventional $\mathrm{CdCl}_{2}$ treatment, which involves $400{ }^{\circ} \mathrm{C} / 30 \mathrm{~min}$ furnace anneal. However, by comparing the $\{220\}$ peak location in the XRD spectrum (Fig.3), it was found that reduced interdiffusion at the CdTe/CdS interface in the RTP cells was probably responsible for the lower $\mathrm{RTP}$ cell efficiency compared with the $\mathrm{CdCl}_{2}$ treated and furnace annealed cells.

\section{Multiple Effects of Cu on Contact Properties and Cell Performance}

The objective of this study was to achieve a better and reliable contact to CdTe solar cells by improving the fundamental understanding of the effects of $\mathrm{Cu}$ on cell efficiency. CdTe solar cells were fabricated by depositing $\mathrm{Au} / \mathrm{Cu}$ contacts with varying thicknesses and deposition conditions on the polycrystalline $\mathrm{CdTe} / \mathrm{CdS} / \mathrm{SnO}_{2} /$ glass structures. It was found that $\mathrm{Cu}$ plays a dual role on the cell performance; on one hand it helps the formation of better ohmic contact and increases the acceptor doping concentration, but on the other hand, excess $\mathrm{Cu}$ diffuses all the way to the $\mathrm{CdTe} / \mathrm{CdS}$ interface to form recombination centers and shunt paths to degrade the cell performance. Both SIMS and C-V measurements confirmed the incorporation of $\mathrm{Cu}$ into the bulk of the CdTe films (Fig.4 and 5). Cd out-diffusion toward the surface of the $\mathrm{CdTe}$ was also observed during the $\mathrm{Au} / \mathrm{Cu}$ deposition. It was found that the thickness of $\mathrm{Cu}$ plays a critical role in dictating the CdTe solar cell performance because both series and shunt resistances decrease with the increase in $\mathrm{Cu}$ thickness. Carrier transport analysis showed that the depletion region recombination dominates the current transport in the CdTe solar cells with $\mathrm{Au} / \mathrm{Cu}$ contact, regardless of the amount of $\mathrm{Cu}$ incorporation in the bulk and near the CdTe/CdS interface. SIMS measurement showed that higher $\mathrm{Au} / \mathrm{Cu}$ deposition rates resulted in a greater pile-up of $\mathrm{Cd}$ near the CdTe surface (Fig.6), generating more Cd vacant sites below the surface and causing a reduction in the cell performance.

\section{Development of Large Grain and Single Crystal CdTe Thin Film Solar Cells}

This study involved the investigation of the effect of crystallinity and grain boundaries on $\mathrm{Cu}$ incorporation in the CdTe films, including the fabrication of CdTe solar cells with larger CdTe grain size. Three different $\mathrm{CdTe}$ structures, $\mathrm{CdTe} / \mathrm{CdS} / \mathrm{SnO}_{2}$ /glass, $\mathrm{CdTe} / \mathrm{CdS} / \mathrm{GaAs}$, and $\mathrm{CdTe} / \mathrm{GaAs}$, were prepared to achieve CdTe films with different degrees of crystallinity and grain size. The $\mathrm{CdTe} / \mathrm{CdS} / \mathrm{GaAs}$ structure gave large $\mathrm{CdTe}$ grains $(\sim 10 \mu \mathrm{m})$ which was used to fabricate a large grain polycrystalline $\mathrm{CdTe}$ thin film solar cell structure for the first time in this study by a combination of selective etching of the GaAs substrate and the film transfer onto a glass substrate (Fig.7). X-ray diffraction and PL measurements on the CdTe films, before and after the transfer, were used to assess lattice mismatch-induced changes in the CdTe lattice constant near the CdTe/GaAs interface (Fig. 8 and 9). SIMS measurement showed that poor crystallinity and smaller grain size $(\sim 2 \mu \mathrm{m})$ of the CdTe film in the conventional polycrystalline $\mathrm{CdTe} / \mathrm{CdS} / \mathrm{SnO}_{2}$ /glass structure promotes $\mathrm{Cu}$ diffusion from the contact 
and decreases cell performance. In the large grain $(\sim 10 \mu \mathrm{m}) \mathrm{CdTe}$ film, Cu concentration was about a factor of 2 lower than the small grain CdTe film, while in the single crystal CdTe film, Cu was virtually undetectable (Fig.10). Therefore, we conclude that grain boundaries are the main conduits for $\mathrm{Cu}$ migration, and larger CdTe grain size or an alternate method of contact formation needs to be developed to mitigate the adverse effect of $\mathrm{Cu}$ and improve the $\mathrm{CdTe}$ cell performance.

\section{Conclusions}

Through a combination of fundamental understanding of the loss mechanisms associated with different layers and interfaces in the cell structure and process optimization, we were able to increase the $\mathrm{CdTe}$ cell efficiency systematically. A $12 \%$ efficient CdTe solar cell was achieved by a combination of optimized $\mathrm{Au} / \mathrm{Cu}$ contact thickness and deposition rate, Te-rich MÖCVD growth of CdTe, and optimized $\mathrm{CdCl}_{2}$ treatment and furnace anneal. Finally, based on the fundamental understanding of defects and loss mechanisms, guidelines were provided for raising the CdTe cell efficiency from $12 \%$ to $\sim 18 \%$ by a combination of thin $\mathrm{CdS}$, large grain $\mathrm{CdTe}$, and reduced $\mathrm{Cu}$ migration and bulk defects.

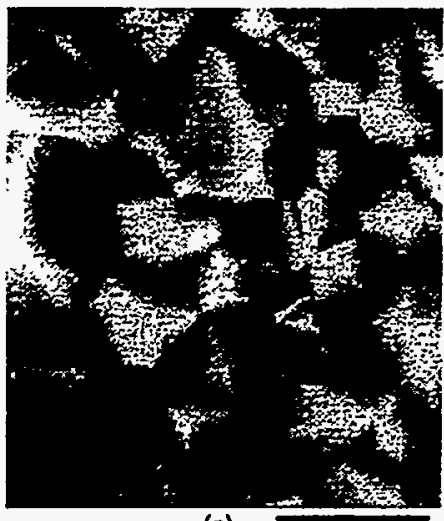

(a)

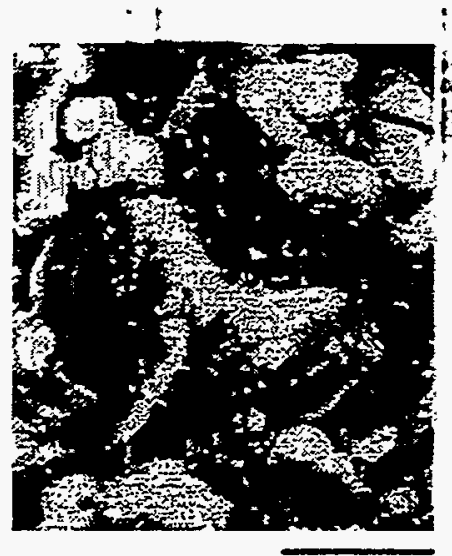

(b)

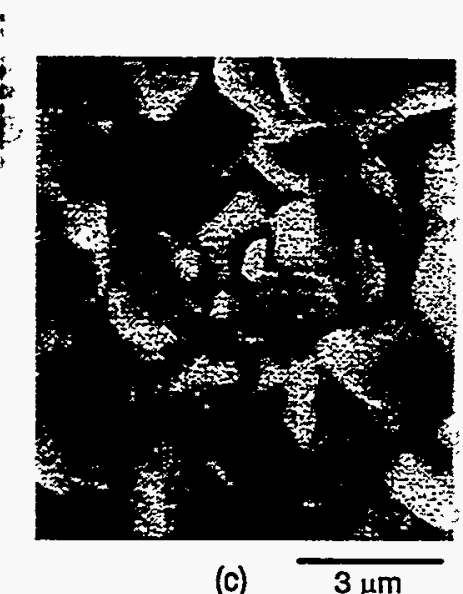

(c) $3 \mu \mathrm{m}$

Fig.l. SEM Microstructure of CdTe (a) as-grown film, (b) after $\mathrm{CdCl}_{2}$ treatment, (c) after RTP $700^{\circ} \mathrm{C}$, $5 \mathrm{sec}$.

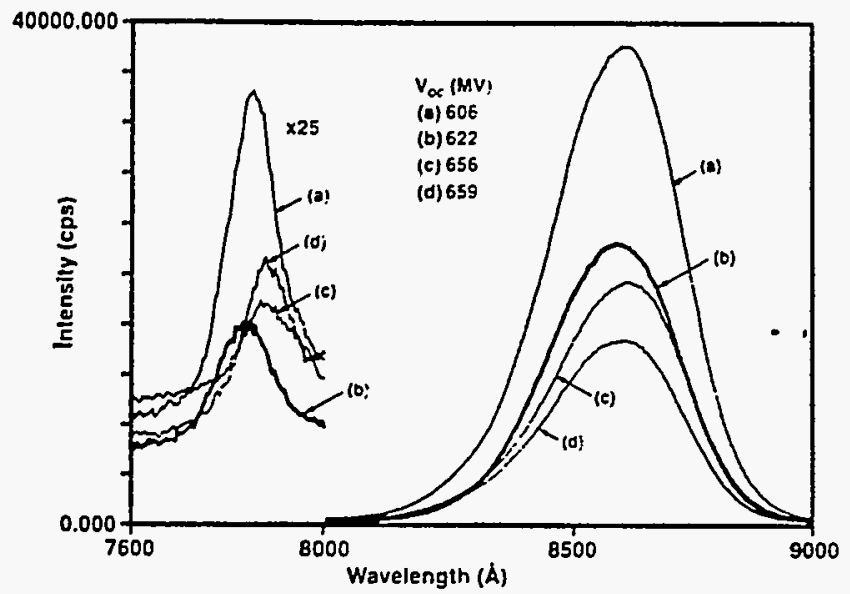

Fig.2. PL spectra for $\mathrm{CdTe}$ treated with various $\mathrm{CdCl}_{2}$ concentration, (a) 1.0 (b) 0.5 , (c) 0.25 , (d) 0.75 . The corresponding $V_{o c}$ also shown
Table 1 Cell data with varying $\mathrm{CdCl}_{2}$ concentration.

\begin{tabular}{ccccc}
\hline $\mathrm{dCl}_{2}$ Concentration & $\mathrm{V}_{\mathrm{oc}}$ & $\mathrm{J}_{\mathrm{sc}}$ & $\mathrm{FF}$ & Eff \\
\hline 0 & 385 & 10.5 & 0.32 & 1.30 \\
0.25 & 694 & 24.4 & 0.528 & 8.89 \\
0.50 & 708 & 25.5 & 0.599 & 10.82 \\
0.75 & 702 & 25.8 & 0.581 & 10.56 \\
1.0 & 665 & 22.9 & 0.483 & 7.38 \\
\hline
\end{tabular}

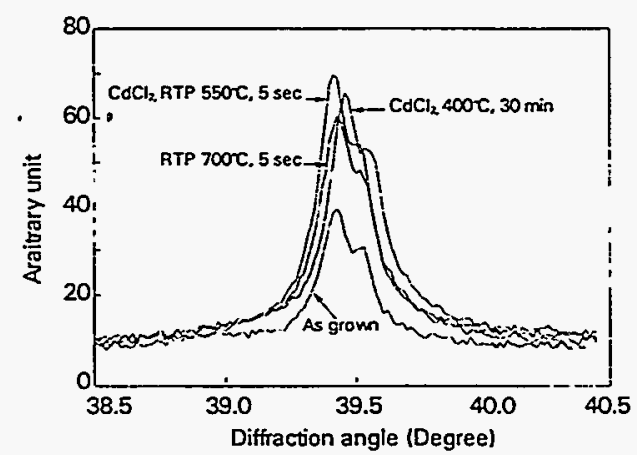

Fig.3. XRD scan on the CdTe/CdS films with RTP or furnace anneal 
Table 2 Cell results for different post-growth treatment conditions.

\begin{tabular}{|c|c|c|c|c|c|}
\hline \multicolumn{2}{|c|}{ Annealing condition } & $\mathrm{V}_{\mathrm{oc}}$ & $J_{s c}$ & FF & Eff \\
\hline Furnace Air & $\mathrm{CdCl}_{2} 400^{\circ} \mathrm{C}$ & 781 & 23.26 & 0.657 & 11.9 \\
\hline $\begin{array}{c}\text { RTP } \\
\text { Forming gas }\end{array}$ & $\mathrm{CdCl}_{2} 600^{\circ} \mathrm{C}$ & 547 & 7.09 & 0.478 & 1.94 \\
\hline \multirow{3}{*}{$\begin{array}{c}\mathrm{RTP} \\
\mathrm{O}_{2}+\mathrm{N}_{2}\end{array}$} & $\mathrm{CdCl}_{2} 600^{\circ} \mathrm{C}$ & 690 & 20.76 & 0.539 & 7.73 \\
\hline & No $\mathrm{CdCl}_{2} 600^{\circ} \mathrm{C}$ & 704 & 18.51 & 0.535 & 6.97 \\
\hline & No $\mathrm{CdCl}_{2} 700^{\circ} \mathrm{C}$ & 746 & 22.24 & 0.644 & 10.7 \\
\hline
\end{tabular}

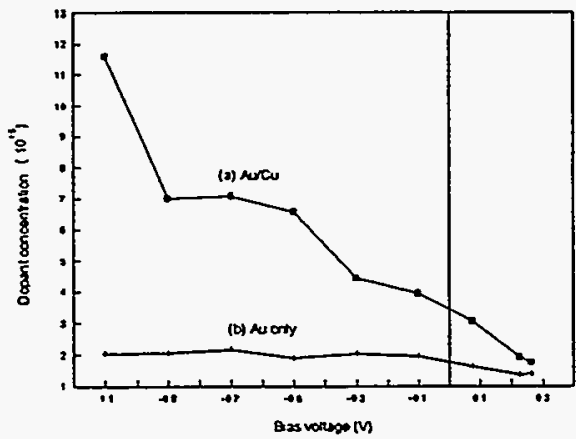

Fig. 5 The acceptor concentration in the CdTe layer by $\mathrm{C}-\mathrm{V}$ measurement with different metal contact on CdTe solar cells.
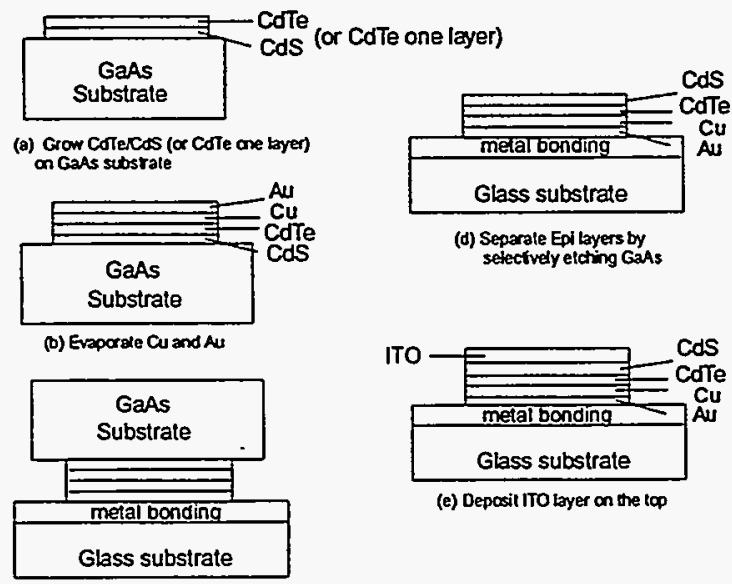

(c) Invert the sample, bond on
metallass substrate

Fig. 7 Large grain thin-film CdTe/CdS solar cell fabrication procedures with a metal bonding technique.

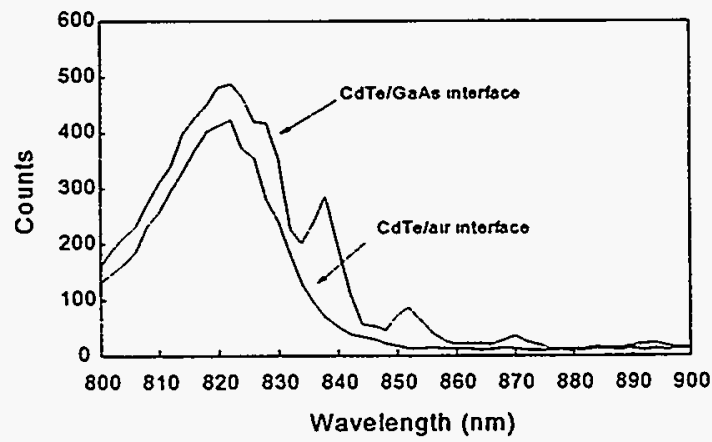

Fig. 9 PL spectrum of CdTe near the CdTe/air interface before lift-off and near the CoTe/GaAs interface after lifl-off.

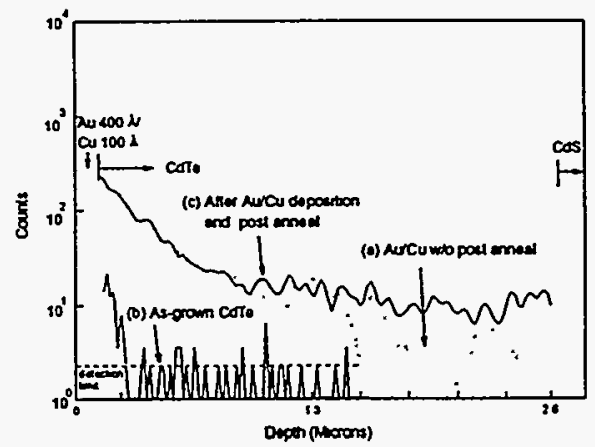

Fig. 4 SIMS record of $\mathrm{Cu}^{+}$ions through the CdTe layer for a $\mathrm{Au} / \mathrm{Cu} / \mathrm{CdT} / \mathrm{CdS}$ cell structure.

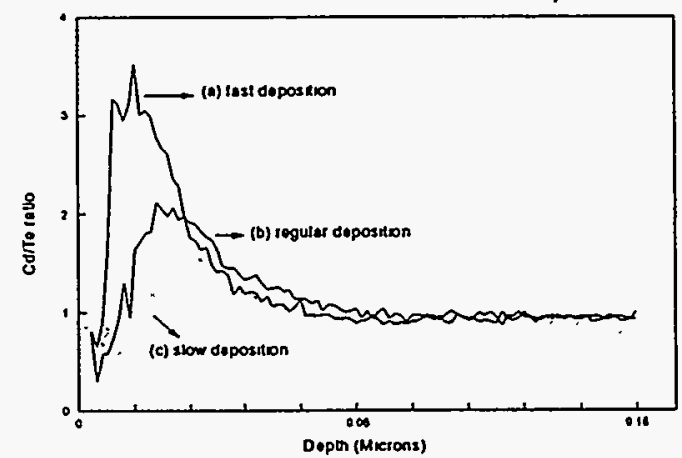

Fig. $6 \mathrm{The} \mathrm{Cd} / \mathrm{Te}$ ratio of SIMS signals near the Cu/CdTe interface for the cells with different $\mathrm{Au} / \mathrm{Cu}$ deposition rate.

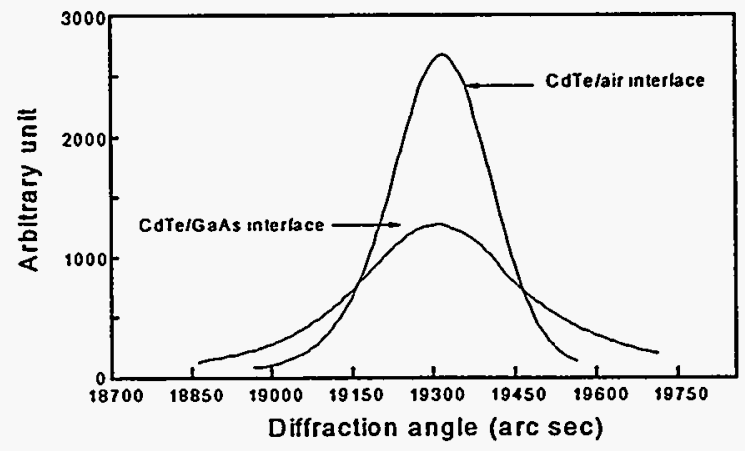

Fig. 8 Rocking curves for the $400 \mathrm{CdTe}$ near the CdTe/air interface and near the CdTe/GaAs interface.

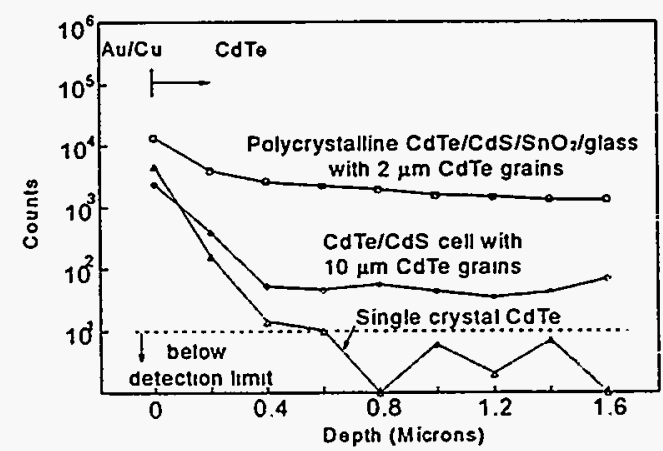

Fig. 10 Comparison of $\mathrm{Cu}$ SIMS profiles in the CdTe layers with different degree of crystallinity. 
Title:

Organization:

Contributors:
Optimization of Transparent and Reflecting Electrodes for Amorphous Silicon Solar Cells

Department of Chemistry, Harvard University, Cambridge, MA.

R. G. Gordon, Principal Investigator;

H. Sato, X. Liu, H. Liang, J. Thornton

\section{Summary of Current Project Objectives}

Transparent conducting materials are essential components of many kinds of solar cells, in which they serve as front-surface electrodes. In tandem cells, back surface electrodes also need to be transparent. Finally, most designs for highly reflective back contacts also call for a transparent conducting layer. The compositions of these transparent conducting layers are usually based on oxides of tin, indium and/or zinc, and are hence referred to as transparent conducting oxides (TCO). In addition to having low electrical resistance and low optical absorption, the structure of a front-surface TCO must minimize reflection losses. The TCO must also resist degradation during cell fabrication and use. Finally, the method for making the TCO must be inexpensive and safe.

Our general objectives are to improve the performance of TCO materials and the methods for their production. We aim to reduce their electrical resistance, optical absorption and reflection losses, and to lower the deposition temperature to avoid thermal degradation of other cell components. For the production method, the prime consideration is to deposit the TCO layers at a high rate with relatively simple apparatus. The method chosen is chemical vapor deposition at atmospheric pressure (APCVD), since it has been demonstrated in the glass-coating industry to be the most cost-effective method for making large areas of TCO coatings.

In view of discussions at the Guidance Team Meeting in May, 1995, and with team members and NREL staff, we have revised the Task Statement for the current phase of the contract to read as follows:

Task 1. Optimize the ratio of the electrical conductivity to the visible absorption for fluorine-doped zinc oxide ( $\mathrm{ZnO}: \mathrm{F})$ films made by a more robust APCVD process from less hazardous precursors. The goal will be to deposit $\mathrm{ZnO}: \mathrm{F}$ films over an area of $10 \mathrm{~cm}$ by $10 \mathrm{~cm}$ with a sheet resistance of less than 12 ohms per square, thickness uniformity $\pm 5 \%$, visible haze of about 10 to $15 \%$, and optical absorption of less than $4 \%$ at $550 \mathrm{~nm}$.

Task 2. Deposit electrically conductive, transparent tin oxide and/or titanium oxide films on textured silver back electrodes, and then deposit amorphous silicon solar cells on these electrodes. Vary the thickness of the tin oxide and titanium oxide in order to optimize the efficiency of the solar cells.

Task 3. Prepare transparent conductors having the structure glass/textured $\mathrm{ZnO}: \mathrm{F} / \mathrm{smooth}$ $\mathrm{TiO}_{2}: \mathrm{Nb}$. Use these structures as substrates for the deposition of amorphous silicon cells having microcrystalline p-layers.

About one-third of the current project resources are allocated to each of these three objectives. More complete information about this project available in its Annual Reports and Quarterly Reports [1]. 
A superstrate amorphous silicon solar cell incorporating these new materials could have the following structure:

\begin{tabular}{c}
\hline glass \\
\hline $\mathrm{ZnQ}: \mathrm{F}$ \\
\hline $\mathrm{TiQ}: \mathrm{Nb}$ \\
\hline$\mu \mathrm{c}-\mathrm{Si}: \mathrm{B}$ \\
\hline $\mathrm{a}-\mathrm{Si}$ \\
\hline$\mu \mathrm{c}-\mathrm{Si}: \mathrm{P}$ \\
\hline $\mathrm{ZnO}$ \\
\hline $\mathrm{Alor} \mathrm{Ag}$ \\
\hline
\end{tabular}

A substrate amorphous silicon solar cell incorporating these new materials could have the following structure:

\begin{tabular}{c}
\hline $\mathrm{ZnO}$ \\
\hline$\mu \mathrm{c}-\mathrm{Si}: \mathrm{B}$ \\
\hline $\mathrm{a}-\mathrm{Si}$ \\
\hline$\mu \mathrm{c}-\mathrm{Si}: \mathrm{P}$ \\
\hline $\mathrm{TiO}: \mathrm{Nb}$ \\
\hline $\mathrm{SnO}_{2}: \mathrm{F}$ or $\mathrm{ZnO}: \mathrm{F}$ \\
\hline $\mathrm{Al}$ or $\mathrm{Ag}$ \\
\hline Stainiess Steel
\end{tabular}

Task 1. Develop improved methods for depositing and using transparent conductors of fluorine-doped zinc oxide in amorphous silicon solar cells

Fluorine-doped zinc oxide has the lowest absorption loss of any known transparent conductor. $\mathrm{ZnO}: \mathrm{F}$ was discovered under our previous NREL contract, and its superior properties documented. Pilot production of $\mathrm{ZnO}: \mathrm{F}$ at Solarex, using diethylzinc and ethanol, had problems reproducing the quality (conductivity and haze level) of $\mathrm{ZnO}: \mathrm{F}$ produced in their Watkins-Johnson belt furnace. Although some samples had outstanding properties, lack of consistent quality has prevented commercial production of this material.

This deposition reaction is easily disrupted by traces of oxygen, which is a common contaminant in the atmosphere inside a belt furnace, which ideally should contain pure nitrogen. Therefore, a more robust manufacturing process must be based on reactants which are less sensitive to oxygen than is diethylzinc.

One less reactive zinc source is zinc acetylacetonate. It does not react with oxygen at temperatures below about $450^{\circ} \mathrm{C}$. Thus we have studied its use as a source for the more reproducible CVD of $\mathrm{ZnO}: \mathrm{F}$. Commercially available zinc acetylacetonate is only partially volatile because it contains water of hydration. Thus we removed the water of hydration by recrystallization from methanol solution, followed by heating. The resulting anhydrous material may be evaporated from a bubbler containing its melt at about $130^{\circ} \mathrm{C}$. Oxygen was found to react with zinc acetylacetonate vapor at substrate temperatures of about 500 to $600{ }^{\circ} \mathrm{C}$ to deposit highly transparent films of pure zinc oxide.

Electrically conductive fluorine-doped zinc oxide films were obtained by CVD from vapor mixtures of zinc acetylacetonate, oxygen and hexafluoropropene. Some films had conductivities as high as 
$1000 \Omega^{-1} \mathrm{~cm}^{-1}$. Experiments varying the hexafluoropropene gas concentration showed that the conductivity decreased for concentrations below about one volume per cent. For concentrations above about five per cent, and for substrate temperatures above about $550^{\circ} \mathrm{C}$, some etching of the glass substrate occurred, and the CVD process was disrupted in those areas. Therefore we coated the glass surface with layers that should resist etching by fluorine-containing gases. Because aluminum oxide and titanium oxide are materials that resist etching by aqueous hydrogen fluoride, we tried precoating the substrate with films of alumina or titania. Such precoating of the glass should also prevent sodium from diffusing out of the glass into the zinc oxide coating. Aluminum oxide coatings were applied by spray pyrolysis from aluminum diisopropoxide ethylacetoacetate, and titanium dioxide coatings were made by CVD from titanium isopropoxide. Deposition of fluorine-doped zinc oxide from zinc acetylacetonate, oxygen and hexafluoropropene was carried out on alumina- or titania-coated glass at $550^{\circ} \mathrm{C}$. The titania-coated glass showed some etching. but no sign of etching was observed on the alumina-coated glass samples.

Task 2. Deposit electrically conductive, transparent tin oxide and/or titanium oxide films on textured back electrodes, and then deposit amorphous silicon solar cells on these electrodes. Vary the thickness of the tin oxide and/or titanium oxide in order to optimize the efficiency of the solar cells.

A diffusion barrier is needed between the amorphous silicon and the back metal (aluminum or silver) electrode, in order to enhance the reflectivity and also to prevent diffusion of metal into the silicon. The most commonly used back diffusion barrier is zinc oxide. However, optical measurements have shown that the silver-zinc oxide interface does not reflect as much light as theory, based on the bulk optical properties of these materials, predicted. The physical origin of this loss of light is not known. Also, some forms of cell degradation may be due to diffusion of silver through the zinc oxide. Thus it may be desirable to replace the zinc oxide with a material which is more resistant to diffusion or reaction, and at the same time is transparent and electrically conductive. Titanium oxide should be a much better diffusion barrier than is zinc oxide, because the structure of titanium oxide is a nearly close-packed arrangement of oxide ions that does not have the open space that is available for diffusion through zinc oxide.

Thus we deposited coatings of titanium oxide on silver back contacts. Textured silver-coated stainless steel was provided by Dr. Subhendu Guha at United Solar Systems. CVD of titanium dioxide was carried out with as little preheating of the silver as possible (about 2 minutes before deposition) in order to avoid oxidation of the silver. The deposition of undoped titanium dioxide was carried out using titanium isopropoxide vapor in nitrogen carrier gas at $500{ }^{\circ} \mathrm{C}$ in an atmospheric pressure CVD system. The samples were moved through the deposition region on a platform in order achieve a uniform thickness of 0.05 micron. The niobium-doped titanium dioxide samples used either 2.5 mole \% or 5 mole \% niobium ethoxide (mole percentages relative to the titanium isopropoxide) and 5 mole \% cyclohexenone, which increases the conductivity.

United Solar Systems deposited samples of their standard zinc oxide on the $\mathrm{TiO}_{2} /$ silver/stainless steel just described. The $\mathrm{ZnO}$ was grown during one deposition run using a large area $\mathrm{DC}$ magnetron sputtering machine to a thickness of about one micron. United Solar Systems next deposited their standard a-SiGe: $\mathrm{H}$ solar cells on the $\mathrm{ZnO} / \mathrm{TiO} /$ silver/stainless steel back contacts in consecutive runs using identical deposition parameters. The solar cells were made in the NIP configuration (i.e. N-layer grown first, light shines through the P-layer). The intrinsic material has a Tauc gap of about $1.45 \mathrm{eV}$; the I-layer thickness is about 0.25 micron. Each sample is then coated with many separate ITO front electrodes having areas of 0.05 or $0.25 \mathrm{~cm}^{2}$.

Table 1 gives some properties measured for these cells. 


\begin{tabular}{|l|lllll|}
\hline Sample Description & $\begin{array}{l}\mathrm{JSC} \\
\mathrm{ma} / \mathrm{cm}^{2}\end{array}$ & FF & $\begin{array}{l}\text { Voc } \\
\text { volts }\end{array}$ & $\begin{array}{l}\mathrm{Rs} \\
\Omega-\mathrm{cm}^{2}\end{array}$ & $\begin{array}{l}\mathrm{Jsc}>630 \mathrm{~nm} \\
\mathrm{ma} / \mathrm{cm}^{2}\end{array}$ \\
\hline no $\mathrm{TiO}_{2}$ & 20.5 & 0.53 & 0.746 & 10.5 & 8.82 \\
$\mathrm{TiO}_{2}$ undoped & 20.7 & 0.51 & 0.738 & 11.9 & 8.71 \\
$\mathrm{TiO}_{2}$ with 5\% niobium & 20.1 & 0.51 & 0.731 & 11.8 & 8.30 \\
\hline
\end{tabular}

Table 1. Properties of solar cells having the structure ITO/ $\mu \mathrm{c}-\mathrm{Si}: \mathrm{B} / \mathrm{a}-\mathrm{Si} / \mu \mathrm{c}-\mathrm{Si}: \mathrm{P} / \mathrm{ZnO} / \mathrm{TiO} \mathrm{O}_{2} / \mathrm{Ag} / \mathrm{SS}$.

The current (Jsc) and voltage (Voc) are hardly changed by the addition of an undoped $\mathrm{TiO}_{2}$ layer. It is difficult to judge whether the small differences in fill factor (FF) and series resistance (Rs) are significant. Addition of a $5 \%$ niobium-doped $\mathrm{TiO}_{2}$ layer results in a slight reduction $\left(0.4 \mathrm{ma} / \mathrm{cm}^{2}\right.$ or $2 \%$ ) in the current, mainly in the red end of the spectrum. Measurements were also made on Voc in room light, which is sensitive to shunting. No statistically significant reduction of shunting was produced by addition of the $\mathrm{TiO}_{2}$ layer.

These results demonstrate that a $\mathrm{TiO}_{2}$ layer may be added to the substrate cell structure with little change in properties. Doping by niobium is not necessary to achieve satisfactory electrical properties for the cell. Future investigations will include the effect of $\mathrm{TiO}_{2}$ thickness on solar cell performance.

\section{Task 3. Deposit and evaluate niobium-doped titanium oxide as a transparent conducting layer on which more efficient and more stable cells can be deposited.}

There are several potential advantages to inserting a transparent, conductive layer of titanium dioxide between the top zinc oxide and the silicon layers of a superstrate solar cell:

1) The titanium dioxide is stable enough so that microcrystalline p-silicon could be deposited directly on it. Cells with this structure are expected to have increased voltage and current.

2) The titanium dioxide layer would reduce the reflection of light, because it has a refractive index (about 2.3) which is intermediate between that of zinc oxide (1.9) and silicon (about 3). This effect should provide increased current.

3) One source of instability in amorphous silicon solar cells is the possible reduction of the transparent metal oxide by the silicon. Such a reduction is possible for tin oxide and zinc oxide, but not for titanium oxide, which should thus provide increased stability.

Polycrystalline titanium dioxide films about 0.05 micron thick were deposited on top of fluorinedoped tin oxide films by CVD from titanium isopropoxide at $500^{\circ} \mathrm{C}$. In order to make a quick test of how protective these films were to chemical attack by hydrogen, we treated these films with nascent hydrogen generated by mixing zinc dust and hydrochloric acid. Bare tin oxide films are dissolved within a few seconds by this treatment. Even after one minute of etching with this mixture, the thin overcoating of titanium dioxide prevented the tin oxide from dissolving. After several minutes of etching, a few spots became etched, presumably by attack through pinholes in the titanium dioxide film. The films did develop a slight gray color during this treatment, which may indicate that some hydrogen diffused through the titanium dioxide film and slightly reduced the tin oxide film.

1. NREL Subcontract XAN-4-11318-05, W. Luft, technical monitor. 


\section{Title: $\quad$ Process Modeling Issues for Thin Film Solar Cell Devices}

Organization: Institute of Energy Conversion, University of Delaware, United States Department of Energy, University Center of Excellence for Photovoltaics Research and Education, (National Renewable Energy Laboratory), Newark, DE 19716

Contributors: R.W. Birkmire (principal investigator), W.A. Buchanan, E. Eser, S.S.Hegedus, H.Hichri, R.Klenk, B.E.McCandless, P.V.Meyers, N.Orbey, J.E.Phillips, TWF Russell, Y.Qu, W.N.Shafarman

\section{Objectives/Technical Highlights}

The objectives of IEC's four year subcontract with NREL are to advance the state-of-the-art of thin film PV modules by improving the thin film deposition, device fabrication, material and device characterization, and related manufacturing technology of solar cells based on $\mathrm{CdTe}, \mathrm{Cu}(\mathrm{InGa}) \mathrm{Se}_{2}$, and a-Si. Accomplishments during this third phase include: fabrication of $15 \%$ efficient $\mathrm{Cu}(\mathrm{InGa}) \mathrm{Se}_{2}$ solar cells with higher $\mathrm{V}_{\mathrm{oc}}$ and $\mathrm{Ga}$ content than previously reported, demonstration of improved and simplified processing of CdTe solar cells using vapor-phase $\mathrm{CdCl}_{2}$ and $\mathrm{HCl}$ treatments leading to $11 \%$ efficient CdTe solar cells, characterization of a variety of textured transparent conductive oxides used as substrates for a-Si devices including thermal and plasma stability and optical enhancement, and significant collaborations with many industrial and university organizations.

\section{Results}

\section{$\underline{\text { High } \mathrm{V}_{o c} \mathrm{Cu}(\mathrm{InGa}) \mathrm{Se}_{2} \text {-Based Solar Cells }}$}

Solar cells were fabricated from $\mathrm{Cu}(\mathrm{InGa}) \mathrm{Se}_{2}$ films which were deposited with no compositional gradients and with $\mathrm{Ga} /(\mathrm{In}+\mathrm{Ga})$ from 0.27 to 0.69 corresponding to bandgaps $\left(\mathrm{E}_{\mathrm{g}}\right)$ from 1.16 to $1.45 \mathrm{eV}$. The $\mathrm{Cu}(\mathrm{InGa}) \mathrm{Se}_{2}$ films were deposited on Mo-coated glass substrates by four source elemental evaporation. A simple two step deposition process consisted of: 1) depositing a Cu-rich $\mathrm{Cu}(\mathrm{InGa}) \mathrm{Se}_{2}$ film with the substrate heated to $450^{\circ} \mathrm{C}$ followed by 2) increasing the substrate temperature to $600^{\circ} \mathrm{C}$ and shutting off the $\mathrm{Cu}$ flux while continuing the same $\mathrm{In}, \mathrm{Ga}$, and Se fluxes. The $\mathrm{Ga} /(\mathrm{In}+\mathrm{Ga})$ ratio was controlled by setting the effusion rates of the In and $\mathrm{Ga}$ sources. X-ray diffraction $(\mathrm{XRD})$ and Auger depth profiles showed that the $\mathrm{Cu}(\mathrm{InGa}) \mathrm{Se}_{2}$ films were single phase with uniform composition and no change in structure, morphology, or compositional uniformity was observed over the range of compositions. The composition was determined by energy dispersive $\mathrm{x}$-ray spectroscopy.

Solar cells were fabricated from the $\mathrm{Cu}\left(\mathrm{InGa}_{\mathrm{N}} \mathrm{Se}_{2}\right.$ films using chemical bath deposited $\mathrm{CdS}$, sputtered $\mathrm{ZnO}$ window layers, evaporated $\mathrm{Ni} / \mathrm{Al}$, and $\mathrm{MgF}_{2}$ anti-reflection layers. Device results listed in Table 1 show that a constant 15\% total area efficiency was achieved for bandgap less than $1.3 \mathrm{eV}$ corresponding to $\mathrm{Ga} /(\mathrm{In}+\mathrm{Ga}$ ) less than 0.5 . Previously the highest efficiency cells were fabricated with $\mathrm{Ga} /(\mathrm{In}+\mathrm{Ga}) \approx 0.25$. $\mathrm{V}_{\text {oc }}$ increased over the entire bandgap range to $788 \mathrm{mV}$. A decrease in device performance with higher $\mathrm{Ga}$ content is caused by a reduced fill factor which, by analysis of the current-voltage (JV) characteristics and quantum efficiency, can be attributed to a voltage dependent current collection. 
TABLE 1. Current-voltage results of devices made from $\mathrm{Cu}(\mathrm{InGa}) \mathrm{Se}_{2}$ films with $\mathrm{Ga} /(\mathrm{In}+\mathrm{Ga})$ from 0.27 to 0.69 corresponding to $\mathrm{E}_{\mathrm{g}}$ from 1.16 to $1.45 \mathrm{eV}$.

\begin{tabular}{cccccc}
\hline \hline $\mathrm{Ga}$ & $\begin{array}{c}\mathrm{E}_{\mathrm{g}} \\
(\mathrm{eV})\end{array}$ & $\begin{array}{c}\mathrm{V}_{\propto} \\
(\mathrm{V})\end{array}$ & $\begin{array}{c}\mathrm{J}_{\text {sc }} \\
\left(\mathrm{mA} / \mathrm{cm}^{2}\right)\end{array}$ & $\begin{array}{c}\mathrm{FF} \\
(\%)\end{array}$ & $\begin{array}{c}\text { eff } \\
(\%)\end{array}$ \\
\hline $\mathrm{In}+\mathrm{Ga}$ & 1.16 & 0.602 & 33.2 & 74.1 & 14.8 \\
0.27 & 1.18 & 0.623 & 32.8 & 73.1 & 14.9 \\
0.30 & 1.23 & 0.639 & 31.9 & 74.3 & 15.1 \\
0.43 & 1.27 & 0.689 & 28.9 & 75.0 & 15.0 \\
0.58 & 1.37 & 0.746 & 25.2 & 69.7 & 13.1 \\
0.69 & 1.45 & 0.788 & 20.3 & 63.4 & 10.1 \\
\hline \hline
\end{tabular}

\section{Single Phase $\mathrm{Cu}(\mathrm{InGa}) \mathrm{Se}_{2}$ Films by Selenization}

Using a process previously developed for reacting $\mathrm{Cu}$-Ga-In metal precursors in a Se containing atmosphere to form single phase $\mathrm{Cu}(\mathrm{InGa}) \mathrm{Se}_{2}$, films with $\mathrm{Ga} /(\mathrm{In}+\mathrm{Ga}$ ) from 0 to 1 have been formed and materials and device properties have been characterized [1]. For this process, $\mathrm{Cu}-\mathrm{In}$ $\mathrm{Ga}$ multilayer films were deposited on Mo-coated glass substrates by sputtering with a $\mathrm{Cu} /(\mathrm{In}+\mathrm{Ga})$ ratio of 0.9 and varying $\mathrm{Ga} /\left(\mathrm{In}+\mathrm{Ga}\right.$ ) ratios. The films were reacted in $\mathrm{H}_{2} \mathrm{Se}$ at $450^{\circ} \mathrm{C}$ for 90 minutes and then annealed at $500^{\circ} \mathrm{C}$ and $600^{\circ} \mathrm{C}$ for 90 minutes in Ar. Details of the process are presented in ref. 1. The XRD data of the films after the $450^{\circ} \mathrm{C}, 90$ minute $\mathrm{H}_{2} \mathrm{Se}$ reaction shows distinct and separate peaks corresponding to $\mathrm{CuInSe} 2$ and $\mathrm{CuGaSe}_{2}$ with very little $\mathrm{Cu}(\mathrm{InGa}) \mathrm{Se}_{2}$ detected. Auger depth profiling showed that the $\mathrm{Ga} /(\mathrm{In}+\mathrm{Ga})$ ratio is higher at the substrate interface than at the surface. The JV and quantum efficiency (QE) measurements of devices made on asreacted films indicate that the devices behave as $\mathrm{CuInSe} 2 / \mathrm{CdS}$ cells. Samples annealed at $500^{\circ} \mathrm{C}$ in Ar retain the characteristics of the as-reacted films, indicating non-uniform Ga distribution. However, XRD measurements indicate that annealing at $600^{\circ} \mathrm{C}$ in Ar produces a single phase structure. EDS and Auger measurements confirm that the $\mathrm{Ga} /(\mathrm{In}+\mathrm{Ga})$ ratio is uniform through the film and consistent with the ratio of the precursors. The $\mathrm{QE}$ measurements are consistent with a $\mathrm{Cu}(\mathrm{InGa}) \mathrm{Se}_{2}$ absorber layer having the same bandgap as expected for $\mathrm{Cu}(\mathrm{InGa}) \mathrm{Se}_{2}$ films having these $\mathrm{Ga} /(\mathrm{In}+\mathrm{Ga})$ compositions .

An evaporation system was constructed with independent $S e$ and $S$ thermal sources for the reaction of $\mathrm{Cu}-\mathrm{In}$ and $\mathrm{Cu}-\mathrm{In}-\mathrm{Ga}$ precursor films to form $\mathrm{Cu}(\mathrm{InGa})(\mathrm{SeS})_{2}$. Also, $\mathrm{CuGaSe} 2$ films were formed by reaction of $\mathrm{Cu}-\mathrm{Ga}$ layers with $\mathrm{H}_{2} \mathrm{Se}$ to determine conditions necessary for good adhesion so time dependent experiments to study the reaction kinetics can be completed.

\section{Mo/CuInSe, Contact Measurements}

Many high efficiency $\mathrm{Cu}(\mathrm{InGa}) \mathrm{Se}_{2}$ solar cells show blocking or non-Ohmic contact behavior in their current voltage characteristic which has been attributed to the Mo back contact. A novel device configuration was developed to allow the JV characteristic of the $\mathrm{Mo} / \mathrm{CuInSe} 2$ junction to be analyzed separately from the rest of the operating solar cell. Direct measurements of the back contact on a device with non-ohmic JV behavior showed that the blocking behavior does not originate at the $\mathrm{Mo} / \mathrm{CuInSe} 2$ contact.

\section{Post Deposition Heat Treatment of CdTe/CdS}

Alternative vapor chloride treatments of $\mathrm{CdTe} / \mathrm{CdS}$ devices have been developed to replace the standard wet $\mathrm{CdCl}_{2}$ process. These vapor treatments allow greater uniformity and reproducibility and do not leave a $\mathrm{CdCl}_{2}$ residue. In one approach, the $\mathrm{CdTe} / \mathrm{CdS}$ structure is reacted with $\mathrm{CdCl}_{2}$ 
vapor generated by heating $\mathrm{CdCl}_{2}$ powder or films. This method has been used routinely at IEC to produce $\mathrm{CdTe} / \mathrm{CdS}$ films having a high degree of preferred (111) orientation, uniform grain size, significantly less bulk interdiffusion, chemically clean surfaces, and uniform device performance at $11 \%$ efficiency [2]. Experiments to determine reaction rate constants and activation energies needed to chemically quantify the $\mathrm{CdCl}_{2}-\mathrm{CdTe} / \mathrm{CdS}$ reactions for scaling up to module dimensions are being conducted. A second approach is reaction of the $\mathrm{CdTe} / \mathrm{CdS}$ structure with $\mathrm{HCl}: \mathrm{O}_{2}$ vapor. Initial results have demonstrated the requisite recrystallization and devices with efficiency of $7 \%$.

\section{Alloying and Interdiffusion of CdTe and CdS}

IEC has previously shown that during the $\mathrm{CdCl}_{2}$ treatment process, $\mathrm{Te}$ diffuses into the $\mathrm{CdS}$ window layer, lowering the bandgap of the window layer thereby reducing $\mathrm{J}_{\mathrm{sc}}$ [3]. Alloys of $\mathrm{CdTe}$ and $\mathrm{CdS}$ were deposited to evaluate properties of the pseudobinary alloy system and to determine solubility limits of $\mathrm{Te}$ in $\mathrm{CdS}$ and $\mathrm{S}$ in CdTe at typical processing temperatures. $\mathrm{CdTe}_{1-x} \mathrm{~S}_{\mathrm{X}}$ films deposited by co-evaporation of CdTe and CdS at substrate temperatures of 200 and $250^{\circ} \mathrm{C}$ were found to be predominately single phase with atomic compositions, $x=[S] /([S]+[\mathrm{Te}])$, ranging from 0 to 0.45 . The films segregated into two phases upon heat treatment at $415^{\circ} \mathrm{C}$ in the presence of $\mathrm{CdCl}_{2}$. The compositions of the zincblende phase and wurtzite phases obtained after heat treatment were $x=0.057 \pm 0.003$ and $x=0.97 \pm 0.01$, respectively, regardless of whether the overall composition of the films was 0.1 or 0.4 . This suggests that the as-deposited films are either metastable or kinetically controlled, and that sufficient heat treatment drives the composition of the films towards equilibrium. Therefore, the compositions of the two phases after heat treatment may be taken as measurements of the solubility limits of $\mathrm{S}$ in $\mathrm{CdTe}$ and Te in CdS at $415^{\circ} \mathrm{C}$.

\section{CdTe Device Stability}

In order to assess long term stability of $\mathrm{CdTe} / \mathrm{CdS}$ solar cells under operating conditions, IEC has initiated a study to identify potential degradation mechanisms in $\mathrm{CdTe} / \mathrm{CdS}$ solar cells.

Preliminary experiments confirm various degrees of reversible and irreversible decreases in $V_{O C}$ and FF which are tentatively attributed to chemical or doping changes occurring at the low resistance $\mathrm{CdTe}$ contact. IEC has set up a stress test station where devices can be subjected to voltage and current bias $\left(-0.5 \mathrm{~V}\right.$ to $\left.+5 \mathrm{~mA} / \mathrm{cm}^{2}\right)$, elevated temperature $\left(60-100{ }^{\circ} \mathrm{C}\right)$, and illumination (0-100 $\left.\mathrm{mW} / \mathrm{cm}^{2}\right)$. This work also supports the CdTe team of the PV Partnership program which has a goal of developing a stability testing protocol for CdTe PV modules.

\section{Evaluation of Textured TCO Substrates and Optical Enhancement for a-Si Devices}

IEC has characterized optoelectronic and structural properties of seven textured TCO materials from different manufacturers used in superstrate a-Si devices. The stability of various textured $\mathrm{SnO}_{2}$ and $\mathrm{ZnO}$ TCOs was evaluated against annealing in air, in vacuum or exposure to hydrogen plasma [4]. Only $\mathrm{ZnO}: \mathrm{F}$ deposited by atmospheric pressure chemical vapor deposition (APCVD) had stable electrical and optical properties under all conditions. Device performance will be optimized for several textured TCO's in IEC's plasma CVD deposition system, which presently yields devices with typical initial parameters of $\mathrm{V}_{\mathrm{OC}}=0.83-0.84 \mathrm{~V}, \mathrm{~J}_{\mathrm{SC}}=13-14 \mathrm{~mA} / \mathrm{cm}^{2}, \mathrm{FF}=71-$ $72 \%$, and efficiency $=8.0-8.4 \%$ for a device structure of glass/textured $\mathrm{SnO}_{2} / \mathrm{p}-\mathrm{b}-\mathrm{i}-\mathrm{n} / \mathrm{Ti} / \mathrm{Ag}$.

The effect of back reflector (BR) reflectivity and texture on optical enhancement to improve $\mathrm{J}_{\mathrm{sc}}$ has been studied on ITO/p-i-n/textured TCO/glass/Ag BR devices deposited at ECD on substrates having haze from 0 to $14 \%$ provided by IEC. Measurements at IEC before and after coupling an external Ag reflector allow separation of the effect of substrate texture (scattering) and back reflection. The $\mathrm{J}_{\mathrm{sc}}$ and $\mathrm{QE}$ at $700 \mathrm{~nm}$ are given in Table 2 . 
Table 2. $J_{\text {sc }}$ and QE data for 2 devices, on smooth (0\%) and textured (14\% haze) substrates, with different external Ag BR.

\begin{tabular}{llll}
\hline \hline Haze & external BR & $\mathrm{J}_{\mathrm{sc}}\left(\mathrm{mA} / \mathrm{cm}^{2}\right)$ & $\mathrm{QE} @ 700 \mathrm{~nm}$ \\
\hline $0 \%$ & no Ag (transparent) & 11.6 & 0.05 \\
$0 \%$ & smooth Ag & 13.0 & 0.07 \\
$0 \%$ & textured Ag & 13.3 & 0.11 \\
$14 \%$ & no Ag (transparent) & 13.9 & 0.22 \\
$14 \%$ & smooth Ag & 15.1 & 0.32 \\
\hline \hline
\end{tabular}

These results show that a textured substrate with a smooth $\mathrm{Ag} B \mathrm{BR}$ has $15 \%$ higher $\mathrm{J}_{\mathrm{SC}}$ than a textured $\mathrm{Ag} B R$ on a smooth device. The red $\mathrm{QE}$ is tripled for the textured substrate $(0.11 \mathrm{vs}$ 0.32). This suggests that the replication of the substrate texture on the top a-Si surface is crucial to enhance multiple scattering from the underlying substrate. However, negligible improvement in red response or $\mathrm{J}_{\mathrm{Sc}}$ was found for haze greater than $5 \%$ for superstrate or substrate devices, suggesting a limit to the increase in scattering.

\section{Tunnel Junction Studies for Tandem a-Si/a-Si Solar Cells}

Current transport in a-Si n/p ("tunnel") junctions was investigated using JV(T) and QE measurements in collaboration with APS [5]: Depositing a thin a-Si(B) $p+$ layer between the $n$ and $\mathrm{p}$-layers and/or replacing either a-Si doped layer with a microcrystalline layer lead to improved interconnect junctions. Tandem solar cells made by APS with these improved junctions had improved initial and stabilized performance.

\section{Collaboration and Participation on NREL Teams}

IEC has been active on the $\mathrm{Cu}(\mathrm{In}, \mathrm{Ga}) \mathrm{Se}_{2}, \mathrm{CdTe}$, and a-Si research teams established by NREL. This has included measurements and analysis on devices from other groups, shared fabrication of devices, and presentations at several team meetings. For example, IEC has measured and analyzed JV (both light and dark, and as a function of temperature and intensity) and QE data on 17 $\mathrm{Cu}(\mathrm{In}, \mathrm{Ga}) \mathrm{Se}_{2}$ substrates fabricated by 8 different groups, and distributed the results to the $\mathrm{Cu}(\mathrm{In}, \mathrm{Ga}) \mathrm{Se}_{2}$ Junction and Absorber team members. During the past year, IEC has had collaborations with many organizations including: Advanced Photovoltaic Systems, AstroPower, Colorado School of Mines, Colorado State University, Energy Conversion Devices, Georgia Institute of Technology, Harvard University, International Solar Electric Technology, Lockheed Martin, National Renewable Energy Laboratory, Penn State University, Purdue University, Siemens Solar, Solarex Thin Film, Solar Cells Inc., Spire Corp., University of Illinois, and University of South Florida. In addition, a graduate student from IEC spent one month at NREL working with their researchers and facilities to characterize $\mathrm{Cu}(\mathrm{In}, \mathrm{Ga}) \mathrm{Se}_{2}$ films.

\section{References}

1. M. Marudachulum, H. Hichri, R. Klenk, R.W. Birkmire, W.N. Shafarman and J.M. Schultz, Appl. Phys.Lett., to be published.

2. R.W. Birkmire, H. Hichri, R. Klenk, M. Marudachalam, B.E. McCandless, J.E. Phillips,

J.M. Schultz and W.N. Shafarman, 13th NREL PV Program Review Meeting (1995), AIP Conf. Proc. 353, 420 (1996).

3. B.E.McCandless and S.S. Hegedus, Proc. $22^{\text {nd }}$ IEEE, 1991, 967-972.

4. S. S. Hegedus, H. Liang, R. Gordon, 13th NREL PV Program Review Meeting (1995), AIP Conf. Proc. 353, 81 (1996).

5. S. S. Hegedus, J. Xi, F. Kampas, Appl. Phys. Lett. 67 (1995), 813. 
Title:

Organization:

Contributors:
Application of CIS to High Efficiency PV Module Fabrication

International Solar Electric Technology (ISET), 8635 Aviation Blvd., Inglewood, CA 90301

B.M. Başol, V.K. Kapur, C.R. Leidholm and A. Halani

\section{Introduction}

ISET has developed processing approaches for the fabrication of high efficiency $\mathrm{CIS}$ solar cells and modules since 1984. As a result of these efforts which were partially funded by the DOE, single cell efficiencies of around $13 \%$ and $1 \mathrm{ft}^{2}$ size module aperture efficiency of $7 \%$ have been demonstrated by an industrial two-stage process. The present project is the first phase of a cost-shared "Thin-Film PV Partnership Program" which was geared towards the development of a novel and low-cost CIS growth technique as well as towards the better understanding of certain issues related to film/substrate interactions, bandgap widening and module integration.

\section{Objective}

The objective of this program is to develop understanding and solutions for certain issues related to economical production of CIS-based modules. The most important task is the development and demonstration of a low-cost, non-vacuum method for CIS film growth. Another task involves study of $\mathrm{Ga}$ addition into $\mathrm{CIS}$ absorber layers. In this report we will present our significant results on these topics.

\section{CIGS Film Growth}

Selenization of $\mathrm{Cu}+\mathrm{In}+\mathrm{Ga}$ metallic precursor layers in $\mathrm{Se}$ or $\mathrm{H}_{2} \mathrm{Se}$ atmosphere always yields absorbers with graded $\mathrm{Ga}$ concentrations. In such films, Ga-rich phase(s) segregate toward the back of the absorber layer, leaving an In-rich surface. In a recent publication, IEC group described a process consisting of a post-selenization annealing step in a Se-free atmosphere, which was found to be essential to initiate the diffusion of $\mathrm{Ga}$ and formation of the quaternary CIGS phase in non-uniform absorbers obtained by selenization. We employed a similar post-selenization step to grow CIGS absorber layers with an overall $\mathrm{Ga} /(\mathrm{Ga}+\mathrm{In})$ ratio of $0.55-0.75$. Soda-lime glass was used as the substrate. Mo layers of $2.0 \mu \mathrm{m}$ thickness were sputter deposited on the soda-lime glass. CIGS absorber films were formed on the Mo/glass sheets by the two-stage selenization technique. In this approach, a precursor layer containing $\mathrm{Cu}$, In, and $\mathrm{Ga}$ was first deposited on the substrate by electron-beam evaporation. The precursor was then selenized in a $\mathrm{H}_{2} \mathrm{Se}$ atmosphere to form the compounds. Thicknesses of the $\mathrm{In}, \mathrm{Cu}$, and $\mathrm{Ga}$ layers were adjusted to obtain films with various compositions and an overall stoichiometric ratio of $\mathrm{Cu} /(\mathrm{In}+\mathrm{Ga})=0.9$. For the precursor of samples $1789-425$ and 1789 - 
575 , the overall $\mathrm{Ga} /(\mathrm{Ga}+\mathrm{In})$ ratio was 0.55 . The $\mathrm{Ga} /(\mathrm{In}+\mathrm{Ga})$ ratio for the precursor of samples $1791-425$ and $1791-575$, on the other hand, was 0.75 . Selenization was carried out for all precursors at $425^{\circ} \mathrm{C}$. A portion of each film was then additionally annealed at $575^{\circ} \mathrm{C}$ in a $\mathrm{N}_{2}$ atmosphere (samples labeled -575 ). Fig. 1 shows the illuminated $\mathrm{J}-\mathrm{V}$ characteristics of solar cells fabricated on three of the absorber layers identified above. The devices fabricated on sample 1791-425 were extremely leaky, and they were not included in Fig. 1.The $\mathrm{J}-\mathrm{V}$ characteristics indicate $a V_{\infty}$ value of $740 \mathrm{mV}$ for sample 1791575 and $625 \mathrm{mV}$ for sample 1789-575. The $V_{o c}$ for sample $1789-425$, on the other hand, is only $400 \mathrm{mV}$ despite the fact that this film contained $55 \% \mathrm{Ga}$. The absolute QE curves were measured for these devices. The solar cell output parameters derived from Fig. 1 as well as the $E_{g}$ values deduced from the long wavelength $Q E$ are summarized in Table I. From this data, the following observations can be made:

(i) The bandgap value of sample 1789-425 deduced from its QE curve was much less than the $1.4 \mathrm{eV}$ expected from a uniform CIGS layer with $55 \% \mathrm{Ga}$. The observed value of $E_{g}=1.07 \mathrm{eV}$ and correspondingly modest $V_{\alpha c}$ value of $400 \mathrm{mV}$, suggest the presence of a CIGS composition with only about $10 \% \mathrm{Ga}$ in the absorber layer. Furthermore, the solar cell fabricated on this absorber is relatively poor with a low fill-factor value.

(ii) High-temperature annealing of sample 1789-425 increased the effective bandgap of this absorber to $1.23 \mathrm{eV}$ and $\mathrm{V}_{o}$ to $625 \mathrm{mV}$, indicating increased $\mathrm{Ga}$ activity. However, this bandgap is still lower than the $1.4 \mathrm{eV}$ expected from a uniform CIGS layer with $55 \% \mathrm{Ga}$. The measured bandgap value of $1.23 \mathrm{eV}$ indicates the presence of a CIGS phase in the absorber with about $32 \% \mathrm{Ga}$.

(iii) The QE data obtained from the cell fabricated on the annealed sample with overall $\mathrm{Ga}$ content of $75 \%$ yielded a bandgap value of $1.39 \mathrm{eV}$ and the device had a $V_{o c}$ of $740 \mathrm{mV}$, which corresponds to a CIGS phase with about $54 \% \mathrm{Ga}$.

Capacitance values of the three devices were measured as a function of frequency. The dispersion in the capacitance was the greatest for sample 1789-425, indicating the presence of a large density of extraneous states in the absorber. The zero bias capacitance value was the smallest for sample 1789-425, indicating the widest depletion width, despite the fact that the built-in potential was expected to be the smallest for this low-voltage device. The spacial variation of the hole density calculated from the slope of the $C-V$ curves indicated that the hole density in all films increased from less than $3 \times 10^{15}$ $\mathrm{cm}^{-3}$ near the highly compensated surface region to about $3 \times 10^{16} \mathrm{~cm}^{-3}$ at about $0.6-0.75$ $\mu \mathrm{m}$ from the junction. Fig. 2 shows the Auger depth profiles and the calculated $\mathrm{Ga} /(\mathrm{Ga}+\mathrm{In})$ ratios of the CIGS films identified in Table I. As seen from these data, the compositions of samples $1789-425$ and $1791-425$, which were not subjected to the hightemperature annealing step after selenization, were highly graded suggesting segregation of in and Ga-rich phases. After the high-temperature annealing step, Ga concentration near the surface increased. However, the resulting absorbers were still graded in terms of their $\mathrm{Ga}$ distribution. According to Fig. $2 a$, the $\mathrm{Ga} /(\mathrm{Ga}+\mathrm{ln})$ ratio for sample $1789-425$ increases from 0.1 at a region about $0.1 \mu \mathrm{m}$ inside the film surface to 0.98 at a depth of $1.4 \mu \mathrm{m}$. The expected $E_{g}$ value of the surface region with $10 \% \mathrm{Ga}$ is 
$1.07 \mathrm{eV}$, which is in excellent agreement with the data of Table I. The Ga/(Ga+ln) ratios near the surface regions of samples $1789-575$ and $1791-575$ are 0.31 and 0.53 , respectively. These values are also in good agreement with the device measurement results that predicted the presence of CIGS phases with $32 \%$ and $53 \% \mathrm{Ga}$, respectively, in these two absorber layers.

\section{Low Cost CIS Film Growth}

ISET has developed a novel CIS film growth technique with the aim of lowering the cost of processing. In this approach, processes requiring high cost equipment such as vacuum evaporators were abandoned and the absorber layer formation was achieved in a totally non-vacuum environment. The feasibility of this novel process was demonstrated by the fabrication of $12.4 \%$ efficient solar cells on CIS layers grown by this technique. We believe that these devices are the highest efficiency cells ever demonstrated on a CIS film grown by a non-vacuum technique. The I-V characteristics of a cell and a submodule made on a similarly prepared film are given in Fig. 3.

\section{Conclusions and Planned Work}

CIGS films could be grown by the two-stage selenization technique provided that a postselenization heat treatment in an inert atmosphere was utilized. Absorbers obtained by this technique were used to fabricate devices with high $V_{o c}$ values. ISET also developed a novel non-vacuum processing approach for the growth of $\mathrm{ClS}$ absorbers. Future work on this topic will include application of this technique to wide bandgap film growth and demonstration of large area submodules.

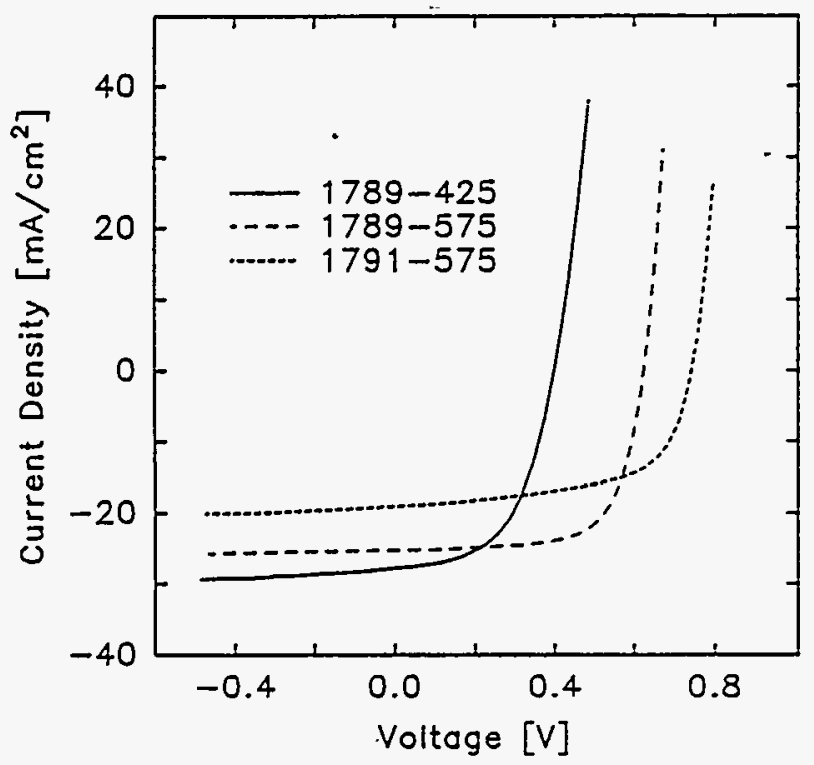

Fig. 1 llluminated $\mathrm{J}-\mathrm{V}$ characteristics of three cells with 0.083 $\mathrm{cm}^{2}$ area.

Table I. Solar cell parameters and bandgap values for the devices of Fig. 1 .

\begin{tabular}{|c|c|c|c|c|c|}
\hline Sample No. & $\begin{array}{c}\eta \\
\text { (active area) }\end{array}$ & $\mathrm{FF}$ & $\begin{array}{c}\mathrm{V}_{\infty} \\
(\mathrm{mV})\end{array}$ & $\begin{array}{c}\mathrm{J}_{\mathrm{g}} \\
\left(\mathrm{mA} / \mathrm{cm}^{3}\right)\end{array}$ & $\begin{array}{c}\mathrm{F}_{3} \\
(\mathrm{eV})\end{array}$ \\
\hline $1789-425$ & 6.4 & 0.54 & 400 & 30 & 1.07 \\
\hline $1789-575$ & 11.7 & 0.69 & 625 & 27.3 & 1.23 \\
\hline $1791-575$ & 9.4 & 0.615 & 740 & 20.6 & 1.39 \\
\hline
\end{tabular}



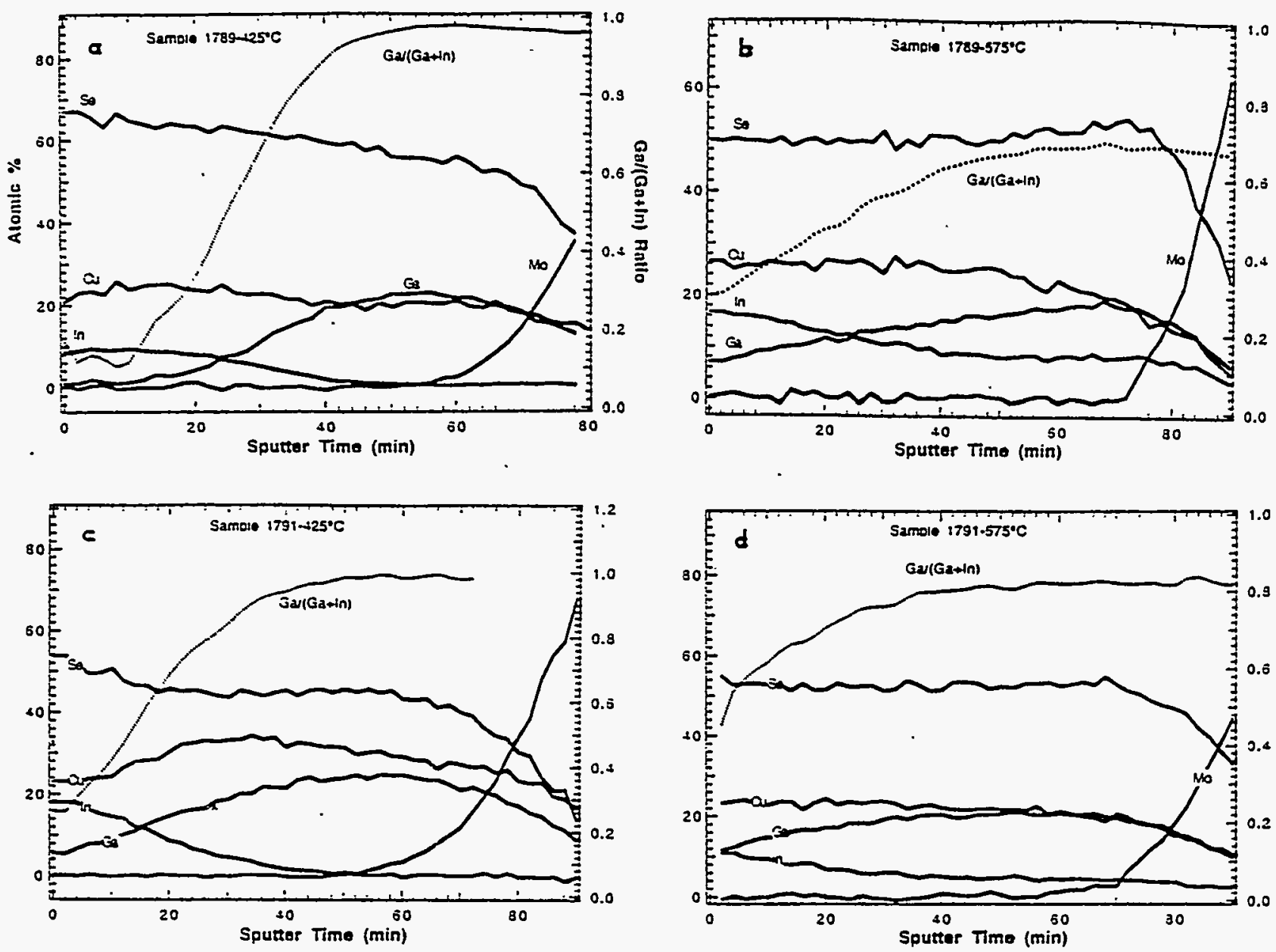

Fig. 2. Auger depth profiles of four CIGS layers showing $\mathrm{Ga}$ distribution.
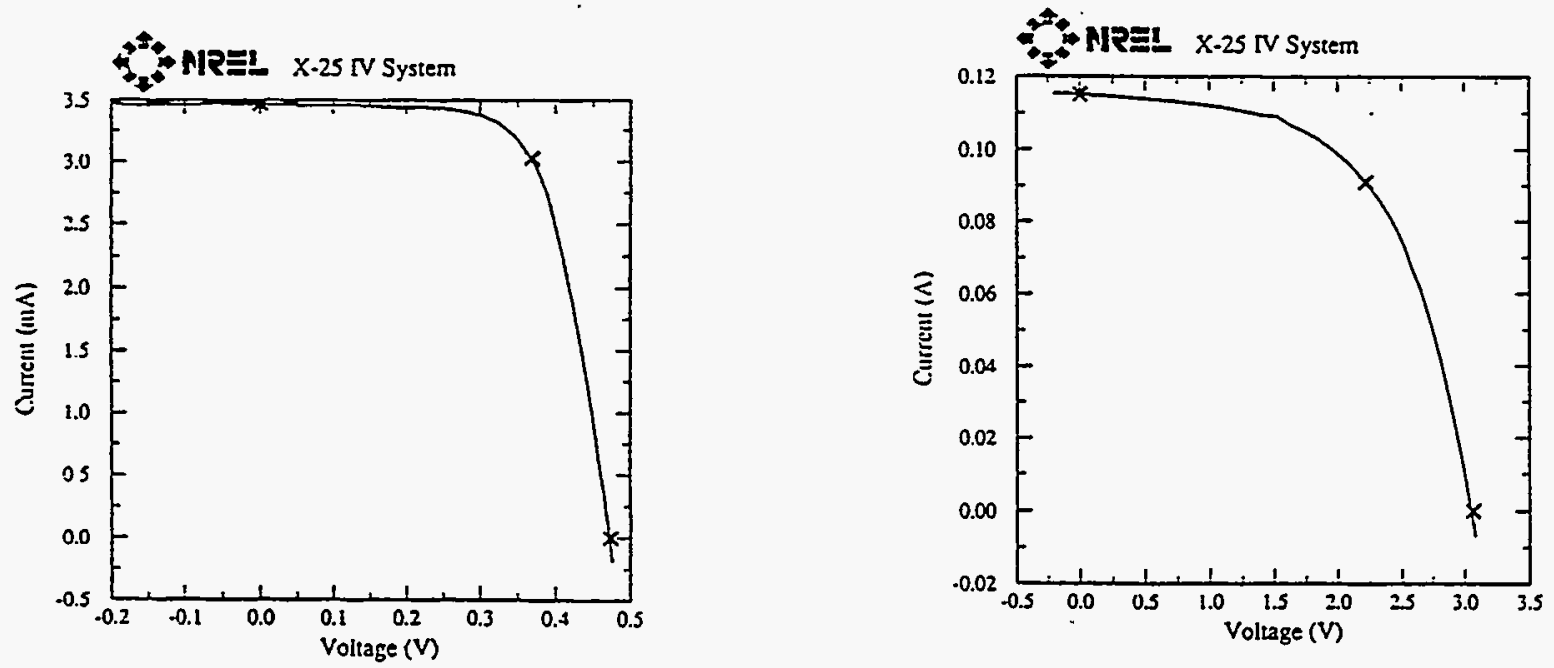

$v_{\infty}=0.4728 \mathrm{~V}$

$I_{x}=3.468 \mathrm{~mA}$

$J_{c}=38.46 \mathrm{mAcm}^{-2}$

Fill Factor $=67.97 \%$

Fig. 3.
$V_{\max }=0.3685 \mathrm{~V}$

$I_{\text {max }}=3.025 \mathrm{~mA}$

$P_{\text {mex }}=1.115 \mathrm{~mW}$

Efficiency $=12.4 \%$

$\begin{array}{ll}V_{c e}=3.063 \mathrm{~V} & V_{\operatorname{cax}}=2.217 \mathrm{~V} \\ I_{x}=0.1150 \mathrm{~A} & I_{\max }=0.09104 \mathrm{~A} \\ J_{x}=\$ .655 \mathrm{mAcm}^{-2} & P_{\max }=201.8 \mathrm{~mW} \\ \text { Fill Factor }=57.27 \% & \text { Efficiency }=8.17 \%\end{array}$

I-V characteristics of a small area $\left(0.09 \mathrm{~cm}^{2}\right)$ cell and $24.71 \mathrm{~cm}^{2}$ submodule fabricated on $\mathrm{CIS}$ layers grown by the non-vacuum technique. 
Title:

organization: Iowa State University

Microelectronics Research Center

Ames, Iowa 50011

Contributors: Vikram L. Dalal, PI

Ralph Knox, Kay Han, Mohan Bhan, Sanjeev Kaushal, Scott DeBoer, E. X. Ping, J. Xu and R. Girvan

\section{Objective:}

The major objective of the program is to develop alternative deposition techniques for growing high quality a-si:H and a$(S i, G e): H$ materials and devices with improved stability.

\section{Material Growth}

\section{I.1 Amorphous $\mathrm{Si}: \mathrm{H}$}

We have discovered that the plasma conditions play a major role in determining the $\mathrm{H}$ content of the films. Using a remote ECR plasma, we can finely control the type and energy of the ions arriving at the growing surface. A low pressure $H$ discharge leads to a high flux of $\mathrm{H}$ ions and radicals which serve to etch the growing film. By controlling the plasma, the microstructure of the films can be changed from amorphous to micro and polycrystalline films [1]. These films typically tend to have $6-8 \%$ hydrogen bonded in them. In contrast, by using a low pressure He discharge, we can reduce the $H$ concentration down to $4.5 \%$ range, while retaining the same electronic qualities. Concomitantly with the reduction in $\mathrm{H}$ content, the Tauc bandgap of the films also shrinks by about 60-70 meV when compared with the bandgap of films made using a low pressure $\mathrm{H}$ discharge. High dilution of $\mathrm{He}$, and a high power, lead to microcrystalline films, a somewhat surprising result, since the standard model for crystallization assumes that some sort of etching of weak bonds by $H$ is necessary to induce microcrystallinity. The electronic properties of He-deposited aSi:H films remain good, with low Urbach energies and low subgap absorption coefficients.

\section{I.2 Amorphous Silicon-Germanium Alloy}

Similar to the results for a-Si:H, plasma conditions play a major role in controlling the properties of $\mathrm{a}-(\mathrm{Si}, \mathrm{Ge}): \mathrm{H}$ alloys. In particular, the use of a low pressure He discharge leads to a significantly lower $\mathrm{H}$ concentration, and to lower Tauc gap than the use of low pressure $\mathrm{H}$ discharge. In Fig. 1 and 2 , we show the results for $\mathrm{H}$ content, and Tauc gap, for the two sets of films. From the figure, we can see that the use of He in the discharge reduces $\mathrm{H}$ content by almost a factor of 2 . The lowering of the Tauc gap is significant, because it means that one can achieve a lower 
gap with a given Ge content using a He plasma than is the case using $H$ plasma. This may lead to better properties of the films.

\section{Device Research}

\section{II.1 Device performance.}

During this year, we have made devices using the same conditions of deposition that we used for achieving the best materials.[2]. This generally involved using temperatures in excess of $350 \mathrm{C}$, which introduced some interesting problems regarding $B$ diffusion. We have successfully solved some of these problems, and achieved devices with high fill factors $(65-68 \%)$. In Fig. 3, we show the device $I(V)$ curve for a device with efficiency of $7.0 \%$. The device did not have a highly reflecting back ITO/Ag contact. Such a reflecting contact is known to improve currents by $25 \%$.

We also measured the stability of our cells, and compared them with the stability of standard glow-discharge produced cells with similar $i$ layer thickness and similar fill factor. In Fig. 4, we show the results for the degradation of fill factors in our cell and the high-performance glow discharge cell. It is clear from this figure that our cell is considerably more stable than the glow discharge cell, even though they both had similar thicknesses of $i$ layer and the similar initial fill factors. That this fact is due to the improved material properties is shown by plotting the ratio of quantum efficiencies under zero and forward bias for our cell and the glow discharge cell. Such a measurement reveals information about the collection of holes. In Fig. 5 we show that the QE ratio for long wavelength photons after light soaking is much lower in the ECR cell than for the GD cell, indicating that the intrinsic material of the ECR cell is degrading less than the intrinsic material of the GD cell.

\section{References}

1. B. Moradi et al, J. Vac. Sci. Tech. A12, 251(1994)

2. V. L. Dalal et al, Appl. Phys. Lett. 64, 1862 (1994) 


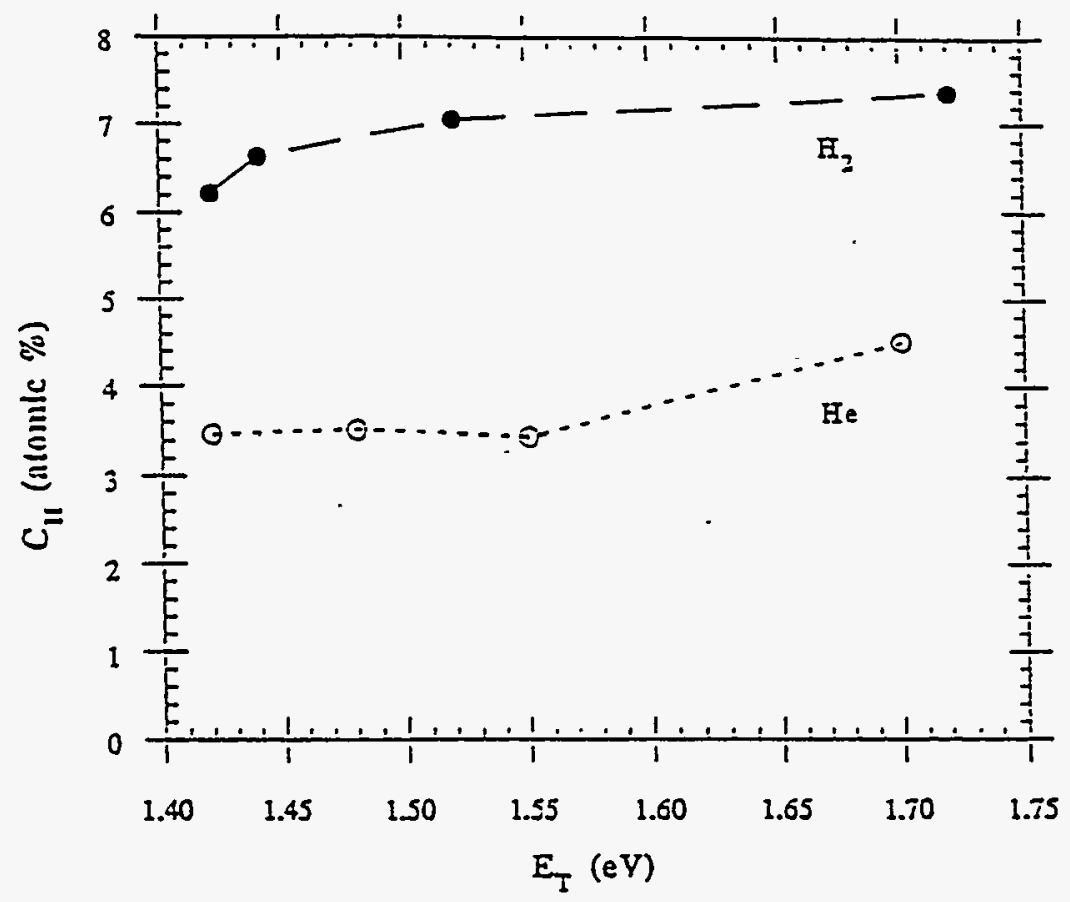

Fig. $1 \mathrm{H}$ content of films deposited using either He or H- ECR discharge plotted as a function of Tauc gap

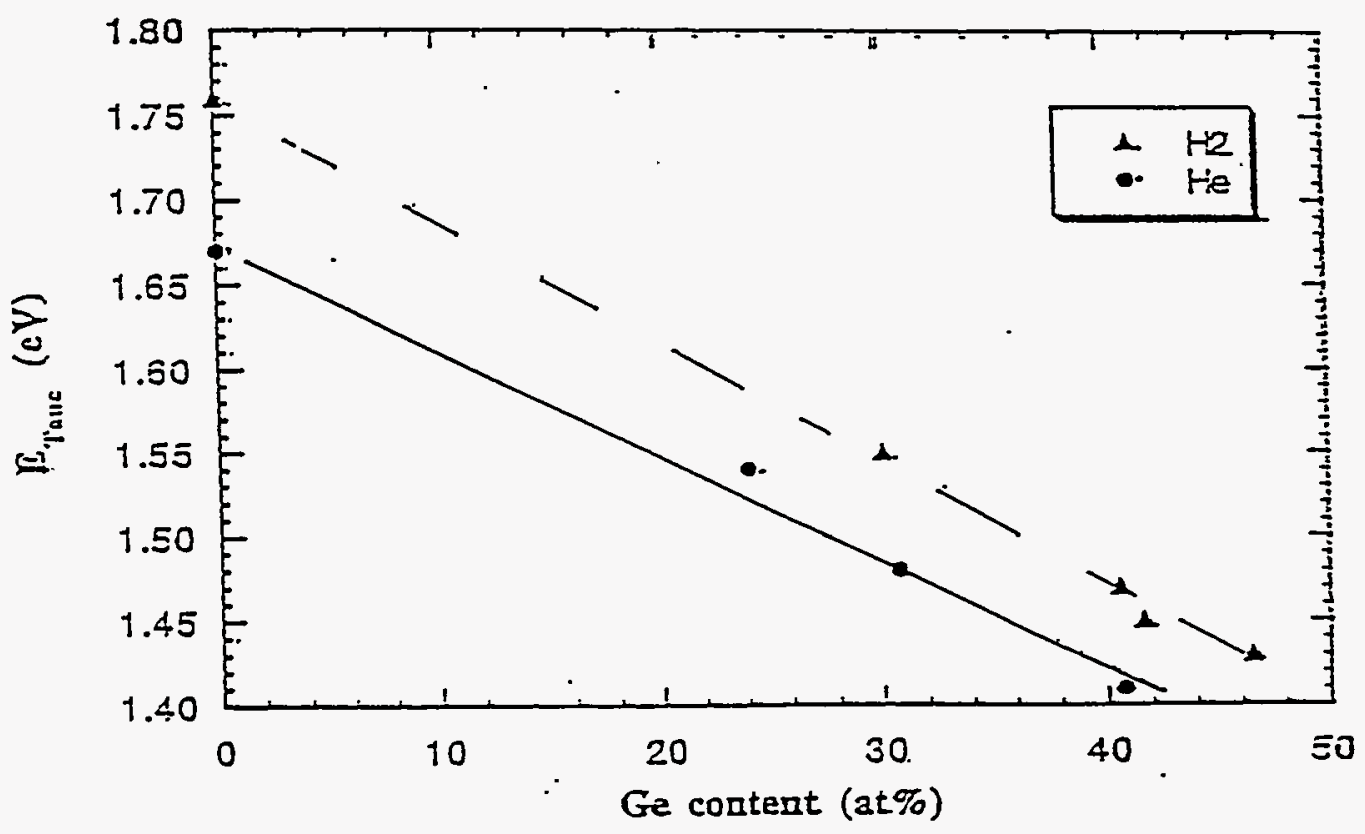

Fig. 2. Tauc gap vs. Ge content for films deposited using either He or H-ECR discharge. 


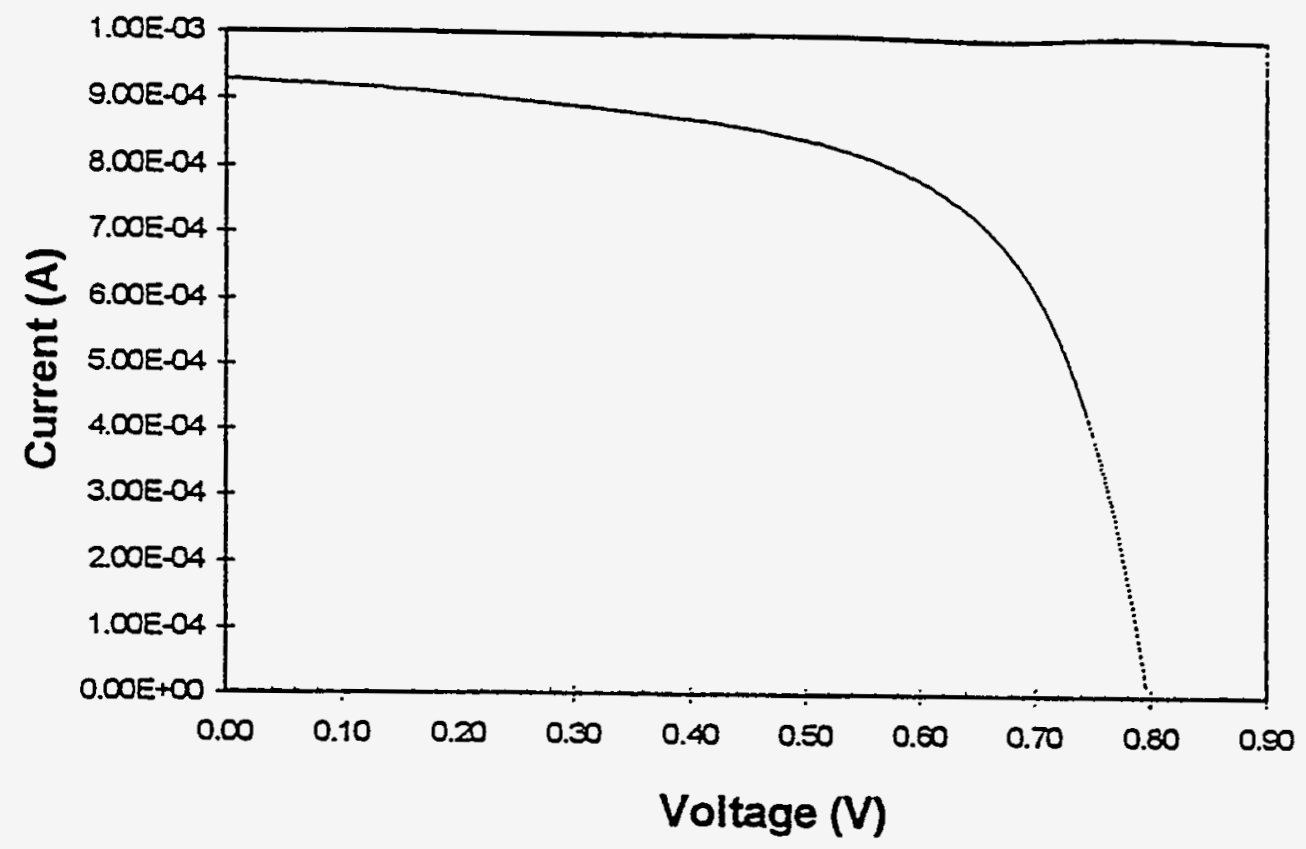

Fig. 3 Device $I(V)$ curve. $J_{s c}=13 \mathrm{~mA} / \mathrm{cm}^{2}, V_{o c}=0.804 \mathrm{~V}, F=0.67$

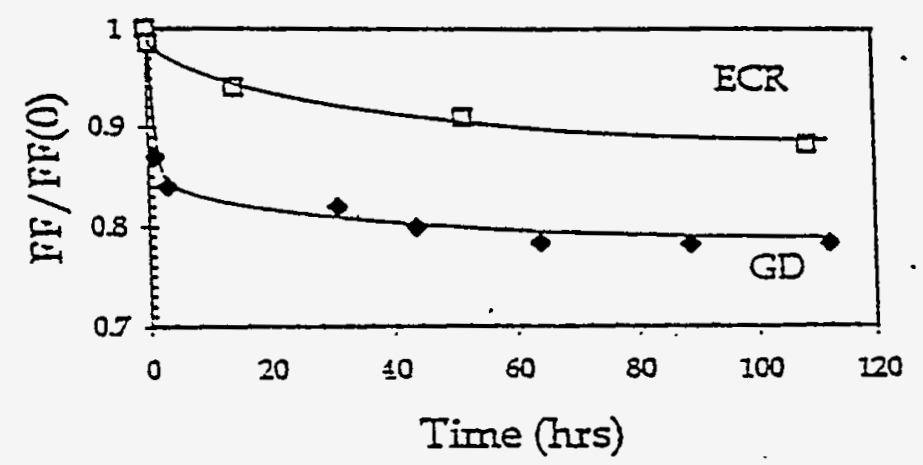

Fig. 4 Relative Degradation in fill factor of ECR and GD cells, measured under 2 xsun intensity.

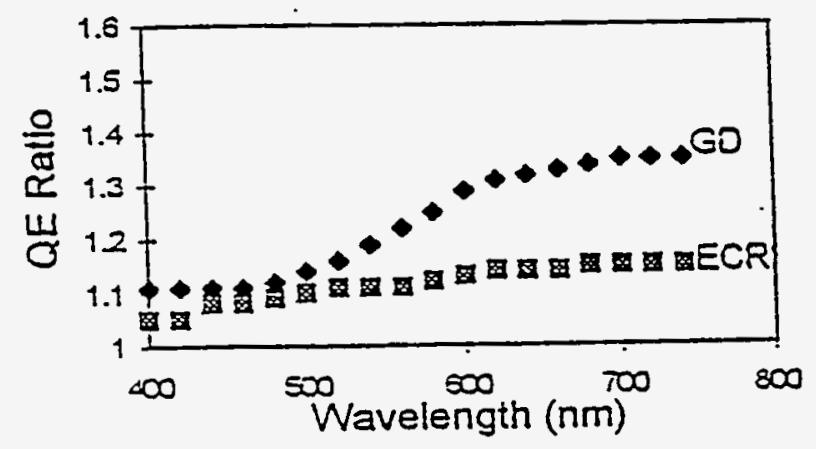

Fig. 5. $Q E$ ratio ( $Q E$ at $O V / Q E$ at $+0.4 \mathrm{~V}$ ) for the $E C R$ and $G D$ cells after degradation 
Title:

Atomic Scale Characterization of Hydrogenated Amorphous Silicon Films and Devices

Organization: National Institute of Standards and Technology (NIST) Boulder, Colorado

Contributors:

A.Gallagher, principal investigator, D.Tanenbaum, A.Laracuente, S.Barzen

\section{Objective}

The overall objective is to assist the development of a cost-effective hydrogenated amorphous silicon (a-Si:H) PV technology. This is done by improving understanding of the mechanisms that limit the efficiency of current PV devices, and devising ways to overcome these limitations.

\section{Technical Approach}

Two types of measurements are being pursued to attain this overall objective. The first results from our recent discovery that small silicon particulates, formed in the discharge, are incorporated into the growing film. This effort is directed toward measuring these particles in the discharge, and finding methods to diminish their growth and incorporation into the PV film. The second measurement is designed to establish the electrical characteristics of cross sectioned PV cells, as a function of illumination and light induced degradation, to establish where losses and degradation are occurring. These will be described below.

\section{1) Particulate incorporation into a-Si:H films}

In recent years many measurements in silane discharges have shown that silicon particulates form in the plasma, and are suspended there due to acquiring a negative charge. This charge, combined with strong sheath electric fields, prevents the particles from reaching the substrate during film growth. However, we have discovered that very small particles are continuously deposited into the film during growth, in sufficient quantity to be a potential explanation for the electrical properties that limit PV efficiency [1]. This occurs for plasma and film parameters that are typical of those used at most facilities for optimized films production, so this is a serious problem. We suspect that as these suspended particles grow in size, but have not yet exceeded $\sim 10 \mathrm{~nm}$ radius, they can become temporarily neutralized, diffuse into the sheath and occasionally become positively there, then the sheath field drives them into the substrate. It is very likely that voids and carrier traps occur within the a-Si: $\mathrm{H}$ film at the particle-film boundaries, with deleterious consequences on PV efficiency. We are now starting studies to see if it is possible to mitigate this particle incorporation.

The easiest way to detect particulate growth and behavior in the discharge is by light scattering, so we are using this method to investigate how the particle sizes and behavior depends on discharge and deposition parameters, and on particle elimination tricks. Light 
scattering detects larger particles than those that we find incorporated into the film, but it provides a detailed and immediate diagnostic of the particles suspended in the plasma. When complemented with scanning tunneling microscope (STM) studies of particles that have deposited into the film, it provides an excellent basis for developing an understanding of particulate behavior and mitigation techniques.

This year we have used light scattering to investigate particle locations in the discharge as a function of discharge power, temperature, gas flow and pressure. The discharge geometry and the typical location of most particle scattering are shown in Fig.1. The qualitative explanation of this scattering pattern is that a positive ion "wind" pushes particles toward the discharge sheaths, which occur a few $\mathrm{mm}$ in front of the electrodes and at the electrode edges. The strong electric field at the sheaths stops the particles near the sheath edge, where they accumulate and grow in size. Thus, they accumulate primarily near the discharge edges when seen in the top view, and in the central region between the electrodes when viewed from the sides or end. The gas flow also drags particles downstream, so they accumulate primarily at that end of the discharge. As the particle size increases, the drag force increases more rapidly than the charge and electric force, until large particles are dragged away toward the pump. However, for some conditions the charged particles have a collective effect on the discharge sheaths and they are dragged away periodically and collectively. The quantitative behavior is very strongly dependent on discharge power and gas flow speed, and somewhat dependent on pressure and temperature. For example, a factor of two in power, which changes the film growth rate from 0.15 to $3 \mathrm{~nm} / \mathrm{s}$, causes more than an order of magnitude increase in particle scattering, from barely perceptible to a very strong signal. Our chamber has a plug flow speed of $50 \mathrm{~cm} / \mathrm{s}$, which is typical of other facilities, so a similar "threshold" would be expected to generally hold.

In addition to the strong light scattering shown diagrammatical in Fig.1, we detect weaker scattering in the middle of the discharge, the region of primary importance. However, light scattering by small particles varies as the sixth power of the diameter (d), so these signals represent primarily $d>200 \mathrm{~nm}$ particles, not the $\mathrm{d}<20 \mathrm{~nm}$ particles that we find are reaching the growing film [1]. In order to understand the behavior of smaller particles, and to investigate the efficiency of particle mitigation techniques for keeping particles out of the film, we need to clearly detect small particles in the presence of larger particles. For this purpose we are constructing a new light scattering apparatus, based on scattering a pulsed, doubled YAG laser beam and detecting the scatter with video equipment. This multiplexed signal should yield the data necessary to assess the full size distribution for $\mathrm{d}>10 \mathrm{~nm}$ particles, versus position in the discharge. Light scattering from $\mathrm{d}<10 \mathrm{~nm}$ particles is too weak, so we must use the STM to detect these in the film after discharge termination.

\section{2) Cross sectioned PV cells}

Hydrogenated amorphous silicon PV cells contain many layers and junctions of a-Si:H plus its alloys with Ge and C. Efficiency and stability depend on many factors, and efficient cell design requires models that incorporate these as correctly as possible. This part of our program is directed toward obtaining a better understanding of the distributed contributions to 

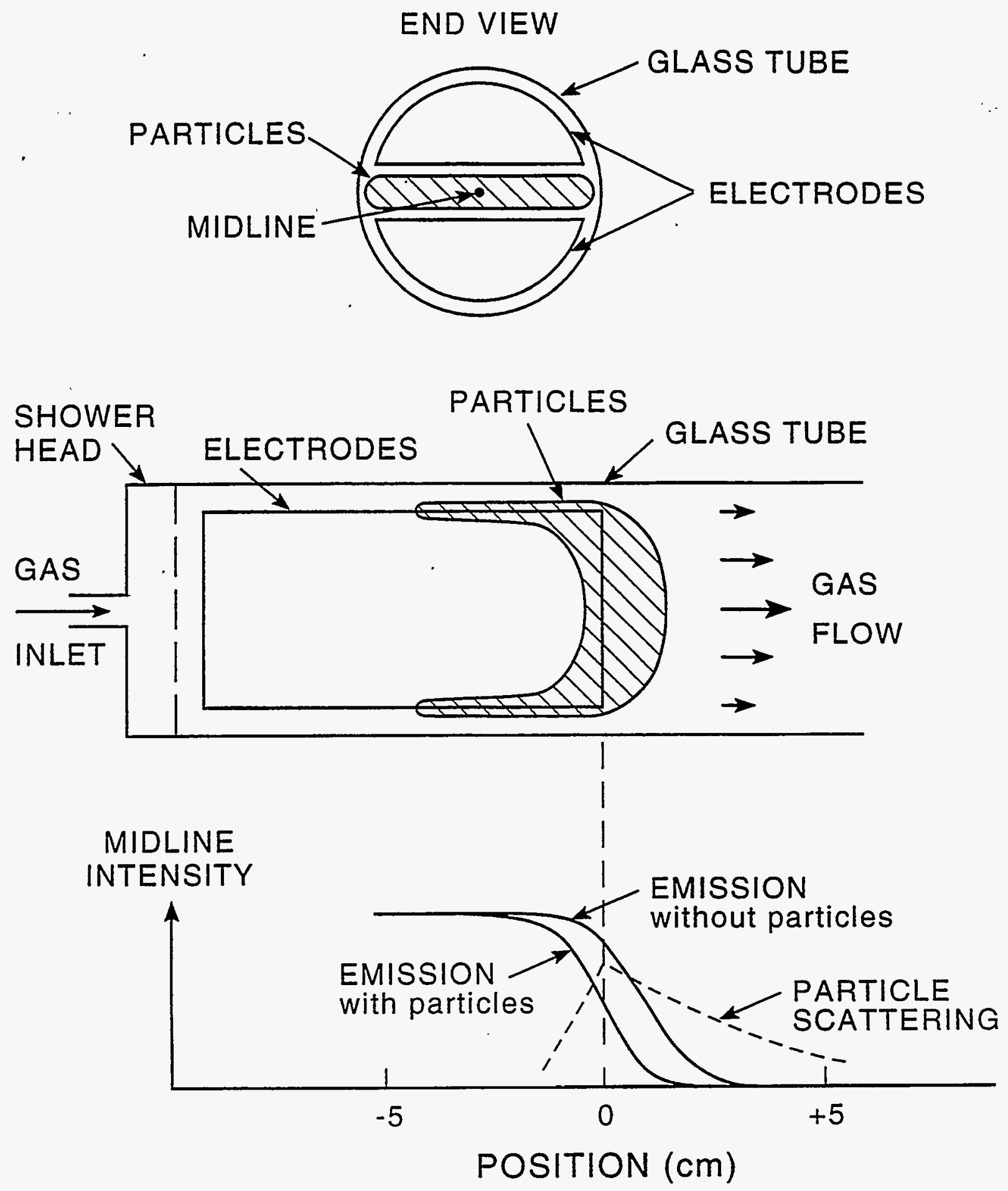

Fig.1. End and top views of the (13.6 Mhz) rf discharge deposition chamber, drawn to scale where the tube diameter is $-6 \mathrm{~cm}$. The position of the strongest particle scattering signals for 30-60 Pa pressure and $~ 3 \mathrm{~nm} / \mathrm{s}$ deposition rate are shown as cross hatching, and the typical intensity distribution is shown in the lower figure. The indicated discharge emission is the dominant, blue $\mathrm{SiH}$ band. 
photo current, photo voltage and light induced degradation in the cells of NREL PV Team manufacturers. We intend to cleave cells in our vacuum chamber and use a STM to measure photo voltages and band properties versus depth through the "cross sectioned" cell. This experiment has been constructed and procedures are being refined. It requires very precise sample positioning under feedback control, over a wide distance range, in order to find the cleaved surface of the cell and achieve tunneling at known positions across the $<1 \mu \mathrm{m}$ thick cell. We have now developed the STM hardware and software needed to achieve these STM manipulations and $\mathrm{i}-\mathrm{V}$ spectroscopy, as well as the optics needed to illuminate the cell surface in the vacuum chamber. We have also developed a STM probe amplifier with IfA current sensitivity, as is required to achieve the band and voltage measurements on the high resistivity intrinsic layer. NREL a-Si:H PV cells are being used to test and trouble shoot the apparatus and procedures.

\section{Conclusions}

It has long been recognized that obtaining high quality a-Si:H PV cells by if discharge deposition from silane requires very low deposition rates, typically ${ }^{-} 0.1 \mathrm{~nm} / \mathrm{s}$. This has serious economic consequences, as it is the primary limit on the throughput of a production line. Efforts to overcome this limitation include using hydrogen diluted silane, higher frequency discharges and thinner layers, and these have been partially successful. However, the reason for this limitation has not been apparent, so it has been difficult to devise major improvements. The data reviewed above shows that increasing the deposition rate above this characteristic value causes a very rapid onset of particle accumulation in the plasma of our deposition chamber, which utilizes typical gas pressures and flow rates. There is a clear implication that particulate growth in the plasma is limiting the acceptable power deposition and resulting film growth rates, particularly when our previous observation of direct particle incorporation into the growing film is noted. This insight should lead to improved deposition rates and perhaps film electronic quality as well, as particle mitigation methods are devised and developed.

\section{References}

1) NREL Phatovoltaic Program EY 1994 Annual Report (June 1995). NREL/TP-410-7993. pp. $187-190$ 


\section{Title: Wide Bandgap Solar Cells With High Stabilized Performance}

\section{Organization: The Pennsylvania State University, University.Park, PA}

Contributors: C.R. Wronski and R.W. Collins: Principal Investigators; Ing-Shin Chen, Lihong Jiao, Sangbo Kim, Joohyun Koh, Yeeheng Lee, Hongyue Liu, and Yiwei Lu: Graduate Assistants

\section{Objectives}

The objectives of this subcontract are to: (i)Develop a cost-effective amorphous silicon PV technology to foster a viable amorphous silicon PV industry in the United States ensuring that this industry remains a world leader in the a-Si technology; (ii)Help the U.S. amorphous silicon PV industry to achieve the U.S./DOE PV Program FY 1995 milestone of $10 \%$ stable efficiency commercial thin-film modules; (iii)Help the U.S. amorphous silicon PV industry to achieve $12 \%$ stable efficiency commercial thin-film modules by 1998; and (iv)Achieve 15\% stable efficiency multi-junction a-Si:H modules for large-scale utility use by the year 2005 .

\section{Scope of Work}

The research in Phase I of this program addresses the following issues:

1. Improved Understanding of Stability in Materials and Solar Cells

2. Intrinsic Materials Optimization

3. Solar Cells Optimized for Intrinsic Layer Performance

4. P-type Layer Optimization

5. Top Cell Interfaces

6. Solar Cell Grading

\section{Results and Discussion}

\section{Improved Understanding of Stability in Materials and Solar Cells}

Real time in situ spectroscopic ellipsometry (RTSE) measurements and ex situ optical and electrical measurements were carried out to optimize a-Si ${ }_{1 \cdot x} \mathrm{C}_{\mathrm{X}}: \mathrm{H}$ and a-Si:H materials for use as the $i$-layer (absorber) in widegap solar cells. Detailed characterization of both the annealed and stabilized degraded state a-Si ${ }_{1-x} C_{X}: H$ films, deposited with hydrogen dilution at substrate temperatures between $50^{\circ} \mathrm{C}$ and $300^{\circ} \mathrm{C}$, were completed.

Progress has been made in the understanding of the relationships between bulk properties of intrinsic materials and $p-i-n$ solar cell performance. Detailed studies were carried out on n-i-metal Schottky barrier devices including i-layers which are thick enough so that the device characteristics are less sensitive to interface effects. Greater 
improvement in self-consistent modeling of different characteristics was obtained with midgap distributions of charged and neutral defects consisting of three Gaussian distributions, rather than with the commonly used two Gaussian distributions, such as those in modeling of $p-i-n$ 's using AMPS. Progress has also been made in obtaining the relationships between bulk parameters and $p-i-n$ solar cell performance.

\section{Intrinsic Materials Optimization}

Widegap materials using conventional PECVD were optimized and the degradation and relative stabilities of different widegap a- $\mathrm{Si}_{1 \cdot \mathrm{x}} \mathrm{C}_{\mathrm{X}}: \mathrm{H}$ materials were compared to those of high H-content materials. RTSE and ex situ photoelectric measurements were employed to optimize $\mathrm{a}_{-} \mathrm{Si}_{1-\mathrm{x}} \mathrm{C}_{\mathrm{X}}: \mathrm{H}$ alloy films for use as the absorber layers in widegap solar cells. The results of measurements on the thickness of surface roughness of $\mathrm{a}-\mathrm{Si}_{1-\mathrm{x}} \mathrm{C}_{\mathrm{X}}: \mathrm{H}$ alloy films by RTSE were independently correlated with ex situ atomic force microscopy (AFM). The results clearly demonstrated that RTSE provides the correct description of the surface thickness of the roughness and is in direct proportion to the rms roughness deduced by AFM.

The growth process for widegap a-Si:H prepared with $\mathrm{H}$-dilution of $\mathrm{SiH}_{4}$ was improved based on the overall conclusion that the best a-Si:H is obtained just within the $\mathrm{H}$-dilution boundary between the growth of amorphous and mixed-phase amorphouscrystalline $\mathrm{Si}$ (as was concluded earlier for $\mathrm{a}-\mathrm{Si}_{1-\mathrm{x}} \mathrm{C}_{\mathrm{X}}: \mathrm{H}$ ).

The studies carried out on $\mathrm{Si}$-rich $\mathrm{a}-\mathrm{Si}_{1-\mathrm{x}} \mathrm{C}_{\mathrm{x}}: \mathrm{H}$ materials fabricated at Solarex and Penn State show that the annealed properties of the films produced with hydrogen dilution over a wide range of $T_{S}$, are close to those of a-Si:H. However their stabilized degraded states are similar to those of the undiluted films. The differences in the degradation between a-Si:H and $\mathrm{a}-\mathrm{Si}_{1 \cdot \mathrm{X}} \mathrm{C}_{\mathrm{X}}: \mathrm{H}$, if accurately modeled, may yield some new insights into the Staebler-Wronski Effect (SWE).

\section{Solar Cells Optimized for Intrinsic Layer Performance}

Procedures were developed for analysis of real time spectroscopic ellispometry (RTSE) data on full $\mathrm{SnO}_{2}: \mathrm{F} / p-i-n$ solar cells prepared in a single-chamber system. In the RTSE analysis, the thickness and optical gaps of the materials are obtained in the actual cell configuration, rather than being inferred from studies on thick layers. In gerenal, results reflect expectations based on thick layer studies, except for an unanticipated $p / i$ interface contaminant layer that forms during single-chamber purging between $p$ - and $i$ layer. We have fabricated a series of solar cells using $\sim 2000 \AA$ widegap a:Si ${ }_{1 \cdot x} \mathrm{C}_{\mathrm{X}}: \mathrm{H}$ $(\sim 1.95 \mathrm{eV}) i$-layers prepared at different $\mathrm{H}$-dilution ratios under otherwise identical conditions. These cells were made with processing procedures similar to those for the midgap baseline cell, the only exception being the use of trimethyboron (TMB) as the $p$ layer doping source gas in an attempt to reduce $p / i$ interface contamination. Cells deposited on specular $\mathrm{SnO}_{2}: \mathrm{F}$ yielded the best combination $\mathrm{V}_{\infty}(0.92 \mathrm{~V})$ and fill factor $(0.67)$. The effective interface contaminant layer for the highest efficiency cell is $\sim 0.6 \AA$, an 
improvement attributed to an optimized interface formation process, including use of TMB as the $p$-layer doping source gas as well as the beneficial effect of striking a $\mathrm{H}_{2}$-rich plasma on the $p$-layer surface.

\section{P-type Layer Optimization}

A TMB capability has also been established for the preparation of our widegap $p$-type layers in both multi-and single-chamber systems. For widegap $p$-layers formed both from diborane and TMB, any increase in gap with carbon incorporation is offset by a roughly equivalent increase in the conductivity activation energy. The only materials that we have observed deviating from such offsetting behavior are a-Si:H:B films whose gaps have been widened by post-hydrogenation with filament-generated atomic hydrogen. We have tried to incorporation such materials into $p$-i-n solar cells on $\mathrm{SnO}_{2}$ :F-coated glass substrates; however, the H-treatment is observed to lead to a degradation of the TCO/p interface. Further work will focus on $\mathrm{ZnO}$ TCO substrates in an attempt to eliminate the problem.

The quality of the standard Penn State TMB a-Si ${ }_{1-x} \mathrm{C}_{x}: \mathrm{H} p$-layers prepared in our multi-chamber system have been compared to those of Solarex using $p-i-n$ structures, without graded layers, deposited on specular TCO substrates. The Penn State cell with a TMB a-Si $1_{1-\mathrm{x}} \mathrm{C}_{\mathrm{X}}: \mathrm{H} p$-layer has $\mathrm{V}_{\mathrm{OC}}=0.92 \mathrm{~V}, \mathrm{~J}_{\mathrm{SC}}=9.6 \mathrm{~mA} / \mathrm{cm}^{2}$, and $\mathrm{FF}=0.69$ under $\mathrm{AM} 1$ light while the corresponding cell incorporating the Solarex $\mathrm{TMB}^{\mathrm{a}}-\mathrm{Si}_{1 \cdot \mathrm{X}} \mathrm{C}_{\mathrm{X}}: \mathrm{H}$ p-layer has $V_{O C}=0.88 \mathrm{~V}, \mathrm{~J}_{S C}=9.6 \mathrm{~mA} / \mathrm{cm}^{2}$, and $\mathrm{FF}=0.58$. The lower $\mathrm{V}_{O C}$ and $\mathrm{FF}$ can be due to the fact that Solarex $p$-layers were exposed to air much longer than Penn State $p$-layers and the H-treatments were not optimized

We have also started to investigate $n-i-p$ solar cell structures prepared on stainless steel and $\mathrm{SnO}_{2}$ substrates in our multi-chamber system. Stainless steel $/ n-i$ structures have been fabricated with our $\mathrm{a}^{-} \mathrm{Si}_{1-\mathrm{x}} \mathrm{C}_{\mathrm{X}}: \mathrm{H} p$-layers or $\mu c$-Si $p$-layers from USSC with both semi-transparent $\mathrm{Cr}$ and specular TCO top contacts. A procedure has been established for obtaining the $\mathrm{V}_{o c}$ with the $10 \%$ transparent $\mathrm{Cr}$ contacts which corresponds to AM1 illumination of the transparent TCO structures. Comparisons have been made between cells with a-Si ${ }_{1-x} C_{X}: H$-layers and $\mu c$-Si $p$-layers and $\mathrm{TCO}$ as top contacts deposited at USSC on the same batch of stainless steel $/ n-i$ structures. Thus far the highest $V_{o x}$ 's were obtained with hydrogen diluted a-Si:H layers deposited at $170^{\circ} \mathrm{C}$. The cell structures, with no graded layers and USSC $\mu \mathrm{c}$-Si $p$-layers had a $\mathrm{V}_{\propto C}$ of $0.96 \mathrm{~V}$, about $90 \mathrm{mV}$ higher than the corresponding $n-i-p(\mathrm{a}-\mathrm{SiC}: \mathrm{H})$ structure. This value is very close to the highest $\mathrm{V}_{\propto C}$ obtained for an all a-Si $i_{1 \cdot x} C_{x}: H$ cell fabricated in a single-chamber system at Penn State and monitored by RTSE. Fabrication of $n-i-p(\mu c-S i)$ solar cells is now being undertaken at Penn State and the stability of the $p-i-n$ and $n-i-p$ structures with different $p$-layers is being investigated.

\section{Top Cell Interfaces}

Techniques are continuing to be developed for analysis of RTSE data collected during the preparation of the top junction $\left(\mathrm{SnO}_{2} / p-i\right)$ of the a-Si:H $p-i-n$ solar cells in our 
single-chamber system. The monolayer-by-monolayer, optical fingerprint of standar cell preparation using a diborane-based $p$-layer on specular $\mathrm{SnO}_{2}$ has been established. The optical fingerprint deduced from RTSE included the evolution of the bulk and roughness layer thicknesses, and the dielectric functions and optical gaps for the $p$ - and $i$-layers, as well as the characteristics of the contaminant layer that forms between the two layers during chamber flushing. Using the optical fingerprint to guide $p / i$ interface formation precedure, optimization of the standard midgap cell has been carried out. The resulting further improvements yielding efficiencies as high as $8.4 \%$ are establishing a baseline for (i)incorporating widegap $i$-layers and (ii) for testing $p$-layer improvements.

The $p / i$ interface structures of the solar cells fabricated with $p$-layers doped with either diborane or TMB, but otherwise prepared similarly, have been compared. Thinner interface layers have been achieved with an optimum $p$-layer doped with TMB $(0.6 \AA)$ versus diborane $(1.7 \AA)$ and the highest efficiency midgap and widegap a-Si ${ }_{1} \times \mathrm{x} \mathrm{C}_{\mathrm{x}}: \mathrm{H}$ cells ( $\sim 8.4 \%$ and $3.9 \%$, respectively), were obtained when the thinnest interface layers were observed. It has been found in the RTSE studies of $\mathrm{SnO}_{2}: \mathrm{F}$ and $\mathrm{a}-\mathrm{Si}_{1 \cdot \times} \mathrm{C}_{\mathrm{x}}: \mathrm{H} p$-layer exposure to $p$-type gases that there is no thermal decomposition in the case of TMB, indicating why TMB has led to further reduction of contamination at the $p / i$ interface.

\section{Solar Cell Grading}

Procedures have been developed to fabricate and characterized layers prepared using continuouly varying $\mathrm{H}_{2}$-dilution. Such layers exhibit a continuously varying void volume fraction with thickness, and because of the non-linear dependence of growth rate on $\mathrm{H}_{2}$-dilution, it is difficult to determine and control the thickness of the deposited layer. Using RTSE, we are able to determine the accumulated thickness vesus time and the void volume fraction vesus thickness, and thus establish the protocol for controlling these layers. Such an approach will be incorporated into either the $p$-layer or buffer layer process in widegap cells, designed so as to minimize TCO damage. Hydrogen-dilution grading has been employed in attempts to improve the open-circuit voltages of our optimum widegap solar cells prepared on specular $\mathrm{SnO}_{2}: \mathrm{F}$. The capability of characterizing the optical gap profile versus thickness for widegap $p / i$-interface buffer layers using RTSE has also been developed. The incorporation of an optimized widegap buffer layer into our widegap a-Si $i_{1 \times x} \mathrm{C}_{\mathrm{X}}: \mathrm{H}$ solar cells on specular $\mathrm{SnO}_{2}: \mathrm{F}$ has led to an increase in $V_{O C}$ from 0.93 to the highest value of $0.96 \mathrm{~V}$. However there was a corresponding drop in the fill factor from 0.62 to 0.60 which resulted in the same initial cell efficiency: We are currently characterizing the carbon concentration profiles and optical gap profiles of buffer layers which have resulted in the increase of initial efficiencies by $20 \%$ for cells prepared on ZnO TCO's 
Organization: $\quad$ School of Electrical and Computer Engineering

Purdue University

West Lafayette, IN 47907-1285

Contributors: $\quad$ J. L. Gray and R. J. Schwartz, principal investigators;

Y. J. Lee, graduate student

\section{Objective}

The purpose of this research program is to develop an accurate numerical model for $\mathrm{CuInSe}_{2}$ (CIS) and CdTe based solar. The model, ADEPT (A Device Emulation Program and Tool), is used to analyze and aid in the design of CIS and CdTe based solar cells and is being released to the photovoltaic community. An additional objective is to use the model to analyze existing devices and to predict performance.

\section{Approach}

ADEPT has been demonstrated to be a useful tool for interpreting the performance of existing solar cells. The accuracy of ADEPT in predicting cell performance depends on several factors. In well understood material systems, such as crystalline, the predictive ability of detailed device simulation codes is quite good. In thin film devices, such as CIS and CdTe based solar cells, the predictive ability of these codes is not as good. Basic characterization of these materials is still an active area of research and is complicated by variations in the material parameters resulting from fabrication methods and even different facilities. In a graded band gap structure these difficulties are multiplied.

ADEPT generates a numerical solution to a set of three coupled partial differential equations commonly referred to as the semiconductor equations. These are Poisson's equation and the hole and electron continuity equations. Coupled with auxiliary equations for the displacement field and carrier currents, these are solved for the electrostatic potential and the hole and electron concentrations on a domain defined by the device structure subject to appropriate boundary conditions and the device operating conditions.

\section{Status}

Version 3.0 of ADEPT for IBM compatibles is presently available. Improvements over the previous versions include graphical display of parameters (in addition to the ability to export parameter tables to other plotting programs), enhanced user definition of absorption coefficients, greatly improved user documentation, as well as correction of reported bugs in previous versions. 
Title:

Qrganization:

Contributors:
Research on High Efficiency, Large Area CuInSe ${ }_{2}$-Based Thin Film Modules

\author{
Siemens Solar Industries \\ Camarillo, California
}

\section{Introduction}

The objective of this research effort is to achieve progress toward the polycrystalline thin-film milestones of the DOE Five Year Plan. SSI began this 3-year, 3 phase cost-shared contract on May 1, 1991 with the overall project goal of fabricating a large area, stable, $12.5 \%$ aperture efficient encapsulated CIS module by scaleable, low-cost techniques on inexpensive substrates. The SSI report in the "NREL Photovoltaic Program FY 1994 Annual Report" (AR94 report) discussed work into November of 1994. That report summarized progress through the entire contract, and discussed conclusions and proposed further directions. This report recaps the progress through the entire contract and provides an update for the period from November of 1994 through the end of the contract on April 15, 1995.

\section{Recap - Results Through FY 1994}

In Phase 1, the emphasis of the research effort was on establishing the baseline large area CIS module fabrication processes including detailed characterization of the cells and interconnects. Environmental durability of laminated and framed large-area CIS modules was also demonstrated [1].

In Phase 2, the initial focus was to implement the processing and characterization methods demonstrated in Phase 1 to meet the contract deliverables schedule and to produce statistically significant quantities of large area modules for outdoor and accelerated environmental testing. Over $23001 \times 4$ foot CIS modules were fabricated. Although the process produced high-efficiency cells and modules, average performance was not adequate for fabrication of representative prototype products. The origins of module reproducibility problems were unclear. Large area module fabrication was temporarily suspended to determine the critical issues affecting yield losses in module fabrication by using small area test devices ( $3.3 \mathrm{~cm}^{2}$ active area cells) and modules $\left(60 \mathrm{~cm}^{2}\right.$ aperture area) as the experimental test bed. This shift greatly enhanced the ability to identify root causes of yield loss in module fabrication. Uniformity and reproducibility of the absorber formation process was addressed through the adoption and further refinement of a graded absorber formation process in which the surface layer of CIS incorporates sulfur [2], combined with the appropriate substrate preparation. Performance losses near interconnects were addressed by developing alternate techniques for fabricating interconnects. Interconnects were made after the formation of the absorber by application of insulating and/or conducting materials [3].

Phase 3 efforts through November 1994 (AR94 report) continued on the critical issues defined in Phase 2. Substrate and base electrode preparation techniques were developed which minimize the number of defects that lead to shunting, areas of poor photoresponse and losses near interconnects. These Phase 3 techniques were distinct from the techniques developed in Phase 2; modifications of the reaction process were necessary to avoid loss of adhesion with Phase 3 substrate and back electrode preparation techniques. The combined effect of improvements in substrate/base electrode preparation techniques and reaction process eliminated the need for the alternate patterning techniques developed in Phase 2. This 
allowed the return to the more production capable interconnect formation method without the need for application of insulating and/or conducting materials.

\section{Continual Progress Update}

As introduced in the AR94 report, the major challenge to move from development to production is to improve reproducibility rather than to improve champion device performance. Therefore, emphasis has been placed on reduction of process variation. The reduction of variation efforts are based on the application of the statistical process control (SPC) discipline, improved diagnostics, and thorough protocols. Application of the SPC discipline is the foundation of progress from the last report (AR94) through the end of this contract. This progress is discussed following a brief overview of the SPC discipline.

Application of Statistical Process Control (SPC) and Analysis of Variation (ANOVA) methods has been the key aspect of the reduction of variation efforts. This approach requires a significant change of orientation, with the focus upon continual reduction of variation in processes rather than upon higher efficiency per se. The SPC discipline provides a means for discerning between the natural random variation inherent in a stable process and variation which indicates that a process has become unstable and that a specific, assignable cause is responsible for the instability. Identifying and eliminating the "special causes" responsible for these observations lead to improved predictability and performance. The first goal is the attainment of stable processes, demonstrated by achieving a good level of statistical control, while further improvement of the average performance level is made easier by the creation of this stable basis. Predictability, as judged by SPC criteria, is a critical issue for accessing production capability. Predictability is necessary for confidence in the results of experiments designed to improve a process, confidence in scale-up of a process, and required for the commitment to move a process from development to production. Accompanying the statistical tools are improved process definition and documentation aimed toward the ISO 9000 standard, improved feedback from individual process steps, and systematic approaches to problem solving and communication of process information. Continued training of all personnel is also a key to successful implementation.

Progress in addressing transient effects and improving the reproducibility of the CdS dip process will be discussed as examples of the SPC discipline. CIS based modules have demonstrated stability in field conditions. However, transient changes in module performance are often observed for the thermal exposures encountered during lamination or accelerated environmental testing. Transient effects induced by lamination and accelerated environmental testing, and effects such as sensitivity to light exposure history and sweep rates during current versus voltage testing, are assumed to be related. These transient effects must also be considered when performing or defining procedures for accelerated environmental testing since accelerated testing may activate mechanisms that are not dominant in service. Any transient effects such as these are an issue when considering predictability as it relates to a production process and commercialization.

Transient changes in module performance are often observed for the thermal exposures encountered during lamination or accelerated environmental testing. Data typical of the early Phase 3 generation of processes indicated that efficiency after lamination may range from $65-85 \%$ of the efficiency prior to lamination. Efficiency changes are dominated by changes in FF. In most cases, efficiency improves with time after lamination and approaches or exceeds the initial efficiency. This "recovery" in efficiency is typically accelerated by light exposure [4]. Lamination procedures with shorter heating times and changes in part handling were developed since the AR94 report. A group of mini-module laminated with these procedures showed a much smaller transient lamination loss averaging 3 to $4 \%$. However, 
statistical process control (SPC) techniques indicate the process is not "in control" (discussed below) as indicated by sporadic results outside the "control limits" in Figure 1.

Within the SPC discipline, proper selection of the right scheme for sampling the output of a process allows data from any process to be charted versus time and "Control Limits" to be calculated from the process data itself. These control limits are the horizontal lines in Figure 1 labeled UCL for Upper Control Limit and LCL for Lower Control Limit. A continued random trend within these limits is a good indicator that the process is stable over time. Since some data in Figure 1 fall outside the control limits the process is not "in control." Low lamination losses have been demonstrated and may be typical but are not-necessarily predictable.

The largest source of process variation reported for work through November (AR94) was due to the block of process steps from CdS dip through final patterning (Figure 2, "Group 0"). The second largest source of variation is from the block of process steps prior to the reaction process. The reaction process and testing introduce relatively low variation for this data set. Based on this initial result, the flow of parts was defined to allow separation or deconvolution of the CdS process from other processes by assuring that $\mathrm{CdS}$ deposition groups were composed of parts from multiple groups for all other process steps. In addition, several aspects of the CdS deposition process were evaluated. ANOVA analysis indicated that a systematic difference in the CdS deposition procedure was introducing variation. When this systematic difference is mathematically removed the variation due to $\mathrm{CdS}$ was reduced as depicted by Group 1 in Figure 2.

A subsequent group of over 100 mini-modules (Figure 2., Group 2) was processed with improved CdS procedures. ANOVA indicates $85 \%$ reduction in variation. This example illustrates how implementation of SPC techniques have been used successfully to identify the source of process variation and then demonstrate reduced process variation. The SPC methodology applies statistical analysis and a framework for systematic experimentation, analysis, and interpretation of data. Process steps with the largest impact on performance variation are addressed before addressing process with less impact on variation. The approach is iterative. Reduction of variation efforts continue with emphasis placed on the next largest source of performance variation.

\section{Contract Summary}

Siemens Solar Industries (SSI) has pursued the research and development of CuInSe ${ }_{2}$ (CIS) thin film PV technology since 1980. Prior to this contract, SSI had demonstrated a $14.1 \%$ efficient $3.4 \mathrm{~cm}^{2}$ active-area $\mathrm{ZnO} /$ thin $\mathrm{CdS} / \mathrm{CIS} / \mathrm{Mo} /$ glass cell, unencapsulated integrated modules with aperture efficiencies of $11.2 \%$ on $940 \mathrm{~cm}^{2}$ and $9.1 \%$ on $3900 \mathrm{~cm}^{2}$ and an encapsulated module with $8.7 \%$ on $3883 \mathrm{~cm}^{2}$ (verified by NREL)[3]. During the present phase of this contract, record unencapsulated mini-module efficiencies of $12.7 \%$ on $69 \mathrm{~cm}^{2}$ (verified by NREL) [5] and encapsulated $12.8 \%$ on $68.9 \mathrm{~cm}^{2}$ have been demonstrated. This result meets the efficiency goal for the contract and is the highest module efficiency for any thin

film technology. Large area $\left(3860 \mathrm{~cm}^{2}\right)$ encapsulated module aperture area efficiency of $10.3 \%$ has also been demonstrated (verified by NREL). Outdoor testing of SSI CIS modules at NREL over a period of more than six years has demonstrated excellent outdoor module stability.

\section{Conclusions and Future Directions}

The foundations have been laid to meet the polycrystalline thin-film milestones of the DOE Five Year Plan. The efficiency goal of the contract has been met with the demonstration of a record encapsulated $12.8 \%$ efficient module on $69 \mathrm{~cm}^{2}$. Outdoor testing has demonstrated excellent intrinsic module 
stability. Future plans include scaling these results to larger areas and emphasizing the reduction of variation methodology to lay the foundation for demonstrating the potential of CIS as a future commercial product.

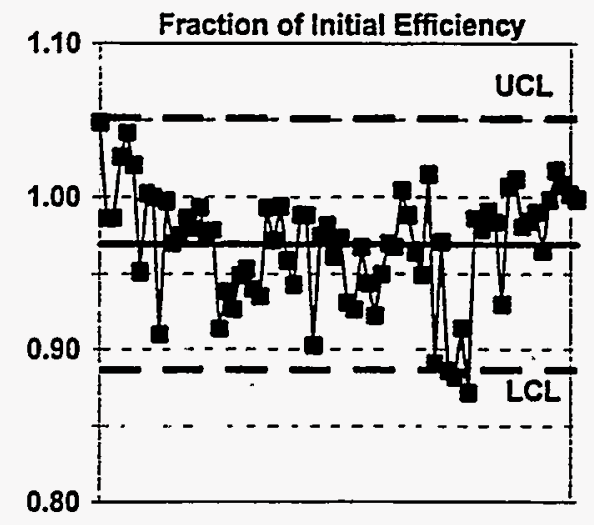

Figure 1.) Statistical process control chart for lamination

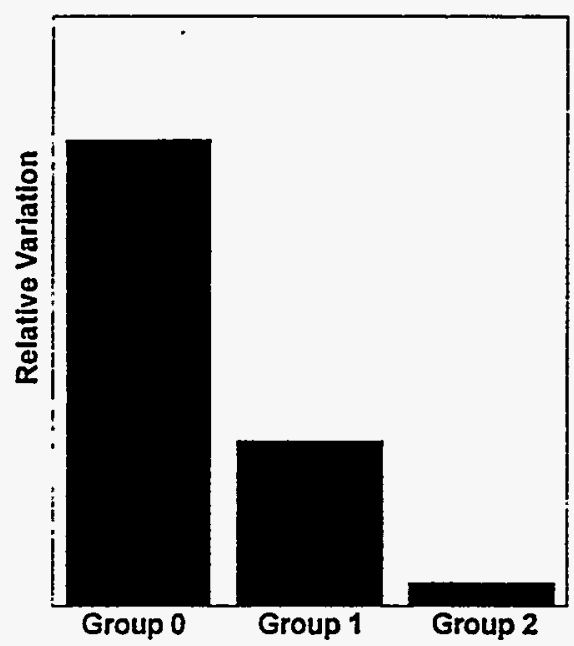

Figure 2.) $85 \%$ reduction in variation illustrating how implementation of SPC techniques has been used successfully to identify the source of process variation and then to reduce process variation.

\section{References}

1.) C. Eberspacher, et al, Annual Report: Photovoltaic Subcontract Program FY 1992, NREL/TP-4105335, March 1993, pp. 138a-138d.

2.) D. Tarrant, J. Ermer, "I-III-VI ${ }_{2}$ Multinary Solar Cells Based on CuInSe ${ }_{2}$ ", 23rd IEEE PVSC, 1993, pp. 372-378.

3.) K. Knapp, et al, "Research on High Efficiency, Large-Area CuInSe 2 -Based Thin-Film Modules", Annual Report, NREL Photovoltaic Program FY 1993, NREL/TP-410-6585, pp 110-113.

4.) D. Willett, S. Kuriyagawa, "The Effects of Sweep Rate, Voltage Bias and Light Soaking on the Measurement of CIS-Based Solar Cell Characteristics", 23rd IEEE PVSC, 1993, pp. 495-500.

5.) R. Gay, et al, "Efficiency and process Improvements in CulnSe ${ }_{2}$-Based Modules", 12th European Photovoltaic Solar Energy Conference, April 1994, pp 935-938. 
Title: Technology Support for Initiation of High Throughput Processing of Thin-Film CdTe PV Modules

Organization: $\quad \begin{aligned} & \text { Solar Cells, Inc. } \\ & \text { Toledo, Ohio }\end{aligned}$

Contributors: R. Sasala, T. Zhou, R. Powell, G. Dörer, G. Rich, M. Steele, K. Bihn, R. Harju, N. Reiter

\section{Objective}

The overall goal of the project is the improvement of process and prototype product improvement of $\mathrm{CdTe}$ based photovoltaic modules. Specifically targeted areas are 1) further simplification and cost reduction of the existing semiconductor deposition process, 2) increased conversion efficiency, and 3) enhancement of cell and module stability.

\section{Approaches}

Process simplification has been approached by 1) transferring laboratory scale results of $\mathrm{HCl}$ post-deposition treatment to pilot production scale processing, and 2) initiating a pilot scale development program aimed at establishing the commercial feasibility of depositing both $\mathrm{CdS}$ and $\mathrm{CdTe}$ at atmospheric pressure. Increases in the conversion efficiency have been approached by reducing the $\mathrm{CdS}$ thickness as well as incorporating an intrinsic $\mathrm{SnO}_{2}$ on top of the TCO in order to maintain open circuit voltage. Cell and module stability was improved by testing a variety of interfacial layers between the CdTe and the back metal and verified by extending monitoring of light soaked samples to times past 15,000 hours.

\section{Results}

Solar Cells INC. has been very successful at reducing the time required to deposit the CdS and CdTe films on $7200 \mathrm{~cm}^{2}$ substrates in less than one minute which is required to boost annual throughputs to the $10 \mathrm{MW}$ level. However, other steps in the production of a finished module conventionally take a significant length of time. One such step is the wet $\mathrm{CdCl}_{2}$ post-deposition heat treatment which involves several time consuming steps that appear to pose difficulties to bring to full-scale production levels. This difficulty has been overcome by the development of a vapor $\mathrm{HCl}$ treatment which has been shown to have a similar influence on device and module performance. The treatment has reduced the process time by a factor of three thus far and has promise for even further reductions as the process is optimized. Figure 1 shows that the $\mathrm{HCl}$ process yields similar product as the $\mathrm{CdCl}_{2}$ treated samples. Batches $0-16$ were treated in the conventional manner and batches 17-26 were treated with $\mathrm{HCl}$. After the initial optimization was complete, the efficiencies are comparable. In addition to reduced processing time, the $\mathrm{HCl}$ treatment is easier to scale up because it is a dry process which lends itself to conventional gas handling equipment and a conventional conveyance system. 
During the past fiscal year, there has been ongoing work on improvement of device efficiency, primarily on the cell and submodule levels. The primary power losses overwhich we have the most direct control is optical loss in the CdS. Samples have been made with progressively thinner $\mathrm{CdS}$ to determine the optimum thickness. The tradeoff of increasing the optical transmission is a loss in open-circuit voltage. One technique which can reduce the voltage loss by as much as $100 \mathrm{mV}$ is to deposit a thin layer of intrinsic $\mathrm{SnO}_{2}$ on the low resistivity $\mathrm{SnO}_{2}$ which is commercially available. It is unclear what the mechanism is that increases the voltage. The best efficiency results that have been achieved in the past year and confirmed at NREL on $1.1 \mathrm{~cm}^{2}$ cells is $12.6 \%$ without an antireflection coating and $12.9 \%$ with a $\mathrm{MgF}$ antireflection coating. The I-V curve taken at NREL is shown in Figure 2. Progress has also been made in increasing submodule efficiency. The best aperture area efficiency achieved was $10.7 \%$ as confirmed by NREL and displayed in Figure 3.

\section{Average Module Performance \\ Solar Cells, Inc.}

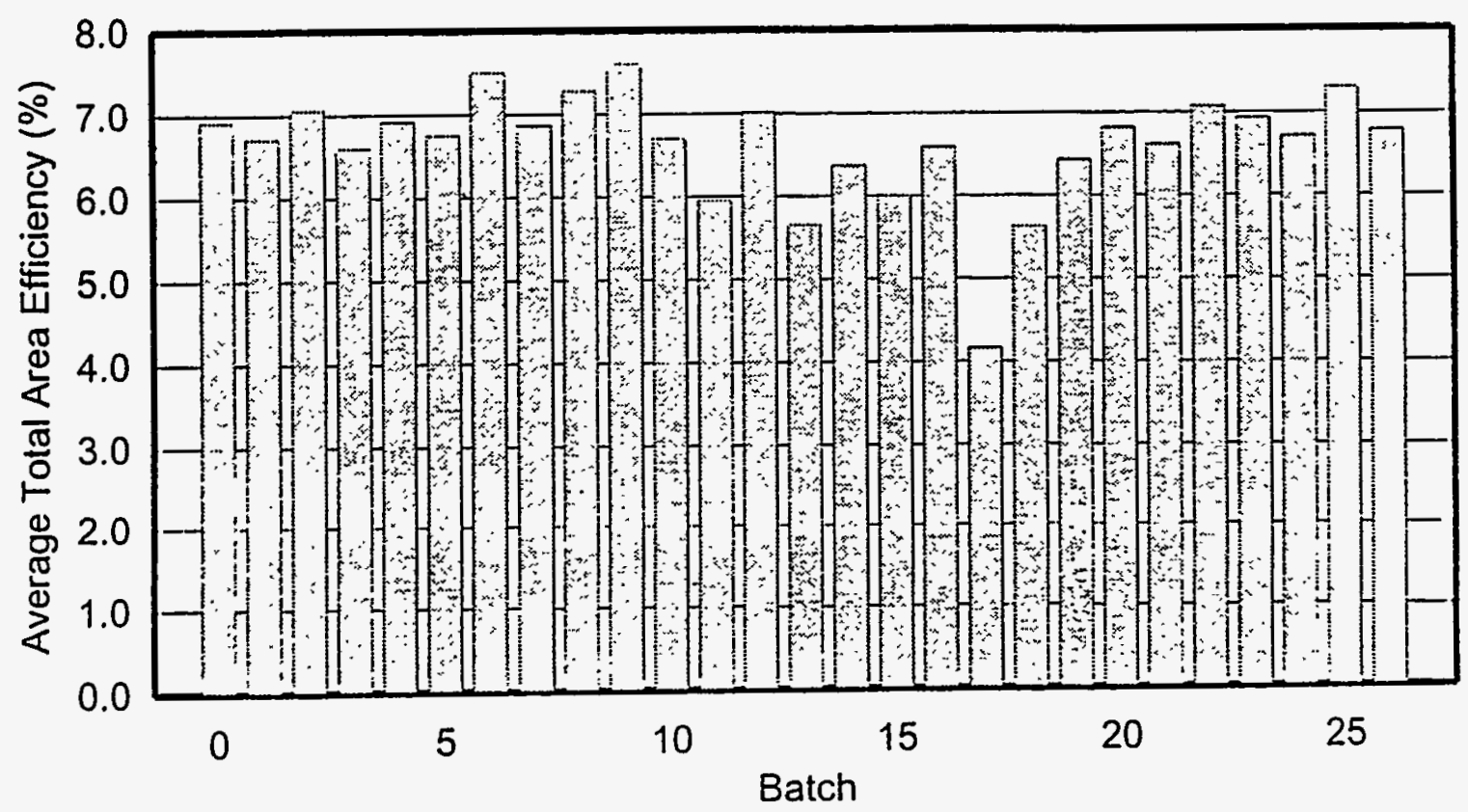

Figure 1. Average module efficiency for modules treated with conventional $\mathrm{CdCl}_{2}$ are shown from batch 0-16 compared to modules treated with $\mathrm{HCl}$ in batch 17-26.

Work has also continued during the past fiscal year on the improvement and monitoring of device and submodule stability. Test samples have been exposed to continuous $80 \mathrm{~mW} / \mathrm{cm}^{2}$ illumination which gives an acceleration factor of about five over actual day / night cycles. Data shown in Figure 4 are for a group of samples that were made by the standard process. The points shown are an average of 6 samples all made in nominally the same manner and were held near the maximum power point while illuminated. The stability shown to 16,000 hours represents about eight years of field use and can be 
extrapolated to maintaining over $90 \%$ of its original efficiency for an equivalent of more than 10 years of field use.

\section{Future Work}

Solar Cells INC. Plans to continue improve cell and module efficiency by further optimization of the thin $\mathrm{CdS}$ for small cells and to modify deposition parameters to achieve better uniformity on large area devices. The optimization of thin $\mathrm{CdS}$ will be aided by experiments designed to further understand the mechanism for voltage degrade and the reason that a high resistivity $\mathrm{SnO}_{2}$ layer partially circumvents the loss. Work will continue on the long-term monitoring of submodules as well as how to extrapolate the data to field use conditions with a higher degree of confidence.

Solar Cells Inc. CdS/CdTe

Sample: B14321H2\#72

May 9, 1995 11:44 AM

ASTM E 892-87 Global
Solar Cells Ine CdTe Minimodule

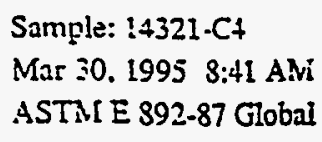

Area $=1.143 \mathrm{~cm}^{2}$ Iradiance: $1000.0 \mathrm{Wm}^{-2}$

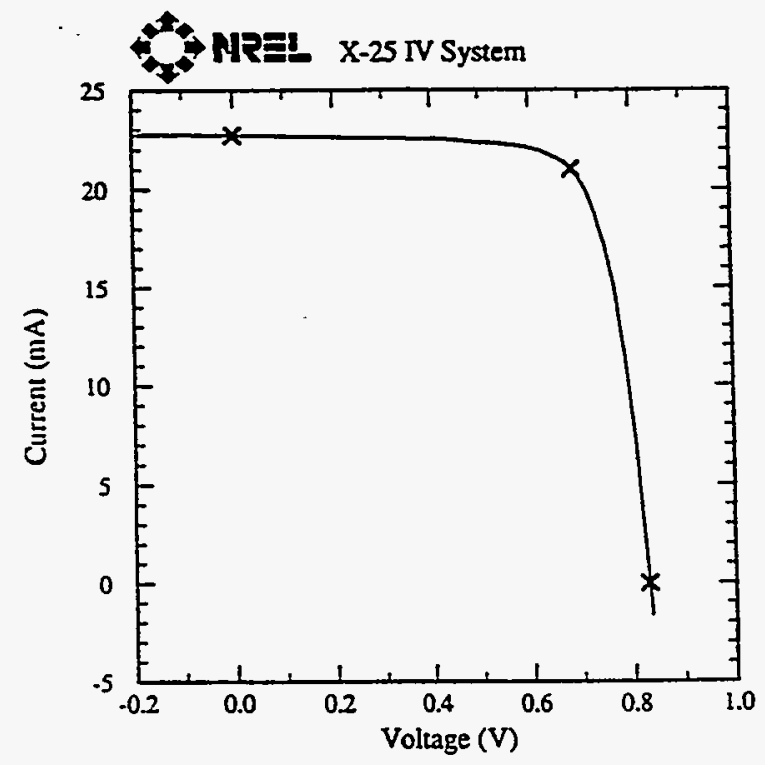

Temp̧enture $=25.0^{\circ} \mathrm{C}$
Area $=63.87 \mathrm{~cm}^{2}$
Imadiance: $1000.1 \mathrm{wm} \cdot 2$

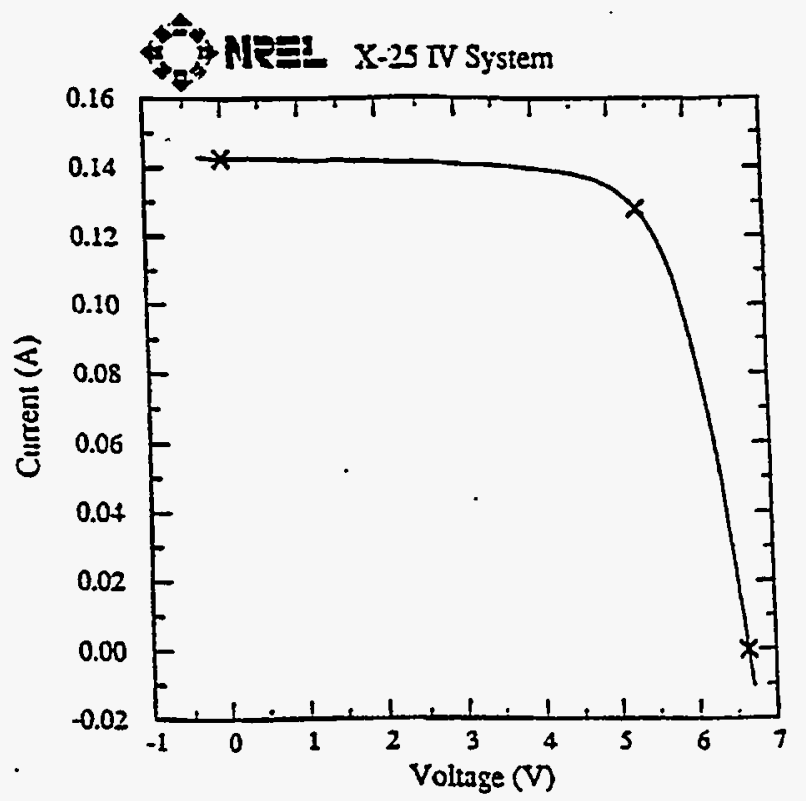

$\mathrm{V}_{\propto}=0.8286 \mathrm{~V}$

$I_{s c}=22.71 \mathrm{~mA}$

$\mathrm{J}_{\mathrm{sc}}=19.87 \mathrm{mAcm}^{-2}$

Fill Factor $=76.56 \%$

$$
\begin{aligned}
& \mathrm{V}_{\max }=0.6854 \mathrm{~V} \\
& I_{\max }=21.02 \mathrm{~mA} \\
& P_{\max }=14.40 \mathrm{~mW} \\
& \text { Efficiency }=12.6 \%
\end{aligned}
$$

$$
\begin{aligned}
& V_{\propto}=6.641 \mathrm{~V} \\
& I_{s c}=0.1425 \mathrm{~A} \\
& J_{s e}=2.231 \mathrm{mAcm}^{-2} \\
& \text { Fill Factor }=72.11 \%
\end{aligned}
$$

Figure 2. I-V curve and critical parameters of highest efficiency $1.1 \mathrm{~cm}^{2}$ total area cell.

Figure 3. I-V curve and critical parameters of highest efficiency $64 \mathrm{~cm}^{2}$ total area submodule. 
Other future work will be geared towards further developing manufacturing processes which can be used to produce high volumes of modules. Specific areas of work include 1) atmospheric pressure deposition of the semiconductors, 2) reduction of time for key postdeposition processing including the $\mathrm{HCl}$ treatment, 3 ) demonstration of a commercially viable alternative interconnection technique which is less dependent on the sheet resistance of the TCO and eliminates the need for laser scribing systems.

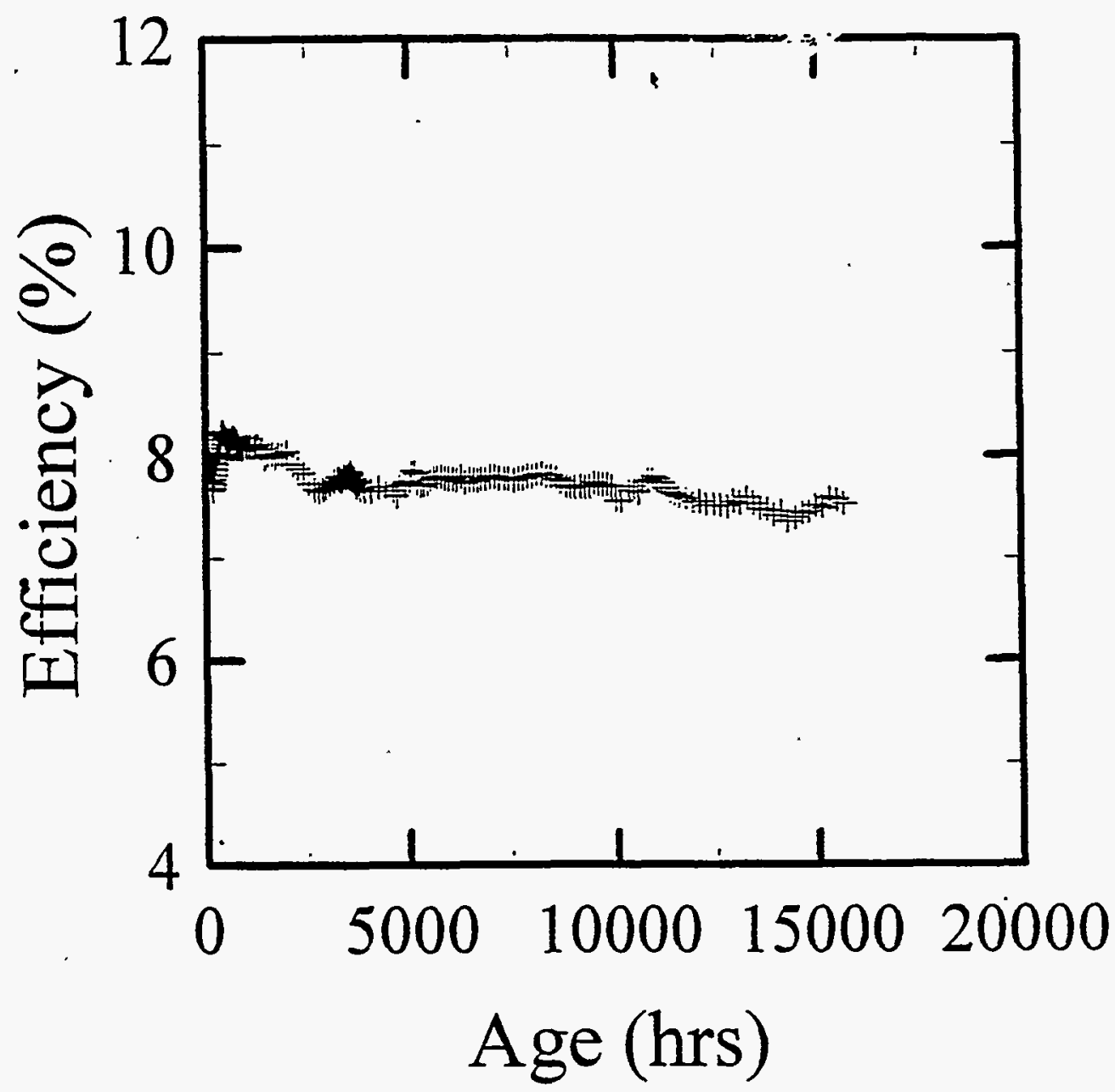

Figure 4. Average efficiency of six submodules exposed to continuous illumination and held near maximum power. 2,000 hours is approximately equal to one year of field use. 
Title: $\quad$ Research on Polycrystalline Thin Film Submodules Based on $\mathrm{CuInSe}_{2}$ Materials

Organization: Solarex Corporation, 826 Newtown-Yardley Rd., Newtown, PA 18940

\author{
Contributors: J. Fogleboch, J. Kessler, L. Russell, S. Skibo, S. Wiedeman, \\ R. Arya and D. Carlson
}

\title{
Objective
}

The objective of this program is the rapid development and understanding of processes and methods for fabricating high efficiency $\mathrm{CuInSe}_{2}$ (CIS) based modules. For the manufacture of PV products on a large scale process speed, process robustness, and yield as well as efficiency, cost, stability and environmental durability are of prime importance. A major goal for this year was the demonstration of a high efficiency submodule, successful development of module formation processes, and a degree of overall process robustness. Further progress in absorber, buffer and window layers, using small area cells, and development of module design and fabrication processes would also be necessary.

\section{Accomplishments}

\section{- Fabrication of an encapsulated CIS based submodule having $13 \%$ aperture area efficiency.}

- Achievement of $15.5 \%$ efficient cells using robust processes.

\section{Background}

CIS based PV exhibits several features which are important for the manufacture of PV on a large scale; it has demonstrated high conversion efficiency for small area cells (in the range of $17 \%$ $[1,2])$, and is potentially a very low cost thin film system. Advantages include a very high optical absorption coefficient, a wide choice of deposition methods for the absorber layer, and the flexibility afforded through modification of electronic and optical properties by using ternary alloys of CIS.

Despite the advantages inherent to the CIS material system, the development of modules has been complicated by several factors. Module structures demand that large areas be uniform and free of shunts. The interconnect operations included after the back contact, absorber layer, and front contact depositions involve scribing and often introduce shunts through debris generation and disruption of the absorber layer. Also, current collection over a large area module structure places demanding requirements on the sheet resistivity and optical transparency of the front contact, and the interconnect resistivity. Moreover, the effect of one process step is often very dependent on the process parameters used for other steps. Thus, development of each process step in isolation is often inappropriate; ultimately entire modules must be fabricated to completion. 


\section{Technical Approach}

In brief, the thin film CIS based structure consists of a molybdenum back contact deposited on a soda-lime glass substrate, on which a $\mathrm{Cu}(\mathrm{In}, \mathrm{Ga}) \mathrm{Se}_{2}$ absorber layer, $\mathrm{CdS}$ buffer layer and $\mathrm{ZnO}$ front contacting layer are deposited. The front and back contact layers are sputtered, the CdS layer is deposited using chemical bath deposition, and the absorber layer is deposited by either sputtering of metals and/or metallic selenides or by coevaporation. Devices are completed using a $\mathrm{Ni} / \mathrm{Al}$ grid on the $\mathrm{ZnO}$ contact. Submodules can be made using a combination of laser and mechanical means to scribe the various layers to form serially interconnected module segments. These processes are described in more detail elsewhere[3]. The device and submodule results achieved are shown in figures $1 \mathrm{a}$ and $1 \mathrm{~b}$.
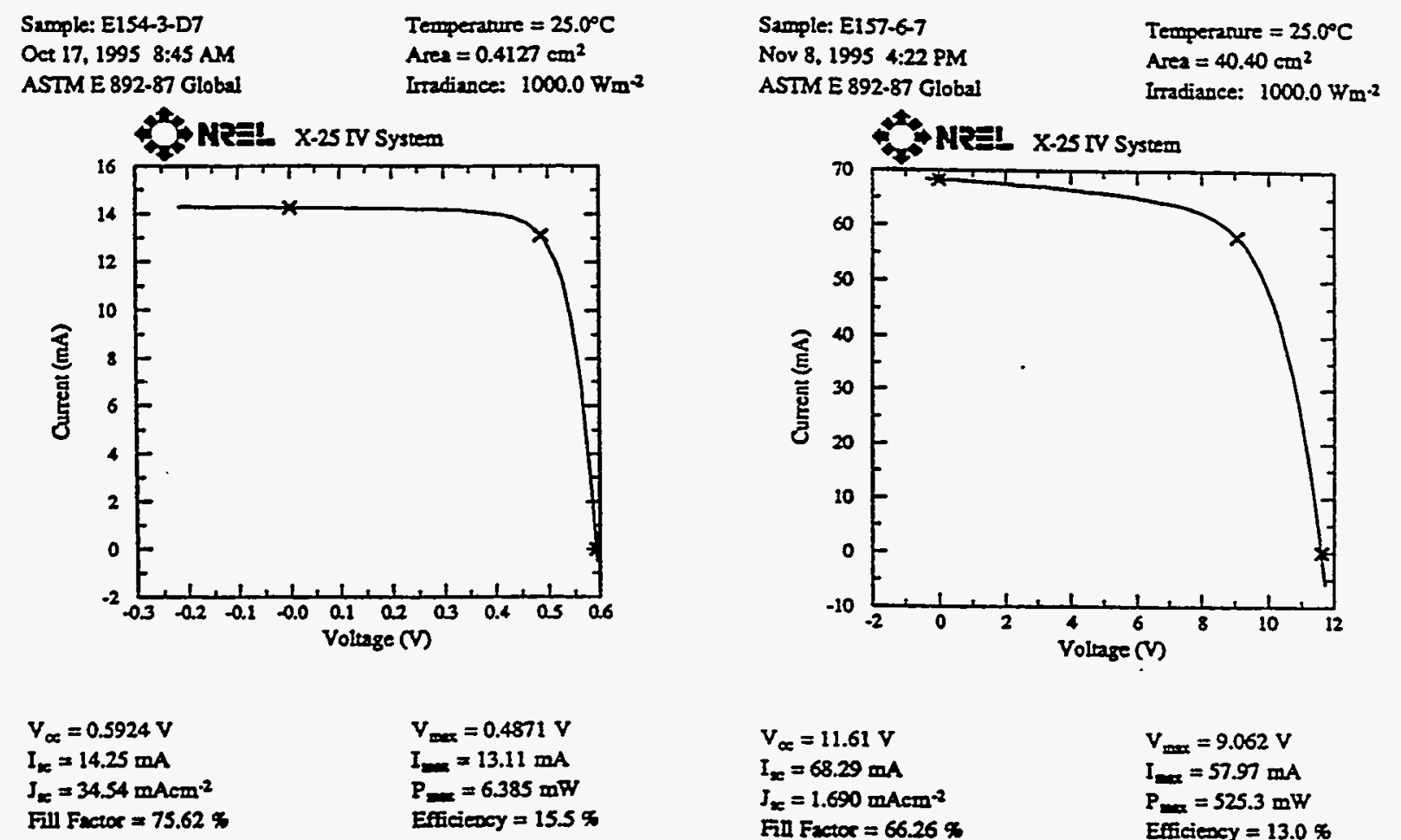
$\mathrm{V}_{\infty}=11.61 \mathrm{~V}$
$I_{x=}=68.29 \mathrm{~mA}$
$J_{x=1.690 \mathrm{mAcm}^{-2}}$

Fill Factor $=66.26 \%$

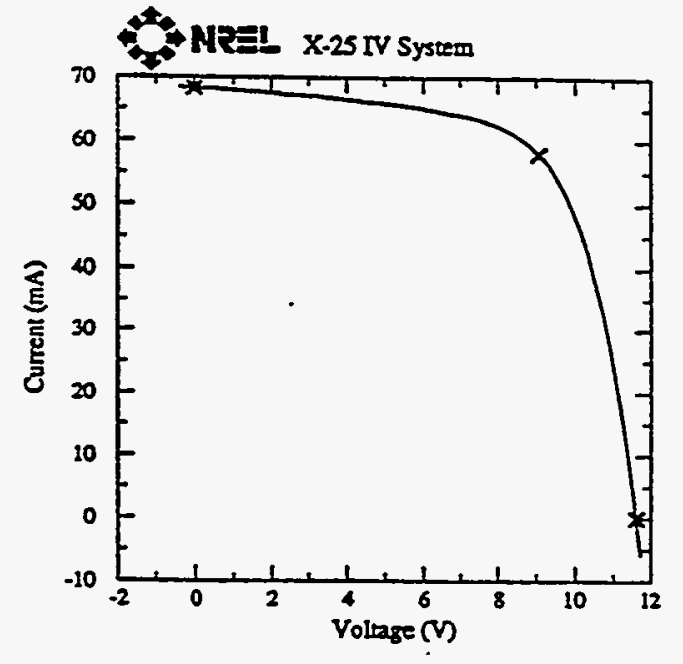

Figure 1. The J-V characteristics measured by NREL of:

(a) a $15.5 \%$ device (total area),

(b) a $13.0 \%$ efficient, 20 segment submodule (aperture area).

During this time period the $\mathrm{ZnO}$ transparent front contact was improved in terms of the optical transparency and sheet resistivity. Significant effort was devoted to process development and improvement of the absorber layer. The J-V characteristics of a $15.5 \%$ efficient (total area) device resulting from these improvements is shown in figure la.

Efforts in several areas were required to improve submodule performance, including back contact deposition, segment formation and interconnection technology, optimization and analysis of module design, evaluation of absorber uniformity, and failure and shunt analysis. The back contact deposition parameters and the subsequent back contact scribing process are highly 
interdependent. These operations were improved significantly with respect to control, reproducibility and debris generation to give very clean, regular scribes of the back contact.

Evaluation of module losses and alternate module design was accomplished using a model to predict module behavior from small area device characteristics. Expected module performance can be calculated as a function of materials properties (such as front and back contact sheet resistivity, front contact optical absorption, interconnect resistivity) and geometric considerations (module segment width, total interconnect width) with representation of the absorber/heterojunction diode behavior taken from actual small area diode measurements. These calculations indicate that submodules having about $13 \%$ efficiency can result by using material layers which yield small area devices of $14.4 \%$ efficiency [4,5], as shown in figure 2 for realistic values of interconnect resistivity and width.

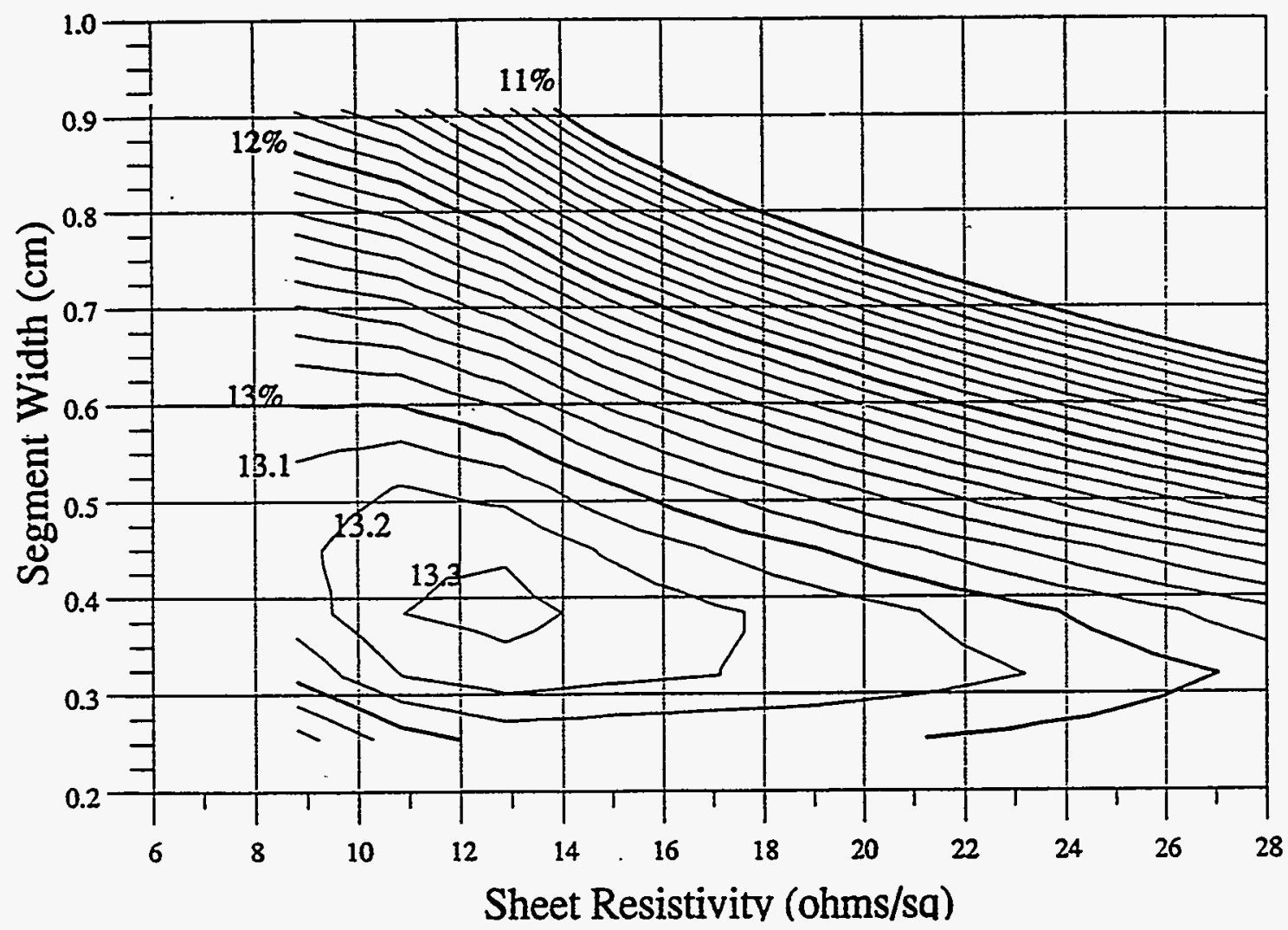

Figure 2. Iso-efficiency contours for submodule performance vs. segment width and front contact sheet resistivity. Interconnect resistivity and width are assumed to be 0 and $0.03 \mathrm{~cm}$ respectively.

Module interconnect processes were also improved to allow interconnects having essentially negligible resistive losses with very little loss of active area [4]. These improvements, coupled with the elimination of some shunting problems and optimal module design described above resulted in the fabrication of a submodule having $13 \%$ aperture area conversion efficiency. To our knowledge, this is the highest confirmed efficiency reported for any thin film monolithic module, as shown in figure $1 \mathrm{~b}$. 


\section{Conclusions}

Significant progress has been made in bringing CIS based, thin film PV toward commercial reality. This is evidenced by the demonstration of a submodule having world record efficiency, and which approaches the efficiency of small area devices produced using the same processes. Future directions for research include further optimization of the front contact properties and scribing operations, statistical analysis of larger quantities of submodules and extension of required processes to fabricate modules of much greater area. In this regard, absorber uniformity has already been demonstrated over $9 " \times 9^{\prime \prime}$ area using the current methods[6], as shown in figure 3 .

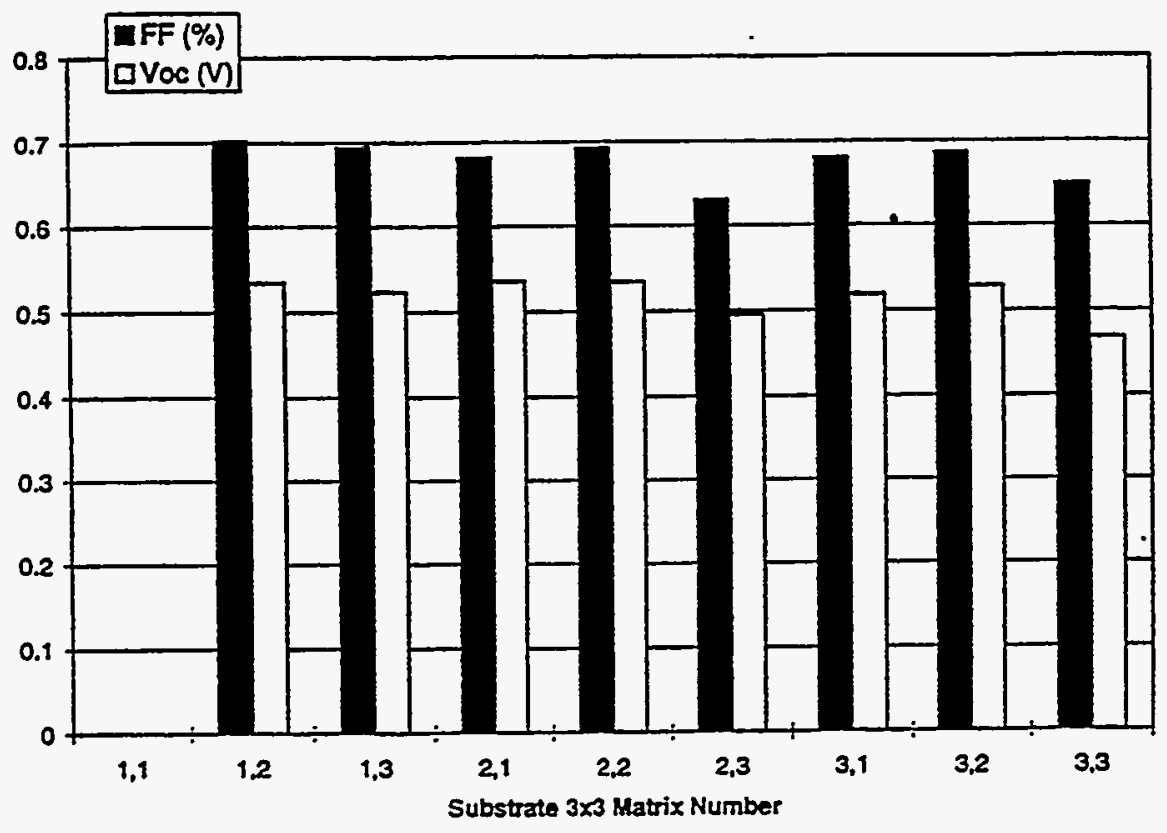

Figure 3. Uniformity of device performance between eight 3" $\times 3$ " substrates made in a single absorber layer deposition. The data shows average Voc and fill factor on each substrate relative to their matrix position in the deposition system. Substrate 1,1 was lost in processing.

\section{References}

1. J. R. Tuttle, A. M. Gabor, M.A. Contreras, A.L. Tennant, K.R. Ramanathan, A. Franz, R. Matson and R. Noufi, (1995) AIP Conference Proceedings 353, p 47.

2. J. Hedström, H. Ohlsén, M. Bodegård, A. Klyner and L. Stolt, Proceedings of 23rd IEEE PVSC, Louisville, KY, May 1993, p 364.

3. J. Fogleboch, J. Kessler, L. Russell, S. Skibo, S. Wiedeman and R. Arya, Solarex Final Technical Report to NREL under Subcontract No. ZN-1-19019-4, 11/11/90-6/30/95.

4. S. Wiedeman, J. Kessler, T. Lommasson, L. Russell, J. Fogleboch, S. Skibo and R. Arya, (1995) AIP Conference Proceedings 353, p 12.

5. S. Wiedeman, J. Kessler, L. Russell, J. Fogleboch, S. Skibo, T. Lommasson, D. Carlson and R. Arya, 13th EC-PVSEC, Nice, France, October, 1995. (to be published)

6. J. Kessler, S. Wiedeman, L. Russell, T. Lommasson, S. Skibo, J. Fogleboch, T. Kloss, R.R. Arya, Proceedings of the 24th IEEE PVSC, Hawaii, 1994 (IEEE, New York, 1994), p 206. 
Organization: Syracuse University, Department of Physics, Syracuse, New York 13244.

Contributors: Eric A. Schiff, principal investigator, Qing Gu and Lin Jiang.

In 1995 our research has focused on wide bandgap materials, in conjunction with our participation in the wide bandgap team of the Amorphous Silicon Research Project. We are working on the following topics:

- Development of novel thin films as possible alternatives to current amorphous and microcrystalline $p^{+}$(boron doped) silicon films. The goal of this research is to develop materials which will increase the open circuit voltage $V_{\propto}$ of a-Si based solar cells incorporating wide bandgap absorber layers $(>1.8 \mathrm{eV}$ using the Tauc criterion).

- Electron and hole mobility measurements in a-Si based materials. In the last year most of our work has involved the "hot wire" a-Si:H deposited by the NREL research group; we made the unexpected finding that the hole drift-mobility of this material is up to five times larger than any we had previously measured in the entire plasma-deposited a-Si based alloy system.

- Electroabsorption studies of a-Si based solar cells to probe their built-in potential. We seek a tool to permit us to directly assess $V_{b i}$ as it varies with differing $n^{+}$and $p^{+}$layers. The built-in potential is one of the key parameters which determines the open circuit voltage obtainable in a solar cell.

Our research contract was acknowledged in the following publications in 1995:

"Fundamental Transport Mechanisms and High Field Mobility Measurements in Amorphous Silicon," Qing Gu, E. A. Schiff, J.-B. Chevrier, and B. Equer, J. Non-Cryst. Solids, in press (1995).

"Diffusion, Drift and Recombination of Holes in a-Si:H," R. Schwarz, F. Wang, S. Grebner, Q. Gu, and E. A. Schiff, in Amorphous Silicon Technology-1995, edited by M. Hack, et al (Materials Research Society, Pittsburgh, 1995), 427.

"Electron Dritt Mobility in a-Si:H Prepared by Hot Wire Deposition," Qing Gu, E. A. Schiff, R. S. Crandall, E. Iwaniczko, and B. Nelson, in Amorphous Silicon Technology-1995, edited by M. Hack, et al (Materials Research Society, Pittsburgh, 1995), 437.

"The Correlation of Open-Circuit Voltage with Bandgap in Amorphous Silicon-Based pin Solar Cells," R. S. Crandall and E. A. Schiff, in 13th NREL Photovoltaics Program Review, edited by H. S. Ullal and C. E. Witt (American Institute of Physics, conf. proc. vol. 353, Woodbury, 1995), p. 101.

"Diffusion-Controlled Bimolecular Recombination of Electrons and Holes in a-Si:H," E. A. Schiff, J. Non-Cryst. Solids 190, pp. i-8 (1995). 
"High-field Electron-Dritt Measurements and the Mobility Edge in Hydrogenated Amorphous Silicon," Qing Gu, E. A. Schiff, Jean-Baptiste Chevrier and Bernard Equer, Phys. Rev. B 52, pp. 5695-5707 (1995).

\section{Amorphous Boron Phosphide}

We deposited a series of films based on diborane/phosphine and trimethylboron/phosphine gas mixtures diluted with hydrogen. We obtained films with better transparency than the standard window layers presented used for a-Si based solar cells, as illustrated in Fig. 1. The films were amorphous, as determined by $\mathrm{X}$-ray scattering measurements. We have not determined the boron/phosphorous stoichiometry nor the hydrogen content.

The electrical properties of the films vary widely. We have adopted as our objective the achievement of $\mathrm{p}$-type conductivity of $10^{-5} \Omega^{-1} \mathrm{~cm}^{-1}$ in these films. This is a reasonable criterion for application of the films as window layers in a-Si based solar cells. Despite promising indications in some films, we have not yet found a way of routinely achieving these electrical properties.

\section{Drift Mobility in Hot-Wire Deposited Amorphous Silicon}

The amorphous silicon research group at NREL has been exploring "hot wire" deposited a-Si:H for some years in an effort to exploit the promising properties of thin films grown by this method in solar cells. Using specimens deposited at NREL, we studed the electron and hole drift mobilities in low hydrogen content (ca. 2 at. \%) a-Si:H prepared using the hot-wire technique. This material had an optical bandgap (Tauc criterion) of about $1.60 \mathrm{eV}$, which should be compared to the $1.75 \mathrm{eV}$ normal for plasma-deposited a-Si:H. The drift mobilities were measured using the well established, photocarrier time-of-flight technique.

The electron drift mobility in the hot-wire material was somewhat different than expected from our previous work on the plasma-deposited a-SiGe:H alloys with comparable bandgaps; a preliminary account of this work is noted in the publication list above. The most noteworthy finding is that the hot-wire material had a hole drift mobility which was up to five times larger (under standard measuring conditions) than any hole mobility we had measured in the entire plasma deposited a-Si:H alloy system. For this system we have found little variation in the hole mobility for optimized system over a wide range of bandgaps.

Some of the temperature-dependent hole drift mobility measurements in hot-wire a-Si:H and conventional plasma-deposited a-Si:H are presented in Fig. 2, where it is clear that the hole mobility in the hot-wire material is enhanced. The most likely origin of the improved hole mobility is a narrower valence bandtail than normal for plasma-deposited a-Si:H. It is generally believed that the open circuit voltage of a-Si:H based solar cells with relatively narrow gap absorber layers is limited by the valence bandtail. The finding of an enhanced hole mobility is thus encouraging for photovoltaic application of the material. 


\section{Electroabsorption and Built-in Potentials}

We have been developing the electroabsorption method $[1,2]$ for determining the built-in potential $V_{\mathrm{bi}}$ in a-Si based solar cells for several years. In this method one passes a light beam through the solar cell, and measures the effect of the cell's bias voltage $V$ on the transmitted light. Without delving into the details, it is sufficient to note that an electroabsorption signal $S$ can be measured which depends linearly on $V: S=a\left(V_{0}-V\right)$. This relationship is easily understood if the parameter $V_{0}$ is interpreted as the built-in potential $V_{\mathrm{bi}}$ of the cell.

The problem which we are working on is that $V_{0}$ depends upon the measuring wavelength in a$\mathrm{Si}: \mathrm{H}$ based cells. We discovered this effect in prior research [2], and proposed that it originates in the differing electroabsorption properties of the $p$ type window layer and the intrinsic absorber layer of pin solar cells.

In the last year we have confirmed this model for the wavelength dependence; in particular the wavelength-dependence effect was not found in Schottky barrier devices, as would be expected if the effect is due to the $p$ layer.

We have developed a new technique for determining the necessary optical properties of the two layers from measurements on a single solar cell. The method is based on using both the first and second harmonics of the electroabsorption signal in conjunction with capacitance measurements. Using this method we have estimated built-in potentials in several solar cells and Schottky barrier diodes from cooperating laboratories (Institute of Energy Conversion, University of Delaware; Solarex Thin Films Division; United Solar Systems Corp.; Energy Conversion Devices, Inc.; Pennsylvania State University). In no case has the built-in potential estimated using electroabsorption exceeded $1.0 \mathrm{~V}$. This result is a surprise, since rough estimates of $V_{B I}$ based on film properties for the individual layers have suggested values several tenths of a Volt higher than we would estimate using electroabsorption. We are still studying the implications of these surprisingly low values for $V_{B I}$.

\section{References}

1. S. Nonomura, H. Okamoto and Y. Hamakawa, Jpn. J. Appl. Phys. 21, 464 (1982).

2. Qi Wang, E. A. Schiff, and S. Hegedus, in Amorphous Silicon Technology - 1994, edited by E. A. Schiff, M. Hack, A. Madan, A. Matsuda, and M. Powell (Materials Research Society, Pittsburgh, 1994), pp. 365--370. 


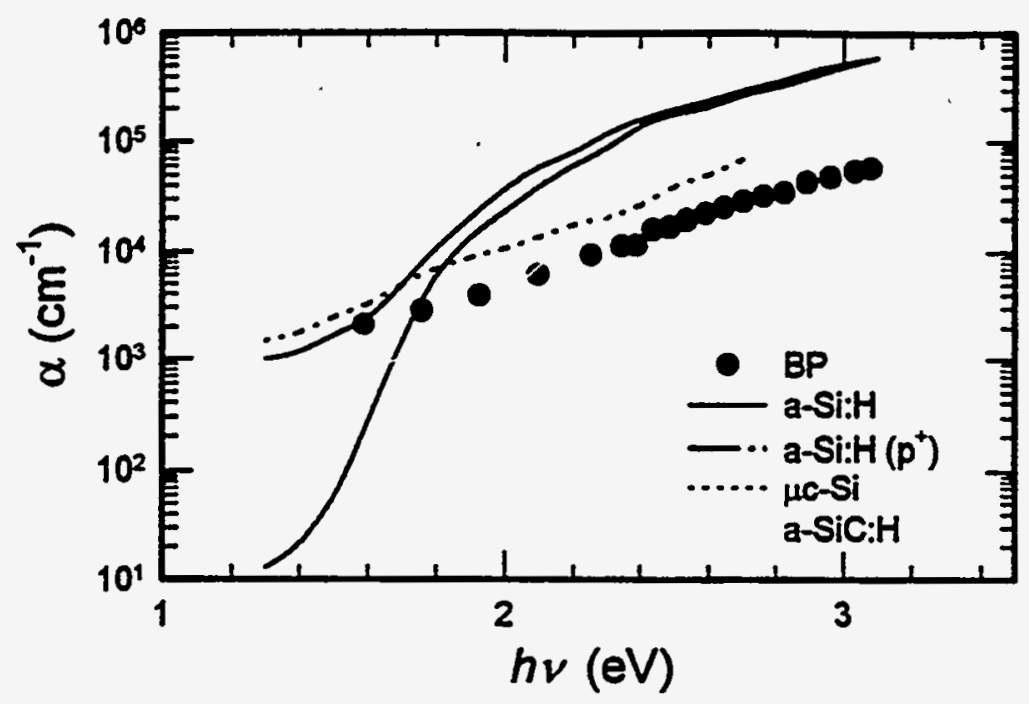

Fig. 1: A comparison of the optical absorption coefficients for several materials used in window layers of a-Si based solar cells. The new results are on the amorphous boron phoshide films, which have better transparency than the alternatives. The electrical properties of the film are not adequate at present for window layer application.

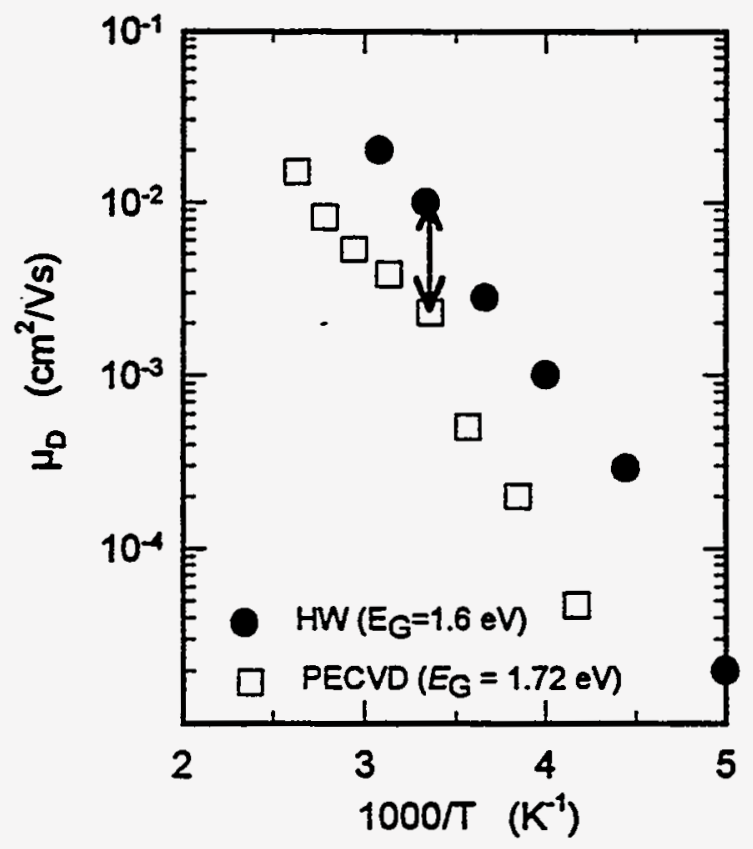

Fig. 2: Hole drift mobility measured using photocarrier time of flight techniques for hot-wire deposited a-Si:H (denoted $\mathrm{HW}$ ) and for conventional plasmadeposited a-Si:H (denoted PECVD). The hot-wire material has an enhanced hole mobility; this result was unexpected given the small variation in hole mobilities across the entire plasma-deposited alloy sytem. 
Title:

Organization:

Contributors:
Amorphous Silicon Research

United Solar Systems Corp. Troy, Michigan

S. Guha, principal investigator, A. Banerjee, E. Chen, T. Glatfelter, M. Haag, G. Hammond, K. Hoffman, M. Hopson, N. Jackett, J. Noch, T. Palmer, D. Wolf, $\mathrm{X}$. Xu, J. Yang, and $\mathrm{K}$ : Younan.

\section{Objective}

The principal objective of this R\&D program is to expand, enhance and accelerate knowledge and capabilities for the development of high-performance, two-terminal multijunction hydrogenated amorphous silicon (a-Si:H) alloy cells and modules. The near-term goal of the program is to achieve $12 \%$ stable efficiency by 1998 using the multijunction approach.

\section{Approach}

The major effort of this program is to develop high efficiency component cells and incorporate them in the triple-junction structure to obtain the highest stable efficiency. The bulk of the effort was directed toward the middle and bottom cell structure. New and improved deposition regimes were investigated to obtain better cell performance. Fundamental studies to obtain better understanding of material and cell performance were undertaken.

\section{Status/Accomplishments}

- Several new deposition regimes/conditions were explored to investigate their effect on material/device performance. No beneficial effect on cell performance could be observed by i) ion bombardment during deposition, ii) preheating the deposition gas mixture and iii) He dilution.

- Hydrogen dilution during deposition is found to improve both the initial and stable performance of a-Si:H and a-SiGe:H alloy cells. No correlation was observed between deep defect density measured by the constant photocurrent method and cell efficiency.

- Use of Schottky barrier structure was demonstrated to be a useful tool for evaluating $p$ in solar cell performance.

- Using internal photoemission method, the electrical bandgap of microcrystalline $p$ layer used in high efficiency solar cell was measured to be $1.6 \mathrm{eV}$. The band discontinuity at the microcrystalline-amorphous interface is found to be predominantly at the valence band edge. Use of these parameters in numerical simulations is found to predict solar cell performance accurately. 
New measurement techniques were developed to evaluate the interface and bulk contribution of losses to solar cell performance.

Using the optimized component cells, a stable active-area efficiency of $11.1 \%$ was achieved in a triple-junction cell of $0.25 \mathrm{~cm}^{2}$ area. The same cell was measured at $10.8 \%$ at NREL using a triple-source simulator (Table $\mathrm{D}$. This is the highest stable cell efficiency measured by NREL for any a-Si:H alloy cell structure. Based on the status of the component cell performance, further improvement is expected in the stable cell efficiency with continued optimization.

The current status at United Solar in terms of initial and stable cell $\left(0.25 \mathrm{~cm}^{2}\right.$ area) efficiencies for different single-, double- and triple-junction structures is shown in Table II. For each of these structures, the stable efficiencies represent the highest reported in the literature. There are several points worth considering. There is a gain from $8.8 \%$ to $11.2 \%$ as we go from a single-junction to a multijunction, multibandgap structure. Use of $\mathrm{Ge}$ in the bottom cell improves the efficiency from $10.1 \%$ to $11.2 \%$. We should note that at this point in time, there is no difference in the stabilized efficiencies between double- and triple-junction cells. As mentioned earlier, optimization of the triple-cell structure is still continuing, and a higher efficiency is expected with the current component cell performance. Moreover, a small improvement in the quality of a-SiGe alloy will have a much greater impact on increasing the stable efficiency of a triplejunction cell. In order to improve the efficiency of the double-junction cell further, the top cell thickness would have to increase to an extent that light-induced degradation will play a major role. The potential for improving the triple-cell efficiency is, therefore, much higher. In fact, computer simulations indicate that using realistic stabilized material parameters, the triple-junction approach is the only.way to reach $15 \%$ efficiency and beyond.

Table I. Characteristics of Triple-Junction a-Si:H Alloy Cells in Initial and Degraded States.

\begin{tabular}{|c|c|c|c|c|c|c|c|c|}
\hline $\begin{array}{l}\text { Sample } \\
\text { No. }\end{array}$ & State & $\begin{array}{c}\eta \\
(\%)\end{array}$ & $\begin{array}{c}\mathrm{J}_{s c} \\
\left(\mathrm{~mA} / \mathrm{cm}^{2}\right)\end{array}$ & $\begin{array}{l}V_{\infty} \\
(V)\end{array}$ & $\mathrm{FF}$ & \multicolumn{3}{|c|}{$\begin{array}{c}\mathrm{Q}\left(\mathrm{mA} / \mathrm{cm}^{2}\right) \\
\text { Top Middle Bottom }\end{array}$} \\
\hline \multirow[t]{4}{*}{ L7358 } & Initial & 13.0 & 7.16 & 2.45 & 0.74 & 7.16 & 7.20 & 8.38 \\
\hline & Degraded & 11.1 & 6.87 & 2.38 & 0.68 & 6.98 & 6.87 & 7.97 \\
\hline & NREL' & 9.93 & 6.25 & 2.38 & 0.67 & & & \\
\hline & $\mathrm{NREL}^{2}$ & 10.8 & 6.75 & 2.38 & 0.67 & & & \\
\hline \multirow[t]{4}{*}{ L7361 } & Initial & 12.9 & 7.15 & 2.45 & 0.74 & 7.15 & 7.15 & 8.35 \\
\hline & Degraded & 11.0 & 6.90 & 2.38 & 0.67 & 6.97 & 6.90 & 8.04 \\
\hline & NREL' & 9.97 & 6.30 & 2.38 & 0.67 & & & \\
\hline & $\mathrm{NREL}^{2}$ & 10.8 & 6.79 & 2.38 & 0.67 & & & \\
\hline L7466 & Initial & 13.3 & 7.36 & 2.45 & 0.74 & 7.38 & 7.36 & 8.31 \\
\hline
\end{tabular}

${ }^{1}$ Total-area, ${ }^{2}$ Calculated active-area 
Table II. Highest Stable Cell Efficiencies at United Solar for Different Junction Configurations.

\begin{tabular}{|c|c|c|c|c|c|c|}
\hline & & $\begin{array}{c}\mathrm{J}_{\mathrm{sc}} \\
\left(\mathrm{mA} / \mathrm{cm}^{2}\right)\end{array}$ & $\begin{array}{l}V_{\infty} \\
\text { (V) }\end{array}$ & $\mathrm{FF}$ & $\prod_{(\%)}^{\eta}$ & Deg. \\
\hline $\mathrm{a}-\mathrm{Si}: \mathrm{H}$ & $\begin{array}{l}\text { Initial } \\
\text { Degraded } \\
\text { (600 hrs) }\end{array}$ & $\begin{array}{l}15.25 \\
15.12\end{array}$ & $\begin{array}{l}0.94 \\
0.91\end{array}$ & $\begin{array}{l}0.72 \\
0.64\end{array}$ & $\begin{array}{r}10.3 \\
8.8\end{array}$ & $14.6 \%$ \\
\hline a-Si:H/a-Si:H & $\begin{array}{l}\text { Initial } \\
\text { Degraded } \\
(1000 \text { hrs })\end{array}$ & $\begin{array}{l}7.9 \\
7.9\end{array}$ & $\begin{array}{l}1.89 \\
1.83\end{array}$ & $\begin{array}{l}0.76 \\
0.70\end{array}$ & $\begin{array}{l}11.4 \\
10.1\end{array}$ & $11.4 \%$ \\
\hline a-Si:H/a-SiGe:H & $\begin{array}{l}\text { Initial } \\
\text { Degraded } \\
(600 \mathrm{hrs})\end{array}$ & $\begin{array}{l}10.67 \\
10.61\end{array}$ & $\begin{array}{l}1.65 \\
1.61\end{array}$ & $\begin{array}{l}0.72 \\
0.66\end{array}$ & $\begin{array}{l}12.6 \\
11.2\end{array}$ & $11.1 \%$ \\
\hline $\begin{array}{c}\text { a-Si:H/a-SiGe:H/ } \\
\text { a-SiGe:H } \\
.\end{array}$ & $\begin{array}{l}\text { Initial } \\
\text { Degraded } \\
\text { ( } 600 \mathrm{hrs})\end{array}$ & $\begin{array}{l}7.16 \\
6.87\end{array}$ & $\begin{array}{l}2.45 \\
2.38\end{array}$ & $\begin{array}{l}0.74 \\
0.68\end{array}$ & $\begin{array}{l}13.0 \\
11.1\end{array}$ & $14.6 \%$ \\
\hline
\end{tabular}

\section{References}

1. A. Banerjee, K. Hoffman, X. Xu, J. Yang, and S. Guha, "Back reflector texture and stability issues in high efficiency multijunction amorphous silicon alloy solar cells," First World Conf. on PV Energy Conversion Proc., 539 (1994).

2. J. Yang, A. Banerjee, T. Glatfelter, K. Hoffman, X. Xu, and S. Guha, "Progress in triplejunction amorphous silicon-based alloy solar cells and modules using hydrogen dilution," First World Conf. on PV Energy Conversion Proc., 380 (1994).

3. X. Xu, J. Yang, A. Banerjee, and S. Guha, "Band edge discontinuities between microcrystalline and amorphous hydrogenated silicon alloys and their effect on solar cell performance," Appl. Phys. Lett. 67, 2323 (1995).

4. X. Xu, A. Banerjee, J. Yang, S. Guha, K. Vasanth, and S. Wagner, "Band discontinuity effect on a-Si:H and a-SiGe:H solar cells," Mat. Res. Soc. Symp. Proc., San Francisco (1995).

5. A. Banerjee, X. Xu, J. Yang, and S. Guha, "Analysis of fill factor losses in a-Si and aSiGe alloy solar cells using a new technique," Mat. Res. Soc. Symp. Proc., San Francisco (1995).

6. S. Guha, J. Yang, A. Banerjee, T. Glatfelter, K. Hoffman, and X. Xu, "Advances in amorphous silicon alloy multijunction cells and modules," PV AR\&D Meeting, Denver (1995).

7. A. Banerjee, X. Xu, J. Yang, and S. Guha, "Carrier collection losses in amorphous silicon and amorphous silicon-germanium alloy solar cells," Appl. Phys. Lett. 67, 2975 (1995).

8. S. Guha, "Material and device consideration for high efficiency a-Si alloy-based multijunction cells," Intl. Conf. on Amorphous Semiconductors Proc., Kobe, Japan (1995). 
Title:

Organization:

Contributors:

\section{Photocharge Transport and Recombination Measurements in Amorphous Silicon Films and Solar Cells by Photoconductive Frequency Mixing}

\author{
Physics Department, University of California at Los Angeles, \\ Los Angeles, CA 90024-1547 \\ R. Braunstein, Principle Investigator; \\ Shriun Dong, research associate.
}

\section{Program Outline}

This research program is concerned with the characterization of the photoconductive properties of $\mathrm{a}-\mathrm{Si}: \mathrm{H}, \mathrm{a}-\mathrm{SiC}: \mathrm{H}$ and a-SiGe:H films from "round robin" sources by the photomixing technique so as to separately determine drift mobility and lifetime. Degradation studies are performed on selected films by in situ light soaking for a quantitative analyses of the transport properties. These studies are to be extended to solar cell devices. The photomixing technique consists of heterodyning two monochromatic laser beams when a dc bias is applied which results in photocurrent composed of a dc and microwave photomixing current; these two photocurrents allow a determination of the drift mobility $\left(\mu_{\mathrm{d}}\right)$ and lifetime $(\tau)$ of the dominant photo generated carriers [1-5].

This report covers the period from $5 / 15 / 95-12 / 15 / 95$

\section{Accomplishments}

Since there is a continued interest in a-Si:H produced by various hydrogen dilution techniques because of the indicated superior quality in overcoming metastable degradation, we have continued our studies on these films by photomixing to determine mobility and lifetime in annealed and under light soaked conditions. We have continued to study the material produced by the hot-wire (HW) deposition technique employed by NREL. As an example of this study we will discuss the results on a representative series of hot-wire samples prepared by Brent Nelson and Eugene Iwaniczko at NREL using substrate heater temperatures of $350 \mathrm{C}, 425 \mathrm{C}, 500 \mathrm{C}$, and $575 \mathrm{C}$. Such samples are expected to have hydrogen contents ranging from over 10 at $\%$ to less than $1 \%$ respectively. The time dependence of light induced degradation of these samples were measured during 5 hours exposure at 4 suns intensity.

I. Electric Field Dependence of Drift Mobility and Lifetime in Annealed and Light Soaked a-Si:H.

We have continued measurements by the photomixing technique to determine the mobility and lifetime in a-Si: $\mathrm{H}$ in as-grown, light-soaked and in the annealed state. These were performed as a function of electric field. The samples consisted of a series of hot-wire, glow discharge and compensated samples. By using the photomixing technique we have found that the drift mobility $\left(\mu_{d}\right)$ of intrinsic hydrogenated amorphous silicon (a-Si:H) films produced by both glow discharge 
and hot-wire techniques increases with increasing field, while the lifetime $(\tau)$ decreases with increasing electric field, and the $\mu_{\mathrm{d}} \tau$ product is essentially independent of electric field indicating that diffusion limited recombination in occurring. We have found that a greater field dependence of the drift mobility of an a-Si:H film in the annealed state indicates a poor stability of the photoconductivity upon light soaking. This empirical relationship is consistent with earlier observations that light soaking decreases the drift mobility in most a-Si:H based materials. This empirical relationship suggest that the Staebler-Wronski degradation of a-Si:H can be linked to defects that are responsible for the field dependence of the drift mobility in the annealed state, Important applications of this empirical relationship include the possibility of assessing the amount of expected light induced degradation without going through time consuming light soaking experiments and possible in situ evaluation of stability, e. g. during sample preparation processes to find the optimal conditions for stable samples. The increase in $\mu_{\mathrm{d}}$ with increasing field as well as the increase in $\mu_{\mathrm{d}}$ with increasing carrier density due to illumination (which we previously observed and reported on in the third quarterly report) can be explained by the existence of long range potential fluctuations.

Figures. 1 show the electric field dependence of the drift mobility for several hot-wire samples. The open dots and solid dots are the experimental points for as-grown and light soaked states respectively. The solid curves were obtained through a curve fitting procedure to a model of transport through potential barriers which we have presented [6]. For purposes of continuity of discussion we summarize the model:

The light induced defects as well as native defects, which serve as recombination centers and trapping centers, can be charged and can form certain potential barriers of fluctuations. In the transport process, the charged carriers can either go over the potential barrier through thermal activation or go around the potential barrier through scattering. If the former dominates the latter, then through simple statistical calculations one can obtain the following electric field (E) dependence of the drift mobility $\mu_{d}(E)$.

$$
\begin{array}{ccc}
\mu_{d}(F)=\mu_{0} \exp \left(-\frac{e V_{P}}{k T}\right) \frac{e L F}{k T\left[1-\exp \left(-\frac{e L F}{k T}\right)\right]} & \left(|L F| \leq V_{P}\right) \\
\mu_{d}(F)=\mu_{0} \frac{\exp \left(-\frac{e V_{P}}{k T}\right)}{\frac{k T}{e L F}+\left(1-\frac{V_{P}}{L F}-\frac{k T}{e L F}\right) \exp \left(-\frac{e V_{P}}{k T}\right)} \quad\left(|L F|>V_{P}\right) &
\end{array}
$$

where $\mu_{0}$ is the drift mobility without the potential fluctuations, while $\mathrm{Vp}$ and $\mathrm{L}$ are an average magnitude and range of the potential fluctuations respectively.

The range $L$ of the potential fluctuations can be determined through curve fitting using the above 
equations to the experimental data shown in Fig. 1; the range of the potential fluctuation in the light soaked state $\mathrm{L}_{\mathrm{LS}}$ and $\mathrm{L}_{\mathrm{An}}$ in the annealed states are given in Table $\mathrm{I}$.

\section{Photoelectric Emission in Air from Amorphous Semiconductors and Transparent Conducting Oxides.}

We have available in our laboratory an apparatus that can perform optically stimulated emission from semiconductors and metal surfaces in air. This apparatus enables us to profile the barrier height on a surface up to 4 inches by 4 inches in area in air; thus it can be employed under normal production environment conditions to monitor possible in-process surface changes of barrier heights. We have performed measurements on a-Si:H, TCO, diamond, porous Silicon and $\mathrm{CuIn}_{\mathbf{x}}$ $\mathrm{Ga}_{1-\mathrm{x}} \mathrm{Se}_{2}$ films. Figure.2 shows an example of the barrier height of a 4 in. by 4 in. surface of a-Si:H. The revealed non-uniformity of barrier height is due possibly to inhomogeneity of composition, resulting perhaps from the positioning of the sample in the glow discharge.

\section{Conclusions}

By employing the photomixing technique we have found that the drift mobility $\left(\mu_{d}\right)$ of intrinsic a-Si:H in both glow discharge and hot-wire samples increases with increasing electric field, and a greater field dependence in the as-grown state indicates a poorer stability upon light soaking and vice versa. This empirical relationship indicates that the amount of light induced degradation my be predictable from the field dependence of the drift mobility in the annealed state. The results were analyzed in terms of a model we developed for transport through long range potential fluctuations. It should be noted that our results suggest that light induced charged dangling bonds may not affect $\mu_{d}$ by increasing recombination, but rather by affecting $\mu_{d}$ through controlling potential fluctuations. In addition we have show that photoemission in air can be employed for the detection of surface contaminants, monitor cleanliness in the production environment, and detect non- uniformities in films.

Measurement of the temperature dependence of the hot-wire samples have enabled us to determine the spread of the band tail of the conduction band and the capture rate. A detailed. analysis will be reported at a later date.

\section{References}

1. E.R.Giessinger, R. Braunstein, and B.G.Martin, J.Appl.Phys. 69, 1469 (1991).

2. Yi Tang, R. Braunstein, B. von Roedern, Mat.Res.Soc.Symp. Proc.258, 735 (1992).

3. Yi Tang, R. Braunstein, B. von Roedern, and F.R.Shapiro, Mat. Res. Soc. Symp. Proc. 297, 407 (1993).

4. R. Braunstein and $Y i$ Tang, Proceedings of the 21st International Conference on the Physics of Semiconductors, Beijing, China (World Scientific, Singapore, 1992). Vol. 1,Pg 269

5. Yi Tang and R. Braunstein, and B. von Roedern, Appl. Phys. Lett. 63,2393 (1993).

6. "Study of Light Induced Instability in Intrinsic Hydrogenated Amorphous Silicon Films by the Photomixing Technique". Yi Tang, S. Dong, R. Braunstein and B. von Roedern ( Submitted to Appl. Phys. Lett. 
Table 1: Experimental and curve fitting results

\begin{tabular}{|c|c|c|c|c|c|}
\hline $\begin{array}{c}\text { Sample } \\
\text { DD }\end{array}$ & Preparation & $\begin{array}{c}\text { Substrate } \\
\text { Temperature }\left({ }^{\circ} \mathrm{C}\right)\end{array}$ & $\begin{array}{c}\mathrm{H} \\
\text { Content }\end{array}$ & $\mathrm{L}_{\mathrm{AN}}(\AA)$ & $\mathrm{L}_{\mathrm{LS}}(\AA)$ \\
\hline \hline THD59 & Hot Wire & 290 & $10-12 \%$ & 278 & 115 \\
\hline THD60 & Hot Wire & 325 & $7-9 \%$ & 144 & 74 \\
\hline THD58 & Hot Wire & 360 & $2-3 \%$ & 310 & 125 \\
\hline THD61 & Hot Wire & 400 & $<1 \%$ & 95 & 68 \\
\hline
\end{tabular}
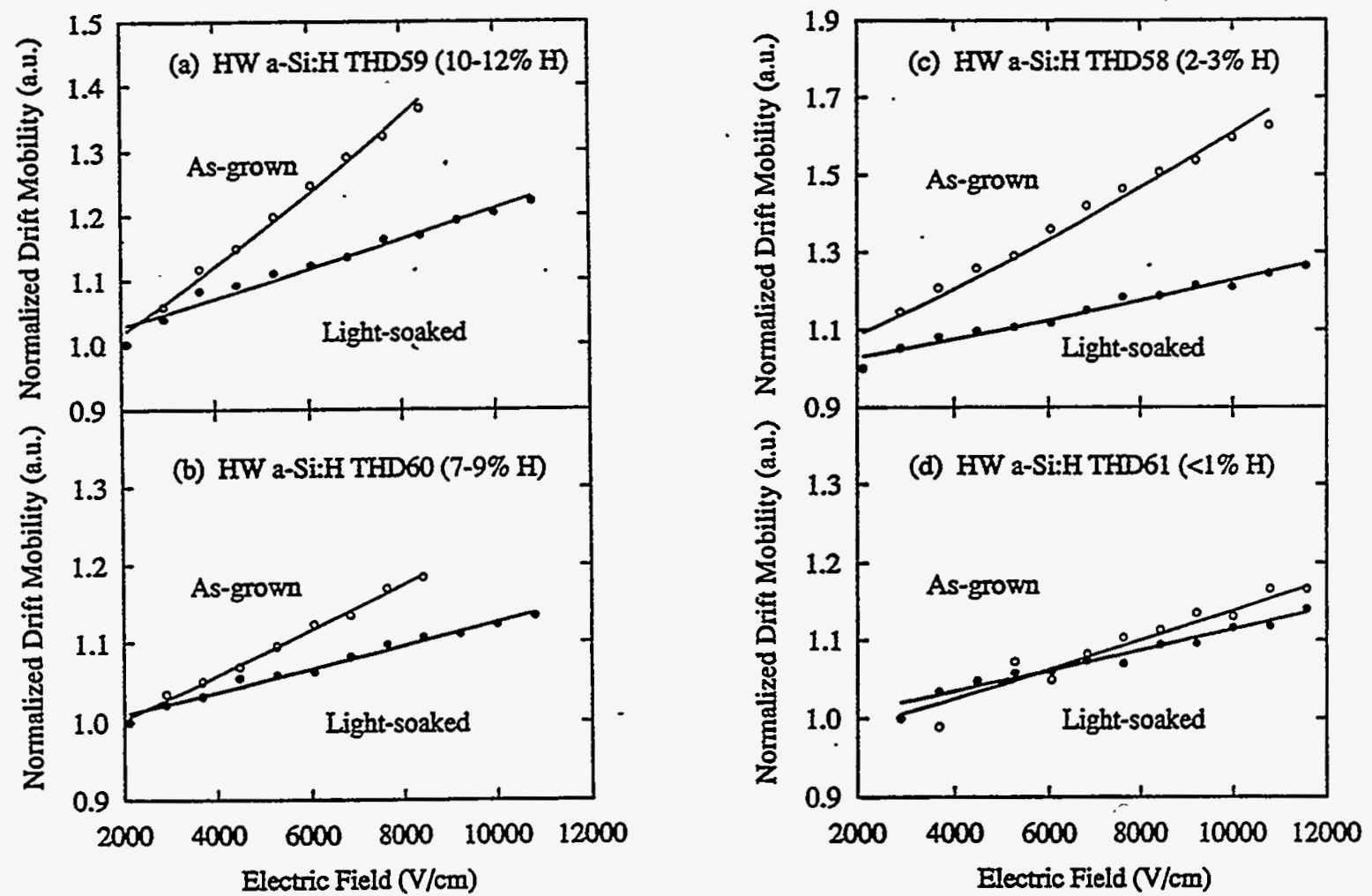

Figure 1. The electric field dependence of the normalized drift mobility. Open circle for as-grown state and solid dot for light-soaked state. a) THD59, b) THD60, c)THD58, d)THD61.

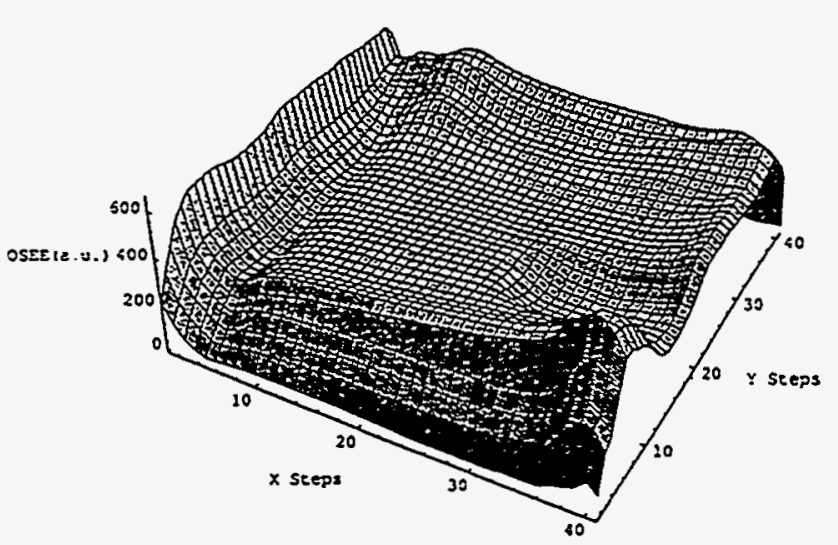

Figure 2. Inhomogeneity of NREL a-Si:H film detected by the Optical Stimulated Electron Emission (OSEE) technique. 


\section{Organization: Department of Physics \\ University of Colorado \\ Boulder, CO 80309-0390}

\section{Contributors: A.M. Hermann (Principal Investigator) and Arturo Fernandez}

The emphasis for the continuation research supported by NREL during the past year related to development of a non-vacuum process -- namely, the use of electrodeposition -- which uses binary precursors, similar to those established in the vacuum process used for the high efficiency CdS/CIGS cell fabrication. Electrodeposition is an inexpensive, non-vacuum process, scalable in principle to large areas. The program was conducted both at NREL (by Arturo Fernandez with input from Rommel Noufi and Raghu Battacharya) and on-site at the University of ColoradoBoulder laboratories of the P.I. (by Arturo Fernandez, Scott Morrison and Jerry Wagner).

OBJECTIVES: The major objective of the electrodeposition work during this period was to deposit pin-hole free smooth films of $\mathrm{Cu}, \mathrm{In}, \mathrm{Cu}+\mathrm{Se}$, and $\mathrm{In}+\mathrm{Se}$ which will provide the base of knowledge needed for a multilayer deposition sequence similar to that used in the successful vacuum evaporation experiments. The formation of CIS and CIGS will then be accomplished in a post-deposition thermal treatment.

TECHNICAL APPROACH: Indium selenide, copper selenide and copper indium selenide thin films have been prepared by electrodeposition techniques on molybdenum-coated glass substrates. Electrodeposited precursors were prepared at varying potentials, $\mathrm{pH}$ and deposition times. The adhesion and uniformity of indium selenide on molybdenum substrates were improved by electrodepositing an initial copper layer $(500 \AA)$ on molybdenum. The plating bath for copper electrodeposition was an aqueous solution of $50 \mathrm{mM} \mathrm{CuSO}_{4}$. The $\mathrm{pH}$ of the solution was adjusted to 1.5 with dilute $\mathrm{H}_{2} \mathrm{SO}_{4}$. The applied potential, current density, and deposition time were $-0.6 \mathrm{~V}$ (vs SCE), $8.5 \mathrm{~mA} / \mathrm{cm}^{2}$, and 20 seconds respectively.

The electrodeposition of In-Se precursor layers on $\mathrm{Mo} / \mathrm{Cu}(500 \AA)$ substrates was obtained using a solution mixture of $25 \mathrm{mM} \mathrm{In} 2\left(\mathrm{SO}_{4}\right)_{3}$ and $25 \mathrm{mM} \mathrm{H}_{2} \mathrm{SeO}_{3}$. The $\mathrm{pH}$ of the solution was adjusted to 1.5 using dilute $\mathrm{H}_{2} \mathrm{SO}_{4}$ ( $10 \%$ by volume) solution. Iterative experiments were performed to optimize the deposition potential and deposition time to attain suitable In-Se layer. As deposited, the In-Se layers were 1-1.5 mm thick, adherent to the substrate and black-reddish in color.

Copper Selenide layers were electrodeposited on Mo substrates using a plating solution of $50 \mathrm{mM}$ $\mathrm{CuSO}_{4}$ and $50 \mathrm{mM} \mathrm{H}_{2} \mathrm{SeO}_{3}$. The $\mathrm{pH}$ was adjusted among 1.7 to 2.4 with $1 \mathrm{M} \mathrm{Na}_{2} \mathrm{SO}_{4}$ solution.

The deposition bath used for co-deposition experiments with $\mathrm{Cu}-\mathrm{In}$-Se was $25 \mathrm{mM} \mathrm{CuSO} 4,25$ $\mathrm{mM} \mathrm{In} 2\left(\mathrm{SO}_{4}\right)_{3}$ and $25 \mathrm{mM} \mathrm{H}_{2} \mathrm{SeO}_{3}$. The $\mathrm{pH}$ was adjusted to 1.5 by dilute $\mathrm{H}_{2} \mathrm{SO}_{4}$ (10\% by volume).

RESULTS AND CONCLUSIONS: Table I lists the atomic ratio of as-deposited and annealed InSe films prepared at different deposition potentials and deposition times. At deposition potentials of -0.9 and -1.1 (vs SCE), the film compositions as analyzed by EPMA were In $1.8 \mathrm{Se} 3$ and $\operatorname{In} 1.9$ - 
2Se3 respectively. At potentials lower than $-0.9 \mathrm{~V}$ the films were selenium-rich, and at potentials higher than $-1.1 \mathrm{~V}$ the films were In rich. The films were also analyzed by ICP and the results were in good agreement with the EPMA compositional analysis data. At deposition potentials of -0.9 and $-1.1 \mathrm{~V}$ (vs SCE), the film compositions analyzed by ICP were $\operatorname{In}_{1.87} \mathrm{Se}_{3}$ and $\mathrm{In}_{1.96 \mathrm{Se}} 3$ respectively.

The XRD of the as-deposited precursor films prepared at $-1.05 \mathrm{~V}$ showed that the films were amorphous in nature and did not show any major peaks (Fig. 1a). The films annealed at $250^{\circ}$, showed the development of $\mathrm{CuIn}_{2} \mathrm{Se}_{3} .5^{15-16}$ and $\mathrm{In}_{2} \mathrm{Se}_{3}$ phases (Fig. 1b). The presence of metallic In and $\mathrm{Cu}$ is also evident in this film. The films annealed at 4500 showed $\mathrm{CuIn} 2 \mathrm{Se}_{3.5}$ as a major phase in the film (Fig. 1c).

The compositions of the In-Se films were sensitive to the types of anions present in the deposition bath. Table II shows the compositional analysis data of the films obtained using InCl3 and $\mathrm{In}_{2}\left(\mathrm{SO}_{4}\right)_{3}$.

Fig. 2 represents the SEM micrograph of a typical In-Se layer. The SEM micrograph shows a homogeneous film without cracks. A detailed micrograph analysis of films annealed at $250^{\circ} \mathrm{C}$ revealed that the individual grain sized in the films increase with increasing potentials. For example, at deposition potential of $-0.76 \mathrm{~V}$ (vs. SCE) the grain size is around $0.75 \mu \mathrm{m}$, and at deposition potential of $-1.2 \mathrm{~V}$ (vs SCE) the grain size is around $1.5 \mathrm{~mm}$. The Auger analysis shows uniform deposition up to a thickness of several microns.

The rate of deposition of the Cu-Se layer was $0.4 \mu \mathrm{m} / \mathrm{min}$ for the first $5 \mathrm{~min}$ of deposition time and varied with the deposition time. The layers adhered well to the Mo substrates. Table III shows the compositional analysis (EPMA) data of the as-deposited $\mathrm{Cu}$-Se layers at different potentials and at two $\mathrm{pH}$ values of 1.7 and 2.4 respectively. At $\mathrm{pH}$ of 1.7 and in the potential range of -0.1 to -0.6 $\mathrm{V}$, the film compositions were close to CuSe. At pH of 2.4 and in the potential range of -0.2 to $-0.8 \mathrm{~V}$ the compositions were near to $\mathrm{Cu}_{2} \mathrm{Se}$. The XRD of the as-deposited films prepared at $\mathrm{pH}$ 2.4 and at $-0.4 \mathrm{~V}$ potential showed $\mathrm{Cu}_{2} \mathrm{Se}$ phase did not change when the films were annealed up to $450^{\circ} \mathrm{C}$. The Auger analysis shows uniform deposition up to a thickness of several microns.

The rate of deposition of $\mathrm{Cu}-\mathrm{In}$-Se layer was $0.16 \mathrm{~mm} / \mathrm{min}$ for the first $5 \mathrm{~min}$ of deposition time and varied with the deposition time. The layers adhered well to Mo substrate. Table IV shows the compositional analysis (EPMA) data of the as-deposited $\mathrm{Cu}$-In-Se layers prepared at different potentials. At potentials between -0.5 to $-0.6 \mathrm{~V}$ the film composition obtained was close to $\mathrm{CuInSe} 2$. The XRD of the as-deposited precursor films prepared at $\mathrm{pH} 1.5$ and at $-0.55 \mathrm{~V}$ potential showed CuIn 2 Se3.5 as a major phase. The XRD of the films annealed at $450^{\circ} \mathrm{C}$ showed only CuIn $2 \mathrm{Se}_{3.5}$ phase. The Auger analysis shows uniform deposition up to a thickness of several microns.

Several preliminary cells of co-deposited, annealed CIS, dip-coated with CdS was completed and studied. The highest efficiency cell was near $8 \%$.

CONCLUSIONS: Electrodeposition shows great promise as a route to the fabrication of efficient large area CIS-based cells. 
Table I

The atomic ratio of as-deposited and anoenled In-Se films prepared at different deposition polentials and deposition times. The compositions are normalized to Se=3.00.

\begin{tabular}{|c|c|c|c|c|}
\hline Precenualiti & $\operatorname{Tr}=(2 e x)$ & Ardepotial film & duaded $=250 \mathrm{C}$ & Anedued if 4500 \\
\hline-0.8 & 300 & $\mathrm{In}_{12 \mathrm{Se}}$ & $\ln _{1,25}$ & $\mathrm{In}_{1 \mathrm{SS}}$ \\
\hline-0.9 & 200 & $\mathrm{In}_{1} \mathrm{Se}_{3}$ & $\ln _{2} \mathrm{Se}_{3}$ & $\ln _{1,5 S}$ \\
\hline-1.1 & 100 & $\ln _{19} S_{9}$ & $\mathrm{In}_{2} \mathrm{Se}_{3}$ & $\mathrm{In}_{2} \mathrm{Se}_{3}$ \\
\hline-1.1 & 130 & $\mathrm{In}_{2 \mathrm{Se}}$ & $\mathrm{L}_{1, x \mathrm{~S}}$ & $\ln _{2, S}$ \\
\hline-1.2 & 300 & $\operatorname{lo}_{112} \mathrm{Se}$ & $\mathrm{Ia}_{1 \mathrm{n}} \mathrm{Se}_{3}$ & $\mathrm{In}_{1 \times 1} \mathrm{Se}$ \\
\hline
\end{tabular}

Table II

The atomic ratio of as-deposited In-Se films prepared asing InCZ, and In $\mathrm{n}_{2}\left(\mathrm{SO}_{3}\right)_{2}$ salts in the deposition bath. The compositions are normalized to $\mathrm{Se}=3.00$.

\begin{tabular}{|c|c|c|c|c|}
\hline Pocenos M & $\operatorname{Tme}(S \operatorname{Sox})$ & Concencretion (M) & $\begin{array}{c}\text { Coupposion ains } \\
\text { LCh }\end{array}$ & $\begin{array}{l}\text { Couposidion esiags } \\
\text { Lexpos }\end{array}$ \\
\hline .0 .9 & 300 & 0.01 & $\ln _{2} S_{5}$ & $\mathrm{lo}_{1,2} \mathrm{Se}_{3}$ \\
\hline-0.9 & 300 & 0.025 & $\mathrm{Io}_{211} \mathrm{SE}_{3}$ & $\mathrm{In}_{21} \mathrm{Se}_{3}$ \\
\hline-1.2 & 300 & 0.025 & $\mathrm{Im}_{2} \mathrm{Se}_{3}$ & $\mathrm{Ia}_{1,1} \mathrm{Se}$ \\
\hline
\end{tabular}

Table III

The compositional analysis (EPMA) data of the as-deposited Ca-Se layers at difterent polentials and at two $\mathrm{pB}$ values of 1.7 and 24 respectirely. The compositions are normalized to Se=l.

\begin{tabular}{|c|c|c|}
\hline Pocosid (v) & PH & Fibu conposition \\
\hline-0.1 & 1.7 & $\mathrm{Cu}_{2 \mathrm{Se}}$ \\
\hline-0.4 & $t .7$ & $\mathrm{Cun}_{\text {ngse }}$ \\
\hline-0.6 & 1.7 & Cnese \\
\hline-0.2 & 2.4 & Cose \\
\hline-0.4 & 2.4 & Cyse \\
\hline-0.6 & 2.4 & $\mathrm{Cu}_{2} \mathrm{Se}$ \\
\hline-0.8 & 2.4 & $\mathrm{Cuse}_{2}$ \\
\hline
\end{tabular}

Table IY

The compositional ibsiysis (EPMA) data of the as-deposited Cn-In-Se layers deposited at diftereat potentials. The compositions are oormalized to $S e=200$.

\begin{tabular}{|c|c|c|c|c|}
\hline Poxential $(V)$ & \multicolumn{4}{|c|}{ Composition } \\
\hline & $\mathrm{Cu}\left(2 L x_{)}\right.$ & $\ln (215)$ & $\operatorname{se}(2 t 5)$ & Or-bese \\
\hline .0 .4 & $30 . \overline{04}$ & 18.46 & 30.7 & $\mathrm{Cu}_{1,12} \sqrt{\mathrm{n}_{2 \pi} \mathrm{Se}}$ \\
\hline .0 .5 & 28.78 & 21.93 & 49.06 & $\mathrm{Cu}_{2,1} \mathrm{I}_{2,1} \mathrm{Se}$ \\
\hline .0 .55 & 29.10 & 21.63 & 48.09 & $\mathrm{Cu}_{2, s} \ln _{2,4} \mathrm{Se}_{2}$ \\
\hline-0.6 & 31.00 & 20.36 & 47.25 & Cu, In Se \\
\hline-0.65 & 27.50 & 22.58 & & $\mathrm{Cu}_{1, \mu} \mathrm{ln}_{\mathrm{en}} \mathrm{Se}$ \\
\hline
\end{tabular}




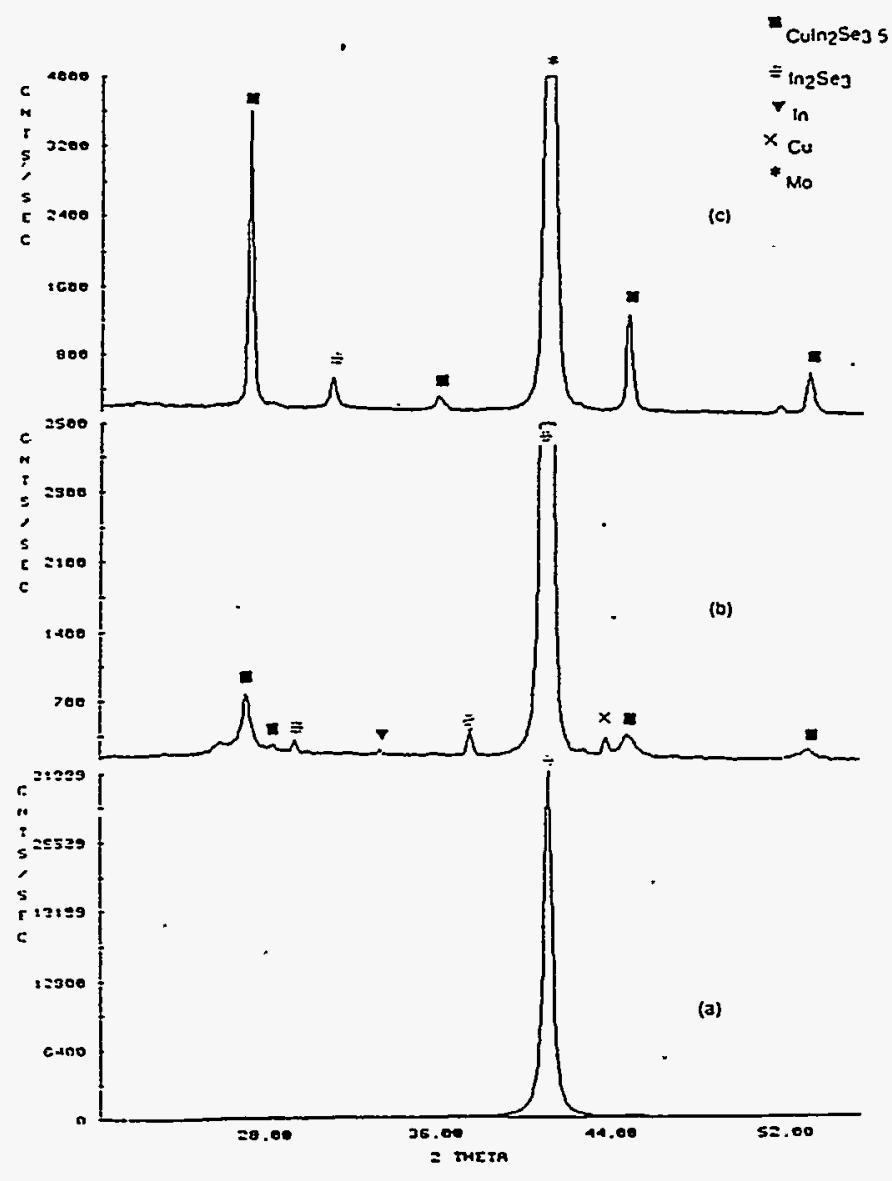

Fig. 1. X-ray diffraction data of In-Se films: (a) As-deposited film on $\mathrm{Mo} / \mathrm{Cu}$; (b) Annealed at $250^{\circ}$ for $30 \mathrm{~min}$ in argon and slow cooled $\left(3^{\circ} \mathrm{C} / \mathrm{min}\right)$; (c) Annealed at $450^{\circ} \mathrm{C}$ for $30 \mathrm{~min}$ in argon and slow cooled $\left(3^{\circ} \mathrm{C} / \mathrm{min}\right)$

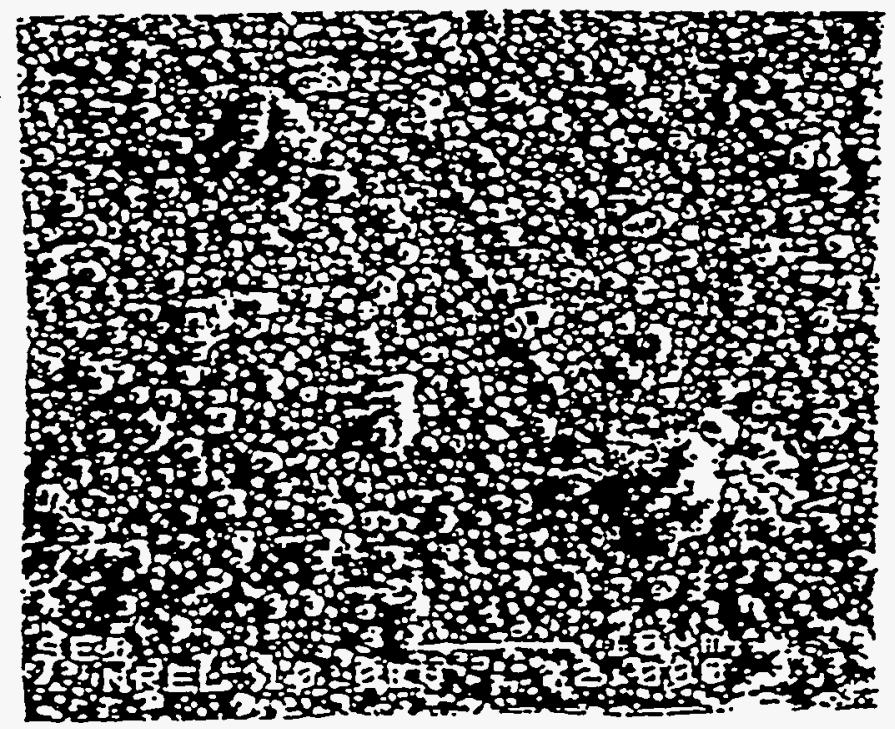

Fig. 2. Scanning electron micrograph (SEM) of a representative as-deposited electrodeposited In-Se film 
Title:

Organization:

Contributors:
Microscopic Origins of Metastable Effects in a-Si:H and

Deep Defect Characterization in a-Si,Ge:H Alloys

\author{
University of Oregon, Eugene, Oregon
}

J. David Cohen, principal investigator; Fan Zhong,

Chintimani Palsule, Daewon Kwon, and Chih-Chiang Chen

The primary research goals of this program are to elucidate the basic mechanisms by which a-Si:H degrades with light exposure, and to study the defect structure in low bandgap a-Si,Ge:H alloys. During the past year we have carried out the following studies: (1) An - evaluation of the electronic properties of a-Si:H samples produced by the hot-wire method at NREL (2) Further work to characterize the properties of the cathodic glow discharge a-Si,Ge:H samples produced by the Harvard group; and (3) The evaluation of the electronic properties of $\mathrm{a}-\mathrm{Si}, \mathrm{Ge}: \mathrm{H} / \mathrm{a}-\mathrm{Si}: \mathrm{H}$ heterojunctions in samples produced by United Solar Systems Corporation.

\section{Approach and Results}

Our research related to the Mid Gap, Metastability Team has focussed on evaluating the electronic properties of hydrogenated amorphous silicon grown by the hotwire deposition method.[1] The series of four samples we evaluated were provided by Brent Nelson and Eugene Iwaniczko at the National Renewable Energy Laboratory and employed stainless steel substrates with deposition temperatures of $290^{\circ} \mathrm{C}, 325^{\circ} \mathrm{C}, 360^{\circ} \mathrm{C}$, and $400^{\circ} \mathrm{C}$. The hydrogen content of these samples varied between over 10at.\% to less than 1at.\%, respectively. Semitransparent Pd Schottky barrier junctions were deposited at the top surface of each sample to allow junction capacitance measurements. For each sample we carried out sub-band-gap photocapacitance spectroscopy to examine the optical gaps and Urbach edge energies, and also drive-level capacitance profiling measurements to deduce overall defect densities of these samples in their as-grown and light soaked metastable states.

Figure 1 shows the photocapacitance data for three of these films in the as-grown state. For the highest hydrogen containing sample (HW290) the sub-band-gap spectrum resembles quite closely corresponding spectra obtained for conventional glow discharge a-Si:H samples. These spectra also indicate quite clearly that, as the hydrogen content decreases, the optical band gap exhibits the expected decrease. However, up to substrate temperatures of $360 \mathrm{~K}$ (a hydrogen content of 2-3at.\%) there is virtually no increase in the deep defect region of these spectra. In addition, the Urbach tail of these spectra also becomes sharper as the hydrogen level is decreased to this level.

Values of the total deep defect density obtained from the drive level profiling measurements are plotted in Fig. 2. These data confirm the findings of the photocapacitance spectra; namely, that the hydrogen level can be decreased to below 3at.\% hydrogen with a modest increase in the overall defect density. Moreover, the light degraded defect density is actually lower in the 3at.\% hydrogen sample compared to the highest hydrogen sample. Such results are quite distinct from what has been found in glow discharge a-Si:H where deep defect densities have universally been found to increase dramatically as the hydrogen content is reduced below 10at.\%. The possibility of reducing the gap of a-Si:H in this way while maintaining a low defect density is likely to be quite important for the improvement of a-Si:H based photovoltaic devices. 


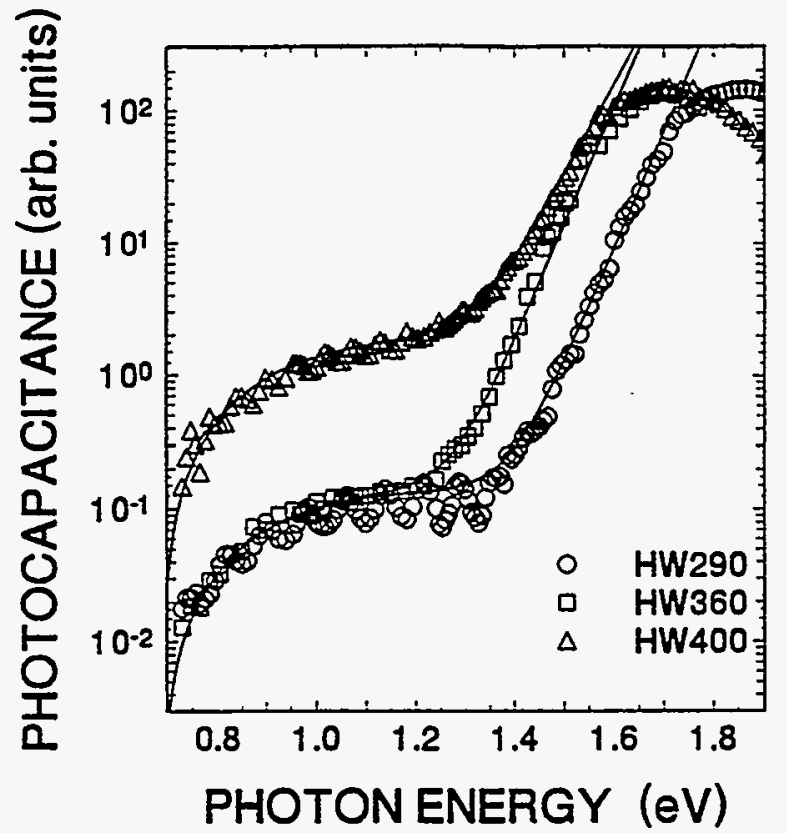

FIG. 1. Comparison of the transient photocapacitance spectra in the as-grown state for hot-wire a-Si:h samples grown with three different substrate temperatures. All data were obtained at $340 \mathrm{~K}$. The thin solid lines are fits based on a density of states consisting of an exponential band tail and a gaussian-shaped deep defect band.

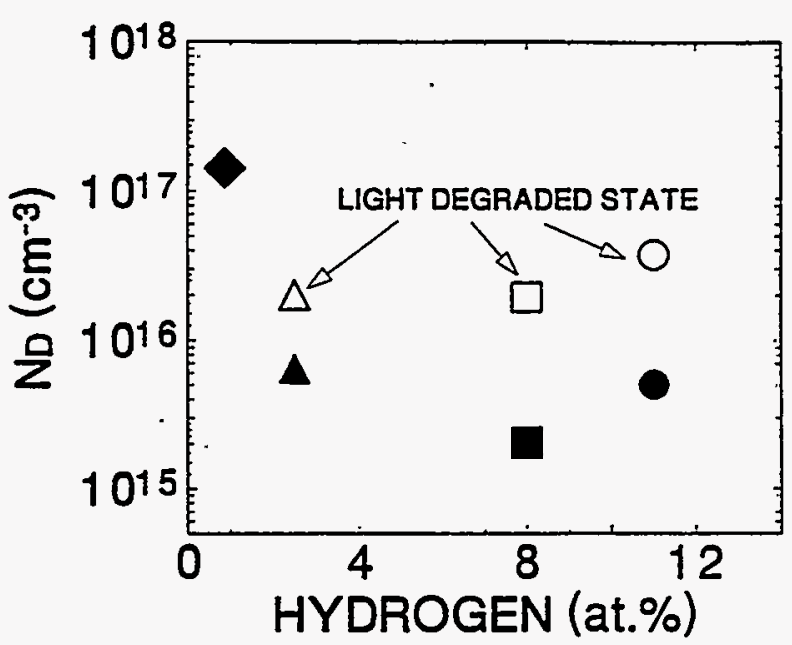

FIG. 2. Summary of total deep defect densities obtained from drive-level capacitance profiling measurements. The filled symbols were obtained in the as-grown state and the open symbols indicate defect densities in the light degraded state.

Our research related to the Low Gap Team has again been focussed on the evaluation of the series of glow discharge $a-\mathrm{Si}_{1-\mathrm{x}} \mathrm{Ge}_{\mathrm{X}}: \mathrm{H}$ cathodic alloy samples obtained from W. Paul's group at Harvard.[2] These had Ge alloy fractions, $x$, ranging between 0.5 to 1.0 and were again evaluated using sub-band-gap transient photocapacitance measurements and drive-level capacitance profiling. Some preliminary results on these samples were included in our report last year and suggested that these alloy samples exhibited significantly lower total defect densities than a-Si,Ge:H samples from other sources..

Figure 3 displays the sub-band-gap spectra for 5 of the cathodic alloy samples. One clearly sees the systematic shift in optical gap to lower energy as the Ge fraction is increased. The Urbach tail for these alloy samples is found to be significantly sharper (with characteristic energies near $45 \mathrm{meV}$ for all samples) than we had found for a-Si,Ge:H alloy samples grown by the photo-CVD method at IEC Delaware[3], or for the device quality glow discharge a-Si,Ge:H samples we had previously obtained from United Solar Systems Corporation.[4] The defect region of these spectra also indicates samples of extremely high quality.

In Figure 4 we plot the measured total defect densities obtained from the drive-level capacitance profiling measurements for the cathodic samples along with those obtained previously for the a-Si,Ge:H alloys from IEC Delaware and from United Solar Systems Corporation. As reported last year, the cathodic samples exhibit significantly lower values. However, qualitative differences in the temperature dependence of the drive-level 
measurements suggested that we might be underestimating the total defect density in the cathodic samples. Therefore, we have also used our fits of the photocapacitance spectra in Fig. 3 to make an independent estimate of these defect densities. Specifically, we assumed a density of states consisting of an exponential valence band tail plus a Gaussian shaped deep defect band. By normalizing the integral of the deep defect band with the integral of the valence bandtail states, we can obtain a fairly accurate estimate. These values are also included in Fig. 4 and generally exceed the drive-level densities by about a factor of 2 . However, even these larger optical estimates are substantially lower than the trend line established for the a-Si,Ge:H samples from the other sources.

Apart from these lower defect densities, we have noted another significant difference between the cathodic alloys and those we had studied previously. In our comparisons between the photocapacitance and transient photocurrent measurements we had observed a substantial difference at higher temperatures which could be attributed to minority carrier processes. In particular, the difference between these two types of subband-gap measurements in the band tail region of the spectrum could be used to estimate a value for the hole $\mu \tau$ product that was related to deep hole trapping. Unlike the case of these previous studies, however, the spectra for the cathodic alloy samples exhibited very little difference. Initially we thought this implied a reduced hole $\mu \tau$. However, a more complete analysis taking into account the different electric field profiles for the cathodic samples now indicates that the $\mu \tau$ values are nearly the same as those in the other samples. However, this is nonetheless surprising since, given the lower deep defect densities, these hole $\mu \tau$ products should actually be improved for the cathodic samples.

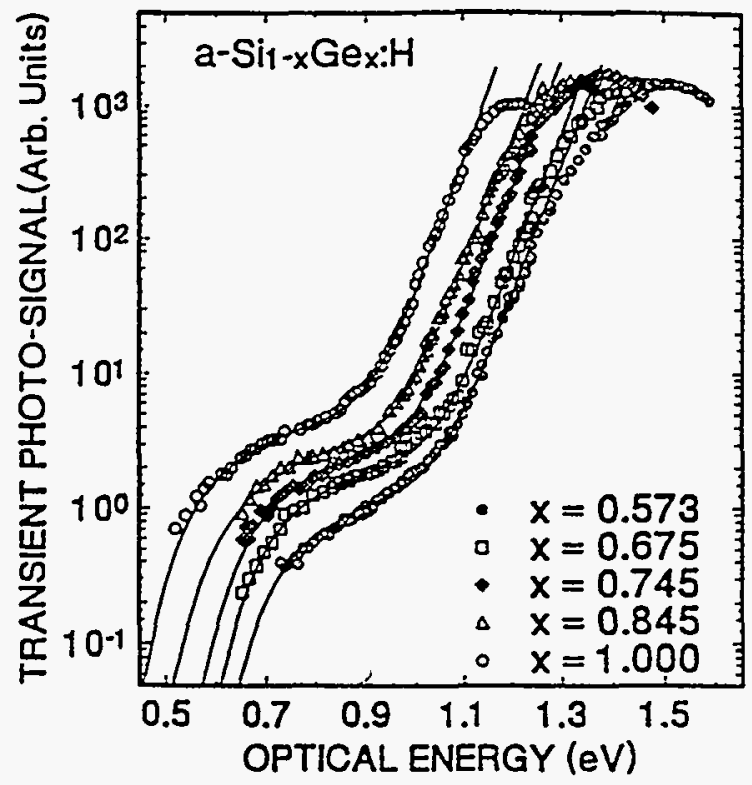

FIG. 3. Sub-band-gap optical spectra for the cathode deposited samples with $\mathrm{Ge}$ content between 57 and 100at.\%. The thin solid lines drawn through each spectrum indicate fits based upon an exponential Urbach tail and a Gaussian shaped defect band.

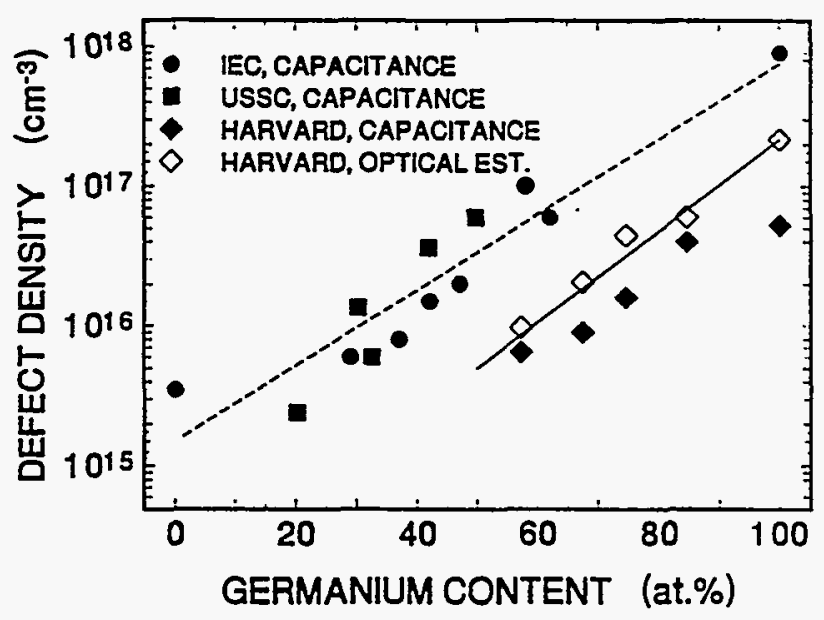

FIG. 4. Comparison of the total defect densities vs. Ge content for the cathodic a-Si,Ge:H alloys to those from two other sources. The solid symbols are values obtained from drive-level capacitance profiling, and the open symbols are estimated from the sub-band-gap spectra. 
Our final area of study has been to examine the electronic properties of the interface between a-Si:H and a-Si,Ge:H material (optical gap near $1.5 \mathrm{eV}$ ). Such interface properties may well play a key role in determining the performance of tandem structures that incorporate such a-Si,Ge:H alloys. A critical question is whether this interface contains a sufficient concentration of defects to affect the overall cell performance. This work is still in somewhat of a preliminary stage, but it appears our junction capacitance methods are particularly well suited to such kinds of studies.

We obtained 4 test device structures from United Solar Systems Corporation. All were deposited on heavily doped $\mathrm{p}^{+}$crystalline Si substrates. Each consisted of either a $3000 \AA$ or a $5000 \AA$ thick layer of a-Si:H next to a much thicker layer (at least 1 micron) of a-Si,Ge:H. The junction was formed at the p+ substrate next to the a-Si:H layer. By subjecting the device to a few volts reverse bias, the a-Si:H/a-Si,Ge:H heterojunction interface would end up lying within the depletion region formed. Thus, charge trapped and emitted from the vicinity of the heterojunction interface could be monitored by capacitance measurements.

The resulting capacitance transients from these kinds of studies indeed indicated that there are large numbers of holes being trapped at the heterojunction interface. These transients indicating hole emission were only obtained for cases where the filling pulse brought the $\mathrm{p}^{+} \mathrm{c}$-Si barrier junction into forward bias. The polarity of these capacitance transients (the fact that the slope of $\mathrm{C}(\mathrm{t})$ is negative) indicates that positive charge is being lost from the depletion region. Such positive charge emission is not observed when the bias during the filling pulse keeps the $\mathrm{p}+$ junction at even a slightly negative bias. This points to the fact that the holes are being injected from the $\mathrm{p}^{+} \mathrm{c}-\mathrm{Si}$ into the depletion region and then being trapped in the vicinity of the heterojunction interface.

Our preliminary analysis indicates that roughly $10^{11}$ holes $/ \mathrm{cm}^{2}$ are being trapped at this interface. Such a large areal density implies this would have a significant effect on the performance of tandem devices that incorporate this type of interface. Further experiments are now underway to test this conclusion by investigating a series of samples in which the interface properties have been modified in different ways during growth.

\section{References}

1. D. Kwon, J.D. Cohen, B.P. Nelson, and E. Iwaniczko, Mat. Res. Soc. Symp. Proc. 377, 301 (1995).

2. F. Zhong, C.-C. Chen, J.D. Cohen, P. Wickboldt, and W. Paul, Mat. Res. Soc. Symp. Proc. 377, 553 (1995).

3. T. Unold, J.D. Cohen, and C.M. Fortmann, Appl. Phys. Lett. 64, 1714 (1994).

4. F. Zhong, J.D. Cohen, J. Yang, and S. Guha, Mat. Res. Soc. Symp. Proc. 336, 305 (1994). 
Title:

Advanced Processing Technology for High Efficiency Thin-

Film CuInSe ${ }_{2}$ and CdTe Solar Cells; Heterojunction

Development and Optimization in Thin-Film Compound

Semiconductor Solar Cells

Organization: Department of Electrical Engineering

Center for Clean Energy and Vehicles

University of South Florida

Tampa, FL

Contributors: $\quad$ D. L. Morel and C. S. Ferekides, principal investigators

G. Attar, P. Bhethanabotla, J. D'Amico, K. Dugan, S.

Karthikeyan, M. Kazi, J. Killian, D. Marinsky, H. Natarajan, D.

Nierman, D. Oman, R. Swaminathan, and S. Zafar, graduate

assistants

CdTe

\section{Objectives}

The primary objective of this project is to prepare state-of-the-art devices using optimized manufacturing friendly conditions. The following key aspects of $\mathrm{CdTe}$ solar cells will be addressed: $\mathrm{TCO} /$ window, CdTe films, CdS films, and back contacts. Device optimization will be based on developing an understanding of the effects of processing variables/changes on device performance.

\section{Technical Approach}

During the last year emphasis has been placed on two areas: improving the efficiency of CdTe cells prepared on cost effective soda lime glass substrates and optimizing the CdS window layer and understanding its importance to the junction formation process. Preliminary work on alternative window layers such as $\mathrm{ZnS}, \mathrm{Cd}_{1-x} \mathrm{Zn}_{\mathrm{x}} \mathrm{S}$, and $\mathrm{ZnSe}$ has also been carried out.

Two processing sequences (listed below) are used for optimizing device performance:

Glass/TCO

Window

CdTe

Back Contact

Tvpe I Soda Lime/SnO,$\quad \mathrm{CBD}$ CdS

Type II Corning $7059 / \mathrm{SnO}_{2} \quad \mathrm{CSS} \mathrm{CdS} \mathrm{(ZnSe,} \mathrm{ZnS)}$

$\operatorname{CSS}\left(T<560^{\circ} \mathrm{C}\right)$

Doped Graphite

Doped Graphite

\section{Results and Discussion}

Low Cost Substrates

At the early stages of this work various soda lime glass substrates were evaluated and a solar cell with efficiency $13.5 \%$ was fabricated ${ }^{l}$. The CSS(CdTe) process was optimized for substrate temperatures in the range of $480-560^{\circ} \mathrm{C}$. Currently, type I devices are exclusively fabricated on Libbey Owens Ford (LOF) $\mathrm{SnO}_{2}$ coated glass which is the industry standard for the fabrication of $\mathrm{CdTe}$ modules. Short-circuit currents are typically low due to the poor optical properties of the glass/TCO substrate (compared to corning 7059 glass), and therefore most efforts have been directed towards utilizing thin CBD CdS in order to enhance the blue response of the cells. This work has recently produced a 13.9\% type I device measured at the National Renewable Energy Laboratory (figure 1.) The increase in the efficiency was due to a $0.8 \mathrm{~mA} / \mathrm{cm}^{2}$ increase in $\mathrm{J}_{\mathrm{sc}}$. 
Window layers

Efforts in this area have been directed towards optimizing the properties of CdS films prepared by various deposition technologies (CSS and if sputtering). Even though, state-of-the-art $V_{\alpha c}$ 's $(840-850 \mathrm{mV}$ ) have been achieved for both CSS and If sputtered CdS, solar cells fabricated with CSS CdS exhibited higher overall efficiencies ${ }^{2}$. The influence of various processing parameters fro the deposition of CSS CdS (temperature profiles, ambient) on the properties of $\mathrm{CdTe} / \mathrm{CdS}(\mathrm{CSS})$ solar cells is currently being studied. The goal is to carefully control the $\mathrm{CdTe} / \mathrm{CdS}$ interface which is critical to device performance. Currently, near $14 \%$ efficiencies can be routinely attained. Typical solar cell parameters are: $V_{\propto c}=830-850 \mathrm{mV}, \mathrm{ff}=70-74 \%$, and $J_{\mathrm{sc}}=22-24 \mathrm{~mA} / \mathrm{cm}^{2}$. Further optimization of the CSS CdS films and better understanding of the influence of subsequent processing on the properties of the $\mathrm{CdTe} / \mathrm{CdS}$ interface should lead to even higher efficiencies.
NREI X-25 IV System

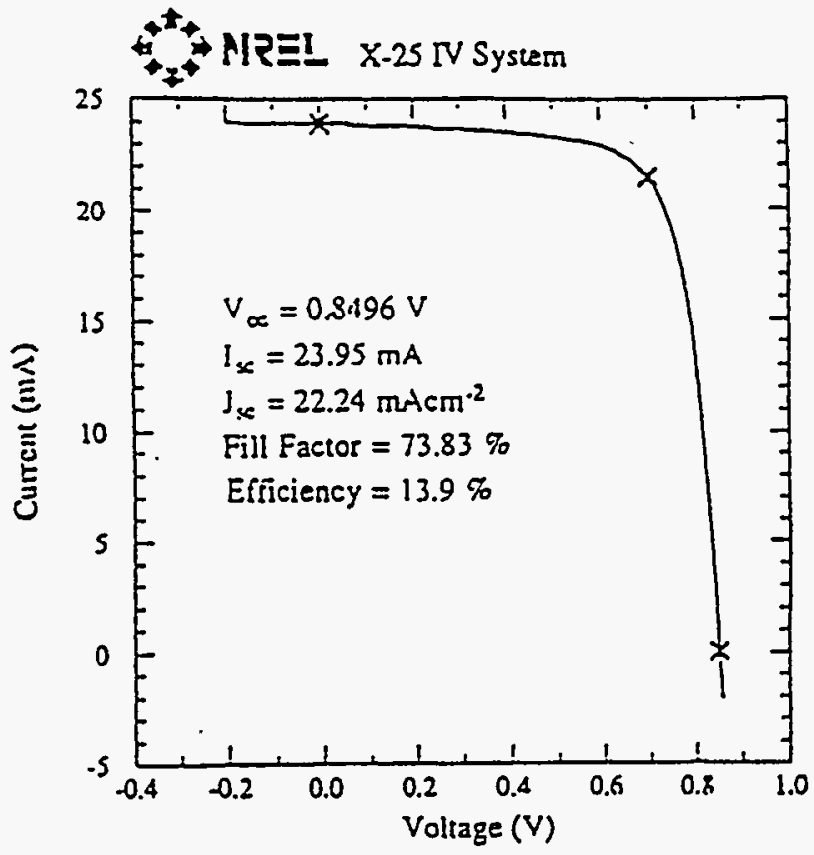

Figure 1. Light I-V for a CdTe/CdS solar cell fabricated on LOF glass/TCO.

CIS

\section{Objectives}

The objectives of this project are to develop improved processing techniques for CIS solar cells and to correlate performance and processing mechanisms.

\section{Results and Discussion}

The deposition process which we have been developing is a two step process involving sequential deposition of metal precursors and Se followed by a high pressure anneal in an Ar ambient. The initial phase of the project focussed on $\mathrm{CuInSe}_{2}$ (no Ga or S). Because of the simplicity of the process, control of individual process parameters is minimal. This makes simultaneous optimization of surface and bulk properties a key challenge. We thus have studied extensively the effect of processing variables on these properties and have developed key correlations and insights to the connections between process variables and performance. At the performance level we have used a phenomenological device model(discussed in the next section) to guide our fabrication efforts. At a fundamental device level the key to performance is controlling the recombination lifetime in the space charge region. The lifetime in tum is determined by the defect structure in the material. It is thus necessary to develop process recipes that minimize the defect density, particularly near the metallurgical junction. The dependence of $V_{o c}$ on recombination center density $(R C D)$ is shown in figure 2. As indicated, the $V_{\infty}$ in CuInSe ${ }_{2}$ devices has been increased to $400 \mathrm{mV}$ by reducing the $R C D$ to the $1 \times 10^{17} / \mathrm{cm}^{3}$ level. This improvement is the result of tuning the selenization/anneal profile to specifically address surface state properties and has led to device efficiencies in the $9-10 \%$ range. The data point at $530 \mathrm{mV}$ for "etched non-Ga devices" was attained by post deposition etching of the film surface. While this significantly reduces the surface 
states which leads to improved $V_{o c}$, control of the etchant has proved difficult and results in losses in $J_{s c}$ and FF and no net gain in efficiency.

With additional effort it will be possible to further tune the process to continue progress on RCD reduction. However, in phase II of the project we have begun introducing $\mathrm{Ga}$ into the process based upon its reputation for generally improving performance. Our approach, however, is driven by the specific expectation that $\mathrm{Ga}$ reduces the defect structure of CIS and thus will lower the RCD. In our initial experiments with $\mathrm{Ga}$ we are already realizing these expectations. As shown in figure 2, CIGS devices have $\mathrm{V}_{\infty}$ 's in the $450-500 \mathrm{mV}$ range corresponding to a reduction in RCD to the low $10^{16} / \mathrm{cm}^{3}$ range. The band gap for these devices is in the 1.0 -

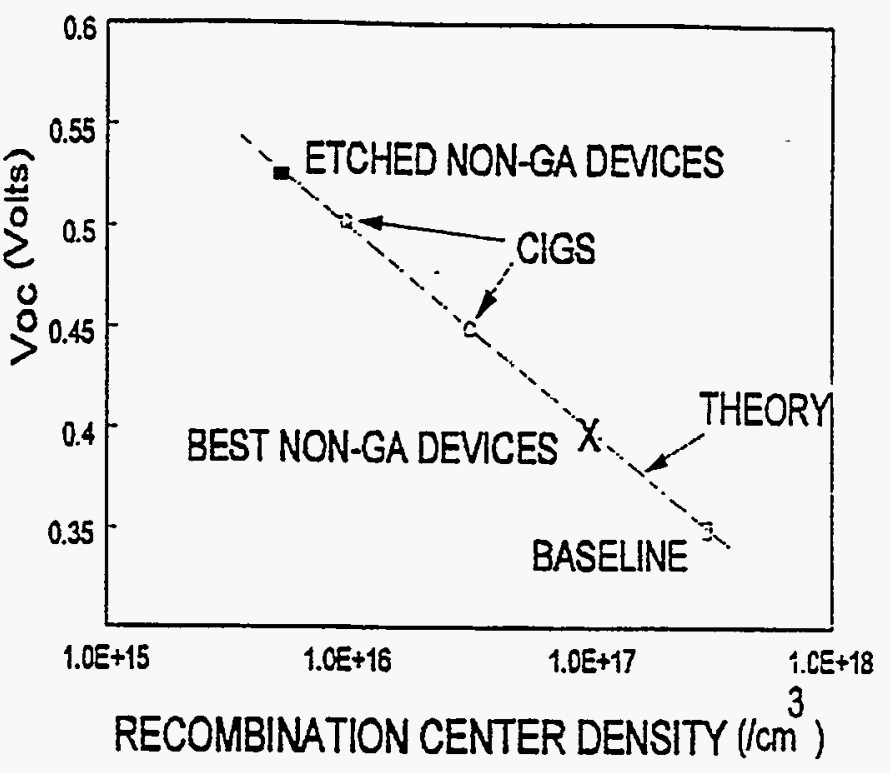
1.1 range suggesting that trace amounts of $\mathrm{Ga}$ are reducing the $\mathrm{RCD}$. For trace levels of $\mathrm{Ga}$ the $\mathrm{J}_{s c}$ 's and FF's are good, but start to diminish as additional $\mathrm{Ga}$ is added. This is similar to tradeoffs we have observed between $J_{s c}$ and $V_{o c}$ in CIS and which we have attributed to bulk/surface interplay ${ }^{3}$ -With further tuning of the process we expect to learn how to effectively incorporate higher levels of $\mathrm{Ga}$ while not adversely affecting either bulk or surface properties. This will result in significant advances in performance.

\section{Heterojunction Characterization and Development}

\section{Objectives}

The objectives of this project are to advance understanding of heterojunction formation and performance in CdTe and CIS devices and to use that understanding to guide further development of the technologies.

\section{Results and Discussion}

We have found the performance of both CIS and CdTe devices to be consistent with Shockley-Read-Hall(SRH) recombination theory. Junction performance is thus dominated by recombination in the space charge layer. The diode factor $A$ and the prefactor $J_{0}$ are both manifestations of the recombination mechanisms. Careful measurement of these parameters for a large cross section of our devices indicates that $A$ is typically in the range $1.3-2.0$. Basic SRH theory involves a single set of recombination centers in the band gap. If the centers are at mid-gap, $A=2$. Moving the centers away from mid-gap results in a lowering of $A$ down to a value of 1 for the most outlying states in energy. While this accommodates the range of $A$ values which we observe, our experimental data can not be consistently fit with the basic theory. In particular basic $\mathrm{SRH}$ theory predicts that $\mathrm{A}$ is voltage dependent, while we see a constant $\mathrm{A}$ over extensive voltage ranges. To overcome this shortcoming we have modified the theory to include a distribution of 
states in the gap which resulted in excellent fit to our experimental data. The view that emerges is one in which the diode properties are determined by the density of states(DOS) profile. Devices with diode factors of 2 are dominated by mid-gap states. Devices with lower $\mathrm{A}$ values have reduced mid-gap states.

In figure 3 we show a plot of $\mathrm{Ln} \mathrm{J}_{\mathrm{o}}$ vs. A from dark IV data for a representative group of our $10-15 \% \mathrm{CdTe}$ devices. Similar results have been attained for CIS as well. Such a correlation is not unexpected since $A$ and $J_{0}$ share a common dependence on carrier lifetime. It is noteworthy, however, that the $\mathrm{A} / \mathrm{J}_{0}$ correlation from the curve which can be written as

$$
\operatorname{Ln} \mathrm{J}_{0}=4 \mathrm{~A}-17.2
$$

predicts only minor variation in. device efficiency with $A$. Experimentally we observe higher efficiency in devices with lower A values ${ }^{5}$. Recombination parameters are modified in the presence of light due to movement of the quasi fermi levels through the DOS profiles. Recent results suggest that devices with high $\mathrm{A}$ factors(and thus dominated by deep states) suffer greater adverse modification of their recombination properties in the presence of light than their low A counterparts. Also, the presence of deep levels which act as traps is

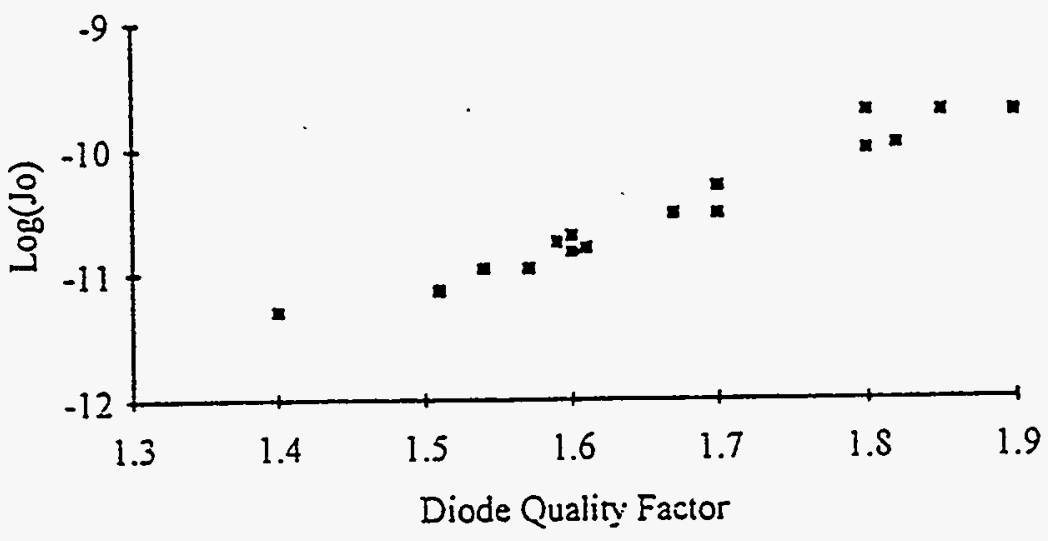

Figure 3. Dependence of $J_{0}$ on $A$ for $C d T e$ devices. seen to influence junction performance as the trap occupancy adjusts to local conditions. This results in time dependent fluctuations in $V_{o c}{ }^{6}$. Thus reduction and control of deep states seems to be the key to improving performance and reliability.

\section{References}

1. "The Effects of CdS Processing and Glass Substrates on the.Performance of CdTe Solar Cells", C. S. Ferekides, K. Dugan, V. Ceekala, J. Killian, D. Oman, R. Swaminathan, and D. L. Morel, Proceedings of the 1st World Conference on Photovoltaic Energy Conversion, Waikoloa, HA, Dec. 1994.

2. D. L. Morel and C. S. Ferekides, NREL Final Report, Contract \# XG-2-11036-1.

3. "The Effect of Interface States on CuInSe, Solar Cells", G. Attar, S. Karthikeyan, H. Natarajan, D.Nierman,s. Zafar, C. S. Ferekides and D. L. Morel, AIP Conference Proceedings 353, p.412(1995).

4. D. L. Morel and C. S. Ferekides, NREL Phase II Report, Contract \# XAD-3-12114-3.

5. D. M. Oman, K. M. Dugan, J. L. Killian, V. Ceekala, C. S. Ferekides and D. L. Morel, Appl. Phys. Lett. 67(13), 1896(1995).

6. R. A. Sasala and I.R. Sites, Proceedings of the 23rd IEEE PV Specialists Conference, Louisville, May 11-14, 1993, p543. 
Title: High Efficiency Thin Film Cadmium Telluride Photovoltaic Cells

Organization: Department of Physics and Astronomy

The University of Toledo

Toledo, $\mathrm{OH} 43606$

Contributors: Alvin D. Compaan, principal investigator, Randy G. Bohn co-investigator; Gerardo Contreras-Puente, Charles N. Tabory (deceased), Meilun Shao, Upali Jayamaha, Andreas Fischer, Zhirong Feng, Chitra Narayanswamy, and Dan Grecu

\section{Objectives}

This project is focussed on improving the fundamental understanding of if sputtering for the deposition of CdTe-related polycrystalline thin film photovoltaics with the goal of exceeding 14\% AM1.5 efficiency for an all-sputtered CdS/CdTe cell on soda-lime glass.

\section{Technical Approaches}

The University of Toledo effort emphasizes if sputtering of both CdS and CdTe from compound semiconductor targets. During the past year, we continued measurements and modifications to improve control of the plasma conditions (such as if power, substrate bias, substrate temperature, and magnetic fields) and to understand their relationships to the film properties and cell performance. Other work utilized laser physical vapor deposition for studies of the ternary alloy materials such as $\operatorname{CdS}_{x} \mathrm{Te}_{1-\mathrm{x}}$ and interdiffused layers important in CdTe-based PV.

For film characterization we use the techniques of Hall effect, optical absorption, photoluminescence, Raman scattering, $x$-ray diffraction, STM, SEM, and EDS. Device characterization included J-V, C-V, and spectral quantum efficiency measurements. We also studied optical emission from the if plasma as well as performed Langmuir probe measurements of the plasma electrical characteristics and their dependence on if power, magnetic field, substrate bias, and gas pressure. We have installed a quadrupole mass spectrometer in our sputter-deposition system to monitor base vacuum as well as gas composition during deposition.

We have continued collaboration with Solar Cells Inc and other PV groups as well as participated in the CdTe PV partnership team.

\section{Real-time and in-situ Monitors of Growth Conditions}

The rf sputtering of CdS and CdTe is being performed in a two-gun turbo-pumped chamber with two opposed 2-inch planar magnetron sputter guns. ${ }^{1.2}$ This system has been designed to permit optical beam monitoring of the film thickness during growth and also to measure glass substrate temperature by optical interference of the back-side reflected signal. ${ }^{3}$ We regularly use these optical monitors for thickness control. Recently we have added a quadrupole mass spectrometer to one port of the six-way stainless cross chamber. This permits the monitoring of the base pressure in the chamber for contaminants. The port is presently being modified to accomodate differential pumping for process vapor monitoring. In addition, we have constructed and calibrated probes for current and voltage monitoring of the power into the sputter guns and slightly modified the matching network to reduce dissipation. 


\section{Target Morphology and Growth Rate}

Target surface morphology has a substantial effect on the the film growth rate for $\mathrm{CdTe}^{4}$ We find that over the first several hours of use, as target conditioning produces microscopic roughness, the growth rate will increase by a factor of 2 to 3 . As a deep erosion track develops, the growth rate increases further. Fig. 1 compares, as a function of substrate temperature, the growth rate for a flat, but conditioned target with the rate for a deeply eroded target. ${ }^{2}$ For higher if powers the rate increases approximately as the square root of if power $^{1}$ above $\sim 10 \mathrm{~W}$ for this Ar pressure of $18 \mathrm{mTorr}$.

\section{Effect of Substrate Bias on Film Microstructure}

In If sputtering, the growing film may be subjected to a substantial ion flux which can be partially controlled with substrate bias. ${ }^{4}$ Using a Langmuir probe $1 \mathrm{~cm}$ from the typically $\sim 50 \mathrm{~cm}^{2}$ substrate we monitored changes in the plasma potential, electron energy, and ion density when bias was applied to the substrate. $^{1,2}$ The ion bombardment energy depends on the voltage across the substrate sheath. When positive bias is applied, the plasma potential rises and the plasma-substrate sheath potential difference is little changed, as shown in Table 1. Negative biases, however, will increase the ion energy since the plasma potential is fixed by the (more positive) chamber walls.

\begin{tabular}{|c|c|c|c|c|c|c||}
\hline \multicolumn{2}{|c|}{ Plasma Parameters in Unbalanced and Balanced Magnetrons $\left(\mathbf{P}_{\mathrm{rf}}=25 \mathrm{~W}\right.$, and $\left.\mathbf{p}=18 \mathrm{mT}\right)$} \\
\hline \hline applied substrate bias & \multicolumn{2}{|c|}{$+32 \mathrm{~V}$} & \multicolumn{2}{|c|}{$0 \mathrm{~V}$} & \multicolumn{2}{|c|}{$-32 \mathrm{~V}$} \\
\hline magnetic field & $\mathrm{UM}$ & $\mathrm{BM}$ & $\mathrm{UM}$ & $\mathrm{BM}$ & $\mathrm{UM}$ & $\mathrm{BM}$ \\
\hline electron temperature (eV) & 6.1 & 5.2 & 5.3 & 4.0 & 5.3 & 4.0 \\
\hline plasma potential & 47.0 & 49.8 & 19.0 & 26.0 & 18.0 & 23.8 \\
\hline saturation ion current density $\left({\left.\mathrm{mA} / \mathrm{cm}^{3}\right)}^{3}\right.$ & 4.58 & & 7.07 & 3.06 & 7.22 & \\
\hline plasma density (cm $\left.{ }^{-3}\right)$ & $4.7 \mathrm{E} 10$ & & $7.6 \mathrm{E} 10$ & $2.8 \mathrm{E} 10$ & $7.9 \mathrm{E} 10$ & \\
\hline ion bombardment energy on substrate (eV) & 15.0 & 17.8 & 22.4 & 17.9 & 50.0 & 55.8 \\
\hline
\end{tabular}

The effect of ion bombardment on the microstructure of CdTe films grown at room temperature is significant. ${ }^{4}$ Without applied substrate bias, the film exhibits a cauliflower-like structure, as shown in Fig. 2a. The film grown with $-50 \mathrm{~V}$ dc substrate bias (Fig. $2 \mathrm{~b}$ ) has a columnar structure as shown by fracture cross-section SEM; its surface is smoother than the surface of the film grown without bias. The film grown with $17 \mathrm{~V}_{\mathrm{rms}}, 60 \mathrm{~Hz}$ ac bias (Fig. 2c) exhibits a mixture of columnar and platelet structure. For the CdTe films deposited at $380 \mathrm{C}$, the energetic particle bombardment due to substrate bias did not cause significant morphology change. This implies that the bombardment-induced mobility is relatively small compared with the thermally-induced mobility at $380 \mathrm{C}$.

Ion bombardment also affects strain in the films. Fig. 3 shows the $<111>$ lattice constant of CdTe films grown with and without dc substrate bias. ${ }^{l}$ Negative substrate bias appears to increase the in-plane compressive stress at deposition temperatures of $\geq 200 \mathrm{C}$. Increasing the Ar sputter gas pressure can reduce this strain and annealing at $380 \mathrm{C}$ after $\mathrm{CdCl}_{2}$ treatment can remove most of the strain as shown in Fig. 4.

Ion bombardment is also affected by the magnetron magnetic field. We have compared a balanced magnetic field planar magnetron (BM) with an unbalanced magnetron (UM). Most electrons emitted from 
the target are trapped close to the target by a balanced magnetic field and move along the $\mathbf{E} \times \mathbf{B}$ drift path. However, in an unbalanced magnetron, magnetic field lines intersect the substrate allowing some energetic electrons to escape and bombard the substrate. Ions diffuse with the electrons to maintain the quasineutrality of the plasma, leading to ion bombardment of the substrate. The effects of the two magnetic field configurations on the plasma parameters are shown in Table 1. The films deposited with the BM show a columnar structure. The films from the UM show a smooth, mirror-like structure. Films deposited with the UM yield much higher photoluminescence, electrical conductivity, and higher cell performance than the films deposited with the BM.

\section{Studies of CdS/CdTe interdiffusion}

Controlling the amount of interdiffusion of sulfur and tellurium across the CdS/CdTe interface appears to be critical to obtaining high efficiency in CdTe-based polycrystalline solar cells. In order to approach the problem systematically we conducted two preliminary series of experiments. First, we prepared a set of films of the ternary alloy $\mathrm{CdS}_{\mathrm{x}} \mathrm{Te}_{1-\mathrm{x}}$ and determined their properties (optical gap, lattice constant, and photoluminescence). Second, we prepared a set of very thin bilayer films of CdS/CdTe on silica. These bilayers are presently being studied by Rutherford backscattering (RBS) and photoluminescence (PL). This information will be correlated with PL and SQE measurements on solar cells subjected to different amounts of post-deposition annealing.

\section{Cell Performance vs. CdS Thickness--CdTe PV Partnership Activity}

In cooperation with the Univ. of South Florida, we have recently prepared eight cell structures, four on substrates of Corning $7059 \mathrm{BSG}$ with $\mathrm{SnO}_{2}$ deposited by USF and four on substrates of $10 \Omega / \square \mathrm{LOF}$ soda lime (SL) glass. The cells had CdS thicknesses of $300,200,100$, and $60 \mathrm{~nm}$, with $2.0 \mu \mathrm{m}$ of CdTe. In each case the two substrates were sputtered side-by-side for both the CdS and CdTe layers at $380^{\circ} \mathrm{C}$. The cells were extensively analyzed at the Colorado State Univ. The BSG/USF substrate cells showed best efficiency (11.9\%) at $100 \mathrm{~nm} \mathrm{CdS}$ and $11.4 \%$ at $60 \mathrm{~nm}$, whereas the SL/LOF substrate cells showed best performance (11.3\%) at $300 \mathrm{~nm}$ of CdS decreasing to $7.1 \%$ for $60 \mathrm{~nm}$ CdS. It is not yet clear whether the drop in performance for thin CdS on LOF glass is due to the rougher texture of the $\mathrm{LOF} \mathrm{SnO}_{2}$ or a difference in chemistry of the $\mathrm{SnO}_{2}$ layers.

\section{References/publications}

1. A. D. Compaan, R.G. Bohn, \& Y. Rajakarunanayake, Subcontract Report: High Efficiency Thin Film Cadmium Telluride Photovoltaic Cells, 20 Jan 1992 - 19 Jan. 95 NREL/TP-451-8120. NREL subcontract no. ZAX-4-14013-4.

2. A.D. Compaan, M. Shao, C.N. Tabory, Z. Feng, A. Fischer, I. Matulionis, \& R.G. Bohn, "RF Sputtered CdS/CdTe Solar Cells: Effects of Magnetic Field, RF Power, Target Morphology, and Substrate Temperature," Proc. First World Conf. on Photovoltaic Energy Conversion, IEEE-PVSC1994, pp. 111-114.

3. Z. Feng, C. N. Tabory, \& A. D. Compaan, "In situ Measurements of Glass Substrate Temperature," Proc. First World Conf. on Photovoltaic Energy Conversion, IEEE-PVSC-1994, pp. 350-353.

4. M. Shao, Ph.D. Thesis, U. of Toledo (1995) "CdTe and CdS Thin Film Preparation Using RF Planar Magnetron Sputtering" (unpublished).

5. R. G. Bohn, C. N. Tabory, C. Deak, M. Shao, A. D. Compaan, \& N. Reiter, Proc. First World Conf. on Photovoltaic Energy Conversion, IEEE-PVSC-1994, pp. 354-357. 


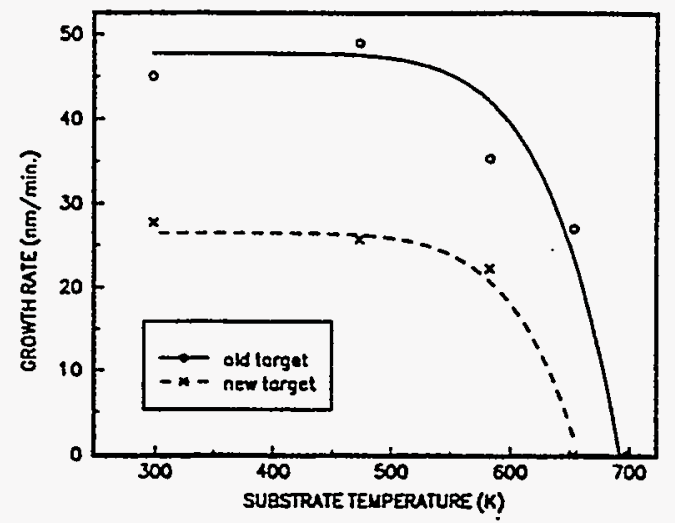

Fig. 1: CdTe film growth rate vs. substrate temperature for if power $=23 \mathrm{~W}$ into 2 " magnetron.
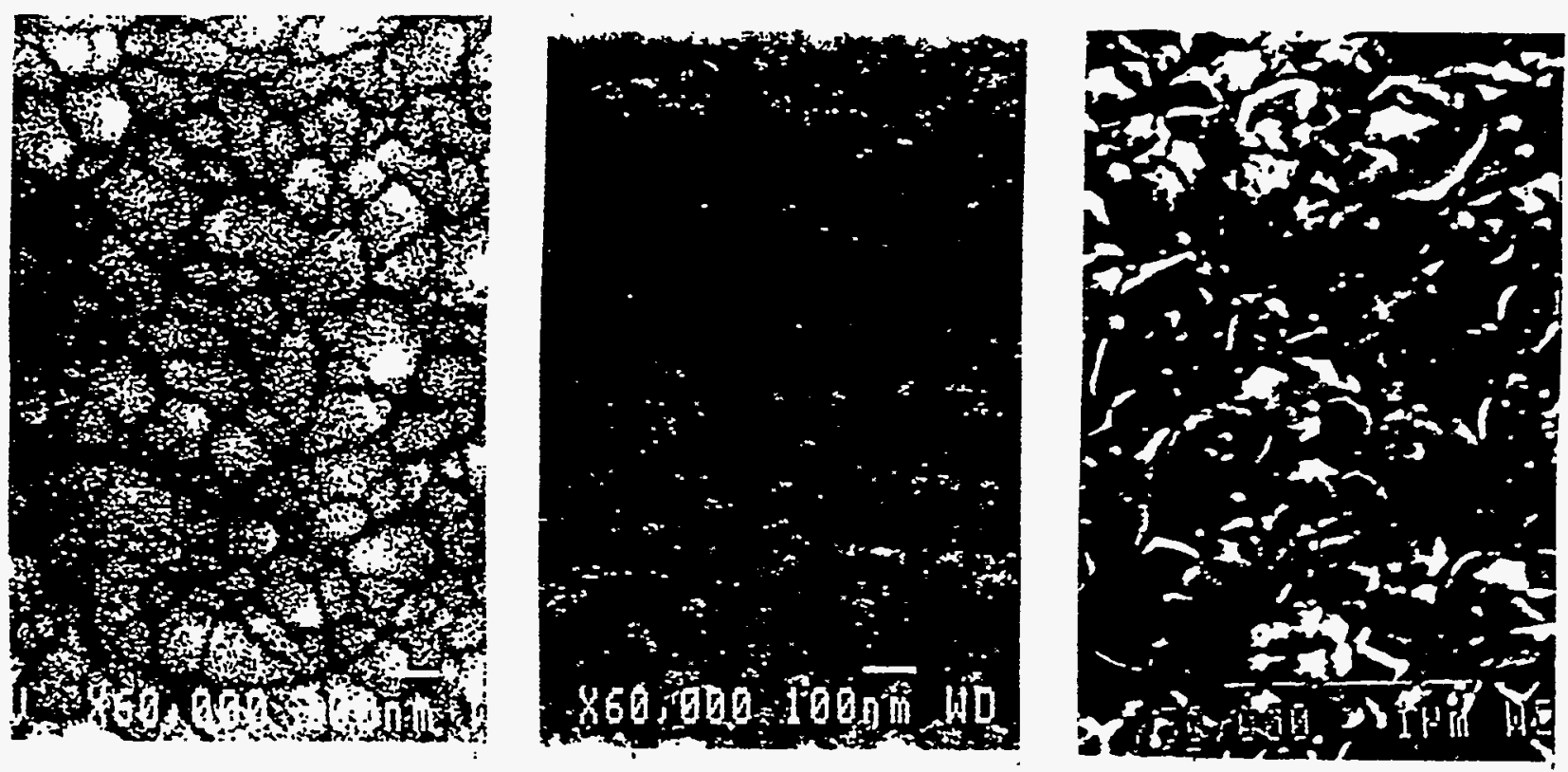

Fig. 2: SEM micrographs of CdTe films grown at room temperature with different substrate electrical bias.

a) self bias $=-2 \mathrm{~V}_{\mathrm{dc}}$, b) $-50 \mathrm{~V}_{\mathrm{dc}}$, c) $17 \mathrm{~V}_{\mathrm{mms}}$. Plasma potential is $+20 \mathrm{~V}_{\mathrm{dc}}$.

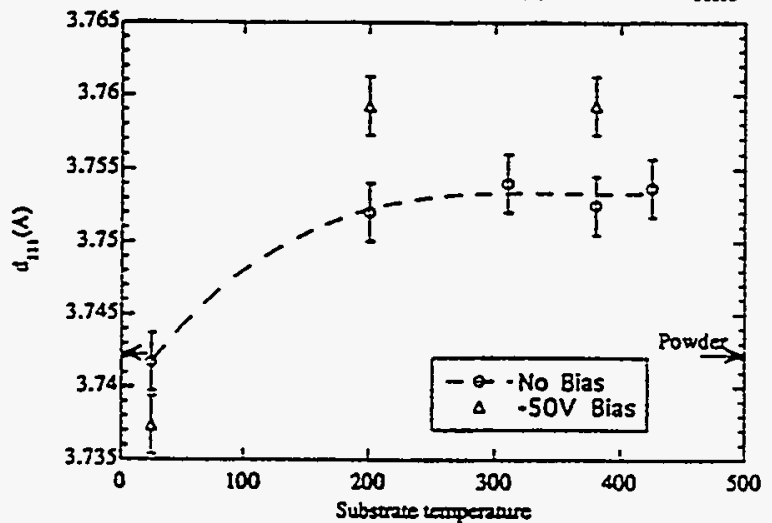

Fig. 3: $<111>$ lattice parameter of CdTe films on BSG using unbalanced magnetron at $25 \mathrm{~W}$ if power and $18 \mathrm{mT}$ Torr or argon gas. Arrow shows the $3.742 \AA$ lattice parameter for CdTe powder.

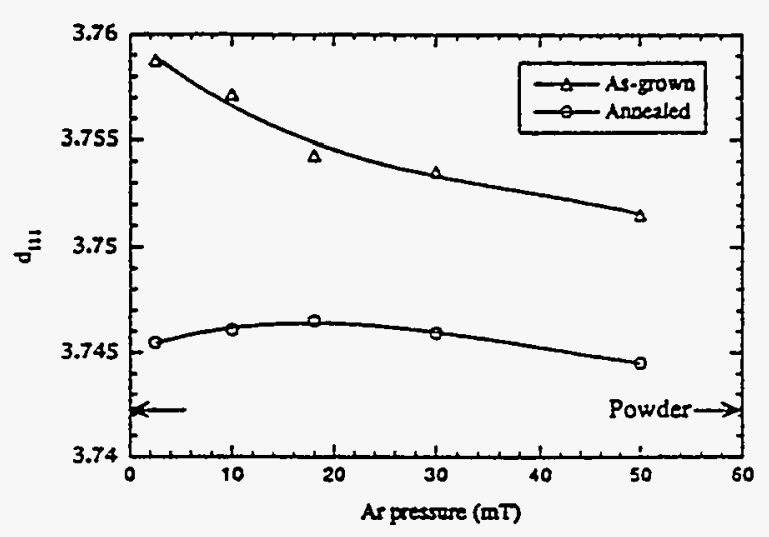

Fig. 4: $<111>$ lattice parameter of CdTe vs. Ar sputter gas pressure at $\mathrm{T}_{s}=310-385^{\circ} \mathrm{C}$. Circles are after treatment with $\mathrm{CdCl}_{2}$ and annealing at $385 \mathrm{C}$. 


\title{
Title: $\quad$ Optimization of Laser Scribing for Thin-film PV Modules
}

Organization: Department of Physics and Astronomy

The University of Toledo

Toledo, OH, 43606

Contributors: Alvin D. Compaan, principal investigator; Upali Jayamaha, research scientist; Ilvydas Matulionis, graduate student

Lower Tier Subcontractors:

\author{
Solar Cells Inc. \\ International Solar Electric Technologies \\ C J Laser Corp.
}

\section{Objectives}

This thin-film $R$ \& $D$ partnership contract is designed to evaluate and optimize new laser scribing processes to improve the reliability, performance, and cost-effectiveness of monolithically integrated polycrystalline thin-film photovoltaic modules. The project is being carried out with support from the lower-tier subcontractors and in collaboration with the CIS- and CdTe-based thin-film PV research teams.

\section{Technical Approach}

Materials for polycrystalline thin-film PV include CdTe, CuIn ${ }_{1-x} \mathrm{Ga}_{x} \mathrm{Se}_{2}, \mathrm{SnO}_{2}: \mathrm{F}, \mathrm{ZnO}: \mathrm{Al}$, molybdenum and other metals. The quality of the laser scribe in these thin-film materials is expected to be strongly dependent on two fundamental properties: 1) pulse duration and 2) wavelength. In order to obtain a wide range of these parameters, we have chosen four laser systems, with wavelengths and pulse durations given in brackets -- the flashlamp-pumped Nd:YAG laser (fp-YAG) $\left[\lambda=532 \& 1064 \mathrm{~nm}, \tau_{\mathrm{p}}=10 \& 200-400 \mathrm{nsec}\right]$, the continuous-lamppumped Nd:YAG laser (cw-pulsed or cwp-YAG) [532/1064 nm, 120-600 nsec], the XeCl excimer laser [308 nm, $15 \mathrm{nsec}]$, and the copper-vapor laser [511/578 $\mathrm{nm}, 50 \mathrm{nsec}]$. Analysis of the scribe lines and ablation spots is being done by optical microscopy, SEM, stylus profilometry, and electrical isolation tests.

In addition to the fundamental properties of pulse duration and wavelength, we will be addressing the issue of cost-effectiveness as determined by laser energy needed per unit scribe length, laser operating efficiency and cost, speed of scribe, suitability for multi-beam handling, optimum focussing (cylindrical vs. spherical lens), pulse-to-pulse amplitude fluctuations and beam profile fluctuations especially as these relate to the fundamentals of laser operation (gaseous vs. solid state media, longitudinal vs. transverse discharge, etc).

\section{Overview of our activities to date}

Since the beginning of this project in April, 1995, we have refurbished our flashlamp-pumped Nd:YAG laser, replaced some laser optics, and had the frequency doubling crystals repolished. 
We assembled a small $x-y$ scanning system with two translation stages and stepper motors. The stepper motor drivers were interfaced to a PC for programmable control. Our system is transportable and designed for scribes up to two inches long with stationary optics and a scanned PV submodule. We also have set up a digitizing scope $(500 \mathrm{Msamples} / \mathrm{sec})$ to record the pulse shapes for all the lasers being studied.

Our work with the $\mathrm{Cu}$ vapor laser has been done in an applications lab of $\mathrm{C} \mathrm{J}$ Laser Corporation in Dayton, Ohio. Our work with the continuous-lamp-pumped YAG has been done at Solar Cells Inc, in Toledo, $\mathrm{OH}$, and we have used their stylus profilometer for quantitative analysis of scribe lines and spots. ISET has supplied us with samples of films of moly on glass, CIGS/moly/glass, and $\mathrm{ZnO} / g l a s s$, and will be assisting with complete CIGS laser-scribed cell fabrication studies. The fp-YAG and the $\mathrm{XeCl}$ excimer lasers are in our lab at the University of Toledo.

\section{Laser systems}

Optically-pumped solid-state lasers---The temporal behavior of the optically pumped Nd:YAG lasers is strongly dependent on the strength of the optical pumping and on the resonator loss. When the fp-YAG laser is operated in the "free-running" mode (without the Q-switch) the pulse durations range from about $400 \mathrm{nsec}$ at threshold flashlamp energy to about $190 \mathrm{nsec}$ at 1.7 times threshold. In this free running mode, the laser emits 50 - $150 \mu$ s long trains of pulses (relaxation oscillations) with about half a dozen spikes near threshold to more than 50 spikes at 1.7 times threshold. Activating the intracavity Q-switch allows the gain to build up much higher so that a single, high power, $\sim 10$ nsec pulse is obtained. The flashlamp-pumped YAG is limited to 30 $\mathrm{Hz}$ PRF, pulse repetition frequency. The cwp-Nd:YAG used in this work is a U. S. Laser model 403PQ adapted for use at SCI. For our measurements it was operated on the frequency-doubled wavelength of $532 \mathrm{~nm}$. The laser is continuously pumped with $\mathrm{Kr}$ lamps and repetitively Qswitched with a Pockels cell. In this case the repetition frequency affects the gain and thereby also the energy per pulse. The pulse duration varies from $120 \mathrm{nsec}$ at $1 \mathrm{kHz}$ to $600 \mathrm{nsec}$ at 30 $\mathrm{kHz}$ PRF.

$\mathrm{Cu}$ vapor laser and the XeCl excimer laser--The copper vapor laser (CJL model MVL-2210) is a pulsed longitudinal discharge with output transitions at $510.6 \mathrm{~nm}$ and $578 \mathrm{~nm}$ (green and yellow) with about $25 \%$ of the 16 watts of power appearing in the yellow transition. PRF is 8 $\mathrm{kHz}$ with a pulse duration of $57 \mathrm{nsec}$. The longitudinal discharge provides the power dissipation to produce the needed $\mathrm{Cu}$ vapor pressure in the Ar buffer gas; thus, the repetition rate of this laser system is not readily adjustable. The $\mathrm{XeCl}$ excimer laser is a transverse discharge system with very high gain and $20 \mathrm{nsec}$ pulses at $\leq 100 \mathrm{~Hz}$ PRF. This system has the advantage of $u v$ wavelength $(308 \mathrm{~nm})$ at which all the PV materials are strongly absorbing.

\section{Ablation thresholds}

In this project we intend to identify ablation thresholds and conditions for most efficient removal of scribe material for each of the laser types and thin-film material types. At this point we have determined approximately several of the thresholds for ablation as measured by production of a visible mark on the surface. These are shown in Table 1. In general the thresholds are low for the strongly absorbing materials and increase with pulse duration. The nearly transparent 
conducting oxides, $\mathrm{SnO}_{2}$ and $\mathrm{ZnO}$, have much higher thresholds due to the weaker optical absorption. Ablation of the TCOs generally shows large spot-to-spot fluctuations for the non-uv wavelengths studied, presumably due to either non-linear absorption processes and/or absorption initiating at defect sites.

\section{Optical micrographs and stylus profilometer results}

The cwp-Nd:YAG laser results--Figure 1a shows a typical micrograph and profilometer trace of a CdTe film. The deepest ablation (the full $2 \mu \mathrm{m}$ film thickness) occurs where there is overlap of two pulses. Very similar results are obtained for the scribe line in CIGS as shown in Fig. 1b. The kerf is marked by a pronounced collar which extends above the plane of the original film. This feature is presumably related to the melt phase formed during the pulse and a splashing effect due to differential surface tension. To avoid module fabrication problems, this collar would need to be removed or, better, avoided entirely.

The $\mathrm{Cu}$ vapor laser results--With the $\mathrm{Cu}$ vapor laser, the scribe lines for $\mathrm{CdTe}$ films are very clean as shown in Fig: 2a. However, in the case of CIGS (Fig. 2b), distinct shoulders are observed along the scribe line which can be even more pronounced than for case of the Nd:YAG laser. Although the data are limited yet, we believe that this effect seen with the $\mathrm{Cu}$ laser on CIGS may result from the relatively slow scan speed rather than from the pulse duration. To illustrate the range of effects in different materials, we show in Fig. 3 an optical micrograph of severe cracking of a Mo film along a $\mathrm{Cu}$ laser track, and in Fig. 4 a profilometer trace of the $\mathrm{Cu}$ laser scribe mark in $10 \Omega \mathrm{LOF} \mathrm{SnO}_{2}$ approximately $0.5 \mu \mathrm{m}$ thick.

\section{Conclusions and future directions}

Data already acquired illustrate that the four laser systems described above will encompass a wide range of ablation behavior and provide a broad basis for understanding how to optimize laser scribing for cost-effectiveness, speed, and reproducibility in thin-film PV modules.

Table 1: THRESHOLDS FOR LASER SCRIBING OF THIN-FILM PV MATERIALS

\begin{tabular}{|c|c|c|c|c|c|c|c|c|c|c|}
\hline \multirow[b]{2}{*}{$\lambda(\mu \mathrm{m}) @ \Delta \tau$} & \multicolumn{2}{|l|}{$\mathrm{SnO}_{2}$} & \multicolumn{2}{|l|}{ CdTe } & \multicolumn{2}{|l|}{$\mathrm{ZnO}$} & \multicolumn{2}{|l|}{ CIGS } & \multicolumn{2}{|l|}{ Moly } \\
\hline & $\begin{array}{l}\text { Abl. Th. } \\
\left(\mathrm{J} / \mathrm{cm}^{2}\right)\end{array}$ & $\begin{array}{l}0.5 \mu \mathrm{m} \\
\mathrm{abl} .\end{array}$ & $\begin{array}{l}\text { Abl. Th. } \\
\left(\mathrm{J} / \mathrm{cm}^{2}\right)\end{array}$ & $\begin{array}{l}2 \mu \mathrm{m} \\
\mathrm{abl} .\end{array}$ & $\begin{array}{l}\text { Abl. Th. } \\
\left(\mathrm{J} / \mathrm{cm}^{2}\right)\end{array}$ & $\begin{array}{l}2 \mu \mathrm{m} \\
\mathrm{abl} .\end{array}$ & $\begin{array}{l}\text { Abl. Th. } \\
\left(\mathrm{J} / \mathrm{cm}^{2}\right)\end{array}$ & $\begin{array}{l}2 \mu \mathrm{m} \\
\text { abl. }\end{array}$ & $\begin{array}{l}\text { Abl. Th. } \\
\left(\mathrm{J} / \mathrm{cm}^{2}\right)\end{array}$ & $\begin{array}{l}2 \mu \mathrm{m} \\
\text { abl. }\end{array}$ \\
\hline $1.064 @ 10 n s$ & 0.31 & & 0.084 & 60 & 0.50 & 4.0 & 0.021 & & 0.052 & $>150$ \\
\hline $0.532 @ 10 \mathrm{~ns}$ & 1.3 & & 0.040 & 122 & 0.66 & 2.4 & 0.024 & & 0.10 & 90 \\
\hline $1.064 @ 150 \mathrm{~ns}$ & & & & & & & & & 1.0 & 2.2 \\
\hline $0.532 @ 180 \mathrm{~ns}$ & $8^{*}$ & $10^{*}$ & 0.3 & 4.0 & 13 & $20^{*}$ & 0.35 & 12 & & \\
\hline \multicolumn{11}{|l|}{$0.308 @ 15 \mathrm{~ns}$} \\
\hline $\begin{array}{l}0.511,0.578 \\
@ 55 \mathrm{~ns}\end{array}$ & 3.4 & 180 & 0.1 & 22 & 0.6 & 75 & 0.1 & 18 & 1.0 & 65 \\
\hline
\end{tabular}

* large shot-to-shot variations 

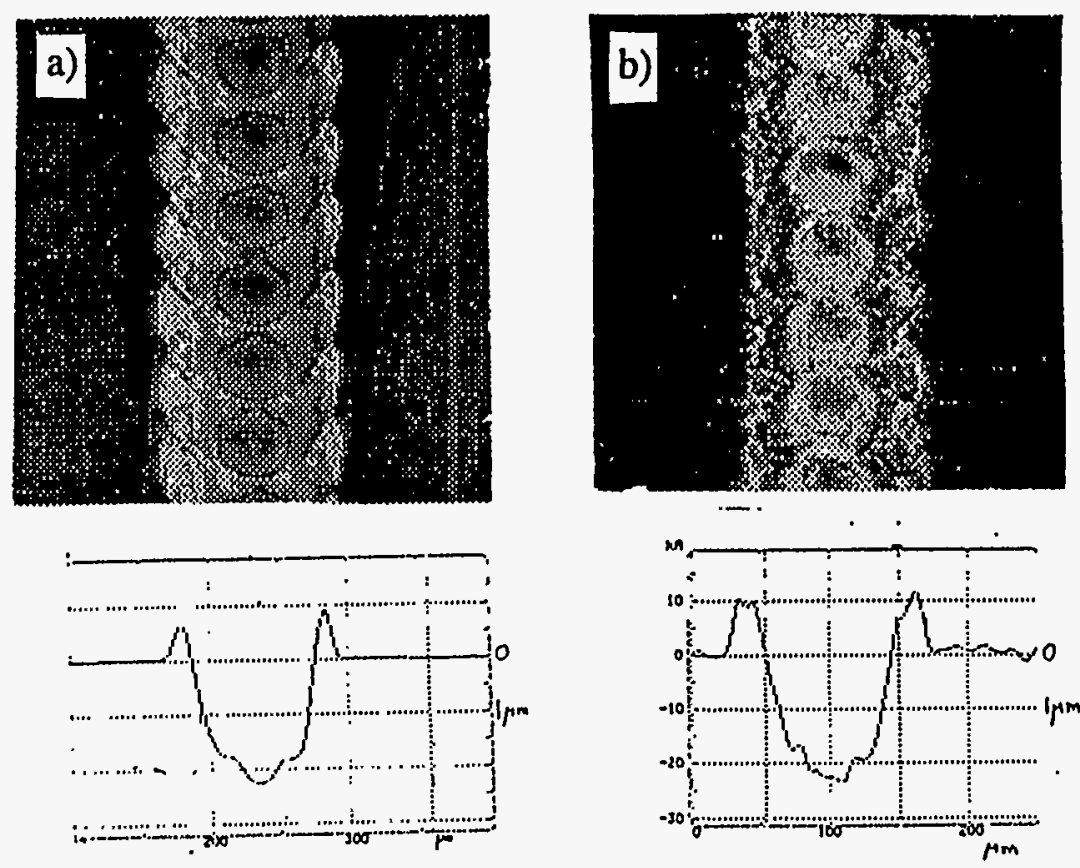

Fig. 1: a) Optical micrograph and profilometer trace of $532 \mathrm{~nm}$ laser scribe in $2 \mu \mathrm{m} \mathrm{CdTe}(P R F=5 \mathrm{kHz}, \mathrm{scan}=250 \mathrm{~mm} / \mathrm{sec}$ ), $1.93 \mathrm{~W}$ of average power. b) Same for $2 \mu \mathrm{m}$ CIGS film.

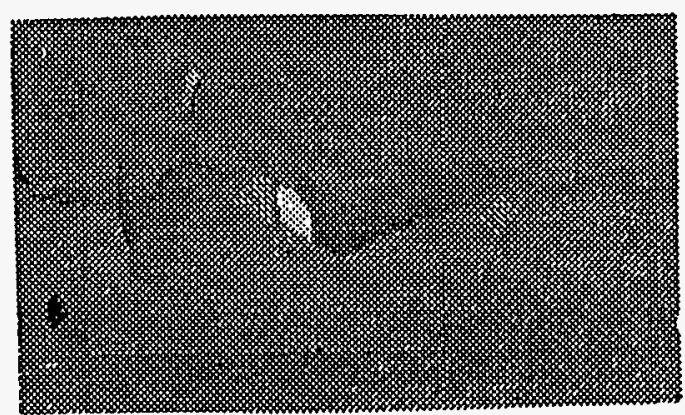

Fig. 3: Optical micrograph of scribe mark from $\mathrm{Cu}$ laser on 2 $\mu \mathrm{m}$ thick molybdenum film showing severe cracking.
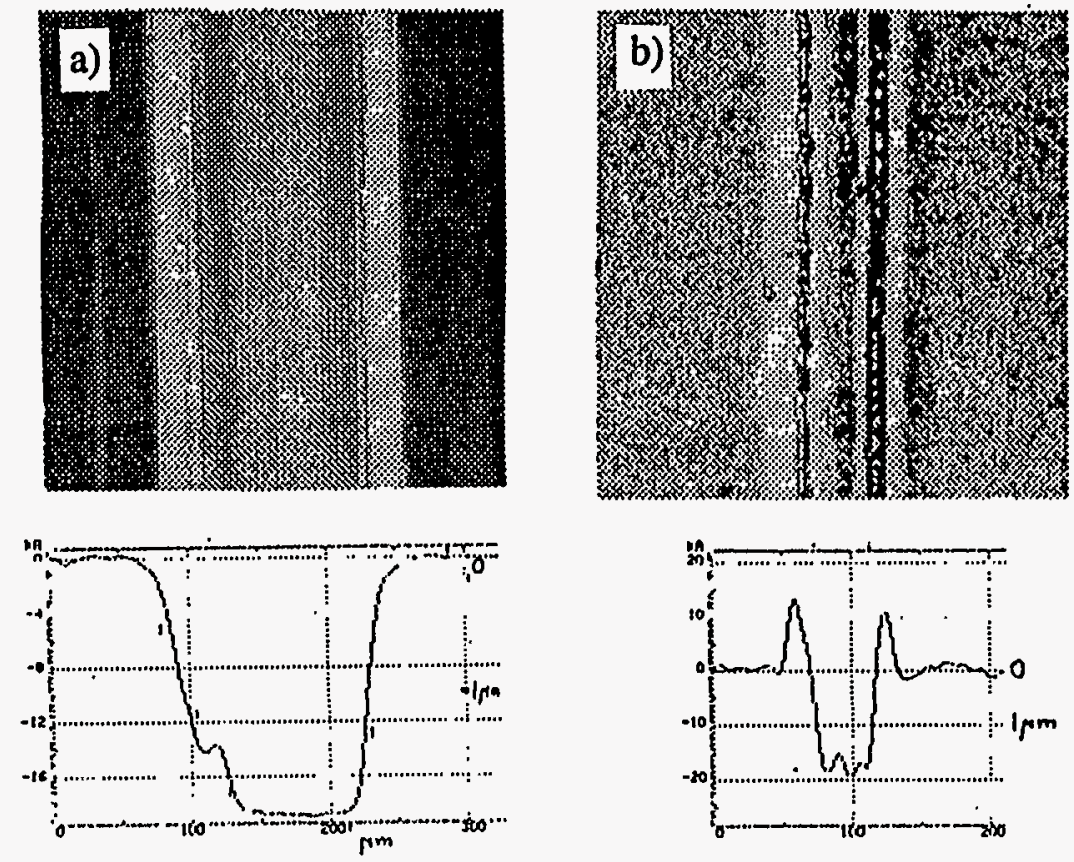

Fig. 2: a) Optical micrograph and profilometer trace of 511/578 $\mathrm{nm}$ laser scribe in CdTe (PRF=8 kHz, $13.5 \mathrm{~mm} / \mathrm{sec})$ with 1.95 $\mathrm{W}$ of average power. (The sample was slightly out of focus.) b) CIGS scribe line with same conditions.

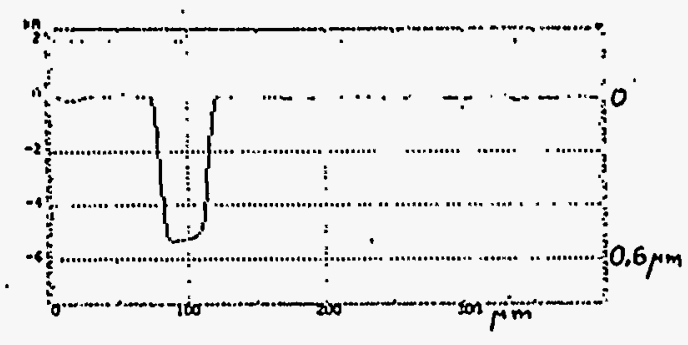

Fig. 4: Profilometer trace of a $\mathrm{Cu}$ laser scribe mark in $10 \Omega$ $\mathrm{SnO}_{2}: \mathrm{F}$ from LOF. 
Title:

Organization:

Contributors:
Investigation Of Polycrystalline Thin Film CuInSe2 Solar Cells Based On ZnSe Windows

\author{
Electronic Materials Laboratory \\ Washington State University / Tri-Cities \\ Richland, Washington
}

Larry C. Olsen, Principal Investigator;

F.W. Addis, Heriberto Aguilar, Phillip Lei, Jun Li and Xiaojun Deng.

\section{OBJECTIVES / APPROACH}

The primary objective of this program is to determine if $\mathrm{ZnSe}$ and/or $\mathrm{ZnO}$ are viable alternatives to $\mathrm{CdS}$ as buffer layer materials for CIS-based and CIGS-based solar cells. To achieve this objective the program has been structured into three tasks: (1) CIS and CIGS cells with $\mathrm{ZnSe}$ and $\mathrm{ZnO}$ buffer layers; (2) Material and device characterization; and, (3) Device modeling. Task 1 comprises the major thrust of the program. The 1995 effort emphasized studies of $\mathrm{ZnO} / \mathrm{CIS}$ cells based on Siemens CIS substrates with $\mathrm{ZnO}$ films grown by MOCVD solution growth methods. Devices are characterized by carrying out I-V analyses and external photoresponse measurements. Physical characterization involves collaboration with NREL. Device modeling studies involves investigation of current loss mechanisms with emphasis on correlation of device performance with cell processing, and the use of PC-1D to carry out modeling calculations. A brief summary of the progress made this past year follows.

\section{ZnO FILM GROWTH}

$\mathrm{ZnO}$ films are being investigated as buffer layers in CIS cells and for the top contact film. Two approaches are utilized for buffer layer formation, namely, chemical vapor deposition (CVD) and chemical bath deposition (CBD). Both CVD and CBD films have been investigated for buffer layers whereas only $\mathrm{CVD} \mathrm{ZnO}$ films are appropriate for top contact layers.

CVD ZnO Film Growth - CVD growth of ZnO is carried out in the WSU MOCVD system which consists of a SPIRE 500XT reactor with added gas handing capabilities. Tetrahydrofuran (THF) is utilized as a source of oxygen, while a zinc adduct formed by reacting dimethylzinc and triethylamine is used as a source of zinc. $\mathrm{ZnO}$ films have been doped with iodine and boron using ethyliodide and diborane as sources. Figure 1 describes results for film resistivity versus substrate temperature.

CBD ZnO Film Growth -- A two-step procedure is used. First, a $\mathrm{Zn}(\mathrm{OH})_{2}$ film is grown on a substrate and then converted to a zinc hydrous oxide film, $\mathrm{ZnO} \times \mathrm{H} 2 \mathrm{O}$, by exposing it to air. The final $\mathrm{ZnO}$ film is obtained by heat treating in air at $180^{\circ} \mathrm{C}$. Test cell studies suggest further work should be done.

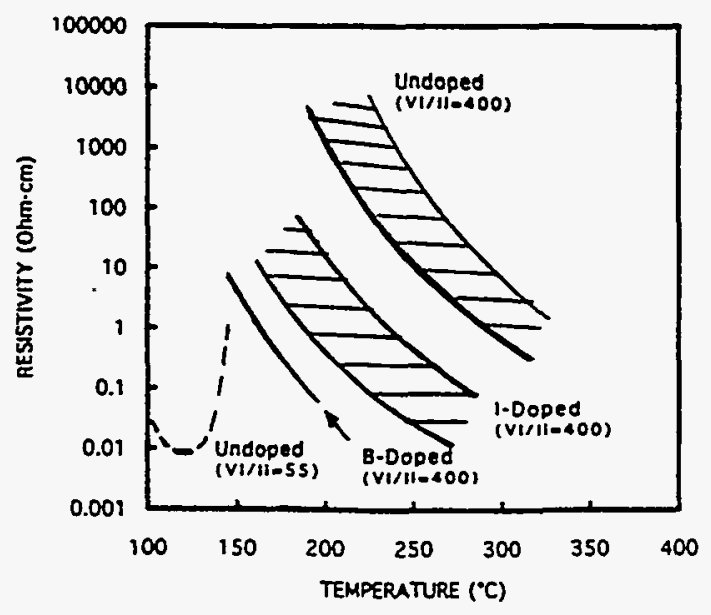

Figure 1. Resistivity of $\mathrm{ZnO}$ films on quartz. 


\section{CIS SOLAR CELLS WITH ZnO BUFFER LAYERS}

CIS solar cells based on a $\mathrm{ZnO}$ buffer layer and $\mathrm{ZnO}$ top contact could lead to simplified cell and module fabrication, and knowledge gained in these studies may lead to an improved understanding of the role of buffer layers in CIS solar cells. Thus, it is desirable to determine optimum ZnO buffer layer properties that will yield the maximum $\mathrm{ZnO} / \mathrm{CIS}$ solar cell efficiency. Although encouraging results have been achieved with $\mathrm{CBD} \mathrm{ZnO}$ buffer layers, this report concentrates on CVD ZnO buffer layers since they have been utilized for completed cells. Substrate temperatures $\left(\mathrm{T}_{\text {sub }}\right.$ ) have been varied from $225^{\circ} \mathrm{C}$ to $350^{\circ} \mathrm{C}$, with the best device performance obtained for $T_{\text {sub }}$ $\approx 225^{\circ} \mathrm{C}$ to $250^{\circ} \mathrm{C}$. Studies of layers grown at lower temperatures are in progress. These studies were conducted with material obtained from Siemens Solar, namely, the so-called 'graded absorber' material. Graded absorber substrates have sulfur near the front face such that the concentration of selenium and sulfur are equal at the surface. The graded absorber material will be referred to as CIS(GA).

Optimization Of Buffer Layers _- $\mathrm{ZnO}$ buffer layers have been grown with the substrate temperature varied from $150^{\circ} \mathrm{C}$ to $350^{\circ} \mathrm{C}$., and with the VI/II ratio varied from 50 to 400 . Determination of optimum processing parameters for $\mathrm{ZnO}$ buffer layers has been aided by fabrication and characterization of $\mathrm{Al} / \mathrm{ZnO} / \mathrm{CIS}$ test cells. After growing a $\mathrm{ZnO}$ film on a CIS substrate, an array of transparent Al circular areas $2.8 \mathrm{~mm}$ in diameter are deposited onto the $\mathrm{ZnO} / \mathrm{ClS}$ structure. Illuminated characteristics of test cells are measured by illuminating the device such that $\mathrm{J}_{\mathrm{Sc}} \approx 40 \mathrm{~mA} / \mathrm{cm}^{2}$. The cell efficiency may be regarded as an estimated active area, AM1.5G efficiency, assuming that one can add a top contact layer and collector grid without degrading the junction properties. Although aluminum is quite reactive, the test cells provide valid information regarding buffer layer properties since the $\mathrm{ZnO}$ films are typically $\geq 300 \AA$ thick, which makes the $\mathrm{ZnO}-\mathrm{Al}$ interface relatively far from the $\mathrm{ZnO}-\mathrm{CIS}$ interface.

Some representative results for test cells and completed cells are tabulated in Table 1. Values of sheet resistance, film thickness and resistivity were obtained for a film grown on a glass witness. As noted above, sheet resistance and thickness values are determined for films grown on glass and Si witnesses, and illuminated properties of test cells were acquired by forcing Jsc = $40 \mathrm{~mA} / \mathrm{cm}^{2}$. The fill factor is particularly sensitive to the buffer layer resistivity, with low values

\section{TABLE 1 -. Effect Of ZnO Buffer Layer Properties On ZnO/CIS(GA) Cell Performance}

\begin{tabular}{|c|c|c|c|c|c|c|c|c|}
\hline Device & $\begin{array}{l}\text { ZnO } \\
\text { Sheet } \\
\text { (ohm/s }\end{array}$ & $\begin{array}{l}\text { Iffer Lay } \\
\text { Thickn } \\
(\AA)\end{array}$ & $\underset{\text { Rhm-cm) }}{\text { Rho }}$ & $\begin{array}{l}\text { Tsub } \\
\left({ }^{\circ} \mathrm{C}\right)\end{array}$ & $\begin{array}{c}\text { Jsc } \\
(\mathrm{mA} / \mathrm{cm} 2)\end{array}$ & $\begin{array}{c}\text { Voc } \\
(\mathrm{mV})\end{array}$ & $\mathbf{F F}$ & $\begin{array}{c}\text { Efficiency } \\
(\%)\end{array}$ \\
\hline Test Cel & & & & & & & & \\
\hline $95-11$ & $6.2 \mathrm{E} 3$ & 987 & 0.06 & 250 & 40 & 407 & 0.31 & 4.5 \\
\hline $95-5$ & $6.0 \mathrm{E} 4$ & 548 & 0.33 & 22 & 40 & 440 & 0.34 & 6. \\
\hline $94-12$ & $3.3 \mathrm{E} 5$ & 380 & 1.2 & 260 & 40 & 417 & 0.38 & 6.8 \\
\hline $94-11$ & $1.0 \mathrm{E} 6$ & 757 & 7.6 & 270 & 40 & 374 & 0.51 & 8.1 \\
\hline $95-2$ & $4.5 \mathrm{E} 8$ & 408 & 1840 & 225 & 40 & 420 & 0.55 & 9.7 \\
\hline $94-83$ & 4.0E8 & 500 & 2000 & 225 & 40 & 43 & 0.58 & 10.5 \\
\hline Aft & Heat $T_{1}$ & nent In Eth & liodide @ & $225^{\circ} \mathrm{C}$ & 40 & 454 & 0.61 & 11.3 \\
\hline nonot & d Solar & & & & & & & \\
\hline $95-100$ & 10 & 10,000 & 0.001 & - & 33.0 & 399 & 0.49 & 6.45 \\
\hline 0111 & 4E9 & 500 & $2 \mathrm{E} 4$ & 255 & 34. & 49 & 0.6 & \\
\hline $94-54$ & $2 \mathrm{E} 10$ & 210 & $4 \mathrm{E} 4$ & 225 & 35.46 & 480 & 0.65 & 11.0 \\
\hline 9480 & $1.2 \mathrm{E} 9$ & 810 & 9700 & 225 & 34.66 & 500 & 0.6 & 11.3 \\
\hline
\end{tabular}

(Included Post Heat Treatment Of Buffer Layer In Ethyliodide) 
of fill factor occurring for low resistivities and larger fill factors resulting when the buffer layer resistivity is greater than $1000 \mathrm{ohm}-\mathrm{cm}$. Best test cell and completed cell performances were achieved when the buffer layer was grown at $225^{\circ} \mathrm{C}$ without doping, and then subsequently heat $r$ treated in ethyliodide to presumably introduce iodine from the top surface of the buffer layer. Properties of three completed cells as measured by NREL are given in Table 1. Results are also given for a completed cell that has the low resistivity $\mathrm{ZnO}$ top contact deposited by Siemens Solar directly in contact with the CIS(GA) substrate. Although much more work must be done before optimum growth parameters can be defined, it is clear that $\mathrm{ZnO}$ buffer layers must be very resistive in order to fabricate efficient solar cells. The buffer layer resistivity may affect device performance because of changes in the electron band structure near the interface and/or effects on the interface recombination velocity (due to added dopants or defects).

$\mathrm{ZnO/CIS} \mathrm{Solar} \mathrm{Cell} \mathrm{Fabrication} \mathrm{And} \mathrm{Performance} \mathrm{--} \mathrm{ZnO} / \mathrm{CIS}$ solar cells were fabricated by first depositing a $\mathrm{ZnO}$ buffer layer, followed by deposition of a low resistivity $\mathrm{ZnO}$ top contact layer and collector grid. To date, structures with the $\mathrm{ZnO}$ buffer layers have been sent to Siemens for deposition of a $\mathrm{ZnO}$ top contact layer with a sheet resistance of 10 to $20 \mathrm{ohm} / \mathrm{sq}$. The cell structure is then returned to WSU for deposition of a Ni/Ag collector grid. Temperature dependent current-voltage (TIV) analyses have been carried out for both test cells and completed solar cells in an effort to characterize current loss mechanisms. Finally, modeling studies of CIS cells with buffer layers are being to conducted to provide guidance for cell processing. Figure 2 gives illuminated I-V characteristics as measured by NREL for Solar Cell 94-80. Details concerning fabrication are given in Table 1 . The total area efficiency is $11.3 \%$ and the active area efficiency is approximately $12 \%$. As far as we know this result represents the best performance reported to date for a $\mathrm{ZnO} / \mathrm{CIS}$ solar cell.. Further optimization of $\mathrm{ZnO}$ buffer layers should lead to larger values of fill factor and thus higher efficiencies. Finally, improved efficiencies are also expected when this approach is applied to CIGS material.

Current Loss Mechanisms -- Forward current (or loss current ) versus voltage data taken under dark and illuminated conditions have been analyzed a many of the devices in Table 1. We find that forward I-V characteristics of devices such as Test Cell 94-12 that have a low resistivity buffer layer are usually characterized by a single mechanism that is probably due to tunneling/recombination. Most importantly, the magnitude of the forward current under illuminated conditions is one to two orders of magnitude larger than under dark conditions. Test

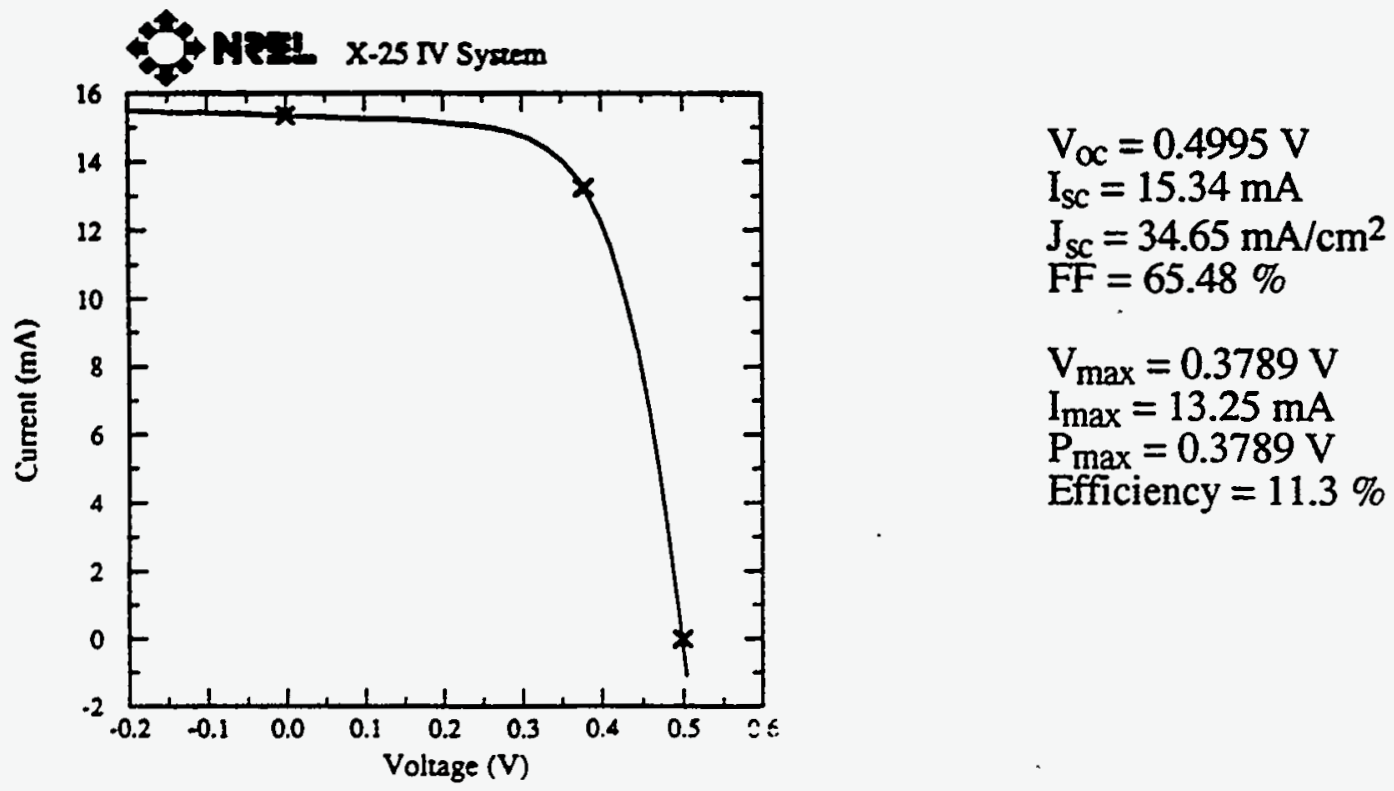

Figure 2 Illuminated I-V characteristics as measured by NREL for a completed $\mathrm{ZnO} / \mathrm{CIS}(\mathrm{GA})$ solar cell with a high resistivity $\mathrm{ZnO}$ buffer layer. 
cells with resistive buffer layers, on the other hand, have forward currents that can be understood in terms of two mechanisms, one dominant at low voltages and one dominant at high voltages ( 0.3 to 0.5 Volts). The high voltage mechanism typically is characterized by space charge recombination. In addition, the change in characteristics between dark and illuminated conditions is much less than in the case of a device with a low resistivity buffer layer.

\section{MODELING THE EFFECTS OF RESISTIVE BUFFER LAYERS}

Modeling calculations have been carried out using PC-1D, a one dimensional computer code based on a finite element numerical approach to solve the semiconductor equations. Up to three regions of different material parameters can be used to define a cell structure, each with its own doping profiles and electronic and optical properties. Recombination of electron hole pairs can be defined in each region by SRH band to band transitions or through user-defined deep level transitions. Surface recombination at interfaces is also taken into account.

CIS Cells With ZnO Buffer Layers -- Modeling calculations have been carried out for CIS cells with $\mathrm{ZnO}$ buffer layers, and with a low resistivity $\mathrm{ZnO}$ top contact layer. These studies concentrated on the effect of buffer layer resistivity and thickness on cell performance. A limited effort has also been devoted to studying the effects of changes in electron affinity for the buffer layer and charged traps in the buffer layer.

The Role Of Resistive Buffer Layers -- It is found that for reasonable values of surface recombination velocity at the CIS/Buffer-Layer interface, the efficiency of a $\mathrm{ZnO} / \mathrm{CIS}$ cell should increase from 10 to $13.3 \%$ as one increases the resistivity of a $300 \AA$ buffer layer from .001 to $10 \mathrm{ohm}-\mathrm{cm}$. The efficiency increases in the same manner if the resistivity is set at a relatively large value, and the buffer layer thickness is increased from zero to $300 \AA$. Most significantly, the open circuit voltage increases from $425 \mathrm{mV}$ to $514 \mathrm{mV}$ as a result of these changes. These results are in general agreement with experimental studies. The basic reasons for the beneficial effect of such layers are illustrated in Figure 4. Figure 4A depicts the electron band diagram of a $\mathrm{ZnO} / \mathrm{CIS}$ structure with a $300 \AA \mathrm{ZnO}$ buffer layer that has a resistivity of $1 \times 10^{5} \mathrm{ohm}-\mathrm{cm}$. A plot of recombination rate per unit volume is plotted versus depth in Figure $4 \mathrm{~B}$ for the condition of maximum power. Two cases are considered, namely, a cell with and without a buffer layer. The effect of the buffer region is to greatly reduce the recombination rate at and near the $\mathrm{ZnO} / \mathrm{CIS}$ interface. The relatively high resistance of the buffer film prevents the CIS from being strongly inverted at the $\mathrm{ZnO} / \mathrm{CIS}$ interface. which results in a low electron concentration $\left(\mathrm{n}_{\mathrm{S}}\right)$ at the interface and a reduced recombination rate.
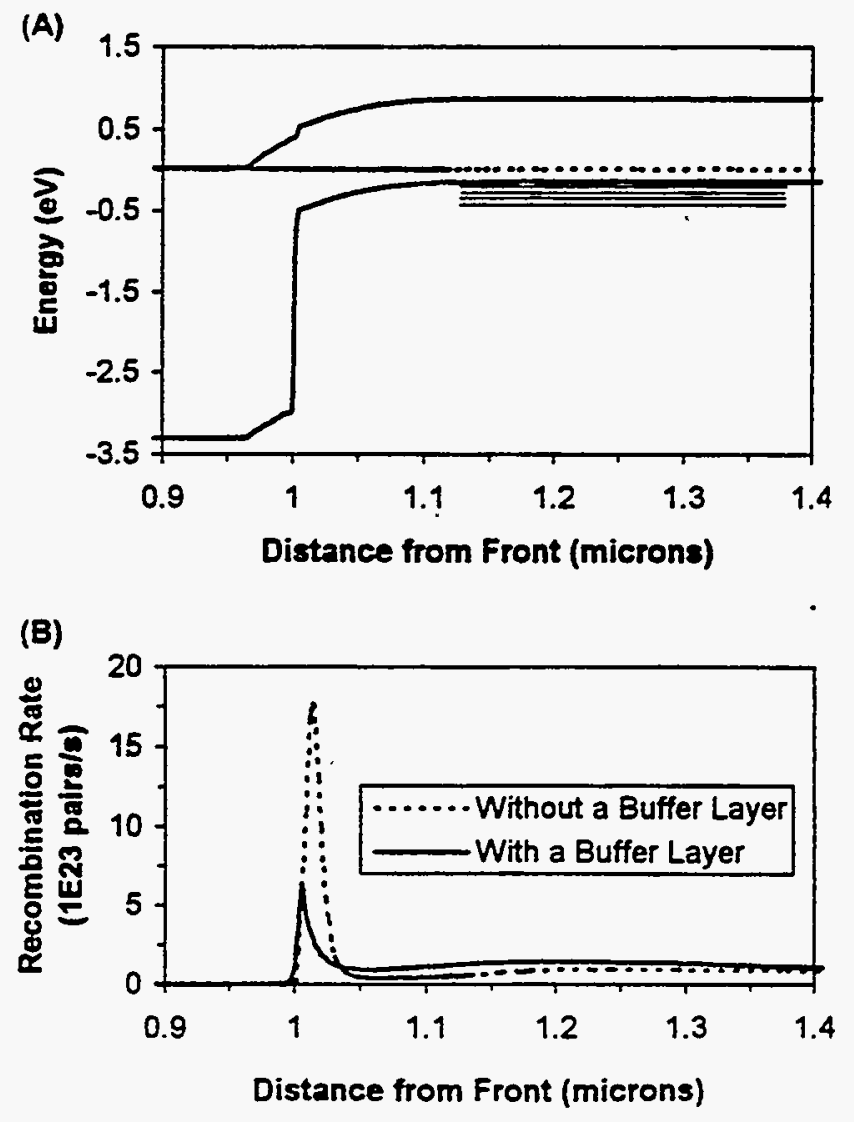

Figure 3. (A) Electron band diagram for CIS cell with a $\mathrm{ZnO}$ buffer layer and a highly conductive $\mathrm{ZnO}$ top contact layer. (B) Recombination rate vs depth. 


\subsection{PHOTOVOLTAIC MANUFACTURING TECHNOLOGY (PVMaT) PROJECT C. Edwin Witt, Manager}

The PVMaT Project was initiated in 1990 to help the U.S. PV industry extend its world leadership role in manufacturing and commercially developing PV modules and systems. It is designed to accomplish this by helping the U.S. PV industry improve manufacturing processes, accelerate manufacturing cost reductions for PV modules, improve commercial product performance, and lay the groundwork for a substantial scale-up in the capacity of U.S.-based PV manufacturing plants.

PVMaT is a government/industry R\&D partnership between the U.S. federal government (through DOE) and members of the U.S. PV industry, which is being conducted in several phases, staggered to support industry progress. It is being carried out in four separate phases that are designed to address separate $R \& D$ requirements. These phases are Phase 1 , Phase $2 \mathrm{~A}$, Phase 2B, Phase $3 \mathrm{~A}$, and Phase 4A. Each phase is aimed at achieving the PVMaT goals and is coordinated to support the specific needs of the PV industry as it makes progress toward module cost reduction and production capacity scale-up. Table 1 identifies the major PVMaT (Phases 2 and 3) awards to date. Phase 1 of this project was a problem-identification phase of about 3 months duration. During Phase 1 , the status and needs of the U.S. PV manufacturing industry were identified, and a Phase 2 procurement responsive to the industry's needs was developed. The Phase 1 subcontracts were completed in 1991.

Phase 2 addresses process-specific module manufacturing problems of individual manufacturers. The first solicitation under this phase (Phase 2A) was open only to organizations that received awards under the Phase 1 solicitation. Seven 3-year subcontracts were implemented in early 1992. The Phase 2A subcontracts through the National Renewable Energy Laboratory (NREL) were costshared between the U.S. government and the U.S. PV industry participants. A description of subcontracted $R \& D$ activities and technical accomplishments under Phase $2 \mathrm{~A}$ is presented below. The next PVMaT Phase 2 effort (Phase 2B) was a solicitation open to all U.S. PV industrial firms. This allowed organizations that were not ready for the earlier Phase $2 \mathrm{~A}$ procurement cycle, or which did not participate in Phase 1, to have a chance to "ramp on" and participate in this process-specific solution phase of the PVMaT Project. Four 3-year subcontracts were awarded under this solicitation in late 1993. They included two subcontracts in CdTe, one on Spheral Solar ${ }^{\mathrm{TM}}$ Cells, and one on cast polysilicon. These subcontracts represented new technology additions to the PVMaT Project.

Phase 3 of the PVMaT Project addresses R\&D problems that are common to the PV industry as a whole through a teamed research approach. Two subcontracts were awarded in January 1993. They focus on module-related R\&D problems.

Subcontracts under the newest phase of the PVMaT Project, Phase 4A, are now being initiated. This phase will emphasize product-driven manufacturing $R \& D$. The intention of Phase $4 A$ is to emphasize improvement and cost reduction in the manufacture of full-system PV products. Subcontracts under this Product-Driven Manufacturing solicitation represent the next step in the PVMaT program and broaden the scope of the PV product as a whole as well as elements that comprise a PV product, such as balance of systems (BOS) components, or system integration. This request for proposals (RFP) solicited proposals from individual U.S. companies and/or U.S. teams working in PV and/or related industries to address the PV manufacturing issues, from modules through packaging and improvement and installation of PV end-use products, including components 
and BOS elements. The goal is to increase the number of U.S. commercial PV products, as well as PV production capacities. This RFP was divided into two categories:

Category 4A1 - Product-Driven System and Component Technology includes manufacturing improvements directed toward innovative, low-cost, high-return, high-impact PV products. Proposals may address manufacturing generally related to PV system components and aspects other than modules, system components such as inverters, and/or system integration efficiency and/or design improvements, with lesser focus on module manufacturing. In addition, proposals may address issues in system/component integration that bring all elements together for a PV product that is offered on the market. This category is more fully described in Enclosure III, Section 3.1.

Category 4A2 - Product-Driven PV Module Manufacturing Technology includes, but is not limited to; improvements in module manufacturing processes and the manufacturing, assembly, and integration of systems to build a PV product, as well as the packaging of that product to meet market requirements. It should include module manufacturing development as a substantial portion ( $50 \%$ or more) of the effort.

The announcement for this solicitation was released in April 1994, with research efforts under both Phase 4A1 and 4A2 initiated in late 1995.

\section{PHASE 2A}

The PVMaT Phase 2A subcontracts awarded in 1992 (listed in Table 1) were cost-shared at a 47.5\% level by the subcontractors and have completed their third and final year of research. The progress and accomplishments of each are discussed in the following pages. 
Table 1. PVMaT Phase 2A Subcontract Awards

Subcontractor

AstroPower, Inc.

Energy Conversion

Devices

Entech, Inc.

Siemens Solar

Industries

Solarex Corporation

Utility Power

Group
Emphasis of Research

Silicon-Film Photovoltaic Manufacturing

Technology

Continuous Roll-to-Roll Amorphous

Silicon Photovoltaic Manufacturing Technology

Photovoltaic Manufacturing Technology

Improvements for Entech's Concentrator Module

Photovoltaic $\mathrm{Cz}$ Silicon Manufacturing

Technology Improvements

Large-Area, Multi-Junction a-Si

Alloy Production Scale-Up Project

Amorphous Silicon Photovoltaic

Manufacturing Technology
Principal

Investigator

Sandi Collins

Robert Hall

Masatsugu Izu

Mark O’Neill

Terry Jester

Robert Oswald

Michael Stern

Gil Duran

AstroPower, Inc. is enhancing its Silicon-Film ${ }^{\mathrm{TM}}$ PV manufacturing production capabilities, optimizing its solar cell processing, and improving the polycrystalline Silicon-Film ${ }^{\mathrm{TM}}$ module assembly portion of its manufacturing line. AstroPower's goals under the PVMaT Project are to:

1. Design, construct, and demonstrate a production machine that generates sheets of SiliconFilm ${ }^{\mathrm{TM}}$ at a wafer production rate equivalent to $3 \mathrm{MW} / \mathrm{yr}$

2. Develop a low-cost fabrication process for 3.15 -W solar cells that are $15 \mathrm{~cm} \times 15 \mathrm{~cm}$, and

3. Develop a large-area module production line that produces $113-\mathrm{W}_{\mathrm{p}}, 0.9-\mathrm{m}^{2}$ modules and $170-\mathrm{W}_{\mathrm{p}}, 1.4-\mathrm{m}^{2}$ modules.

At the completion of the last phase, AstroPower's accomplishments under this subcontract included the following:

- Demonstrated a $14.6 \%$-efficient, small-area cell based on the Silicon-Film ${ }^{\mathrm{TM}}$ material

- Introduced the first AstroPower Silicon-Film ${ }^{\mathrm{TM}}$-based product, the $15-\mathrm{cm} \times 45-\mathrm{cm} \mathrm{AP-225}$ cell into production

- Completed the optimization of the gettering sequence 
- Completed the development of a continuous surface-preparation/gettering/diffusion process, and

- Completed the development of very large sheets of solar cells (up to $18,000 \mathrm{~cm}^{2}-2-\mathrm{ft}^{2}$ ).

Energy Conversion Devices (ECD) directed its research efforts toward the development of the continuous, roll-to-roll amorphous silicon (a-Si) PV manufacturing technology. ECD's overall goals under this subcontract are to:

1. Support production of $0.3-\mathrm{m} \times 1.2-\mathrm{m}(1-\mathrm{ft} \times 4-\mathrm{f})$ triple-junction a-Si:Ge alloy modules with $10.2 \%$ stable efficiency

2. Enhance the effective production throughput by developing advanced serpentine deposition techniques, and

3. Reduce material and labor costs. Successful implementation of this project will allow ECD to work toward an ultimate goal of building a 100-MW/yr roll-to-roll, automated a-Si module manufacturing facility. At this anticipated production capacity, ECD expects to reduce the cost of PV modules to less than $\$ 1.00 / \mathrm{W}_{\mathrm{p}}$.

At the completion of the last phase, ECD's accomplishments under this subcontract included the following:

- Demonstrated a 2,500-ft. production run of dual-junction solar cells with a yield of $99.7 \%$ on material having an initial subcell conversion efficiency of $10.2 \%$

- Fabricated the world's first roll-to-roll, 4- $\mathrm{ft}^{2}$, triple-junction, two-bandgap a-Si module (initial aperture-area efficiency of $9.5 \%$ )

- Demonstrated a triple-junction a-Si solar cell, with the top cell deposited in the serpentine deposition chamber having an initial efficiency of $9.5 \%$

- Completed the conceptual design for an automated, high-volume (100-MW/yr) PV manufacturing plant for the production of PV modules, with an expected material cost reduction of $71 \%$.

ENTECH, Inc, has directed research under its PVMaT subcontract at PV manufacturing technology improvements for ENTECH's $21 \mathrm{X}$ linear concentrator module. The specific ENTECH goals under this project are to

1. Enhance its concentrator module quality

2. Increase the ENTECH concentrator module performance

3. Improve product durability

4. Expand the ENTECH manufacturing capacity to $10 \mathrm{MW} / \mathrm{yr}$

5. Address environmental, safety, and health issues 
6. Reduce ENTECH module manufacturing costs

7. Increase ENTECH manufacturing-line automation.

Successful completion of this research is expected to result in a linear concentrator module manufacturing plant with the capability of producing PV modules at a rate of $10 \mathrm{MW} / \mathrm{yr}$.

At the completion of the last phase, ENTECH's accomplishments under this subcontract included the following:

- Developed and tested over 20,000 cell packages fabricated with the semi-automated prism cover application station, using the new prism-cover tape, which resulted in high yields and cell packages with an $>17 \%$ lot-average conversion efficiency

- Eliminated over 300-parts/receiver through the integration of a new dry-film process for bonding cell packages to the new extruded ENTECH module heat sink

- Installed two 100-kW power plants, each using 288 fourth-generation ENTECH modules developed under the PVMaT project.

Siemens Solar Industries is investigating improvements to its Czochralski $(\mathrm{Cz})$ Silicon PV manufacturing technology. Its goals for this research include

1. Improving $\mathrm{Cz}$ Si-ingot quality

2. Increasing the materials use efficiency for crystalline $\mathrm{Si}(\mathrm{c}-\mathrm{Si})$ wafer sawing

3. Investigating improvements in device processing

4. Investigating the introduction of automation to a significant portion of its $\mathrm{c}-\mathrm{Si}$ module manufacturing lines

5. Reducing the amount of hazardous waste generated. The successful completion of this project was expected to result in a decrease in Siemens' c-Si module costs of more than half while doubling its U.S. manufacturing capability.

At the completion of the last phase, Siemens' accomplishments under this subcontract included the following:

- Achieved a $4 \%$ shift in electrical yield of the solar cells fabricated with improved PVMaT processes

- Decreased the cost of production per solar cell by $65 \%$

- Achieved an overall improvement in cell mechanical yield of $10 \%$ throughout the Siemens line

- Completed the overall implementation of wire saws in the Siemens manufacturing line 
- Completed the commercialization of the new 75-W module developed under the PVMaT project

- Reduced the caustic waste from the Siemens PV production line by an additional $20 \%$ by volume.

Solarex Corporation is focusing on the large-area, multi-junction, a-Si alloy production scale-up. The goals of this effort include

1. Improving the deposition and quality of its transparent front contact

2. Optimizing its laser-patterning process

3. Scaling up the semiconductor deposition process

4. Improving the back-contact deposition and

5. Scaling up and improving the encapsulation and testing of its a-Si:H modules.

Successful completion of this 3-year project will result in Solarex producing a-Si:H modules of $\sim 0.37 \mathrm{~m}^{2}\left(4 \mathrm{ft}^{2}\right), 10 \%$ stabilized efficiency, $50 \mathrm{~W}$, and a total overall module yield of $275 \%$. At a proposed production capacity of $10 \mathrm{MW} / \mathrm{yr}$, Solarex believes this would result in a potential module cost reduction from the present price of $\$ 11.05 / \mathrm{W}_{\mathrm{p}}$ to $\$ 1.14 / \mathrm{W}_{\mathrm{p}}$.

At the completion of the last phase, Solarex's accomplishments under this subcontract included the following:

- Reported an $8.0 \pm 0.2 \%$ stabilized efficiency distribution on 100 out of 107 of their $4-\mathrm{ft}^{2}$ $\mathrm{Si} / \mathrm{SiGe}$ modules

- Completed the development of a low-cost zinc-oxide/aluminum rear contact

- Environmentally qualified the 4- $\mathrm{ft}^{2}$ modules/EVA-tedlar encapsulation

- Developed a cost-effective process for the production of stable, $8 \%$-efficient tandem modules, and

- Initiated construction of a 10-MW/yr factory based on these processes developed under the PVMaT subcontract.

Utility Power Group (UPG) and its major lower-tier subcontractor, Advanced Photovoltaic Systems (APS), have focused their research on a-Si PV manufacturing technology. UPG's goals under the PVMaT Project are to:

1. Significantly advance its PV manufacturing technologies

2. Reduce module production costs 
3. Increase average module performance, and

4. Increase the production capacity of UPG's existing manufacturing facility.

After identifying two specific process steps with the greatest potential for cost reduction in PVMaT Phase 1, UPG chose to concentrate its research efforts on two areas: encapsulation, with consideration given to approaches that do not require a second glass layer; and automation of electrical termination.

At the completion of the last phase, the accomplishments by UPG under this subcontract included the following:

- Completed installation of the advanced encapsulation and termination systems in the UPG production line

- Demonstrated over $95 \%$ manufacturing yields and $80 \%$ reduction in manufacturing costs with the UPG advanced encapsulation and termination systems

- Received 2 U.S. Patent covering the UPG advanced termination system, and

- Supported the evaluation of incorporating the UPG's advanced encapsulation system into the manufacturing lines of two major thin-film PV module manufacturers.

Advanced Photovoltaic Systems, as a lower-tier subcontractor to UPG, has focused its research on improving its PV manufacturing technology, primarily by improving process control, increasing automation of the manufacturing line, and improving the quality of its product. During the beginning of this last phase as a lower-tier subcontractor under the UPG/NREL subcontract, APS found it necessary to request that UPG issue them a Stop Work Order in order to suspend their activities under the subcontract. UPG has taken over a small portion of these activities directed at integrating the UPG's advanced encapsulation system into the manufacturing line of other PV module manufacturers. 


\section{PHASE 2B}

Under Phase 2B, NREL awarded four 3-year subcontracts in late 1993. These subcontracts (listed in Table 2) are cost-shared at a 51.3\% level by the subcontractors and are completing their second year. The results of research under these subcontracts are outlined below.

\section{Table 2. PVMaT Phase 2B Subcontract Awards}

Subcontractor

Golden Photon, Inc.

Solar Cells, Inc.

Solarex Corporation

Texas Instruments
Emphasis of Research

Commercial Scale-Up of Advanced ThinFilm Photovoltaic Technologies

High-Throughput Manufacturing of Thin-Film CdTe Photovoltaic Modules

Cast Polycrystalline Silicon Photovoltaic Cell and Module Manufacturing Technology Improvements

Photovoltaic Manufacturing Technology Phase 2B - Spheral Solar Technology
Principal Investigator

Steve Johnson

Dan Sandwisch

John Wohlgemath

Jim Skelly

Rusty Schmit

Golden Photon, Inc. is concentrating its activities on the commercial scale-up of advanced thinfilm CdS/CdTe PV modules produced using spray deposition. The goals set by Golden Photon for this subcontract include the development and implementation of:

1. Improved production processes and subsequent equipment designs that allow incremental scale-up to a 2-MW/yr manufacturing capacity

2. An employee exposure monitoring plan that will ensure employee safety from $\mathrm{Cd}$ exposure, and

3. Environmental, safety, and health (ES\&H) programs that meet the requirements of all local, state, and national authorities.

At the completion of the subcontract, these goals are expected to result in a $2-\mathrm{MW} / \mathrm{yr} \mathrm{CdTe}$ module manufacturing line capable of production costs of about $\$ 1.50-\$ 2.00 / \mathrm{W}_{\mathrm{p}}$.

Golden Photon's major accomplishments during the second year of this subcontract included the following: 
- Demonstrated (1,000 substrate run) a $95 \%$ yield on their high-resistance tin deposition line

- Showed a significant increase in the average process yield for each of the film deposition processes

- Demonstrated a production line average of over 20-W/panel for 3 consecutive months, and

- Demonstrated a 28-W module.

Solar Cells, Inc. (SCI) has directed its research at developing high-throughput manufacturing of thin-film CdTe PV modules. The goals of SCI under the PVMaT Project are to:

1. Advance its PV manufacturing technologies

2. Reduce module production costs

3. Increase module performance, and

4. Provide the groundwork for SCI to expand its commercial production capacities.

$\mathrm{SCI}$ plans to meet these objectives by designing and debugging a 20-MW/yr, automated, continuous PV manufacturing line that produces $60-\mathrm{cm} \times 120-\mathrm{cm}$ CdTe modules.

SCI's accomplishments after the second phase of this subcontract included the following:

- Demonstrated a 8.1\%-efficient $60-\mathrm{cm} \times 120-\mathrm{cm}$ CdTe fully encapsulated module

- Demonstrated a 120 -module fabrication run on their 100-kW CdTe pilot production line, resulting in average module efficiency of $7.0 \%$

- Completed Quality-Assurance/Quality-Control program manuals and an ES\&H program and procedures manuals for $\mathrm{SCI}$ plant operation

- Completed the design and specification for the scribe 1 laser system and CdS/CdTe deposition systems

- Completed the 20-MW plant layout.

Solarex Corporation (Crystalline Silicon Division) has focused its research under this subcontract on advancing its cast-ingot polycrystalline Si PV module manufacturing technology. Solarex's goals under PVMaT Phase 2B are to:

1. Increase automation in module assembly and handling of larger wafers

2. Improve the module manufacturing throughput, yield, and process control

3. Reduce PV module production labor and production costs to less than $\$ 1.20 / \mathrm{W}$ 
4. Achieve an increase in solar cell performance from $12.8 \%$ to $15 \%$

5. Increase wafer production by using wire saws to reduce center-to-center cut distances from $600 \mu \mathrm{m}$ to $400 \mu \mathrm{m}$

6. Develop larger wafers through increased ingot casting sizes, and

7. Expand commercial module manufacturing capacity by a factor of three.

Solarex's accomplishments after the second phase of this subcontract included the following:

- Developed a process for casting larger ingots that increased the amount of useable silicon obtained from each cast by $125 \%$

$\therefore$ Operated the wire saw in a production mode, demonstrating its ability to produce as many wafers as sixteen ID saws at a lower per-wafer cost

- Completed two pilot runs of aluminum paste back-surface field process, which verified a greater than $5 \%$ increase in cell efficiency and qualified modules with these cells to IEEE 1262 and IEC 1215 standards

- Doubled the capacity of the Solarex module assembly area

- Qualified a module electrical termination system that costs less than $\$ 1.00$ per module.

Texas Instruments, Inc. (TI) has directed its subcontract activities under PVMaT toward research on Spheral Solar ${ }^{\mathrm{TM}}$ Si PV technology. TI's goals under this subcontract are to:

1. Optimize existing processes

2. Investigate alternative approạches for reducing material waste, material handling (labor) costs, and cycle times of its Spheral Solar ${ }^{\mathrm{TM}}$ technology in order to demonstrate a module manufacturing process with costs of $\$ 2.00 / \mathrm{W}$

3. Increase manufacturing yield while reducing cost and improving logistics through sphere fabrication processes

4.... Improve cell yields through optimization of bonding pressures, temperatures, and foil configuration parameters, and

5. Increase Spheral Solar ${ }^{\mathrm{TM}}$ cell efficiencies from $8.5 \%$ to greater than $11 \%$.

At the beginning of the second phase of their PVMaT subcontract, Texas Instruments found it necessary to request a termination of their Spheral Solar ${ }^{\mathrm{TM}}$ program effective January 26, 1995. This decision to terminate the Spheral Solar ${ }^{\mathrm{TM}}$ program was made by the senior management of TI based upon its strategic decision to focus its investments in its core businesses and a current lack of external private funding to invest in a large-scale factory. 


\section{PHASE 3A}

The PVMaT Phase 3A subcontracts both began in early January 1993 with a 25\% cost sharing by the subcontractors. They have focused on module-related R\&D problems that are common to several PV manufacturing groups. The resuits of the second year of research under these subcontracts (listed in Table 3 ) are outlined below.

\section{Table 3. PVMaT Phase 3A Subcontract Awards}

Subcontractor

Spire Corporation

Springborn Laboratories
Emphasis of Research

Automated Solar Cell Assembly

Teamed Process Research

Photovoltaic Manufacturing Technology
Principal Investigater

Mike Nowlan

Bill Holley

Spire Corporation has focused its research on the development of high-throughput, automated processes for interconnecting silicon solar cells that are generic to the PV industry. Specifications were that the equipment be capable of processing cells to $200-\mu \mathrm{m}$ thickness and achieve higher yields. The equipment was to be capable of handing cell sizes up to $15 \mathrm{~cm}$ across, in square or round shape, and capable of processing up to $5 \mathrm{MW} / \mathrm{year}$.

At the completion of the subcontract, Spire achieved these goals, and the result is the SPIASSEMBLERTM 5000 . This fully automated solar cell assembler combines the steps of silicon cell interconnection into strings, string testing through a flash I-V test, and string placement onto prepared module substrate for assembly into a module. The equipment reduces manufacturing cost through increased silicon utilization efficiency, higher product yields, improved process control, increased processing rates, and flexible automation. The assembler features flexibility through software control of several stringing options, including: tab length and bend location; number of cells/string in strings up to $2 \mathrm{~m}$ long; number of strings/module, up to $1.4 \mathrm{~m}$ across; series or parallel string arrangement; and cell and string inspections. The labor requirement for the assembler is one operator compared to four for other interconnect equipment (Spire's SPI-TAB 1000 and SPICONNECT 1000). Based upon test runs, the assembler can handle $5 \mathrm{MW} /$ year, using $1.25-\mathrm{W}$ cells, three shifts/day, and allowing $20 \%$ non-operating time for maintenance, cleaning, and repair. A cost comparison was conducted at the completion of this subcontract. Spire compared costs among a manual process, the semi-automated modification of the assembler, and the assembler. Their analysis shows that the added cost of the stringing process is $\$ 0.446 / \mathrm{W}$ for manual operations, $\$ 0.164 / \mathrm{W}$ for the semi-automated process, and $\$ 0.088 / \mathrm{W}$ for the fully automated assembler.

Springborn Materials Science Corporation has focused its research on understanding the degradation of EVA-based encapsulants in PV modules. Springborn's goals under the PVMaT Project are to: 
1. Pursue the advanced development of a PV module encapsulation sheet

2. Reformulate the EVA-based elastomer, and

3. Formulate alternative elastomer-based encapsulants that will more effectively resist degradation when exposed to high-temperature and ultraviolet (UV) insolation in the field. Yellowing or browning of some EVA-based encapsulants has occurred at a number of installations worldwide, where both temperature and UV radiation are comparatively high.

The subcontract with Springborn is now nearing completion, with testing of full-size modules the remaining task to be accomplished. Over the nearly three phases, Springborn surveyed most of the examples of discolored modules in the United States and compiled these observations in a Task 1 report. Springborn then initiated a series of tests using an Atlas Ci35A Weather-Ometer Xenon Exposure System, operated at $100^{\circ} \mathrm{C}$ and $95 \%$ relative humidity to simulate accelerated aging. Through a series of tests, the company has identified four reformulated, EVA-based encapsulant materials which have demonstrated little discoloration in both in the Weather-Ometer and at DSET Laboratories noutdoor testing using EMMA (Equatoral Mount with Mirrors for Acceleration) conditions, mirrot concentration nominally 5 suns. These materials are prepared using the same methods and equipment as the materials already in use. Three of these new formulations are standard cure, and one is a fast cure. Springborn has shipped sample encapsulant sheets of these candidate materials to module manufacturers to be made into mini-modules for bench-level accelerated testing and full-size modules for outdoor aging studies.

\section{PHASE 4A}

Under Phase 4A, the most recently subcontracted phase of the PVMaT Project, NREL awarded eight 2-year Phase 4Al and five 3-year Phase 4A2 subcontracts in late 1995. The Phase 4A ProductDriven Manufacturing research represents the next step in the PVMaT program and broadens the scope to the PV product as a whole as well as elements that comprise a PV product, such as BOS components or system integration.

\section{PHASE 4A1}

These subcontracts (listed in Table 4) represent the Product-Driven System and Component Technology portion of the PVMaT Phase 4A research and are cost-shared at a $23 \%$ level by the subcontractors. They include manufacturing improvements directed toward innovative, low-cost, high-return, high-impact $P V$ products. The results of research under these subcontracts are outlined below. 


\section{Table 4. PVMaT Phase 4A1 Subcontract Awards}

Subcontractor

Solar Design

Associates

Trace Engineering

Omnion Power

Corporation

Utility Power Group .

Ascension Technology

Evergreen Solar

Advanced Energy

Systems
Emphasis of Research

Development of Standardized, Low-Cost AC PV Systems

Modular DC to AC Power Inverter Module for PV Applications

Three-Phase Power Conversion System

for Utility-Interconnected PV Applications

Development of a Low-Cost Integrated

20-kW AC Solar-Tracking Sub-array for

Grid-Connected PV Power System Applications

Manufacture of an AC Photovoltaic Module

Advanced Polymer PV System

Next-Generation Three-Phase Inverter
Principal

Investigator

Steven Strong

Christopher Frietas

Hans Meyer

Mike Stern

Edward Kern

Jack Hanoka

Robert Wills

Solar Design Associates, Inc. (SDA) and lower-tier subcontractor Solarex Corporation, have directed their research at "The Development of Standardized, Low-Cost AC PV Systems." SDA will work with Solarex to create standard, certified modular "building blocks" utilizing the new Solarex AC-PV MSX-240 module. These building blocks will be designed so identical units can then be combined to create PV systems of virtually any capacity to suit a particular application. SDA's approach is to conduct a system-level review of PV systems' component design and installation, then develop "kits" incorporating innovative mounting systems, enhanced modular inverters, and pre-manufactured wiring systems. These units will first be developed and tested for U.S. applications, then modified as necessary for export to Japanese and European markets. Units will be deployed at utility team member locations in the United States, Europe, and Japan, and performance data will then be collected.

Solar Electric Specialties, Inc. has directed its research at the "Design, Fabrication and Certification of Advanced Modular PV Power Systems." Two different, packaged integrated systems will be developed, fabricated, and tested. One is the Modular Autonomous Photovoltaic Power Supply or MAPPS. This unit is a modular, pole-mounted PV system autonomous from any electric grid and with no generator backup. The second is a $1-\mathrm{kW}$ Photogenset, a containerized PV system including a back-up generator suitable for off-grid electricity applications and services. Both systems will incorporate technical innovations and low-cost manufacturing techniques, and will then 
be submitted for UL certification. The advantages of these products are: a) shorter production lead times, b) higher overall quality and system reliability, c) decreased management and engineering time, d) lower overhead and inventory costs, and e) lower material and installation labor. These products will be designed for a variety of market applications such as telecommunications and telemetry, remote homes and pumping systems, and utility sales related to stand-alone systems for utility use. The approach will be to fabricate prototypes, then submit them for safety certification and conduct functionality testing.

Trace Engineering has directed its research at developing a "Modular DC to AC Power Inverter Module for PV Applications." Trace will use its previous experience to develop a next-generation inverter, with a modular design that will meet a wide range of performance requirements. The new units will be easily manufactured and be lower cost, with higher efficiency and improved serviceability in the field. The $2-5-\mathrm{kW}$ DC to AC power inverter units will utilize as much of the existing, tested technology as possible. The universal design will allow for cost reductions through the increased manufacturing volume of a single unit design, with sufficient versatility for use in parallel or in series, and will then be applied in readily expandable units. The approach is to develop the inverter's topology and improve efficiency, then improve control and protection systems and design the packaging to meet requirements for the intended market segments.

Ominion Power Corporation has directed its research at "Three-Phase Power Conversion System for Utility-Interconnected PV Applications." Omnion will develop a prototype 100-kilowatt, threephase power conversion system (PCS) and supporting manufacturing processes to achieve improvements in cost, reliability, and performance. Omnion will apply user comments and needs identified by a team of representatives from utilities and system integrators to refine the PCS specifications and design a standard "point design" product. The PCS product will be designed for high reliability (40,000 hours mean time between failures) and an improved converter efficiency of $96 \%-97 \%$, while meeting FCC standards for electromagnetic interference and reducing audible noise. Manufacturing costs will be reduced by facilitating the flow of materials and components to the assembly line, incorporating jigs and fixtures to ease product handling, and implementing semiautomated product testing. A PCS prototype and several pre-production units will be tested to verify the design of both the product and the manufacturing process.

Utility Power Group and its lower-tier subcontractor, Siemens Solar Industries, have directed their research at the "Development of a Low-Cost Integrated 20-kW AC Solar-Tracking Sub-array for Grid-Connected PV Power System Applications." UPG will focus on the design, fabrication, and testing of a modular panel and an integrated power processing unit. These are to be combined with UPG's proven PV system technology to create a fully integrated and modular $20-\mathrm{kW}$ AC PV solar-tracking sub-array. This sub-array will then be deployed in a grid-connected, customer-driven application for which UPG expects to demonstrate a $40 \%$ reduction in area-related BOS costs and a 50\% reduction in power-related BOS losses. UPG will work with secondary team member Siemens Solar Industries to develop an advanced PV module laminate for the modular panels.

Ascension Technology, Inc. (ATI) and its lower-tier subcontractor, ASE Americas Corporation (ASE), have directed their research at the "Manufacture of an AC Photovoltaic Module." ATI will refine and integrate its module-scale inverter (MSI) with a large-area 300- $\mathrm{W}_{\mathrm{p}}$ PV module manufactured by ASE to develop a new product for utility-interactive PV systems. The approach will be to develop an advanced version of the MSI based on performance enhancement goals, optimized circuit topology and layout, and compact construction. The overall layout of the MSI will 
be designed for enhanced performance, lower weight, high reliability, and compliance with applicable Underwriters Laboratory (UL) standards. These will be integrated with the ASE modules and subjected to accelerated testing and outdoor performance characterization. ATI will complete the process to develop a complete system package for the AC PV Module with a new version of the $\cdots$ ATI PV Source Circuit Protector, an updated RoofJack mounting system, and a utility interconnection unit. These will then be installed at the locations of $20 \mathrm{co-funding} \mathrm{electric} \mathrm{utilities}$ for testing.

Evergreen Solar, Inc. has directed its research at the development of an "Advanced Polymer PV System." Evergreen Solar plans to introduce improvements in PV module materials and assembly methods to reduce both factory and field BOS costs associated with interconnection, module framing, and mounting the PV array. To accomplish this, Evergreen will develop an Innovative Mounting System (IMS) which will incorporate quick-mount modules. To implement this design, Evergreen will develop: a) a candidate backskin material that accommodates the IMS concept, eliminates the module frame, and integrates the junction box; b) an alternative lamination method for the new backskin; and c) a new encapsulant suitable for the alternative lamination method, resulting in improved module service life and performance. The company's approach is to develop conceptual designs and materials, test them, and implement modifications as necessary.

Advanced Energy Systems, Inc. (AESI, formerly Skyline Engineering), and lower-tier subcontractors are conducting research to develop the "Next-Generation Three-Phase Inverter." The AESI goal is to reduce the manufacturing cost of inverters for medium-scale (50-100-kW) gridinteractive and stand-alone/hybrid PV applications. To achieve its cost goals, AESI will evaluate digital control, incorporate smart power components and use soft switching techniques. The units will also be designed for ease of manufacturing and economies of production, and will incorporate remote monitoring, control, and service communications capabilities. AESI will design, develop, produce and test a prototype 50-kW utility-interactive and stand-alone/hybrid inverter. This work will result in production-model power conditioning systems that will meet the requirements for utility-interconnected PV and stand-alone/hybrid power applications. AESI will team with a lowertier subcontractor to develop high-volume manufacturing plans for manufacturing of these new products.

\section{PHASE 4A2}

These subcontracts (listed in Table 5) represent the Product-Driven PV Module Manufacturing Technology portion of the PVMaT Phase 4A research and are cost-shared at a $41.5 \%$ level by the subcontractors. They include improvements in module manufacturing processes and the manufacturing, assembly, and integration of systems to build a PV product, as well as the packaging of that product to meet market requirements. The results of research under these subcontracts are outlined below. 
Table 5. PVMaT Phase 4A2 Subcontract Awards

Subcontractor

AstroPower

Iowa Thin Film

Technologies

Solar Engineering

Applications .

Siemens Solar

Industries

ASE Americas
Emphasis of Research

Large-Area Silicon-Film Panels and

Solar Cells

PVMaT Monolithic a-Si Modules on

Continuous Polymer Substrates

Manufacturing of the SEA Power Grid

Photovoltaic $\mathrm{Cz}$ Silicon Module

Improvements

Market-Driven EFG Modules
Principal

Investigator

James Rand

Frank Jeffrey

Neil Kaminar

Kim Mitchell

Mike Kardauskas

AstroPower, Inc., under a 3-year subcontract, will address the establishment of a low-cost manufacturing capability for Silicon-Film ${ }^{\mathrm{TM}}$ and solar cells and panels by taking advantage of the continuous processing capability of the Silicon-Film ${ }^{\mathrm{TM}}$ technology. Under this effort, entitled "Large Area Silicon-Film ${ }^{\mathrm{TM}}$ Panels and Solar Cells," AstroPower will be developing a continuous/in-line facility by focusing on each process component contributing to the SiliconFilm ${ }^{\mathrm{TM}}$ panel manufacturing process and addressing the first commercial-scale product to emerge from the development of the Silicon-Film ${ }^{\mathrm{TM}}$ process. The effort is expected to lead to accelerated reduction of PV manufacturing costs for installed systems, provide the foundation for significantly increased production capacity, and reduce handling and waste streams. To meet these goals, AstroPower will focus on:

1. Extending continuous processing from the Silicon-Film ${ }^{\mathrm{TM}}$ sheet fabrication step through the solar cell fabrication steps

2. Developing a very-large-area solar cell (target size of $30 \mathrm{~cm} \times 60 \mathrm{~cm}$ ), and

3. Applying the large-area solar cell to a utility-scale panel, thereby eliminating the step of combining modules into panels.

AstroPower will also address the development of new panel products and processes; the improvement in solar cell performance; a reduction in the cost of critical baseline solar cell processes; the development of processes for the manufacturing of a solar cell based on $31-\mathrm{cm}$-wide sheet; the reduction of manufacturing costs of the existing $16.5-\mathrm{cm}$-wide sheet process; and the production of a $31-\mathrm{cm}$-wide Silicon-Film ${ }^{T M}$ sheet at a production capacity of $20 \mathrm{MW} / \mathrm{yr}$. The longterm goals include the development of a $24-\mathrm{W}, 30-\mathrm{cm} \times 60-\mathrm{cm}$ Silicon-Film ${ }^{T M}$ solar cell and a 
manufacturing capability for a 384-W, $4^{\prime} \times 8^{\prime}$ Silicon-Film ${ }^{\mathrm{TM}}$ panel for deployment in utility-scale applications.

Iowa Thin Film Technologies Inc. (ITF), under a 3-year subcontract, is focusing on its long-term goals of increasing the throughput of its metallization, a-Si deposition, and laser-scribing and welding processes, and reducing the overall module-manufacturing costs on the ITF production line by $68 \%$. Efforts to accomplish these goals under this subcontract, entitled "Monolithic Amorphous Silicon Modules on Continuous Polymer Substrates," are directed at replacing the ITF TiN layer with a less absorbing $\mathrm{ZnO}$ layer and investigating alternative feedstocks for the supply of $\mathrm{Zn}$ and $\mathrm{O}$ in the $\mathrm{ZnO}$ growth process. Additionally, ITF will design and implement web steering system and machine control programs; identify new laser operating parameters to optimize the laser beam scan speed; study alternative processes for the scribing and methods of welding shunts in cell-interconnects; and complete automation of the final process steps of busbar attachment and web cutting. These task efforts, and studies of the development of a new water-based insulating ink printing and roll-based laminating processes for the ITF production line, along with the design and implementation of baffles for the isolation of deposition regions, will support the ITF PVMaT subcontract goals.

Solar Engineering Applications Corporation (SEA), under a 3-year subcontract, will address the establishment of a low-cost manufacturing capability for linear concentrator modules by taking advantage of its continuous-processing capability. Under this effort, entitled "Manufacturing of the SEA Powergrid, "SEA will take advantage of the continuous-processing capability of its lens and side-panel extrusion technology. SEA will develop a continuous, in-line, high-volume production technology for extruded lenses ( $50 \mathrm{MW} / \mathrm{yr})$ with a goal of $88 \%$ lens transmission. Other goals include module side panels ( $25 \mathrm{MW} / \mathrm{yr}$ ) and a parallel process for an automated receiver assembly. Additionally, SEA will develop a high-volume roll-forming technology for the fabrication of panel frame members, and a high-volume automated technology for the bonding of plastic collector components without the use of solvent adhesives. This effort is expected to lead to an accelerated reduction of PV manufacturing costs for installed systems, and provide the foundation for significantly increased production capacity. The long-term goals include a 50-MW/yr manufacturing capacity for panels at $\$ 2 / W_{p}$.

Siemens Solar Industries, under a 3-year subcontract, will address improvements in its $\mathrm{Cz}$ silicon module manufacturing technology to achieve an $18 \%$ reduction in module cost per watt. Under this effort, entitled "Photovoltaic $\mathrm{Cz}$ Silicon Module Improvements," Siemens will focus on module cost reduction and improved $\mathrm{PV}$ module manufacturing process technology in its $\mathrm{Cz}$ crystalline silicon (c-Si) module manufacturing. These goals will be addressed through the identification of alternative $\mathrm{Cz}$ module designs, material sources and processes which lower module component costs and by improving manufacturing process yields by $15 \%$, reducing labor costs in $\mathrm{Cz}$ module manufacturing, and increasing productivity by $15 \%$. Cost reductions in direct and indirect materials will be targeted through reduced materials usage or lower cost alternative materials or processes in the areas of crystal growing, wafer slicing, $\mathrm{Cz}$ cell fabrication and $\mathrm{Cz}$ module fabrication. An additional objective of this PVMaT subcontract will be to improve the $\mathrm{Cz}$ module reliability through a careful statistical study of controlling parameters.

ASE Americas, Inc., under a 3-year subcontract, will focus on advancing its manufacturing technology by reducing edge-defined, film-fed growth (EFG) wafer, cell, and module manufacturing costs relative to pre-PVMaT Phase 4A levels. Under this effort, entitled "Market-Driven EFG 
Modules," ASE will establish a low-cost manufacturing capability for ASE solar cells and modules fabricated with $250-\mu \mathrm{m}$ thick EFG wafers. ASE will address EFG wafer, cell, and module manufacturing to produce EFG cells with an average efficiency of $15.5 \%$ and modules with a $25 \%$-reduced manufactured cost. Manufacturing line improvements will consist of a further decrease in the EFG wafer thickness to $250-\mu \mathrm{m}$ in mass production; an increase in as-grown EFG material electronic quality to meet the demands for higher-efficiency solar cells; improvements in solar cell fabrication technology to produce EFG cells averaging $15.5 \%$ for $10-\mathrm{cm} \times 10-\mathrm{cm}$ area wafers, which will include demonstrations of a front-surface texturing layer for light trapping; development of an environmentally safe and reduced-cost diffusion glass removal process; and development of an integrated interconnect, lamination, and fabrication method. The final objective of this subcontract is to lower the EFG wafer, cell, and module manufacturing costs by $25 \%$ relative to pre-PVMaT Phase 4A levels.

\section{PROGRESS IN THE PV INDUSTRY}

It is important to put the technical progress that the U.S. PV industry is making into perspective. Based on the improvements in manufacturing processes, reductions in manufacturing costs, introduction of new products, and improvements in product performance, it would be expected that the U.S. PV industry would exhibit both a scale-up of its U.S.-based PV manufacturing capacity and a reduction in its production costs. This has indeed been the case.

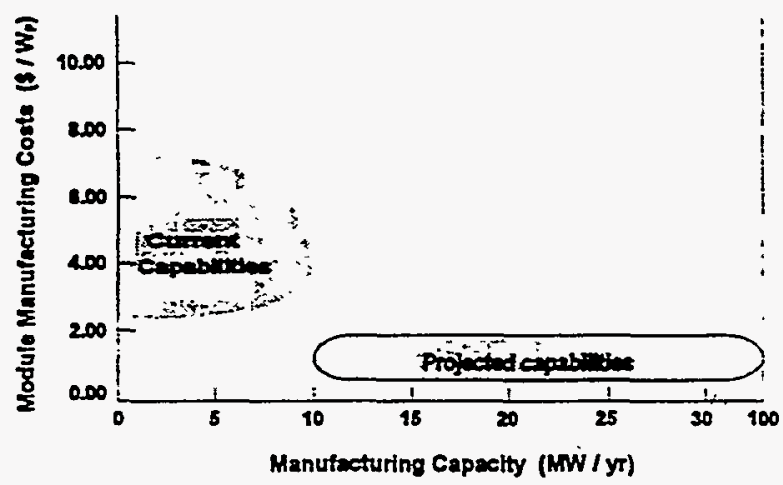

Figure 1 - 1995 PVMaT Projections
At the beginning of Phase 2A subcontracted research in the PVMaT Project, information was collected to establish the status of both the current and projected capacities and module costs for the eight subcontractors (APS was included as a lower-tier to UPG). These data, shown in Fig. 1, represented both the diverse status of a still-maturing industry and the optimistic speculation of its members regarding the effects that the PVMaT Project would have after PVMaT efforts were allowed to take effect. Additional information was collected as these subcontractors neared the completion of their individual subcontracts in late 1995.

These recent data, shown in both Figures 1 and 2, represent an update of the previous projections regarding these subcontracted efforts. Data projected in these figures are based on each manufacturer's maximum production capacity during a given year, assuming they were to operate "full-out." Module cost estimates were then based on these manufacturing levels and included only those costs directly associated with the manufacturing of the modules (not marketing, administration, sales, etc.). It should be noted that the data associated with any particular point in 
time represent a potential capability. Actual manufacturing production levels may be lower (and concomitant costs higher) due to other considerations such as market conditions, available labor, etc.

The data for establishing the 1996 module manufacturing costs have a relatively high degree of accuracy, since capital equipment for 1996 plant installations is generally already in place or on order. This lends significant credibility to the trend represented in Fig. 2 for the PVMaT manufacturers. When compared to the 1992 "Current Capabilities" and the "Projected Capabilities" in Fig. 1, the Phase 2A subcontractors appear to be on track to achieving their original goals.

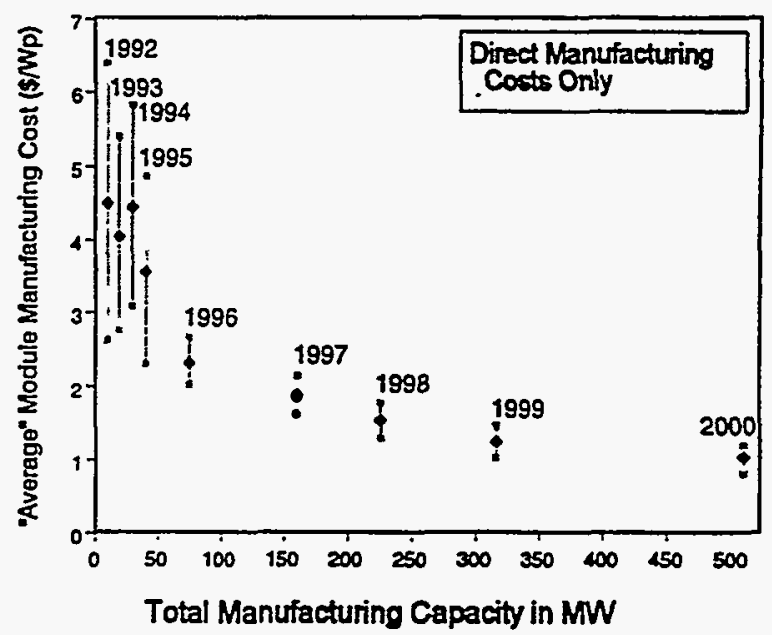

PVAaT participents wth active manutectirto lines (12)

Figure 2 - PVMaT Manufacturing Cost/Capacity

It is apparent, from Figs. 1 and 2, that the U.S. PV industry involved in the PVMaT Project has made significant progress toward reducing manufacturing costs and increasing PV module manufacturing capacity. It has also indicated in both its future Cost/Capacity data and its technical projections that its optimism for continuing these improvements is high.

An indication of an industry's strength is the relative market share. Figure 3 shows that U.S. market share on a world basis suffered a significant decline in the early 1980s. Although the U.S. PV market share began declining in 1982, the marked decline of production in 1985 clearly reflects the end of energy tax credits in the United States. Since that time, the U.S. production has hovered between $30 \%$ and $36 \%$ of the world total. It is promising, however, that the U.S. market share has been strengthening since 1993, achieving $41 \%$ of the world shipments in 1995. With PVMaT manufacturers representing $79 \%$ of the U.S. PV production in 1994, we believe an element of this strengthening position is the result of a new generation of PV modules with improved performance and durability, developed with the help of government-industry partnerships that are the comerstone of the PVMaT Project.

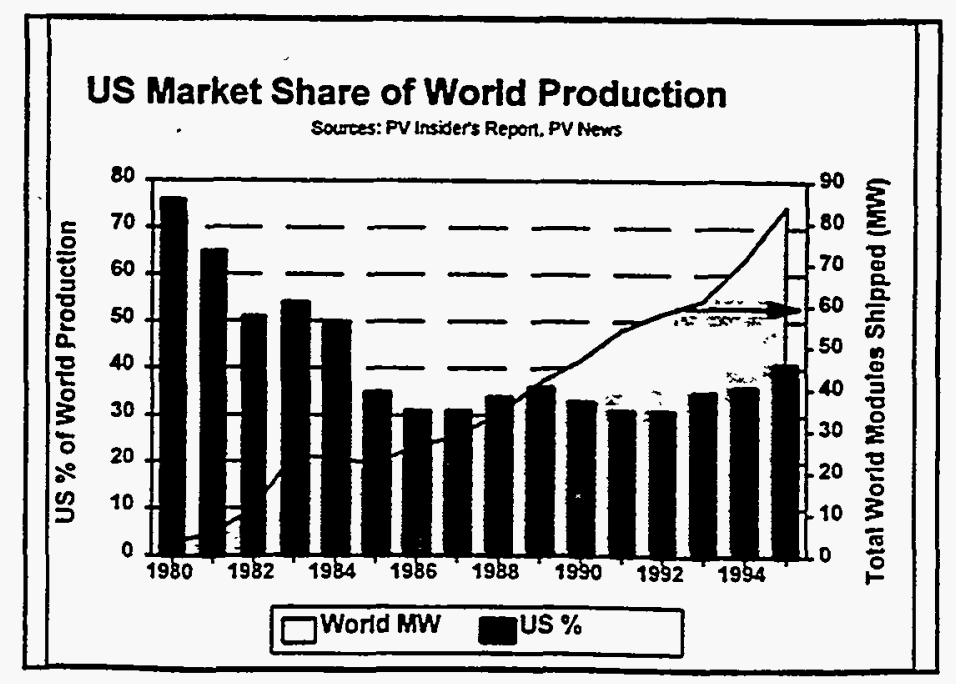

Figure. 3 - U.S. Market Share of World Production 
The PVMaT Project is currently in the first year of its subcontracted efforts under Phase 4A, with research in Phases 2B and 3A in their final year.

The members of the U.S. PV manufacturing industry that have been participating in the DOE PVMaT government/industry R\&D partnership have made progress toward achieving their individual goals. They have been successful in increasing the performance and reliability of their products, increasing the yield and throughput of the production lines, reducing PV product manufacturing costs, introducing new product lines, and scaling up PV manufacturing plant capacity. It is believed that these activities, supported by the PVMaT government/industry R\&D partnership, have had an effect on the strength and stability of the U.S. PV manufacturing industry as a whole, and this partnership is providing the U.S. industry with a solid base for a significantly improved and less costly renewable energy technology. 


\section{PVMaT Subcontracts}



Organization:

AstroPower, Inc.

Solar Park

Newark, DE 19716-2000

Contributors:

J.A. Rand, A.M. Barnett, J.C. Checchi, J.S. Culik, S.R. Collins,

D.H. Ford, R.B. Hall, E.L. Jackson, C.L. Kendall, A.M. Trabant

\section{Introduction}

AstroPower is addressing the establishment of a low cost manufacturing capability for SiliconFilm ${ }^{\mathrm{TM}}$ solar cells and panels by taking advantage of the continuous processing capability of the Silicon-Film ${ }^{\mathrm{TM}}$ technology. Under this effort a continuous/in-line manufacturing process is being developed by focusing on each process component contributing to the Silicon-Film ${ }^{\mathrm{TM}}$ panel manufacturing process. The effort is expected to lead to accelerated reduction of PV manufacturing cost for installed systems, provide the foundation for significantly increased production capacity, and reduce handling and waste streams. The process development will be based on a new $31-\mathrm{cm}$ wide Silicon-Film ${ }^{\mathrm{TM}}$ sheet. The long-term target is to generate the new wide sheet at a production rate of $20 \mathrm{MW} / \mathrm{yr}$ from one machine. Other long-term goals include the development of a $24 \mathrm{~W}, 30 \mathrm{~cm} \times 60 \mathrm{~cm}$ Silicon-Film ${ }^{\mathrm{TM}}$ solar cell and a manufacturing capability for a $384 \mathrm{~W}, 4^{\prime} \times 8^{\prime}$ Silicon-Film ${ }^{\mathrm{TM}}$ panel for deployment in utility-scale applications.

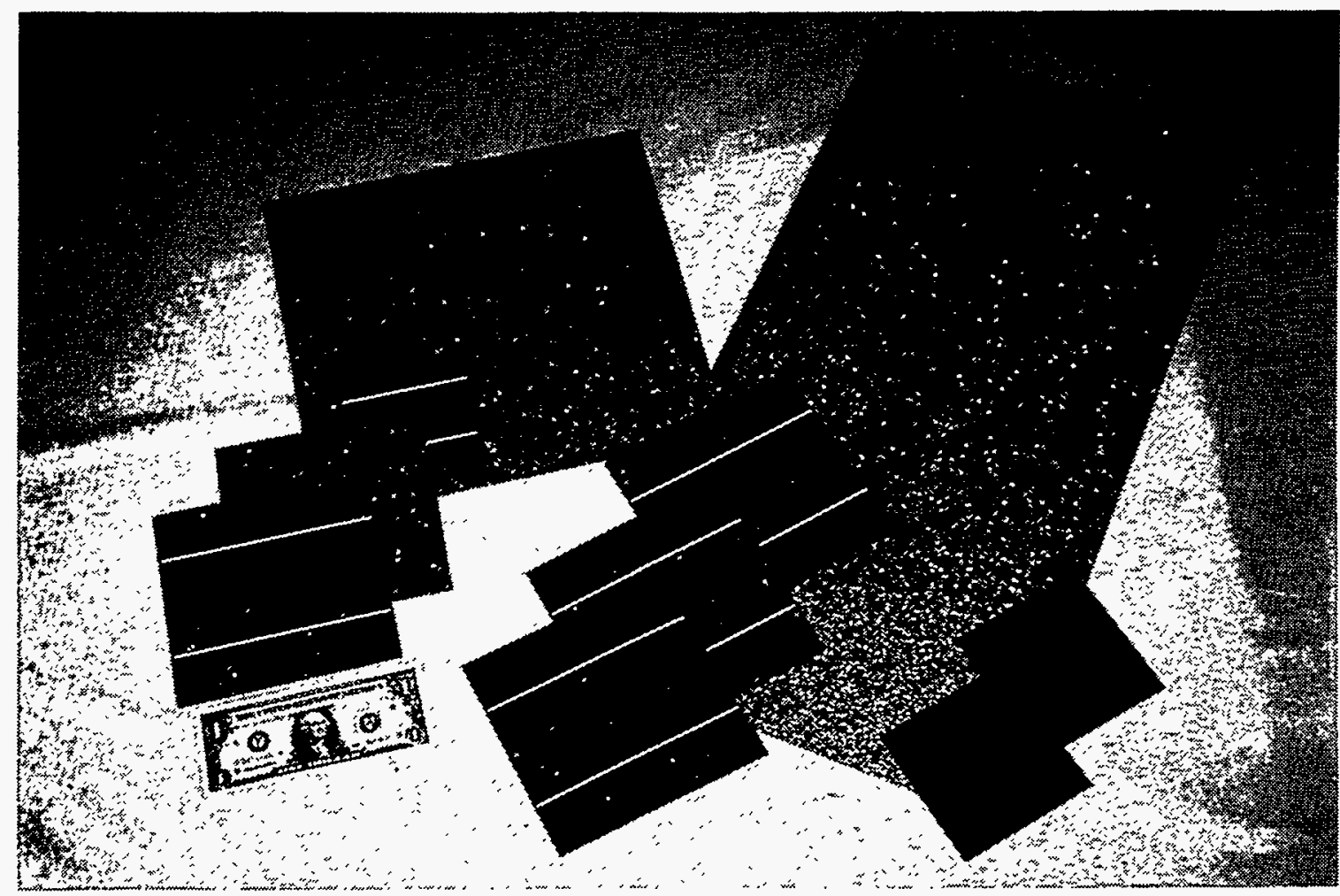

Figure 1. Photograph demonstrating the different sizes of Silicon-Film ${ }^{\mathrm{TM}}$ sheet and solar cells. Included are $100 \mathrm{~cm}^{2}$ and $240 \mathrm{~cm}^{2}$ solar cells, and sheets $30 \mathrm{~cm} \times 60 \mathrm{~cm}$ and $60 \mathrm{~cm} \times 60 \mathrm{~cm}$. 


\section{Continuous Processing}

The achievement of a continuous sheet process which produces high-quality material provides an opportunity to propagate continuity down the process stream. Figure 2 illustrates a continuous process that generates solar cells that are nominally $30 \mathrm{~cm} \times 60 \mathrm{~cm}$ in size. It is envisioned that all the solar cell processes could be carried out while the silicon substrate is in sheet form. The solar cell is then sized at the last step before incorporation into a $4^{\prime} \times 8^{\prime}$ panel or smaller module. The achievement of continuous processing will lead to lower cost per watt by reducing labor, while improving control, quality, and yield.

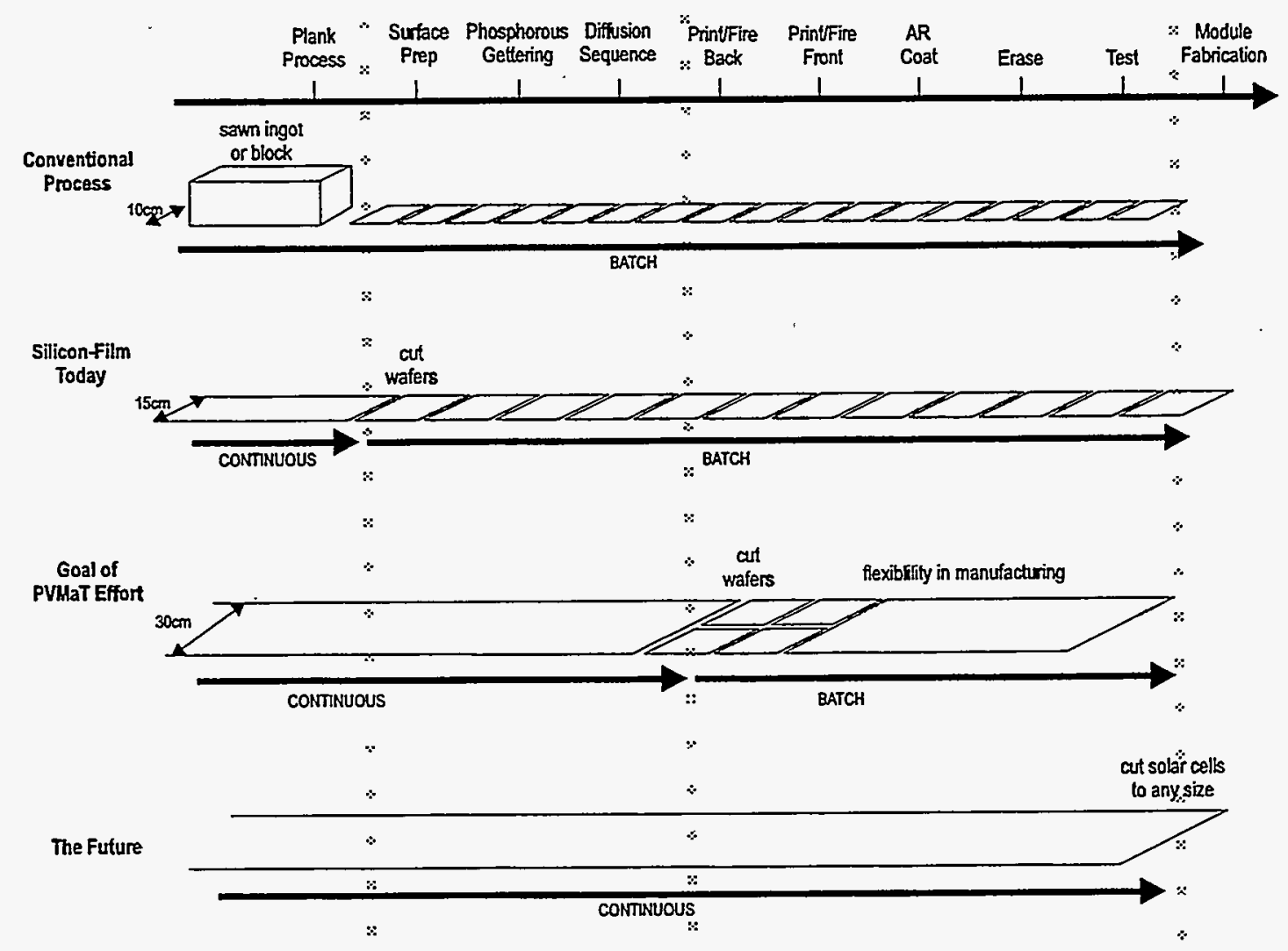

Figure 2. History and plan for the development of in-line continuous manufacturing technologies at AstroPower.

The issue of optimal solar cell size will be addressed during the proposed project. The final selection of a solar cell size will be determined by the economics of the manufacturing process. The point at which a sheet is sized to a discrete solar cell will be analyzed from an economic perspective.

There are multiple steps required to fabricate a solar cell from the starting silicon substrate sheet, each requiring process development and engineering. It is an objective of the proposed work to develop and engineer continuous manufacturing processes for preparing the silicon substrate sheet 
surface, and for diffusing the emitter layer. The steps in the envisioned continuous process will be developed in a modular fashion so that they can be joined together when completed.

The development work directed toward continuous Silicon-Film ${ }^{\mathrm{TM}}$ production includes an advanced diffusion process. Design rules require developing coating and dopant drive-in processes compatible with both discrete wafer and continuous sheet processing. Under this effort, AstroPower is investigating phosphorous dopant sources with key advantages in reducing processing costs and minimizing environmental impact. Like all Silicon-Film ${ }^{\mathrm{TM}}$ manufacturing efforts, the diffusion process and associated equipment are being engineered using advanced design and development systems (e.g. response surface methodologies and computer aided design) to allow immediate adaptability to large-scale production settings.

\section{Large Area Solar Cell}

The flexibility of the fully developed Silicon-Film ${ }^{\mathrm{TM}}$ solar cell product is illustrated in Figure 3 . The solar cell process will operate continuously and fabricate processed material that will result in solar cell sizes up to $30 \mathrm{~cm} \mathrm{x} 60 \mathrm{~cm}$. The actual size of the solar cells and panels will depend on the specific customer applications. As indicated, the market segments addressed include a 384 watt utility-scale panel, a 100 watt commodity power module, and a broad range of specialty modules.

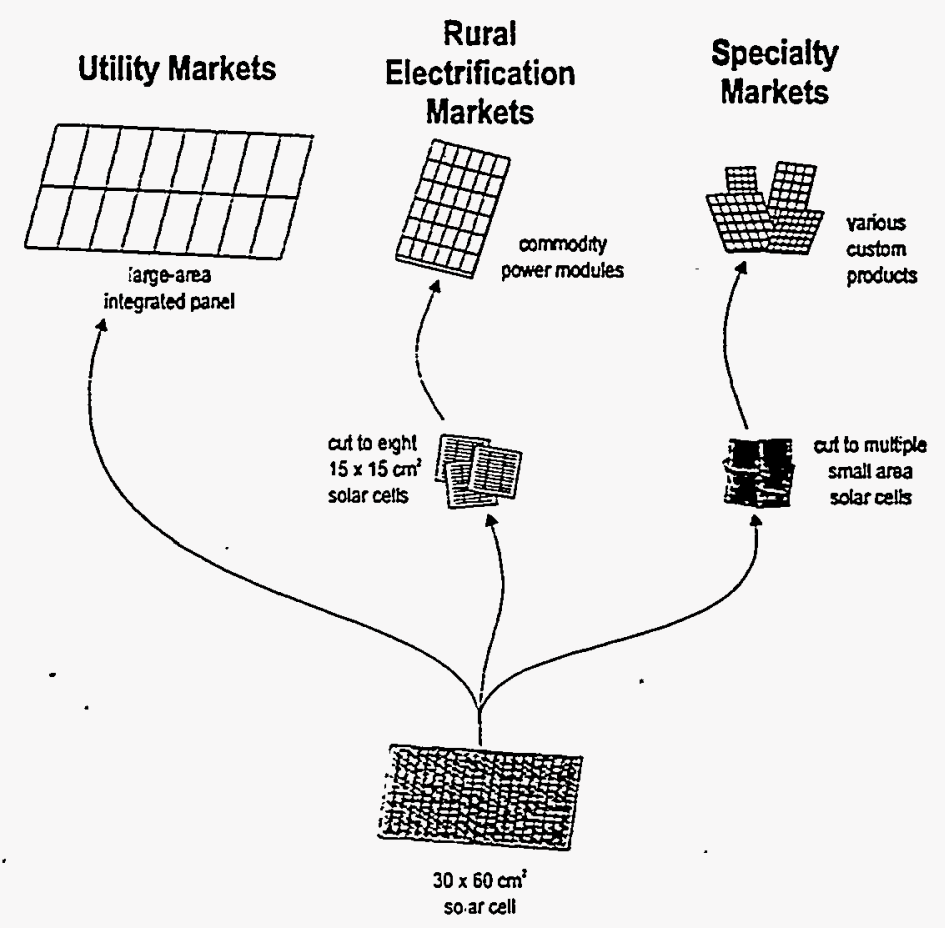

Figure 3. Flexibility of a $30 \mathrm{~cm} \times 60 \mathrm{~cm}$ solar cell in meeting several different $P V$ markets. 


\section{Solar Cell Performance}

The quality of Silicon-Film ${ }^{\mathrm{TM}}$ material and the maturity of the solar cell processing have continued to progress, and is reflected in increased solar cell efficiencies. Under the present effort, our work is organized to 1) evaluate advanced processing techniques such as bulk gettering and surface passivation in laboratory-sized solar cells $\left(1.0 \mathrm{~cm}^{2}\right)$, then 2) transfer those results to productionsized solar cells. A laboratory optimization effort has recently been completed and an efficiency of $14.6 \%$ was verified at NREL on a $1.0 \mathrm{~cm}^{2}$ solar cell (see Figure 4). That solar cell was fabricated with an experimental post growth treatment that removed impurities from the grown material.

Performance on production-sized solar cells $\left(240 \mathrm{~cm}^{2}\right)$ has reached a verified efficiency of $12.2 \%$ [1]. An effort is now underway to evaluate transferring technology learned on the laboratorysized solar cells to increase performance on the larger wafers.

In preparation for even larger solar cells, novel processing techniques are being investigated. A sheet diffusion process, as mentioned earlier, and new metallization techniques are being investigated that will lend themselves to a large area solar cell fabrication process. Very large prototype solar cells $\left(1800 \mathrm{~cm}^{2}\right)$ are expected in late 1996.

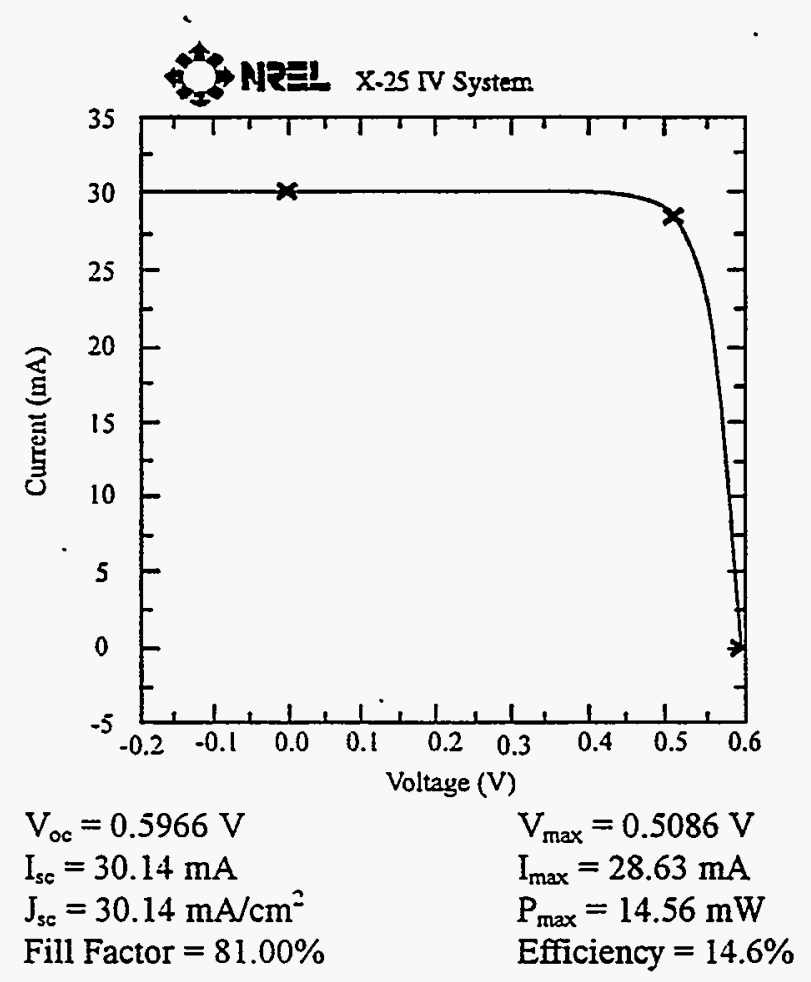

Figure 4. I-V curve for a laboratory-sized $1.0 \mathrm{~cm}^{2}$ solar cell fabricated with Silicon-Film ${ }^{T M}$ material fabricated with an atvanced process sequence.

[1] 13TH NREL Photovoltaics Program Review, (May 1995), AIP Conference Proceedings 353, American Institute of Physics, Woodbury, New York, 1995, p.283. 
Title:

Organization:

Contributors:
Continuous Roll-To-Roll a-Si

Photovoltaic Manufacturing Technology
Energy Conversion Devices, Inc. 1675 West Maple Road

Troy, Michigan 48084

M. Izu, Principal Investigator, S.R. Ovshinsky, X. Deng, H.C. Ovshinsky, and S.J. Jones

\section{Objectives and Goals}

The overall objective of this three-year program, completed in July 1995, was to advance roll-to-roll, triplejunction photovoltaic (PV) manufacturing technologies, to reduce the module production costs, to increase the stabilized module performance, and to expand commercial production capacity utilizing such roll-to-roll technology.

The specific three-year goal was to develop advanced large-scale manufacturing technology incorporating ECD's earlier research advances with the capability of producing modules with stable $10.2 \%$ efficiency at a cost of approximately $\$ 1.00$ per peak watt.

Major efforts during this subcontract include: Task I: Optimization of the high performance back-reflector system; Task II: Optimization of the Si-Ge Narrow Bandgap Solar Cell; Task III: Optimization of the Stable Efficiency of Photovoltaic Modules; Task IV: Demonstration of Serpentine Web Continuous Roll-to-Roll Deposition Technology; Task V: Material Cost Reductions; and Task VI: Improving the Module Assembly Process.

\section{Approaches}

We have performed manufacturing technology development work utilizing two advanced continuous roll-toroll amorphous silicon (a-Si) alloy solar cell manufacturing machines, both were engineered and manufactured by ECD at ECD's expense [1-9].

\section{1. $2 \mathrm{MW}$ Production Line}

This 2 MW production plant consists of a continuous roll-to-roll substrate washing machine, a continuous roll-to-roll back-reflector deposition machine, a continuous roll-to-roll amorphous silicon alloy deposition machine and a continuous roll-to-roll transparent conductor deposition machine.

The production line produces triple-junction two bandgap a-Si alloy solar cells consisting of an a$\mathrm{Si} / \mathrm{a}-\mathrm{Si} / \mathrm{a}-\mathrm{SiGe}$ structure on a 5 mil. thick, $14 \mathrm{in}$. wide, $2500 \mathrm{ft}$. long stainless steel roll at a speed of $1 \mathrm{ft} . / \mathrm{min}$. This production line represents the world's first commercial production line of high efficiency a-Si alloy solar cells utilizing a multi-junction spectrum-splitting cell design and high performance back-reflector. 


\section{2. $200 \mathrm{~kW}$ Pilot Line}

This multipurpose continuous roll-to-roll a-Si alloy solar cell deposition machine consists of $n-i-p$ chambers to deposit a-Si n-i-p solar cells, a sputtering chamber to deposit textured $\mathrm{Ag} / \mathrm{ZnO}$ backreflector and TCO top conductor, and a serpentine deposition chamber to develop new high throughput serpentine deposition processes.

\section{Major Accomplishments}

- Successful incorporation of a high-performance $\mathrm{Ag} / \mathrm{Z} \mathrm{nO}$ back-reflector system into our continuous rollto-roll commercial production operation.

- Incorporation of high quality a-SiGe narrow bandgap solar cells into a commercial continuous roll-toroll manufacturing process.

- Demonstration that the continuous roll-to-roll production of high efficiency triple-junction two bandgap solar cells is consistent and uniform throughout a $2500 \mathrm{ft}$. run with high yield. The average initial subcell efficiency and yield were $10.21 \%$ and $99.7 \%$, respectively.

- Achievement of $11.1 \%$ initial subcell efficiency of triple-junction two bandgap a-Si alloy solar cells manufactured in continuous roll-to-roll production.

- Production of the world's first $4 \mathrm{ft}^{2}$ PV modules utilizing triple-junction two bandgap solar cells manufactured in a commercial, continuous roll-to-roll production line. These $4 \mathrm{ft}^{2}{ }^{2}$ modules had $9.5 \%$ initial efficiencies and $8 \%$ stable aperture-area module efficiencies.

- Demonstration of the long-term stability of ECD's $4 \mathrm{ft.}^{2}$ production module. The stable module efficiency after 2,380 hours of sunlight soaking at $50^{\circ} \mathrm{C}$ is $7.9 \%$. Light soak testing of ECD modules performed at NREL under one sun at $50^{\circ} \mathrm{C}$ showed $15 \%$ degradation after 600 hours. ECD modules have passed heat and humidity/freeze cycles of NREL recommended module reliability testing procedure.

- Process optimization to reduce the layer thickness and to improve gas utilization resulted in a $77 \%$ material cost reduction for germane and a $58 \%$ reduction for disilane.

- Design, construction (at ECD's expense) and completion of initial optimization of a $200 \mathrm{~kW}$ multipurpose continuous roll-to-roll a-Si alloy solar cell deposition machine having upgraded machine and construction specifications;

- Design and construction, at ECD's expense, of a serpentine deposition chamber which has demonstrated a compact, low-cost deposition machine design with improved throughput and gas utilization factor.

- Demonstration of triple-junction solar cells with $9.5 \%$ initial efficiency with top a-Si cells deposited in the serpentine deposition chamber.

- Development of a new back-reflector evaluation technique using PDS to effectively analyze the optical losses of textured back-reflector and develop an improved textured $\mathrm{Ag} / \mathrm{ZnO}$ back-reflector system demonstrating $26 \%$ gain in $J_{s e}$ over previous textured Al back-reflector systems. 
- Development of a new grid/busbar design utilizing thin wire grids which improved the efficiency by approximately $3-4 \%$ and reduced the grid/busbar cost by approximately $50 \%$.

- Development of a concept design for an automated high volume (100 MW/yr) PV manufacturing plant for producing a low-cost, large-area PV module with an expected material cost reduction of $71 \%$. The module manufacturing cost is estimated to be $\$ 1.00$ per peak watt.

\section{References}

1. Izu, M.; Deng, X.; Krisko, A.;Whelan, K.; Young, R.; Ovshinsky, H.C.; Narasimhan, K.L.; Ovshinsky, S.R. (1993). "Manufacturing of Triple-Junction $4 \mathrm{ft}^{2}$ a-Si Alloy PV Modules." Proc. of 23rd IEEE PVSC, 919.

2. Izu, M.; Ovshinsky, S.R.; Deng, X.; Krisko, A.; Ovshinsky, H.C.; Narasimhan, K.L.; Young, R. (1993). "Continuous Roll-To-Roll Amorphous Silicon Photovoltaic Manufacturing Technology." AIP Conf. Proc. 306, 12th NREL PV Program Rev., 198.

3. Guha, S.; Yang, J.; Banerjee, A.; Glatfelter, T.; Hoffman, K.; Ovshinsky, S.R.; Izu, M.; Ovshinsky, H.C.; Deng, X. (1994). "Amorphous Silicon Alloy Photovoltaic Technology - From R\&D to Production." Proc. of MRS Spring Meeting.

4. Deng, X.; Izu, M.; Narasimhan K.L.; Ovshinsky, S.R. (1994). "Stability Test of $4 \mathrm{ft}^{2}$ "TripleJunction a-Si Alloy PV Production Modules." Proc. of MRS Spring Meeting.

5. Izu; M.; Ovshinsky, H.C.; Deng, X.; Krisko, A.; Narasimhan, K.L.; Crucet, R.; Laarman, T.; Myatt, A.; Ovshinsky, S.R. (December 1994). "Continuous Roll-To-Roll Serpentine Deposition for High Throughput a-Si PV Manufacturing." Proc. of the First World Conference on Photovoltaic Energy Conversion, Hawaii, 820.

6. Deng, X.; Narasimhan, K.L., (December 1994). "New Evaluation Technique for Back-Reflector Using Photothermal Deflection Spectroscopy." Proc. of the First World Conference on Photovoltaic Energy Conversion, Hawaii, 555.

7. X. Deng; Narasimhan, K.L.; Evans, J.; Izu, M.; Ovshinsky, S.R. (December 1994). "Dependence of a-Si Solar Cell $\mathrm{V}_{\infty}$ On Deposition Temperature. Proc. of the First World Conference on Photovoltaic Energy Conversion, Hawaii, 678.

8. Izu, M.; Deng, X; Ovshinsky, H.C.; Ovshinsky, S.R. (April 2-7, 1995). "Roll-To-Roll RF PECVD Machine for a-Si Solar Cell Manufacturing." Proc. of Annual Conference of Society of Vacuum Coaters, Chicago, IL.

9. Izu, M.; Ovshinsky, S.R.; Deng, X.; Ovshinsky, H.C.; Jones, S.J.; Doehler, J. (May 16-19, 1995). "Continuous Roll-To-Roll a-Si PV Module Manufacturing." Proc. of 13th NREL PV Program Review, Lakewood, CO. 
Title:

Organization:

Contributors:
PV Metal Roofing and Shingle Modules

Energy Conversion Devices, Inc. (ECD) Troy-Michigan 48084

Energy Conversion Devices

Masat Izu, program manager; Tim Ellison, Larry Fatalski, Herb Ovshinsky, Ramona Souleyrette, and Ken Whelan

United Solar Systems Corporation (United Solar)

Troy Glatfelter, Kais Younan, Kevin Hoffman,

Arindam Banerjee, Jeff Yang, and Subhendu Guha

National Assoc. for Home Builders Research Center (NAHB) Joe Wiehagen

\section{Summary}

Under DOE/NREL's PV:BONUS program, ECD and United Solar are developing and commercializing two new Building-Integrated Photovoltaic (BIPV) modules: (1) a metal roofing module developed by ECD, and (2) a shingle module developed by United Solar. Key features of both these modules include:

- These modules are roofing materials, designed to be aesthetically and mechanically integrated into residential and commercial buildings.

- The modules are installed directly over standard roof sheathing using standard installation techniques already available in the roofing industry. Consequently the modules can be installed by roofing contractors and electricians without any special training thus minimizing the installation and balance-of-system costs.

- These lightweight and flexible polymer-encapsulated modules are fabricated from multijunction, multi-bandgap a-Si alloy solar cells on a thin flexible stainless steel substrate using a proprietary roll-to-roll process developed by ECD and United Solar.

During the last year these module designs were refined and prototypes were built, tested on outdoor prototype arrays, and qualified. In early 1996 these modules will be showcased on demonstration projects and the products will be refined for high volume commercial production using high efficiency triple junction PV cells. It is expected that these modules can be produced for less than $2.5 \mathrm{~S} / \mathrm{W}_{\mathrm{pk}}$ in large volume. These PV systems will greatly accelerate the acceptance and penetration of PV into the enormous building and utility markets. In the following two sections we describe these two new novel modules.

\section{ECD's Metal Roofing Module}

ECD's PV metal roofing module is an exact replacement for a commercially available standing seam metal roofing product produced by the ATAS Aluminum Corporation. Triple-junction multi-bandgap a-Si alloy solar cells produced in a continuous roll-to-roll process on a thin stainless steel substrate are processed and assembled into a large area cell module. This stainless steel module is encapsulated 
with EVA/Tefzel and laminated onto a galvalume metal roof panel produced by ATAS. These PV metal roofing panels are then roll-formed into "pans" with $3 / 4$ inch standing seams on each side so that the modules can accept the standard metal roofing mounting hardware. Depending upon the application, the width of the module may be varied from 1 to $2 \mathrm{ft}$, and the length may be varied from a few feet to over $22 \mathrm{ft}$. This module is shown in Fig. 1. Table I summarizes the proposed specifications for standard PV metal roofing modules with varied lengths. The installation process is summarized below:

- The roof is prepared using standard architectural and roofing construction techniques.

- The modules are then installed using the same exact method as conventional metal roofing:

- The pans/modules are trimmed to length.

- The first pan/module is located and mounted with a standard ridge vent bracket and two fasteners at the top of the pan/module.

- Standard clips are attached along the standing seam.

- Additional pans/modules are installed to cover the roof

- Standard ends and flashing are added to complete the roof installation.

- The module leads fall through the ridge vent at the top of the roof. An electrician, working in the attic area, completes the electrical installation.

To ensure the water-tight integrity of the system:

- The PV metal roofing module has no horizontal seams.

- The metal roofing vertical seams are covered by standard metal roof battens.

- All the wiring goes through the ridge vent on top of the roof underneath the ridge cap and thus requires no penetrations to made in the roof.

These features also result in ease of installation and reliability of the system, and low installed costs.

A $2 \mathrm{~kW}$ rooftop system will be installed a townhouse outside of Washington DC build by the NAHB Research Center. The system will be grid-connected and have battery storage. The technical and economic performance of the system shall be tested by NAHB and ECD in conjunction with the local utility.

\section{United Solar System's Shingle Module}

United Solar has produced amorphous silicon alloy based PV material on long rolls of thin flexible stainless steel for several years. The advantages of this flexible PV technology have been exploited in the development of a new shingle roofing module, illustrated in Fig. 2, designed to emulate the conventional asphalt shingle in form, structural function, and installation. The shingle module can be installed at the same time the building is roofed with standard asphalt shingles, using similar techniques and achieving an aesthetically appealing integration between the PV and asphalt shingles.

The installation procedures developed for this module allows separation of the roofing and electrical trades: a roofer installs the PV shingle using essentially identical procedures as for conventional asphalt shingles; later an electrician working under the roof completes the electrical interconnection of the PV modules. This key advantage offers the opportunity of introducing PV into the market without the need to develop a new trade. The roofer is required make a penetration into the roof for each 10-tab shingle module (see inset of Fig. 2) with the aid of a template, followed by insertion of a plastic grommet. The module's wires are inserted through the grommet. The electrical 
interconnections are made at an unspecified later time by a licensed electrician, working under the roof. These design concepts were exposed to experts in both the roofing and home electrical trades. There was consensus that the design is sound, in terms of ability of the roofing tradesmen to perform installations and in terms of compliance with the building and National Electrical Codes.

The modules have passed all the tests contained in the SERI Interim Qualification Tests and Procectures (Doc. SERI/TR-213-3624) as well as additional tests patterned after procedures used in the asphalt roofing industry (e.g. tests in standing water to ensure leak integrity).

The program also included installation and outdoor exposure tests in two mini-roof installations: one at United Solar and another at the NAHB Research Center. The weather at both facilities provided exposure of the modules to the elements (snow, ice, rain, sleet, freeze-thaw cycles, hot sunlight, etc.) under actual deployment conditions. We now have over 1 year of outdoor data that shows stabilized conversion efficiency without electrical or mechanical problems.

A $2 \mathrm{~kW}$ array will be installed on an ecological demonstration townhouse next to the Science and Technology Museum in the Olympic Village, Atlanta. This is a joint effort of United Solar, ECD, the DOE, and the Southface Energy Institute. The array will be mechanically installed by roofing tradesmen; the electrical interconnections will be made later under the eaves of the roof by an electrician. The specifications for this array are outlined in Table II. PV shingles will be integrated with conventional asphalt shingles in this installation.

Table I: Specifications for possible standard metal roofing modules. The modules use only two types of cells: $12^{\prime \prime} \mathrm{L} \times 123^{3 / 4} \mathrm{~W}$ cells for the $21^{\prime}$ and $13^{\prime}$ foot modules, and $9^{\prime \prime} \mathrm{L} \times 12^{3 / 4}$ " W cells for the $17^{\prime}$ and $9^{\prime}$ modules. Each module has either 16 (AM-20 and AM-16), or 8 (AM-12 and AM-8) cells; two of the longer modules, and 4 of the shorter modules are connected in series for charging a $48 \mathrm{~V}$ system, a good compromise between low voltages and small conductor sizes. Each module has 4 feet of dead space which can be trimmed in the field.

\begin{tabular}{|c|c|c|c|c|c|c|c|}
\hline Module & $\begin{array}{l}\text { Length } \\
\text { [ft/m] }\end{array}$ & $\begin{array}{l}\text { Weight } \\
\text { [lb/kg] }\end{array}$ & $\begin{array}{c}\text { Power } \\
\text { [W] }\end{array}$ & $\begin{array}{c}\mathbf{V}_{\text {Maxpwr }} \\
{[\mathrm{V}]}\end{array}$ & $\begin{array}{c}\text { I }_{\text {MerPWT }} \\
\text { [A] }\end{array}$ & $\begin{array}{l}V_{O C} \\
{[V]}\end{array}$ & $\begin{array}{l}I_{S C} \\
{[A]}\end{array}$ \\
\hline $\mathbf{A M}-\mathbf{8}$ & $9 / 2.8$ & $16 / 7$ & 59 & 14.2 & 4.2 & 18.6 & 5.2 \\
\hline AM - 12 & $13 / 4$ & $24 / 11$ & 79 & 14.2 & 5.5 & 18.6 & 6.9 \\
\hline AM -16 & $17 / 5.2$ & $32 / 14$ & 118 & 28.5 & 4.2 & .37 & 5.2 \\
\hline AM - 20 & $21 / 6.4$ & $39 / 18$ & 158 & 28.5 & 5.5 & 37 & 6.9 \\
\hline
\end{tabular}

Table II. Specifications for PV shingle array system to be installed in early 1996 at the Olympic Village, Atlanta.

\begin{tabular}{|lcl|}
\hline Parameter & Value & Units \\
\hline \hline Number of Modules & 120 & \\
Area of PV Coverage & 503 & $\mathrm{ft}^{2}$ \\
Array Operating Voltage & 50 & $\mathrm{~V}_{\mathrm{DC}}$ \\
Array Operating Current & 45 & $\mathrm{~A}$ \\
Arrav Operating Power & 2.25 & $\mathrm{~kW}$ \\
\hline
\end{tabular}




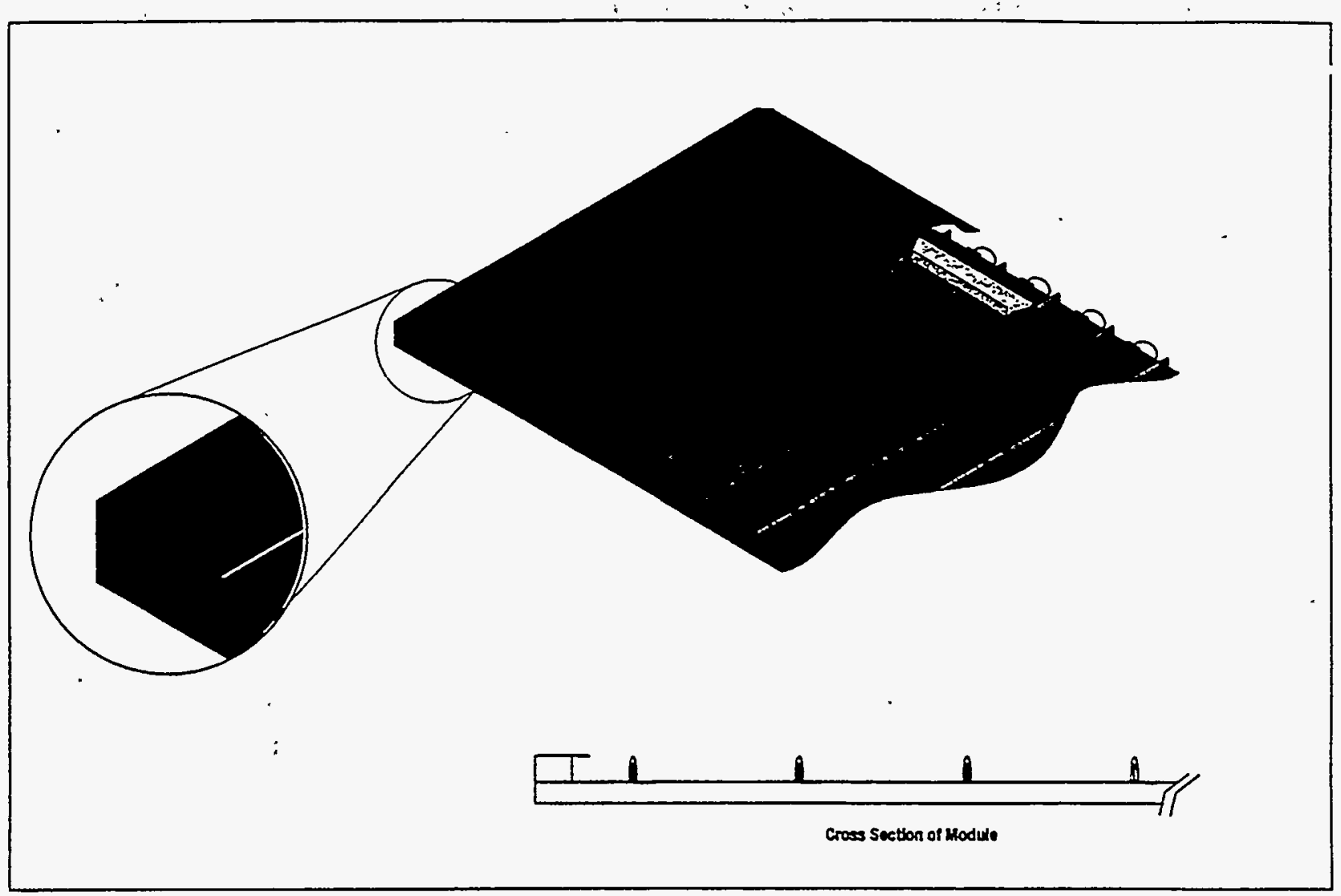

Figure 1. Graphic illustration of the ECD metal roofing module.

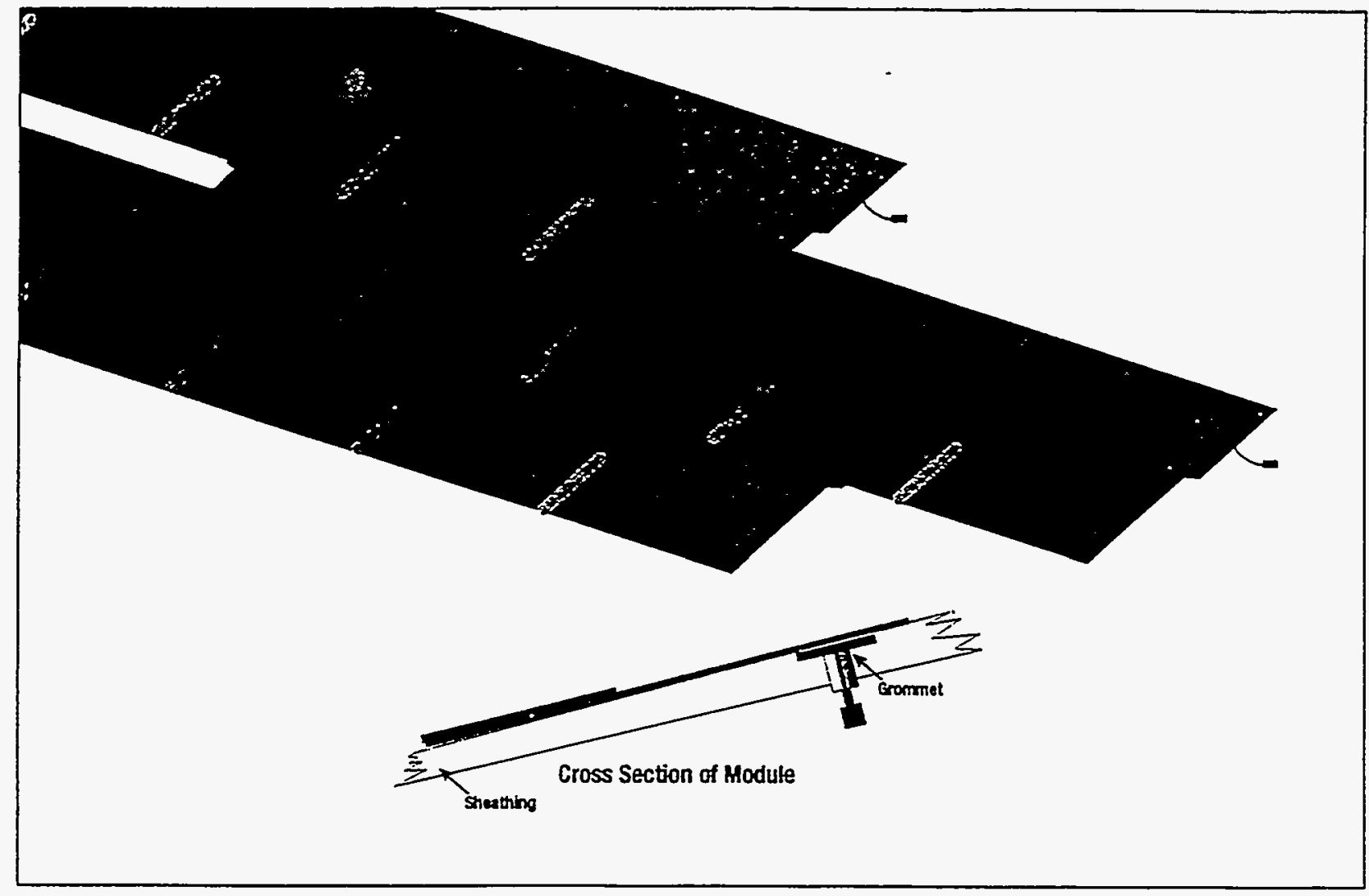

Figure 2. Graphic illustration of the United Solar shingle modules. A cross section illustrating installation on a roof deck is also shown. 

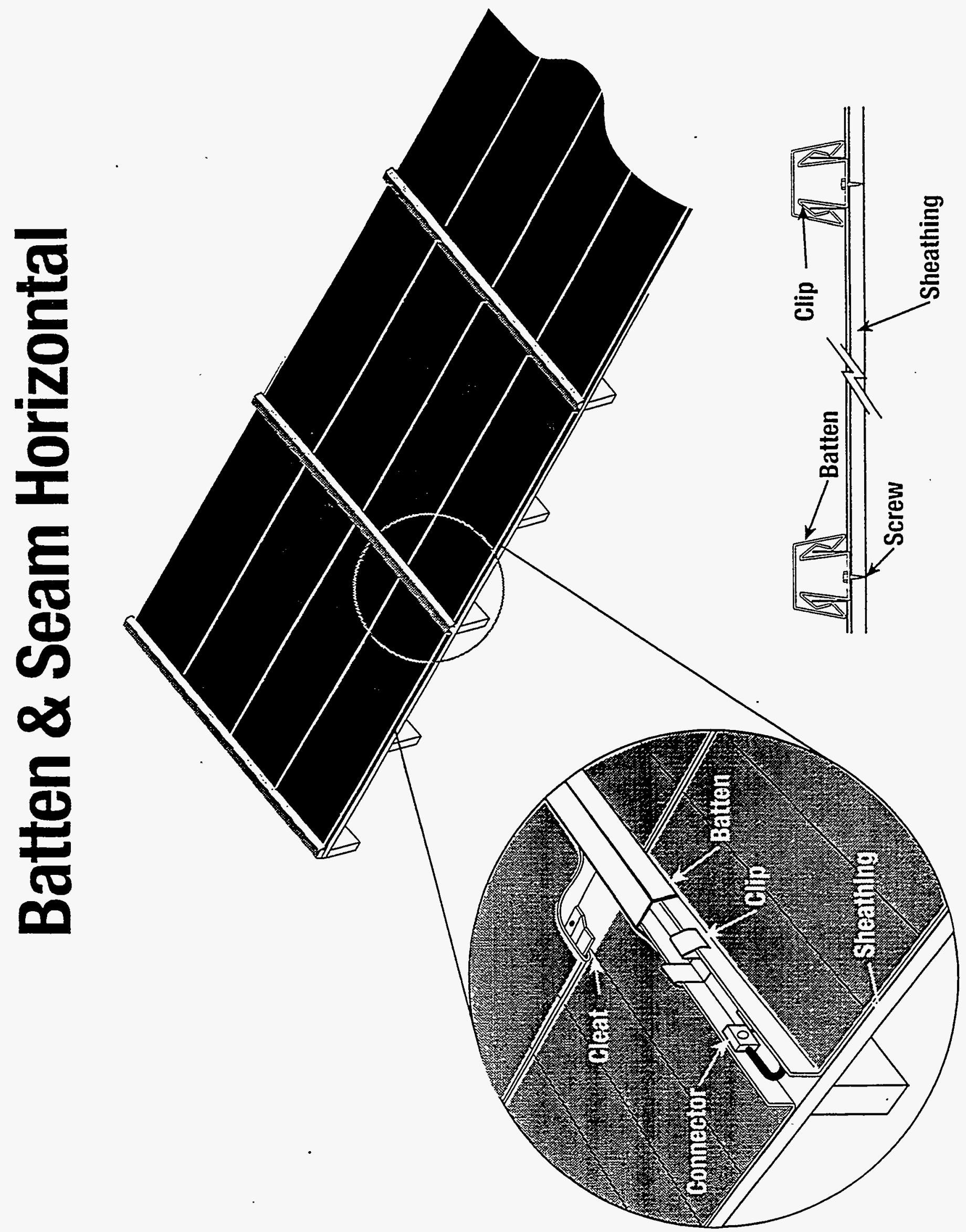


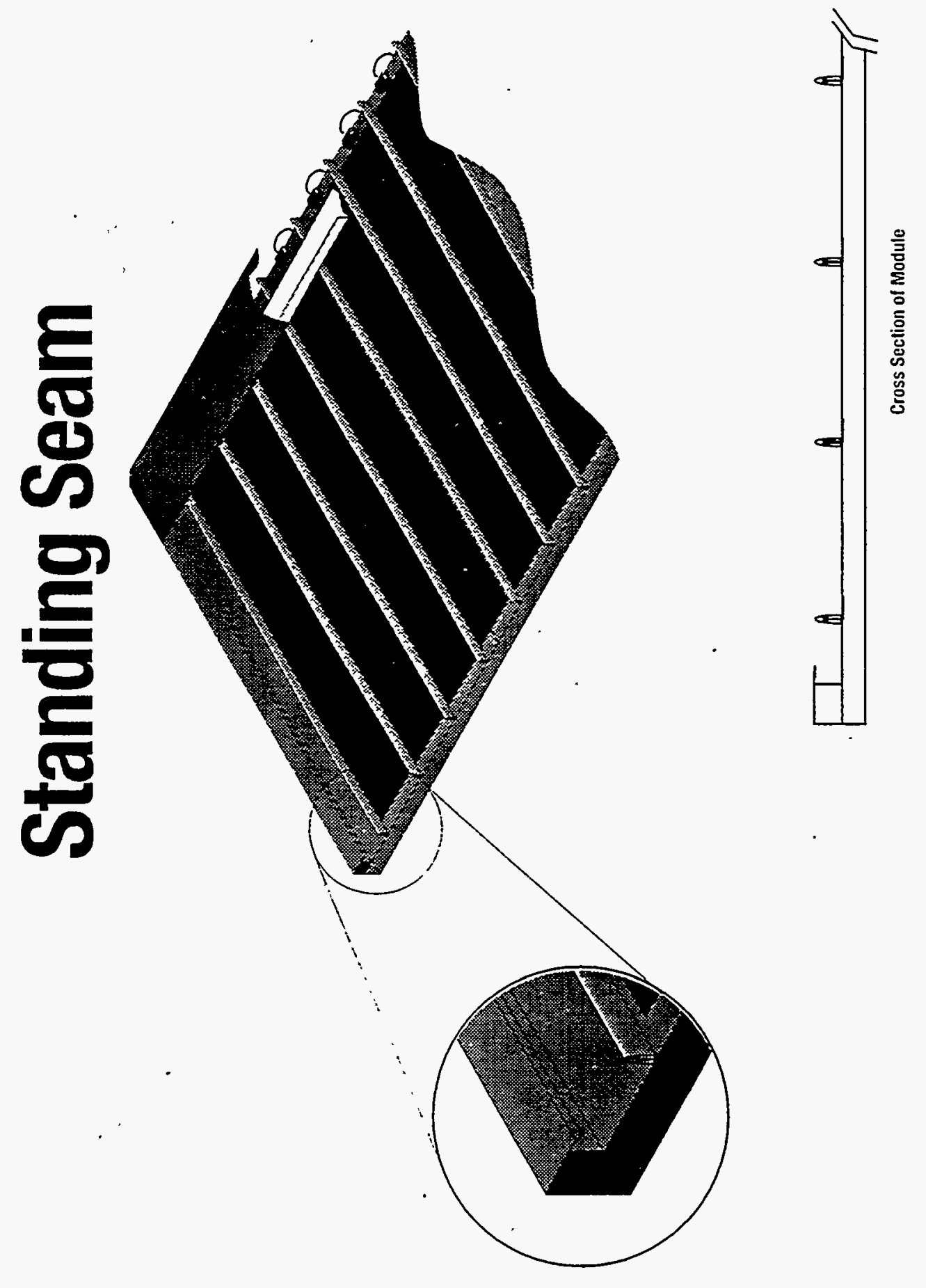




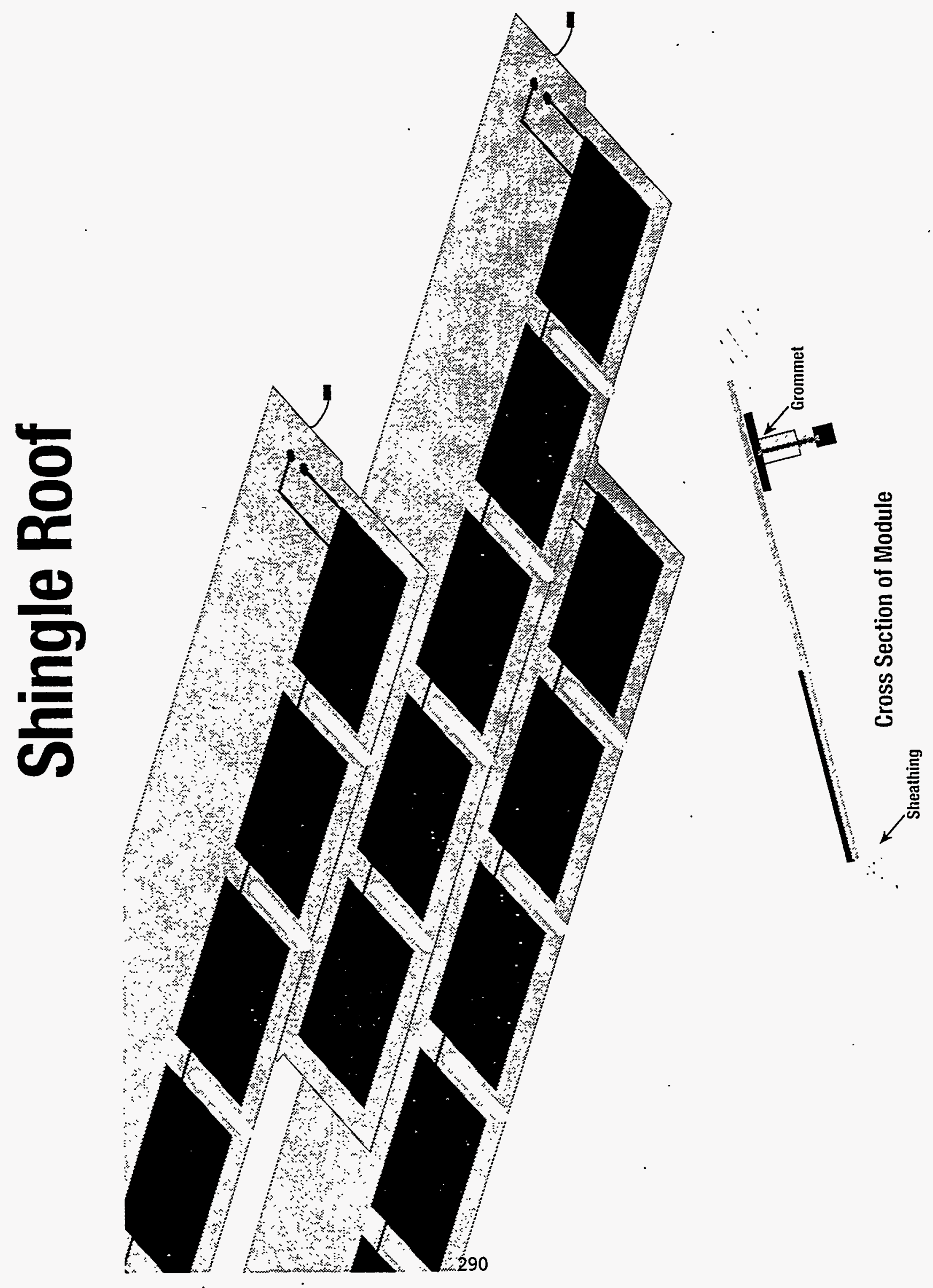


Title: $\quad$ Photovoltaic Manufacturing Technology (PVMaT) Improvements for ENTECH's Concentrator Module

Organization: - ENTECH, Inc., Dallas-Fort Worth Airport, Texas

Contributors: M.J. O'Neill, Program Manager

A.J. McDanal, Principal Investigator

\section{Introduction}

With significant assistance from the U.S. Department of Energy (DOE), Sandia National Laboratories (Sandia), and the National Renewable Energy Laboratory (NREL), the ENTECH technical team has been developing, field-testing, refining, and commercializing linear Fresnel lens photovoltaic concentrator technology for the past 17 years. Under this PVMaT project, ENTECH is developing the manufacturing technology required to mass-produce our fourth-generation concentrator module.

\section{Objective}

The key objective of ENTECH's PVMaT Program is to design, develop, and implement improved manufacturing processes for ENTECH's fourth-generation concentrator module. The improved processes are being engineered to simultaneously achieve enhanced product quality (i.e., improved module efficiency, reliability, and field lifetime), higher production volume (10 MW/year initial rate), and lower production costs $\left(\$ 1.25 / \mathrm{W}_{\text {peak }}\right.$ goal).

\section{Approach}

An outstanding international technical team has been assembled to improve the manufacturing processes associated with our fourth-generation linear Fresnel lens concentrator module. This team includes technical personnel from NREL (Ed Witt, Rick Mitchell, John Benner, et al.), Sandia (Alex Maish, Dave King, et al.), 3M (Paul Jaster, DuWayne Radke, Char Thill, et al.), Consumers, Inc. (Arnie Kapitz), BP Solar (Tim Bruton), ASE (Wilfried Schmidt), Siemens Solar (Kim Mitchell and Richard King), Solarex (John Wohlgemuth and Mohan Narayanan), Spire Corporation (Mike Nowlan), AIT (Ed Chalupa and Hugh Wilson), Klos Technologies (Rick Simpson), Columbia Aluminum (Walt Brown), DuPont/Clear Solutions (Stan Levy), and key component suppliers, in addition to ENTECH's in-house staff.

\section{Module Description}

Our line-focus photovoltaic concentrator modules have been described in many previous publications ${ }^{1-10}$. Fig. 1 shows a cross-sectional sketch of our fourth-generation module. The lens aperture is $84 \mathrm{~cm}$ ( $33 \mathrm{in}$.) wide by $366 \mathrm{~cm}$ (144 in.) long, for a total sun-capturing area of $3.1 \mathrm{~m}^{2}$. Our production lenses from $3 \mathrm{M}$ provide a net optical efficiency of $90 \%$ while focussing sunlight onto 37 series-connected solar cells, each having active area dimensions of $4.1 \mathrm{~cm}$ (1.6 in.) wide by 9.65 $\mathrm{cm}$ (3.8 in.) long. The geometric concentration ratio of the module is $85 \mathrm{~cm}$ divided by $4.1 \mathrm{~cm}$, which equals $21 \mathrm{X}$. Each cell is covered with a prismatic cell cover to eliminate gridline shadowing losses. Waste heat from the cell string is rejected via the world's largest extruded aluminum heat sink. 
The module peak efficiency at standard test conditions $\left(\mathrm{STC}=25 \mathrm{C}\right.$ cell temperature and $1 \mathrm{~kW} / \mathrm{m}^{2}$ direct normal irradiance) is equal to the product of lens optical efficiency $(90 \%)$, cell-to-receiver packing factor (98\%), receiver encapsulating layer transmittance (94\%) and cell efficiency. Three of our cell suppliers (ASE, BP Solar, and Solarex) have already achieved about $19 \%$ peak efficiency for developmental cells delivered under this program, as verified in recent tests at Sandia. With such developmental cells, our peak module efficiency is presently about $16 \%$, corresponding to a module power rating of about $500 \mathrm{~W}_{\text {peak }}$. For a production run of 21,000 cells, ASE achieved over $17 \%$ lotaverage cell efficiency. With such production cells, our peak module efficiency is presently about $14 \%$, corresponding to a module power rating of about $430 \mathrm{~W}_{\text {peak }}$, as verified by Sandia. Clearly, our fourth-generation module is the largest photovoltaic module of any type yet produced, in terms of both aperture area and power output.

\section{Significant Results During FY 1995}

Cell Package Improvements: During FY 1995, we verified the producibility of our new solar cell package. The new cell package, shown in Fig. 2, incorporates die-cut prism cover tape, with integral pressure-sensitive adhesive (PSA), bonded to the upper surface of the cell to eliminate gridline obscuration losses. The new prism cover tape is delivered by our team member, $3 \mathrm{M}$, on rolls containing 3,500 prism covers each. Each prism cover is aligned and bonded to a solar cell using a semi-automated prism cover application work station, which allows covers to be applied to cells at rates faster than one per minute. Each cell package also includes two integral bypass diodes, for cell shading protection. Each cell package also includes four solder-plated copper ribbons, for carrying the cell current into and out of the cell package. Over 20,000 of these new cell packages have been produced with excellent yields and over $17 \%$ lot-average conversion efficiency.

Dielectric Improvements: During FY 1995, we verifed a simple new dry-film process for bonding the cell packages to the heat sink, and for encapsulating the resultant cell string. This new process (patent approved) incorporates two mass-produced polymer film layers, the first between the cells and heat sink, and the second between the cells and surrounding atmosphere. The selected film is DuPont Tefzel $^{\circledast}$, which provides excellent dielectric strength, high-temperature survivability, outstanding toughness, ease-of-use, and reasonable cost. The new dielectric process uses off-theshelf tape and film dispensing equipment to attach the film layers to the heat sink and completed receiver assembly, respectively. The new process also eliminated over 300 parts per receiver.

Independent Module Testing: During FY 1995, Sandia correlated their cell operating temperature data for our module and for one-sun modules, mounted on the same outdoor sun-tracker. Fig. 3 shows the side-by-side cell temperature comparison for one of our modules versus a one-sun module made by Siemens Solar. Note that the cell temperatures are the same within about $10 \mathrm{C}$. These data confirm the thermal effectiveness of the new cell laydown approach, discussed under Dielectric Improvements above, and the new heat sink, discussed under Module Description above.

System Production and Deployment: During FY 1995, two utility-scale, $100 \mathrm{~kW}$ power plants, each using 288 of our new concentrator modules, were dedicated. One plant, owned by Central \& South West (CSW), is deployed at the CSW Solar Park near Ft. Davis, Texas. The other plant, owned by TU Electric (TUE), is deployed at the TUE Energy Park in Dallas, Texas. The modules 
used in these two production systems were manufactured using all of the new processes developed under this PVMaT program. Fig. 4 shows the measured performance of the four SolarRows comprising the solar array at the CSW site. Each SolarRow is an integrated, two-axis sun-tracking structure, containing 72 fourth-generation modules. Four SolarRows comprise each $100 \mathrm{~kW}$ power plant. Note the consistently high performance of the four SolarRows.

\section{Conclusions and Future Plans}

Substantial progress has been made in developing the manufacturing technology needed to produce efficient, reliable, high-quality, low-cost concentrator modules. Unlike many other emerging photovoltaic technologies, our fourth-generation concentrator technology requires no further breakthroughs in materials, efficiency, stability, or basic manufacturing processes, to meet the Department of Energy's electrical energy cost goals. High-volume procurement of vendor-furnished parts, coupled with automated assembly of these parts into concentrator modules, will enable this technology to provide cost-effective electrical power in sunny regions of the world.

\section{References}

1. M.J. O'Neill et al., "Photovoltaic Manufacturing Technology Improvements for ENTECH's Concentrator Module: Phase 1 Final Technical Report," NREL/TP-214-4486, Denver, November 1991.

2. M.J. ONeill et al., "ENTECH's Fourth-Generation Linear Concentrator Module," 1992 DOE/Sandia Crystalline Photovoltaic Technology Project Review Meeting, SAND92-1454, Albuquerque, July 1992.

3. T.M. Bruton et al., "Recent Developments in Concentrator Cells and Modules Using Silicon Laser Grooved Buried Grid Cells," 11 th European PVSEC, Montreaux, Switzerland, October 1992.

4. M.J. ONeill, "Fourth-Generation, Line-Focus, Fresnel Lens Photovoltaic Concentrator," 4th SunshineWorkshop on Crystalline Silicon Solar Cells, Tokyo, Japan, November 1992.

5. M.J. O'Neill and A.J. McDanal, "Manufacturing Technology Improvements for a Line-Focus Concentrator Module," 23rd IEEE-PVSC, Louisville, May 1993.

6. M.J. O'Neill, "Photovoltaic Manufacturing Technology (PVMaT) Improvements for ENTECH's Concentrator Module," 12th NREL PV Program Review Meeting, Denver, October 1993.

7. M.J. O'Neill and A.J. McDanal, "Fourth-Generation Concentrator System: From the Lab to the Factory to the Field," 1st WCPEC, Hawaii, December 1994.

8. M.J. O'Neill, "Chapter 10, Silicon Low-Concentration, Line-Focus, Terrestrial Modules," in the book, Solar Cells and their Applications, Edited by L.D. Partain, John Wiley, ISBN 0-471-57420-1, 1995.

9. M.J. O'Neill and A.J. McDanal, "Fourth-Generation Photovoltaic Concentrator System Development," Sandia Report No. SAND95-1553, Albuquerque, October 1995.

10. M.J. O'Neill and A.J. McDanal, "Photovoltaic Manufacturing Technology Improvements for ENTECH's Concentrator Module: Final Report," NREL Report No. NREL/TP-41 1-20277, Denver, Nov. 1995. 

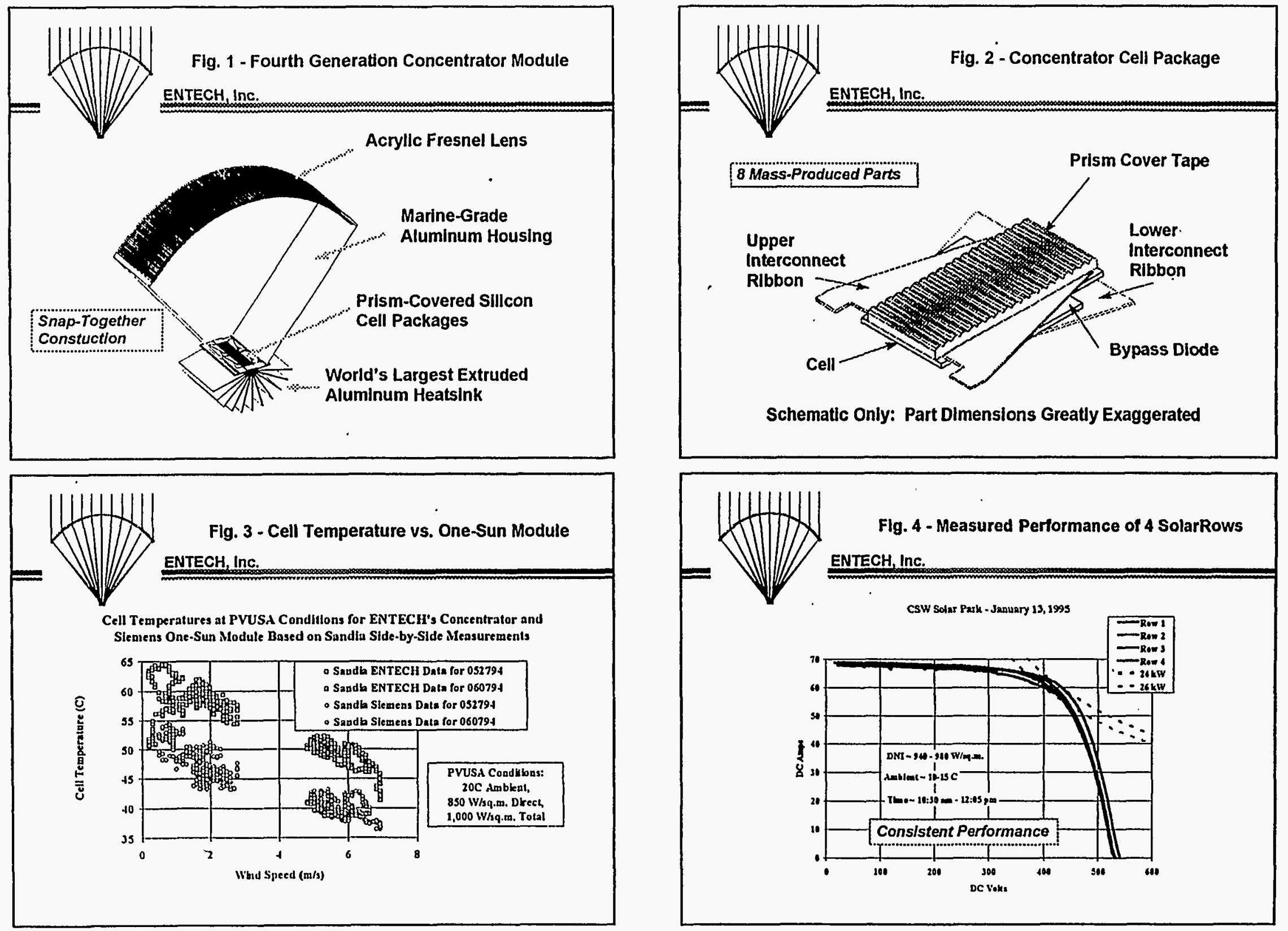
Title: High-Efficiency, Large-Area CdTe Panels

Organization: Golden Photon, Inc., Golden, Colorado

Contributors: S. P. Albright, Principal Investigator; John Kester, D. Zubia

\section{Objectives and Focuis}

The objectives of this multi-year Subcontract are "The improvement of Near-term CdTe Manufacturing and Product Capabilities and Establishment of Next Generation CdTe Technology".

The GPI objectives in Photovoltaics are covered through continuing advancement in three major areas; specifically, module efficiency, reliability, and cost:

Most of the efforts in 1995 under this Subcontract has been devoted to Product enhancement, qualification, and certification. This focus was adopted in order to better certify the stability objective: To achieve greater than 20-year module life with no greater than $10 \%$ efficiency degradation.

Also, during 1996, much of the developmental foundation was laid for the implementation of "next-generation" processing methods for reducing the overall costs of selected process steps. These efforts are expected to continue in 1996 at increased rates.

\section{Important Milestones and Results}

Manufacturing Facility

During 1994 and 1995, Golden Photon, Inc. constructed a 2_MW facility in Golden Colorado for proving the feasibility of the commercialization of CdTe-based photovoltaic systems. Initial sales of modules have been accomplished. Achieving further market, operations, and development milestones will continue to be the primary focus at GPI in 1996.

\section{Manpower and Team Building}

In order to accelerate efforts in a variety of areas, a new Manager of R\&D, John Kester, (Ph.D., Physics) and a new Scientist (M.S., EE) have joined the team to provide improved focus on experimental design, characterization, measurement, and experimental execution.

\section{Qualification/Certification Testing}

All critical "Pre-Qualification" tests ((based on the requirements for IEEE PAR1262, but not in full sequence, nor certified) on GPI modules has been successfully completed. Although not yet fully completed, Certification tests for UL Listing, and a Qualification test sequence (IEEE PAR1262) is underway at Arizona State University under Bob Hammond.

\section{Module Characterization}

Temperature coefficients were determined at GPI by ASTM methods during June. The following values were determined: $\mathrm{NOCT}=56 \mathrm{C},-0.0028 \% / \mathrm{C}$ for Voc, $+0.0002 \% / \mathrm{C}$ for Isc, and $-0.0022 \% / C$ for Wmp. 
Morphology Improvement

Morphology improvement of the device layers is one of the needs to improve the longer term efficiency of this technology. Negotiations are nearing completion for a joint development arrangement with NREL to address some of these issues. Lead by D. Ginley from NREL, it is believed that significant improvements in morphology will result from this joint effort.

\section{Surface Recombination/Passivation}

Device Passivation efforts have been somewhat successful in that NREL has measured Minority Carrier Lifetimes of $2.2 \mathrm{nS}$ on GPI CdTe layers. This is believed to be a record for the industry. See Fig. 1.

\section{Environmental and Employee Health and Safety Issues}

A large investment in equipment, controls, and policies exists in the GPI 2MW facility. A great deal of effort has been placed on ensuring that methods and policies for Employee Health and Safety have been properly evaluated, implemented, and monitored. An excellent safety record has resulted. Further technology and policy needs to ultimately be developed in order to fully minimize the costs associated with the present methods. The continuing development and monitoring of such implemented methods is believed to be necessary in order to safely ensure lowest cost recycling of such waste, consistent with the commitment of Golden Photon as a successful, safe, and environmentally friendly, commercial venture.

\section{Summary and Future Activities}

Ensuring that the new $2 \mathrm{MW}$ Golden, $\mathrm{CO}$ facility continues to progress successfully through the initial commercialization phase remains the important company focus. Quality Control improvements are expected to be the primary improvement vehicle, and have been accelerated greatly by the existence of the PVMAT Subcontract through NREL. It is the intention of GPI to begin expansion to a larger plant size in 1997, assuming that the critical Marketing, Manufacturing, and Developmental milestones for 1996 are met.

Developmentally, future efforts will focus on further enhancing process and product technology. Process and Product development will continue at even higher rates in 1996 in order to provide lower cost, higher quality modules to photovoltaic markets. Some of these enhancements include:

- Improved interconnection methods with optical losses of less than 5\%,

- Large reductions in module framing and mounting costs,

- Implementation of faster throughput methods for encapsulation

- Higher rates of deposition using lower cost methods for CdS and other depositions 
Major Project Reports

1. Albright, S.P.; Singh, V.P.; Jordan, J.F. "Junction Characteristics of CdS/CdTe Solar Cells", Presented at the 9th Photovoltaic Advanced Research and Development Project Review Meeting, Denver, CO, November 15-18, 1987., Published in Solar Cells, Elsevier Sequoia, The Netherlands, 24 (1988) 43-56.

2. Ackerman, B.; "Methods and Models for Life Testing of CdS-CdTe Solar Cells", From Photovoltaic Thin Film Module Reliability Testing and Evaluation Workshop, Sponsored by SERI under DOE, August 1987, Lakewood,CO.

3. Albright, S.P.; Jordan, J.F.; Ackerman, B.; Chamberlin, R.R. "Developments on CdS/CdTe Photovoltaic Panels at Photon Energy, Inc.", Presented at the 9th AR\&D Meeting in Lakewood, Colorado; Published in Solar Cells, Elsevier Sequoia, The Netherlands, 27 (1989) 77-90.

4. Ackerman, B.;Albright, S.P. "Encapsulation and Life Testing Issues for One Foot Square Cds/CdTe Modules" Presented at Photovoltaic Thin Film Module Reliability Testing and Evaluation Workshop, Sponsored by SERI, June 1989, Golden, Colorado; Published in Solar Cells.

5. Albright, S.P.; Ackerman, B.; Chamberlin, R.R.; Jordan, J.F. "Progress on Polycrystalline Thin Film CdTe Modules,"Presented at Polycrystalline Thin Film Program Meeting, Lakewood, Colorado, August 16-17,1989.

6. Albright, S.P.; Ackerman, B.; Jordan, J.F. "Efficient CdTe/CdS Solar Cells and Modules by Spray Processing" IEEE Transactions on Electron Devices, Vol. 37, No 2, February 1990, pp. 434-437.

7. Albright, S.P.; Chamberlin, R.R.; Jordan, J.F.; "High-Efficiency Large- Area CdTe Panels", Final Technical Progress Report from Photon Energy, Inc., Prepared under SERI Subcontract ZL-7-06031-3, Covering the period from June, 1987 to July,1990.

8. Albright, S.P.; Chamberlin, R.R.; Jordan, J.F. "Cadmium Telluride Module Development", Presented at PVAR\&D meeting in Lakewood,CO, October 1990. (To be Published in Solar Cells).

9. Albright, S.P.; Chamberlin, R.R.; Ackerman, B.; Jordan, J.F.; "Performance Measurement Irregularities on CdS/CdTe Devices and Modules", Int. J. Solar Energy, Harwood Academic Publishers GmbH, Malaysia, Vol.12, 1992, pp. 109-120 


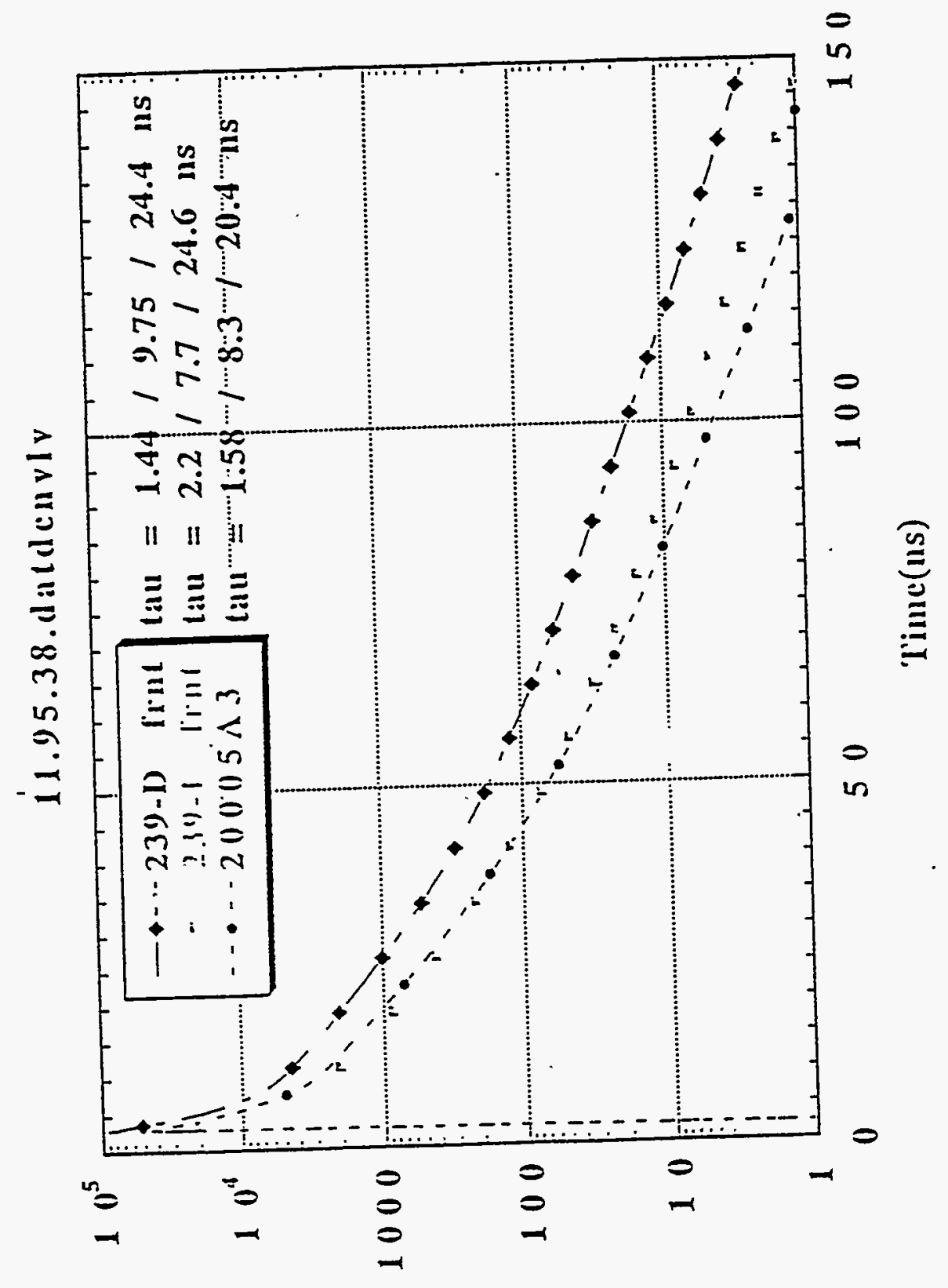

7นIJ $Q-6 \varepsilon 己$ 
Title:

Organization:

Contributors:
Monolithic Amorphous Silicon Modules on Continuous Polymer Substrates

\author{
Iowa Thin Films Technologies, Inc., Ames, IA
}

F.R. Jeffrey, Principal Investigator; D. Grimmer; S. Martens, H. Shanks, and $M$. Noack, Investigators

\section{Objective}

The objective of this subcontract over its three year duration is to improve overall module performance, -increase the throughput of the metalization, a-Si deposition, laser-scribing, and welding processes and to reduce the overall module manufacturing costs of the ITF production line by $68 \%$.

\section{Approach}

This subcontract consists of three one-year phases beginning July 1995. Phase I project activities are divided into four tasks and processes: replace the ITF TiN layer with a less absorbing $\mathrm{ZnO}$ layer; design and implement a web steering system for feeding the web onto the printer platen; establish new laser operating parameters to optimize the laser beam scan speed; and develop a new insulating ink printing and roll based laminating process for the ITF production line

\section{Research Results (7/15/95-9/30/95)}

Task 1 is aimed at back reflector improvement and deposition throughput improvement. Zinc Oxide is being researched as an alternative to titanium nitride as the intermediate layer between the aluminum base metal/ reflector/ electrical conductor and the silicon layers for photovoltaic cells. $\mathrm{ZnO}$ has optical benefits that can enhance the reflectance of light from the base of the cell improving the overall efficiency of the cell. However, silicon may cause adhesion and electrical shorting problems in the $\mathrm{ZnO}$ layer. A first step was to develop operating parameters (pressure, voltage, etc.) that produce transparent conductive $\mathrm{ZnO}$ thin film for the improved back reflector.

Initially, a Zinc, $2 \%$ aluminum target was install in the PTS for deposition onto a polyester web. Polyester was used because it is transparent to the wavelengths of light of interest and allows for transmission spectra tests. Zinc oxide is deposited from a metal target using a reactive sputtering technique in an argon/oxygen atmosphere. Deposition parameters were optimized for optical and electrical performance. 
Table 1 below shows selected sample results indicating the $\%$ oxygen, total pressure, target potential and resistance.

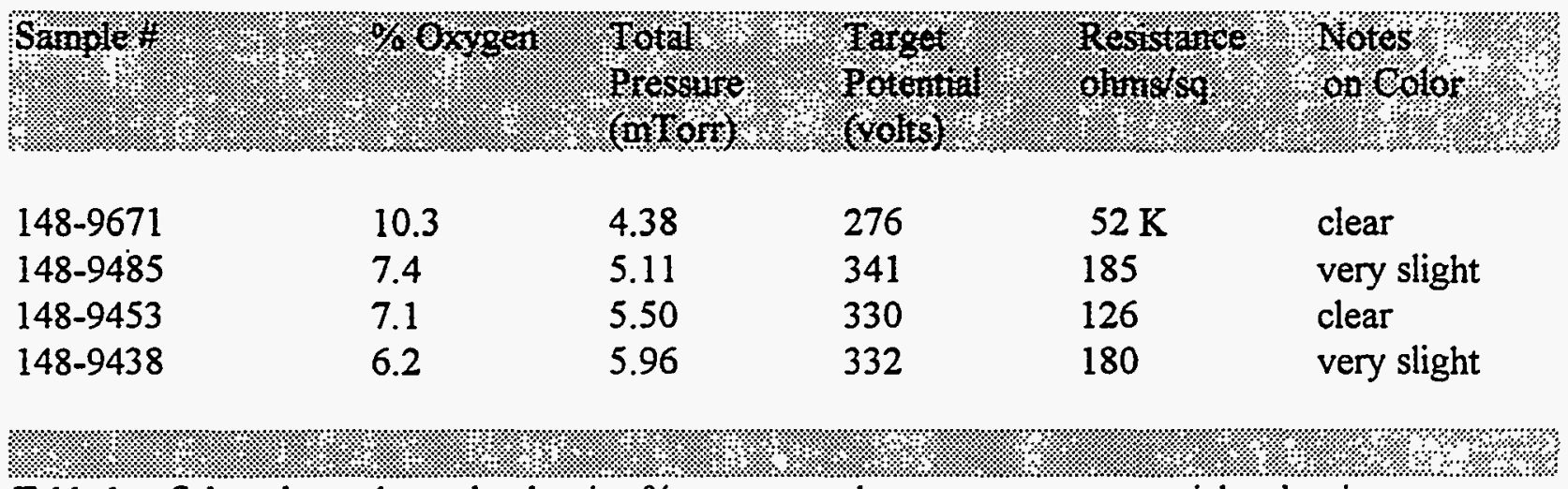

Table 1. Selected sample results showing \% oxygen, total pressure, target potential and resistance.

Thickness measurements were made on samples \#148-9485, -9453 , and -9438 . These were found to be $1 \mathrm{~K} \mathrm{~A}, 2.4 \mathrm{~K} \mathrm{~A}$, and $2.2 \mathrm{~K}$ A respectively.

From these measurements, the conditions of $5.5 \mathrm{mT}$ and $7.1 \%$ oxygen, sample \#-9453, was deemed the most favorable, even though sample \#-9485 had a lower bulk resistivity. \#-9453 had the highest deposition rate and was very transparent were-as the lower resistance of \#-9485 was probably due in part to the metallic Zinc in the film as noted by its brownish coloration.

The conductivity of the sputter deposited $\mathrm{ZnO}$ films show a strong dependence on oxygen partial pressures as indicated in the table of selected samples above. This indicates that the conductivity may be due to the generation of oxygen vacancies and/or zinc interstitials during depositions. In either case, the conductivity of the films would be unstable. Another possibility is that when sputtering with an aluminum enriched target, the reduced oxygen pressure allows the aluminum to occupy zinc sites in the crystal lattice rather than being fully oxidized to $\mathrm{Al}_{2} \mathrm{O}_{3}$ and remaining inert. To test this idea, transmission spectroscopy of samples \#-9671 and \#-9453 was performed using an expanded scale of 320 to $2000 \mathrm{~nm}$ wave length light. These two samples were chosen because they exhibited widely varying electrical conductivity but both were optically clear.

The upper plot in Figure 1 on the following page shows a comparison between the two samples over the full range of wave lengths. This plot shows that transmission through sample \#-9453 decreases with increasing wavelengths when compared with sample \#-9671, indicating the presence of free charge carriers. The lower plot shows an enlargement of the region from 320 to $500 \mathrm{~nm}$ wavelengths. This plot shows a blue shift in the transmission curve for sample \#-9453 as compared with \#-9671, indicating a change in the band gap energy probably due to the incorporation of aluminum atoms in lattice sites. 
K: \$B9453; abec 2689.8- 388.8; pto 1781; int 1.88; ond 23.790-258.88; 1

inf: $11: 21: 15 \quad 95 / 12 / 21$

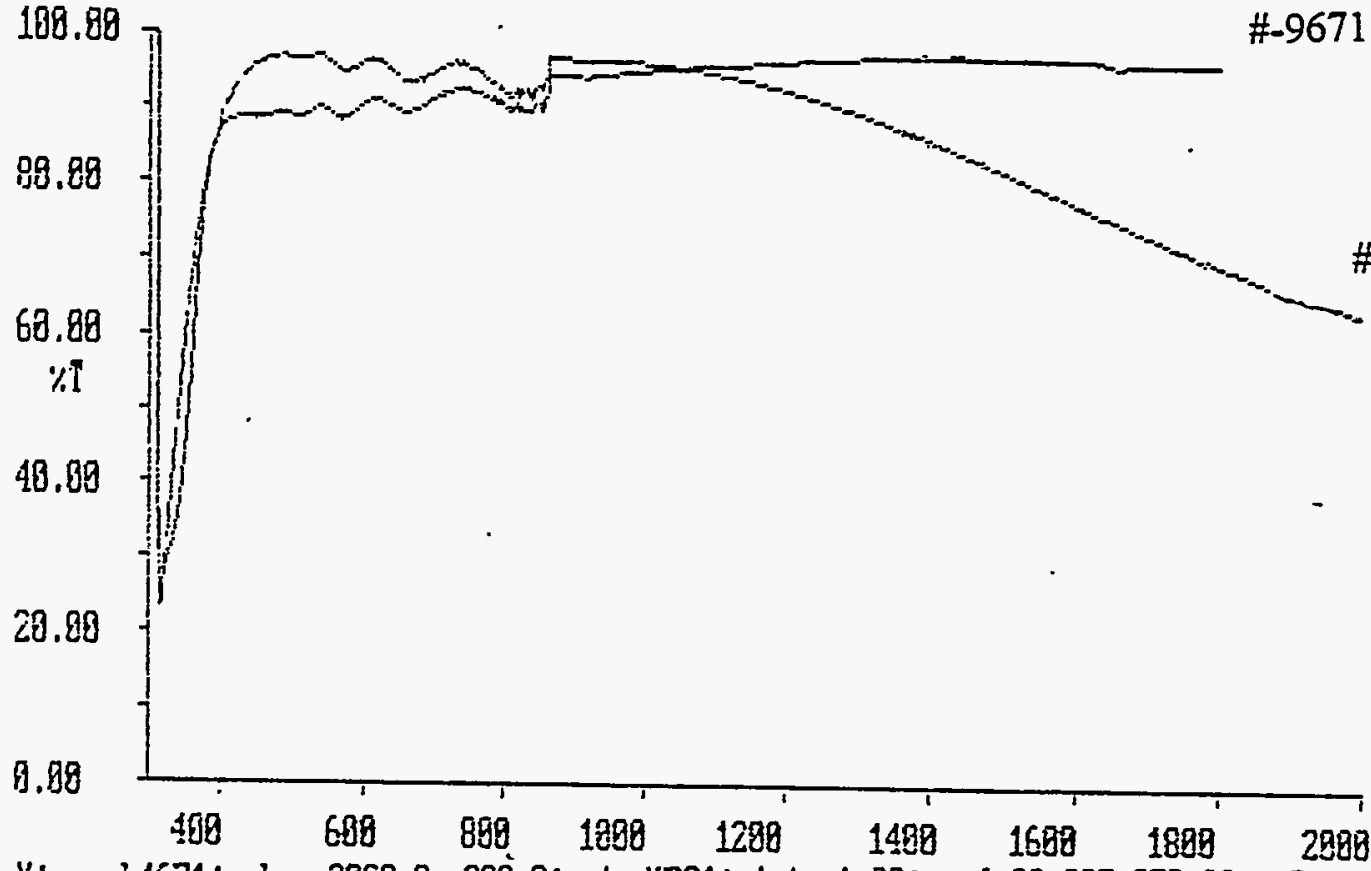

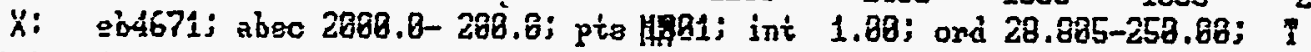

inf: 11:11:85 95/12/21

198.98

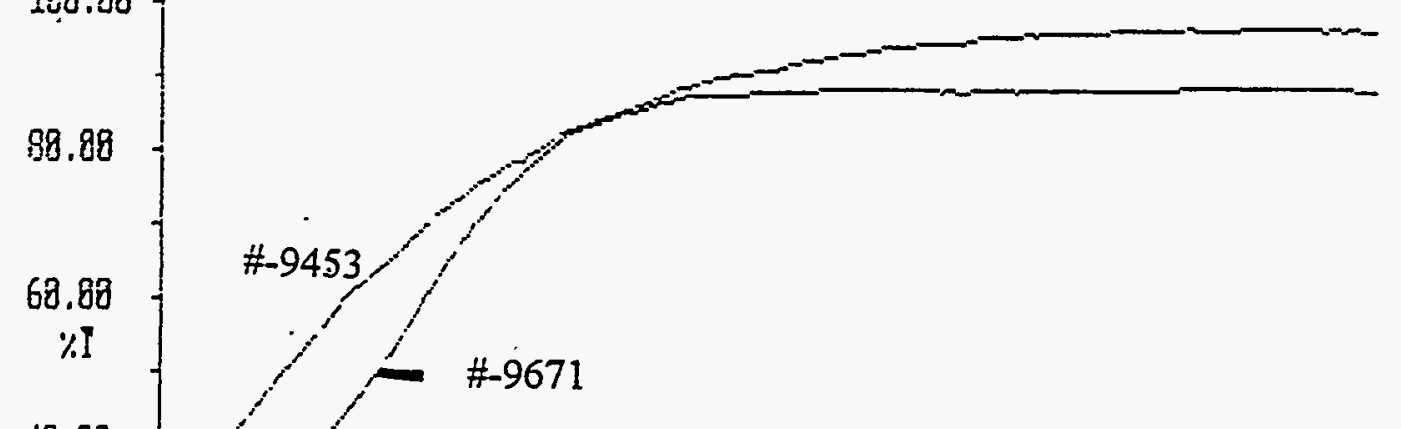

40.80

20.89

6.89

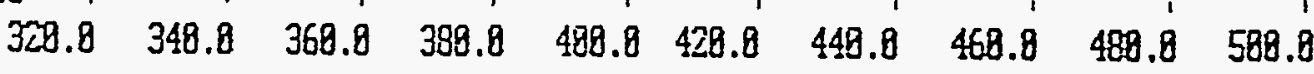

แกก

Figure 1. Comparison between the two samples \#-9453 and \#-9671 over the full range of wave lengths. 
This information indicates that the conductivity may be due to aluminum acting as a dopant. In addition, a preliminary thermal stability test on samples of sputter deposited $\mathrm{ZnO}$ shows a slight increase in conductivity and long term stability when the samples are heated to $200 \mathrm{C}$, again indicating the aluminum doping is the source of the charge carriers.

Task 2 is aimed at improvements in laser-scribing, welding, and printing process throughput. In relation to this task, contact was made with a number of web guide system suppliers and a suitable supplier was identified. The new system for feeding the web onto the printer platen should insure increased accuracy in the position of the web edge to an approximate $0.5 \mathrm{~mm}$ variation.

Identification of parameters necessary for improved scriber throughput has resulted in a new list of parameters for the control software. One of the more important determinations made was in scribing speed. Scribe rates up to $99 \mathrm{~mm} / \mathrm{sec}$ are possible. "Clean" scribes have been achieved at $90 \mathrm{~mm} / \mathrm{sec}$, a $50 \%$ improvement in speed. Use of the higher speeds awaits experiments when layers do not change during a run. Higher rates would be prone to a reduced yield from minor fluctuations in laser power.

Task 3 is aimed at substrate and materials cost reduction. Information has been gathered on new and less expensive inks, both conducting and insulating; improved screen materials; improved and environmentally friendly ink reclaiming materials. Each of these efforts is directed at improving material and lowering production costs.

Task 4 involves the development of a roll based laminating system. Design of a roll-based laminating machine is being considered as part of an integrated end-manufacturing process to convert submodules into finished modules complete with electrical leads. A complete survey of roll based laminating equipment vendors has been conducted towards meeting this end. A preliminary design of a roll based laminator system was completed

Twenty-three converting technology companies were contacted regarding what they could provide in the areas of die-cutting, sheeting, carrier webs, pick-and-place, island placement, tabbing, or roll-to-roll lamination on at least a 13" web. Of these it was determined that eleven would not be of help. We will continue investigating the other twelve and obtain final quotations prior to the end of December 1995.

\section{Conclusions}

Although this subcontract began only two and one half months ago, milestones due were met and progress was made in each of the project task areas. During the next twelve months we will proceed in replacing the ITF TiN layer with a less absorbing $\mathrm{ZnO}$ layer; designing and implementing a web steering system for feeding the web onto the printer platen; establishing new laser operating parameters to optimize the laser beam scan speed; and developing a new insulating ink printing and roll based laminating process for the ITF production line. 
Title: $\quad$ Advanced Development of PV Encapsulants

$\begin{array}{ll}\text { Organization: } & \text { Springborn Materials Science Corp. } \\ & \text { Enfield, Connecticut } 06082\end{array}$

Contributors: W.H. Holley, principal investigator; J.P. Galica, administrative manager; S.C. Agro, R.S. Yorgensen; M. Ezrin and

P. Klemchuk, Institute of Materials Science, University of Connecticut

Objectives: EVA resin-based encapsulant is employed extensively by module manufacturers worldwide, has been in commercial use since $1981^{1}$, and has proven dependable from the standpoint of production, module fabrication, and end-use. Despite the widespread acceptance of EVA resin-based A9918P and similar formulations for PV, some module producers and end-users have reported cases of browning with field-aged modules. While isolated, these reports raised serious concern as to the long term reliability of EVA resin-based encapsulants.

The first goal of this PVMaT program was problem definition, using accelerated U.V. aging studies (AAS) to evaluate the influence of compositional, processing, and operating parameters on A9918P discoloration. Also, instrumental analysis was used to verify suspected chemical mechanisms. EVA-based encapsulant from field-aged and Weather-Ometer-aged modules and laminates was analyzed by GC/MS (gas chromatography/mass spectrometry) and GC/FID (flame ionization detection) for UV absorbers, FTIR (fourier transform infrared spectroscopy) and Raman spectroscopy for unsaturation and oxidation, and TGA (thermogravimetric analysis) and XPS (X-ray photoelectron spectroscopy) for vinyl acetate content. Based on results of these studies, the next goal was to develop approaches for stabilizing the EVA-based encapsulant against discoloration/degradation, including reformulated systems. These "stabilization strategies" were evaluated by AAS using xenon-arc Weather-Ometer and EMMA (equatorial mount with mirrors for acceleration; outdoor weathering) with periodic measurement of Yellowness Index.

Technical Approach: Sample Preparation: For AAS, vacuum-laminated coupon-size laminates, measuring $2.7 \times 2.75$ inches were used. A commercial cure schedule was used for all laminates. For most of this work, glass/EVA-based encapsulant/glass laminates were used to facilitate colorimetric, and spectrographic measurements.

Superstrates: Superstrates were commercially-used low iron glasses to allow maximum transmission of UV-B light (ie. 285 to $350 \mathrm{~nm}$ ), that wavelength region suspected as being responsible for encapsulant discoloration. Glasses included Solite (AFG - samples from the mid 1980s), Starphire (PPG), Solatex II (AFG - containing cerium oxide, estimated at less than $4 \%$ ), and Solarphire (PPG - also with cerium oxide). During development work it was speculated that Solarphire and Solatex II would benefit this application by filtering most of the UV-B radiation.

Sample Exposure and Evaluation: Laminates were exposed to 0.55 watts $/ \mathrm{m}^{2} / \mathrm{nm}$ ( @ $340 \mathrm{~nm}$ ), $95 \%$ R.H., and $100^{\circ} \mathrm{C}$ in an Atlas Ci35A Weather-Ometer with quartz/borosilicate glass filters. The nominal lower end U.V. cut-off was $285 \mathrm{~nm}$. Coupons were monitored for Yellowness Index 
during AAS per ASTM D-1925, and percent light transmission (\%T) between 250 and $900 \mathrm{~nm}$ by U.V.-VIS spectrophotometer. Some analysis of additive and vinyl acetate contents was also done.

Results: Additives are Key to Browning: One hypothesis for browning of EVA encapsulants is long sequences of conjugated double bonds (polyenes), resulting from loss of acetate groups. ${ }^{2}$

However, a sequence of eight or more conjugated double bonds is required to absorb visible light, a known cause of discoloration of polyvinyl chloride. ${ }^{3}$ The EVA resin used in A9918P contains 33 weight percent or 15 mole percent vinyl acetate. As the reactivity ratios for this copolymer are unity during polymerization, sequences of eight units of more, be required for color when thermolyzed to conjugated unsaturation, seem unlikely.

It is noteworthy that when Sung and Noggle conducted carbon-13 NMR studies of a 40 percent vinyl acetate content EVA they found that for sequences of three and five VA units, the intensity of the peak was "too weak to be observed."

When instrumental supported the opinion that polyenes in the EVA backbone did not appear the cause of browning, other possible contributors were examined, specifically additives and their interactions in the presence of temperature and UV-B light." The following was observed:

a) Neither IR nor Raman spectroscopy indicate the presence of measurable levels of unsaturation in discolored EVA-based encapsulant; b) The acetate contents of numerous samples, unaged as well as aged in the field and the Weather-Ometer, were virtually identical and at the expected levels (Table ' 1), j.6 c) When treated with peracetic acid, a very brown EVA-based sample lost none of its color. But a PVC control, which had been purposely degraded to generate conjugated unsaturation and a dark color, was bleached to translucent white when the unsaturation was oxidized using the same method. This result again supports the hypothesis that browning of EVA-based encapsulant arises from cause(s) other than polyenes in the polymer backbone, likely the additives; d) EVA samples without additives, when laminated between low iron glass, had almost no browning after extended AAS; ${ }^{\circ}$ e) There was little loss of Cyasorb UV 531 after AAS of a laminate of A9918P without Lupersol 101, while A9918P with the normal level of Lupersol 101 exhibited a $40 \%$ reduction in Cyasorb after 12 weeks AAS. And the later showed significant yellowing while the former did not. ${ }^{\varsigma 6}$

Reformulated EVA-Based Encapsulants Resist Browning: Using the above analyses as a guide, four reformulated encapsulants were developed. After 34 weeks AAS, glass/encapsulant/glass laminates prepared with all four have evidenced Yellowness Indexes of only 2.6 to 5.3 , while controls with A991SP or "fast cure" 15295 have had Yellowness Indexes of 80.0 and 71.2, respectively (Figure 1)." Yellowness becomes visible at an index of approximately 10.

Glass Superstrates Containing Cerium-Oxide Reduce Browning: After 30 weeks exposure in the Weather-Ometer, glass/encapsulant/glass laminates prepared with "fast cure" $15295 \mathrm{P}$ and cerium-oxide-containing glass had a Yellowness Index of 5.3, not readily detectable by eye, and one year exposure produced a 13 Index. By comparison, after the same 30 weeks AAS, a $15295 \mathrm{P}$ control with Starphire ${ }^{\mathrm{TM}}$ low-iron glass had an Index of 64.4. 
In addition, a year of accelerated outdoor EMMA exposure in Phoenix resulted in essentially no color for samples with cerium-oxide-containing glass, versus a 32.7 Index with Solite ${ }^{\text {TM }}$ glass and 31.8 with Starphire glass when each was combined with "standard-cure" A9918P encapsulant.

Tefzel Superstrate Reduces Encapsulant Browning: After 60 weeks of AAS, a Tefzel ${ }^{\mathrm{TM}} / \mathrm{A} 9918 \mathrm{P} /$ glass laminate had a Yellowness Index of 2.0 , which is undetectable by eye, while 44 weeks of accelerated outdoor EMMA resulted in no measurable yellow.

Conclusions: From extensive analysis of field and Weather-Ometer aged EVA-based encapsulants, it appears that browning arises from some.other cause than thermolysis of repeating vinyl acetate groups leading to conjugated polyenes of 8 sequences or more in the EVA backbone. No conjugated unsaturation or loss of acetate group could be detected.

Instead, there is strong correlational AAS data and analysis results to support the hypothesis that interaction of additives is a key step in browning. Fully formulated A9918P. encapsulant browns significantly on extended AAS, while EVA without additives shows almost no browning.

In addition, when EVA with stabilizing additives and peroxide crosslinker was subjected to AAS the stabilizing additives were significantly depleted and the laminates had measurable yellow. On the other hand, when the peroxide was removed from the system and the encapsulant exposed in the same manner, there was no loss of stabilizers and almost no color development.

We are very pleased to report that development work has yielded three "stabilization strategies" which greatly limit photothermal browning: Use of cerium-oxide-containing glass superstrates, especially with "fast-cure" 15295P, use of Tefzel cover film in place of glass, and most importantly, use of new EVA-based encapsulants, three "standard-cure" (X9903P, X9923P, and $\mathrm{X} 9933 \mathrm{P}$ ) and one that processes under "fast-cure" conditions (X15303P). For added protection of these encapsulants, a cerium-oxide-containing glass, either Solatex II ${ }^{\mathrm{TM}}$ or Solarphire ${ }^{\mathrm{TM}}$ may be used as the superstrate. These glasses should contain a sufficient quantity of cerium oxide to provide a lower end light transmission cut-off of approxinately $325 \mathrm{~nm}$.

\section{References}

1. Wohigemuth, J.H., and R.C. Petersen, Solar Cells: Their Science, Technology, Applications and Economics, "Solarex Experience with Ethylene Vinyl Acetate Encapsulation," Elsevier Sequoia, Lausanne, 30, May 1991, pp. 383-387.

2. Pern, F.J. and A.W. Czanderna, "Characterization of EVA Encapsulant: Effects of Thermal Processing and Weathering Degradation on its Discoloration," Solar Energv Materials and Solar Cells, 25, 1992, pp. 3-23.

3. Abbas, K.B. and E.M. Sorvik, "On the Thermal Degradation of Poly(vinyl chloride) III," Journal of Applied Polymer Science, 19, 1975, pp. 2991-3006. 
4. Sung, H.N. and J.H. Noggle, "Carbon-13 NMR of Poly(vinyl Acetate) and Ethylene-Viny.l Acetate Copolymer," Journal of Pol. Science: Pol. Physics Edition, 19, 1981, pp. 1593-1602.

5. Ezrin, M., P Klemchuk, et. al., "Discoloration of EVA Encapsulation Sheet in Photovoltaic Modules," Proceedings, Society of Plastics Engineers Annual Technical Conference, May 1995.

6. Holley, W.H. "Advanced Development of EVA-Based PV Encapsulants," NREL PVMaT Report NREL/TP-411-7693, covering work under subcontract ZAG-3-11219-02-105661 by Springborn Laboratories, for the period 31 Mar. 1994 to 30 Sept. 1994.

7. Holley, W.H., et. al., "Investigation into the Causes of Browning in EVA Encapsulated Flat Plate PV Modules," Proceedings of 1994 IEEE First World Conference on Photovoltaic Energy: Conversion. Dec. 5-9, Hawaii, pp. 893-896.

8. Galica, J., et. al., "Advanced Development of Non-Discoloring EVA-Based PV Encapsulants," Proceedings, $13^{\text {th }}$ European P.V. Solar Energy Conference, Oct. 1995, Nice (to be published).

34 Weeks Xenon-arc Exposure
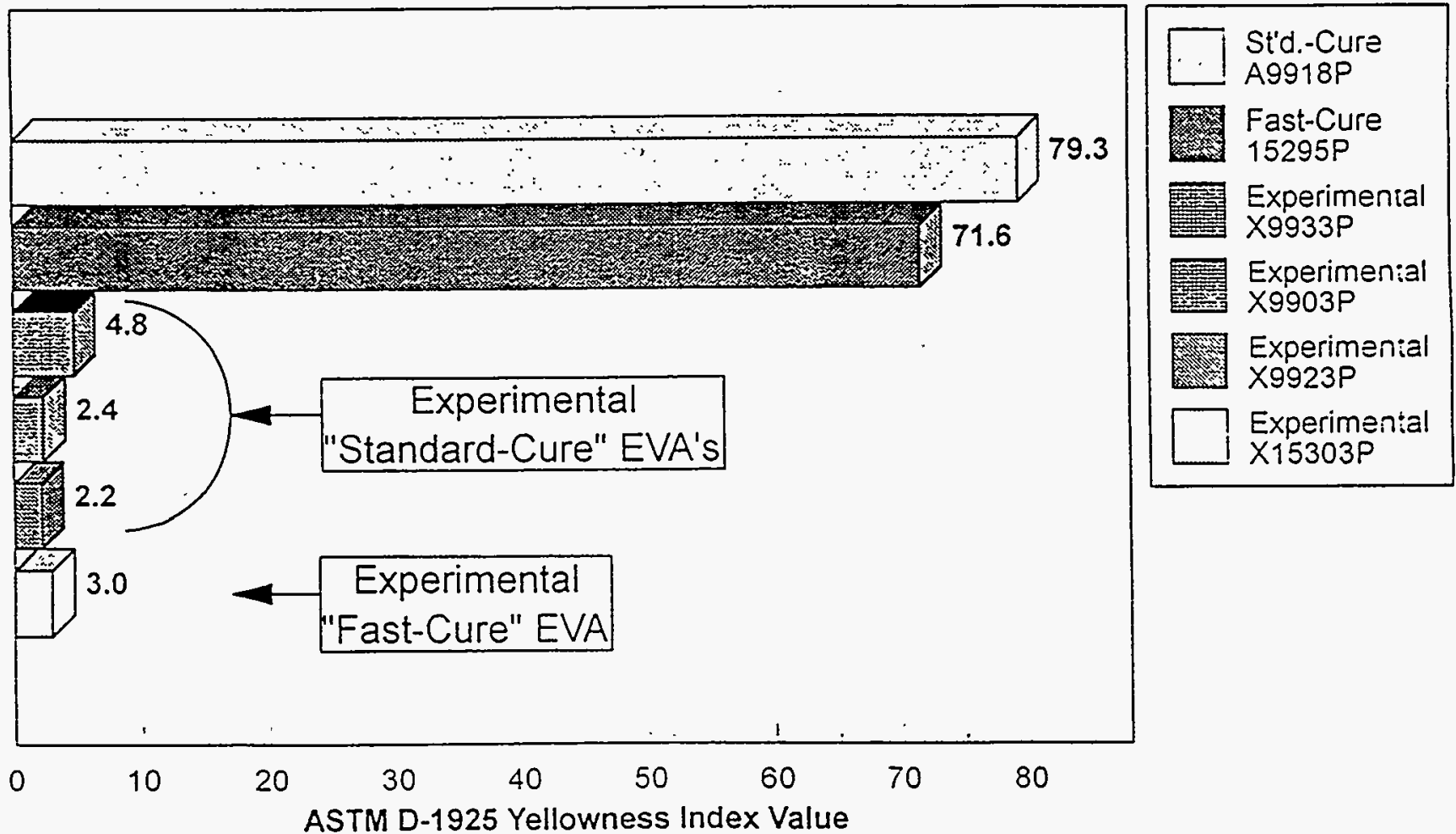

Figure 1. Effect of Accelerated Aging on Relative Discoloration of EVA-Based Encapsulants Using Non-U.V. Screening Glass (Starphire) 
Title:

Organization:

Contributors:
PV Cz Silicon Manufacturing Technology Improvements

Siemens Solar Industries, Camarillo, CA 93011

Theresa Jester, Kim Mitchell, Sergio Vasquez

\section{Introduction}

Siemens Solar Industries (SSI) began a three-year, three-phase cost shared contract in March 1992 to demonstrate significant cost reductions and improvements in manufacturing technology. The work has focused on near-term projects for implementation in the SSI Czochralski $(\mathrm{Cz})$ manufacturing facility in Camarillo, California. The work has been undertaken to increase the commercial viability and volume of photovoltaic manufacturing by evaluating the most significant cost categories and then lowering the cost of each item through experimentation, materials refinement, and better industrial engineering. The program has concentrated in the areas of crystal growth, wafer technology, module development and environmental, safety and health issues.

\section{Objective}

The objective of the program is to reduce costs in photovoltaic manufacturing by approximately $10 \%$ per year. The program consists of three focused tasks relating to cost reduction. The silicon wafer itself contributes about half of the total module cost and has the most potential for cost reduction. The cell processing costs are about a quarter of the module costs, with cell efficiency results being most important. Module assembly and packaging costs are the balance, with the module design, both materials and labor, contributing significantly.

\section{Approach}

Task 1: Silicon Crystal Growth and Thin Wafer Technology. Crystal growing costs are driven by material growing yields and indirect manufacturing costs such as electricity and machine parts used each time an ingot is fabricated. Wafering costs are driven by labor and the number of good slices yielded per length of crystal processed. This first task of reducing the wafer costs has focused on the polysilicon material used for ingot growth, and the implementation of wire saw machines to improve the yielded wafers per inch of ingot.

Task 2: Silicon Cell Processing. Cell processing costs are driven by the electrical contacts used, and the labor required for the process steps to clean the wafer, form the semiconductor junction, and the contacts. The cell tasks have been focused on better junction formation and reduced contact resistance. Process automation for lower labor costs has been implemented.

Task 3: Silicon Module Fabrication and Environmental, Safety, and Health Issues. Module costs are highly sensitive to labor and materials. The module design tasks are driven by high reliability in the field and lower costs. Environmental, safety and health issues govern the ability to 
manufacture products commercially. This program has focused on chlorflorocarbon (CFC) elimination and caustic chemical waste reduction.

\section{Results and Discussion}

During FY 1994 (10/01/93-9/30/94), several significant manufacturing technology improvements were achieved.

The Crystal Growing operation increased production significantly during the last three years. The increase is attributable to several factors including improved polysilicon quality and cleaning, upgrades to the growing equipment, specifically diameter controls, and upgrades to the hotzone graphite parts which have increased reliability and reduced cycle time. A wide range of poly silicon starting materials have been evaluated. Crystal Growing yields, cell performance and impurity analysis have been conducted. Overall crystal growing yields improved by $8 \%$ as a result of the study.

Wiresaws were tested and a process developed for manufacturing silicon wafers for photovoltaic use. The average number of wafers per inch obtained to date on the wiresaw is 44 , which is a $50 \%$ yield improvement over the results obtained for $\mathbb{D}$ sawn wafers (see Figure 1). Several critical issues have been identified for high yield of the wiresaw process. These include:

- Wire material specification, such as strength and temper

- Slurry and solvent usage

- Wafer cleaning

- Ingot mounting

- Operator Training

Complete conversion of the manufacturing process from $\mathbb{D}$ saws to wire saws has been accomplished under this contract.

Cell processing costs are driven by the amount of silicon used per cell (wafer thickness), the electrical performance of the cells, and the labor required for the processing of wafers into active cell devices. Work in this area has been focused on the reduction of labor through automation of various material transfer steps and the improvement of the electrical output of the cells processed by improving the contact resistance and diffusion processes. The increased yield of wafers per inch with wire saws is a significant improvement in cost.

The cell electrical distribution has improved significantly as a result of improved contact and diffusion processes. Through these process studies, coupled with contact firing process changes, contact coverage re-design and diffusion changes, a greater than $4 \%$ shift in the electrical performance was effected (see Figure 2).

Larger area cells have been developed and implemented which have an area improvement of almost $40 \%$. Full commercial implementation of a larger cell, 75 watt module has been accomplished under this contract. In addition, a semi-automated assembly line was designed, built and installed. 
Safety, health and environment issues have been addressed with the complete elimination of CFC's during May 1993. Significant reduction in costs and volume of caustic chemicals have also been achieved. Costs per cell have been reduced $60 \%$ and volume by over $20 \%$.

\section{Summary}

Reduction in $\mathrm{Cz}$ photovoltaic manufacturing costs are driven by several parameters. Low wafer cost, high electrical and mechanical yields in fabricating cells, low labor in cell fabrication and module fabrication, and materials used for making modules are all significant factors. Siemens Solar has reduced costs by over $30 \%$ in three years by addressing each of these items. Summary Cost Reduction shows the categories and total savings for manufacturing cost improvement under this contract (see Table 1).

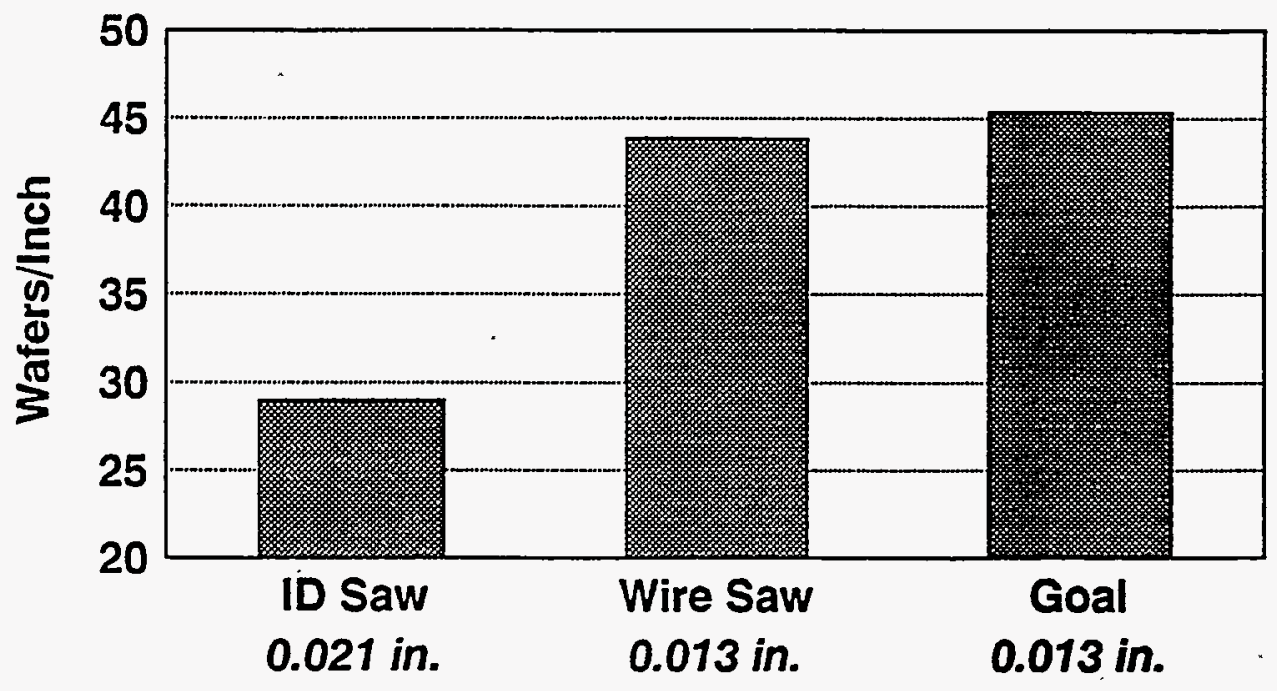

Figure 1 - ID vs. Wire Sawn Wafers Per Inch 


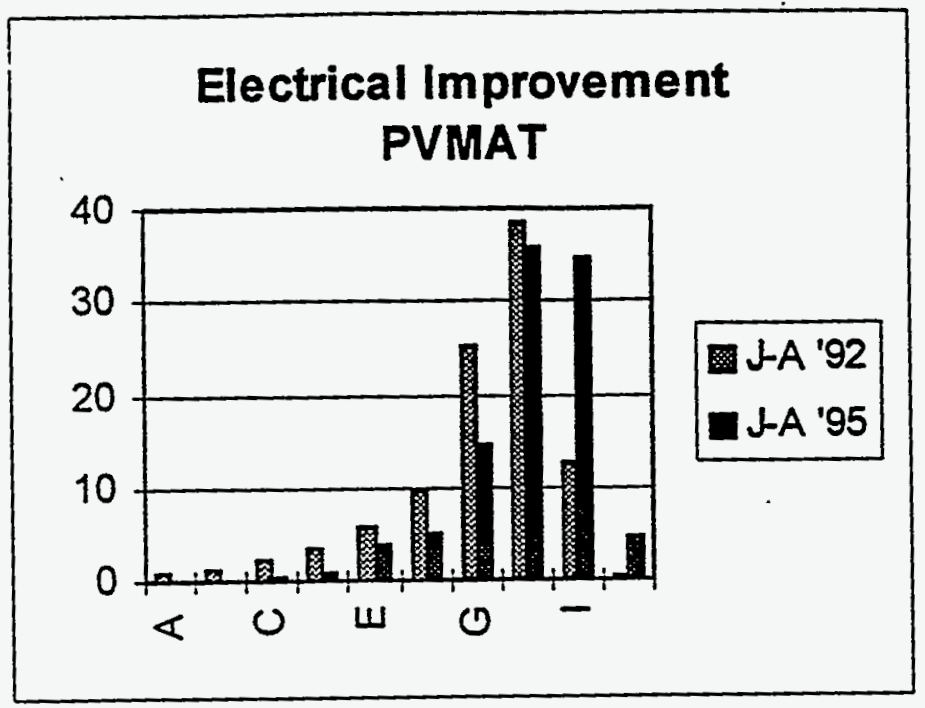

Figure 2 - Electrical Improvement

Table I - Summary Cost Reduction

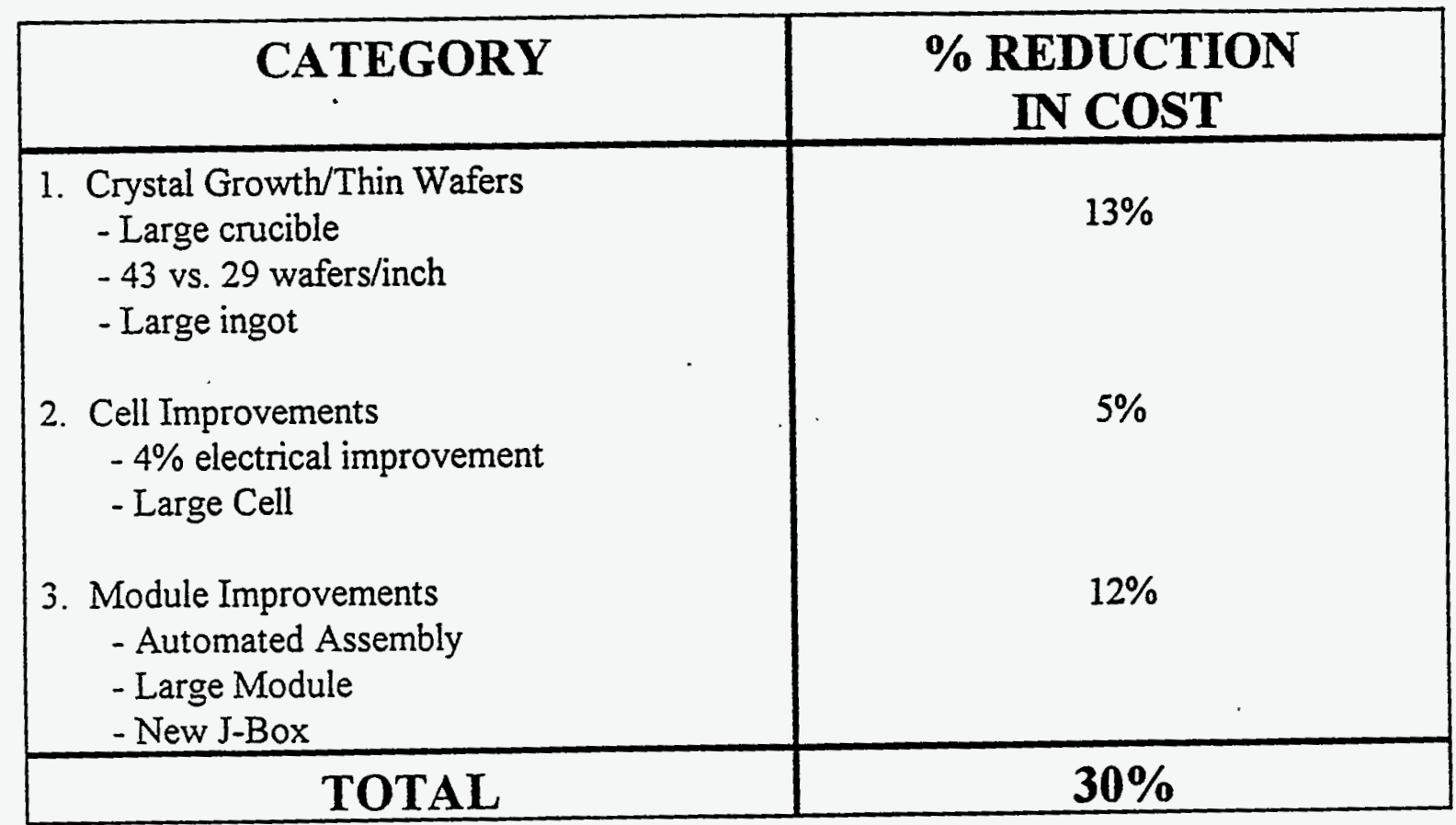


Title:

High Throughput Manufacturing of Thin-Film CdTe Photovoltaic Modules

Organization: $\quad$ Solar Cells, Inc. Toledo, Ohio

Contributors: $\quad$ D. Sandwisch, Principal Investigator, J. Bohland, J. Brossia, S. Collins, S. Cox, L. Crosser, L. Deutsch, G. Dorer, T. Firsdon, M. Flis, J. Foote, R. Harju, S. Kaake, W. Kazan, J. Kem, G. Khouri, K. Klaus, D. Malkuian, K. Miller, R. Nicholson, R. Powell, N. Reiter, R. Sandefur and T. Zhou

\section{Objective and Technical Approach}

The objective of this subcontract over its three year duration is to advance SCI's PV manufacturing technologies, reduce module production costs, increase module performance and .provide the groundwork for SCI to expand it's commercial production capacity. SCI shall meet these objectives by designing, debugging, and operating a $20 \mathrm{MW} /$ year automated, continuous manufacturing line that produces $60 \mathrm{~cm} \times 120 \mathrm{~cm}$ thin-film CdTe PV modules.

The second year effort under this subcontract focused on equipment design, quality programs, and environmental, safety, and health programs. SCI utilized a $100 \mathrm{~kW}$ pilot production line to demonstrate and improve manufacturing processes. The pilot line also provided a manufacturing environment in which to develop employee training and EH\&S programs. Over $40 \mathrm{~kW}$ of thin-film CdTe modules have been produced and deployed as part of this development effort (see Fig. 1).

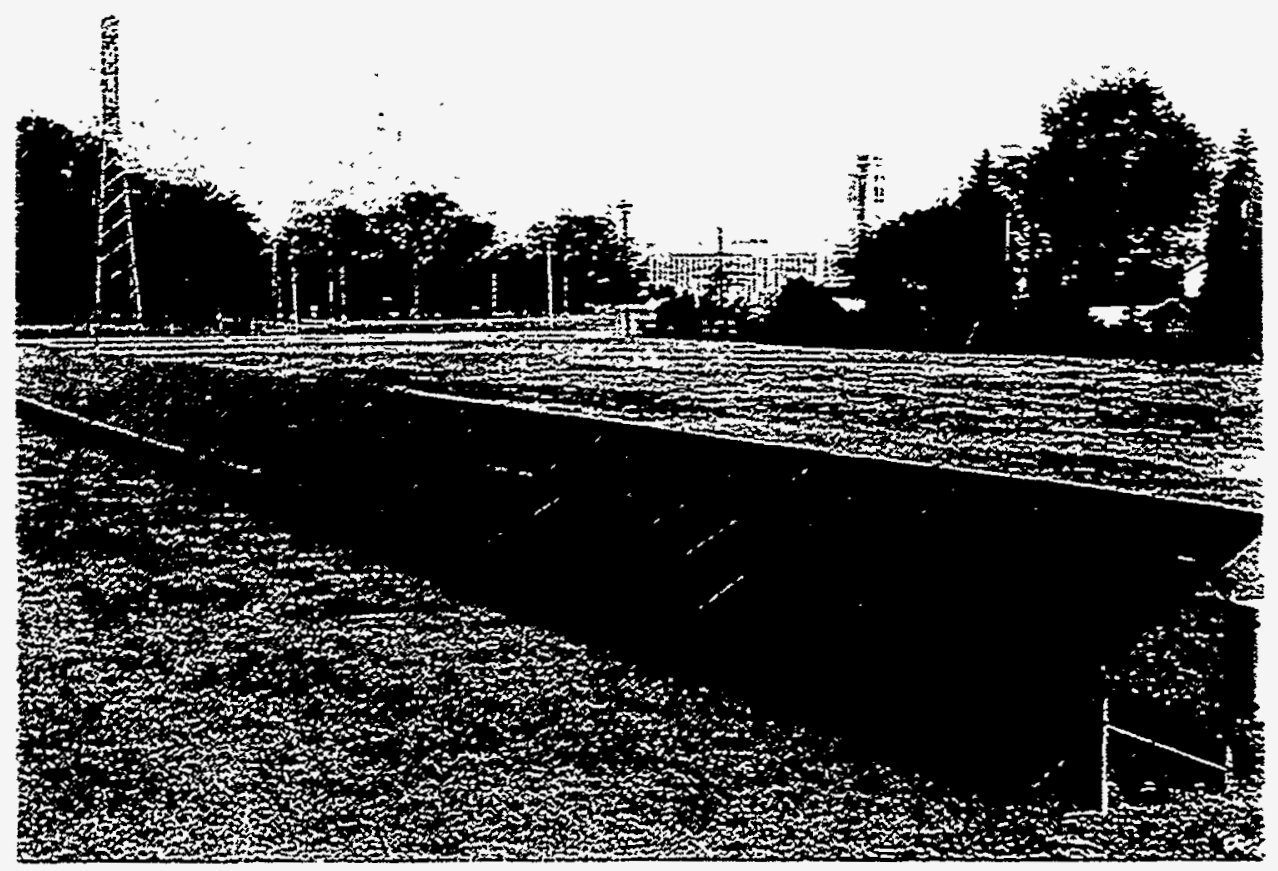

Figure 1. 12kW grid-connected array in Toledo, Ohio. 


\section{Equipment Design and Debug}

All critical equipment for the manufacturing line was designed or specified. Substrate preparation and panelization equipment was demonstrated at multi-megawatt levels. A prototype semiconductor treatment system was brought on-line. This system could potentially eliminate four processing steps and reduce environmental issues related to the process ${ }^{[1]}$. The system has demonstrated superior performance over previous systems. Two laser ablation systems were installed as part of the linear interconnect systems requirements.

A major part of the effort has been focused on refinement of the semiconductor deposition process. Specifically, a prototype deposition system was utilized to improve film thickness uniformity over the $60 \mathrm{~cm} \times 120 \mathrm{~cm}$ substrate. Figure 2 shows improvement in semiconductor film thickness uniformity due to better process control and process parameter refinements.
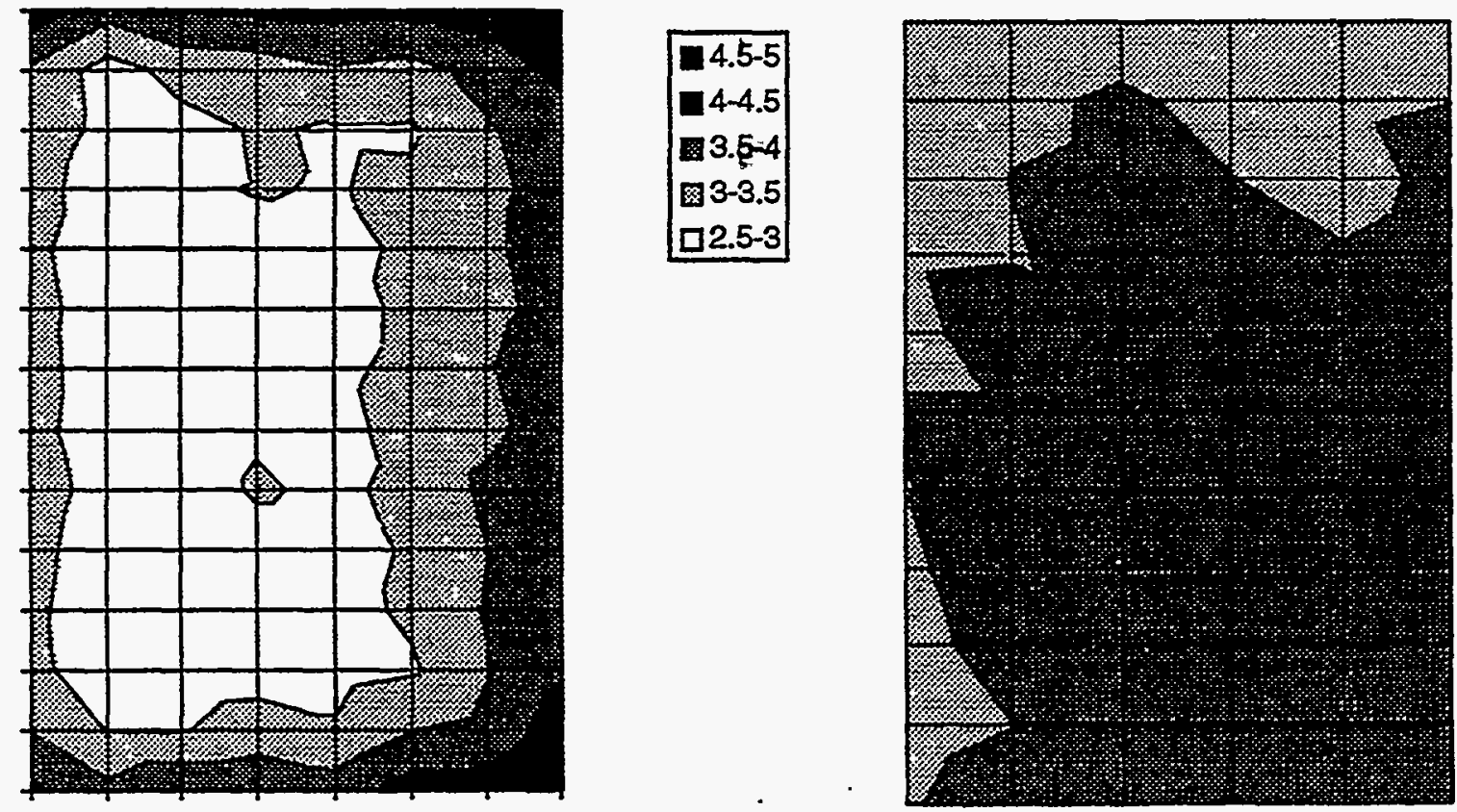

Figure 2. Semiconductor deposition process improvement - film thickness uniformity improved significantly through process control measures (substrate on the right shows improved uniformity).

\section{Quality Assurance/Quality Control}

SCI has initiated a quality assurance program specifically focused on the 20MW manufacturing line. This program is under evaluation and refinement on a $100 \mathrm{~kW}$ pilot production line. These efforts have significantly improved process performance.

Figure 3 shows advances in semiconductor deposition process control and throughput. The chart reflects a fourfold increase in throughput for a single deposition run. These increases were achieved due to two critical enhancements: 1) improved operator training and 2) in-line, nondestructive film thickness measurement. This progress resulted in more consistent module performance and substantially improved raw material utilization. In addition, the cycle time was 
decreased by $20 \%$. These advancements were achieved with nominal modifications to the deposition equipment.

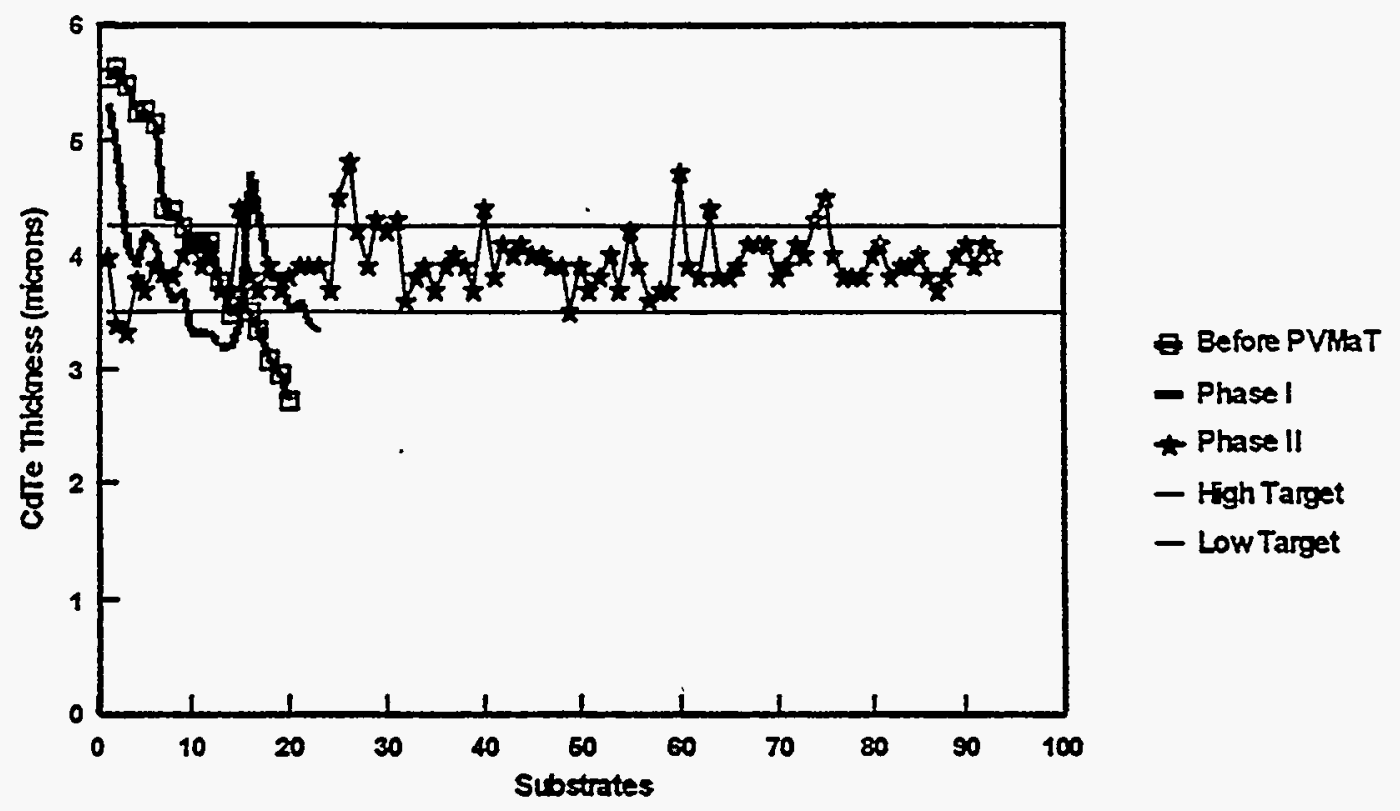

Figure 3. Semiconductor deposition process control -- film thickness and throughput.

Product reliability was also evaluated through stress testing based on the protocols established in "Interim Qualification Tests and Procedures for Terrestrial Photovoltaic Thin-Film Flat-Plate Modules" [2]. Pass rates of $100 \%$ have been demonstrated. Extended stress testing, such as longterm damp-heat, has been incorporated to further understand the durability of the modules in field environments.

\section{Environmental, Health and Safety Programs}

Production of thin-film CdTe photovoltaic modules involves regulated materials. An important part of the development effort is to establish programs and procedures which effectively handle safety, health and environmental issues that accompany the production, deployment and disposal of these modules. These programs include basic employee training as well as specific operational programs such as industrial hygiene and biological monitoring.

These programs and procedures have proven to be more than sufficient to control regulated materials in the work environment. Engineering controls such as ventilation have been implemented to assure that hygiene sampling (air and surfaces) and biological test results are maintained within allowed and expected limits.

Two prototype on-line effluent handling systems were demonstrated on the $100 \mathrm{~kW}$ pilot production line. These systems reduce waste streams by concentrating regulated materials or neutralizing effluents. These systems have led to a $98 \%$ volume decrease in waste materials. 


\section{Conclusions}

SCI has proven that thin-film CdTe provides a technology vehicie by which significant advantages can be obtained over current technologies. Key results and comments are:

1) SCI has produced a 60 Watt, $8.4 \%$ efficient module - the most powerful thin-film, polycrystalline module on record.

2) SCI has defined and demonstrated cost effective thin-film CdTe modules capable of achieving installed costs of less than $\$ 3.00 /$ Watt by 2000 .

3) Module durability has been demonstrated.

4) The process for making these modules has been proven on a 100kW pilot line.

5) No technology barriers have been identified including safety, health, and environmental issues.

\section{Next:Steps}

Throughout the duration of the subcontract, efforts will be focused on the expansion of manufacturing capacity. The process will be demonstrated at a multi-megawatt level providing for commercialization of the product. The major tasks will include the installation and startup of the manufacturing line and the roll out of the quality and EH\&S programs.

\section{Acknowledgments}

The author is pleased to acknowledge the efforts of the SCI development team. The extraordinary capability and dedication of this team was essential in achieving the results described above. This work bas been jointly funded by NREL and Solar Cells, Inc. under Subcontract Number ZAI-411294-02.

\section{References}

[1] T. Zhou, et al., "Vapor Chloride Treatment of Polycrystalline CdTe/CdS Films", Proceedings of the 1994 First IEEE World Conference on Photovoltaic Energy Conversion, pp. 103-106, 1994.

[2] "Interim Qualification Tests and Procedures for Terrestrial Photovoltaic Thin-Film Flat-Plate Modules", SERI/TR-213-3624, 1990. 
Organization Solarex, A Business Unit of Amoco/Enron Solar

Contributors J. H. Wohlgemuth, Program Manager; F. Artigliere, M. Brisson, M.

Conway, J. Creager, T. Duquaine, T. Koval, M. Narayanan, M. Roy, T.

Tomlinson and D. Whitehouse of Solarex; B. Huff, M. Diver, R.

Fernandez and D. Vanecek of ARRI

\section{Objectives}

The objectives of this three-year program are to advance Solarex's cast polycrystalline silicon manufacturing technology, reduce module production cost, increase module performance and expand Solarex's commercial production capacities. Two specific objectives of this program are to reduce the manufacturing cost for polycrystalline silicon PV modules to less than $\$ 1.20$ /watt and to increase the manufacturing capacity by a factor of three.

\section{Approach and Accomplishments}

\section{Casting}

The original goal of the casting task was to develop the ability to cast ingots that yield four bricks with a cross-section of $15 \mathrm{~cm}$ by $15 \mathrm{~cm}$ with at least equivalent material quality as was achieved for the standard $11.4 \mathrm{~cm}$ by $11.4 \mathrm{~cm}$ bricks. This represents a $73 \%$ increase in the useable silicon obtained from each casting.

During the second year of the program, the process to cast larger ingots was developed. The initial large ingots had well-behaved runs, but had cracks extending upward from the bottom with cell efficiencies that averaged approximately $90 \%$ of standard cast material. Modifications to the insulation configuration and optimization of the process program lead to successful casting of full size ingots for $4-15 \mathrm{~cm}$ by $15 \mathrm{~cm}$ bricks without cracking, yielding solar cells with average efficiency equivalent to that of standard cast material.

Most of Solarex's products are still based on the use of $11.4 \mathrm{~cm}$ by $11.4 \mathrm{~cm}$ solar cells, so an effort is underway to develop casting of ingots large enough to produce $9 \times 11.4 \mathrm{~cm}$ by $11.4 \mathrm{~cm}$ bricks. Such an ingot represents a $125 \%$ increase in the useable silicon obtained from each cast, requiring approximately $20 \%$ more silicon than the PVMaT 15 $\mathrm{cm}$ by $15 \mathrm{~cm}$ ingot. We were able to load the added charge of silicon feedstock into the PVMaT crucible and successfully pour and freeze out the larger ingots. Efforts are now underway to optimize the process for casting these larger ingots and to increase casting capacity by modifying all of the casting stations to produce the larger ingots. 


\section{Wire Saw}

The goal of the wire saw task is to develop the wire saw technology for cutting $15 \mathrm{~cm}$ by $.15 \mathrm{~cm}$ polycrystalline wafers on $400 \mu \mathrm{m}$ centers at lower cost per cut than achieved today on the ID saws. This represents a 50\% increase in the useable silicon obtained from each cast and a 50\% increase in the yield of wafers per purchased kilogram of Si feedstock.

Solarex is utilizing an HCT wire saw for this program. During the first year of the program the saw was used to successfully demonstrate the ability to cut $11.4 \mathrm{~cm}$ by 11.4 $\mathrm{cm}, 11.4 \mathrm{~cm}$ by $15.2 \mathrm{~cm}$ and $15 \mathrm{~cm}$ by $15 \mathrm{~cm}$ wafers on $500 \mu \mathrm{m}$ and $400 \mu \mathrm{m}$ centers. The major efforts during the second year were to 1) gain experience operating the saw in a semi-production mode, 2) reduce the cost of consumables, spare parts and waste disposal and 3) develop an improved method for demounting and cleaning the wafers after they are cut on the wire saw. This wire saw has shown that it can produced as many wafers as 16 ID saws at better yield and lower per wafer cost than the ID saws.

\section{Cell Process}

The goal of the cell task is to increase cell efficiencies to $15 \%$, while decreasing the cost per watt at the module level. The developed process must be compatible with automated manufacturing at large volumes.

During the first year of the program a cost effective aluminum paste back surface field was developed that increased cell efficiencies by 5 to $6 \%$. During the second year several large production pilot runs were completed. These runs yielded an average encapsulated cell efficiency of $13.77 \%$, which was a $5.3 \%$ increase in efficiency over the standard process during the same time period: Cells from the pilot runs were made into modules that were then successfully qualified to the IEC 1215 and IEEE 1262 environmental test procedures for PV modules.

Mechanical texturing with a tool was used to produce a 2.5 to $3.5 \%$ increase in encapsulated cell efficiency over a planar cell with a single layer antireflective coating. Independent measurements at Sandia have confirmed a weighted front reflectance under AM1.5G illumination of $6.7 \%$, with a minimum of $4.8 \%$ for an encapsulated solar cell. These numbers include losses from the front glass surface of approximately $4 \%$ and reflection off of the grid lines. Achieving such a low reflectance indicates significant light trapping within the encapsulated device including trapping of light initially reflected off of the grid lines.

Phosphorous gettering can be used to increase the efficiency of polycrystalline silicon cells. In a number of experiments with Solarex cast polycrystalline material, a small but significant increase in short circuit current and efficiency has been achieved. If phosphorous gettering can be integrated into a process sequence without adding additional processes like etch-back or etch-off, it can be a cost effective method for increasing cell efficiency. 
Mechanical texturing, phosphorous gettering and an aluminum paste back surface field have now been combined into an integrated cell process sequence with screen printed $\mathrm{Ag}$ paste contacts and a $\mathrm{TiO}_{2}$ antireflective coating. Encapsulated cell efficiencies as high as $14.88 \%$ have been achieved on $11.4 \mathrm{~cm}$. by $11.4 \mathrm{~cm}$. cast polycrystalline silicon solar cells using this integrated process.

Efforts are now underway to scale the cell process for larger $(15.2 \mathrm{~cm}$. by $15.2 \mathrm{~cm}$.) cells.

\section{Module Assembly}

The initial goal of this task was to modify Solarex's present module assembly system to increase throughput by $100 \%$ and decrease the labor requirement by $50 \%$. This has now been modified by Solarex requirements to increase the assembly capacity by $200 \%$ instead of $100 \%$.

Solarex subcontracted with the Automation and Robotics Research Institute (ARRI) at the University of Texas at Arlington to assist in modeling the assembly process and equipment and to help plan the expansion of the facility. ARRI has utilized AT\&T's discrete event simulation package called Witness to evaluate the present production line and the new concepts proposed to expand the facility. The initial efforts involved identifying simple changes that could increase production without major capital expense. This effort resulted in improvements that increased capacity by $40 \%$.

Solarex and ARRI then developed a module assembly factory concept to meet Solarex's production goals through the year 2000. Implementation of this plan is now underway. The changes and additions made to date have increased the assembly capacity by $100 \%$, meeting the initial goal of this PVMaT task.

\section{Frameless Module Development}

The goal of this task is to develop and qualify a frameless module design incorporating a lower cost back sheet material (less than $\$ 0.05 /$ square foot) and user friendly, low cost electrical termination (less than $\$ 1.00 /$ module). Since PVMaT is designed for large systems, modules are designed to mount directly onto the support structure without integral frames. A low cost support structure has been designed and 3M Very High Bond Tape chosen as the adhesive for mounting the modules to the support structure. Several large arrays have now been fabricated using this system.

A number of candidate materials were selected for evaluation as a back sheet material to replace the 3 part back sheet used by Solarex at the start of the PVMaT program. Only one candidate, single sheet Tedlar, has proven to meet all of the technical requirements. Solarex switched to this back sheet for all of its commercial products. While this material does not meet the $\$ 0.05 /$ square foot goal, it represents at least a $70 \%$ reduction in back sheet cost. 
For electric termination, we need a system that has low material cost, but also does not require appreciable labor for field assembly. Junction boxes and weather tight quick connects are not available in the price range of interest. We selected and have qualified a system with pig tail wires that are connected via a butt crimp connector and protected with polyolefin shrink tubing. This system meets the $\$ 1.00 /$ module.goal.

\subsection{Automated Cell Handling}

The goal of the automated cell handling task is to develop automated handling equipment for $200 \mu \mathrm{m}$ thick $15 \mathrm{~cm}$ by $15 \mathrm{~cm}$ polycrystalline silicon wafers and cells with a high yield (less than $0.1 \%$ breakage per process handling step) at a throughput rate of at least 12 cells or wafers per minute. ARRI is supporting Solarex in this task. They began this effort by studying the strength and fracture behavior of Solarex cast polycrystalline wafers. A four point bend test and a cantilever test were devised and used to measure the mechanical strength of the wafers. The scatter in measured strengths was modeled using Weibull statistics, and a distribution of the probability of failure as a function of strength was determined. The ultimate strength in bending for the polycrystalline wafers was found to be $119.3 \mathrm{MPa}$. In addition, the Young's modulus was found to be $168.8 \mathrm{GPa}$ and the Weibull modulus was calculated to be 9.56 .

A finite element model was then developed to determine the distribution of stress and deflection in a wafer based on the applied load. This model along with the Weibull modulus can be used to determine the probability of breakage of the wafer under the specified load. The model was used to simulate a typical wafer handling situation, to estimate the maximum load that can be applied during handling and the corresponding probability of failure. This model is now being used to guide the development of wafer handling equipment.

\section{Conclusions}

This PVMaT program has lead to many process, product and equipment improvements that either have been implemented or are planned for implementation over the next two years. Our preliminary analysis indicates that through the year 2000, Solarex will save $\$ 5.00$ for every dollar it invested in PVMaT and our customers will save approximately $\$ 7.00$ for every dollar that NREL invested in this PVMaT.

\section{References}

1. S. Narayanan, J. Creager and J. Wohlgemuth, "Impact of Phosphorus Gettering on Large Area Polycrystalline Silicon Solar Cells with Back Surface Field", 13th PVSEC, 1995.

2. Cast Polycrystalline Silicon Photovoltaic Cell and Module Manufacturing Technology Improvements, Annual Subcontract Report- FY 1994, NRELTP-411-8244. pp. 22. Available NTIS: Order No. DE95009285. 
Organization: $\quad$ Spire Corporation, Bedford, Massachusetts

Contributors: G. Darkazalli, program manager, M. J. Nowlan, principal investigator, S. J. Hogan, J. S. Patterson, S. F. Sutherland, J. M. Murach, and W. F. Breen

\section{Introduction}

Spire successfully completed a 34 month program entitled "Automated Solar Cell Assembly Teamed Process Research" as part of NREL's Photovoltaic Manufacturing Technology (PVMaT) Phase 3A project. The objective of this work was to reduce the cost and improve the quality of terrestrial photovoltaic modules by developing automated high-throughput (5 MW/year) processes for interconnecting standard and thin crystalline silicon solar cells. A completely new automated system was developed in this program that demonstrated a high-throughput, high-yield solar cell interconnection process. This system, designated the SPI-ASSEMBLER ${ }^{\mathrm{TM}} 5000$, is now offered as a commercial product to the photovoltaics industry. Extensive processing evaluations were done on the Assembler with a range of different cell types in FY 1995.

\section{Approach}

The Assembler, shown in Figure 1, automatically produces strings of interconnected solar cells from stacks of cells, reels of copper ribbon, and liquid flux. New automated processes were developed for cell loading, cell aligning, cell inspection, ribbon handling (ribbon feeding, forming, cutting to length), flux application, ribbon-to-cell soldering, cell string handling, and in-line string I-V testing. ${ }^{1}$ Labor content was significantly reduced by combining tabbing and interconnecting into a single automated process.

Low stress processes were developed for obtaining high yields with thin and standard solar cells. For example, a machine vision system aligns and inspects cells without handling cell edges, while conductive preheating combined with a high-intensity light source solders a cell's front and back contacts in a single low-stress heating step. Flexible automation techniques were incorporated to enable production of a variety of module designs with minimal mechanical adjustments or tooling changes.

\section{Results Obtained in 1995}

Extensive processing evaluations were done on the Assembler during this period. More than 15,000 solar cells fiorn six major module manufacturers were processed through the system, including cells made from single-crystal $\mathrm{Cz} \mathrm{Si}$, polycrystalline cast $\mathrm{Si}$, and polycrystalline $\mathrm{EFG}$ ribbon $\mathrm{Si}$. Yields over $98 \%$ vesre achieved with both thin $(200 \mu \mathrm{m}) \mathrm{Cz}$ Si cells from Solec and standard thickness (300 to $400 \mu \mathrm{m}) \mathrm{Cz} \mathrm{Si}$, cast $\mathrm{Si}$, and ribbon $\mathrm{Si}$ cells from a range of manufacturers. 
Solar cell performance measurements made before and after processing showed no cell degradation. Measurement data taken for $200 \mu \mathrm{m}$ Solec cells show that the power increased slightly (2\%), mainly due to an increase in fill factor. This may be caused by an increase in conductivity along the contact busses provided by the interconnect ribbons.

Both Siemens Solar Industries and Solarex made modules from cell strings fabricated on the assembly system which they subjected to accelerated environmental testing per IEC 1215 and IEEE 1262 standards. ${ }^{2}$ These tests consisted of thermal cycling, thermal and humidity-freeze cycling, and damp heat soaking. The maximum allowable power loss is 5\% for the IEC 1215 testing and $10 \%$ for the IEEE 1262 testing. All modules passed both qualification tests, with an average power loss of only $2.3 \%$. Test results are provided in Table 1 .

In June, 1995, processing evaluations were concluded at Spire and the Assembler was shipped to ASE Americas to evaluate the Assembler in a production setting. An evaluation run was done in which 119 9-cell strings were fabricated (1071 cells). The cell yield (percentage of non-cracked cells) was excellent at $99.3 \%$ over all 119 strings. Excellent solder joint pull strengths were recorded on cells selected at random from the evaluation run. The average front and back contact pull strengths were $357 \mathrm{~g}$ and $329 \mathrm{~g}$, respectively, when pulled at a $90^{\circ}$ angle. These values are particularly noteworthy given the narrow ribbon width $(1.5 \mathrm{~mm})$ used for these strings.

As a result of the technology developed in this program, Spire has two significant new products to offer the PV industry: the SPI-ASSEMBLER 5000 and the SPI-STRINGER 500. The Stringer is a semi-automated version of the Assembler, designed for manufacturers with smaller production throughput requirements. Several of these products have already been sold to PV manufacturers.

A detailed cost analysis was done to compare the cost of the Assembler's automated processes to the semi-automated Stringer's processes and to manual processes. ${ }^{3}$ The costs, not including cells, were calculated to be $\$ 0.445 / \mathrm{W}$ for the manual case, $\$ 0.163 / \mathrm{W}$ for the Stringer, and $\$ 0.087 / \mathrm{W}$ for the Assembler.

\section{References}

1. M. J. Nowlan, S. J. Hogan, G. Darkazalli, S. F. Sutherland, W. F. Breen, J. M. Murach, and J. S. Patterson, "Advanced Automation Techniques for Interconnecting Thin Silicon Solar Cells," proc. IEEE First World Conf. on Photovoltaic Energy Conversion (1994) 828.

2. S.J. Hogan, M.J. Nowlan, T.L. Jester, and J.J. Hummel, "Performance and Reliability Testing of Crystalline Silicon Cell Strings Fabricated with a Spi-Assembler 5000 Apparatus," NREL PV Performance and Reliability Workshop, Lakewood, CO, Sep. 21, 1994.

3. M. J. Nowlan, S. J. Hogan, and G. Darkazalli, "Cost Analysis of an Automated Process for Interconnecting Crystalline Silicon Solar Cells," proc. 13th NREL PV Program Review Meeting, Lakewood, CO, May 16-19, 1995. 


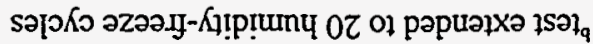

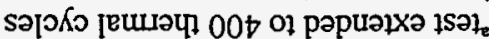

\begin{tabular}{|c|c|c|c|}
\hline $\begin{array}{l}I^{\cdot} \varepsilon^{-} \\
\varsigma^{\prime} I^{-} \\
9^{\cdot} I^{-} \\
t^{\prime} Z^{-}\end{array}$ & 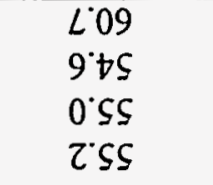 & 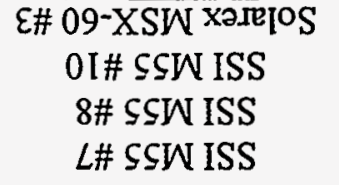 & 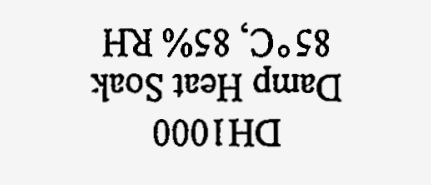 \\
\hline $\begin{array}{l}\varsigma^{*} \varepsilon^{-} \\
8 \cdot \varepsilon^{-} \\
\nabla^{-} \varepsilon^{-} \\
I^{\cdot} \varepsilon^{-}\end{array}$ & $\begin{array}{l}609 \\
I \subseteq \varsigma \\
\varepsilon \bullet \varsigma \\
\tau \cdot \varsigma \varsigma\end{array}$ & $\begin{array}{c}\text { I\# 09-XSW Xəre] } \\
\text { q6\# SSW ISS } \\
\text { qS\# SSW ISS } \\
{ }_{q} \varepsilon \# \text { SSW ISS }\end{array}$ & 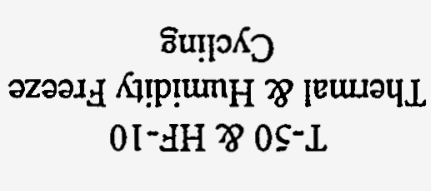 \\
\hline $\begin{array}{l}8 I^{-} \\
\angle 0^{-} \\
S^{-} T^{-} \\
S^{-} T^{-}\end{array}$ & $\begin{array}{l}\angle 09 \\
8 \circ S \\
6 \circ S \\
I^{\circ} S S\end{array}$ & $\begin{array}{l}\text { ¿Z\# 09-XSW XәIе[OS } \\
\text { t\# SSW ISS } \\
\text { Z\# SSW ISS } \\
\text { I\# SSW ISS }\end{array}$ & 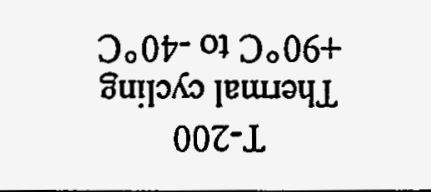 \\
\hline 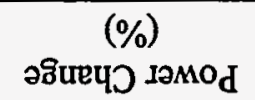 & $\begin{array}{c}(M) \\
\text { JəMOd [ए!?!חI }\end{array}$ & 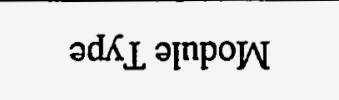 & ${ }^{1 S O J}$ \\
\hline
\end{tabular}

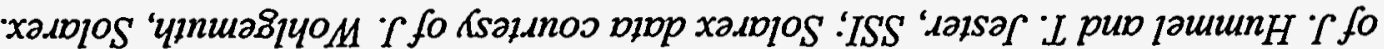

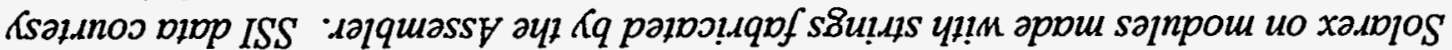

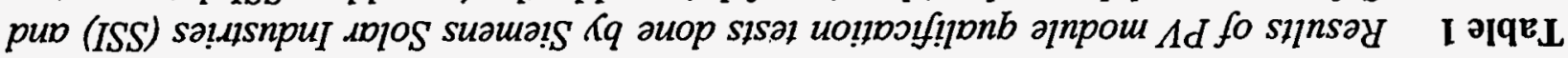

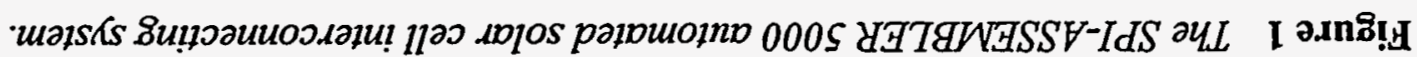

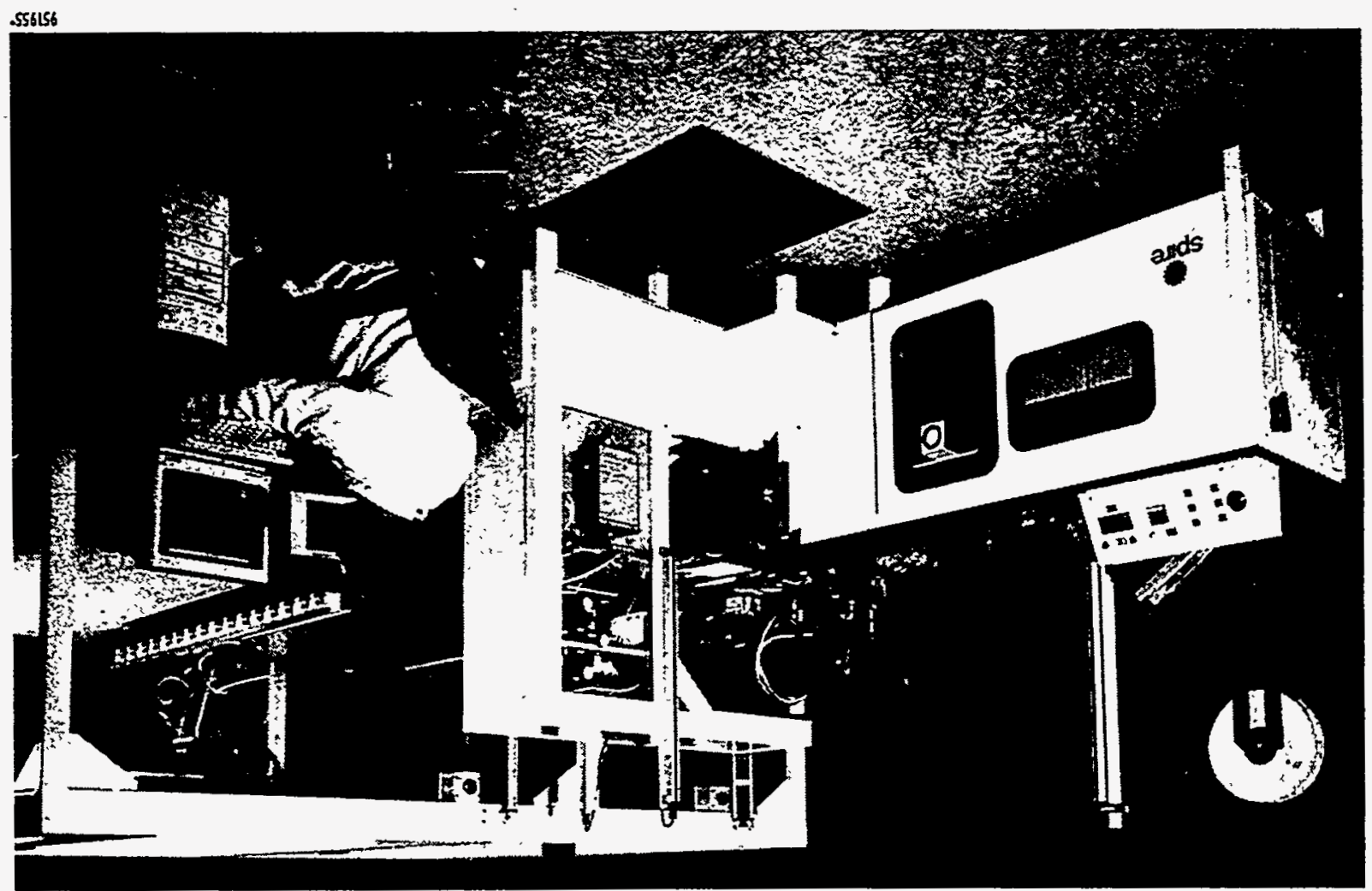


Title:

Organization:

Contributors:
Amorphous Silicon

Photovoltaic Manufacturing Technology

Utility Power Group

Los Angeles, California

G. Duran, Principal Investigator;

K. Mackamul, D. Metcalf

Introduction:

Photovoltaic modules based on thin-films create challenges as well as opportunities with regard to the encapsulation of the module. The first generation amorphous silicon based PV modules were characterized by a lack of reliability due to the encapsulation of the modules. Efforts were made to address these reliability issues and modules were produced with reliability equal to those based on crystalline and polycrystalline silicon. The maturity of a-Si PV module manufacturing has reached a stage where cost and manufacturability, as opposed to reliability, are now the major issues. Even so, the dependable glass/EVA/glass package is not a low cost nor lightweight encapsulation system.

Unfortunately, the PV modules based on polycrystalline thin-films have not reached the same level of maturity of the a-Si based modules. The polycrystalline thin-film modules are just now going through the first generation of development and have shown signs of failure during the module qualification testing procedure. In order for these materials to make an impact on the long-term PV market, the reliability of the packaging must be addressed. Utility Power Group has addressed the issues concemed with producing a cost effective, high throughput, reliable encapsulation system designed with the thin-film module in mind.

\section{Advanced Encapsulation Material}

Utility Power Group (UPG) has evaluated a wide variety of encapsulation materials to be utilized on thinfilm modules. The encapsulation materials have been evaluated in terms of the following characteristics:

1. Substrate adhesion

2. Scratch resistance

3. Chemical resistance

4. Water and water-vapor resistance

5. Compatibility with module fabrication techniques

6. Air quality concerns

7. Cost

8. Safe application

9. Application speed

After careful analysis the materials judged to be the most attractive as the replacement encapsulation material are silicone based. 
Substrate Adhesion:

These silicone materials are generally described as structural adhesives. The primerless silicone adhesive is a flowable silicone elastomer that develops a strong, self-priming adhesive bond to many substrates when properly applied and cured, The adhesive, when heated, cures to a strong, flexible elastomer that is ideally suited for adhesive applications.

Scratch Resistance:

The candidates for advanced substitute encapsulation materials all showed a weakness in the scratch resistance test as outlined in the NREL/TR-213-3624 qualification test procedure when compared to the glass/EVA/glass package. None of the thin (less than $0.025^{\prime \prime}$ ) coatings used as candidate encapsulation materials (polyurethanes, epoxies, or silicones) could consistently pass the scratch test. UPG was able to modify the silicone material to decrease its susceptibility to being cut. The modification to the silicone material included adding a component to the silicone after the application of the encapsulation material to the module. The material found to be the best additive are glass beads in a size range of 0.003 " to 0.006 ". Glass beads are chemically stable and mechanically robust. The crust formed on the surface of the encapsulation material allows the material to pass the surface-cut susceptibility test and the smooth surfaces of the beads will not scratch the thin-films under the encapsulant.

Chemical Resistance:

The cured modified silicone adhesive is capable of being submerged in a hot solution of sodium hydroxide for periods in excess of one hour without any sign of chemical attack to the encapsulated aluminum thinfilm. This special formulation silicone rubber has extended resistance to weather, sunlight, moisture, radiation, oxidation, and ozone.

ADVANCED ENCAPSULATION MATERIAL

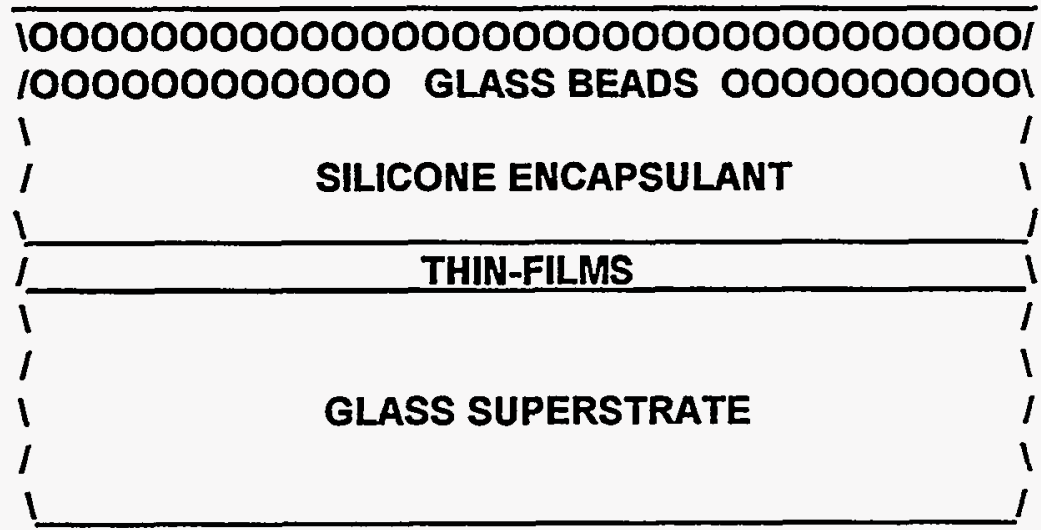

Figure 1. The Advanced Encapsulation Material composed of the modified silicone structural adhesive with the addition of the glass beads for enhanced mechanical strength. 


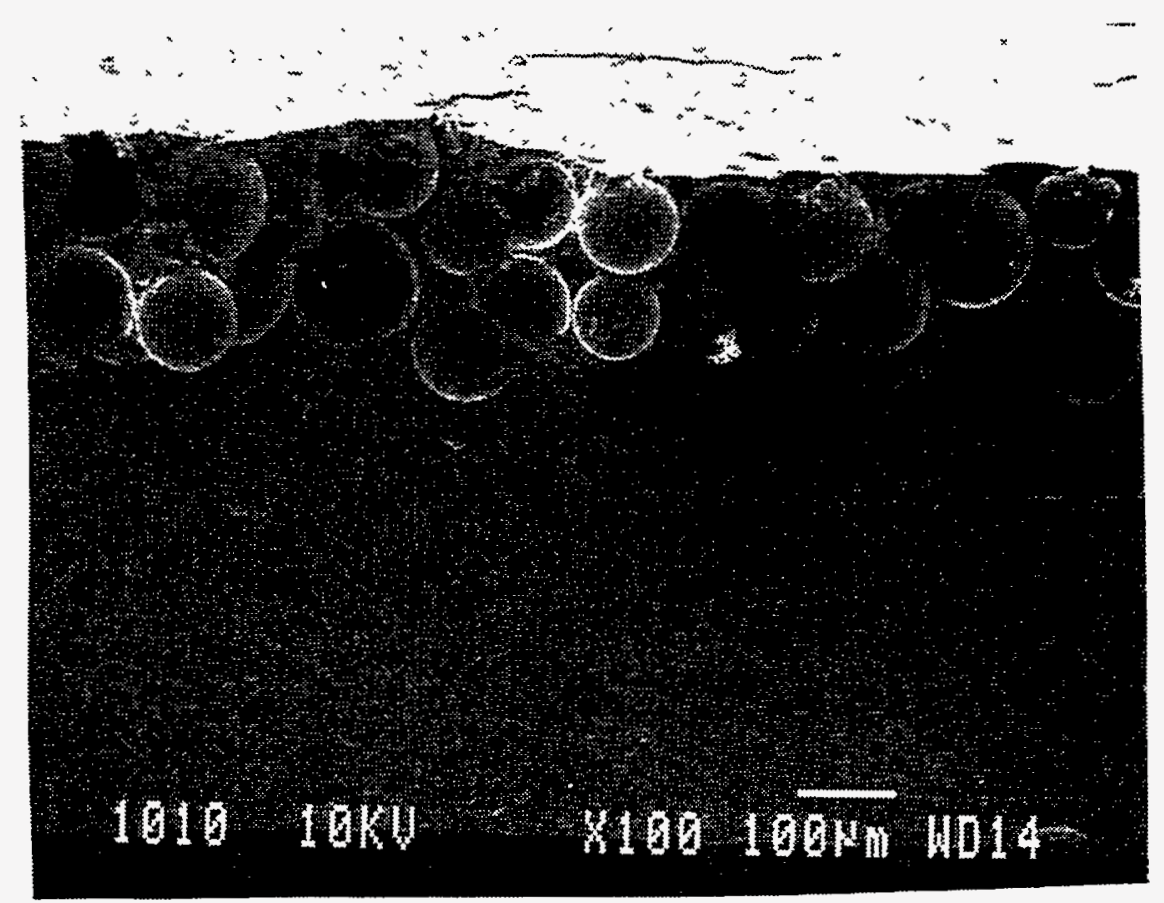

Figure 2. SEM micrograph illustrating glass beads embedded in the modified silicone adhesive. Notice that while the glass beads are fully submerged in the encapsulant, there is no tendency to drift down through the encapsulant to come in contact with the thin-films.

Water and Water-Vapor Resistance:

The encapsulation material cures to a flexible rubber that demonstrates superior resistance to liquid water or water-vapor attack of the protected thin-films.

Compatibility with Module Fabrication Techniques:

The application, and more importantly, the curing of the modified silicone formulation on the PV module has not demonstrated any incompatibilities with the processing of the PV module.

Air Quality Environmental Concerns:

The primerless silicone material formulation includes no solvents or cure by-products.

Cost:

At the thickness being applied as an encapsulant material $(0.012 ")$, the cost of the modified silicone formulation remains within the goal of the PVMaT Project, approximately $\$ 0.40 /$ sq.ft.

Safe Application:

Toxicity studies of primerless silicone adhesive formulations have shown minimal industrial handling problems.

Application Speed:

The silicone material has been modified to be applied with a roller coater at a speed of 10 feet per minute. 


\section{Advanced Encapsulation System}

After evaluating various application techniques for the encapsulation material, the technique determined to be the most cost effective in terms of initial cost, throughput, ease of setup and operation, and minimizing waste material production was roller coating.

Roller coating operates with three rolls; the coating roll, doctor roll, and backup roll.

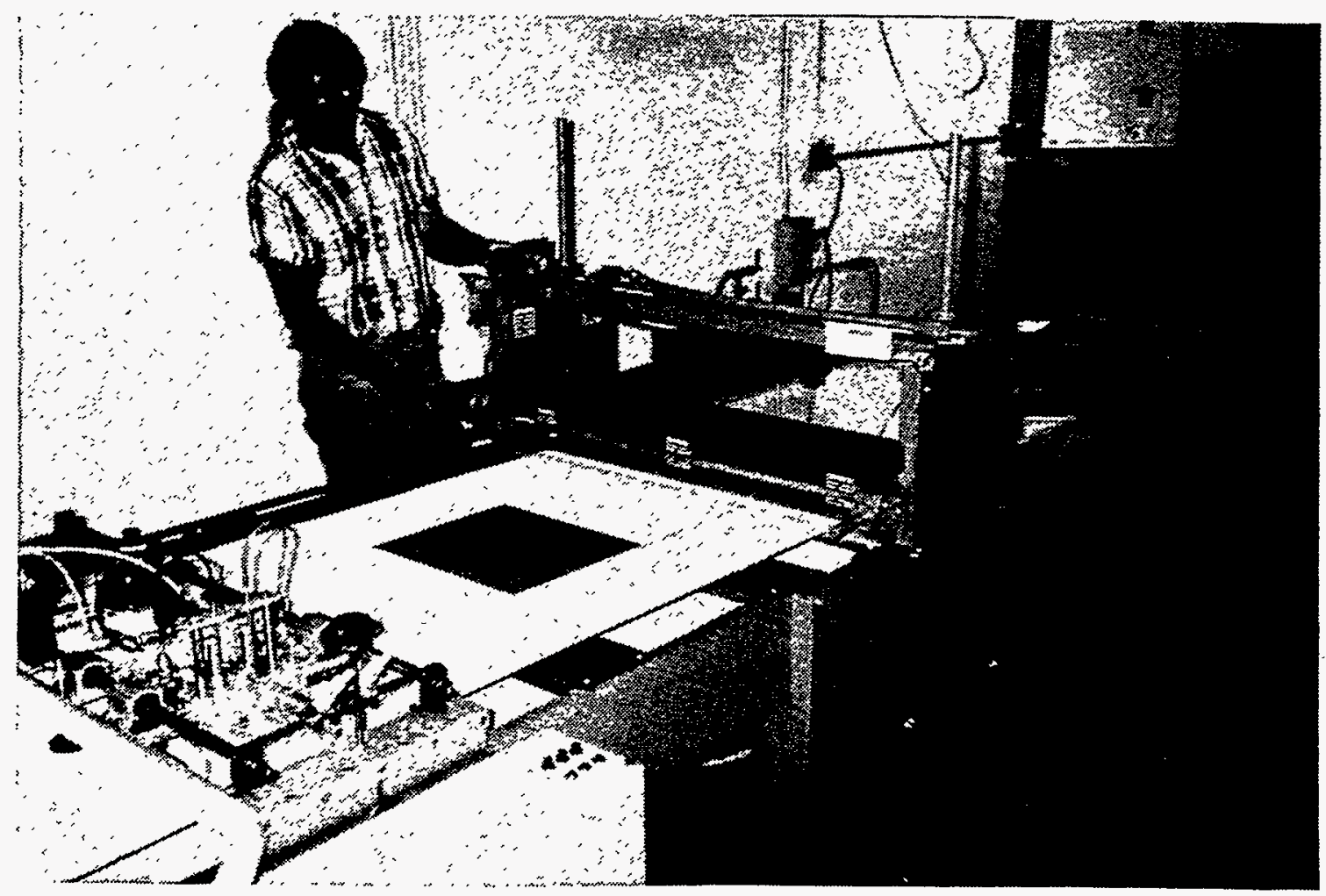

Figure 3. An operator adjusts the two parameters which control the roller coating process; the coating roll/doctor roll distance, and the coating roll/backup roll distance. These two parameters determine the thickness of the coating and the quality of the coating. 
TITLE:

DEVELOPMENT OF A LOW COST INTEGRATED $20 \mathrm{KW}$

A.C. SOLAR TRACKING SUB-ARRAY FOR GRID CONNECTED PV POWER SYSTEM APPLICATIONS

ORGANIZATION: Utility Power Group

Los Angeles, California

CONTRIBUTORS: M. Stern, Principal Investigator;

G. Duran, K. Mackamul, R. West

\section{Introduction}

This PVMaT Phase 4A1 work effort focuses on improving the cost effectiveness of Utility Power Group's ('UPG") PV solar tracking sub-array system and component technology. While it is well understood that PV modules now represent over $60 \%$ of the total cost of an installed grid-connected PV power system, efforts to substantially reduce their cost per watt through conversion efficiency improvements or through the use of low cost thin film materials are out of the scope of this project. The remaining $40 \%$ of the system cost and thus the focus of UPG's work effort encompasses the balance of the system's mechanical and electrical components and hardware as well as the system's installation and is known by the acronym "BOS" (ㅁalance- $\underline{-}$ - - System).

\section{Objective}

The objective of UPG's two year effort is to reduce the BOS costs associated with single axis tracking grid connected PV power systems by 50\%. Based upon today's average cost of \$8.00/watt PTC for an installed ground mounted 200kWAC system with $10 \%$ efficient PV modules representing $\$ 4.80 /$ watt or $60 \%$ of the cost, today's BOS costs are $\$ 3.20 /$ watt or $40 \%$ of the total. UPG's goal is to reduce the BOS cost by $50 \%$ to $\$ 1.60 /$ watt such that BOS costs represent only $25 \%$ of the total system cost subject to further reductions in PV module cost.

\section{Approach}

UPG's approach is to eliminate the redundancy and superfluous features associated with a number of discrete PV system components while reducing field installation labor in favor of factory production labor. Specifically, during the first year, UPG will design, fabricate, and test a Modular Panel and an Integrated Power Processing Unit which shall then be combined with UPG's proven PV system technology to create a fully integrated and 
modular $20 \mathrm{~kW}$ A.C. PV Solar Tracking Sub Array. During the second year, UPG will field deploy and test the Sub-Array in a grid-connected utility customer driven appiication.

UPG's Modular Panel is a structurally and electrically modular, factory assembled, field deployable component utilizing high volume manufactured PV module laminates.

UPG's Integrated Power Processing Unit integrates all dc and ac power collection, control, processing, and switching functions, as well as solar tracking functions within a single outdoor enclosure.

\section{$\underline{\text { Results }}$}

Only the first three months of this project took place during FY1995 and therefore significant results cannot be reported. Various PV module laminate designs and configurations have been analyzed for their use in the Modular Panel, and preliminary functionality design of the Integrated Power Processing Unit has been completed. 


\title{
5.0 PHOTOVOLTAIC MODULE AND SYSTEM PERFORMANCE AND ENGINEERING PROJECT
}

\author{
Richard DeBlasio, Manager
}

\section{Objectives}

The objective of this project is to conduct module and systems development through state-of-theart photovoltaic module and system research, engineering, testing, evaluation, and analysis tasks. The objective includes providing technical results, solutions to technical issues, technical assistance, and coordination for the development of PV standards, codes, and criteria for test and evaluation procedures. The objective further includes maintaining and enhancing supporting facilities and capabilities that are consistent with the DOE National Photovoltaics Program, that are complementary to other DOE PV projects, and that will ensure project capabilities and facilities are available resources for cooperative research and utilization by the U.S. PV research and development community.

\section{Technical Approach}

The project primarily includes NREL in-house activities and a small amount of subcontracts. The project is organized under the primary areas shown in Figure 1. Each group supports PV standards and codes activities. The following paragraphs provide an introductory description of the PV Module and System Performance and Engineering Project activities.

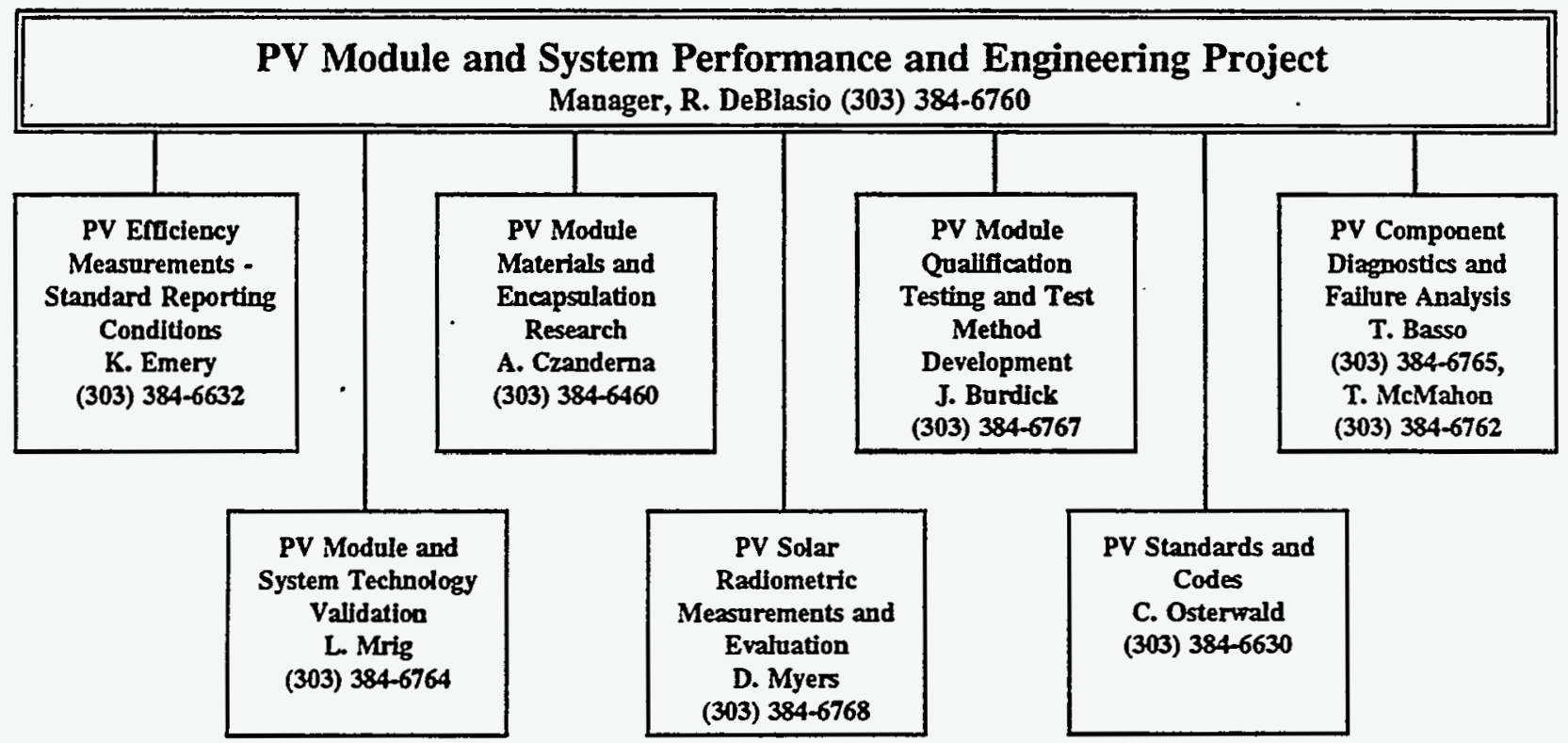

Figure 1. PV Module and System Performance and Engineering Project Contacts 
NREL Outdoor Test Facility. This past year, the U.S. Department of Energy completed the construction of the Outdoor Test Facility (OTF) laboratory building at NREL. The 10,000-square-foot OTF accommodates nearly all the laboratories for the PV Module and System Performance and Erigineering Project, colocating and consolidating activities and equipment from four other NREL locations. The OTF lies adjacent to the existing PV outdoor test site, and those field-deployed PV modules and systems are now connected to monitoring and control systems housed in the OTF Outdoor Test and Measurement Laboratory. The OTF also has an adjacent south-facing pad and roof-mounted array structures for additional experiments and measurement systems.

PV Efficiency Measurements-Standard Reporting Conditions. The performance of PV devices of all sizes and technologies is evaluated by providing secondary calibrations of PV cells and modules, efficiency measurements with respect to standard reporting conditions, efficiency verification of subcontract deliverables, and, current-voltage (I-V) measurements under varying temperature, spectral irradiance, and total irradiance. Capabilities also support the entire PV community by providing information on PV measurement equipment and systems appropriate for the end user, I-V measurement procedures, and measurement uncertainty analysis, and by being committed to obtaining the lowest possible uncertainty in the measurement of the standardized PV performance of single- and multi-junction cells and modules. Included in the uncertainty analysis are the determination of potential artifacts in the I-V results because of equipment or procedures, and realistic estimates of the elemental error sources.

PV Module Materials and Encapsulation Research focuses on polymeric materials and formulations that will provide optimal module performance and protection from the environment for a 30-year life. The objectives are to identify, understand, and mitigate the causes of materials change, and to identify new or improved materials. The objectives include using accelerated life testing, real-time testing, and developing data analysis methods for correlation. Also included are identifying and testing superstrate materials for improving photostability, and testing commercially available adhesive films for simplifying the encapsulation process.

PV Module Qualification Testing and Test Method Development objectives are to investigate, understand, and improve PV module long-term performance and reliability. The approach includes developing and validating test methods and procedures, and performing module exploratory/qualification and reliability tests on PV modules, as well as on R\&D prototypes and contract deliverables, with special emphasis on thin-film photovoltaic technologies. The team is working to establish a correlation between indoor (artificial, accelerated) test results and outdoor (natural, real-time) exposure as related to reliability/stability/weathering issues. In addition, theoretical and experimental studies are undertaken on PV module lifetime prediction from accelerated testing. Further, the work with the NREL failure analysis group is aimed at developing methods and techniques to perform failure analysis on PV modules, establishing means of determining the mechanisms of failure, and then obtaining possible solutions to these module performance and/or reliability problems.

PV Component Diagnostics and Failure Analysis is concerned with conducting and documenting module and system reliability and failure activities. Data are obtained from operating systems, demonstration or prototype units, qualification and exploratory tests, research results, dedicated studies, and from the literature. Dedicated activities are pursued to uncover 
and reproduce identified failure mechanisms, to validate proposed hypotheses, to explore areas that are believed to be of high priority for long-term PV reliability, to recommend new tests for screening or qualification, and to develop better understanding for improved reliability and design. The cause of PV module failure is determined through nondestructive and destructive evaluation procedures. From these evaluations, more basic materials determinations can be confirmed and probable causes and mechanisms can be proposed. System-level concerns are addressed, with emphasis extending outward from the module interface.

PV Module and System Technology Validation encompasses activities for testing, monitoring, and evaluating state-of-the-art and emerging PV technologies on the module and system level. Technology development and validation are also supported by analyzing the performance and reliability of PV modules and systems in support of the DOE National Photovoltaics Program. The approach includes maintaining a database on performance and reliability, performing thinfilm module stability testing outdoors with comparison to indoor light-soak experiments, and researching and developing performance evaluation methodologies for modules (for example, seasonal performance, spectral measurements, and techniques for evaluating modules on instantaneous power output and energy production). System performance activities also include supporting the Photovoltaics for Utility-Scale Applications (PVUSA) Project, the Federal Energy Management Program, PVMaT and PV:BONUS projects, and international commitments such as the DOE-South Africa and DOE-India activities.

PV Solar Radiometric Measurements and Evaluation directly supports characterizing, measuring, testing, designing, and understanding the performance of PV cells, submodules, modules, and systems. That is pursued by providing scientific and engineering understanding of incident solar and artificial irradiance, and through developing instruments and/or measurement methods. The approach focuses on providing integrated technical support (for example, developing and providing broadband and spectral radiometric measurements, and instrument calibrations, characterization, and utilization to assist PV performance measurements). The approach includes operation of reference radiometric sensors, calibration of solar simulators and other optical instrumentation, and development and operation of a reference meteorological and irradiance system at NREL.

PV Standards and Codes focus is on coordinating the consensus development of PV standards, codes, and, criteria for test and evaluation procedures. For example, NREL holds an annual PV Standards and Codes Forum. The coordination includes participating and performing major leadership roles in the IEEE Standards Coordinating Committee 21 and in the ASTM Subcommittee E44.09, conducting round-robin cell and module test method verification, developing criteria for module certification and test facility accreditation through the PV community with balanced industry representation and subcontract support, revising the National Electrical Code Article 690, developing PV energy rating standards, and working in the international arena (for example, through the NREL-managed Solar Energy Industries Association subcontract for International Electrotechnical Commission TC-82 secretariat and U.S. Technical Advisory Group coordination of international PV standards development). Additionally, NREL staff have coordinated the PEP '93 intercomparison of reference cell calibrations and cell performance measurements. 


\section{PV Module and System}

Performance \&Engineering

\section{In-House Research}


Organization: NREL PV Module and Systems Performance and Engineering Project

\author{
Contributors: $\quad$ K.A. Emery, team leader; S. Rummel, Y. Caiyem, H. Field, D. Duniavy
}

\title{
Objectives
}

The performance of photovoltaic devices of all sizes and technologies is evaluated by this activity. This team supports the entire PV community by providing secondary calibrations of PV modules and cells, efficiency measurements with respect to a given set of standard reporting conditions, efficiency verification of contract deliverables, current versus voltage (I-V) measurements under varying temperature, spectral irradiance, and total irradiance. Support is provided for in-house programs in device fabrication, module stability, module reliability, PV systems, and alternative rating methods by performing baseline testing, specialized measurements, and other assistance when required. This activity also supports the entire PV community by providing information on PV measurement equipment and systems that are appropriate for the end user, I-V measurement procedures, and uncertainty analysis. Included in the uncertainty analysis are the determination of potential artifacts in the I-V results because of equipment or procedures, and realistic estimates of the elemental error sources. This activity is committed to obtaining the lowest possible uncertainty in the measurement of the standardized PV performance of single- and multi-junction cell and modules.

\section{Technical Approach}

The photovoltaic current versus voltage characteristics are measured with respect to standard terrestrial reporting conditions $\left(25^{\circ} \mathrm{C}\right.$ temperature, $1000 \mathrm{Wm}^{-2}$ total irradiance and ASTM E892 global reference spectrum). The intensity of the Spectrolab X-25 solar simulator (30-cm-square beam) is adjusted until the measured short-circuit current of a reference cell is equal to its calibration value corrected for spectral mismatch. The current versus voltage characteristics are then measured using 4-terminal Kelvin connections to the PV device with a custom data acquisition system designed to give a random error of less than $\pm 0.1 \%$ and a non-random error of less than $\pm 1 \%$. The $\mathrm{I}-\mathrm{V}$ system has a voltage range of $\pm 50 \mathrm{~V}(0.1-\mu \mathrm{V}$ resolution $)$ and $\pm 8 \mathrm{~A}$ to $\pm 1 \mathrm{pA}$. These procedures have been shown to be valid for any given tabular reference spectrum including AMO and the ASTM direct normal reference spectrum. Because of the wide current and voltage range, the system is also used for concentrator and dark I-V measurements. For two-terminal multi-junction devices the spectrum of the Spectrolab model X-25 solar simulator is adjusted, using a special filter plate developed at NREL, until each junction is producing the correct photo-current. The I-V system is also used for examining the effects of pre-measurement conditions, bias rate, maximum power versus illumination time, $\mathrm{V}_{\mathrm{oc}} v s$. time, and $\mathrm{I}_{\mathrm{sc}} v s$. time on the $\mathrm{PV}$ performance.

The filter spectral response system uses periodic monochromatic light directed through one of 51 10 -nm bandwidth interference filters covering the spectral range from 290 to $1900 \mathrm{~nm}$. The system is capable of providing steady-state light bias levels up to $2 \mathrm{~A}$ and voltage bias levels from $\pm 1 \mathrm{mV}$ to $\pm 40 \mathrm{~V}$. The intensity of the bias light is normally adjusted to give the short-circuit current under standard reporting conditions. The custom operational-amplifier-based data acquisition system allows for a wide range of gains $\left(1\right.$ to $\left.10^{6}\right)$. The intense light from the $1000-\mathrm{W}$ lamp illuminating the 5-cm-diameter filters allows for small cells to large modules to be illuminated with adequate signal to noise. The uncertainty in the relative spectral response as a function of wavelength is less 
than $\pm 5 \%$. The data can be made absolute by forcing the integrated quantum efficiency to agree with the measured spectral response. The absolute spectral response can also be measured directly with this system, giving an uncertainty of $\pm 10 \%$. The limiting factor in the uncertainty is $: 1.0 \pm 5 \%-10 \%$ spatial nonuniformity of the monochromatic beam. The second grating monork-ometer-based system has a wavelength range from $300-10,000 \mathrm{~nm}$ with a $1-\mathrm{nm}$ wavelength resoli: $\cdot .1$ and $\sim 5-\mathrm{nm}$ bandwidth. The grating system was designed for accurate absolute spectral response measurements by illuminating a small $1 \mathrm{~mm}$ by $3 \mathrm{~mm}$ rectangular area and measuring the power of the entire beam. The system uses all reflective optics so chromatic aberrations and beam wander with wavelength are not present. The grating system uses an operational amplifier for the current-to-voltage conversion, allowing a $\pm 15-\mathrm{V}$ voltage bias range with $0 \pm 1 \mathrm{mV}$ being the normal configuration. A lock-in amplifier is used to detect the ac signal. The system is capable of broadband or filtered light bias for multi-junction or nonlinear devices. Both systems rely on accurate pyroelectric detectors for the measurement of the light power.

The efficiency versus concentration measurements are measured with an unfiltered $1000-\mathrm{W}$ Xe-arc light source that is focused to a small area and the 1-sun data acquisition system described above. Using the cell's $I_{s c}$ measured at 1 sun (ASTM E891-87 direct normal reference spectrum at a total 1-sun irradiance of $1000 \mathrm{Wm}^{-2}$ ) and assuming linearity in the current with total irradiance, the I-V characteristics are measured. Using a technique developed at NREL, the temperature of the space charge region temperature can be accurately set to a given value even though large temperature gradients $\left(>10^{\circ} \mathrm{C}\right)$ may exist between the plate temperature and junction temperature. The procedure involves setting the sample temperature to the reference temperature (for example, $25^{\circ} \mathrm{C}$ without illumination [no heat load]). Using a high-speed shutter and voltmeter, the open-circuit voltage is sampled (1000 readings/sec). The highest measured $V_{o c}$ is then taken to be the $V_{o c}$ under concentration. The thermoelectrically controlled vacuum plate is then cooled until this $V_{o c}$ is reached. This same procedure is used in the Spectrolab X-25 system for samples on thermally insulating substrates where temperature gradients of $5^{\circ}-10^{\circ} \mathrm{C}$ are typical.

The primary reference cell calibration procedure involves measuring the short-circuit current, total irradiance, and spectral irradiance at the same time outdoors with the same $5^{\circ}$ field of view. The total irradiance is measured with a primary absolute-cavity radiometer, and the spectral irradiance is measured with a LICOR LI- 1800 spectroradiometer. The uncorrected average calibration value $\left\langle\mathrm{CV}_{\mathrm{u}}\right\rangle$ is calculated for the 30 Isc and Etot readings taken during the 30 seconds required to measure $E_{s}(\lambda)$. The atmospheric constraint for a valid calibration value is that the range (maximum to minimum) over the 30 -second time period in Isc be less than $0.25 \%$, in Etot be less than $0.07 \%$, and in $\left\langle\mathrm{CV}_{\mathrm{u}}\right\rangle$ be less than $0.1 \%$. Once a valid $\left\langle\mathrm{CV}_{\mathrm{u}}\right\rangle$ is obtained, the short-circuit current is corrected for temperature to $25^{\circ} \mathrm{C}$ and the spectrally corrected calibration value $\mathrm{CV}$ is computed. Since the measurement of $E_{s}(\lambda)$ does not encompass the limits of the reference spectrum, the measured spectrum is extended using a computer model developed by the group to encompass the range of the reference spectrum ( $300-4000 \mathrm{~nm})$. The calibration value $C V$ is computed at least 20 times for at least 3 separate days giving a single primary calibration value. This procedure has been shown to have a total uncertainty of less than $\pm 1 \%$ by rigorous uncertainty analysis, intercomparison with primary AMO standards, and other intercomparisons.

The I-V characteristics of modules are routinely evaluated using the Spire $240 \mathrm{~A}$ Solar Simulator. This system has a $0-100-\mathrm{V}$ and $0-20-\mathrm{A}$ range for a $61-$ by $122-\mathrm{cm}$ area. The Spectrolab Large-Area Pulsed Solar Simulator (LAPSS) produces a 1 -sun beam of light $(2-\mathrm{m}$ by $2-\mathrm{m}$ area and could 
illuminate a 4-m by 4-m area). The custom I-V system of the LAPSS has three modes of operation: capacitive sweep from $\mathrm{I}_{\mathrm{sc}}$ towards $\mathrm{V}_{\mathrm{oc}}$, current at a fixed power supply voltage, and bi-directional sweeping over a user-defined or automatically determined voltage range. The systcin is designed to handle $\pm 200-\mathrm{V}$ maximum ( $1-\mu \mathrm{V}$ minimum), $\pm 30-\mathrm{A}$ maximum $(0.01-\mathrm{mA}$ minimum) with 10 voltage and current ranges to ensure accuracy. The Spectrolab data acquisition system has a $0-100-V, 0-40-A$ measurement range. The system has been used to evaluate the $\mathrm{I}-\mathrm{V}$ characteristics as a function of concentration for several $\mathrm{GaInP} / \mathrm{GaAs}$ concentrator cells fabricated at NREL to 1500 suns. The concentrator lamp housing allows measurement to 2000 suns.

During FY 1995 standardized Outdoor module I-V measurements were performed on a variety of 1 -sun and concentrator modules using one of two fixed-tilt platforms or a 2-axis Martin Marietta Tracker. For flat-plate modules the total irradiance was measured with an Eppley pyranometer and a crystal-Si reference cell in a module package mounted in the plane-of-array. A normal incidence pyrheliometer calibrated against NREL's cavity radiometers is used to measure the direct normal irradiance for concentrator modules. The module temperature is measured with a platinum RTD temperature sensor attached to the back surface of the module. The spectral irradiance is measured during the I-V measurement using a LICOR LI-1800 spectroradiometer with a Teflon dome and temperature-controlled detector. Meteorological parameters at the time of measurement including direct to diffuse ratio, wind speed, wind direction, barometric pressure, air temperature, and relative humidity are stored with the data. The outdoor I-V measurement system is capable of handling devices with a voltage and current range of $\pm 100 \mathrm{~V}$ and $\pm 40 \mathrm{~A}$ maximum to $\pm 0.01 \mathrm{mV}$ and $\pm 1 \mu \mathrm{A}$ minimum.

\section{Results}

During FY 1995 the team calibrated 1684 cells (I-V under standard reporting conditions), and performed over 2728 module measurements on the SPIRE 240A Solar Simulator (1881 module measurements) and outdoors under clear-sky prevailing conditions (total irradiance $>950 \mathrm{~W} / \mathrm{m}^{2}$ ). There measurements were on representative cells and modules of all PV technologies being investigated at NREL; in the United States and worldwide. Approximately 45 papers acknowledged the support of this group during FY 1995. This team has assisted nearly all module manufacturers with their QA/QC programs by providing telephone consultations on measurement strategy and procedures, and a calibration tractability path for their cells and modules. The technology of PV I-V and spectral response measurements was transferred to a variety of university and manufacturing groups through phone conversations and visits. The group also supported work in spectral irradiance modeling and energy rating methods, resulting in several co-authorships. The move of the module measurement activities from the trailers to OTF was completed with a minimum of disruption to customers.

\section{Publications}

1. I. Eisgruber, R. Matson, J. Sites, and K. Emery, "Interpretation of Laser Scans from ThinFilm Polycrystalline Photovoltaic Modules," Proc. 24th IEEE Photovoltaic Specialists Conf., Waikoloa, HI, December 5-9, 1994, pp. 283-286, IEEE, New York, 1994.

2. T. Strand, L. Mrig, R. Hansen, and K. Emery, "Technical Evaluation of a Dual-Junction Same-Band-Gap Amorphous Silicon Photovoltaic System at NREL," Proc. 24th IEEE Photovoltaic Specialists Conf., Waikoloa, HI, December 5-9, 1994, pp. 850-853, IEEE, INew York, 1994. 
3. B. Kroposki, K. Emery, D. Myers, and L. Mrig, "A Comparison of Photovoltaic Module Performance Evaluation Methodologies for Energy Ratings," Proc. 24th IEEE Photovoltaic Specialists Conf., Waikoloa, HI, December 5-9, 1994, pp. 858-862, IEEE, New York, 1994.

4. M. Wanlass, J. Ward, K. Emery, T. Coutts, "Ga $\mathrm{In}_{1-\mathrm{x}}$ As Thermophotovoltai: - Onverters," Proc. 24th IEEE Photovoltaic Specialists Conf., Waikoloa, HI, December 5-9, 1994, pp. 1685-1691, IEEE, New York, 1994.

5. M.W. Wanlass, J.S. Ward, K.A. Emery, A. Duda, and T.J. Coutts, "Improved, Large-Area, Two-Terminal InP/Ga ${ }_{0.47} \mathrm{In}_{0.53}$ As Tandem Solar Cells," Proc. 24th IEEE Photovoltaic Specialists Conf., Waikoloa, HI, December 5-9, 1994, pp. 1717-1720, IEEE, New York, 1994.

6. S. Kurtz, K. Emery, and J. Olson, "Methods for Analysis of Two-Junction, Two-Terminal Photovoltaic Devices," Proc. 24th IEEE Photovoltaic Specialists Conf., Waikoloa, HI, December 5-9, 1994, pp. 1733-1737, IEEE, New York, 1994.

7. K. Emery and H. Field, "Artificial Enhancements and Reductions in the PV Efficiency," Proc. 24th IEEE Photovoltaic Specialists Conf., Waikoloa, HI, December 5-9, 1994, pp. 1833-1838, IEEE, New York, 1994.

8. R. Venkatasbramanian, M. Timmons, J. Hutchby, H. Field, and K. Emery, "GaAs and $\mathrm{Al}_{0.2} \mathrm{Ga}_{0.8}$ As Solar Cells with an Indirect-Bandgap $\mathrm{Al}_{0.8} \mathrm{Ga}_{0.2}$ As Emitter; Heterojunction Cells-A Novel Approach to Higher Performance?,"Proc. 24th IEEE Photovoltaic Specialists Conf., Waikoloa, HI, December 5-9, 1994, pp. 1839-1842, IEEE, New York, 1994.

9. C.C. Shen, P.T. Chang, and K. Emery, "Two-Terminal Monolithic InP/GaInAsP Tandem Solar Cells with Tunneling Intercell Ohmic Connections," Optoelectronics-Devices and Technologies, Vol. 9, pp. 551-560, 1994.

10. M.A. Green, K. Emery, K. Bücher, and D.L. King, "Solar Cell Efficiency Tables (version 5)," Progress in Photovoltaics Research and Applications, Vol. 3, pp. 51-55, 1995.

11. K. Emery, J. Caiyem, D. Dunlavy, H. Field, L. Ottoson, and S. Rummel, "Cell and Module Standardized Characterization Performance," presented at 13th NREL Photovoltaics Program Review Meeting, Lakewood, C., May 16-19, 1995.

12. K. Emery, "PV Reference Cell-Construction, Calibration, and Use," presented at the PV Radiometric Workshop, Vail, CO, July 24, 25, 1995, NREL Tech. Rep. NREL/CP-411120008 , September 1995, pp. 35-42.

13. H. Field, "Radiometry for Characterizing Photovoltaic Devices in the Laboratory," presented at the PV Radiometric Workshop, Vail, CO, July 24, 25, 1995, NREL Tech. Rep. NREL/CP4111-20008, September 1995, pp. 107-114. 
14. M.A. Green, K. Emery, K. Bücher, and D.L. King, "Solar Cell Efficiency Tables (version 6)," Progress in Photovoltaics Research and Applications, Vol. 3, pp. 229-233, 1995.

15. K. Emery, J. Caiyem, D. Dunlavy, H. Field, L. Ottoson, and S. Rummel, "I esixjerature and Irradiance Behavior of Photovoltaic Devices," presented at the Photovoltaic Performance and Reliability Workshop, Golden, CO, September 7-8, 1995.

338 
Organization: PV Module and System Performance and Engineering Project NREL PV Program

Contributors: A.W. Czanderna, Leader; F. J. Pern, S. H. Glick

\section{Objectives:}

The task goals are to develop and demonstrate innovative flat-plate PV module encapsulation materials that will provide optimum module performance and protection from the environment for a 30-year operational life in the field. The long-term objectives of this research are (1) to identify, understand, and then mitigate the causes of changes in PV cell and module materials that alter crucial component materials properties and reduce the performance and/or limit the service life of encapsulated cells and modules and (2) to identify or develop new or improved materials, incorporate them into minimodules with different encapsulation schemes, and determine if greater promise is offered for a module service lifetime of 30 years or more.

The primary objectives for the task during FY 1995 are to (a) identify, test and screen new or improved polymeric materials and formulations that offer greater promise than ethylene vinyl acetate (EVA) copolymer for a module life expectancy of 30 years and meet low-cost goals, (b) use accelerated life testing (ALT) to narrow the potential candidate materials for larger scale evaluation, including real-time testing (RTT), (c) develop a data analysis method (procedure) to correlate the results from various spectrophotometric measurements (FA, UV-vis, and color index) for ALT-degraded polymers, (d) identify and test various superstrate materials (polymers and glass) for improving the EVA photostability, and (e) test commercially available adhesive films for simplifying the PV module encapsulation process.

\section{Technical Approach:}

The five objectives are being addressed primarily for contemporary crystalline silicon flat-plate PV module technologies, with the expectation that the same methodologies will be applicable to thinfilm PV modules when service life becomes an important issue for them. The technical approach consists of conducting experimental work, interpreting the results, establishing test methods, and seeking solutions to module reliability and materials durability problems. The background and problems associated with EVA degradation in PV modules have been summarized. ${ }^{1-4}$ A critical review of using EVA as a pottant in PV modules is in press. ${ }^{5}$

The generic module materials durability studies are being performed primarily on polymeric pottant materials using the recent results obtained for degraded EVA. Efforts to modify the present formulation of EVA with the optimum concentrations of Cyasorb UV 531, Tinuvin 770, and Naugard $\mathrm{P}$ and with improved UV and thermal stabilizers have been completed and the modified materials are being ranked from ALT against the existing formulations (A9918, 15295, and 15295P), all of which use Cyasorb UV 531. The modified formulations that rank the best versus A9918 and/or 15295 and equivalents are being prepared in sufficient quantity for more quantitative ALT and deployment for real-time testing in minimodules. In addition, samples of NREL-modified EVA are being n.ade, other candidate commercially available or proprietary pottants are being procured, 
and all samples will be studied as separate materials, materials in contact with other module components, and in complete minimodules. The materials are being subjected to both RTT using field deployment and ALT using DSET Suntest units, Oriel solar simulators, and other existing light sources for test-size specimens; in FY 1996, we will finally be able to use WeatherCmeters $B$ for ALT. Designed experiments are being used to separate mechanisms thought to irulude UV, $T$, humidity, and possibly metal ion catalyzed effects.

The degradation will be and is being monitored from characterization measurements made before, during, and after testing, as described. ${ }^{1-7}$ Non-destructive measurements of degradation that can be used in the field will be sought. Degradation mechanisms will be identified to assist in selecting new or modified materials options. The mechanisms will eventually be necessary for projecting module service lifetime. As time permits, characterization measurements will be made on field-degraded EVA secured from ASE Americas, Siemens Solar Industries, Solarex, USSC, SWTDI, Ben Gurion University in Israel, and other industrial partners requesting assistance.

Efforts are being continued to identify an appropriate low-cost cover glass or polymer (e.g., modified Tefzel and/or PMMA) that will serve as an effective UV screen for EVA. UV-vis measurements will be made for the candidate materials, which will be laminated with EVA and added to our ALT matrix of experiments. The potential will be assessed for using self-assembled monolayers in surface modification schemes to inhibit degradative reactions, particularly at metallization/polymer interfaces.

\section{Results: EVA and Alternative Polymer Pottants in Crystalline Silicon Modules}

EVA as a Pottant: We have had accepted for publication a chapter for SEM/SC entitled "Encapsulation of PV Modules Using Ethylene Vinyl Acetate Copolymer as the Pottant: A Critical Review," by Czanderna and Pern (NREL/TP-412-3957, February, 1995). ${ }^{5}$ This document (120 pages) is a critical review of the literature on EVA dating to the 1950s. The three principal foci are on the development of the encapsulant by JPL and Springborn, their accelerated life testing of it and comparisons with deployment in the field, and the extensive and more complete studies of EVA degradation at NREL since 1990. With the task work now focusing on advanced encapsulants that are either improved EVA or new polymers, this chapter will provide the worldwide PV community with the benefit of the NREL work. The review also places in context the reasons for the erroneous projections of service lifetime for EVA (one author projected 750 years!) within the framework of the fundamental limitations of EVA photothermal degradation in an encapsulated system without any UV filtering. It further addresses the issue of the completeness of R\&D that needs to be done to permit valid projections for service lifetime of an encapsulated PV system. We conclude that alternative polymers must be sought for assuring a ">30-year pottant" will be available to the PV industry.

Degradation Mechanisms: Summaries of the known degradation mechanisms are available. ${ }^{25,6}$ Although 15295 EVA discolors and degrades by the same mechanism as A9918 EVA ${ }^{6}$, it does so at a slower rate, as our studies have shown. ${ }^{7}$

EVA Discoloration Rate: Several factors that affect the discoloration rate of EVA copolymer encapsulants used in C-Si PV modules upon accelerated exposure have been investigated by employing UV-visible spectrophotometry, spectrocolorimetry, and fluorescence analysis 
measurements. A variety of film samples including the two typical (unprimed) EVA formulations, A9918 and 15295, were studied. The films were laminated, cured, and exposed to either a concentrated $1-\mathrm{kW}$ Xe or an enhanced-UV light source. The results indicate that the tiant of EVA discoloration can be affected by both chemical and physical factors. The degraistive chemical factors include (1) EVA formulation, (2) presence and concentration of ccrny generated, UV-excitable chromophores that depend on the type of curing agent used, (3) loss rate of the UV absorber, Cyasorb UV 531, (4) curing agent and curing conditions, and (5) film thickness. The degradative physical factors include the (6) UV light intensity, (7) UV-filtering effect of glass superstrates, (8) gas permeability of polymeric superstrates, (9) lamination-delamination, and (10) photobleaching rate due to diffusion of air into the laminated films. Details are published. ${ }^{3.6,8}$

Which EVA and Superstrate to Use? We made an unequivocal recommendation to the PV industry that if they continue using EVA, the formulation 15295 (fast-cured with Lupersol TBEC) be used in place of A9918 for a slower discoloration rate. This recommendation followed slightly over 3 years of work in which field-degraded specimens were analyzed, the mechanisms of degradation of EVA were elucidated, and laboratory investigations were used to understand the causes of yellowing and how the processes are retarded. The recommendation was also made to a leading c-Si manufacturer in April, 1994 and NREL has been advised they are in the process of changing from their use of A9918 to 15295. Mobil Solar began work in the summer of 1991 to change to a different polymer, which was a direct result of interactions with NREL, and announced the change in November, 1993. Solarex began using 15295 EVA exclusively in late 1987, and their modules are widely thought to discolor at the slowest rate of the known EVA encapsulated modules. The reasons for the slower discoloration rate are included in the review. ${ }^{5}$

The discoloration of both A9918 and 15295 EVA is slowed further by the use of a cerium-containing glass, which was recommended for consideration by industry in 1991 by NREL (Czanderna). However, at that time, it was considered by industry to be too costly as an addition so the idea was not pressed by NREL. To the dismay of the PV industry, one of the major suppliers of the glass for PV modules began delivering the UV-filtering glass ca. 1990 !

Potential Elvax 150 Replacement(s): We have identified several combinations of stabilizers other than Tinuvin 770 and/or Naugard $P$ that showed when tested in the form of thin films, an improvement factor ranging from 4.2 to 14.5 for stabilizing Cyasorb UV 531 or other UV absorbers against discoloration. The modified Elvax 150 formulations are currently being tested as thin films and laminated thick films; a patent application has been filed.

Efficiency Losses: We have continued to collaborate on projects in which the efficiency losses from yellowed EVA in modules or minimodules are being monitored. Details are available in reports from SWDTI, the literature, ${ }^{10}$ and NREL. ${ }^{1,7}$

Equipment Acquisitions: We began using a custom-made laminator for making minimodules from $2^{\prime \prime} \times 2^{\prime \prime}$ up to l' $^{\prime} \mathrm{l}^{\prime}$. In addition, we ordered three major pieces of capital equipment necessary for the preparation, ALT, and characterization of encapsulated PV cells and mini-modules. These include a regular WeatherOmeter (UV, $\mathrm{T}$, and $\mathrm{RH}$ control), a custom-made, prototype WeatherOmeter (WOM) that will permit in situ I-V measurements during the ALT, and a custom-made, prototype, portable degradation monitor. With the monitor, the transmittance or 
absorbance, reflectance, color indices (yellowness) and fluorescence of EVA or other polymers will be measured for encapsulated PV cells/modules in field-deployed specimens.

The equipment, when combined with the FY 1996-approved acquisition of a mir:-extruder, will permit NREL to make high-quality, compounded polymer films on a laboratory scale, laminate PV cells into an encapsulated system of contemporary or advanced design, subject them to extended ALT in the WOMs, and to use (mostly) existing characterization capabilities before, during, and after the ALT to establish causes of degradation. From the latter, revised formulations/designs will be devised until the further ALT, modeling, diagnostic, and mechanistic work will permit valid service lifetime projections. ${ }^{11,12}$

Industrial Interactions: We have interacted extensively with over 50 personnel from the $P V$ and chemical industries to exchange information and/or secure samples of various polymer encapsulant candidates and glass superstrate materials for improving EVA photostability.

Other Activities: (1). A patent was granted in August 1995 related to stabilizing interfaces using organized molecular assemblies. ${ }^{13}$ (2). Two invention disclosures were also filed based on results of testing modified EVA formulations and analyzing discolored EVA from laboratory minimodules and field-exposed modules. (3). C. M. Lampert, editor, Solar Energy Materials and Solar Cells (SEM/SC), invited A. Czanderna to serve as guest editor for selecting and having reviewed expanded versions of about 50 significant papers presented at the WCPEC for a special issue of the international journal. Czanderna accepted and formed an editorial team consisting of $\mathrm{T}$. Ciszek (NREL), H. W. Schock (U. Stuttgart, FRG), A. Luque (Madrid, Spain), A. Hamakawa (U. Osaka, Japan), and himself. Fitty-five reviewed manuscripts have been delivered to Elsevier and the special issue should be available in early 1996 . We also made a minor contribution to a chapter on PV performance and reliability. ${ }^{14}$

\section{References}

1. A.W. Czanderna. (1994) "Current Status of EVA Degradation in Si Modules and Interface Stability in CdTe/CdS Modules." AIP Conference Proceedings, R. Noufi and H. Ullal, eds., Vol. 306, Woodbury, NY: Am. Inst. Physics; pp. 147-155.

2. A.W. Czanderna. (October 1993) Conference Record of Photovoltaic Performance and Reliability Workshop. L. Mrig, ed., NREL/CP-410-6033. Golden, CO: National Renewable Energy Laboratory; pp. 311-357.

3. A.W. Czanderna, F.J. Pern, and S. Glick. (1995) "PV Module Materials and Encapsulation Research." AIP Conference Proceedings, H.S. Ullal and C.E. Witt, eds., Vol. 353, 13th NREL Photovoltaics Program Review. Woodbury, NY: American Institute of Physics; pp. 197-206.

4. F.J. Pern, S.H. Glick, A.W. Czanderna, and R. DeBlasio. (1995) "Alternative PV Module Encapsulation Materials and Schemes." AIP Conference Proceedings, H.S. Ullal and C.E. Witt, eds., Vol. 353, 13th NREL Photovoltaics Program Review. Woodbury, NY: American Institute of Physics; pp. 569-580. 
5. A.W. Czanderna and F.J. Pern. (February 1995) "Encapsulation of PV Modules Using Ethylene Vinyl Acetate Copolymer as a Pottant: A Critical Review." NREL/TP-412-7359, Solar Energy Materials and Solar Cells; 4x, In Press.

6. F.J. Pern and A.W. Czanderna. (1992) AIP Conference Proceedings, R. Noufi, ed. 268. Photovoltaic Advanced Research and Development Project. Woodbury, NY: American Institute of Physics, pp. 445-451.

7. F.J. Pern. (October 1994). Conference Record of a Photovoltaic Performance and Reliability Workshop. L. Mrig, ed. (National Renewable Energy Laboratory; Golden, CO: pp. 329-347.

8. F.J. Pern. (1994): Conference Record of the 24th IEEE Photovoltaic Specialists Conference-1994, Piscataway, NJ: IEEE Service Center; pp. 897-900 (also In Press in Ref. 5).

9. A. Rosenthal and S. Durand, SWTDI; summarized elsewhere in this report.

10. D. Berman, S. Biryukov, and D. Faiman. (1995) Solar Energy Materials and Solar Cells; Vol. 36, p. 421.

11. A.W. Czanderna and F.J. Pern. (May 1995) "Estimating Lifetimes of a Polymer Encapsulant for Photovoltaic Modules from Accelerated Testing." NREL/TP-412-7195, Durability Testing of Nonmetalic Materials. R.J. Herling, ed. ASTM STP 1294. Philadelphia, PA: American Society for Testing and Materials; 1996, In Press.

12. H.M. Kim, G.J. Jorgensen, D.E. King, and A.W. Czanderna. (May 1995) "Development of Methodology for Service Lifetime Prediction of Renewable Energy Devices." Durability Testing of Nonmetalic Materials. R.J. Herling, ed. ASTM STP 1294. Philadelphia, PA: American Society for Testing and Materials, 1996, In Press.

13. Patent Allowed. "Molecular Assemblies as Protective Barriers and Adhesion Promoter Interlayer:" D.E. King, A.W. Czanderna, and C. Kennedy (August, 1995).

14. R. DeBlasio, J. Stone, and T. Surek, with K. Emery, D. Myers, B. Kroposki, L. Mrig, J. Burdick, A.W. Czanderna, T. Strand, and C. Osterwald. (1995) "Photovoltaic Performance and Reliability." Advances in Solar Energy. K. Boer, ed. Boulder, CO: American Solar Energy Society; Vol. 10, Boulder, CO: pp. 247-346. 
Title: PV Module Qualification Testing and Test Method Development

Organization: PV Module and System Performance and Engineering Project NREL PV Program

Contributors: Joseph Burdick, Team Leader

Jim Pruett, Master Technician

Elvira Beck, Research Associate

\section{Objectives}

The research team objectives are to investigate, understand, and improve photovoltaic module longterm performance and reliability by developing test methods and procedures and performing exploratory/qualification and reliability tests on PV modules in order to evaluate long-term module reliability as well as to validate ASTM and IEEE test methods, procedures, and standards; to establish a correlation between indoor (artificial, accelerated) test results and outdoor (natural, realtime) exposure as related to reliability/ stability/weathering issues; to help identify module failure mechanisms and work to obtain solutions to reliability problems; and to develop methods to help predict PV module service lifetime in the field.

\section{Technical Approach}

Our research team's approach is to design, develop and perform tests on photovoltaic modules and interact with the PV industry (manufacturers, test facilities, end users, etc.) with the intent of helping to improve PV modules in terms of both long-term performance and reliability. This is accomplished by performing module exploratory/qualification tests on all commercially available PV modules, as well as on R\&D prototypes and contract deliverables, with special emphasis on thinfilm photovoltaic technologies. These tests include environmental, electrical, optical, thermal, mechanical and other physical stresses which we apply to the specimens for either accelerated or long-term periods of exposure. We are attempting to establish a correlation between indoor (artificial, accelerated) test results and outdoor (natural, real-time) exposure as related to reliability/stability/weathering issues. We also evaluate, with the goal of validating, ASTM and IEEE test methods, procedures, and standards. In addition, we are exploring theoretical and experimental studies on PV module lifetime prediction from accelerated testing. Further, our work with the failure analysis group is an attempt to develop methods and techniques to perform failure analysis on PV modules, establishing means of determining the mechanisms of failure, and then obtaining possible solutions to these module performance and/or reliability problems. Finally, we interact with the PV community through written and verbal communications, as well as reports and publications, to exchange ideas and results with the goal of improving the long-term performance and reliability of photovoltaic modules.

\section{Results}

Module Qualification Testing:

MQT Sequence Testing: A test program is under way for testing commercially available modules and $R \& D$ prototypes from various manufacturers by running them through the complete MQT 
sequence. This test method involves the Initial, Final, and A-D Sequences of the IEEE qualification test procedure for PV modules [1]. The first batch of modules has completed all but the Sequence B group of tests; the second batch will be run when Batch $\# 1$ is completed. The test plan involves modules of several different PV technologies from the following companies: Batch \#1-a-Si (APS and Solarex), a-Si/a-Si (UPG and USSC), CIS (Siemens Solar), mono x-Si (Siemens Solar), multi x-Si (Solarex), Spheral-Si (Texas Instruments); Batch \#2-a-Si/a-Si/a-Si:Ge (ECD), CdTe (Golden Photon and Solar Cells, Inc.), Silicon-Film (AstroPower). The following tests have been completed thus far: Initial Tests (Ground Continuity, Dry and Wet Hi-Pot, Wet Insulation-Resistance), Sequence A (200 Thermal Cycles, Surface-Cut Susceptibility), Sequence C (Damp Heat, Dry HiPot), Sequence D (Outdoor Exposure), and Final Tests (Ground Continuity, Dry and Wet Hi-Pot, Wet Insulation-Resistance). The remaining tests will be completed in the near future.

The key MQT results to date were published in a technical paper presented at the 1st World Conference on Photovoltaic Energy Conversion in December, 1994 [2]. Further results were published at the 13th NREL Photovoltaics Program Review meeting [3].

Electrical Integrity Testing: A new cage was constructed at the new Outdoor Test Facility Module Testing Laboratory to house the dry and wet hi-pot, ground-continuity and wet insulation-resistance tests. The cage was completed and these tests were set up to the appropriate safety standards. Now completed, these electrical integrity tests have been performed on all the modules going through Batch \#1, listed above.

Thermal Cycling, Humidity-Freeze Cycling, Damp-Heat Testing: Both the old and new BMA environmental test chambers have been modified to allow the monitoring of the temperature of each module under test, as well as to electrically identify if and when an open-circuit or short-to-ground failure occurs during thermal or humidity-freeze cycling, or during the damp-heat test. The old BMA chamber is primarily used for the damp-heat test ( 1000 hours at $85^{\circ} \mathrm{C}, 85 \%$ R.H.). The new BMA environmental chamber has a large internal capacity, allowing us to test many more modules simultaneously, as well as larger modules. This chamber has been successfully used to run the 200 thermal-cycle test $\left(-40^{\circ} \mathrm{C}\right.$ to $\left.+90^{\circ} \mathrm{C}\right)$, and has performed very well. This chamber has been modified to enable us to perform the UV/thermal test with fluorescent lamps (see below).

Hail Impact Test System (H.I.T.S.): This apparatus has been used to test several products, prototypes, and modules, including: Golden Photon, Utility Power Group and Mobil Solar. The tests helped these manufacturers redesign and improve their PV modules.

Mechanical Flex Tester (MFT): This machine has been set up, tested, and is now fully operational. This particular unit applies a cyclic pressure load $\left(35 \mathrm{lbs} / \mathrm{ft}^{2}\right)$ alternately to each face of a flat-plate module in order to simulate wind and other dynamic mechanical loads that a module would experience in field-installed applications. Once the final instrumentation has been installed-flat pressure transducers to measure the pressure on the front and back of the module during testing - the apparatus will be used in the MQT sequence.

UVTThermal Test Apparatus: This apparatus has been designed and constructed and has been installed in the new BMA environmental chamber. This apparatus consists of PV module test racks, as well as UV/ fluorescent lamps and ballasts. We will be testing the proposed IEEE UV/thermal test in the future with both UV-A and UV-B lamps to investigate the validity of this test procedure. 
Static Load Test Apparatus: This apparatus was designed and construction has now been completed. This unit will allow static mechanical loads $\left(50 \mathrm{lbs} / \mathrm{ft}^{2}\right)$ to be applied to each face of a PV module to simulate snow or other such static mechanical loads which a module might experience in the field. Modifications to this apparatus have been completed which allow the monitoring of the temperature of each module under test, as well as to electrically identify if and when an open-circuit or short-to-ground failure occurs during testing.

New Qualification Test Method Development: New module qualification tests and methods are being developed. The following tests have been designed and set up thus far: (1) thermal and humidity-freeze cycling, (2) hail impact test, (3) mechanical flex test (dynamic), (4) scratch or surface-cut test, (5) ground-continuity test, (6) dry and wet hi-pot tests, (7) wet insulation-resistance (megger) test, (8) damp-heat test, (9) saltwater immersion and corrosion test, and (10) static load test. Additional tests presently under development are: (1) non-intrusive hot-spot endurance test, (2) UV/thermal test using fluorescent lamps, (3) accelerated weathering (UV/solar radiation/ thermal/moisture) test using filtered-xenon lamps in an envir-onmental test chamber, (4) mechanical twist/termination robustness test, and (5) bypass-diode thermal test.

\section{Photostability Studies:}

Environmental Chamber/Nortek Argon-arc Lamp: Having made several improvements [3] to this chamber/ light-source exposure system, including: (1) the improvement of the lamp's spectral irradiance in the near-infrared region of the spectrum $(\sim 700-1300 \mathrm{~nm})$ to be closer to that of the sun; (2) improving the irradiance uniformity at the test plane from $\pm 20 \%$ to $\pm 5 \%$; (3) achieving more uniform module temperatures during long-term exposure $\left(50 \pm 4^{\circ} \mathrm{C}\right)$; and (4) further improvements in the test structure and wiring of the test area to enable electrical and thermal monitoring of each of the PV modules under test, we continued our light-soak testing program.

Light-Soak Testing: Light-Soak Test \#4 was performed in 1995. It involved the light-soaking of multi-junction a-Si PV modules from various manufacturers (APS, Solarex, USSC and ECD, etc.). They were exposed to 1-sun illumination under an argon-arc lamp at $50^{\circ} \mathrm{C}$, loaded at Pmax. Some modules were placed outdoors for further testing. Since the modules have "stabilized," further exposure will be performed at successively lower module temperatures (e.g., $40,30,20^{\circ} \mathrm{C}$, etc.), to try to better simulate the photostability that the modules exhibit out-doors. Light-Soak Test \#Cl was also performed. This test involved 1-sun exposure under metal-halide lamps in the SolarClimatic at $50^{\circ} \mathrm{C}$. The samples used in this study were mini-modules of CIS and CdTe from Siemens Solar and Solar Cells, Inc., respectively.

Indoor/Outdoor Photostability Correlation Study: This correlation study involves a comparison of the long-term outdoor photostability of commercial a-Si PV modules with various types of indoor testing performed on identical modules, such as: (1) continuous illumination and constant temperature, and (2) cycled illumination and temperature, (3) lower exposure temperatures (as mentioned above). The improvement in the spectrum of the Vortek argon-arc lamp as well as exposure under more realistic temperatures will hopefully help to enhance the correlation between indoor and outdoor results of this testing. 
New Accelerated-Weathering System (AWS): The procurement of this equipment was completed in 1995. The separate parts of this system consist of: (A) an indoor large-area test chamber that will provide accelerated weathering-UV/Vis/IR radiation, thermal and humidity cycling. and specimen water spray - on PV modules: the Atlas XR260 was chosen for this portion of the system, and was delivered in November and is now operational; (B) an outdoor solar-tracking concentrator apparatus for accelerated weathering of PV modules: the American SunCo OATS apparatus (Qutdoor $\Delta$ ccelerated-weathering Iracking System) was purchased for this portion of the system, and was delivered and installed at the outdoor PV test site in August; and (C) a multiplexing module I-V curve tracer to obtain I-V characteristics of modules under test: the Daystar RD-1200 Multi-Tracer was chosen for this portion of the system, and was delivered and calibrated in November. This entire area of testing will help us to observe the synergistic effects of various outdoor weather conditions (solar radiation, temperature and humidity) on PV modules in an accelerated environment. This, in turn, will help us to correlate the indoor, accelerated test results with outdoor, realtime exposure testing. The various experiments using this equipment are being developed and will begin in 1996.

\section{Module Performance Characterization:}

Multi-Reference-Cell Package: A multiple reference-cell package in a module enclosure has been designed to enable us to instantaneously and simultaneously measure various portions of the incident spectrum during outdoor or indoor testing. Several packages will be fabricated: one of these will be used with PV systems installed at the outdoor test site, two will be used with the Standardized Outdoor Measurement System (SOMS), one will be used with the outdoor experimental test rack and module energy ratings study, one will be used with the Reference Irradiance/Meteorology System (RMS), and two will be used for indoor module testing.

Irradiance/Temperature Coefficients and PERMM Study: Using the SolarClimatic 1600, which was procured from Atlas Electric Devices and delivered and installed at NREL in October, 1994, we are able to measure the I-V characteristics of various PV modules at different illuminations and temperatures, thereby providing us with the irradiance and temperature coefficients of voltage, current, fill factor, power, and efficiency. We performed an extensive study of five different PV technologies-dual- and triple-junction a-Si, CIS, mono $\mathrm{x}$-Si and multi $\mathrm{x}$-Si-at six irradiances $\left(200,400,600,800,1000,1100 \mathrm{~W} / \mathrm{m}^{2}\right)$ and six temperatures $\left(0,15,25,40,60,80^{\circ} \mathrm{C}\right)$. We obtained $\mathrm{I}-\mathrm{V}$ curves for each of these modules at each of these irradiances and temperatures. With these results, we wrote a computer program to acquire and convert existing weather data (solar irradiation, ambient temperature, and wind speed) for eight sites (Boston, Boulder, Detroit, Miami, Phoenix, San Francisco, Seattle, and St. Louis) and five different years (1961, 1968, 1975, 1982, 1990). We then interpolated our I-V vs irradiance and temperature data to obtain the power, energy and efficiency for each module for each site and for each year. This work-called the PERMM (PV Energy Rating Method for Modules) study - was submitted to the Technical Review Committee evaluating new energy rating methods in November, 1994 and March, 1995, and was published at the 13th NREL PV Program Review [3] and the PV Radiometric Workshop [4]. 


\section{Eacilities:}

FTLB Laboratury \#156: This laboratory has now been decommissioned and is no longer part of our project. All the test equipment and instrumentation-Vortek light source, Tenney and both BMA environmentai test chambers-were successfully moved to and set up in the new Outdoor Test Facility (OTF) in October and November, 1995.

Joyce Street Facility (JSF): The Module Testing Laboratory at the JSF is now being utilized primarily as a storage facility. The test equipment formerly stationed there-the Hail Impact Test System (H.I.T.S.), the Mechanical Flex Tester (MFT), the static load apparatus, the hi-pot, groundcontinuity, insulation-resistance, and other electrical-integrity tests, and the SolarClimatic 1600, have all been successfully moved to and set up in the new OTF in October and November, 1995.

Outdoor Test Facility (OTF): The OTF was completed, and we moved into the office area in May, 1995. As mentioned above, the test equipment, instrumentation, etc., were moved in and set up in October and November. Maintaining and improving the equipment, running tests, updating the Safe Operating Procedures (SOPs), etc., are all part of the ongoing, daily functions of our research team.

\section{Relevant Conferences:}

Ist World Conference on Photovoltaic Energy Conversion: Waikoloa, Hawaii, December, 1994. PV Energy Rating Methods Technical Review Committee Meeting: Golden, CO, March, 1995. 13th NREL Photovoltaic Program Review Meeting. Lakewood, CO, May, 1995.

NREL Standards and Codes Forum: Golden, CO, June, 1995.

Photovoltaic Radiometric Measurements Workshop: Vail, CO, July, 1995.

Photovoltaic Performance and Reliability Workshop: Lakewood, CO, September, 1995.

\section{Photovoltaic Standards:}

ASTM: The ASTM Standard entitled, "Test Method for Measurement of the Electrical Performance and Spectral Response of Multijunction Photovoltaic Cells and Modules," is presently being written. Draft 200R2 was presented to the Standards and Codes Forum at NREL in June, 1995.

IEEE : The final draft of the IEEE Document entitled, "Recommended Practice for Qualification of Photovoltaic Modules," which outlines the test sequence and procedures for module qualification testing, was submitted to the PV community for review and comments in early 1995 and then went to ballot. It has now been approved and accepted for publication.

\section{Subcontract Monitoring:}

Spire PVMaT Contract ("Automated Solar Cell Assembly"): Technical Monitoring Team member. PV Module Certification/Laboratory Accreditation Criteria Development: Technical Review Team member. 


\section{References / Publications}

1. "Rerorrnended Practice for Qualification of Photovoltaic (PV) Modules," PAR 1262, IEEE: s:anisiards Coordinating Committee 21, 1995.

2. "Qualification Testing of Thin-Film and Crystalline Photovoltaic Modules," 1st World Conference on Photovoltaic Energy Conversion, Waikoloa, Hawaii, December, 1994, and Advances in Solar Energy, 1995.

3. "Photovoltaic Module Qualification Testing, Light-Soak Test Equipment Improvements, and Module Performance Characterization," 13th NREL PV Program Review Meeting, Lakewood, CO, May, 1995.

4. "An Evaluation of Regional Differences in Module Performance for Various PV Technologies Using the National Solar Radiation Data Base," PV Radiometric Measurements Workshop, Vail, CO, July, 1995. 
Title: Photovoltaic Module and System Technology Validation

Organization: Photovoltaic Module and System Performance and Engineering.Project

Contributors: L. Mrig, team leader; T. Strand, staff engineer; B. Kroposki, staff engineer; R. Hansen, master technician; E. van Dyk, visiting researeh associate

\section{Objectives}

The objective of this research activity is to test, monitor, evaluate, and analyze state-of-the-art and advanced PV technologies at the module and system levels. The team assists in the development and validation of $\mathrm{PV}$ technologies by analyzing the performance and reliability of PV modules and systems in support of the DOE National PV Program goal of 30-year lifetime for PV modules. The results of this activity are then disseminated to the PV community in the form of publications, test reports and presentations. In addition, this team interacts with PV manufacturers (through cooperative research agreements) and other test facilities to exchange information, results, and experience. The team also provides engineering support to other in-house PV projects (e.g., PVMaT, international projects, and thin-film partnerships). Finally, the team aids in the development of PV standards and codes for use by the PV community.

\section{Technical Approach}

Module and System Testing for Performance, Reliability, and Stability

The team maintains a database of flat-plate PV technologies performance and reliability, at both the module and system levels. The team continues to perform module and system stability testing on all thin-film and advanced PV technologies. Long-term outdoor exposure tests and indoor controlled light-soaking experiments are conducted to address the issue of module stability. Module performance is measured periodically during the tests. The team monitors and evaluates the outdoor performance of PV modules and small systems (approximately $1 \mathrm{kWp}$ each) at NREL's PV Outdoor Test Site. This activity focuses on emerging PV module technology evaluation/validation, flat-plate module and system development, and failure analysis. Photovoltaic and data acquisition systems are designed and deployed at this test site to conduct in-situ technical evaluations of the PV array, system performance, and reliability. The team also designs and deploys small $(\sim 100-\mathrm{W})$ stand-alone systems. These small systems demonstrate the feasibility of thin-film PV technologies for remote or stand-alone applications (e.g., telecommunication, lighting, etc.).

This testing is being conducted at NREL to assist PV manufacturers and system integrators in evaluating array/system performance and identifying technical issues, if any, by fielding the modules and PV systems under actual operating conditions. This research furnishes independently tested data to the PV community on module and system performance and reliability. The PV array and system performance data collected form the basis of technical publications and reports. A major aspect of this cooperative array/system research activity at NREL is the technical exchange of results obtained from these evaluations with manufacturers and industry. 
Module and System Performance Evaluation Studies

The team is researching and developing performance evaluation methodologies for modules and systems. These studies include seasonal performance, spectral measurements, and techniques for evaluating instantaneous power output and energy production of the modules and systems. This team has established a technical review committee (TRC) made up of representatives from PV manufacturers, PV users, and government labs to resolve the issues associated with module energy ratings and performance evaluation methodology development.

\section{Standards and Codes}

The team has members participating and working on IEEE SCC21, NEC Article 690 TRC, and IEC $\mathrm{TC} / 82$ committees, to help in the development of standards and codes, through consensus, for the PV industry.

\section{Support for Internal Projects}

The team provides technical support for PVMaT, PVBONUS, PVUSA, FEMP, and international projects (e.g., India and South Africa).

\section{Results}

\section{Outdoor Module Stability and Performance Testing}

The team has over 100 modules under study at the PV Outdoor Test Site. These modules include single-, dual-, and triple-junction a-Si, CdTe, CIS, single crystalline $\mathrm{Si}$, multicrystalline $\mathrm{Si}$, and spherical-Si modules. Long-term outdoor performance data are collected by periodically taking I-V curves. From these data temperature coefficients are determined. To study the module's stability over time the data are normalized to $1000 \mathrm{~W} / \mathrm{m}^{2}$ and corrected to $25^{\circ} \mathrm{C}$. Figures 1 and 2 illustrate Siemens CIS and Solar Cells, Inc. CdTe normalized and temperature-corrected performance vs. time for two modules. These graphs show that the modules are essentially stable over the time period.

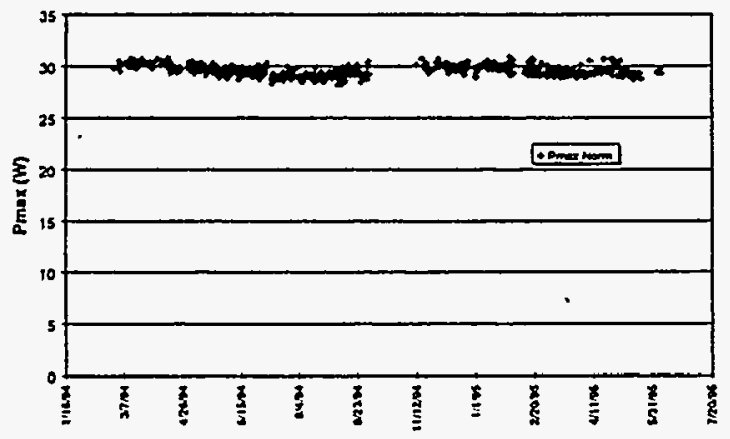

Figure 1. Siemens Solar CIS Module Performance

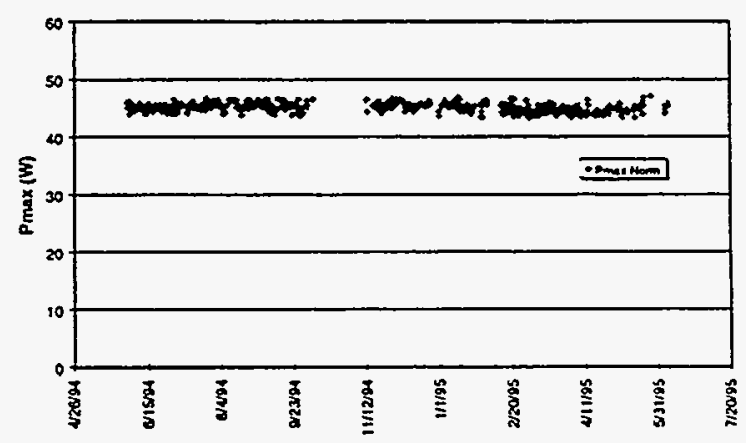

Figure 2. Solar Cells, Inc. CdTe Module Performance

\section{Indoor Controlled Light-Soak Testing}

Amorphous Silicon: Light Soak 4 was started in FY 1995. This test exposed 8 sets of multi-junction a-Si modules from different manufacturers to simulated solar irradiance at $1000 \mathrm{~W} / \mathrm{m}^{2}$ and $50^{\circ} \mathrm{C}$ for 
800 hours. The modules have reached the stability criteria and will continue to be tested at lower temperatures. This will allow for comparisons of stabilized performance with modules from the same sets that are $t=$ ing exposed outdoors. Status reports have been provided to the manufacturers and program managers.

Cadmium Telluride and Copper Indium Diselenide: Light Soak C1 was conducted on a set of minimodules from Solar Cells, Inc.(CdTe), Golden Photon (CdTe), and Siemens Solar (CIS). This test exposed the modules to $1000 \mathrm{~W} / \mathrm{m}^{2}$ at $50^{\circ} \mathrm{C}$ for 1000 hours. Damp Heat and UV tests were also conducted on full-size modules from Golden Photon. Test reports were distributed to the manufacturers.

\section{Module Performance Evaluation Methodology Development}

A study was conducted on the accuracy of different module energy rating techniques. The results can be found in a paper presented at the 24th IEEE [3]. Using inputs from a technical review committee, a methodology for rating modules based on energy production was developed through a subcontract with Endecon Engineering. A report on this work was presented at the 13th NREL PV Program Review [5].

\section{System Evaluation Results}

In-situ testing of advanced thin-film PV systems has been in progress since December 1992. PV systems currently under evaluation include advanced thin-film PV technologies of a-Si, CIS, and $\mathrm{CdTe}$ as well as EFG x-Si and $\mathrm{Czx}$-Si. The effects of weather, temperature, solar spectrum, soiling, and age on array/system performance are being studied. Work currently in-progress includes indepth studies of performance versus temperature and spectrum, system energy ratings, and determination of temperature coefficients.

The team designed, installed, and began monitoring several systems in FY 1995 at the PV Outdoor Test Site. These systems include a $1.4-\mathrm{kW}_{\mathrm{dc}} \mathrm{PV}$ array employing ASE Americas (formerly Mobil Solar) GP300DG PV modules, a 1-kW Solarex a-Si/a-Si:Ge PV array, a 1.2-kW de SCI CdTe PV array (replacing the SCI 400-W de PV array), a Small-Systems Test Bed (capable of testing three small stand-alone PV system configurations simultaneously), and a meteorological system.

Preliminary temperature coefficients for $\mathrm{P}_{\max }$ were calculated for two thin-film polycrystalline PV arrays being evaluated by this team. Using these coefficients, the normalized array output power was corrected for temperature. Figure 3 shows the Siemens Solar CIS array performance, normalized to $1000 \mathrm{~W} / \mathrm{m}^{2}$ and corrected to $25^{\circ} \mathrm{C}$ back-of-module temperature. A strong inverse correlation between array power and back-of-module temperature was observed at both the module and array levels. This is mainly due to the narrow band gap of the CIS material, which results in a strong inverse correlation between voltage and temperature. The temperature-corrected CIS array power was shown to be relatively stable over the test period. Figure 4 shows dc power corrected for temperature and normalized to $1000 \mathrm{~W} / \mathrm{m}^{2}$ versus time for the Solar Cells, Inc. $400-\mathrm{W}_{\mathrm{dc}} \mathrm{CdTe}$ $\mathrm{PV}$ array. This figure shows that the temperature-corrected CdTe array power was relatively stable over the test period. 


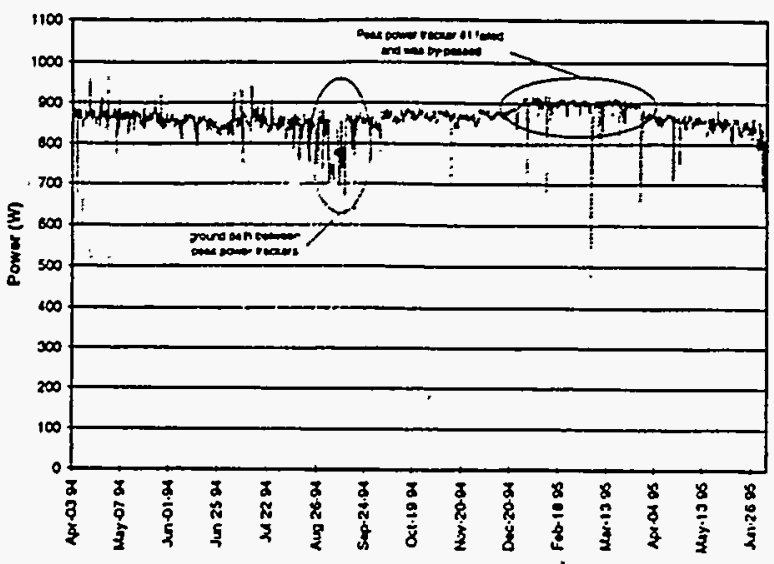

Figure 3. Siemens Solar CIS Array Performance

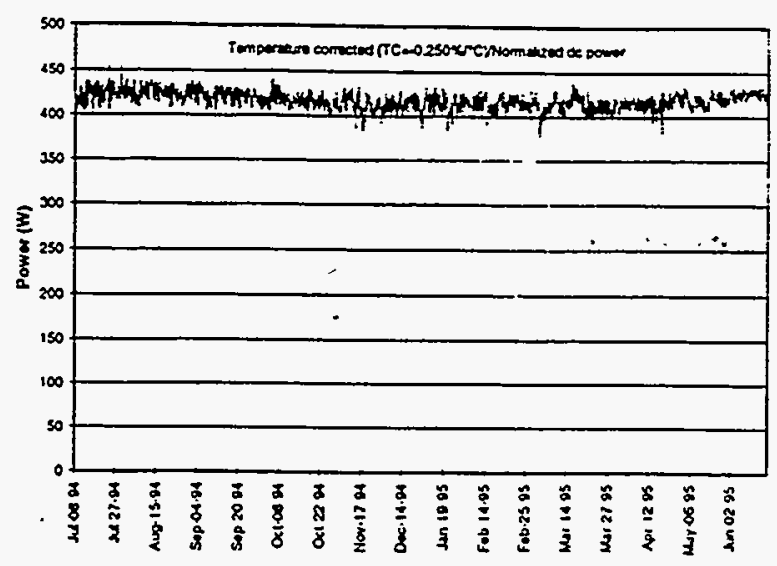

Figure 4. Solar Cells. Inc. Array Performance

Test Reports Delivered to Industry

Proprietary test reports specific to the various PV modules and systems under evaluation by the team have been compiled and delivered to industry. The test reports delivered in FY 1995 include:

1. Final Status Report on modules from Light Soak 3, 12/94

2. MTR95SSI001, report detailing performance of Siemens Solar CIS Modules, 2/95

3. MTR95SCI001, report detailing performance of Solar Cells, Inc. CdTe Modules, 3/95

4. Initial Status Report on modules from Light Soak 4, 3/95

5. MTR95SCI002, report detailing performance of Solar Cells, Inc. CdTe Modules, 8/95

6. MTR95SSI002, report detailing performance of Siemens Solar CIS Modules, 8/95

7. Report detailing the status of the Siemens Solar CIS PV Array, 12/94

8. STR95SCI.001, report detailing the final performance of the SCI 400-W CdTe PV Array and the initial performance of the SCI 1- kW CdTe PV Array, 8/95

9. STR95SSI.001, report detailing the performance of the Siemens Solar CIS PV Array, 9/95

10. Report detailing the initial performance of the Solarex a-Si/a-Si:Ge 1- kW PV Array, 11/95

\section{Standards and Codes}

The team completed work on IEEE standard 1262 "Recommended Practice for Qualification of PV Modules". This document was submitted and accepted by the IEEE Standards Board. Members of this team are also on two IEEE SCC 21 committees working on field testing (PAR 1373) and system safety (PAR 1374) draft standards. The team completed work on the 1996 and began work on the 1999 NEC code cycle for Article 690.

\section{Support for Internal/DOE Projects}

The team supported PVMaT as Technical Monitoring Team Members for the following Phase 4Al subcontracts: Utility Power Group, Solar Design Associates, Omnion, Ascension Technologies, Solar Electric Specialties, Advanced Energy Systems, Inc., and Trace Engineering. Engineering support was provided to the Sustainable Rural Economic Development Ramakrishna Mission Initiative, West Bengal, India. The team coordinated, conducted tests, and provided results to the CdTe PV Partnership Team. 
Dr. Ernest van Dyk, a visiting research associate from South Africa, has been working with the team in analyzing the performance of the two $6-\mathrm{kW}_{\mathrm{ac}}$ grid-connected PV systems located on the roof of the SERF building. A long abstract covering the results of this effort has been submitted for publication at the $26^{\text {th }}$ IEEE PVSPC. Preliminary results of this work have shown. in addition to expected seasonal trends, that system monitoring is a valuable tool in assessing performance and detecting faulty equipment. The systems were both found to be operating at approximately $7 \%$ to $8 \%$ below their estimated rating, which was based on PVUSA test conditions. This may be attributed to the design inverter efficiency being estimated at $95 \%$ compared to measured values of approximately $87 \%$. The continuous monitoring also revealed faulty peak-power point tracking equipment. Furthermore, methods applied for this analysis may be used to evaluate and compare systems employing different $\mathrm{PV}$ technologies.

This team supported the Federal Energy Management Program (FEMP) by designing and installing four PV systems for the 1994 Pageant of Peace in Washington, D.C. Further engineering support was given to FEMP through a feasibility study for PV-powered area lights at the $9^{\text {th }}$ Street park in Washington, D.C. Engineering support was also given to the DOE National PV Program by conducting a feasibility study for a utility-interconnect PV system at NREL's FTLB building and by providing a conceptual design for use of photovoltaics on the White House.

\section{Publications List}

1. Outdoor Performance Stability and Controlled Lightsoak Testing of Multijunction Amorphous Silicon PV Modules at NREL, L. Mrig, et al., $1^{\text {st }}$ WCPEC, Waikoloa, Hawaii, December, 1994.

2. Technical Evaluation of a Dual-Junction Same-Band-Gap Amorphous Silicon Photovoltaic System at NREL, T. Strand, L. Mrig, R. Hansen, and K. Emery, $1^{\text {st }}$ WCPEC, Waikoloa, Hawaii, December, 1994.

3. A Comparison of Photovoltaic Module Performance Evaluation Methodologies for Energy Ratings, B. Kroposki, L. Mrig, and K. Emery, $1^{\text {st }}$ WCPEC, Waikoloa, Hawaii, December, 1994.

4. Results of Module and System Testing at NREL, L. Mrig, R. Hansen, B. Kroposki, and T. Strand, 13th NREL PV Program Review, Lakewood, Colorado, May 1995.

5. Development of a Photovoltaic Module Energy Ratings Methodology, B. Kroposki, L. Mrig, C. Whitaker, and J. Newmiller, $13^{\text {th }}$ NREL PV Program Review, Lakewood, Colorado, May 1995.

6. Technical Evaluation of a USSC Integrated/Direct Mount PV Roofing Module System at NREL, T. Strand, R. Hansen, and L. Mrig, $13^{\text {th }}$ NREL PV Program Review, Lakewood, Colorado, May 1995.

7. Contributing authors for "Advances in Solar Energy" Volume 10, Chapter 5, Sections 5.4, 5.6, and 5.7 .

8. Completed an expansion paper for publication of the paper titled Technical Evaluation of a Dual-Junction Same-Band-Gap Amorphous Silicon Photovoltaic System, July, 1995. This work is in press and will appear in Volume 4X of the "Solar Energy Materials and Solar Cells" 1996 publication.

9. Polycrystalline Thin-film Module and System Performance, T. Strand, B. Kroposki, R. Hansen, and L. Mrig, 1995 PV Performance and Reliability Workshop, September, 1995.

10. Proceedings for the 1995 PV Performance and Reliability Workshop, editor L. Mrig. 
11. New Insights into the Staebler-Wronski Degradation Mechanism from Analysis of Solar Cell and Module Degradation Data, B. Von Roedern, B. Kroposki, T. Strand, and L. Mrig, $13^{\text {th }}$ European PVSEC, Nice, France, October, 1995. 
Title: Photovoltaic Component Diagnostics and Failure Analysis

Organization: NREL PV Module and System Performance and Engineering Project

Contributors: T. J. McMahon, Module Lead; and T. S. Basso, System Lead

\section{Objectives}

The objectives are to conduct and document module and system reliability and failure activities. The purposes are to provide technical results, recommendations or solutions to technical issues, technical assistance, and technical inputs. These are intended for the development of PV standards, codes, criteria for test and evaluation procedures, and design input. This activity aims to establish, develop, enhance, and provide support facilities, equipment, and approaches that are complementary to, or extensions of, other PV means.

\section{Technical Approach}

Module- and system-level concerns will be addressed through working relationships with the industry, users and other colleagues. Results are generally considered confidential, usually only disclosing generic data in the open literature. System level concerns are addressed with emphasis extending outward from the module interface. We will build upon ongoing and past activities, since minimal resources are available for new thrusts.

Module and system reliability performance data is obtained from operating systems, demonstration or prototype units, qualification and exploratory tests, research results, dedicated studies, and, from the literature. This is used for establishing a comprehensive database for individual module and array/system component performance with emphasis on reliability and operational characteristics. Analyses will be performed, including the correlation of real time exposure testing, accelerated testing, and deployed system results. Dedicated tasks are pursued to uncover and reproduce identified failure mechanisms, to validate proposed hypotheses, to explore areas that are believed to be of high priority for long term PV reliability, to recommend new tests for screening or qualification, and to develop better understanding for improved reliability and design.

The cause of PV module failure will be determined through nondestructive and destructive evaluation procedures. Nondestructive testing (NDT) includes measuring individual cells of modules using methods such as the newly devised two-terminal shunt resistance measurement technique, the chopped light cell output determination, and, laser scanning. After NDT, module de-encapsulation may be done, or, samples cored or cut from the module, to allow further evaluation of the suspect areas. This allows further characterization such as chemical analyses, microscopy, spectroscopy, etc. From these evaluations, more basic materials determinations can be confirmed and probable causes and mechanisms can be proposed.

\section{Results}

Our non-intrusive "two-terminal method" for measuring the shunt resistance of the individual cells within a series-connected module [References 1-3] was submitted by NREL for the 1995 
R\&D 100 Award. Although the award was denied, the method is being used more and more to measure the individual shunt resistances of cells within series-connected modules. Low shunt resistance values indicate which cells may have manufacturing flaws or have degraded after qualification testing. These values allow you to know at any light level which. if any, of the cells in the series-connected module will degrade the module output due to low shunt resistance. In a paper presented at the NREL Photovoltaics Program Review [Reference 4], we explained how the cell shunt resistances are measured and how the energy rating of a module is ultimately impacted since the efficiency of a module is reduced even more at lower illumination levels.

Baseline shunt resistance measurements of cells in representative modules being deployed in the - field are now being made. This allows us to separate manufacturing flaws that affect the initial performance from flaws that degrade the performance during environmental stress tests. This past year we baselined modules from Solarex, Texas Instruments (TI), Solar Cells Inc, and Advanced Photovoltaic Systems (APS). Solar Cells Inc. and APS modules showed manufacturing flaws and TI's modules showed serious flaws that showed up after flex testing. We also measured Utility Power Group degraded modules from the NREL PV field site that had efficiencies of less than half of their initial values. We provided test reports to manufacturers or NREL colleagues.

In a second paper [Reference 5] presented at the NREL Photovoltaics Program Review, we show how shunt resistance affects module efficiency vs intensity. A Spire simulator is used to measure module efficiency and the intensity is varied with sheets of vellum. The PSpice circuit simulation and analysis program, which was being used to understand the equivalent circuitry that can be used to represent a module, is now used in diagnosing cell failures within a module, especially electrical failures such as hot-spot burn-outs, and is used here to quantitatively predict the effect of cell shunt resistance on module performance at different light levels (Figure 1).

Many modules have been characterized in this way, but because of the proprietary nature of these kind of data, only data on a monocrystal silicon test module can be presented here. We studied the peak-power performance of a 12-cell, series-connected monocrystalline Si module before and after connecting shunt resistors across each cell. Intrinsic $R_{\mathrm{sh}}$ values are between 38 and $272 \mathrm{ohms} / \mathrm{cell}$. We chose to connect shunt resistors having values of $0.5,1$, and $2 \mathrm{ohms}$ across each cell in this study because we have observed these values on cells in modules supplied to NREL. These low $R_{s b}$ values can be the result of new emerging technologies or the result of degradation in $R_{s h}$ for a module undergoing stress testing. These modules, as well as the 12 cell test module, show losses in the experimentally observed peak-power module efficiencies vs light intensity, which can be explained by low $R_{\mathrm{sh}}$ values.

The test module was configured to have access to each cell in the string. The measured peakefficiency data were compared with the shunt and series resistance loss behavior calculated for this module using the PSpice circuit simulation and analysis program. No fitting parameters were used for the situation where the intrinsic module and three shunt resistors were added to simulate emerging technologies. Figure 1 shows the excellent agreement. 


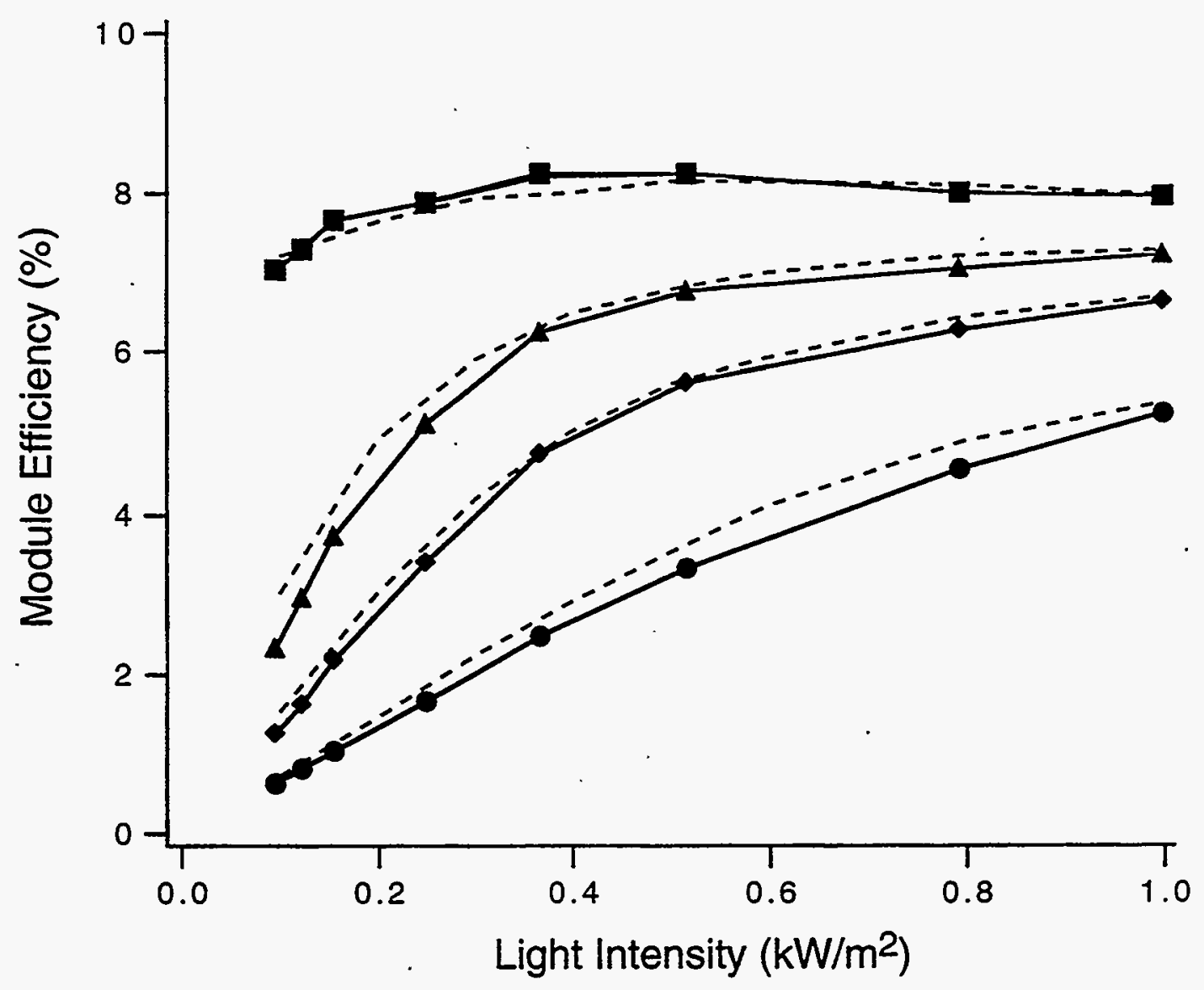

Figure 1. Measured (points) and calculated (dashed line) peak-power efficiencies vs light intensity for the intrinsic module-top, and for the 2-, 1-, and $0.5-\mathrm{ohm}$ cell shunt conditions-lower curves.

Additionally, data of this kind were measured on five 3' by 3' modules supplied to the PV Module and System Engineering Project and given to us before deployment for initial cell shunt resistance determinations. Each module had 36,6 " by 6 " cells connected in series. The dark resistance of four of the poorer performing modules ranged from $43 \mathrm{ohms}$ to $60 \mathrm{ohms}$ and the cells within the modules ranged from $0.25 \mathrm{ohm}$ to $4 \mathrm{ohms}$. As anticipated, these modules do perform poorly, especially under reduced sunlight conditions. The fifth module used improved cell-processing techniques and has a dark resistance of $380 \mathrm{ohms,} \mathrm{with} \mathrm{cell} \mathrm{resistances} \mathrm{between}$ 4 and 20 ohms.

As above, to see what efficiencies would be expected as a result of shunt losses, the measured cell shunt resistances were used in the SPICE circuit simulator calculation. Figure 2 shows the calculated and measured peak-power efficiencies for these modules before and after improved cell processing. The two lower curves are the calculated (dashed) and measured peak-power efficiencies (full diamonds) for an earlier module for which cell shunt resistances were between 


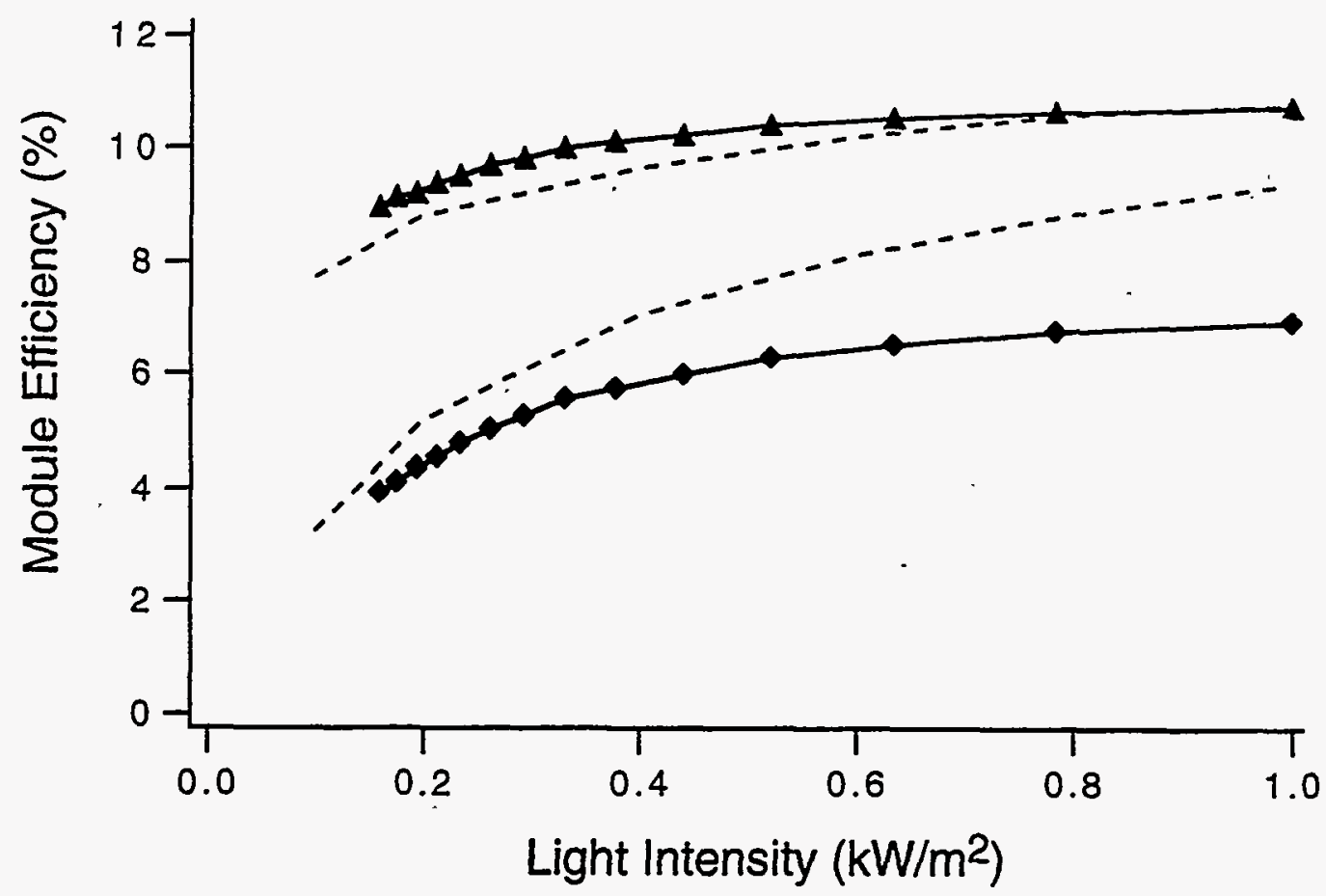

Figure 2. Measured (points) and calculated (dashed line) peak-power efficiencies vs light intensity for the improved module with 4 to 20 ohms cell shunt resistance-top, and for the 0.33 to 4 ohms cell shunt resistance-lower curves.

0.33 and 4 ohms. The upper pair are calculated (dashed) and measured values (full diamonds) for an earlier module for which cell shunt resistances were between 0.33 and 4 ohms. The upper pair are calculated (dashed) and measured values (full triangles) for a later, much improved version where the cell shunt resistances were between 4 and 20 ohms. Calculated efficiencies on both modules are normalized for the $1-\mathrm{kW} / \mathrm{m}^{2}$ point on the better module.

We see that the better module agrees with calculated shunt loss behavior. The poorer module agrees only at lower intensity, where shunt losses usually dominate. The measured behavior is poorer than predicted at high intensity. More than likely, this is due to recombination or series resistance losses that weren't included in the calculation.

\section{Conclusions}

We have continued to apply our two-terminal, nondestructive, module cell shunt resistance measurement technique, showing how it is useful for supporting qualification testing, failure analysis, and module cell performance characterization. This shunt resistance measurement technique will continue to be used at NREL and is readily available for others to implement. 
Title: Photovoltaic Solar Radiometric Measurements and Evaluation

Organization: NREL PV Module and Systems Performance and Engineering Project

Contributors: D.R. Myers, Senior Staff Engineer and Team Leader; T.W. Cannon, Senior Engineer; D. Trudell, Master Technician

\section{Objectives}

The Photovoltaic (PV) Solar Radiometric Measurements and Evaluation team is a part of the PV Module and System Performance and Engineering Project. The team provides engineering measurements and expert scientific evaluation of optical radiation for characterization and performance testing of PV cells, modules, and systems. High-quality broadband ( 300 nanometers $[\mathrm{nm}]$ to $2400 \mathrm{~nm}$ ) and spectral (wavelength-dependent) instrumentation, measurement and uncertainty analysis techniques, and calibrations quantify uncertainty in PV device and system performance testing and evaluation. The team contributes technical information to industry and NREL PV engineers and researchers concerning radiometric measurements, instrumentation, analysis, and indoor and outdoor solar radiation data and measurements. The team also provides optical radiometer calibrations in support of the DOE/NREL PV program and other program activities and areas at NREL.

\section{Technical Approach}

The team performance radiometric calibrations at the Outdoor Test Facility (OTF) Radiometric Characterization Laboratory, outdoors at the field test site, and in the Optical Metrology Laboratory, shared with NREL's Metrology Laboratory. The team maintains spectral and total irradiance reference standard sources, purchased directly from the National Institute of Standards and Technology (NIST), absolute cavity radiometers compared with and traceable to the World Radiometric Reference, and quality electronic equipment that supports radiometric calibrations, measurements, and research to improve radiometry applied to photovoltaic performance evaluation.

Optical radiometric instrumentation is calibrated, maintained, operated, and loaned to researchers to satisfy measurement needs of NREL internal and external PV program participants. NREL engineers and subcontractors obtain technical consultation on radiometric measurement problems and data analysis critical to PV performance calibrations, testing, and evaluation. This includes the NREL PV Reference Meteorological and Irradiance System (RMIS), monitoring radiometric and atmospheric parameters with respect to standard reporting conditions during outdoor PV testing, five spectroradiometer systems, and an array of broadband radiometers for measuring natural and artificial optical radiation sources.

The team contributes to the PV optical radiometry field by working with photovoltaic manufacturers, researchers in the NREL/DOE PV program, the American Society for Testing and Materials (ASTM), Institute of Electrical and Electronics Engineers (IEEE), the Council for Optical Radiation Measurements (CORM), and the NIST Radiometric Physics Division. T. Cannon is a member of the CORM board of directors, and D. Myers and Cannon are members of ASTM Committee E-44 on Solar, Thermal, and Aiternative Energy Sources. 


\section{Results}

Team accomplishments in fiscal year 1995 include:

- Conducted PV Solar Radiometric Measurements Workshop, July 24-25, 1995 :

Twenty-six attendees from the PV industry, utilities, and national laboratories addressed the current status of and areas of concern for optical radiation research and needs to improve PV performance evaluation and testing. Team objectives to meet needs and concerns were identified and summarized. Published proceedings [1] and summary [2] of discussions at the 1995 Photovoltaic Performance and Reliability Workshop.

- Participated in Module Energy Rating (MER) Technical Review Committee:

Identified standard data sets (reference days, test data) and processing algorithms (spectral, power, irradiance). Technical review and guidance on approach, implementation, and validation for MER subcontractor and NREL technical monitor $[3,4]$.

- Calibrated 61 different radiometers and sources for NREL PV research and industry, and for several other NREL/DOE programs:

Transferred World Radiometric Reference to two PV cavity radiometers through NREL Working Group that participated International Pyrheliometric Comparisons, Davos, Switzerland.

Calibrated 6 spectral and 11 broadband radiometers, and 3 accelerated testing and cell performance solar simulators, for NREL PV program. Calibrated and characterized 31 broadband radiometers for Arizona Public Service Company, Arizona State University PV testing applications, 4 spectral radiometers, and 4 artificial light sources for other DOE/NREL programs.

- Contributed to standards and codes, technical publications:

Technical comments on improved radiometric and uncertainty aspects of IEEE, ASTM, International Standards Organization draft and ballot documents $[5,6,7]$ for PV qualification and accelerated weathering testing. Myers and Cannon members of ASTM Committee E-44. Myers drafted two chapter sections, 5.3.4 Total Irradiance Measurements and 5.3.5 Spectral Irradiance Measurements, Chapter 5 (Photovoltaic Performance and Reliability) of Volume 10 of Advances in Solar Energy. [8]

- Relocated, maintained routine operation of RMIS at NREL test site:

We relocated RMIS data acquisition archiving systems to the OTF, maintaining best practice radiometric and environmental data for PV performance evaluation, calibrations, data quality control, and MER analysis. High-reliability backup and highly time-resolved (5-second) data supplement routine monitoring for special tests (system and module I-V traces, spectral effects [9.10]). 
- Invention disclosures and patent awards:

T. Cannon developed designs for a UV/broadband spectral radiome: $\because$. innitor and circumsolar radiometric monitor. He developed and implemented two si:: $\ldots$..ii $e$ packages for radiometric data processing and archiving (Pulse Analysis Spectroradicisn ::ic System, Quiklook Spectral data processing).

Major technical interactions during FY 1995 included:

- Enron/Amoco-Solarex, Houston, TX: Radiometric and meteorological data and software for Nevada Solar Enterprise Zone.

- Sunset Technology, Highlands Ranch, CO: MER and translation equations.

- Energy Photovoltaics, Inc.; Princeton, N.J.: Cumulative frequency distributions for irradiance and temperature for CIS module design and rating.

- Fraunhofer Institute for Solar Energy Systems, Freiburg, Germany: Radiometric data base description and data for MER applications.

- European Space Agency, Spain: Simple spectral model and reference spectral distributions for PV cell calibrations.

- Florida Solar Energy Center, Cape Canaveral, FL: Spectroradiometer calibration and troubleshooting.

- Solar Cells, Inc., Toledo, OH: Radiometric monitoring instrumentation and specifications.

\section{Conclusions}

PV test engineers conducting system performance and stability monitoring activities at the NREL PV outdoor test site compare plane of array (POA) radiometric data with high-quality data from the RMIS. Archive 1-minute time resolution data is used for system and MER analysis. Calibrations of POA sensors provide traceability and known uncertainty for POA measurements.

Technical contributions to draft standards for PV performance, Qualification, and durability testing were made at the 1995 Standards and Codes Forum, and the September 1995 Utility Photovoltaic Group (UPVG) Team-up meeting to draft 1995 UPVG procurement specifications.

Future work includes continuing technical contributions to IEEE and ASTM standards; developing a PV radiometric measurements guide; continuing calibration of radiometers; performing radiometric data quality assurance; and assisting in validation of MER methods.

Team members presented papers and technical contributions at the following workshops and conferences: 
- Recent Advances in Atmospheric Radiometry, 1995 Annual CORM meeting, May 16-18, 1995, Ottawa, Ontario, Canada

- 13th NREL Photovoltaic Program Review, May 16-18, 1995, Denver, CO

- 1995 Photovoltaic Standards and Codes Forum, June 26-28 1995, Golden, CO

- Photovoltaic Solar Radiometric Measurements Workshop, July 24-25, 1995, Vail, CO

- Utility Photovoltaics Group (UPVG) Team-up, 1995, specifications review meeting, September 4-5, 1995, Golden, CO

- PV Performance and Reliability Workshop, September 21-23, 1994, Golden, CO.

\section{References}

1. Myers, D.R., Chairman (September 1995). PV Radiometric Workshop Proceedings. NREL/CP-411-20008. Golden, CO: National Renewable Energy Laboratory.

2. $\quad$ Myers, D.R. (November 1995). "PV Solar Radiometric Issues and Needs." In proceedings Photovoltaic Performance and Reliability Workshop, L. Mrig, ed., NREL/CP-411-20379. Golden, CO: National Renewable Energy Laboratory.

3. Whitaker, C. (November 1995). "NREL Module Energy Rating Methodology." In proceedings, Photovoltaic Performance and Reliability Workshop, L. Mrig, ed., NREL/CP411-20379. Golden, CO: National Renewable Energy Laboratory.

4. Kroposki, B., L. Mrig, D.R. Myers, K. Emery (1994). "A Comparison of Photovoltaic Module Performance Evaluation Methodologies for Energy Ratings." First World Conference on Photovoltaic Energy Conversion, December 5-6, 1994, Waikoloa, Hawaii.

5. IEEE Standards Coordinating Committee 21, Photovoltaics (April 1995). "Recommended Practice for Qualification of Photovoltaic (PV) Modules." Draft.

6. ASTM Subcommittee G3.09 (November 1995). Draft "Standard Tables for Reference Solar Spectral Irradiance at Air Mass 1.5: Direct Normal and Hemispherical for $37^{\circ}$ Tilted Surface." West Conshohocken, PA: American Society for Testing and Materials.

7. IEC Document 82/146/CDV (August 1995). "Reference Solar Day." Draft International Electrochemical Commission Technical Committee 82 procedure. International Electrochemical Commission.

8. Böer, K., ed. (1995). Advances in Solar Energy. Boulder, CO: American Solar Energy Society, Vol. 10, pp. 260-266.

9. Strand, T., K. Emery, R. Hansen, L. Mrig (1994). "Technical Evaluation of a Dual Junction Same Band Gap Amorphous Silicon Photovoltaic System at NREL." First World Conference on Photovoltaic Energy Conversion, December 5-6, 1994, Waikoloa, Hawaii.

10. Myers, D.R., T.W. Cannon (1995). "Photovoltaic Solar Radiometric Measurements and Evaluation." Proceedings of 13th NREL Photovoltaic Program Review, May 16-18, 1995, Denver, Colorado. Edited by H. Ullal, E. Witt. American Institute of Physics Press. 
Organization: NREL PV Module and System Performance and Engineering P: $: \cdots:$ it

Contributors: C.R. Osterwald

\section{Objectives:}

The objective of this work was to provide coordination for the development of PV standards, codes, and criteria for test and evaluation procedures that are consistent with the DOE National Photovoltaics Five-Year Plan. Included in this objective is the development of criteria for implementation of a possible third-party module certification/laboratory accreditation program.

\section{Technical Approach:}

Technical approaches used for these objectives included participation with the Institute of Electrical and Electronics Engineers (IEEE) Standard Coordination Committee 21 and U.S. Technical Advisory Group for International Electrotechnical Commission (IEC) Technical Committee 82, and chairing American Society for Testing and Materials (ASTM) subcommittee E44.09, titled 'Photovoltaic Electric Power Conversion." The module certification (emphasizing qualification testing) and test facility accreditation criteria development effort was continued through a subcontract extension to Arizona State University. Other efforts included coordination of the Photovoltaic Energy Project (PEP) '93 Intercomparison of reference cell calibrations and cell performance measurements. To facilitate these activities, a third NREL PV Standards and Codes Forum was held to provide individual and joint meetings at the same location and establish coordination of efforts and flow of information between committees on standards issues.

\section{Results}

The PEP '93 Intercomparison coordination continued with the circulation of the sample sets. Two separate sample sets are being circulated. The first is composed entirely of $2 \times 2 \mathrm{~cm}$, singlecrystal Si reference cells that each participant will calibrate with their best calibration method. The results of these calibrations will be analyzed and used to establish the World PV Scale of primary reference cells. The second sample set consists of devices from newer PV technologies that have unique measurement problems, such as narrow bandgap cells, large-area cells, and series-connected tandem cells. Twelve laboratories from throughout the world are participating in the intercomparison. The sample circulation was scheduled to be completed on June 30, 1995, but shipping delays in some countries have caused the completion date to slip by about 8 months.

PV standards activities within ASTM during FY 1995 dealt with several issues of importance to the PV industry. Development of draft standards for mechanical integrity of PV modules, electrical performance of multijunction devices, and wet insulation integrity testing of PV modules have continued. Two existing reference cell calibration standards, E 1039 and E 1125 were reapproved and have been published by ASTM. Revisions of E 1143 on PV device linearity and E 1328 on PV terminology were also completed [1]. Work on a new draft standard for visual inspections of PV modules was initiated.

The Arizona State University (ASU) subcontract, titled "PV Module Certification and Laboratory Accreditation Criteria Development," was completed on November 30, 1994. The major objective for this subcontract was to develop and document criteria and recommendations that 
specify guidelines to be used to certify a PV module for performance and reliability, along with the guidelines to be used to certify a PV module for performance and reliability, along with the specific equipment, facilities, quality assurance procedures, and technical exper isi an accredited laboratory needs in order to perform PV module certification. Development of - tsic ititeria and the documentation took pläce through a consensus committee, formed by ASU. ${ }^{2}$, ingresentatives of PV manufacturers, end users, standards and codes organizations, and testiriy laboratories. Thirty organizations committed to work on the project. The final repoit for the subcontract was developed into an NREL technical report that was published in April 1995 [2]. A 1-year subcontract extension was awarded to ASU to continue the certification/accreditation work by development of the legal papers and criteria that a certification body may need to initiate a program.

The 1995 PV Standards and Codes Forum was held on June 26 through June 28. Over 30 representatives from manufacturers, test laboratories, end users, and the national laboratories attended meetings of IEEE Standards Coordinating Committee 21, the U.S. Technical Advisory Group to IEC Technical Committee 82, and ASTM subcommittee E44.09. A report on the efforts to propose changes to the National Electric Code Article 690 for the 1996 review cycle was also presented during the Forum. Three draft standards were reviewed during the ASTM E44.09 meeting, including the module wet insulation integrity test and the two new drafts for module mechanical integrity testing and multijunction device performance testing. IEEE SCC21 held simultaneous working group meetings for document development, including PV module qualification sequence, PV system battery sizing, PV system safety, and PV system field acceptance testing.

\section{Conclusions:}

The two FY 1995 milestones for this subtask were both met. The first was to organize and lead the PV Standards and Codes Coordination Forum, which will include consensus standards and codes organizations (meeting, 6/95). Completion of the NREL technical report on the module certification/accreditation work fulfilled the second deliverable.

\section{References:}

1. ASTM standards may be obtained from: ASTM, 100 Barr Harbor Dr., West Conshohoken, PA, 19428-2959, USA.

2. Photovoltaic Module Certification/Laboratory Accreditation Criteria Development (April 1995). NREL/TP-412-7680, available from NTIS. 


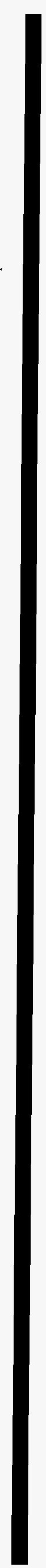




\section{PV Module and System Performance \& Engineering Subcontracts}



Title:

Photovoltaic Module Certification/

Laboratory Accreditation Criteria Development

Organization: Arizona State University

Tempe, Arizona

Contributors: $\quad$ R. L. Hammond, B. D. Wood, C. E. Backus, R.L. Sears; Principal Investigators (ASU)

G. A. Zerlaut, Consultant, SC-International Inc.

R. V. D'Aiello, RD Associates

C. R. Osterwald, NREL Contract Monitor

R. DeBlasio, NREL Project Manager

\section{Background}

\section{The Need For A PV Certification Program}

As the Photovoltaic (PV) industry continues to mature, there is a growing need to instill confidence in users and customers, on a national and international basis, that PV products meet minimum standards for performance, reliability and durability. Such confidence is generally instilled by product certification and labeling (e.g., UL listing and labeling).

At present, $P V$ module manufacturers essentially self-certify modules through product warranties and, in most cases, in-house qualification testing. Because no formal certification program is currently in existence, buyers of PV modules must either have their own specifications for qualifying a product prior to purchase, or utilize the specifications of past purchasers. This situation requires buyers to have an intimate knowledge of PV module testing, which for most buyers is not practicable.

The majority of module qualification testing has been performed by the manufacturers (as opposed to independent testing laboratories), primarily because of a) economic reasons, b) a lack of standardization and c) a lack of qualified laboratories to perform the tests. Also, some manufacturers have developed in-house qualification testing capabilities as part of product development. Independent testing laboratories would allow these manufacturers to concentrate on product development and reduce their need for facilities to perform routine testing, thereby reducing costs. Manufacturer's costs associated with certification may also be reduced as liability is shared by manufacturers and the product Certification Body.

It is expected that a certification/laboratory accreditation program would enable manufacturers to certify products through a single process, avoiding the current situation of the multiple standards required for different markets and/or different customers. This would reduce the cost to the manufacturers and ultimately to the buyers of PV modules.

The initial step toward the establishment of a certification/accreditation program in the PV industry is the development of the criteria that specify what testing needs to be done in order to provide assurance that a particular PV module product shall perform reliably. Along with this, the equipment and facilities required by a laboratory for module 
certification testing needs to be identified. The second step in this process is to develop the documents required for a formal certification/accreditation program.

\section{Phase 1.}

In March 1993 the National Renewable Energy Laboratory (NREL) issued a Request for Proposal to develop the criteria for photovoltaic module certification and laboratory accreditation. The NREL-stated objective was to:

...produce a document detailing the equipment, facilities, quality assurance procedures and technical expertise an accredited laboratory needs for performance and reliability testing of concentrating and non-concentrating PV modules. This document shall also detail the specific test standards necessary for a module design to be certified.

The statement of work consisted of two tasks, Criteria Development Committee Formation and Certification/Accreditation Criteria Development, all to be completed within 12 months.

Arizona State University (ASU) was awarded a subcontract in October 1993 to develop the required criteria. The Phase 1 work was completed in November 1994 with a final report delivered to $\mathrm{NREL}^{1}$.

\section{Phase 2}

The contract was expanded in scope in October 1994 to include an implementation phase; Phase 2.

The goal of Phase 2 was to "Retain the existing Criteria Develop Committee and recruit additional industry members to form a Certification/Accreditation Implementation Committee. Maintain the continuity and momentum of the Phase 1 program. Develop an implementation plan. Motivate senior level managers of PV manufacturers to endorse and participate in the implementation plan.

The objectives of Phase 2 were to:

- Perform as much as possible of the development and groundwork needed prior to the actual initiation of a certification/accreditation program.

- Retain the existing Criteria Development Committee and recruit additional industry members, including as many PV manufacturers as practicable, to form a.

Certification/Accreditation Implementation Committee.

- Maintain the continuity and momentum of the current criteria development effort.

- Develop documentation and recommend procedures to:

a) form a certification body

b) implement a certification program using the criteria in documents PV-1, PV-2 and PV-3 (developed in Phase 1)

c) recommend the selection of a particular laboratory accreditation body

d) develop a pro forma budget for a certification body

e) develop draft corporation papers for a certification body

- Develop recommendations for obtaining seed money to implement a certification program - how much, potential sources, and potential commitments (i.e., industry).

- Document PV-3: Identify and evaluate possible refinements to the performance testing procedures. 
- Motive senior level managers of PV manufacturers to endorse and participate in the implementation plan.

- Educate and motivate senior level managers of PV manufacturers and PV industry as a whole.

\section{Objective Of The Phase 2 Report}

The objective of the Phase 2 report is to provide all of the fundamental draft documents that may be required to establish and operate a PV module certification/laboratory accreditation program. The documents contained herein are intended only as draft documents that could be easily modified to meet the needs of a Certification Body.

In order to meet the intent of this objective (i.e., having all pertinent documents in one report), four documents from the Phase 1 final report are repeated in this report: Section 2 Terminology, and documents PV-1, PV-2, and PV-3 (see Annexes 1, 2, and 3).

\section{Methodology}

The approach used to develop the required documents in Phase 1 and Phase 2 was:: a) enlist the services of a team of local experts to develop the first draft of the required documents, assist with project strategy and provide general guidance, b) establish a 30-member Criteria Development Committee of manufacturers, users and testing laboratories, c) make extensive use of telephone, facsimile and the US Postal Service to communicate with all participants and develop consensus criteria documents, and, d) hold one committee meetings in conjunction with a related standards meeting in order to minimize the cost and time burden on committee members and other participants.

\section{Conclusions}

All basic draft documents needed to implement a PVModule Certification' Laboratory Accreditation program have been developed and are included in the Phase 2 final report (the first draft was delivered to NREL on January 3, 1996). A draft five-year operating budget and a preliminary educational marketing program were also included in the report.

The next step in the implementation program is for one or more individuals to file the Articles of Incorporation to establish the legal framework for the Certification Body. Following that, the incorporators should recruit participants who are willing to provide the first year of funding for the Certification Body and serve on the board of directors.

Recruiting participants to fund the Certification Body is the only remaining barrier to implementing a formal PV Module Certification/Laboratory Accreditation program.

\footnotetext{
${ }^{1}$ NREL (Carl Osterwald) developed the Contractors Final Report into a Technical Report, Photovoltaic Module Certification/Laboratory Accreditation Criteria Development (April 1995). NREL/TP-412-7680. Available NTIS and OSTI.
} 
Title: NREL Module Energy Rating Methodology Project

Organization: Endecon Engineering, San Ramon, CA

Contrihuluis: · C. Whitaker, Principal Investigator; J. Newmiller; W. Howard; L. Mrig. TRC Chairman; B. Kroposki; D. Myers; K. Emery; J. Anderson. Also, industry representatives that comprise the Technical Review Committee.

\section{Objective}

Develop a simple measure of PV performance that will provide a realistic estimate of how one or more modules will perform in a user's specific applications.

\section{Approach}

Develop a strawman approach to a module energy rating.

Convene an industry-representative Technical Review Committee (TRC) to provide feedback and guidance on the strawman and subsequent documents.

Develop and validate a PV performance model of sufficient accuracy.

Define weather data profiles that will describe the range of operating conditions in the U.S. and emphasize performance différences between various module types.

\section{Project Status}

As described in the FY 1994 Report [1], the first meeting of the TRC reached the following conclusions concerning the general characteristics of this module energy rating:

- Target audience is primarily the end user (consumer, system integrator, OEM, distributor, utility engineer).

- Rating should provide stabilized single-module energy values in Watt-hours.

- Energy values should be provided for both peak-power and fixed voltage operation.

- Flat plate modules should be modeled with fixed latitude-tilt orientation.

- Concentrator modules should be modeled with two-axis tracking orientation.

- Energy values should be based on four extreme condition days and one average day.

- Input data should include irradiance (global and direct), ambient temperature, wind speed, location and date (for sun position), and spectrum.

These goals were used to develop a strawman modeling approach that was presented to the TRC in March 1995. At this meeting, discussions regarding the inadequacy. (for the purposes of the project) of the existing PG\&E data led to the conclusion that further module characterization and additional outdoor validation data were needed. Specifically, concerns were raised about the accuracy of the thermopile pyranometer measurements, and the need for concurrent measurement of spectrum during validation data collection.

A meeting was held in conjunction with the TRC meeting with NREL climatologists concerning how best to represent the U.S. climate in five "representative" days covering reasonable or nominal extremes of irradiance and temperature. Five actual days were selected from the National Solar Resource Database (NSRDB) for use in the MER calculation.

The MER modeling approach consists of irradiance models (determination of plane-of-array (POA) incidence angle from location and time, and POA diffuse irradiance from horizontal diffuse irradiance measurements)

To obtain the appropriated characterization and validation data, experimental arrangements were made and data collected between May and July of 1995. Indoor characterization of five sample modules with respect to temperature and irradiance levels was performed, including cross-sensitivity testing for those parameters. 
Sample spectral responses were collected from cells of similar manufacturing technologies to those represented by the sample modules. Outdoor testing (conducted on July 28 through 31,1995 ) yielded IV curves, module temperature, weather data. and spectra for use in model validation. These data include both clear and cloudy conditions, and morierate to cold ambient temperatures.

Model validation ind sample calculations were performed between August and December 1995: at which point : : the draft final report was sent out to the TRC [2].

\section{Conclusions}

This project set out to address three specific goals:

- Define a Module Energy Rating Methodology,

- Define specific conditions for evaluating module performance representative of the US climate,

- Define procedures and techniques for measuring module characteristics and estimating module performance at the representative conditions.

The first of these goals has been squarely met. A general method for describing a PV module energy rating has been developed and agreed upon by an industry-representative Technical Review Committee.

The second goal has been met in principle with the definition of five representative daily weather profiles. These profiles are further defined by the source of weather data (the National Solar Radiation Database) and specific locations and dates within the database. While these suggested weather profiles appear to adequately represent a broad range of geographic locations and climatic conditions, they are subject to change under further scrutiny.

The third goal has not been satisfactorily met. The combined errors in characterizing module performance, in translating indoor measurements to outdoors, in measuring ambient conditions and module performance, and in accounting for all of the various parameters affecting module performance exceed the accuracy required to compare one module to another under a variety of conditions.

Among the issues identified in the draft final report, the following significant work needs to be addressed:

- Indoor module characterization and outdoor validation data should span the range of conditions defined in the five reference days. Extrapolation error is significantly greater than interpolation error for all of the tested models. This constraint must be weighed against the accuracy with which these conditions can be measured. For example, reduced irradiance levels during characterization should not introduce significant spectral changes in the light source.

- Additional evaluation of the power models chosen for this project is needed. With the exception of the original development work on the power models, no other independently corroborating studies of them are known to the participants. Also, an additional model or models will likely be required in order to handle multi-bandgap technologies.

\section{References}

1. Annual Report. Photovoltaic Program. FY 1994, (September 1994). NREL/TP-410-7993. 426 pp. Available NTIS: Order No. DE92000092.

2. Photovoltaic Module Energy Rating Procedure, Draft Final Report, (December 1995). 
Title: $\quad$ PV Module Degradation--EVA Pore Volume

Organization: Polymer Science Dept., Penn State Univ., University Park, PA

Contributors: D. L. Allara, principal investigator

\section{Objective:}

The objectives of this effort are (i) to conduct a methodical evaluation of the feasibility of using spectroscopic ellipsometry (SE) as a non-destructive means of detecting the pore or void volume changes in EVA as a result of increased curing of the polymer, and (ii) to use data from objective (i) to evaluate the potential of using SE for correlating the extent of degradation in field-degraded samples with the expected increase in gel content from prior NREL studies of clear, light yellow, yellow, yellow brown, and brown EVA.

\section{Technical Approach:}

As background, the long term degradation of modules fabricated with ethylene vinyl acetate (EVA) copolymer has become a concern to the DOE PV Program and to PV manufacturers. Evidence indicates that the EVA degradation is related to the formulation of EVA as well as the EVA copolymer itself. A need exists for measuring the extent of degradation of EVA for field deployed modules as well as for laboratory test specimens. One measure of the degradation is the gel content of the EVA, and how this changes with time. The increase in gel content will of necessity cause a change in the pore volume in the polymer from cross linking. The latter alters the physical properties of the polymer that may cause the EVA to lose important components of their physical properties.

EVA was prepared with varying gel content as specimens that are suitable for making state-of-theart, SE measurements of the pore or void volume. The gel content is being varied from $50 \%$ to at least $100 \%$ and determined by an extraction procedure. When a suitable correlation is established for the EVA thickness that is optimal for SE measurements, the thickness will be varied to approach commercial reality and to determine the feasibility of SE measurements for determining pore sizes in EVA of increasing thicknesses.

SE will then be used to establish the feasibility of obtaining a correlation between the pore size and distribution in EVA prepared by NREL staff. The EVA, which is being degraded in various stages from criteria already known to NREL staff, has been prepared on substrates and in the configuration to provide optimum results from the SE measurements. Ultimately, the samples will be prepared at several thicknesses that approach reality to establish how well the SE measurements still correlate with the extent of degradation.

\section{Results and Discussion:}

Curing of EVA thin films. Thin films of ethylene vinyl acetate (EVA) copolymer were prepared in the thickness range $20 \mathrm{~nm}$ to $200 \mathrm{~nm}$, and then cured under vacuum at $145 \pm 2^{\circ} \mathrm{C}$ for 1 $\mathrm{h}$. The samples were placed in a vacuum oven equipped with a Varian direct drive vacuum pump which was used to maintain continually the oven pressure at $50 \mathrm{mTorr}$. Before use, the oven was thoroughly cleaned with ethanol and kept under vacuum at $100^{\circ} \mathrm{C}$ for $12 \mathrm{~h}$. After the $1 \mathrm{~h}$ cure, the samples were kept under vacuum and returned to room temperature over the course of $12 \mathrm{~h}$.

Single wavelength ellipsometry data taken on the samples before and after the curing process were virtually identical for the entire thickness range of the films. However, the beam from the heliumneon laser $(632.8 \mathrm{~nm})$ in the ellipsometer was observed to scatter on some parts of the cured wafers indicated the existence of a roughness above $\sim 1 \mu \mathrm{m}$. Further, the film surfaces were 
inhomogeneous by visual inspection. It would appear that the film surfaces roughened during the cure in localized regions. This same phenomenon was observed during spectroscopic ellipsometry (SE) measurementi: : ind will be discussed later.

Infrared (IR) Spectra. Polarized IR spectra of a $91.8 \mathrm{~nm}$ EVA film have been obtained ànd" analyzed as difference spectra by subtracting the IR spectrum taken for an uncured sample from the corresponding cured one. Using a kinematic sample holder, the exact same portion of the film was analyzed in obtaining both spectra. The difference spectrum shows that the curing process results in a slight reduction in the methyl $\mathrm{C}-\mathrm{H}$ and the carbonyl stretching intensities $(-5 \%)$ but does not reveal the presence of any new absorptions nor any significant peak frequency shifts. In the case of a $53.2 \mathrm{~nm}$ film, the observed changes were even smaller. For all film thicknesses, the small decreases in the IR peak intensities are consistent with small changes in the film density possibly arising from evaporation of residual solvent and/or physisorbed organic impurities (the cure temperature is over $200^{\circ} \mathrm{C}$ above $\mathrm{T}_{\mathrm{g}}$ ). A polarization difference spectrum was obtained by placing the cured sample at two orthogonal orientations relative to the polarizer transmission axes (while maintaining normal incidence) and then subtracting the two spectra obtained. The spectrum shows that the films are completely amorphous with no net orientation of the IR-active functional groups in the copolymer.

Spectroscopic Ellipsometry (SE). In the spectroscopic ellipsometer used in these studies, a broad-band output of a xenon arc lamp is directed along a set of optical rails mounted on a goniometer. For the present measurements the angle of incidence was fixed at $69.5^{\circ}$. To maximize the source intensity at the sample, the source is collimated by focusing the light to a point, using a short focal length lens placed adjacent to the Xe lamp, adjusting the size of the point with an iris and then passing the diverging beam from the point into another lens, placed at a distance equal to its focal length away from the iris, to generate a final collimated output beam. The latter is then sent through a calcite polarizer, which is rotating at a fixed frequency and in this case is about $55 \mathrm{~Hz}$. The speed of rotation need not be constant, and may be set to any speed that the encoder circuit and feedback electronics can accept. The effect of the rotating polarizer is to create rotating, linearly polarized light, which then reflects off the sample. The sample has $x, y, z$, and $\theta$ degrees of freedom. After reflection from the sample, the light is directed through a calcite polarizer (analyzer) for which the polarization direction is held fixed at $30^{\circ}$ with respect to the optical bench. The linearly polarized output light is then directed into a Czerny-Turner double monochromator with a resolution of $0.5 \mathrm{~nm}$. The detector is a $5 \mathrm{amp}$ Hammatsu PMT. The output signal, modulated by the rotation frequency of the polarizer, is transformed by Hadamard analysis to the desired quantities $\psi$ and $\Delta$. These variables completely describe the change in the polarization state of the light upon reflection from the sample (e.g., see R.M.A. Azzam and N.M. Bashara, Ellipsometry and Polarized Light, North Holland, Amsterdam, 1977, Chapter 3).

The SE instrument was calibrated using a $200 \mathrm{~nm}$ gold film evaporated on a silicon wafer. Gold is chosen for its high reflectivity and extreme sensitivity to the angle incidence. Therefore, it is a good test to verify that the optical alignment has not significantly drifted over time. The simulation included is for the simple 2-layer air-gold model, and shows a very good fit to the experimental spectrum.

The SE data for three EVA films of thicknesses $21.5 \mathrm{~nm}, 52.3 \mathrm{~nm}$, and $91.8 \mathrm{~nm}$ were plotted as an overlay of difference spectra for the uncured film at various locations on the wafer. This provides information about the spot to spot thickness gradients of the film. These spectra were then compared to simulated spectra of $\mathrm{SiO}_{2}$ films of the same thicknesses on $\mathrm{Si}(111)$. The $\mathrm{SiO}_{2}$ spectra are similar to the EVA ones and showed the same peaks and overall curve shape; however, the peak locations were shifted in energy to higher and/or lower values. These simulations verify that the peaks observed in the EVA spectra are caused by film thickness interference effects, and not to electronic features in the films. 
A second spectrum, which was obtained for an average of 2 to 5 spots on the sample, included for each thickness an overlay plot of the cured and uncured samples. The standard deviation of the two data sets was similar in each case; however, the analysis time was longer for the cured films because diffuse scattering of the light beam reduces the intensity at the detector with a consequent decrease in signal to noise. It appears that the changes observed are about twice the magnitude of the sample spot-to-spot variation. A thorough error analysis of this effect is in progress. In contrast to the uncured samples, the source beam spot was visible on the cured films (it was approximately a $1 \mathrm{~mm} \times 3 \mathrm{~mm}$ ellipse). A third plot was taken as a difference spectrum of the data found for the cured and uncured EVA. In all cases, the observed changes are located near peaks in the $\psi$ and $\Delta$ spectra. This is reasonable since these are the wavelength regions that are most sensitive to the particular wafer thickness.

\section{Conclusions:}

As a potential non-intrusive test for polymer degradation in field-degraded modules, the investigators are using IR and SE as tools in which SE has the potential of being used for measuring changes in the pore volume in the polymer. Their initial characterization with both SE and IR show that A9918 EVA films suffer no obvious chemical or orientational changes after exposure to the standard thermal curing conditions, although visual inspection shows that the surfaces have become inhomogeneous and rougher. The IR data show that the films remain amorphous with no evidence for forming crosslinking or conjugated double bonds at the sensitivity of the IR probe. Small changes in thickness develop based on the SE data. The samples have been sent to NREL for degradation in UV and T for about three months to induce changes that are known to cause gel content changes. They will be returned to Penn State for SE and IR evaluation in 1996.

Future Work. The samples analyzed were sent to NREL for a series of degradation treatments and gel-content analyses. A full analysis of the present IR and SE data of the cured films is in progress and will be completed when the complete data sets, including those from the fully degraded films, are in hand. 
Title:

Organization.

Contributurs:
Long Term Effects on Roof-Mounted Photovoltaic Modules

Southwest Technology Development Institute (SWTDI)

A. Rosenthal and S. Durand

\section{Objectives}

The objectives of this effort were (i) to conduct a methodical monitoring of roof-mounted, PV module performance degradation, and (ii) to document module operating temperatures as a function of roof-mount design.

\section{Technical Approach}

The long term degradation of modules fabricated with EVA is a concern to the DOE PV Program and to PV manufacturers. The rate of EVA degradation is related to module operating temperature, which raises concerns about roof-mounted modules since they operate hotter than ground- or rackmounted modules.

In coordination with NREL staff, SWTDI selected 12 modules of a specific design from each of 4 manufacturers that have been dedicated to a long term evaluation effort. Modules with a significant exposure history (installed prior to 1985) were selected. Both crystalline and thin film modules were chosen for evaluation. All of the modules available for this project had already been mounted on the rooftops of prototype buildings at the SWTDI. Selected modules on each prototype building were rewired so that each module is electrically terminated at a terminal strip to facilitate taking individual module IV curves. Each of these modules has a thermocouple bonded to its back surface and terminated at the terminal strip. For the modules selected, SWTDI records were reviewed to retrieve available data on the "as delivered" module efficiencies. Manufacturers were also contacted to determine if records of individual module IV curves are available and if they would be able to retrieve IV curve data for the selected modules. When individual module efficiencies could not be obtained, measured array efficiencies were used to estimate individual module efficiencies.

For the selected modules, SWTDI measured the baseline individual module efficiencies at the contract initiation, and obtained IV curves (light only) at a measured module temperature. SWTDI developed a detailed measurement protocol that was reviewed by NREL prior to beginning the measurements. SWTDI then made the measurements according to their planned protocol at intervals from Feb. 1992 through April, 1994; the project was then extended from November, 1994 through February, 1996 with the same technical approach, except dark I-V curves were also requested by NREL. SWTDI provided NREL with a copy of the data as well as with a summary report after data collection. Following baseline efficiency measurements, one module of each type was packaged and shipped to NREL for destructive analysis. The remaining modules remained mounted at SWTDI for continued, real-time, exposure testing in the Las Cruces, NM solar environment.

For objective (ii), SWTDI prepared a summary report documenting all module mounting designs installed at SWTDI along with an evaluation of the module operating temperatures. The summary report included a commentary on the various mounting methods that discusses the design tradeoffs such as ease of installation, ease of maintenance, water tightness, and the design impact on module operating temperature. Results of a 1985 SWRES study of module temperature vs. module spacing above the roof were also included. This summiry report was delivered to NREL in 1992, about three months after the contract was initiated. For objective (i), a final report was delivered to NREL in May, 1994, for the period 2/1992-4/1994; an interim report covering 11/94 through 2/95 was received in $3 / 95$. 


\section{Hardware Limitations in the Long-Term Evaluation of Modules}

The project test plan requires I-V curves to be taken on $48 \mathrm{PV}$ modules every two weeks for a multiple of years. Prior to the implementation of testing, dedicated hardware was develop for this purpose. The hardware, which was designed by engineers on the staff of SWTDI, was also reviewed by a professor of power electronics at New Mexico State University.

The I-V curve tracer that was developed uses the following instruments to measure the necessary parameters:

Table 1. Sensor Hardware

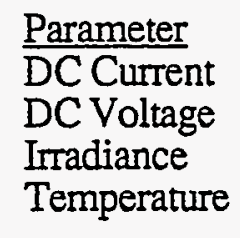

Sensor

Current Shunt

Voltage Divider

LiCor Pyranometer

Type T Thermocouple

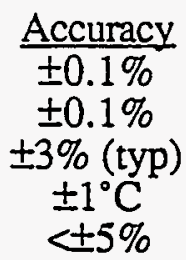

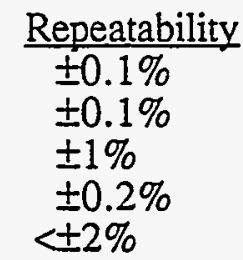

In addition to the sensors, the I-V curve tracer uses a 14 bit analog to digital converter (A/D) with an accuracy of \pm 1 LSB. This represents a possible error of only 1 part in about 16,000 .

For this project, the absolute accuracy of a given module I-V curve is not as important as the ability to resolve changes recorded in successive I-V curves for each module, so the repeatability figure is extremely important. The repeatability figure of $\pm 2 \%$ (Table 1 ) indicates that changes in module performance can only be unambiguously resolved when they exceed $2 \%$.

Unfortunately, raw I-V curves cannot be compared directly. Since module performance is a function of both irradiance and module temperature, I-V curves acquired under various conditions must be normalized (translated) to a set of standard conditions for comparisons to be meaningful. Normalization is performed according to the procedure given in ASTM 1036-85 in which a set of standard algorithms is used in conjunction with module specific coefficients to translate an I-V curve acquired under one set of conditions to its analog under another (standard) set of conditions. There are limits to the accuracy and repeatability associated with the use of these normalizing equations. These limits are a function of how accurately the normalizing coefficients have been derived and how large the difference is between the actual and standard conditions. In general use, normalization according to the ASTM standard is probably accurate to only $\pm 5 \%$ for any given I-V curve. With careful practice, $\pm 2 \%$ is a reasonable estimate of the accuracy of the normalization.

\section{Results and Discussion:}

The criteria for selecting the four arrays/module types used in this study are as follows. All candidate modules had to have been encapsulated with EVA (ethylene vinyl acetate) and passed JPL Block V specifications. Preference was given to the arrays and modules that had achieved the longest cumulative exposure to sunlight and for which early performance documentation was available.

The four arrays/module types selected for the study are (a) Solarex model MIT\#1002967 polycrystalline silicon modules installed in July 1981, (b) Mobil Solar model Ra-180 single crystal silicon modules installed in October 1983, (c) Solarex model SA-20 amorphous silicon modules installed in May 1988, and (d) Sovonics model P-101 amorphous silicon modules installed in January 1989. All performance reports presented mean ratings (normalized to conditions of 1000 $\mathrm{W} / \mathrm{m}^{2}$ and $45^{\circ} \mathrm{C}$ module temperature) for the modules in each of the four arrays. Normalization was achieved using coefficients derived according to the ASTM standard E1036-85. That method utilizes I-V data obtained over a range of temperatures. The coefficients for each module type are 
presented for each module in the final report to the technical monitor. The mean module rating is presented in Table 1 for each array under test since the project was begun in 1992.

Table 1. Normalized Mean Module Ratings In Watts (W)

$\begin{array}{lrrrrrrr} & (7 / 92) & (1 / 93) & (7 / 93) & (12 / 93) & (4 / 94) & (3 / 95) & \text { Loss } \\ \text { Array } & (W) & (W) & (W) & (W) & (W) & (W) & \frac{\%}{(W)} \\ \text { Solarex MIT } & 49.05 & 49.60 & 48.19 & 49.96 & 47.62 & 47.4 * & 3.7 \% \\ \text { Mobil Ra-180 } & 129.45 & 128.45 & 131.10 & 128.53 & 127.63 & 130.2 & \ldots \\ \text { Solarex SA-20 } & 12.21 & 11.31 & 12.5 & 10.75 & 11.81 & 11.16 & -* * * \\ \text { Sovonics P-101 } & 10.07^{* * *} & 7.50 & 8.72 & 7.5 & 7.61 & 7.59 & \ldots\end{array}$

* One module was removed for destructive analysis in April, 1994, with a rating of $43.3 \mathrm{~W}$ and $9.9 \%$ decline from its output in $11 / 1992$. It was projected to be $41.6 \mathrm{~W}$ (4\%/year) for the purposes of this Table [1].

**A $10 \%$ decline occurred in one year for one of the eight modules.

***Inaccurate first rating reflects improper normalization.

After three years of study, some of the modules demonstrate an unambiguous decline in performance, as can be seen by the loss in percent over the 2 -year period. For example, the Solarex MIT modules exhibit a collective loss of $3.7 \%$ per module. However, a detailed analysis on a module by module basis for the ten Solarex modules deployed in 1981 show declines of $3 \%$ to $10 \%$ for 5 modules, but $1.5 \%$ or less for the other 5 . While the average of all ten modules is $3.7 \%$ or $1.2 \%$ per year, three of the modules exhibited declines in excess of $2.3 \% / y e a r$. The EVA in all of the modules has a yellow-brown color. The widening variances in the module power output can be a source of mismatch losses in large arrays, but the only possible mismatch losses in the present study is for differences in the output of each individual cell in series with each other. Additional comments on the four module types were reported for the period 2/1992 through 4/1994 [1]. These comments will be updated after monitoring through 2/1996.

\section{Future:}

The initial SWTDI subcontract expired in April, 1994, but a new subcontract was placed to cover an additional 15 months of monitoring from November, 1994 through February, 1996. The work reported by SWTDI and NREL analysis of the data demonstrate that monitoring individual modules is essential to identify which modules undergo accelerated degradation. The modules that degrade the most can be more gainfully subjected to post mortem analyses to learn more about the causes of the degradation.

The only other systematic monitoring of performance losses of individual modules in an array was published recently by D. Faiman and coworkers of Ben Gurion U. (BGU), Israel [2]. Both the SWDTI and BGU studies would be better if I-V data had been gathered on each individual module at the time of deployment and periodically thereafter, and if dark I-V data were also collected during the present and ongoing monitoring. The latter has been specified in the SWTDI contract extension.

1. NREL Photovoltaic Program FY 1994 Annual Report, K.A. Summers, Coordinator, NREL/TP-410-7993, June, 1995, pp. 349-352.

2. D. Berman, S. Biryukov, and D. Faiman, Solar Energy Materials and Solar Cells, 36, 421 (1995). 
Title:

PV Translation Equations and PV Module Energy Rating Concept

Organization: Jerry Anderson, Program Manager, Sunset Technolog: Corporation, 3388 W. Oak Leaf Place, Highlands Ranch, CO. 80126

Objective: $\quad$ Two tasks were involved in this research program. New photovoltaic translation equations were developed for translating photovoltaic I-V performance characteristics from one temperature and irradiance. condition to another such condition.

A second unrelated task was to develop a-concept for an energy rating system for PV modules.

Summary:

Translation Equations: New equations have been developed for the purpose of evaluating the performance of photovoltaic cells, modules, panels and arrays. These equations enable the performance values determined at one temperature and irradiance condition to be translated to any other condition of temperature and irradiance.

Energy Rating: An approach has been defined for evaluating module energy output in a simple manner. The concept consists of evaluating a module on a solar simulator to derive a matrix of performance characteristics as a-function of irradiance and temperature, and then using that data to determine energy output for 5 reference days.

Discussion:

Translation Equations: New translation equations were developed and extensively checked against actual test data. Compared to the currently-used translation equations:

- The new equations more accurately translate $I_{s c}$ and $V_{o c}$.

- The new equations simply and accurately translate I-V curve data points.

- Two new equations have been developed for translating maximum power.

- The new equations utilize dimensionless coefficients such that $\alpha$ and $\beta$ have units of ${ }^{\circ} \mathrm{C}^{-1}$, and not, for example, amps ${ }^{\circ} \mathrm{C}^{-1}$ or volts $\cdot{ }^{\circ} \mathrm{C}^{-1}$.

- The new dimensionless coefficients are constant, and do not change with cell size, module internal circuitry or external series-parallel arrangements.

- The new equations are accurate over a wide range of conditions $\left(25^{\circ} \mathrm{C}\right.$ to $75^{\circ} \mathrm{C}$ device temperature and 100 to $1000 \mathrm{~W} / \mathrm{m}^{2}$ irradiance).

Results from over 100 translations show good agreement between the analytical predictions and actual test measurements, and superior performance when compared to the current translation equations. A comparison of the currently-used equations and the new equations is presented in Table 1. 


\section{TABLE 1}

COMPARISON OF THE CURRENT TRANSLATION EQUATIONS AND THE NEW EQUATIONS

\begin{tabular}{|c|c|}
\hline CURRENT EQUATIONS & NEW EQUATIONS \\
\hline 1). $\begin{aligned} I_{S C 2} & =I_{S c 1}+I_{S c 1} \cdot\left(\frac{E_{2}}{E_{1}}-1\right)+\alpha \cdot\left(T_{2} \cdot T_{1}\right) \\
\Delta I_{s c} & =I_{s c 2}-I_{s c 1}\end{aligned}$ & 1). $I_{S C 2}=\frac{I_{S C 1}}{\left[1+\alpha \cdot\left(T_{1}-T_{2}\right)\right] \cdot\left[E_{1} / E_{2}\right]}$ \\
\hline 2). No equation available for direct computation of $V_{o c 2}$ & 2). $V_{O C 2}=\frac{V_{O C 1}}{\left[1+\beta \cdot\left(T_{1}-T_{2}\right)\right] \cdot\left[1+\delta \cdot \ln \left(E_{1} / E_{2}\right)\right]}$ \\
\hline $\begin{array}{l}\text { 3). I-V Data Pair Translation: } \\
\qquad \begin{array}{l}\text { A. } \cdot V_{2}=V_{1}-\beta \cdot\left(T_{2}-T_{1}\right)-\Delta I_{s c} \cdot R_{s}-k \cdot\left(T_{2}-T_{1}\right) \cdot I_{2} \\
\text { B. } I_{2}=I_{1}+\Delta I_{s c}\end{array}\end{array}$ & $\begin{array}{l}\text { 3). I-V Data Pair Translation: } \\
\qquad \begin{array}{l}\text { A. } V_{2}=V_{1} \cdot\left(\frac{V_{O C 2}}{V_{O C 1}}\right) \\
\text { B. } I_{2}=I_{1} \cdot\left(\frac{I_{S C 2}}{I_{S C 1}}\right)\end{array}\end{array}$ \\
\hline 4). $\quad P_{2}=I_{2} \cdot V_{2}$ & 4). $\quad P_{2}=I_{2} \cdot V_{2}$ \\
\hline 5). No equations available for $P_{\max }$ & 5). $\begin{aligned} P_{\max 2} & =P_{\max 1} \cdot\left(\frac{I_{S c 2}}{I_{S c 1}}\right) \cdot\left(\frac{V_{O C 2}}{V_{O C 1}}\right) \text {, or } \\
P_{\max 2} & =\frac{P_{\max 1} \cdot\left(E_{2} / E_{1}\right)}{\left[1+\gamma \cdot\left(T_{1}-T_{2}\right)\right] \cdot\left[1+\delta \cdot \ln \left(E_{1} / E_{2}\right)\right]}\end{aligned}$ \\
\hline
\end{tabular}


Energy Rating: A wide variety of photovoltaic module energy rating methods are possible. Many ideas have been proposed in the past, and many more will probably be offered in the future.

Invariably, the energy rating topic evokes thoughts of historical weather data, Solmet irradiance tabulations, weather maps with irradiation and ambient temperature isobars, and complex analytical expressions for module performance. It is often viewed as a complicated system-level subject wheres'i concerns for such things as soiling factors, wiring losses, tracking, rack-mounting vs roof-mounting, sto are believed to be needed for the evaluation. Even worse, the energy rating issue becomes entangled wit: 'he desire for sitespecific and even seasonal performance predictions. This thinking has lead to frusiration and to the eventual abandonment of any energy evaluation or energy rating program.

It is the belief of this author that these complex approaches are unnecessary, and that a very simple test method and rating system based on well defined reference days can be developed. The proposed approach is based on the following philosophy:

- energy rating should be viewed as a module issue

- the energy rating program should be based on test data

- the test program should be performed by an independent laboratory

- the energy evaluation should be based on a few reference days

- the reference days should include moderate and extreme days

- both battery charging and maximum power loads should be considered

- the rating numbers should be easy to understand :

- for battery charging operation amp-hrs should be specified

- for maximum power operation watt-hours should be specified

- only single-day energy values should be stated

- module designs should be rewarded with better ratings if they have:

- unique optical characteristics (reflective control features)

- improved thermal performance features

- improved spectral response characteristics

- low battery leakage current loss (nighttime)

- superior low-light performance

The energy rating concept developed has 4 basic parts having the easy-to-remember "authoritative" acronyms UMP, REF, WIFE and COP:

UMP: Universal Map of Performance: This is a matrix of module I-V performance characteristics versus irradiance and module temperatures which is measured using a solar simulator. A simple heating pad and neutral filters are employed to control module temperature and incidenf irradiance.

REF: Reference Environmental Functions: This is a group of five reference days which are defined in terms of irradiance, ambient temperature, wind speed, solar incidence angles, and spectral irradiance distribution; all of which are tabulated versus time of day. For battery charging applications the battery voltage versus time characteristics are also given. Four of these reference days represent generic extreme days and the fifth reference day represents a nominal (moderate) type of day.

WIFE: Wattage Influence Factor Effects: This segment consists of modifiers which take into account module temperature factors such as wind speed, ambient temperature and irradiance; spectral irradiance distribution effects caused by air mass or overcast conditions; spectral differences between the reference cell and module (and between the simulator versus the reference solar spectrum); the module spectral response change with temperature; and the effect of solar incident angle losses such as surface reflections.

COP: Coefficients of Performance: The results of the evaluation of the UMP module data versus the five REF "reference day" characteristics (as modified using the WIFE factors) are presented in a graphical manner. Coefficients would be oresented for both battery charging (amp-hrs) and maximum power (watt-hrs) operation. 
The results can be shown in tabular form, or can be illustrated in some sort of symbolic form. One such label which presents the battery charging amp-hours and the maximum power operation watt-hrs for 5 reference days is illustrated as follows:

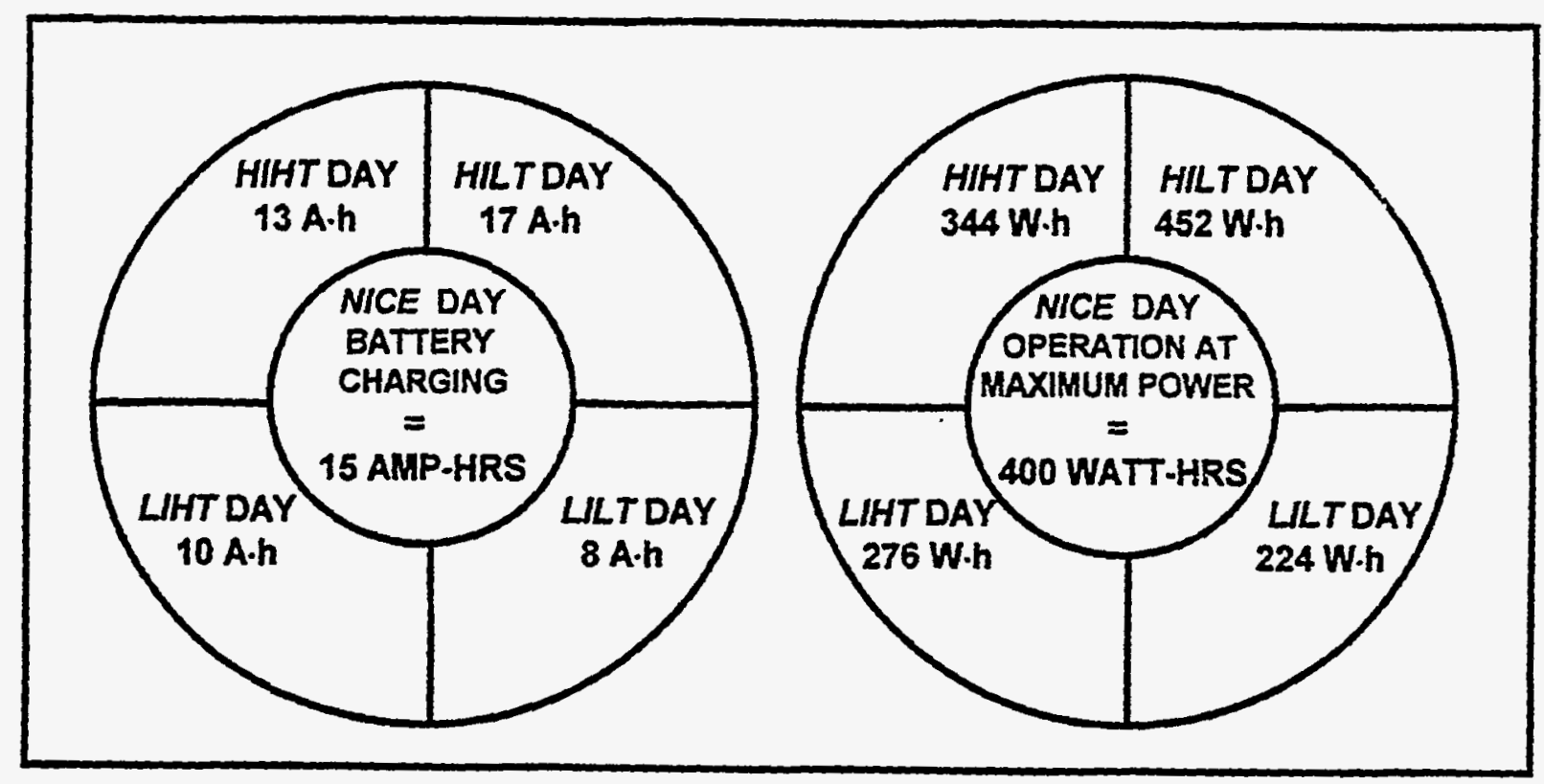

The following five types of reference days denoted by the acronyms HIHT, HIIT, LIHT, LILT, and NICE are defined with hourly tabulations of irradiance (direct with incidence angle and diffuse), ambient temperature, wind speed, spectral energy distribution, etc.

- High Irradiance, High Temperature (HIHT): This might be typical of a summer day in a desert southwest location. The peak irradiance is $1200 \mathrm{~W} \cdot \mathrm{m}^{-2}$, the peak temperature is $45^{\circ} \mathrm{C}\left(113^{\circ} \mathrm{F}\right)$, sunrise is at $5: 00 \mathrm{AM}$, and sunset is at 7:00 PM.

High Irradiance, Low Temperature (HIL I): This reference day is typical of a spring day in the mountain states. The peak irradiance is $1000 \mathrm{~W} \cdot \mathrm{m}^{-2}$, the peak temperanure is $6^{\circ} \mathrm{C}\left(43^{\circ} \mathrm{F}\right)$, sunrise is at $6: 00 \mathrm{AM}$, and sunset is at 6:00 PM.

- Low Irradiance, High Temperature (LIHT): This might be typical of a light overcast, but hot summer day in the plains states. The peak irradiance is only $600 \mathrm{~W} \cdot \mathrm{m}^{-2}$, the peak temperature is $30^{\circ} \mathrm{C}\left(95^{\circ} \mathrm{F}\right)$, sunrise is at $5: 00 \mathrm{AM}$, and sunset at 7:00 PM.

- Low Iradiance, Low Temperature (LIT): This reference day is typical of a winter day in the midwest with full overcast and cold ambient temperature. The peak irradiance is only $200 \mathrm{~W} \cdot \mathrm{m}^{-2}$ (all diffuse), the peak temperature of $0{ }^{\circ} \mathrm{C}\left(32^{\circ} \mathrm{F}\right)$, sunrise is at 7:00 AM, and sunset at 5:00 PM.

- Normal Irradiance, Cool Environment (NICE): This might be typical of an early summer day in a cool coastal region. The peak irradiance is $1000 \mathrm{~W} \cdot \mathrm{m}^{-2}$, the peak temperature is $18^{\circ} \mathrm{C}\left(66^{\circ} \mathrm{F}\right)$, sunrise is at $5: 00 \mathrm{AM}$, and sunset at 7:00 PM. Table 5 presents a sample illustrative tabulation for the NICE reference day.

Acknowledgments:

The author is grateful for the foresight, interest and guidance of Laxmi Mrig, the NREL program manager for this work. The author also wishes to thank Richard DeBlasio and Ben Kroposki of NREL for their technical assistance. In addition, a special thanks is extended to Steve Chalmers, Vice-Chairman of the IEEE SCC 21 committee for sparking this author's interest in the translation equation subject. This work was funded under NREL Subcontract No. TAD-4-14166-01. 


\title{
6.0 PV APPLICATIONS AND MARKET DEVELOPMENT
}

\author{
R. Taylor, Manager
}

During FY 1995 the PV Applications and Market Development Project continued to provide an NREL focus for support of the analytical, applications, and market development efforts of the DOE Photovoltaics Program and the Office of Solar Energy Conversion. The overall objective of this project is to conduct a coordinated set of market conditioning and project development activities (through NREL in-house research, NREL subcontracted research, and technical support to DOE Golden Field Office [GO] contracted efforts) that lead toward sustainable domestic and international markets for photovoltaic systems.

The project is divided into seven interrelated areas of activity, each led by an experienced NREL staff member, as shown in the table below. In many instances, the specific activities implemented within the PV Applications and Market Development Project cross both the activity and personnel boundaries shown below. This cross-fertilization is encouraged by both PV Program and NREL line management and in every case leads to better activities and products. The delineations listed below are largely for administrative convenience and to identify a "lead contact."

\begin{tabular}{|c|c|c|}
\hline \multicolumn{3}{|c|}{ Project Activity Areas } \\
\hline Project Activity & Contact Name & Telephone \\
\hline $\begin{array}{l}\text { NREL Project Management } \\
\text { DOE Headquarters staff support }\end{array}$ & $\begin{array}{l}\text { Roger Taylor } \\
\text { Doug Arent }\end{array}$ & $\begin{array}{l}(303) 384-6432 \\
(202) 651-7541 \\
\end{array}$ \\
\hline $\begin{array}{l}\text { Domestic Markets \& Utilities } \\
\text { PV-DSM Analysis } \\
\text { Utility support (UPVG, PV4U, SCE) }\end{array}$ & $\begin{array}{l}\text { Christy Herig } \\
\text { William Wallace }\end{array}$ & $\begin{array}{l}(303) 384-6546 \\
(303) 384-6476\end{array}$ \\
\hline $\begin{array}{l}\text { PV-Buildings } \\
\text { PV:BONUS } \\
\text { EPA Data Analysis }\end{array}$ & $\begin{array}{l}\text { Roger Taylor } \\
\text { Cecile Warner }\end{array}$ & $\begin{array}{l}\text { (303) } 384-6432 \\
(303) 384-6466 \\
\end{array}$ \\
\hline Sunrayce 95 & Byron Stafford & (303) 384-6426 \\
\hline PV Education, Assistance, and Training & John Thornton & (303) 384-6469 \\
\hline $\begin{array}{l}\text { International Markets } \\
\text { Brazil } \\
\text { China } \\
\text { India } \\
\text { South Africa } \\
\text { Russia } \\
\text { Egypt } \\
\text { Indonesia } \\
\text { Village Power Hybrid Team }\end{array}$ & $\begin{array}{l}\text { Roger Taylor } \\
\text { Cecile Warner } \\
\text { William Wallace } \\
\text { Jack Stone } \\
\text { Robert McConnell } \\
\text { Ken Touryan } \\
\text { Larry Kazmerski } \\
\text { Roger Taylor } \\
\text { Cecile Wamer }\end{array}$ & $\begin{array}{l}(303) 384-6432 \\
(303) 384-6466 \\
(303) 384-6476 \\
(303) 384-6470 \\
(303) 384-6419 \\
(303) 275-3009 \\
(303) 384-6600 \\
(303) 384-6432 \\
(303) 384-6466\end{array}$ \\
\hline
\end{tabular}

The accomplishments and ongoing efforts in each of these activity areas are briefly discussed in the following sections. 


\section{NREL Project Management}

Overall management of the PV Applications and Market Development project includes provision of full-time support to the DOE Office of Solar Energy Conversion and the DOE PV'Program.

\section{Domestic Markets and Utilities}

\section{PV-DSM Analysis}

NREL continues to lead the U.S. and international interest in analysis of PV as a demand-side management (DSM) option. Coordination and management of two key subcontracts, along with many meetings and interactions with the Utility Photovoltaic Group (UPVG) and others, has established NREL leadership in this area. Coupled with the product development activities under Photovoltaics Building Opportunities in the United States (PV:BONUS), PV as a DSM option is moving closer to reality.

The Center for Energy and Environmental Policy Research at the University of Delaware is conducting a project entitled: "Evaluation of DSM Opportunities for Photovoltaics." The objectives of this ongoing project are: 1) to evaluate existing residential, commercial and industrial DSM incentive programs with regard to incentive levels and utility avoided costs; 2) to analyze the interactions between incentive programs, avoided costs, and regulatory influences; 3) to develop a spreadsheet evaluation model to describe the economic costs and benefits to electric utilities and their customers; and 4) to develop and promote the role PV can play in residential and commercial DSM programs.

The Atmospheric Sciences Research Center. State University of New York at Albany continued a project entitled: "Solar Resource/Utility Load Matching Assessment." The objectives of this study are to 1) investigate the viability of using insolation data measured by geostationary satellites and to 2) infer the load matching capability of PV systems in selected electric utilities across the United States. By comparing PV load-matching capability estimated from the satellite data with that obtained from ground measurements, it has been shown that satellite data can be used to provide a good indication of PV effective load-carrying capability.

\section{DOE-GO Utility Support}

The PV Applications and Market Development group provides technical support to the DOE-GO activities related to commercialization of PV in the utility industry. During 1995, primary efforts went into critical review and evaluation of the UPVG/Technology Experience to Accelerate Markets in Utility Photovoltaics (TEAM-UP)/Photovoltaic Collaborative Market Project to Accelerate Commercial Technology (PV-COMPACT) proposals. In addition, activities conducted by Southern California Edison and Georgia Tech (for the 1996 Olympics) are supported by the group. An NREL staff addition during the first part of FY 1995 considerably enhanced this support to DOE-contracted programs. 


\section{PV_Buildings}

During 1993 a major multiyear, three-phase procurement was initiated that is directe.: at supporting the development of cost-effective PV products and product-supply/product-user relai:onships that provide a sustainable market for PV in the buildings sector. Titled PV:BONUS, the 5-year, $\$ 25$ million (DOE), government/industry cost-shared initiative is being contractually administered by DOE-GO as a financial assistance (cooperative agreement) program. The PV:BONUS program was fully under way during 1995 with Phase 2 product development efforts.

Four cooperative agreements constituted the PV:BONUS program.

Energy Conversion Devices (ECD) (along with subtier organizations United States Solar Systems Corporation (USSC), Arizona Public Service Company, Bechtel, Detroit Edison, MERRA, Michigan Public Service Company, Minoru Yamasaki Associates, National Association of Home Builders Research Center, Oakland CC, and the Solar Design Association) is developing two product lines: a standing seam metal roofing system and a shingle-like roofing system based on the ECD/USSC amorphous silicon/stainless-steel PV technology.

Delmarva Power \& Light (DP\&L) (along with subtier organizations University of Delaware, Ascension Technologies, and AC Battery Corp.) is conducting economic evaluations and product testing for a dispatchable PV system that may be marketed by DP\&L to commercial electric utility customers.

Solar Design Associates (along with subtier organizations Solarex Corporation, and Kawaneer) is developing a high-power (250-300 watt) AC PV module that will be totally self- contained, generating $120 \mathrm{~V}, 60 \mathrm{~Hz}$ power. Such a module can be paralleled together, individually power tracked, and connected directly into a building's electric service entrance or other appropriate electrical interface.

Fully Independent Residential Solar Technology, Inc. (along with subtier organizations Bradley Builders and Avis America, among others) is developing integrated PV energy systems for the modular home industry. These systems and homes will be marketed as stand-alone remote homes and as grid-connected units.

In addition to the direct support of GO for the PV:BONUS procurement, NREL has contracted with the American Institute of Architecture (AIA)/Research Council to expand the previously funded activity entitled: "Design for Photovoltaics: Curriculum Development for North American Architecture School Faculty" into a self-study course that will be available through AIA as part of their new continuing education requirement for AIA members. 


\section{Sunrayce 95}

Sunrayce is a DOE-sponsored biennial university design competition for solar - in wed electric vehicles. It is dedicated to the educational development of university science ist: engineering students with its themes of Education, Energy, and the Environment. A successful race from Indianapolis to Denver was held in June 1995.

\section{PV Education, Assistance, and Training}

The technical assistance, education and training activity provides for the continuation and enhancement of specific actions directed at accelerating the commercialization of currently costeffective PV products, supporting utilities and other organizations investigating the possible purchase of a PV system to meet the electrical needs of a particular site, and developing and providing educational materials for student training.

\section{International Market Development}

\section{Brazilian Rural Electrification Pilot Project}

The U.S. Department of Energy, Office of Solar Electric Conversion, through NREL, is undertaking a cooperative joint pilot project with eight states together with the Centro de Pesquisas de Energia Eletrica (CEPEL) in the Federal Republic of Brazil. The objective of the effort is to establish and assess the efficiency, operability, and reliability of solar-energy-based rural electrification. During 1995, a Phase 2 expanded program in six additional states was implemented that included two $50-\mathrm{kW}$ hybrid power systems. Completion of these hybrid systems is expected in 1996.

\section{China}

During 1995, NREL devoted considerable effort to assisting DOE in the establishment of project opportunities in China including development of four annexes to the DOE/Chinese State Science and Technology Commission protocol agreement. A project was launched in the province of Gansu and plans were made for additional cooperative efforts. NREL has also provided support to a World Bank mission focused on developing a renewable energy project in China.

\section{India}

During 1995, NREL devoted considerable effort to assisting DOE in the establishment of project opportunities in India, and an initial project with the Ramakrishna Mission was launched.

\section{$\underline{\text { Russia }}$}

At the request of DOE's Office of Technical and Financial Assistance (OTFA), project personnel travelled to Moscow to investigate possible collaborative project opportunities between DOE/NREL and Russian scientists and engineers. Project funds were provided by OTFA to investigate hybrid power system opportunities at several Russian sites during 1995, and to facilitate further collabor?tion and demonstrations with the InterSolarCenter in Moscow. 


\section{$\underline{\text { South Africa }}$}

At the request of DOE, NREL facilitated the development of a new black-owns $\wedge \mathrm{V}$ module manufacturing plant in cooperation with Spire Corporation and numerous South Africur agencies. The plant began operation in the fall of 1995.

\section{Village Power Hybrid team}

As part of the growth in village power opportunities, largely focused on diesel fuel displacement, an NREL Director's Development Fund award was provided to help establish a sustainable village power focus and test capability at the NREL National Wind Technology Center. The PV Applications and Market Development project has participated in this effort, which closely aligns with several ongoing (Brazil) and future (India, China, and Indonesia) hybrid project opportunities. 


\section{PV Applications and}

Market Development Subcontracts 

Photovoltaics in Buildings: Design Guidance for Practicing Architects and Design Professionals (AAX-5-14408-01)

\author{
AIA Research \\ 1735 New York Avenue, NW \\ Washington, DC 20006
}

Contributors: Deane Evans, Principle Investigator, AlA Research

Stephanie Vierra, Program Manager, ALA Research

Steven Strong and Robert Erb, Principal Instructors

\begin{abstract}
Program Objectives
In response to renewed interest in energy and the environment among architects in North America, AIA Research, under a grant from the National Renewable Energy Laboratory, conducted a series of 6 workshops in 1995 for architects in the U.S. on Building-integrated Photovoltaics. Concern for resource efficient, sustainable design had already risen dramatically in the early ' $90 \mathrm{~s}$. As a result, information on energy conservation strategies and techniques was available to the architectural profession. However, before the series of workshops was developed, there was little or no specific data on photovoltaics and their potential role in buildings. The goal of the workshops was to meet this information gap by developing and disseminating timely, stateof-practice guidance on PV in buildings to architects.
\end{abstract}

\title{
Approach to Developing the Program
}

To accomplish this goal the program built directly on the knowledge gained from the "Design for PV" curriculum course developed by AIA Research for architecture schools in the U.S. Specific components of the curriculum were incorporated into the workshops - specifically the existing slide set and several publications. A team of experts, Steven Strong, Principal and Robert Erb, Project Manager, both of Solar Design Associates in Harvard, Massachusetts was then identified to conduct the series of workshops. Mr. Strong has earned an international reputation for the pioneering integration of renewable energy systems - especially PV - with environmentally responsive building design. Mr. Erb, trained as an architect and planner is responsible for the detail design and construction oversight of building-integrated PV for the firm.

Each workshop covered basic information on: PV cell and module technology, PV systems, PV integrated into buildings, utility interfaces, economics and case studies. A set of resources was given to each workshop attendee including: Photovoltaic Fundamentals, Solar Radiation Data Manual, Building-Integrated Photovoltaics, and Building-Integrated Photovoltaics: $A$ Case Study.

The workshops were specifically designed to elicit feedback from the participants which aided in the refinement process of each workshop. The feedback was also intended to help inform and guide the possible development of a self-study course for architects as a follow-on effort to the workshops. The workshops also met the requirements of mandatory continuing education established in 1995 for AIA members.

Workshop locations were selected to ensure regional diversity and to represent a good cross section of locations throughout the U.S. Support was solicited from local AIA chapters and Committees on the Environment, local utilities, PV manufacturers and other interest groups identified as promising sources of "early adopters" of the technology. Utility involvement was 
especially important and resulted in increased linkages and synergy between the utilities and their local architectural communities.

The first workshop took place in Sacramento at the Sacramento Municipal Utility District service center. This workshop brought in local experts from SMUD (whose utility PV program is internationally respected) and from the local Committee on the Environment. A unique feature of the workshops was the introduction of a "real world" design problem, where the participants were asked to apply the knowledge they gained during the day to the design of a PV array. The audience of architects, many of whom were familiar with PV and SMUD's programs, provided critical feedback and commentary on the content and structure of the presentations. Questions and evaluations from the audience were recorded and used to tailor the following workshops.

Additional workshops took place in Atlanta, GA., Stamford, CT., Phoenix, AZ., Honolulu, HI., and Los Angeles, CA. At each workshop refinements to the program were made and new information was introduced as it became available from the industry and NREL.

\section{Program Results}

The workshops were decidedly a success. Over 300 architects and other building professionals attended, and their formal evaluations were consistently high. Many new linkages were formed in the process of the workshops including those of the utilities and PV industry to the architectural community. In addition, telephone calls and mail requests to AIA Research indicate continued demand within the profession for information and documentation on PV in buildings. To meet this demand, and to continue our efforts at keeping architects informed about PV, AIA Research has just begun a new design competition for architects and design professionals. on buildingintegrated PV. AIA Research hopes to incorporate the results of the competition, combined with core information from the workshops, into a self-study course on Building-Integrated Photovoltaics to be made available through the AlA's Continuing Education System. 
Title:

Organization:

Contributors:
Solar Resource Utility Load Matching . . ;essment

Atmospheric Sciences Research Center. Thic University at Albany, Albany, New York

Richard Perez, principal investigator, and Rober Seals

\section{OBJECTIVES}

The project was initiated in August 1991. Its initial objective was to estimate the effective capacity of photovoltaics (PV) for a sample of US utilities. This investigation required time coincident hourly electric load and local PV output data obtained via simulation from satellite remote sensing. Hence a secondary, but fundamental, objective of this work was to demonstrate the accuracy of satellite remote sensing for the considered application.

The project's objective was updated to include a larger sample of US utilities as well as "sub-utilities" -- buildings and substations. The rationale was to confirm and refine the relationships observed between load shapes and the effective capacity of PV-- i.e., knowing load shape parameters would be sufficient to estimate PV's effective capacity.

The current and future objectives this projects are:

(1) To continue increasing the experimental load sample in order to validate observed load shape/PV capacity relationships, with emphasis on small sub-utility loads. In this respect, data from nine EPA PV sites operated by Ascension Technology are being investigated.

(2) To make use of the above relationship to map PV's effective capacity in the United States.

(3) As a follow-on to (2), to study the year-to-year variability of PV's effective capacity maps as well as long term trends, applying of the observed load shape-based relationships to commercial utility data packages.

(4) To validate and apply this studies' finding internationally through the International Energy Agency's upcoming task on grid-connected PV.

\section{RESULTS AND DISCUSSION OF ACTIVITIES COMPLETED IN FY 95}

\section{Recap on past findings}

The following results have been documented during initial phases of this study:

- Satellite-based site/time hourly solar radiation data from climatologically representative years have been shown to be effective for addressing PV-utility interaction issues at arbitrary locations in the US.

- The effective capacity of PV was found to be substantially greater its capacity factor for many US utilities. PV's effective capacity was not found to be correlated with the solar resource. On the other hand, a strongly defined relationship between PV's effective capacity and simple load parameters was identified. 


\section{Update on current investigations}

A considerable amount of our effort this year was spent on producing the first PV capacity map of the United States. We hereby report on this investigation.

Effective Capacity Metrics: For a given utility or sub-utility, effective capacity is a measure of the availability of PV power that is available at peak time, and therefore, that can effectively contribute to localized and/or utility-wide generating capacity. The main parameter we use for quantifying PV capacity is the Effective Load Carrying Capability or ELCC originally introduced by [1]. We use a relative, normalized (generation mix-independent) version of the ELCC (see [2]) for our investigation. ELCC may range from $0 \%$ (no effective capacity) to $100 \%$ (effective capacity equals, installed capacity). Other parameters, such as the Minimum Buffer Energy Storage (MBES) have been introduced throughout this project to quantify load matching but are not considered for this initial mapping exercise. ELCC is dependent on the considered PV array geometry and level of load penetration [2]. For this preliminary map, we focus our attention on ideally tracking flat plate collectors, at a "modest" $2 \%$ load penetration.

Load Shape - Effective Capacity Relationship Between: A well defined relationship was observed between load shapes and effective capacity. This relationship is shown in Fig. 1. The load shape is characterized in terms of its "modified summer-to-winter peak load ratio" (a composite parameter based primarily on the load's summer-to-winter peak load ratio, but also accounting for time of peak, extent of evening shoulder, daily excursion, off-season load and load size). By contrast, ELCC was not found to be well correlated with the magnitude of the local solar resource as can be seen in Fig. 2.

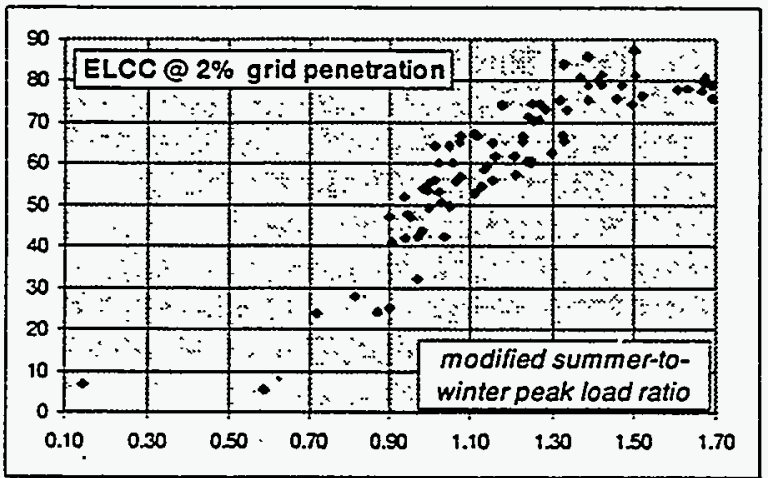

Figure 1: ELCC of $P V$ vs. load shape parameter based on the analysis of 82 utility and substation load-years

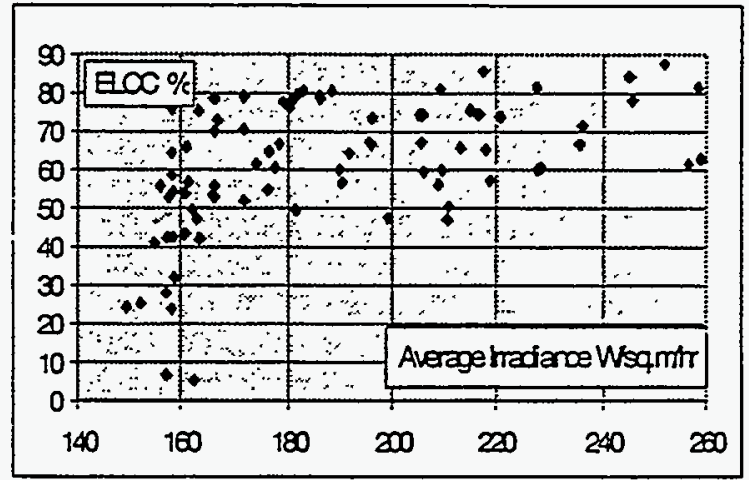

Figure 2: Effective capacity of $P V$ vs. overall solar resource

Mapping Methodology: Basic load shape data were obtained for over 500 utilities with peak loads in excess of $75 \mathrm{MW}$ for the year 1993. Direct application of the relationship observed between the summer-to-winter peak load ratio and PV capacity [2], provides an initial estimate of PV's effective capacity for each utility for the year 1993

Utility loads were gridded on a $2^{\circ}$ latitude-by $-2^{\circ}$ longitude map. Each utility was distributed into one or more grid cells, depending on the size and shape of its service territory and the location its major points of use (i.e., large cities). The resulting effective capacity of each grid cell was then estimated from a weighted average of utilities (or portion thereof) composing each cell. Finally, for display purposes, the $2^{\circ}$ resolution map was interpolated into a $1^{\circ}$ resolution map.

Results: A preliminary rendering of the map is given in Fig. 3 . The map features three main regions of high PV capacity: 


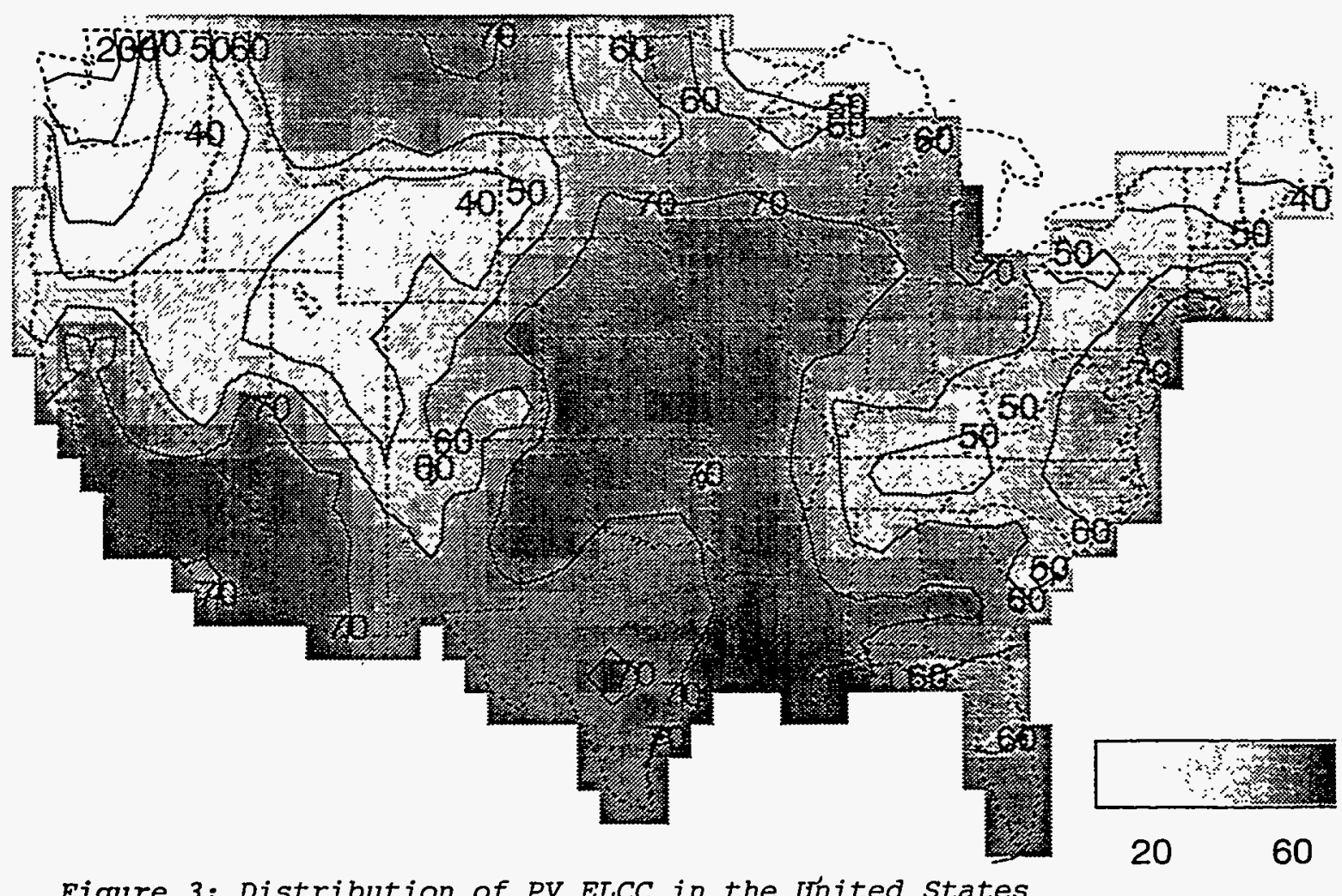

Figure 3: Distribution of PV ELCC in the United States

- The largest region covers the central US from the northwestern great plains and the metropolitan areas of Chicago and Detroit down to the lower Mississippi Valley. The core of this region features the highest capacities observed in the United States.

- The second region includes California, western Arizona, southern Nevada.

- The third region, the smallest geographically but very large in terms of installed utility capacity, spans the eastern metropolitan area extending from North Carolina to Boston.

The features of this map are fully consistent with the partial evidence gathered from the test utilities for which ELCC was formally derived. That is, regions of highest PV effective capacity do not always overlap with regions traditionally associated with solar energy development. For instance, there is overlap for California and Western Arizona, for the Pacific Northwest, the extreme northeast and of the north end of the Great Lakes. However, this is not the case for much of the western US and the extreme southeastern US, two traditional solar regions. There is no overlap either for the northem heartland and the eastern seaboard which had not been considered to be regions of high solar potential

The map does reflect key climatic and socio-economic realities, the combination of which is highly relevant to the effective capacity of PV. The regions of high effective capacity tend to match areas associated with strong summer heat waves (particularly in the case of the central US region). These regions may also be associated with areas where utility demand is driven by commercial air conditioning (particularly in the case of the eastern and western metropolitan regions), and where the electrical heating load is not significant (i.e., absence of cold weather conditions and/or use of other energy sources such as oil or gas). 

Significance: The map should constitute a useful source of information for the economic and technical assessment of PV's opportunity in the US. An example of possible application is sketched in Fig. 4 , merging the present capacity map, and the traditional solar resource (energy) map, with maps of capacity and energy value to identify the distribution of customer-sited grid-connected PV markets.

Beyond this preliminary map: The map in Fig. 3 is based only on 1993 data and could well reflect unusual [climatic or other] circumstances in some areas of the country, although it is doubtful that its overall structure should be questioned, given the consistency with of the present results the partial experimental evidence from

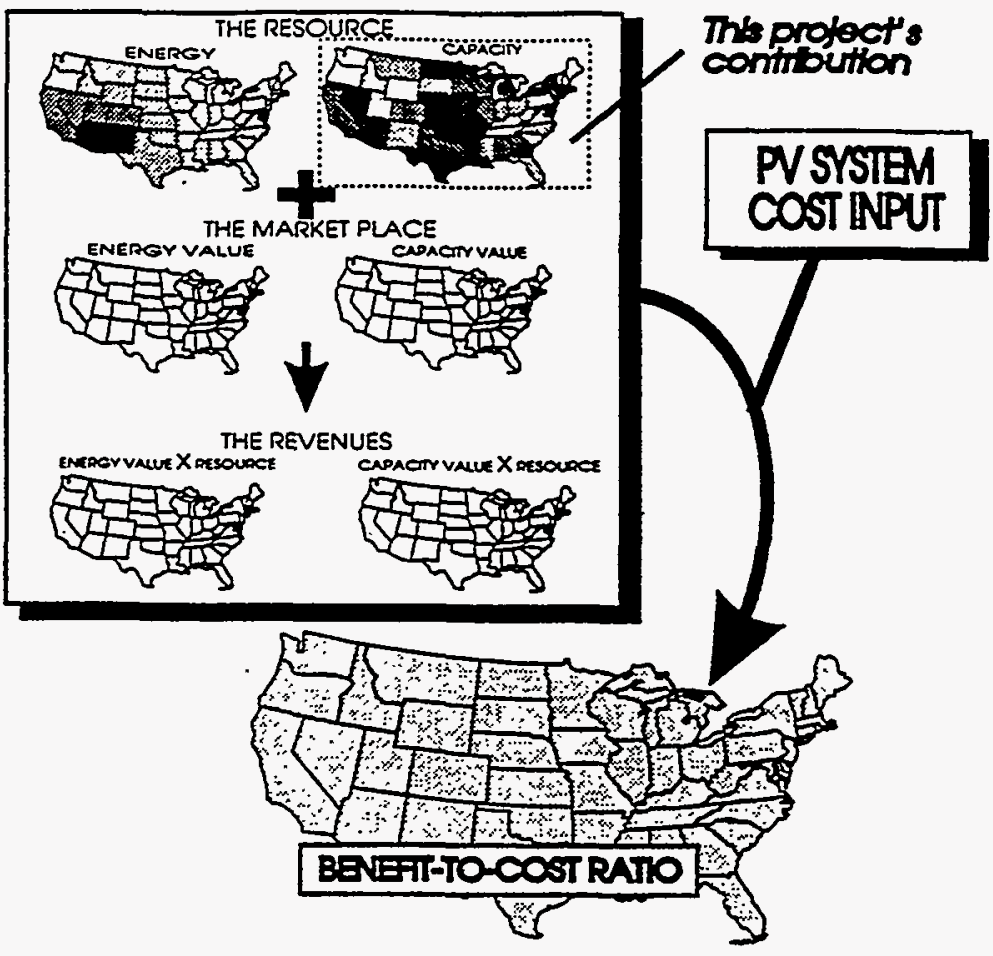

Figure 4: An example of how the information produced in this paper could contribute to a nation-wide assessment of $P V$ development opportunities. 1987 and 1988. Nevertheless, it would be important (and relatively straightforward) to repeat the exercise over several years in order to: (1) eliminate the risk of unusual circumstances, (2) observe year-to-year variability, and (3) identify possible trends toward (or away from) increased PV's effective capacity.

ACKNOWLEDGMENTS: This project was undertaken as a part of NREL contract XR-1-11168-1 with Roger Taylor, William Wallace, and Christy Herig as project managers. Thanks to David Renné and his team (NREL) and Dan Tarpley (NOAA) for their help throughout the load matching project. Thanks to Mike Bergman and the UPVG staff who played a key role in providing peak load data.

\section{REFERENCE}

1. L. Garver, (1966): Effective Load Carrying Capability of Generating Units. IEEE Transactions, Power Apparatus and Systems, Vol. Pas- 85 , no. 8

2. R. Perez, R. Seals and R. Stewart, (1993): Solar Resource - Utility Load Matching Assessment, Interim Subcontract Report no. NREL/TP-411-6292, NREL, Golden, CO and R. Perez, R. Seals and R. Stewart, (1994): Matching Utility Peak Loads with Photovoltaics, Proc. RENEW-94. (NESEA), Stamford, CT, USA 


\section{Partnership Financing}

CEEP elaborated three partnership financing options (Table 1). The first option assumes that the utility would make funds available to the customer to invest in the PV-DSM system. The customer would be charged an interest rate equal to the utility's after-tax weighted cost of capital. In addition, the utility would be paid $3 \%$ of the loan to cover any administrative expenses that the utility might incur in making the loan. The customer would utilize their electric bill savings and tax benefits to repay the loan. In this partnership case, the benefits of the system operated in the Mid-Atlantic region of the U.S. cover $85 \%$ of its costs-9 percentage points higher than the customer-owned and operated system. This calculation was based solely on traditional benefits.

If the system is sited in an area with a particularly high cost of service, the utility may receive "non-traditional" benefits. Because the magnitude of distributed benefits is extremely site-specific, we chose a conservative value of $\$ 150$ per kilowatt-year, based on results from five case-studies that estimated the value of distributed benefits for PV technologies. The full $16.3 \mathrm{~kW}$ credited capacity of a $10 \mathrm{~kW}$ PV system with storage was used to determine total distributed benefits over the 25-year life of the system. In addition, an environmental benefit was calculated by using the value of sulfur dioxide allowances being traded under the 1990 Clean Air Act Amendment (\$250/ton). We assumed that the utility would offer these non-traditional benefits to the customer as an incentive for investing in PV-DSM. Under this arrangement, PV-DSM would be cost-effective for Delmarva Power \& Light's commercial customers.

Table 1. Utility-Customer Partnership Options: ${ }^{a}$ Dispatchable PV-DSM for Delmarva Power \& Light

\begin{tabular}{|c|c|c|c|}
\hline \multicolumn{2}{|l|}{ Benefits (\$) } & \multicolumn{2}{|c|}{ Costs $(\$)$} \\
\hline \multicolumn{4}{|c|}{ Without Non-traditional Benefits (16.3 kW Credited Pealk-Shaving Capacity) } \\
\hline $\begin{array}{l}\text { Bill Savings } \\
\text { Tax Benefits and Deductions }\end{array}$ & $\begin{array}{l}\$ 40,250 \\
\$ 63,120\end{array}$ & $\begin{array}{l}\text { Capital Costs } \\
\text { O\&M Costs } \\
\text { Loan Servicing Costs }\end{array}$ & $\begin{array}{r}\$ 95,740 \\
\$ 23,510 \\
\$ 2,900\end{array}$ \\
\hline \multicolumn{4}{|c|}{ Benefit-Cost Ratio $=0.85$} \\
\hline \multicolumn{4}{|c|}{ With Non-traditional Benefits (16.3 kW Credited Peak-Shaving Capacity) ${ }^{b}$} \\
\hline $\begin{array}{l}\text { Bill Savings } \\
\text { Environmental Benefits } \\
\text { T\&D Benefits } \\
\text { Tax Benefits and Deductions } \\
\quad \text { Total }\end{array}$ & $\begin{array}{r}\$ 40,250 \\
\$ 300 \\
\$ 26,120 \\
\$ 63,120 \\
\$ 129,790 \\
\text { Benefit-Co }\end{array}$ & $\begin{array}{l}\text { Capital Costs } \\
\text { O\&M Costs } \\
\text { Loan Servicing Costs }\end{array}$ & $\begin{array}{r}\$ 95,740 \\
\$ 23,510 \\
\$ 2,900\end{array}$ \\
\hline \multicolumn{4}{|c|}{ With Efficiency Improvement and Cost Reduction (25.6 kW Credited Peak-Saving Capacity) ${ }^{c}$} \\
\hline $\begin{array}{l}\text { Bill Savings } \\
\text { Tax Benefits and Deductions }\end{array}$ & $\begin{array}{l}\$ 63,360 \\
\$ 43,810\end{array}$ & $\begin{array}{l}\text { Capital Costs } \\
\text { O\&eM Costs } \\
\text { Loan Servicing Costs }\end{array}$ & $\begin{array}{r}\$ 73,520 \\
\$ 32,000 \\
\$ 2,210\end{array}$ \\
\hline Total & $\begin{array}{c}\$ 107,170 \\
\text { Benefit-Cos }\end{array}$ & $\begin{array}{r}\text { Total } \\
\text { atio }=1.00\end{array}$ & $\$ 107,730$ \\
\hline
\end{tabular}

Notes: ${ }^{\mathrm{a}}$ : Benefits and costs are discounted at the rate of $7.99 \%$, assuming that the utility, or its subsidiary, would provide financing at the same rate. The loan rate now becomes the customer's opportumity cost of capital, thereby becoming the castomer's appropriate discount rate.

b: In the TRC test, the B/C ratio is also greater that one if tax savings are included as benefits.

c: Improved conversion efficiency (from 10 percent to 15 percent) and cost rectuctions for modules allow the purchase of a larger PV array (from $10 \mathrm{~kW}$ to $15.5 \mathrm{~kW}$ ). The system's credited penk-shaving capacity increases from $16.3 \mathrm{~kW}$ to $25.6 \mathrm{~kW}$, which requires additional storage $(84 \mathrm{kWh}$, instead of $50 \mathrm{kWh})$. O\&M expenses are higher due to the increased storage needs.

Source: Center for Energy and Environmental Policy. University of Delaware 
The third option considers the impacts of technological improvements and PV system cost reductions on the economic viability of PV-DSM. In particular, the PV AC-conversion efficiency was assumed to increase from 10 to 15 percent, while the cost of the PV array would decline by one-third. The efficiency gain would result in more peak-shaving capacity from the $105 \mathrm{~m}^{2} \mathrm{PV}$ array assumed in our base-case analysis (the system would now have a credited peak-shaving capacity of $25.6 \mathrm{~kW}$ and a rated capacity of $15.75 \mathrm{~kW}$ ). Under these assumptions, PV-DSM would be cost-effective even without a utility incentive.

\section{Task B: $\quad$ Spreadsheet Applications - Case Studies}

CEEP, in cooperation with NREL staff, identified five utilities in 1994 to participate as case studies. Utility-specific data were utilized in the PV-DSM SAT created by CEEP for the purpose of evaluating the economics of PV-DSM in the service territories of the five-study case utilities. The five utilities under investigation are diverse in terms of their geographic location, size, and rate structure. The participating utilities are Niagara Mohawk Power Corporation, Austin Electric, Sacramento Municipal Utility District, Delmarva Power \& Light, and an East-Coast Urban Utility. The performance of a 10 kW PV-DSM system with a 25-year life was assessed for use in the service territories of each of the casestudy utilities.

Our analysis showed that dispatchable PV had higher customer and utility value than nondispatchable systems in all five service territories. For this report, therefore, we have focused on the economic performance of dispatchable PV systems. The benefit-cost ratios of dispatchable systems for three investment options were compared: customer-owned; utility-owned; and Green Investment Fund (GIF). GIF calculations assumed that the customer contributes 80 percent of the potential bill savings and 100 percent of the tax savings available from the operation of the system. The utility was assumed to pay a rebate to the Fund equivalent to the avoided capacity costs (at zero capacity factor) of a gas-fired combustion turbine based on the credited capacity of the PV system. In the five utility service territories, the economics of dispatchable PV-DSM are more favorable for customer-owned than utility-owned systems (due largely to the tax treatment of capital investments afforded to customers). The GIF was the most economic vehicle for developing dispatchable PV-DSM.

\section{Task C: $\quad$ PV-DSM Workshop}

CEEP organized a PV-DSM Workshop with the objective of assembling the best available information on PV-DSM utility applications and transferring that information throughout the utility sector and to PV manufacturers. The workshop was closely coordinated with NREL and complemented the PV BONUS workshop, also held at the University of Delaware. The workshop concept was co-initiated by, and received enthusiastic support from, the PV BONUS program managers. The overall result of the workshop was to increase synergy between the PV-DSM and PV BONUS programs combining PV-DSM and PV building integration approaches to identify near-term applications.

The workshop was held on October 19-20, 1994 at the University of Delaware, and the contents were published in a proceedings titled Forging PV-Buildings Bridges: $A$ Joint NRELIDOE, PV-DSM and PV BONUS Workshop. The PV-DSM workshop included information on: i) the economics of PV-DSM, ii) the technical potential and performance of PV-DSM systems, iii) regulatory and other policy issues related to the commercialization of PV-DSM systems, iv) the status and use of the PV-DSM Spreadsheet Analysis Tool, v) a discussion of case study results from Task $B$, and vi) methodological and conceptual issues in the assessment of PV-DSM. 
CEEP staff have been working closely with researchers in the Institute of Policy and Management (IPM) at the Chinese Academy of Sciences in the collection of data and information needed for the study. So far, CEEP has collected the nation's and region's overall renewable resource data, including the geographical distribution of solar and wind resources, solar insolation and wind speed. CEEP has also collected site data in Inner Mongolia for both community- and household-based PV, wind, and PV-wind hybrid systems. These data include information on resource characteristics, population, appliance stock, and load profile (for both residential and commercial loads). In addition, system-level data were also obtained: technical and initial cost information, financial information for purchasing and operating renewable energy systems, and equivalent data on competing alternatives such as diesel generators and grid-extension systems.

In addition to data collection, during a visit to China in May 1995, CEEP staff interviewed several officials from both the central and local governments to discuss policy issues relevant to PV and other renewable energy utilization in China. CEEP staff have also had several research meetings with the IPM researchers in Beijing to review the collected data and to discuss additional data needs. In collaboration with IPM, CEEP also organized a one-day workshop in Beijing on May 12 with the objective of assembling Chinese expert evaluations and suggestions regarding the study's data collection strategy and research design.

\section{References}

1. J. Byrne, C. Govindarajalu, W. Kempton and Y-D. Wang. 1994. Emergency Power As an Additional Value From Dispatchable PV Peak Shaving. Prepared for the National Renewable Energy Laboratory. July. Center for Energy and Environmental Policy, University of Delaware.

2. J. Byrne, Y-D. Wang, R. Nigro and S. Letendre. 1994. "Photovoltaics in a Demand-Side Management Role." Proceedings of the ACEEE 1994 Summer Study on Energy Efficiency in Buildings. August. American Council for Energy-Efficient Economy. Washington, DC.

3. J. Byrne, S. Letendre, R. Nigro, C. Govindarajalu, W. Wallace and Y-D. Wang. 1994. "Photovoltaics For Demand-Side Management Utility Markets: A Utility/Customer Partnership Approach." Proceedings of the First World Conference on Photovoltaic Energy Conversion. December 4-9, Waikoloa, Hawaii.

4. J. Byrne, Y-D. Wang, S. Letendre, C. Govindarajalu, R. Nigro and W. Bottenberg. 1994. "Deployment of a Dispatchable Photovoltaic System: Technical and Economic Results." Proceedings of the First World Conference on Photovoltaic Energy Conversion. December 4-9, Waikoloa, Hawaii.

5. J. Byrne, A.M. Barnett, et al. 1994. "Dispatchable Commercial Building Photovoltaic DemandSide Management System Tests." Proceedings of the 12th European Photovoltaic Specialists Conference.

6. J. Byrne, S. Letendre, C. Govindarajalu and Y-D. Wang. 1995. "PV-DSM: Opportunities for Early Commercialization." Proceedings of the American Solar Energy Society's Solar 1995 Conference. Minneapolis, Minnesota.

7. J. Byrne, Y-D. Wang, R. Nigro and S. Letendre. 1995. "Photovoltaics: A Dispatchable PeakShaving Option." Public Utilities Fortnightly. September 1.

8. J. Byrne, S. Letendre, R. Nigro and Y-D. Wang. 1995. "Evaluating the Economics of Photovoltaics in a Demand-Side Management Role." Energy Policy. Forthcoming. 


\subsection{LIST OF ACTIVE SUBCONTRACTS}

\begin{tabular}{|c|c|c|c|c|}
\hline \multicolumn{5}{|c|}{ Subcontracts for FY 1995} \\
\hline $\begin{array}{l}\text { Contractor, Principal } \\
\text { Investigator, Address }\end{array}$ & $\begin{array}{c}\text { Work Title } \\
\text { (Research Activity) }\end{array}$ & $\begin{array}{l}\text { Contract } \\
\text { Number }\end{array}$ & $\begin{array}{l}\text { FY } 1995 \\
\text { Funding } \\
\text { (\$K) }\end{array}$ & $\begin{array}{l}\text { Start/End } \\
\text { Dates }\end{array}$ \\
\hline \multicolumn{5}{|c|}{ CRYSTALLINE SLICON AND ADVANCED DEVICES PROJECT } \\
\hline $\begin{array}{l}\text { California Institute of } \\
\text { Technology } \\
\text { H. Atwater } \\
\text { Pasadena, CA } 91125 \\
\end{array}$ & $\begin{array}{l}\text { Novel Low-Temperature } \\
\text { Substrate Technology for } \\
\text { Compound Semicon- } \\
\text { ductor Solar Cells }\end{array}$ & XAL-4-13357-01 & 75.0 & $\begin{array}{l}7 / 94 \\
7 / 97\end{array}$ \\
\hline $\begin{array}{l}\text { Duke University } \\
\text { T. Tan } \\
\text { Durham, NC } 27708\end{array}$ & $\begin{array}{l}\text { Influence of Self- } \\
\text { Interstitials Injected by } \\
\text { Phosphorus Diffusion on } \\
\text { Defect Structures and } \\
\text { Electronic Properties in } \\
\text { Crystalline Silicon }\end{array}$ & XD-2-11004-1 & 0.0 & $\begin{array}{l}3 / 92 \\
1 / 96\end{array}$ \\
\hline $\begin{array}{l}\text { Georgia Institute of Technology } \\
\text { A. Rohatgi } \\
\text { Atlanta, GA } 30332\end{array}$ & $\begin{array}{l}\text { Post-Growth Quality } \\
\text { Enhancement }\end{array}$ & XD-2-11004-2 & 64.8 & $\begin{array}{c}4 / 92 \\
12 / 95\end{array}$ \\
\hline $\begin{array}{l}\text { Johns Hopkins University } \\
\text { Dr. Searson } \\
\text { Baltimore, MD } 21218 \\
\end{array}$ & $\begin{array}{l}\text { Solar Electric Conversion } \\
\text { at Dye-Sensitized } \\
\text { Nanostructured Electrodes }\end{array}$ & $\mathrm{XAD}-3-12114-4$ & 175.6 & $\begin{array}{l}7 / 93 \\
7 / 96\end{array}$ \\
\hline $\begin{array}{l}\text { MIT } \\
\text { L. Kimerling } \\
\text { Cambridge, MA } 02139\end{array}$ & $\begin{array}{l}\text { Role of Point Defects and } \\
\text { Impurities in Processing : } \\
\text { and Performance of } \\
\text { Crystalline Silicon Solar } \\
\text { Cells. }\end{array}$ & $\begin{array}{l}\text { XD-2-11004-4 } \\
\therefore \therefore\end{array}$ & 96.5 & $\begin{array}{l}7 / 92 \\
7 / 95\end{array}$ \\
\hline $\begin{array}{l}\text { North Carolina State University } \\
\text { D. Han } \\
\text { Raleigh, NC } 27695\end{array}$ & $\begin{array}{l}\text { New Approaches for High- } \\
\text { Efficiency Cascade Solar } \\
\text { Cells }\end{array}$ & $\mathrm{XAD}-3-12114-5$ & 160.0 & $\begin{array}{l}8 / 93 \\
7 / 97\end{array}$ \\
\hline $\begin{array}{l}\text { North Carolina State University } \\
\text { G. Rozgonyi } \\
\text { Raleigh, NC } 27695\end{array}$ & $\begin{array}{l}\text { Electrical and Structural } \\
\text { Characterization of PV } \\
\text { Silicon Substrates }\end{array}$ & $\mathrm{XD}-2-11004-06$ & 41.5 & $\begin{array}{l}5 / 92 \\
6 / 95\end{array}$ \\
\hline $\begin{array}{l}\text { Princeton University } \\
\text { S. Forrest } \\
\text { Princeton, NJ } 08544\end{array}$ & $\begin{array}{l}\text { Crystalline Organic } \\
\text { Multiple Quantum-Well } \\
\text { Solar Cells }\end{array}$ & XAI-3-11167-03 & 0.0 & $\begin{array}{l}7 / 93 \\
1 / 96\end{array}$ \\
\hline
\end{tabular}




\begin{tabular}{|c|c|c|c|c|}
\hline \multicolumn{5}{|c|}{ Subcontracts for FY 1995} \\
\hline $\begin{array}{l}\text { Contractor, Principal } \\
\text { Investigator, Address }\end{array}$ & $\begin{array}{c}\text { Work Title } \\
\text { (Research Activity) }\end{array}$ & $\begin{array}{l}\text { Contract } \\
\text { Number }\end{array}$ & $\begin{array}{l}\text { FY } 1995 \\
\text { Funding } \\
\text { (\$K) }\end{array}$ & $\begin{array}{l}\text { Start/End } \\
\text { Dates }\end{array}$ \\
\hline $\begin{array}{l}\text { Research Triangle Institute } \\
\text { R. Vankatasubramanian } \\
\text { Research Triangle Park, NC } \\
27709\end{array}$ & $\begin{array}{l}\text { High-Efficiency GaAs } \\
\text { Solar Cells }\end{array}$ & YAL-4-13357-03 & 160.0 & $\begin{array}{l}8 / 94 \\
8 / 97\end{array}$ \\
\hline $\begin{array}{l}\text { Spire Corporation } \\
\text { S. Vernon } \\
\text { Bedford, MA } 01730\end{array}$ & $\begin{array}{l}\text { High-Performance } \\
\text { (Porous) Silicon Solar } \\
\text { Cells }\end{array}$ & XAI-3-11670-2 & 0.0 & $\begin{array}{l}9 / 93 \\
9 / 95\end{array}$ \\
\hline $\begin{array}{l}\text { SUNY at Buffalo } \\
\text { W. Anderson } \\
\text { Buffalo, NY } 14260 \\
\end{array}$ & $\begin{array}{l}\text { Thin-Film Silicon by } \\
\text { Constitutional } \\
\text { Supercooling }\end{array}$ & AAI-3-11167-01 & 9.8 & $\begin{array}{l}6 / 93 \\
6 / 95\end{array}$ \\
\hline $\begin{array}{l}\text { Texas Tech. University } \\
\text { S. Eistreicher } \\
\text { Lubbock, TX } 79409\end{array}$ & $\begin{array}{l}\text { Theoretical Analysis of } \\
\text { Hydrogen-Vacancy } \\
\text { Complex Formation and } \\
\text { Diffusion in Silicon }\end{array}$ & XAI-4-14195-01 & 0.0 & $\begin{array}{l}3 / 94 \\
3 / 95\end{array}$ \\
\hline $\begin{array}{l}\text { Texas Tech. University } \\
\text { S. Eistreicher } \\
\text { Lubbock, TX } 79409\end{array}$ & $\begin{array}{l}\text { Theoretical Analysis of } \\
\text { Hydrogen-Vacancy- } \\
\text { Impurity Formation and } \\
\text { Dissociation }\end{array}$ & $\mathrm{XAX}-5-15230-01$ & 17.0 & $\begin{array}{l}6 / 95 \\
4 / 96\end{array}$ \\
\hline $\begin{array}{l}\text { University of California at } \\
\text { Berkeley } \\
\text { E. Weber } \\
\text { Berkeley, CA } 94720\end{array}$ & $\begin{array}{l}\text { Identification and Control } \\
\text { of Lifetime-Reducing } \\
\text { Defects }\end{array}$ & $\mathrm{XD}-2-11004-3$ & 61.6 & $\begin{array}{l}7 / 92 \\
7 / 95\end{array}$ \\
\hline $\begin{array}{l}\text { University of California at } \\
\text { Los Angeles } \\
\text { E. Yablonovitch } \\
\text { Los Angeles, CA } 90095\end{array}$ & $\begin{array}{l}\text { Ultra-Efficient Epitaxial } \\
\text { Liftoff Solar Cells } \\
\text { Exploiting Optical } \\
\text { Confinement in the Wave } \\
\text { Limit }\end{array}$ & XAL-4-13357-02 & 106.0 & $\begin{array}{l}7 / 94 \\
7 / 97\end{array}$ \\
\hline $\begin{array}{l}\text { University of Florida at } \\
\text { Gainesville } \\
\text { T. Anderson } \\
\text { Gainesville, FL } 32611 \\
\end{array}$ & $\begin{array}{l}\text { New Plasma Source of } \\
\text { Hydrides }\end{array}$ & XCG-4-14194-01 & 0.0 & $\begin{array}{l}6 / 94 \\
8 / 95\end{array}$ \\
\hline $\begin{array}{l}\text { University of Illinois } \\
\text { A. Rockett } \\
\text { Urbana, } \mathbb{I L} 61801\end{array}$ & $\begin{array}{l}\text { Effects of Crystal Defects } \\
\text { on CuInSe }\end{array}$ & $\mathrm{XAD}-3-12114-1$ & 154.5 & $\begin{array}{l}6 / 93 \\
6 / 96\end{array}$ \\
\hline $\begin{array}{l}\text { University of South Florida } \\
\text { L. Jastrzebski } \\
\text { Tampa, FL } 33620\end{array}$ & $\begin{array}{l}\text { Optimization of Gettering } \\
\text { Processes for PV Silicon }\end{array}$ & $\mathrm{XD}-2-11004-05$ & 498.1 & $\begin{array}{l}5 / 92 \\
3 / 96\end{array}$ \\
\hline
\end{tabular}




\begin{tabular}{|c|c|c|c|c|}
\hline \multicolumn{5}{|c|}{ Subcontracts for FY 1995} \\
\hline $\begin{array}{l}\text { Contractor, Principal } \\
\text { Investigator, Address }\end{array}$ & $\begin{array}{c}\text { Work Title } \\
\text { (Research Activity) }\end{array}$ & $\begin{array}{l}\text { Contract } \\
\text { Number }\end{array}$ & $\begin{array}{c}\text { FY } 1995 \\
\text { Funding } \\
(\$ K)\end{array}$ & $\begin{array}{l}\text { Start/End } \\
\text { Dates }\end{array}$ \\
\hline $\begin{array}{l}\text { University of South Florida } \\
\text { D. Morel } \\
\text { Tampa, FL } 33620\end{array}$ & $\begin{array}{l}\text { Heterojunctions in Thin- } \\
\text { Film Solar Cells }\end{array}$ & $\mathrm{XAD}-3-12114-03$ & 182.4 & $\begin{array}{l}5 / 93 \\
5 / 96\end{array}$ \\
\hline $\begin{array}{l}\text { University of Utah } \\
\text { C. Taylor } \\
\text { Salt Lake City, UT } 84112\end{array}$ & $\begin{array}{l}\text { Electronic Processes in } \\
\text { Thin-Film PV Materials }\end{array}$ & $\mathrm{XAD}-3-12114-02$ & 218.8 & $\begin{array}{l}4 / 93 \\
6 / 96\end{array}$ \\
\hline $\begin{array}{l}\text { University of Washington } \\
\text { B. ORegan } \\
\text { Seattle, WA } 98105\end{array}$ & $\begin{array}{l}\text { Electrochemical } \\
\text { Fabrication of Dye- } \\
\text { Sensitized Heterojunction } \\
\text { for PV Energy Conversion }\end{array}$ & XCR-5-15234-01 & 39.9 & $\begin{array}{l}6 / 95 \\
6 / 96\end{array}$ \\
\hline \multicolumn{5}{|c|}{ THIN FILM PV TECHNOLOGIES PROJECT } \\
\hline $\begin{array}{l}\text { Astropower, Inc. } \\
\text { J. Rand } \\
\text { Newark, DE } 19716\end{array}$ & $\begin{array}{l}\text { Light-Trapped, } \\
\text { Interconnected, Silicon- } \\
\text { Film Modules }\end{array}$ & ZAF-5-14142-02 & 749.9 & $\begin{array}{l}11 / 94 \\
5 / 95\end{array}$ \\
\hline $\begin{array}{l}\text { Colorado School of Mines } \\
\text { J. Trefny } \\
\text { Golden, CO } 80401\end{array}$ & $\begin{array}{l}\text { Process of } \\
\text { Electrochemically } \\
\text { Deposited CdTe-Based } \\
\text { Solar Cells } \\
\end{array}$ & XAF-5-14142-11 & 216.8 & $\begin{array}{l}5 / 95 \\
4 / 98\end{array}$ \\
\hline $\begin{array}{l}\text { Colorado School of Mines } \\
\text { D. Williamson } \\
\text { Golden, CO } 80401\end{array}$ & $\begin{array}{l}\text { Microstructure of a-Si- } \\
\text { Based Solar Cell Materials } \\
\text { by Small-Angle X-ray } \because \therefore \\
\text { Scattering }\end{array}$ & $\begin{array}{l}\mathrm{XAN}-4-13318-04 \\
\therefore\end{array}$ & 41.0 & $\begin{array}{l}4 / 94 \\
4 / 97\end{array}$ \\
\hline $\begin{array}{l}\text { Colorado School of Mines } \\
\text { D: Williamson } \\
\text { Golden, CO } 80401\end{array}$ & $\begin{array}{l}\text { Process of Electro- } \\
\text { chemically Deposited } \\
\text { CdTe-Based Solar Cells }\end{array}$ & XAF-5-14142-11 & 216.8 & $\begin{array}{l}4 / 95 \\
4 / 98\end{array}$ \\
\hline $\begin{array}{l}\text { Colorado State University } \\
\text { J. Sites } \\
\text { Fort Collins, CO } 80525\end{array}$ & $\begin{array}{l}\text { Device Physics of Thin- } \\
\text { Film Polycrystalline Cells } \\
\text { and Modules }\end{array}$ & $\mathrm{XAX}-4-14000-01$ & 104.0 & $\begin{array}{l}12 / 93 \\
12 / 96\end{array}$ \\
\hline $\begin{array}{l}\text { Energy Conversion Devices } \\
\text { X. Deng } \\
\text { Troy, MI } 48084\end{array}$ & $\begin{array}{l}\text { Amorphous Silicon } \\
\text { Multijunction Cells }\end{array}$ & ZAN-4-13318-11 & 318.0 & $\begin{array}{l}7 / 94 \\
7 / 97\end{array}$ \\
\hline $\begin{array}{l}\text { Energy Photovoltaics } \\
\text { A. Delahoy } \\
\text { Princeton, NJ } 08543\end{array}$ & $\begin{array}{l}\text { Ultra-thin CIS Devices } \\
\text { with Non- } \mathrm{H}_{2} \mathrm{Se}\end{array}$ & XG-2-12051-1 & 43.1 & $\begin{array}{l}3 / 92 \\
2 / 95\end{array}$ \\
\hline
\end{tabular}


Subcontracts for FY 1995

\begin{tabular}{|c|c|c|c|c|}
\hline $\begin{array}{l}\text { Contractor, Principal } \\
\text { Investigator, Address }\end{array}$ & $\begin{array}{c}\text { Work Title } \\
\text { (Research Activity) }\end{array}$ & $\begin{array}{l}\text { Contract } \\
\text { Number }\end{array}$ & $\begin{array}{l}\text { FY } 1995 \\
\text { Funding } \\
\text { (\$K) }\end{array}$ & $\begin{array}{l}\text { Start/End } \\
\text { Dates }\end{array}$ \\
\hline $\begin{array}{l}\text { Energy Photovoltaics } \\
\text { A. Delahoy } \\
\text { Princeton, NJ } 08543\end{array}$ & $\begin{array}{l}\text { Amorphous Silicon } \\
\text { Multijunction Cells }\end{array}$ & ZAF-5-14142-04 & 953.4 & $\begin{array}{l}3 / 95 \\
1 / 98\end{array}$ \\
\hline $\begin{array}{l}\text { Florida Solar Energy Center } \\
\text { N. Dhere } \\
\text { Cocoa, FL } 32922\end{array}$ & $\begin{array}{l}\text { Polycrystalline } \mathrm{CuInSe}_{2} \\
\text { and CdTe PV Solar Cells }\end{array}$ & XG-2-11036-05 & 24.7 & $\begin{array}{c}4 / 92 \\
12 / 95\end{array}$ \\
\hline $\begin{array}{l}\text { Georgia Institute of Technology } \\
\text { A. Rohatgi } \\
\text { Atlanta, GA } 30332\end{array}$ & MOCVD CdTe Sputtering & XG-2-11036-03 & 63.7 & $\begin{array}{c}2 / 92 \\
11 / 95\end{array}$ \\
\hline $\begin{array}{l}\text { Golden Photon } \\
\text { S. Albright } \\
\text { Golden, CO } 80403 \\
\end{array}$ & $\begin{array}{l}\text { High-Efficiency, Large- } \\
\text { Area CdTe Panels }\end{array}$ & ZAF-5-14142-06 & 267.8 & $\begin{array}{l}8 / 95 \\
2 / 96\end{array}$ \\
\hline $\begin{array}{l}\text { Harvard University } \\
\text { R. Gordon } \\
\text { Cambridge, MA } 02138\end{array}$ & $\begin{array}{l}\text { Optimization of } \\
\text { Transparent and Reflecting } \\
\text { Films for a-Si Solar Cells }\end{array}$ & XAN-4-13318-05 & 163.8 & $\begin{array}{l}5 / 94 \\
5 / 97\end{array}$ \\
\hline $\begin{array}{l}\text { Harvard University } \\
\text { R. Gordon } \\
\text { Cambridge, MA } 02138 \\
\end{array}$ & $\begin{array}{l}\text { Examination of Devices } \\
\text { Incorporating Improved } \\
\mathrm{a-S \textrm {Si } _ { 1 1 - x }} \mathrm{Ge}_{x}: \mathrm{H} \text { of large } \mathrm{x}\end{array}$ & XCR-5-15253-01 & 21.0 & $\begin{array}{l}6 / 95 \\
12 / 95\end{array}$ \\
\hline $\begin{array}{l}\text { Iowa State University } \\
\text { V. Dalal } \\
\text { Ames, IA } 50011\end{array}$ & $\begin{array}{l}\text { Stability and Electronic } \\
\text { Properties of a-Si:H and } \\
\text { a-SiGe:H Alloys }\end{array}$ & XAN-4-13318-08 & 356.0 & $\begin{array}{l}6 / 94 \\
5 / 97\end{array}$ \\
\hline $\begin{array}{l}\text { ISET } \\
\text { V. Kapur } \\
\text { Inglewood, CA } 90301\end{array}$ & $\begin{array}{l}\text { Novel Two-Stage } \\
\text { Selenization Methods for } \\
\text { Fabrication of CIS Cells } \\
\text { and Submodules }\end{array}$ & YI-2-12069-1 & 0.0 & $\begin{array}{l}3 / 92 \\
4 / 95\end{array}$ \\
\hline $\begin{array}{l}\text { ISET } \\
\text { V. Kapur } \\
\text { Inglewood, CA } 90301\end{array}$ & $\begin{array}{l}\text { Application of CIS to } \\
\text { High-Efficiency PV } \\
\text { Module Fabrication }\end{array}$ & ZAF-5-14142-07 & 496.4 & $\begin{array}{l}4 / 95 \\
4 / 98\end{array}$ \\
\hline $\begin{array}{l}\text { National Institute of Standards } \\
\text { and Technology } \\
\text { A. Gallagher } \\
\text { Boulder, CO } 80303\end{array}$ & $\begin{array}{l}\text { Growth Mechanisms and } \\
\text { Characterization of a-Si:H } \\
\text { Alloy Films }\end{array}$ & DAD-4-14084-1 & 170.0 & $\begin{array}{l}4 / 94 \\
4 / 97\end{array}$ \\
\hline $\begin{array}{l}\text { Pennsylvania State University } \\
\text { R. Collins } \\
\text { University Park, PA } 16802\end{array}$ & $\begin{array}{l}\text { Wide-Band-Gap Solar } \\
\text { Cells with High Stabilized } \\
\text { Performance }\end{array}$ & XAN-4-13318-03 & 256.3 & $\begin{array}{l}7 / 94 \\
7 / 97\end{array}$ \\
\hline
\end{tabular}


Subcontracts for FY 1995

\begin{tabular}{|c|c|c|c|c|}
\hline $\begin{array}{l}\text { Contractor, Principal } \\
\text { Investigator, Address }\end{array}$ & $\begin{array}{c}\text { Work Title } \\
\text { (Research Activity) }\end{array}$ & $\begin{array}{l}\text { Contract } \\
\text { Number }\end{array}$ & $\begin{array}{c}\text { FY } 1995 \\
\text { Funding } \\
\text { (\$K) }\end{array}$ & $\begin{array}{l}\text { Start/End } \\
\text { Dates }\end{array}$ \\
\hline $\begin{array}{l}\text { Purdue University } \\
\text { J. Gray } \\
\text { West Lafayette, IN } 47907\end{array}$ & $\begin{array}{l}\text { Development and } \\
\text { Application of a Computer } \\
\text { Model for CdTe and } \\
\text { CuInSe }_{2}\end{array}$ & XG-2-11036-2 & 54.5 & $\begin{array}{l}3 / 92 \\
10 / 95\end{array}$ \\
\hline $\begin{array}{l}\text { Solar Cells, Inc. } \\
\text { T. Zhou } \\
\text { Toledo, OH } 43607\end{array}$ & $\begin{array}{l}\text { Technology Support for } \\
\text { Initiation of High- } \\
\text { Throughput Processing of } \\
\text { Thin-Film CdTe PV } \\
\text { Modules }\end{array}$ & ZAF-5-14142-05 & 300.0 & $\begin{array}{l}3 / 95 \\
8 / 95\end{array}$ \\
\hline $\begin{array}{l}\text { Solar Cells, Inc. } \\
\text { T. Zhou } \\
\text { Toledo, OH } 43607\end{array}$ & $\begin{array}{l}\text { Fabrication of Stable } \\
\text { Large-Area Thin-Film } \\
\text { CdTe PV Modules }\end{array}$ & ZR-1-11059-1 & 0.0 & $\begin{array}{l}5 / 91 \\
2 / 95\end{array}$ \\
\hline $\begin{array}{l}\text { Solarex Corporation } \\
\text { R. Arya } \\
\text { Newtown, PA } 18940\end{array}$ & $\begin{array}{l}\text { Amorphous Silicon } \\
\text { Multijunction Cells }\end{array}$ & ZAN-4-13318-01 & 400.0 & $\begin{array}{l}9 / 94 \\
9 / 97\end{array}$ \\
\hline $\begin{array}{l}\text { Solarex Corporation } \\
\text { R. Arya } \\
\text { Newtown, PA } 18940\end{array}$ & $\begin{array}{l}\text { Research on } \\
\text { Polycrystalline Thin-Film } \\
\text { Submodules Based on } \\
\text { CuInSe. }\end{array}$ & ZN-1-1019-4 & 0.0 & $\begin{array}{l}11 / 90 \\
8 / 95\end{array}$ \\
\hline $\begin{array}{l}\text { Syracuse University } \\
\text { E. Schiff } \\
\text { Syracuse, NY } 13244:\end{array}$ & $\begin{array}{l}\text { Research on High- } \\
\text { Bandgap Materials and } \\
\text { Amórphous-Silicon-Based } \\
\text { Solar Cells }\end{array}$ & $\begin{array}{c}\mathrm{XAN}-4-13318-06 \\
\cdots\end{array}$ & 103.0 & $\begin{array}{l}5 / 94 \\
4 / 97\end{array}$ \\
\hline $\begin{array}{l}\text { United Solar Systems } \\
\text { Corporation } \\
\text { S. Guha } \\
\text { Troy, MI } 48084 \\
\end{array}$ & $\begin{array}{l}\text { Amorphous Silicon } \\
\text { Research }\end{array}$ & ZAN-4-13318-02 & 420.0 & $\begin{array}{l}7 / 94 \\
7 / 97\end{array}$ \\
\hline $\begin{array}{l}\text { United Solar Systems } \\
\text { Corporation } \\
\text { S. Guha } \\
\text { Troy, MI } 48084\end{array}$ & $\begin{array}{l}\text { Thin Film a-Si Alloy } \\
\text { Research Partnership }\end{array}$ & ZAF-5-14142-01 & 583.0 & $\begin{array}{l}2 / 95 \\
6 / 96\end{array}$ \\
\hline $\begin{array}{l}\text { University of California at } \\
\text { Los Angeles } \\
\text { R. Braunstein } \\
\text { Los Angeles, CA } 90024\end{array}$ & $\begin{array}{l}\text { Amorphous Silicon } \\
\text { Photocharge Transfer }\end{array}$ & XAN-4-13318-10 & 15.0 & $\begin{array}{l}5 / 94 \\
5 / 97\end{array}$ \\
\hline
\end{tabular}




\begin{tabular}{|c|c|c|c|c|}
\hline \multicolumn{5}{|c|}{ Subcontracts for FY 1995} \\
\hline $\begin{array}{l}\text { Contractor, Principal } \\
\text { Investigator, Address }\end{array}$ & $\begin{array}{c}\text { Work Title } \\
\text { (Research Activity) }\end{array}$ & $\begin{array}{l}\text { Contract } \\
\text { Number }\end{array}$ & $\begin{array}{c}\text { FY } 1995 \\
\text { Funding } \\
\text { (\$K) }\end{array}$ & $\begin{array}{l}\text { Start/End } \\
\text { Dates }\end{array}$ \\
\hline $\begin{array}{l}\text { University of Central Florida } \\
\text { N. Dhere } \\
\text { Orlando, FL } 32816\end{array}$ & $\begin{array}{l}\text { Polycrystalline Thin-Film } \\
\text { CuInSe, and CdTe PV } \\
\text { Solar Cells }\end{array}$ & $X G-2-11036-05$ & 72.8 & $\begin{array}{l}10 / 95 \\
12 / 95\end{array}$ \\
\hline $\begin{array}{l}\text { University of Colorado } \\
\text { A. Hermann } \\
\text { Boulder, CO } 80309\end{array}$ & $\begin{array}{l}\text { Novel Thin-Film CuInSe } \\
\text { Fabrication }\end{array}$ & XC-0-10012-1 & 0.0 & $\begin{array}{c}3 / 90 \\
10 / 95\end{array}$ \\
\hline $\begin{array}{l}\text { University of Delaware-IEC } \\
\text { R. Birkmire } \\
\text { Newark, DE } 19716\end{array}$ & $\begin{array}{l}\text { Thin-Film Materials and } \\
\text { Devices Processing and } \\
\text { Modeling Issues for Thin- } \\
\text { Film Solar Cell Devices }\end{array}$ & XAV-3-13170-01 & $1,415.5$ & $\begin{array}{l}12 / 93 \\
1 / 97\end{array}$ \\
\hline $\begin{array}{l}\text { University of Florida } \\
\text { T. Anderson } \\
\text { Gainesville, FL } 32611\end{array}$ & $\begin{array}{l}\text { Processing of CIS-Based } \\
\text { Solar Cells: } \\
\text { Characterization of } \\
\text { Deposition Processes in } \\
\text { Terms of Chemical } \\
\text { Reaction Analyses } \\
\end{array}$ & XAF-5-14142-10 & 250.0 & $\begin{array}{l}5 / 95 \\
5 / 98\end{array}$ \\
\hline $\begin{array}{l}\text { University of North Carolina } \\
\text { H. Daxing } \\
\text { Chapel Hill, NC } 27514\end{array}$ & $\begin{array}{l}\text { The Factors Governing the } \\
\text { Staebler-Wronski } \\
\text { Photodegradation Effect in } \\
\text { a-Si:H Solar Cells/ } \\
\text { Theoretical and } \\
\text { Experimental }\end{array}$ & XAN-4-13318-09 & 103.0 & $\begin{array}{l}7 / 94 \\
5 / 97\end{array}$ \\
\hline $\begin{array}{l}\text { University of Oregon } \\
\text { D. Cohen } \\
\text { Eugene, OR } 97403\end{array}$ & $\begin{array}{l}\text { Identifying Electronic } \\
\text { Properties Relevant to } \\
\text { Improving Stability in } \\
\text { a-Si:H-Based Cells and } \\
\text { Overall Performance in } \\
\text { a-SiGe:H-Based Cells }\end{array}$ & XAN-4-13318-07 & 123.0 & $\begin{array}{l}4 / 94 \\
4 / 97\end{array}$ \\
\hline $\begin{array}{l}\text { University of South Florida } \\
\text { D. Morel } \\
\text { Tampa, FL } 33620\end{array}$ & $\begin{array}{l}\text { Advanced Processing of } \\
\text { CdTe and CuInGaSe } e_{2}^{-} \\
\text {Based Solar Cells and } \\
\text { Submodules }\end{array}$ & XAF-5-14142-09 & 286.6 & $\begin{array}{l}4 / 95 \\
4 / 98\end{array}$ \\
\hline $\begin{array}{l}\text { University of Toledo } \\
\text { A. Campaan } \\
\text { Toledo, OH } 43606\end{array}$ & $\begin{array}{l}\text { High-Efficiency Thin-Film } \\
\text { Cadmium-Telluride-Based } \\
\text { Solar Cells }\end{array}$ & $Z A X-4-14013-01$ & 125.0 & $\begin{array}{l}1 / 94 \\
1 / 97\end{array}$ \\
\hline
\end{tabular}


Subcontracts for FY 1995

\begin{tabular}{|c|c|c|c|c|}
\hline $\begin{array}{l}\text { Contractor, Principal } \\
\text { Investigator, Address }\end{array}$ & $\begin{array}{c}\text { Work Title } \\
\text { (Research Activity) }\end{array}$ & $\begin{array}{l}\text { Contract } \\
\text { Number }\end{array}$ & $\begin{array}{c}\text { FY } 1995 \\
\text { Funding } \\
\text { (\$K) }\end{array}$ & $\begin{array}{l}\text { Start/End } \\
\text { Dates }\end{array}$ \\
\hline $\begin{array}{l}\text { University of Toledo } \\
\text { A. Campaan } \\
\text { Toledo, OH } 43606\end{array}$ & $\begin{array}{l}\text { Optimization of Laser } \\
\text { Scribing for Thin-Film PV } \\
\text { Modules }\end{array}$ & $Z A F-5-14142-08$ & 125.0 & $\begin{array}{r}4 / 95 \\
4 / 97\end{array}$ \\
\hline $\begin{array}{l}\text { Washington State University } \\
\text { L. Olsen } \\
\text { Richland, WA } 99352 \\
\end{array}$ & $\begin{array}{l}\text { CuInSe } e_{2} \text { Solar Cells Based } \\
\text { on } \mathrm{ZnSe} \text { Windows }\end{array}$ & XAG-2-11036-06 & $\begin{array}{c}35.0 \\
\quad: \ldots\end{array}$ & $\begin{array}{c}2 / 92 \\
10 / 95\end{array}$ \\
\hline \multicolumn{5}{|c|}{ PHOTOVOLTAIC MANUFACTURING TECHNOLOGY (PVMaT) PROJECT } \\
\hline $\begin{array}{l}\text { Ascension Technology, Inc. } \\
\text { E. Kern } \\
\text { Waltham, MA } 01773\end{array}$ & $\begin{array}{l}\text { Manufacture of an AC } \\
\text { Photovoltaic Module }\end{array}$ & ZAF-5-14271-05 & 189.4 & $\begin{array}{l}7 / 95 \\
9 / 97\end{array}$ \\
\hline $\begin{array}{l}\text { Astro Power, Inc. } \\
\text { J. Rand } \\
\text { Newark, DE } 19711 \\
\end{array}$ & Silicon-Film ${ }^{\mathrm{TM}}$ PVMaT & ZM-2-11040-5 & $1,158.8$ & $\begin{array}{l}1 / 92 \\
7 / 95\end{array}$ \\
\hline $\begin{array}{l}\text { Astro Power, Inc. } \\
\text { J. Rand } \\
\text { Newark, DE } 19711\end{array}$ & $\begin{array}{l}\text { Large-Area Silicon-Film }{ }^{T M} \\
\text { Panels and Solar Cells }\end{array}$ & ZAF-5-14271-03 & 841.3 & $\begin{array}{l}6 / 95 \\
12 / 95\end{array}$ \\
\hline $\begin{array}{l}\text { Energy Conversion Devices } \\
\text { M. Izu } \\
\text { Troy, MI } 48084\end{array}$ & $\begin{array}{l}\text { Continuous Roll-to-Roll } \\
\text { a-Si PVMaT }\end{array}$ & ZM-2-11040-7 & 0.0 & $\begin{array}{l}1 / 92 \\
11 / 95\end{array}$ \\
\hline $\begin{array}{l}\text { ENTECH, Inc. } \\
\text { M. ONeill } \\
\text { Dallas/Ft. Worth Airport, TX } \\
75261\end{array}$ & $\begin{array}{l}\text { PVMaT Improvements for } \\
\text { ENTECH's Concentrator } \\
\text { Module }\end{array}$ & $Z M-2-11040-4$ & 0.0 & $\begin{array}{c}2 / 92 \\
12 / 95 \\
\vdots \\
0\end{array}$ \\
\hline $\begin{array}{l}\text { Golden Photon, Inc. } \\
\text { J. Brines } \\
\text { Golden, CO } 80401\end{array}$ & $\begin{array}{l}\text { Commercial Production of } \\
\text { Thin-Film CdTe PV } \\
\text { Modules }\end{array}$ & ZAI-4-11294-203 & 14300 & $\begin{array}{r}1 / 94 \\
4 / 95\end{array}$ \\
\hline $\begin{array}{l}\text { Iowa Thin Film Technology } \\
\text { F. Jeffrey } \\
\text { Ames, IA } 50010 \\
\end{array}$ & $\begin{array}{l}\text { PVMaT Monolithic a-Si } \\
\text { Modules on Continuous } \\
\text { Polymer Substrates }\end{array}$ & ZAF-5-14271-04 & 485.3 & $\begin{array}{l}7 / 95 \\
1 / 96\end{array}$ \\
\hline $\begin{array}{l}\text { Omnion Power Engineer Corp. } \\
\text { D. Porter } \\
\text { East Troy, WI } 53120\end{array}$ & $\begin{array}{l}\text { Three-Phase Power } \\
\text { Conversion System for } \\
\text { Utility Interconnected PV } \\
\text { Applications }\end{array}$ & ZAF-5-14271-02 & 371.9 & $\begin{array}{l}8 / 95 \\
8 / 97\end{array}$ \\
\hline $\begin{array}{l}\text { Siemens Solar Industries } \\
\text { T. Jester } \\
\text { Camarillo, CA } 93011\end{array}$ & $\begin{array}{l}\text { Photovoltaic } \mathrm{Cz}-\mathrm{Si} \\
\text { Manufacturing Technology } \\
\text { Improvements }\end{array}$ & ZM-1-11040-1 & 0.0 & $\begin{array}{l}3 / 92 \\
8 / 95\end{array}$ \\
\hline
\end{tabular}




\begin{tabular}{|c|c|c|c|c|}
\hline \multicolumn{5}{|c|}{ Subcontracts for FY 1995} \\
\hline $\begin{array}{l}\text { Contractor, Principal } \\
\text { Investigator, Address }\end{array}$ & $\begin{array}{c}\text { Work Title } \\
\text { (Research Activity) }\end{array}$ & $\begin{array}{l}\text { Contract } \\
\text { Number }\end{array}$ & $\begin{array}{l}\text { FY } 1995 \\
\text { Funding } \\
\text { (\$K) }\end{array}$ & $\begin{array}{l}\text { Start/End } \\
\text { Dates }\end{array}$ \\
\hline $\begin{array}{l}\text { Solar Cells, Inc. } \\
\text { D. Sandwisch } \\
\text { Toledo, OH } 43607\end{array}$ & $\begin{array}{l}\text { High-Throughput } \\
\text { Manufacturing of Thin- } \\
\text { Film CdTe PV Modules }\end{array}$ & ZAI-4-11294-02 & 100.0 & $\begin{array}{l}11 / 93 \\
11 / 96\end{array}$ \\
\hline $\begin{array}{l}\text { Solar Electric Specialties } \\
\text { Company } \\
\text { Willits, CA } 95450\end{array}$ & $\begin{array}{l}\text { Design, Fabrication, and } \\
\text { Certification of Advanced } \\
\text { Modular PV Power } \\
\text { Systems }\end{array}$ & ZAF-5-14271-07 & 155.2 & $\begin{array}{l}9 / 95 \\
9 / 97\end{array}$ \\
\hline $\begin{array}{l}\text { Solarex Corporation } \\
\text { R. Oswald } \\
\text { Newtown, PA } 18940\end{array}$ & $\begin{array}{l}\text { Large-Area Triple- } \\
\text { Junction a-Si Alloy } \\
\text { Production Scale-up } \\
\text { Project }\end{array}$ & ZM-2-1 1040-2 & 0.0 & $\begin{array}{l}3 / 92 \\
12 / 95\end{array}$ \\
\hline $\begin{array}{l}\text { Solarex Corporation } \\
\text { J. Wohlgemuth } \\
\text { Frederick, MD } 21754\end{array}$ & $\begin{array}{l}\text { PVMaT Phase 2B- } \\
\text { Process-Specific Issues }\end{array}$ & ZAI-4-11294-01 & $1,134.3$ & $\begin{array}{l}12 / 93 \\
12 / 96\end{array}$ \\
\hline $\begin{array}{l}\text { Spire Corporation } \\
\text { M. Nowlan } \\
\text { Bedford, MA } 01730\end{array}$ & $\begin{array}{l}\text { Automated Solar Cell } \\
\text { Assembly Teamed Process } \\
\text { Research }\end{array}$ & ZAG-3-11219-01 & 127.0 & $\begin{array}{c}1 / 93 \\
10 / 95\end{array}$ \\
\hline $\begin{array}{l}\text { Springborn Laboratories } \\
\text { W. Holley } \\
\text { Enfield, CT } 06082\end{array}$ & $\begin{array}{l}\text { Advanced Development of } \\
\text { PV Encapsulants }\end{array}$ & ZAG-3-11219-02 & 312.5 & $\begin{array}{l}12 / 92 \\
9 / 96\end{array}$ \\
\hline $\begin{array}{l}\text { Texas Instruments } \\
\text { K. Schmit } \\
\text { Dallas, TX } 75243\end{array}$ & $\begin{array}{l}\text { PVMaT, Phase 2B- } \\
\text { pheral Solar Technology }\end{array}$ & ZAI-4-11294-04 & 0.0 & $\begin{array}{l}1 / 94 \\
1 / 95\end{array}$ \\
\hline $\begin{array}{l}\text { Trace Engineering } \\
\text { C. Freitas } \\
\text { Arlington, WA } 98223\end{array}$ & $\begin{array}{l}\text { 2-kW DC to AC Power } \\
\text { Inverter Module for PV } \\
\text { Applications }\end{array}$ & ZAF-5-14271-08 & 83.7 & $\begin{array}{l}9 / 95 \\
9 / 97\end{array}$ \\
\hline $\begin{array}{l}\text { Utility Power Group } \\
\text { G. Duran } \\
\text { Chatsworth, CA } 91311\end{array}$ & $\begin{array}{l}\text { Amorphous Silicon PV } \\
\text { Manufacturing } \\
\text { Technology-2A }\end{array}$ & ZM-1-11040-6 & 0.0 & $\begin{array}{l}4 / 92 \\
12 / 95\end{array}$ \\
\hline $\begin{array}{l}\text { Utility Power Group } \\
\text { M. Stern } \\
\text { Chatsworth, CA } 91311\end{array}$ & $\begin{array}{l}\text { Low-cost Integrated } \\
20-\mathrm{kW} \text { AC Solar-Tracking } \\
\text { Subarray for Grid- } \\
\text { Connected PV Power } \\
\text { System Applications }\end{array}$ & ZAF-5-14271-06 & 247.7 & $\begin{array}{l}7 / 95 \\
1 / 96\end{array}$ \\
\hline
\end{tabular}




\begin{tabular}{|c|c|c|c|c|}
\hline \multicolumn{5}{|c|}{ Subcontracts for FY 1995} \\
\hline $\begin{array}{l}\text { Contractor, Principal } \\
\text { Investigator, Address }\end{array}$ & $\begin{array}{c}\text { Work Title } \\
\text { (Research Activity) }\end{array}$ & $\begin{array}{l}\text { Contract } \\
\text { Number }\end{array}$ & $\begin{array}{l}\text { FY } 1995 \\
\text { Funding } \\
\text { (\$K) }\end{array}$ & $\begin{array}{l}\text { Start/End } \\
\text { Dates }\end{array}$ \\
\hline \multicolumn{5}{|c|}{ PV MODULE AND SYSTEM PERFORMANCE AND ENGINEERING PROJECT } \\
\hline $\begin{array}{l}\text { Arizona State University, } \\
\text { R. Hammond } \\
\text { Tempe, AZ } 85282\end{array}$ & $\begin{array}{l}\text { Lab Accreditation Module } \\
\text { Certification Criteria }\end{array}$ & AAF-4-13301-01 & 85.0 & $\begin{array}{l}10 / 94 \\
11 / 95\end{array}$ \\
\hline $\begin{array}{l}\text { Colorado Piping and Mechanical } \\
\text { A. White } \\
\text { Denver, CO } 80210\end{array}$ & $\begin{array}{l}\text { Outdoor Test Facility } \\
\text { Outfitting }\end{array}$ & ACL-5-15644-01 & 353.9 & $\begin{array}{l}8 / 95 \\
10 / 95\end{array}$ \\
\hline $\begin{array}{l}\text { Endecon } \\
\text { C. Witaker } \\
\text { San Ramon, CA } 94583\end{array}$ & $\begin{array}{l}\text { PV Energy Rating } \\
\text { Methodology } \\
\text { Development }\end{array}$ & AAI-4-14192-01 & 0.0 & $\begin{array}{l}5 / 94 \\
8 / 95\end{array}$ \\
\hline $\begin{array}{l}\text { ISCE, Inc. } \\
\text { J. Leckenby } \\
\text { Englewood, CO } 80112\end{array}$ & $\begin{array}{l}\text { Purchase and Installation } \\
\text { of Casework and Fume } \\
\text { Hoods for OTF }\end{array}$ & ACM-5-15454-01 & 50.6 & $\begin{array}{l}7 / 95 \\
10 / 95\end{array}$ \\
\hline $\begin{array}{l}\text { New Mexico State University } \\
\text { A. Rosenthal } \\
\text { Las Cruces, NM } 88003\end{array}$ & $\begin{array}{l}\text { Long-Term Environ- } \\
\text { mental Effects on Roof- } \\
\text { Mounted PV Modules }\end{array}$ & XAX-5-14448-01 & 0.0 & $\begin{array}{l}11 / 94 \\
2 / 96\end{array}$ \\
\hline $\begin{array}{l}\text { Pennsylvania State University } \\
\text { D.L. Allara } \\
\text { University Park, PA }\end{array}$ & $\begin{array}{l}\text { PV Module Degradation-- } \\
\text { EVA Pore Volume }\end{array}$ & $\mathrm{XAX}-4-13471-01$ & 22.0 & $\begin{array}{c}11 / 93 \\
6 / 96\end{array}$ \\
\hline $\begin{array}{l}\text { SEIA } \\
\text { K. Sheinkopf } \\
\text { Washington, DC } 20001\end{array}$ & $\begin{array}{l}\text { National Electric Code } \\
\text { (NEC Support) }\end{array}$ & HI-2-12006-27 & 0.0 & $\begin{array}{l}6 / 94 \\
6 / 95\end{array}$ \\
\hline $\begin{array}{l}\text { SEIA } \\
\text { K. Sheinkopf } \\
\text { Washington, DC } 20001\end{array}$ & $\begin{array}{l}\text { Management and } \\
\text { Administration of } \\
\text { IEC/PV/TEC-83 } \\
\text { Secretariat and } \\
\text { International Standards } \\
\text { Development }\end{array}$ & HII-2-12006-9 & 0.0 & $\begin{array}{l}6 / 94 \\
6 / 95\end{array}$ \\
\hline $\begin{array}{l}\text { Sunset Technology } \\
\text { J. Anderson } \\
\text { Thousand Oaks, CA } 91362\end{array}$ & PV Performance Modeling & TAD-4-14166-01 & 0.0 & $\begin{array}{l}3 / 94 \\
3 / 95\end{array}$ \\
\hline $\begin{array}{l}\text { SWTDI/SWRES } \\
\text { S. Durand } \\
\text { Las Cruces, NM }\end{array}$ & $\begin{array}{l}\text { Long-Term Effects on } \\
\text { Roof-Mounted } \\
\text { Photovoltaic Modules }\end{array}$ & $\mathrm{XAX}-5-14448-01$ & 23.5 & $\begin{array}{l}11 / 94 \\
2 / 96\end{array}$ \\
\hline
\end{tabular}


Subcontracts for FY 1995

Contractor, Principal

Investigator, Address

Work Title
(Research Activity)

Contract

Number

FY 1995

Start/End

Funding

Dates

(\$K)

PV APPLICATIONS AND MARKETS DEVELOPMENT PROJECT

\begin{tabular}{|c|c|c|c|c|}
\hline $\begin{array}{l}\text { AIA/ACSA Research Council } \\
\text { D. Evans } \\
\text { Washington, DC } 20006\end{array}$ & $\begin{array}{l}\text { PV in Buildings Design } \\
\text { Guidance for Architects }\end{array}$ & AAX-5-14408-01 & 68.6 & $\begin{array}{l}10 / 94 \\
8 / 95\end{array}$ \\
\hline $\begin{array}{l}\text { American Wind Energy } \\
\text { Association } \\
\text { K. Rackstraw } \\
\text { Washington, DC } 20001\end{array}$ & $\begin{array}{l}\text { Brazilian Anemometer } \\
\text { Loan Program }\end{array}$ & ACO-5-15224-1 & 43.2 & $\begin{array}{l}7 / 95 \\
7 / 96\end{array}$ \\
\hline $\begin{array}{l}\text { Ascension Technology, Inc. } \\
\text { E. Kern } \\
\text { Lincoln Center, MA } 01773\end{array}$ & $\begin{array}{l}\text { Brazil Meteorological } \\
\text { Instrumentation Data } \\
\text { Collection, and EPA PV } \\
\text { Analysis }\end{array}$ & ACR-5-15285-01 & 262.1 & $\begin{array}{l}8 / 95 \\
2 / 96\end{array}$ \\
\hline $\begin{array}{l}\text { Bergey Windpower Company } \\
\text { M. Bergey } \\
\text { Norman, OK } 73069\end{array}$ & $\begin{array}{l}\text { Brazilian Rural } \\
\text { Electrification Pilot } \\
\text { Project_Phase } 2\end{array}$ & AAD-5-13504-05 & 4.3 & $\begin{array}{l}10 / 94 \\
10 / 95\end{array}$ \\
\hline $\begin{array}{l}\text { Centro De Pesquisas De Energia } \\
\text { Electrica (CEPEL) } \\
\text { A. Mosse } \\
\text { Rio De Janeiro, Brazil 20001- } \\
970\end{array}$ & $\begin{array}{l}\text { Brazilian Rural } \\
\text { Electrification Pilot Project }\end{array}$ & AAD-3-13179-01 & 0.0 & $\begin{array}{l}12 / 92 \\
12 / 96\end{array}$ \\
\hline $\begin{array}{l}\text { Innovative Design } \\
\text { M. Nicklas } \\
\text { Raleigh, NC } 27603 \\
\end{array}$ & $\begin{array}{l}\text { Improving PV-Buildings } \\
\text { Economics by Capturing } \\
\text { Thermal Energy }\end{array}$ & AAT-5-14451-01 & 39.6 & $\begin{array}{l}1 / 95 \\
7 / 95\end{array}$ \\
\hline $\begin{array}{l}\text { Institute of Policy and } \\
\text { Management } \\
\text { L. Xiuguo } \\
\text { Beijing, China } 100080\end{array}$ & $\begin{array}{l}\text { Renewable Energy Policy } \\
\text { Development and } \\
\text { Commercial Project } \\
\text { Identification in China }\end{array}$ & AAT-5-15002-01 & 21.8 & $\begin{array}{l}3 / 95 \\
3 / 96\end{array}$ \\
\hline $\begin{array}{l}\text { Interstate Renewable Energy } \\
\text { Council (IREC) } \\
\text { J.M. Weissman } \\
\text { Latham, NY } 12110\end{array}$ & $\begin{array}{l}\text { PV-Renewable Energy } \\
\text { Procurement Workshop in } \\
\text { a Box Program }\end{array}$ & AAX-5-15227-01 & 10.0 & $\begin{array}{c}6 / 95 \\
10 / 95\end{array}$ \\
\hline $\begin{array}{l}\text { New Resources Group } \\
\text { D. Eberle } \\
\text { Freeman, MO } 64746\end{array}$ & Sunrayce & $\begin{array}{l}\text { TAT-5-15182-01 } \\
\end{array}$ & 357.0 & $\begin{array}{l}3 / 95 \\
9 / 95\end{array}$ \\
\hline
\end{tabular}




\begin{tabular}{|c|c|c|c|c|}
\hline \multicolumn{5}{|c|}{ Subcontracts for FY 1995} \\
\hline $\begin{array}{l}\text { Contractor, Principal } \\
\text { Investigator, Address }\end{array}$ & $\begin{array}{c}\text { Work Title } \\
\text { (Research Activity) }\end{array}$ & $\begin{array}{l}\text { Contract } \\
\text { Number }\end{array}$ & $\begin{array}{l}\text { FY } 1995 \\
\text { Funding } \\
\text { (\$K) }\end{array}$ & $\begin{array}{l}\text { Start/End } \\
\text { Dates }\end{array}$ \\
\hline $\begin{array}{l}\text { New World Village Power } \\
\text { L. Mott } \\
\text { Waitsfield, VT } 05673\end{array}$ & $\begin{array}{l}\text { Brazilian Rural } \\
\text { Electrification Pilot Project }\end{array}$ & AAD-5-13504-06 & 11.7 & $\begin{array}{l}10 / 94 \\
10 / 95\end{array}$ \\
\hline $\begin{array}{l}\text { Neos Corporation } \\
\text { C. Lane } \\
\text { Lakewood, CO } 80224 \\
\end{array}$ & $\begin{array}{l}\text { Photovoltaic Circuit Rider, } \\
\text { Phase II }\end{array}$ & AAX-4-14410-01 & 0.0 & $\begin{array}{l}6 / 93 \\
3 / 95\end{array}$ \\
\hline $\begin{array}{l}\text { NE Sustainable Energy } \\
\text { Association } \\
\text { P. Lipke } \\
\text { Greenfield, MA } 01301\end{array}$ & $\begin{array}{l}\text { IEA Task } 16 \text { Final } \\
\text { Conference }\end{array}$ & ACR-5-15233-01 & 55.0 & $\begin{array}{l}6 / 95 \\
5 / 96\end{array}$ \\
\hline $\begin{array}{l}\text { NRECA International, Inc. } \\
\text { D. Waddle } \\
\text { Washington, DC } 20036\end{array}$ & $\begin{array}{l}\text { Brazil and Central } \\
\text { America Electrification } \\
\text { Support Program }\end{array}$ & TAT-5-14430-01 & 72.8 & $\begin{array}{l}5 / 95 \\
5 / 96\end{array}$ \\
\hline $\begin{array}{l}\text { Pacific Energy Group } \\
\text { H. Wenger } \\
\text { Walnut Creek, CA } 94596\end{array}$ & $\begin{array}{l}\text { Renewable Energy } \\
\text { Applications and } \\
\text { Economic Analysis for } \\
\text { Electric Power }\end{array}$ & AAX-5-15330-01 & $82 . \dot{5}$ & $\begin{array}{l}8 / 95 \\
8 / 96\end{array}$ \\
\hline $\begin{array}{l}\text { Photocomm, Inc. } \\
\text { S. McCarney } \\
\text { Scottsdale, AZ } 85260\end{array}$ & $\begin{array}{l}\text { Brazilian Rural } \\
\text { Electrification Pilot } \\
\text { Project-Phase } 2 \\
\end{array}$ & AAD-5-13504-03 & 0.0 & $\begin{array}{l}10 / 94 \\
4 / 95\end{array}$ \\
\hline $\begin{array}{l}\text { Siemens Solar Industries } \\
\text { E. Prokopovych } \\
\text { Camarillo, CA } 93011\end{array}$ & $\begin{array}{l}\text { Brazilian Rural } \\
\text { Electrification Pilot } \\
\text { Project-Phase } 2 \\
\end{array}$ & AAD-5-13504-01 & 0.0 & $\begin{array}{l}10 / 94 \\
4 / 95\end{array}$ \\
\hline $\begin{array}{l}\text { SEIA } \\
\text { R. Sellers } \\
\text { Washington, DC } 20001 \\
\end{array}$ & N/A & HAI-2-12006-95 & 20.0 & N/A \\
\hline $\begin{array}{l}\text { SEIA } \\
\text { R. Sellers } \\
\text { Washington, DC } 20001 \\
\end{array}$ & $\begin{array}{l}\text { Solar Industry Outreach } \\
\text { and Development }\end{array}$ & HI-2-12006-57 & 38.0 & $\begin{array}{l}5 / 94 \\
5 / 95\end{array}$ \\
\hline $\begin{array}{l}\text { SEIA } \\
\text { R. Sellers } \\
\text { Washington, DC } 20001 \\
\end{array}$ & $\begin{array}{l}\text { Solar Energy Industry } \\
\text { Support }\end{array}$ & HII-2-12006-89 & 61.9 & $\begin{array}{l}6 / 95 \\
2 / 96\end{array}$ \\
\hline $\begin{array}{l}\text { Service Engineering Laboratory } \\
\text { T. Service } \\
\text { Greenfield, MA } 01301\end{array}$ & $\begin{array}{l}\text { Sunrayce } 95 \text { Structural } \\
\text { Analysis Report Reviews }\end{array}$ & AAX-5-15070-01 & 14.6 & $\begin{array}{l}1 / 95 \\
6 / 95\end{array}$ \\
\hline
\end{tabular}




\begin{tabular}{|c|c|c|c|c|}
\hline \multicolumn{5}{|c|}{ Subcontracts for FY 1995} \\
\hline $\begin{array}{l}\text { Contractor, Principal } \\
\text { Investigator, Address }\end{array}$ & $\begin{array}{c}\text { Work Title } \\
\text { (Research Activity) }\end{array}$ & $\begin{array}{l}\text { Contract } \\
\text { Number }\end{array}$ & $\begin{array}{l}\text { FY } 1995 \\
\text { Funding } \\
\text { (\$K) }\end{array}$ & $\begin{array}{l}\text { Start/End } \\
\text { Dates }\end{array}$ \\
\hline $\begin{array}{l}\text { Solar Design Associates } \\
\text { S. Strong } \\
\text { Harvard, MA } 01451\end{array}$ & $\begin{array}{l}\text { The Development of } \\
\text { Standardized, Low-Cost } \\
\text { AC PV Systems }\end{array}$ & ZAF-5-14271-01 & 444.9 & $\begin{array}{l}9 / 95 \\
9 / 97\end{array}$ \\
\hline $\begin{array}{l}\text { Solar Electric Light Fund, Inc. } \\
\text { R. Freling } \\
\text { Washington, DC } 20009\end{array}$ & $\begin{array}{l}\text { Gansu PV Rural } \\
\text { Electrification Program } \\
\text { Report }\end{array}$ & AAX-5-15301-01 & 0.0 & $\begin{array}{l}8 / 95 \\
9 / 95\end{array}$ \\
\hline $\begin{array}{l}\text { Solarex Corporation } \\
\text { Roy Gibson } \\
\text { Frederick, MD } 21701\end{array}$ & $\begin{array}{l}\text { Brazilian Rural } \\
\text { Electrification }\end{array}$ & AAD-5-13504-02 & 0.0 & $\begin{array}{l}10 / 94 \\
4 / 95\end{array}$ \\
\hline $\begin{array}{l}\text { Spire Corporation } \\
\text { M. Nowlan } \\
\text { Bedford, MA } 01730\end{array}$ & $\begin{array}{l}\text { South Africa PV Module } \\
\text { Assembly Development } \\
\text { Program }\end{array}$ & YAN-5-15246-01 & 694.0 & $\begin{array}{l}5 / 95 \\
5 / 96\end{array}$ \\
\hline $\begin{array}{l}\text { SUNY at Albany } \\
\text { R. Perez } \\
\text { Albany, NY } 12205\end{array}$ & $\begin{array}{l}\text { Solar Resource Utility } \\
\text { Load-Matching } \\
\text { Assessment }\end{array}$ & XAR-1-11168-1 & 50.0 & $\begin{array}{l}9 / 91 \\
12 / 94\end{array}$ \\
\hline $\begin{array}{l}\text { University of Delaware } \\
\text { J. Byrne } \\
\text { Newark, DE } 19616\end{array}$ & $\begin{array}{l}\text { Evaluation of DSM } \\
\text { Incentive Opportunities for } \\
\text { PV }\end{array}$ & XR-2-11248-1 & 100.0 & $\begin{array}{l}10 / 91 \\
6 / 96\end{array}$ \\
\hline $\begin{array}{l}\text { United States Export Council for } \\
\text { Renewable Energy (US/ECRE) } \\
\text { H. Sherr } \\
\text { Washington, DC } 20001 \\
\end{array}$ & $\begin{array}{l}\text { U.S./Russia Cooperative } \\
\text { Program in Renewable } \\
\text { Energy }\end{array}$ & KAI-3-13012-00 & 85.0 & $\begin{array}{l}7 / 95 \\
1 / 96\end{array}$ \\
\hline \multicolumn{5}{|c|}{ HISTORICALLY BLACK COLLEGES AND UNIVERSITIES } \\
\hline $\begin{array}{l}\text { Clark Atlanta University } \\
\text { L. Lewis } \\
\text { Atlanta, GA } 30314 \\
\end{array}$ & $\begin{array}{l}\text { HBCU PV Research } \\
\text { Associates Program }\end{array}$ & $\mathrm{XAX}-5-15021-02$ & 77.8 & $\begin{array}{l}9 / 95 \\
9 / 98\end{array}$ \\
\hline $\begin{array}{l}\text { Wilberforce University } \\
\text { R. Nadella, E. Asikele, T. Habash } \\
\text { Wilberforce, OH } 45384\end{array}$ & $\begin{array}{l}\text { HBCU PV Research } \\
\text { Associates Program }\end{array}$ & $\mathrm{XAX}-5-15021-01$ & 62.0 & $\begin{array}{l}9 / 95 \\
9 / 98\end{array}$ \\
\hline $\begin{array}{l}\text { Solar Cells, Inc. } \\
\text { T. Zhou } \\
\text { Toledo, OH } 43607\end{array}$ & HBCU Internship & HAX-5-15266-01 & 8.4 & $\begin{array}{l}7 / 95 \\
9 / 95\end{array}$ \\
\hline $\begin{array}{l}\text { University of Delaware-IEC } \\
\text { S. Hegedus } \\
\text { Newark, DE } 19716\end{array}$ & HBCU Internship & HAX-5-15269-01 & 4.3 & $\begin{array}{l}6 / 95 \\
8 / 95\end{array}$ \\
\hline
\end{tabular}




\begin{tabular}{|l|l|c|c|c|}
\hline \multicolumn{1}{|c|}{ Subcontracts for FY 1995 } \\
\hline $\begin{array}{c}\text { Contractor, Principal } \\
\text { Investigator, Address }\end{array}$ & $\begin{array}{c}\text { Work Title } \\
\text { (Research Activity) }\end{array}$ & $\begin{array}{c}\text { Contract } \\
\text { Number }\end{array}$ & $\begin{array}{c}\text { FY 1995 } \\
\text { Funding } \\
\text { (\$K) }\end{array}$ & $\begin{array}{c}\text { Start/End } \\
\text { Dates }\end{array}$ \\
\hline $\begin{array}{l}\text { University of South Florida } \\
\text { D. Morel }\end{array}$ & HBCU Internship & HAX-5-15265-01 & 4.2 & $6 / 95$ \\
Tampa, FI, 33620 & & & & $8 / 95$ \\
\hline
\end{tabular}




\subsection{NREL PHOTOVOLTAIC PROGRAM FY 1995 BIBLIOGRAPHY}

October 1994-September 1995

Abelson, J. R. Research on Silicon-Carbon Alloys and Interfaces: Final Subcontract Report, 15 February 1991 - 31 July 1994. July 1995; 82 pp. NREL/TP-411-8111. NTIS Order No. DE95009243.

Ahrenkiel, S. P.; Bode, M. H.; Al-Jassim, M. M.; Luo, H.; Xin, S. H.; Furdyna, J. K. Microcharacterization of Composition Modulations in Epitaxial $\mathrm{ZnSe}_{1 .-\mathrm{x}}$ Tex. Joumal of Electronic Materials. April 1995; 24(4); pp. 319-325.

Albin, D. S.; Tuttle, J. R.; Noufi, R. Formation of Large-Grain CuInSe, Films by Selenization by High-Rate Se Transport Under Moderate Vacuum Conditions. Journal of Electronic Materials. April 1995; 24(4); pp. 351-357.

Basol, B.; Kapur, V.; Halani, A.; Leidholm, C.; Minnick, A. Novel Two-Stage Selenization Methods for Fabrication of Thin-Film CIS Cells and Submodules: Final Subcontract Report, 1 March 1993 - 31 March 1995. June 1995; 28 pp. NREL/TP-413-8008. NTIS Order No. DE95009229.

Bertness, K. A.; Friedman, D. J.; Kurtz, S. R.; Kibbler, A. E.; Kramer, C.; Olson, J. M. High Efficiency GaInP/GaAs Tandem Solar Cells. IEEE Aerospace and Electronic Systems Magazine. December 1994; 9(12); pp. 12-17.

Bhattacharya, R. N.; Paranthaman, M. Alternating-Current Electrodeposition (Metafuse) Process for Forming Thallium-Oxide Superconductors. Physica C. 1 September 1995; 251(1-2); pp. 105109.

Birkmire, R. W.; Phillips, J. E.; Buchanan, W. A.; Hegedus, S. S.; McCandless, B. E.; Shafarman, W. N. Processing and Modeling Issues for Thin-Film Solar Cell Devices: Annual Subcontract Report, 16 January 1994 - 15 January 1995. June 1995; 110 pp. NREL/TP-451-8017. NTIS Order No. DE95009237.

Branz, H. M.; Asher, S.; Xu, Y.; Kemp, M. Evidence for Exchange between Free and Deep Hydrogen (Deuterium) During Diffusion. Hack, M. et al., eds. Amorphous Silicon Technology 1995: Proceedings of the Materials Research Society Symposium, 18-21 April 1995, San Francisco, California. Materials Research Society Symposium Proceedings, Vol. 377. Pittsburgh, PA: Materials Research Society; 1995; pp. 331-336.

Broman, L. Thermophotovoltaics Bibliography. Progress in Photovoltaics: Research and Applications. January 1995; 3(1); pp. 65-74.

Bullock, J. N.; Xu, Y.; Benson, D. K.; Branz, H. M. Wide-Gap a-SiC:H Alloys for Novel Photovoltaic-Electrochromic Window Coatings. Hack, M. et al., eds. Amorphous Silicon Technology 1995: Proceedings of the Materials Research Society Symposium, 18-21 April 1995, 
San Francisco, California. Materials Research Society Symposium Proceedings, Vol. 377. Pittsburgh, PA: Materials Research Society; 1995; pp. 589-594.

Cannon, T. W. Advances in Radiometric Instrumentation for PV Performance Evaluation. (Viewgraphs from oral presentation). Mrig, L. ed. Proceedings of the Photovoltaic Performance and Reliability Workshop, 21-23 September 1994, Lakewood, Colorado. NREL/CP-411-7414. Golden, CO: National Renewable Energy Laboratory; 1995; pp. 31-56. NTIS Order No. DE95000251.

Cannon, T. W. Spectral Radiometric Instrumentation and Modeling for PV Applications. PV Radiometric Workshop Proceedings, 24-25 July 1995, Vail, Colorado. NREL/CP-411-20008. Golden, CO: National Renewable Energy Laboratory; September 1995; pp. 93- 106.

Carlen, M. W.; Xu, Y.; Crandall, R. S. Observation of Slow Dangling-Bond Relaxation in p-type Hydrogenated Amorphous Silicon. Physical Review. B, Condensed Matter. 15 January 1995- II; 51(4); 2173-2179.

Carr, K. F.; Carlson, N.; Weitzman, P.; Sopori, B. L.; Marshall, C.; Allen, L. Applications of Scanning Defect Mapping System for Semiconductor Characterization. Ashok, S. et al., eds. Defect and Impurity Engineered Semiconductors and Devices: Proceedings of the Materials Research Society Symposium, 17-21 April 1995; San Francisco, California. Materials Research Society Symposium Proceedings, Vol. 378. Pittsburgh, PA: Materials Research Society; 1995; pp. 579-583.

Compaan, A. D.; Bohn, R. G.; Rajakarunanayake, Y. High-Efficiency, Thin-Film Cadmium Telluride Photovoltaic Cells: Annual Subcontract Report, 20 January 1994 - 19 January 1995. August 1995; 53 pp. NREL/TP-451-8120. NTIS Order No. DE95009256.

Contreras, M. A; Gabor, A. M.; Tennant, A. L.; Asher, S.; Tuttle, J.; Noufi, R. 16.4\% Total-Area Conversion Efficiency Thin-Film Polycrystalline $\mathrm{MgF}_{2} / \mathrm{ZnO} / \mathrm{CdS} / \mathrm{Cu}(\mathrm{In}, \mathbf{G a}) \mathrm{Se}_{2} / \mathrm{Mo}$ Solar Cell. Progress in Photovoltaics: Research and Applications. October 1994; 2(4); pp. 287-292.

Contreras, M. A.; Webb, J.; Tennant, A.; Noufi, R. Room Temperature Infrared Electroluminescence from Thin-Film Polycrystalline Chalcopyrite $\mathrm{Cu}(\mathrm{In}, \mathrm{Ga}) \mathrm{Se}_{2}-\mathrm{Based}$ Diodes. Ashok, S. et al., eds. Defect and Impurity Engineered Semiconductors and Devices: Proceedings of the Materials Research Society Symposium, 17-21 April 1995, San Francisco, California. Materials Research Society Symposium Proceedings, Vol. 378. Pittsburgh, PA: Materials Research Society; 1995; pp. 803-808.

Crandall, R; Luft W. Future of Amorphous Silicon Photovoltaic Technology. June 1995; 26 pp. NREL/TP-411-8019. NTIS Order No. DE95009235.

Czanderna, A. W.; Jung, D. R.; Herdt, G. C. Bonding and Penetration at Metal/Self-Assembled Organic Monolayer Interfaces. ANTEC '95: Proceedings of the 53rd Annual Technical Conference, 9-11 Moy 1995, Boston, Massachusetts. Brookfield, CT: Society of Plastics Engineers; 1995; Part 2: pp. 2734-2738. 
San Francisco, California. Materials Research Society Symposium Proceedings, Vol. 377. Pittsburgh, PA: Materials Research Society; 1995; pp. 589-594.

Cannon, T. W. Advances in Radiometric Instrumentation for PV Performance Evaluation. (Viewgraphs from oral presentation). Mrig, L. ed. Proceedings of the Photovoltaic Performance and Reliability Workshop, 21-23 September 1994, Lakewood, Colorado. NREL/CP-411-7414. Golden, CO: National Renewable Energy Laboratory; 1995; pp. 31-56. NTIS Order No. DE95000251.

Cannon, T. W. Spectral Radiometric Instrumentation and Modeling for PV Applications. PV Radiometric Workshop Proceedings, 24-25 July 1995, Vail, Colorado. NREL/CP-411-20008. Golden, CO: National Renewable Energy Laboratory; September 1995; pp. 93- 106.

Carlen, M. W.; Xu, Y.; Crandall, R. S. Observation of Slow Dangling-Bond Relaxation in p-type Hydrogenated Amorphous Silicon. Physical Review. B, Condensed Matter. 15 January 1995- II; 51(4); 2173-2179.

Carr, K. F.; Carlson, N.; Weitzman, P.; Sopori, B. L.; Marshall, C.; Allen, L. Applications of Scanning Defect Mapping System for Semiconductor Characterization. Ashok, S. et al., eds. Defect and Impurity Engineered Semiconductors and Devices: Proceedings of the Materials Research Society Symposium, 17-21 April 1995, San Francisco, California. Materials Research Society Symposium Proceedings, Vol. 378. Pittsburgh, PA: Materials Research Society; 1995; pp. 579-583.

Compaan, A. D.; Bohn, R. G.; Rajakarunanayake, Y. High-Efficiency, Thin-Film Cadmium Telluride Photovoltaic Cells: Annual Subcontract Report, 20 January 1994 - 19 January 1995. August 1995; 53 pp. NREL/TP-451-8120. NTIS Order No. DE95009256.

Contreras, M. A; Gabor, A. M.; Tennant, A. L.; Asher, S.; Tuttle, J.; Noufi, R. 16.4\% Total-Area Conversion Efficiency Thin-Film Polycrystalline $\mathrm{MgF}_{2} / \mathrm{ZnO} / \mathrm{CdS} / \mathrm{Cu}(\mathbf{I n}, \mathbf{G a}) \mathrm{Se}_{2} / \mathrm{Mo}$ Solar Cell. Progress in Photovoltaics: Research and Applications. October 1994; 2(4); pp. 287-292.

Contreras, M. A.; Webb, J.; Tennant, A. Noufi, R. Room Temperature Infrared Electroluminescence from Thin-Film Polycrystalline Chalcopyrite $\mathrm{Cu}(\mathrm{In}, \mathrm{Ga}) \mathrm{Se}_{2}-\mathrm{Based}$ Diodes. Ashok, S. et al., eds. Defect and Impurity Engineered Semiconductors and Devices: Proceedings of the Materials Research Society Symposium, 17-21 April 1995, San Francisco, California. Materials Research Society Symposium Proceedings, Vol. 378. Pittsburgh, PA: Materials Research Society; 1995; pp. 803-808.

Crandall, R.; Luft, W. Future of Amorphous Silicon Photovoltaic Technology. June 1995; 26 pp. NREL/TP-411-8019. NTIS Order No. DE95009235.

Czanderna, A. W.; Jung, D. R.; Herdt, G. C. Bonding and Penetration at Metal/Self-Assembled Organic Monolayer Interfaces. ANTEC '95: Proceedings of the 53rd Annual Technical Conference, 9-11 May 1995, Boston, Massachusetts. Brookfield, CT: Society of Plastics Engineers; 1995; Part 2: pp. 2734-2738. 
Czanderna, A. W.; Tillman, N. T.; Herdt, G. C. Polymers as Advanced Materials for Desiccant Applications, Part 3: Alkali Salts of PSSA and PolyAMPSA and Copolymers of PolyAMIPSASS. ASHRAE Transactions 1995: Technical and Symposium Papers Presented at the 1995 Winter Meeting of the American Society of Heating, Refrigerating and Air-Conditioning Engineers, Inc., 25-29 Jamuary 1995, Chicago, Illinois. ASHRAE Transactions, Vol. 101, Part 1. Atlanta, GA: American Society of Heating, Refrigerating and Air-Conditioning Engineers, Inc.; 1995; pp. 697-712.

Dalal, V. Comprehensive Research on the Stability and Electronic Properties of a-Si:H and a-SiGe:H Alloys and Devices: Final Subcontract Report, 10 March 1991 - 30 August 1994. April 1995; 108 pp. NREL/TP-411-7695. NTIS Order No. DE95004088.

Deng, X.; Sopori, B. L. Low-Temperature Diffusivity of Hydrogen in Different Silicon Substrates. Ashok, S. et al., eds. Defect and Impurity Engineered Semiconductors and Devices: Proceedings of the Materials Research Society Symposium, 17-21 April 1995, San Francisco, California. Materials Research Society Symposium Proceedings, Vol. 378. Pittsburgh, PA: Materials Research Society; 1995; pp. 359-364.

Dhere, N. G. Polycrystalline CuInSe, and CdTe PV Solar Cells: Annual Subcontract Report, 15 April 1993 - 14 April 1994. November 1994; 43 pp. NREL/TP-451-7189. NTIS Order No. DE95000244.

Duran, G.; Mackamul, K.; Metcalf, D. Amorphous Silicon Photovoltaic Manufacturing Technology - Phase 2A: Annual Subcontract Report, 1 May 1993 - 30 April 1994. January 1995; 87 pp. NREL/TP-411-7472. NTIS Order No. DE95000298.

Field, H. Radiometry for Characterizing Photovoltaic Devices in the Laboratory. PV Radiometric Workshop Proceedings, 24-25 July 1995, Vail, Colorado. NREL/CP-411-20008. Golden, CO: National Renewable Energy Laboratory; September 1995; pp. 107-114.

Fifth Workshop on the Role of Impurities and Defects in Silicon Device Processing: Extended Abstracts from the Workshop, 13-16 August 1995, Copper Mountain, Colorado. August 1995; 166 pp. NREL/SP-413-8250. NTIS Order No. DE95009278.

Franceschetti, A.; Wei, S. H.; Zunger, A. Absolute Deformation Potentials of Al, Si, and NaCl. Physical Review. B, Condensed Matter. 15 December 1994-II; 50(24); pp. 17797-17801.

Franceschetti, A; Zunger, A. Pressure Dependence of Optical Transitions in Ordered GaP/InP Superlattices. Applied Physics Letters. 5 December 1994; 65(23); pp. 2990-2992.

Friedman, D. J.; Kurtz, S. R.; Bertness, K. A.; Kibbler, A. E.; Kramer, C.; Olson, J. M.; King, D. L.; Hansen, B. R.; Snyder, J. K. 30.2\% Efficient GaInP/GaAs Monolithic Two-Terminal Tandem Concentrator Cell. Progress in Photovoltaics: Research and Applications. January 1995; 3(1); pp. 47-50.

Gallagher, A.; Tanenbaum, D.; Laracuente, A.; Jelenkovic, B. Atomic-Scale Characterization of Hydrogenated Amorphous-Silicon Films and Devices: Annual Subcontract Report, 14 
February 1994 - 14 April 1995. August 1995; 24 pp. NREL/TP-411-8246. NTIS Order No. DE95009287.

Gray, J. L.; Schwartz, R. J.; Lee, Y. J. Numerical Modeling of CuInSe and CdTe Solar Cells: Annual Subcontract Report, 1 March 1994 - 28 February 1995. July 1995; 22 pp. NREL/TP-451-8117. NTIS Order No. DE95009263.

Guha, S. Research on Stable, High-Efficiency Amorphous Silicon Multijunction Modules: Final Subcontract Report, 1 January 1991 - 31 August 1994. October 1994; 96 pp. NREL/TP-411-7190. NTIS Order No. DE95000215.

Hall, R. B.; Rand, J. A.; Cotter, J. E. Development of Large-Area Monolithically Integrated Silicon-Film TM Photovoltaic Modules: Final Subcontract Report, 1 May 1991 - 31 December 1994. April 1995; 33 pp. NREL/TP-413-7702. NTIS Order No. DE95004089.

Han, D. X.; McNeil, L. E.; Wang, K. D.; Yeh, C. N. Experimental Study of the Factors Governing the Staebler-Wronski Photodegradation Effect in a-Si:H Solar Cells: Annual Subcontract Report, 1 March 1994 - 31 March 1995. August 1995; 31 pp. NREL/ TP-411-8116. NTIS Order No. DE95009272.

Herdt, G. C.; Czanderna, A. W. Metal Overlayers on Organic Functional Groups of Self-Organized Molecular Assemblies. V. Ion Scattering Spectroscopy and X-Ray Photoelectron Spectroscopy of Ag/COOH Interfaces. Journal of Vacuum Science and Technology. A, Vacuum, Surfaces and Films. May/June 1995; 13(3); pp. 1275-1280.

Herdt, G. C.; Czanderna, A W. Penetration of Silver into Self-Assembled Organic Monolayers. Holubka, J. W., ed. Proceedings of the 18th Anmual Meeting of the Adhesion Society, 19-22 February 1995, Hilton Head, South Carolina. Blacksturg, VA: The Adhesion Society, Inc.; 1995; pp. 224-226.

Herdt, G. C.; Jung, D. R.; Czanderna, A. W. Weak Interactions Between Deposited Metal Overfayers and Organic Functional Groups of Self-Assembled Monolayers. Progress in Surface Science. 1995; 50(1-4); pp. 103-129.

Holley, W. Photovoltaic Manufacturing Technology (PVMaT): Semiannual Subcontract Report, 31 March 1994 - 30 September 1994. April 1995; 31 pp. NREL/TP-411-7693. NTIS Order No. DE95004093.

Holley, W.; Agro, S.; Galica, J.; Thoma, L.; White, R.; Yorgensen, R. Advanced Development of PV Encapsulants: Annual Subcontract Report, 30 December 1992 - 31 March 1994. November 1994; 82 pp. NEEL/TP-411-7352. NTIS Order No. DE95000246.

Izu, M. Continuous Roli-to-Roll Amorphous-Silicon Photovoltaic Manufacturing Technology: Annual Subcontract Report, 8 April 1993 - 7 April 1994. November 1994; 92 pp. NREL/TP-411-7188. NTIS Order No. DE95000243.

Jackson, W. B.; Johnson, N.; Nickel, N.; Schumm, G.; Street, R. A.; Thompson, R.; Tsai, C. C.; Van de Walle, C.; Walker, J. Researcin on the Stability, Electronic Properties, snä Structure of 
a-Si:H and Its Alloys: Final Subcontract Report, 1 June 1991 - 31 May 1994. July 1995; 57 pp. NREL/TP-451-8101. NTIS Order No. DE95009238.

Jester, T. Photovoltaic Czochralski Silicon Manufacturing Technology Improvements: Annual Subcontract Report, 1 April 1993 - 31 March 1994. March 1995; 27 pp. NREL/TP-411-7498. NTIS Order No. DE95004066.

Jester, T. PV Cz Silicon Manufacturing Technology Improvements: Semi-Annual Subcontract Report, 1 April 1994 - 30 September 1994. September 1995; 18 pp. NREL/TP-411-8248. NTIS Order No. DE95009288.

Jung, D. R; Czanderna, A. W.; Herdt, G. C. Bonding and Penetration at Metal/Self-Assembled Organic Monolayer Interfaces. Drzal, L. T., et al., eds. Polymer/Inorganic Interfaces II: Proceedings of the Materials Research Society Symposium, 18-20 April 1995, San Francisco, California. Materials Research Society Symposium Proceedings Vol. 385. Pittsburgh, PA: Materials Research Society; 1995; pp. 11-25.

Jung, D. R.; Czanderna, A. W. Metal Overlayers on Organic Functional Groups of Self-Assembled Monolayers. VL. X-Ray Photoelectron Spectroscopy of $\mathbf{C r} / \mathbf{C O O H}$ on 16-Mercaptohexadecanoic Acid. Journal of Vacunm Science and Technology. A, Vacuum, Surfaces and Films. May/June 1995; 13(3); pp. 1337-1344.

Jung, D. R.; Czanderna, A. W. X-Ray Photoelectron Spectroscopy of $\mathrm{Cr} / \mathrm{COOCH}_{3}$ Interfaces on Self-Assembled Monolayers of Mercaptohexadecanoate. (Abstract No. 542). Extended Abstracts from the 187th Meeting of The Electrochemical Society, 21-26 May 1995, Reno, Nevada. Vol. 95-1. Pennington, NJ: The Electrochemical Society, Inc.; 1995; pp. 839-840.

Khattak, C. P.; Schmid, F.; Cudzinovic, M.; Symko, M.; Sopori, B. L. Analysis and Control of the Performance-Limiting Defects in HEM-Grown Silicon for Solar Cells. Ashok, S. et al., eds. Defect and Impurity Engineered Semiconductors and Devices: Proceedings of the Materials Research Society Symposium, 17-21 April 1995, San Francisco, California. Materials Research Society Symposium Proceedings, Vol. 378. Pittsburgh, PA: Materials Research Society; 1995; pp. 767-776.

King, D. E. Oxidation of Gold by Ultraviolet Light and Ozone at 25 Degrees C. Journal of Vacurm Science and Technology. A, Vacuum, Surfaces, and Films. Proceedings of the 41st National Symposium of the American Vacuum Society, 24 October 1994, Denver, Colorado. May/June 1995; 13(3); Part 2: pp. 1247-1253.

Kroposki, B.; Mrig, L.; Whitaker, C.; Newmiller, J. Development of a Photovoltaic Module Energy Ratings Methodology. May 1995; 10 pp. NREL/TP-411-8005. NTIS Order No. DE95009221. Presented at the 13th NREL PV Program Review Meeting, 16-19 May 1995, Lakewood, Colorado.

Kroposki, B. D.; Mrig, L. Thin-Film Module Stability Testing at NREL. Mrig, L. ed. Proceedings of the Photovoltaic Performance and Reliability Workshop, 21-23 September 1994, Lakewood, Colorado. NREL/CP-411-7414. Golden, CO: National Renewable Energy Laboratory; 1995; pp. 149-160. NTIS Order No. DE95000251. 
Leboeuf, C.; Taylor, R. W.; Corbus, D.; Moszkowicz, M.; Lima, J.; Ribeiro, C. Joint U.S./Brazilian Hybrid Power System (Wind-PV-Diesel) on Marajo Island. Musial, W. D.; Hock, S. M.; Berg, D.E., eds. Wind Energy 1995: Proceedings of the Energy and Environmental Expo '95, The Energy-Sources Technology Conference and Exhibition, 29 Jamuary - 1 February 1995, Houston, Texas. SED-Vol. 16. New York: American Society of Mechanical Engineers; 1995; p. 47.

Lu, Z. W.; Zunger, A.; Fox, A. G. Comparison of Experimental and Theoretical Electronic Charge Distributions in Gamma-TiAl. Acta Metallurgica et Materialia. 1 December 1994; 42(12); pp. 3929- 3943.

Luft, W.; Branz, H. M.; Dalal, V. L.; Hegedus, S. S.; Schiff, E. A. Progress in Amorphous Silicon PV Technology: An Update. July 1995; 22 pp. NREL/TP-411-8108. NTIS Order No. DE95009242. Prepared for the 13th NREL Photovoltaics Program Review Meeting, 16-19 May 1995, Lakewood, Colorado.

Luo, J. S.; Olson, J. M.; Kurtz, S. R.; Arent, D. J.; Bertness, K. A.; Raikh, M. E.; Tsiper, E. V. Optical Anisotropy and Spontaneous Ordering in $\mathbf{G a}_{0.5} \mathbf{I n}_{0.5} \mathbf{P}$ : An Investigation Using Reflectance-Difference Spectroscopy. Physical Review. B, Condensed Matter. 15 March 1995-II; 51(12); pp. 7603-7612.

Luo, J. S.; Olson, J. M. Reflectance Anisotropy and the Ordering Mechanism in GaInP at High Growth Temperatures. Goronkin, H.; Mishra, U. Compound Semiconductors 1994: Proceedings of the 21st International Symposium on Compound Semiconductors, 18-22 September 1994, San Diego, California. Institute of Physics Conference Series No. 141. Bristol, UK: Institute of Physics Publishing; 1995; pp. 201-206.

Mader, K. A.; Zunger, A. Short- and Long-Range-Order Effects on the Electronic Properties of III-V Semiconductor Alloys. Physical Review. B, Condensed Matter. 15 April 1995-II; 51(16); pp. $10462-10476$.

Mahan, A. H.; Johnson, E. J.; Crandall, R. S.; Branz, H. M. Measurement of Two Deep H Bonding Levels in Device Quality Glow Discharge a-Si:H. Hack, M. et al., eds. Amorphous Silicon Technology 1995: Proceedings of the Materials Research Society Symposium, 18-21 April 1995, San Francisco, California. Materials Research Society Symposium Proceedings, Vol. 377. Pittsburgh, PA: Materials Research Society; 1995; pp. 413-418.

McCormack, R; de Fontaine, D.; Wolverton, C.; Ceder, G. Nonempirical Phase Equilibria in the W-Mo-Cr System. Physical Review. B, Condensed Matter. 1 June 1995-II; 51(22); pp. 1580815822.

McMahon, T. J.; Basso, T. S. Two Terminal Diagnostics for Cells in Series Connected Photovoltaic Modules. Stine, W. B.; Tanaka, T.; Claridge, D. E. ,. eds. Solar Engineering 1995: Proceedings of the ASME/JSMEJJSES International Solar Energy Conference, 19- 24 March 1995, Maui, Hawaii. New York: American Society of Mechanical Engineers; 1995; Vol. 2: pp. 1191-1194.

McNutt, P. Experiences with Commercially Available Stand-Alone Photovoltaic Area Lighting Systems at NREL. Mrig, L. ed. Proceedings of the Photovoltaic Performance and Reliability 
Workshop, 21-23 September 1994, Lakewood, Colorado. NREL/CP-411-7414. Golden, CO: National Renewable Energy Laboratory; 1995; pp. 643-656. NTIS Order No. DE95000251.

Moutinho, H. R.; Hasoon, F. S.; Kazmerski, L. L. Studies of the Micro- and Nanostructure of Polycrystalline CdTe and CuInSe, Using Atomic Force and Scanning Tunneling Microscopy. Progress in Photovoltaics: Research and Applications. January 1995; 3(1); pp. 39-46.

Murphy, R.; Sopori, B. L.; Rose, D. Influence of Dislocations on the I-V Characteristics of Silicon Solar Cells. Ashok, S. et al., eds. Defect and Impurity Engineered Semiconductors and Devices: Proceedings of the Materials Research Society Symposium, 17-21 April 1995, San Francisco, California Materials Research Society Symposium Proceedings, Vol. 378. Pittsburgh, PA: Materials Research Society Symposium; 1995; pp. 749-756.

Myers, D. R. Photovoltaic Radiometric Measurements Workshop Introduction and Overview. PV Radiometric Workshop Proceedings, 24-25 July 1995, Vail, Colorado. NREL/CP-411-20008. Golden, CO: National Renewable Energy Laboratory; September 1995; pp. 1-8.

Nelson, A. J. Photoemission Investigation of the $\mathrm{ZnSe} / \mathrm{CdTe}$ Heterojunction Band Discontinuity. Journal of Applied Physics. 15 August 1995; 78(4); pp. 2537-2540.

Nelson, A. J.; Frigo, S. P.; Rosenberg, R. Surface Type Conversion of CuInSe, with $\mathrm{H}_{2} \mathrm{~S}$ Plasma Exposure: A Photoemission Investigation. Journal of Vacuum Science and Technology. A, Vacuum, Surrfaces and Films. July/August 1995; 13(4); pp. 1990- 1993.

Nelson, A. J.; Sinha, K.; Moreland, J. Growth and Characterization of the Binary Defect Alloy $\mathrm{Cu}_{2-5} \mathrm{Se}$ and the Relation to II-VI/I-III-VI Heterojunction Formation. Ashok, S. et al., eds. Defect and Impurity Engineered Semiconductors and Devices: Proceedings of the Materials Research Society Symposium, 17-21 April 1995, San Francisco, California. Materials Research Society Symposium Proceedings, Vol. 378. Pittsburgh, PA: Materials Research Society; 1995; pp. 249-254.

Niles, D. W.; Li, X.; Sheldon, P.; Hochst, H. Photoemission Determination of the Band Diagram of the Te/CdTe Interface. Journal of Applied Physics. 1 May 1995; 77(9); pp. 4489-4493.

Nowlan, M. Automated Solar Cell Assembly Teamed Process Research: Semiannual Subcontract Report, 6 December 1993 - 30 June 1994. January 1995; 33 pp. NREL/TP-411-7473. NTIS Order No. DE95000299.

Olsen, L. C. Investigation of Polycrystalline Thin Film CuInSe, Solar Cells Based on ZnSe Windows: Annual Subcontract Report, 15 February 1993 - 14 February 1994. March 1995; 23 pp. NREL/TP- 413-7684. NTIS Order No. DE95004068.

Osterwald, C. R.; Hammond, R. L.; Wood, B. D.; Backus, C. E.; Sears, R. L.; Zerlaut, G. A.; D'Aiello, R. V. Photovoltaic Module Certification/Laboratory Accreditation Criteria Development. April 1995; 181 pp. NREL/TP-412-7680. NTIS Order No. DE95004063. 
Oswald, R.; Morris, J. Large-Area Triple-Junction a-Si.Alloy Production Scaleup: Annual Subcontract Report, 17 March 1993 - 18 March 1994. November 1994; 67 pp. NREL/ TP-411-7350. NTIS Order No. DE95000245.

Oswald, R.; Morris, J. Large-Area, Triple-Junction a-Si Alloy Production Scale-Up: Semi-Annual Subcontract Report, 17 March 1994 - 18 September 1994. September 1995; 28 pp. NREL/TP-411- 8245. NTIS Order No. DE95009286.

PV Raçiometric Workshop Proceedings, 24-25 July 1995, Vail, Colorado. September 1995; 193 pl. NREL/CP-411-20008. NTIS Order No. DE95009297.

Schulz, D. L.; Parilia, P. A.; Gopalaswamy, H.; Swartzlander, A.; Duda, A.; Blaugher, R. D.; Ginley, D. S. Fiapid Furnace Annealing of $\mathrm{YBa}_{2} \mathrm{Cu}_{3} \mathrm{O}_{\mathbf{x}}$ Thick Films on $\mathrm{Ni} / \mathrm{NiO}$ and Inconel Substrates Prepared by Spray Pyrolysis of $\mathbf{Y B a}_{2} \mathbf{C u}_{3} \mathbf{O x}$ Powder. Materials Research Bulletin. 1995; 30(6); pp. 689-6.97.

Schulz, D. L.; Parilla, P. A.; Ginley, D. S.; Voigt, J. A.; Roth, E. P. Thick C-Axis Textured $(\mathrm{Tl}, \mathrm{Pb})(\mathrm{LSa}, \mathrm{Sr})_{2} \mathrm{Ca}_{2} \mathrm{Cu}_{3} \mathrm{O}_{9} / \mathrm{Ag}_{0.37}$ Superconducting Tapes by an Ink Spray Pyrolysis Method Using a 21-1Free Precursor. Applied Physics Letters. 7 November 1994; 65(19); pp. 2472-2474.

Scofield, J. H.; Duda, A; Albin, D.; Ballard, B. L.; Predecki, P. K. Sputtered Molybdenum Bilayer Back Contact for Copper Indium Diselenide-Based Polycrystalline Thin-Film Solar Cells. Thin Solid Films. 1 May 1995; 260(1); pp. 26-31.

Sharps, P. R. Growth and Development of GaInAsP for Use in High- Efficiency Solar Cells: Final Subcontract Report, 1 July 1991 - 30 December 1993. October 1994; 38 pp. NREL/TP-451-7166. NTIS Order No. DE95000210.

Silverman, A.; Zunger, A.; Kalish, R.; Adler, J. Atomic-Scale Structure of Disordered $\mathbf{G a}_{1-x} \mathbf{I} \mathbf{n}_{\mathbf{2}} \mathbf{P}$ Alloys. Physical Review. B, Condensed Matter. 15 April 1995-II; 51(16); pp. 10795-10816.

Sinha, K.; Mascarenhas, A.; Kurtz, S. R.; Olson, J. M. Determination of Free Carrier Concentration in n-GaInP Alloy by Raman Scattering. Journal of Applied Physics. 15 August 1995; 78(4); pp. 2515-2519.

Sites, J. R. Device Physics of Thin-Film Polycrystalline Cells and Modules: Annual Subcontract Report, 6 December 1993 - 5 December 1994. May 1995; 37 pp. NREL/TP-451-7709. NTIS Order No. DE95009209.

Strand, T.; Hansen, R.; Mrig, L. Technical Evaluation of a USSC Integrated/Direct Mount PV Roofing Module System at NREL. May 1995; 11 pp. NREL/TP-411-8006. NTIS Order No. DE95009217. Presented at the 13th NREL PV Program Review Meeting, 16-19 May 1995, Lakewood, Colorado.

Strand, T.; Mrig, L.; Hansen, R.; Emery, K. Technical Evaluation of a Dual-Junction Same-Band-Gap Amorphous Silicon Photovoltaic System at NREL, December 1994; 6 pp. NREL/TP-411-7402. NTIS Order No. DE95000277. Prepared for the First Wi Gild Cnuference on Photovoltaic Energy Conversion, 5-9 December 1994, Waikoloa, Hawaii. 
Strand, T. R.; Mrig, L.; Hansen, R. Technical Evaluation of Photovoltaic Systems at NREL. Mrig, L. ed. Proceedings of the Photovoltaic Performance and Reliability Workshop, 21-23 September 1994, Lakewood, Colorado. NREL/CP-411-7414. Golden, CO: National Renewable Energy Laboratory; 1995; pp. 559-568. NTIS Order No. DE95000251.

Sunrayce 95: Race for the Future (Brochure). February 1995; 8 pp. NREL/MK-411-7500.

Tan, T.; Sopori, B.; Estreicher, S.; Jastrzebski, L. Fifth Workshop on the Role of Impurities and Defects in Silicon Device Processing: Summary of Panel Discussions, 13-16 August 1995, Copper Mountain, Colorado. September 1995; 15 pp. NREL/TP-413-20005. NTIS Order No. DE95009298.

Tarrant, D. E.; Gay, R. R. Research on High-Efficiency, Large-Area CuInSe-Based Thin-Film Modules: Final Subcontract Report, 16 August 1993 - 30 June 1995. July 1995; 99 pp. NREL/TP-413- 8121. NTIS Order No. DE95009257.

Tuttle, J. R; Contreras, M. A.; Gillespie, T. J.; Ramanathan, K. R.; Tennant, A. L.; Keane, J.; Gabor, A. M.; Noufi, R. $\mathbf{1 7 . 1 \%}$ Efficient $\mathbf{C u}(\mathrm{In}, \mathrm{Ga}) \mathrm{Se}_{2}-\mathrm{Based}_{\mathrm{T}}$ Thin-Film Solar Cell. Progress in Photovoltaics: Research and Applications. July/August 1995; 3(4); pp. 235-238.

Tuttle, J. R.; Contreras, M.; Bode, M. H.; Niles, D.; Albin, D. S.; Matson, R.; Gabor, A. M.; Tennant, A.; Duda, A; Noufi, R. Structure, Chemistry, and Growth Mechanisms of Photovoltaic Quality Thin-Film $\mathrm{Cu}(\mathrm{In}, \mathrm{Ga}) \mathrm{Se}_{2}$ Grown from a Mixed-Phase Precursor. Journal of Applied Physics. 1 January 1995; 77(1); pp. 153-161.

Tuttle, J. R.; Sites, J. R.; Delahoy, A.; Shafarman, W.; Basol, B.; Fonash, S.; Gray, J.; Menner, R.; Plillips, J.; Rockett, A.; Scofield, J.; Shapiro, F. R.; Singh, P.; Suntharalingam, V.; Tarrant, D.; Walter, T.; Wiedeman, S.; Peterson, T. M. Characterization and Modeling of $\mathbf{C u}(\mathbf{I n}, \mathbf{G a})$ (S,Se) $)_{2}$-Based Photovoltaic Devices: A Laboratory and Industrial Perspective. Progress in Photovoltaics: Research and Applications. March 1995; 3(2); pp. 89-104.

Unold, T.; Branz, H. M. Monte Carlo Simulations of Defect Relaxation in Amorphous Silicon. Hack, M. et al., eds. Amorphous Silicon Technology 1995: Proceedings of the Materials Research Society Symposium, 18-21. April 1995, San Francisco, California. Materials Research Society Symposium Proceedings, Vol. 377. Pittsburgh, PA: Materials Research Society; 1995; pp. 293-298.

von Roedern, B. Concept of the Mobility Edge, a Detriment Toward the Understanding of Disordered Semiconductor Devices. Applied Physics Communications. November 1994; 13(3-4); pp. 177-186. Presented at the 15th International Conference on Amorphous Semiconductors, 6-10 September 1993, Cambridge, United Kingdom.

Wang, L. W.; Zunger, A. Local-Density-Derived Semiempirical Pseudopotentials. Physical Review. B, Condensed Matter. 15 June 1995-II; 51(24); pp. 17398-17416.

Wei, S. H.; Franceschetti, A.; Zunger, A. E1, E2, and E'0 Transitions and Pressure Dependence in Ordered $\mathbf{G a}_{0.5} \mathbf{I n}_{0.5} \mathrm{P}$. Physical Review. B, Condensed Matter. 15 May 1995-I; 51(19); pp. 13097-13102. 
Wei, S. H.; Zunger, A. Band Offsets and Optical Bowings of Chalcopyrites and Zn-Based II-VI Alloys. Journal of Applied Physics. 15 September 1995; 78(6); pp. 3846-3856.

Wei, S. H.; Zunger, A. Theory of Reflectance-Difference Spectroscopy in Ordered III-V Semiconductor Alloys. Physical Review. B, Condensed Matter. 15 May 1995-II; 51(20); pp. 1411014114.

Williamson, D. L. Microstructure of Amorphous-Silicon-Based Solar Cell Materials by Small-Angle X-Ray Scattering: Annual Subcontract Report, 6 April 1994 - 5 April 1995. August 1995; 40 pp. NREL/TP-411-8122. NTIS Order No. DE95009273.

Witt, C. E.; Herwig, L. O.; Mitchell, R. L.; Thomas, H.; Sellers, R.; Ruby, D. Recent Progress in the Photovoltaic Manufacturing Technology Project (PVMaT). December 1994; 10 pp. NREL/TP-411- 7416. NTIS Order No. DE95000279. Prepared for the First World Conference on Photovoltaic Energy Conversion, 5-9 December 1994, Waikoloa, Hawaii.

Wohlgemuth, J. Cast Polycrystalline Silicon Photovoltaic Module Manufacturing Technology Improvements: Semiannual Subcontract Report, 8 December 1993 - 30 June 1994. March 1995; 38 pp. NREL/ TP-411-7497. NTIS Order No. DE95004065.

Wohlgemuth, J. Cast Polycrystalline Silicon Photovoltaic Cell and Module Manufacturing Technology Improvements: Annual Subcontract Report, 1 December 1993 - 30 November 1994. September 1995; 32 pp. NREL/TP-411-8244. NTIS Order No. DE95009285.

Wolverton, C.; Zunger, A. First-Principles Theory of Short-Range Order, Electronic Excitations, and Spin Polarization in Ni-V and Pd-V Alloys. Physical Review. B, Condensed Matter. 15 September 1995 - II; 52(12); pp. 8813-8828.

Wolverton, C.; Zunger, A. Short- and Long-Range Order of the Binary Madelung Lattice. Physical Review. B, Condensed Matter. 15 March 1995-I; 51(11); pp. 6876-6891.

Yeh, C. Y.; Zhang, S. B.; Zunger, A. Confinement, Surface, and Chemisorption Effects on the Optical Properties of Si Quantum Wires. Physical Review. B, Condensed Matter. 15 November 1994-I; 50(19); pp. 14405-14415.

Zhang, S. B.; Zunger, A. Structure and Formation Energy of Steps on the GaAs(001)-2 x 4 Surface. Materials Science and Engineering. B, Solid State Materials for Advanced Technology. March 1995; 30(2-3); pp. 127-136.

Zhang, Y.; Mascarenhas, A. Conduction- and Valence-Band Effective Masses in Spontaneously Ordered GaInP ${ }_{2}$. Physical Review. B, Condensed Matter. 15 May 1995-I; 51(19); pp. 13162-13173.

Zhou, T. X. Fabrication of Stable, Large-Area Thin-Film CdTe Photovoltaic Modules: Final Subcontract Report, 10 May 1991 - 28 February 1995. June 1995; 32 pp. NREL/TP-413-7991. NTIS Order No. DE95009222. 
Zhu, J. G.; Al-Jassim, M. M.; Huffman, M. Microstructure and Domain Configurations in Ferroelectric $\mathrm{PbTiO}_{3}$ and $\mathrm{Pb}(\mathrm{Zr}, \mathrm{Ti}) \mathrm{O}_{3}$ Thin Films. Joumal of Electronic Materials. 1995; 24(7); pp. 885-891.

Zweibel, K. Thin Films: Past, Present, Future. April 1995; 18 pp. NREL/TP-413-7486. NTIS Order No. DE95004084. 


\section{REPORT DOCUMENTATION PAGE}

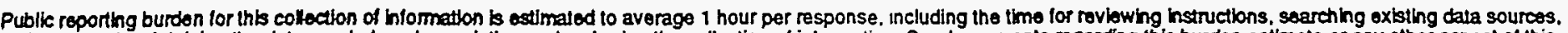

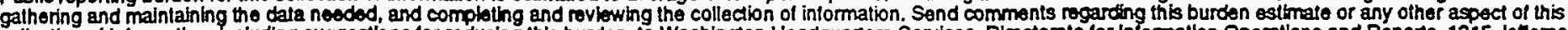

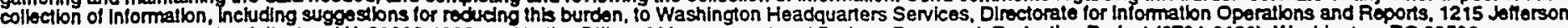
Davis Highway, Sulie 1204, Ankgton, VA $22202-402$, and to the Ottce of Management and Budget. Paperwork Redudion Proled (0704-0188), Washington, DC 20503.

\begin{tabular}{|l|l|l|}
\hline 1. AGENCY USE ONLY (Leave blank) & $\begin{array}{l}\text { 2. REPORT DATE } \\
\text { June1996 }\end{array}$ & $\begin{array}{l}\text { 3. REPORT TYPE AND DATES COVERED } \\
\text { Annual Report }\end{array}$ \\
\hline
\end{tabular}

\section{TITLE AND SUBTITLE}

NREL Photovoltaic Program FY 1995 Annual Report

6. AUTHOR(S) Annual Report

Stuart Smoller, Coordinator

7. PERFORMING ORGANIZATION NAME(S) AND ADDRESS(ES)

B. PERFORMING ORGANIZATION REPORT NUMBER

9. SPONSORINGMONITORING AGENCY NAME(S) AND ADDRESS(ES)

10. SPONSORINGMONTORING AGENCY REPORT NUMBER

National Renewable Energy Laboratory

1617 Cole Blvd.

NRELTP-410-21101

Golden, CO 80401-3393

\section{DE96007869}

\section{SUPPLEMENTARY NOTES}

NAEL Tochnical Monitor: NA

12a. DISTRIBUTIONAVAILABILTTY STATEMENT

National Technical Information Service

U.S. Department of Commerce

5285 Port Royal Road

Springtield, VA 22161 12b. DISTRIBUTION CODE

UC-1250

13. ABSTRACT (Maximum 200 words)

This report summarizes the in-house and subcontract research and development (R\&D) activities under the National Renewable Energy Laboratory (NREL) Photovoltaics (PV) Program from October 1, 1994 through September 30, 1995 (fiscal year [FY] 1995). The NREL PV Program is part of the U.S. Department of Energy's (DOE) National Photovoltaics Program, as described in the DOE Photovoltaics Program Plan, FY 1991-FY 1995. The mission of the DOE National Photovoltaics Program is to: "Work in partnership with U.S. industry to develop and deploy photovoltaic technology for generating economically competitive electric power, making photovoltaics an important contributor to the nation's and the world's energy use and environmental improvement. The two primary goals of the national program are to (1) maintain the U.S. PV industry's world leadership in research and technology development and (2) help the U.S. industry remain a major, profitable force in the world market. The NREL PV Program provides leadership and support to the national program toward achioving its mission and goals.

14. SUBJECT TERMS

photovoltaics ; solar colls
15. NUMBER OF PAGES 440

16. PRICE CODE
17. SECURTYY CLASSIFICATION OF REPORT Unclassified
18. SECURTY CLASSIFICATION OF THIS PAGE Unclassified
19. SECURTY CLASSIFICATION OF ABSTRACT Unclassified
20. LIMITATION OF ABSTRACT UL

Standard Form 298 (Fiov. 2-89) Prescribed by ANSI SId. Z38-18 298-102 